UNITED STATES DEPARTMENT OF THE INTERIOR

U.S. GEOLOGICAL SURVEY

PRELIMINARY DETERMINATION OF EPICENTERS
MONTHLY LISTING

JULY - SEPTEMBER 1996

NATIONAL EARTHQUAKE INFORMATION CENTER ${ }^{1}$

Open-File Report

$96-600-\mathrm{C}$

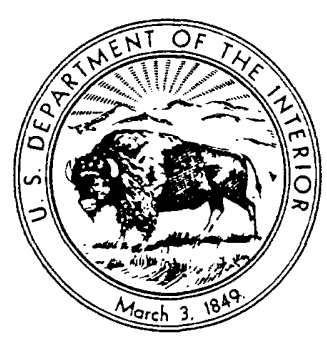

This report is preliminary and has not been reviewed for conformity with U.S. Geological Survey (USGS) editorial standards 


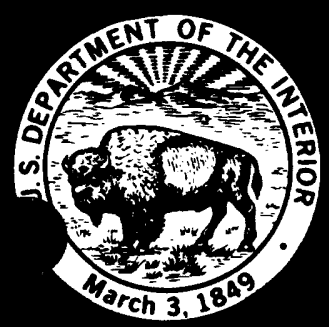

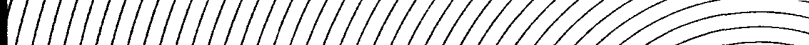 PRELLMINAR EPICE

\section{JULY 1996}

\begin{tabular}{|c|c|c|c|c|c|c|c|}
\hline & $I G I$ & $\begin{array}{l}\text { IN } \\
\text { UTC }\end{array}$ & IIME & $\begin{array}{r}\mathrm{GE} \\
\mathrm{COO}\end{array}$ & & $\begin{array}{l}\text { PHIC } \\
\text { IATES }\end{array}$ & \\
\hline DAY & HR & $\mathbf{M N}$ & SEC & LAT & & LONG & \\
\hline 01 & $\begin{array}{l}00 \\
01\end{array}$ & $\begin{array}{l}21 \\
06\end{array}$ & $\begin{array}{l}11.2 * \\
15.2 *\end{array}$ & $\begin{array}{l}51.722 \\
53.869\end{array}$ & $\begin{array}{l}\mathbf{N} \\
\mathbf{N}\end{array}$ & $\begin{array}{l}159.779 \\
142.167\end{array}$ & $\begin{array}{l}\mathbf{E} \\
\mathbf{E}\end{array}$ \\
\hline $\begin{array}{l}01 \\
01 \\
01 \\
01 \\
01 \\
01 \\
01\end{array}$ & $\begin{array}{l}01 \\
01 \\
01 \\
02 \\
03 \\
03 \\
04 \\
04\end{array}$ & $\begin{array}{l}16 \\
29 \\
42 \\
12 \\
21 \\
57 \\
24 \\
33\end{array}$ & $\begin{array}{l}32.8 * \\
50.4^{*} \\
12.8 \\
37.4 * \\
31.38 \\
10.2 ? \\
40.9 * \\
05.08\end{array}$ & $\begin{array}{r}51.725 \\
2.722 \\
79.449 \\
51.733 \\
60.296 \\
8.40 \\
4.932 \\
37.713\end{array}$ & $\begin{array}{l}\text { N } \\
\text { S } \\
\mathbf{N} \\
\mathbf{N} \\
\mathbf{N} \\
\mathbf{S} \\
\mathbf{S} \\
\mathbf{N}\end{array}$ & $\begin{array}{r}159.999 \\
139.042 \\
2.465 \\
159.623 \\
153.324 \\
123.91 \\
129.878 \\
122.546\end{array}$ & $\begin{array}{l}E \\
E \\
E \\
E \\
W \\
E \\
E \\
\text { W }\end{array}$ \\
\hline & $\begin{array}{l}04 \\
04\end{array}$ & $\begin{array}{l}42 \\
52\end{array}$ & $\begin{array}{l}12.5 \\
11.7\end{array}$ & $\begin{array}{l}51.623 \\
12.479\end{array}$ & $\begin{array}{l}\mathbf{N} \\
\mathbf{N}\end{array}$ & $\begin{array}{l}159.370 \\
125.541\end{array}$ & \\
\hline
\end{tabular}

DEPTH MAGNITUDE SD NO. MB $\mathrm{MSz}$

$33 \mathrm{~N} 4.0 \quad 1.1$ $33 \times 4.14 .0 \quad 0.9$

$37 \mathrm{D} 3.8$

$33 \mathrm{~N} 4.4$

10 G 4.2

$33 \mathrm{~N} 4.0$ 126

100 G 4.3 200 G 3.8 11

$33 \mathrm{~N} 4.1$ $33 \mathrm{~N} 5.05 .31 .1$

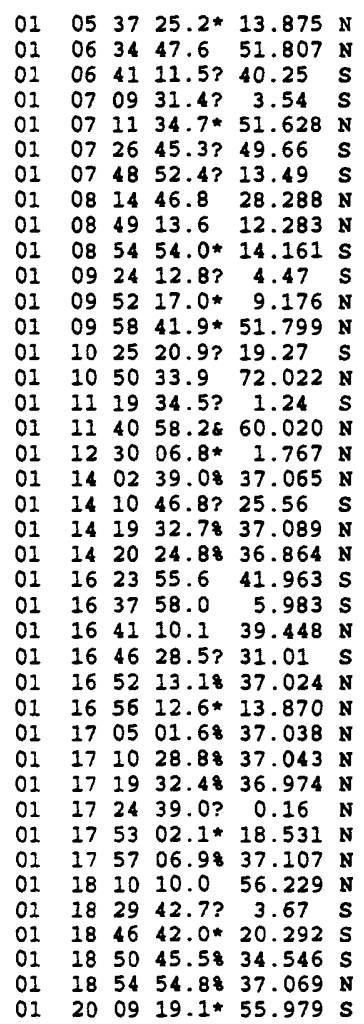

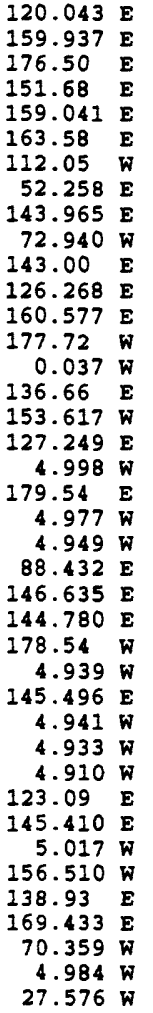

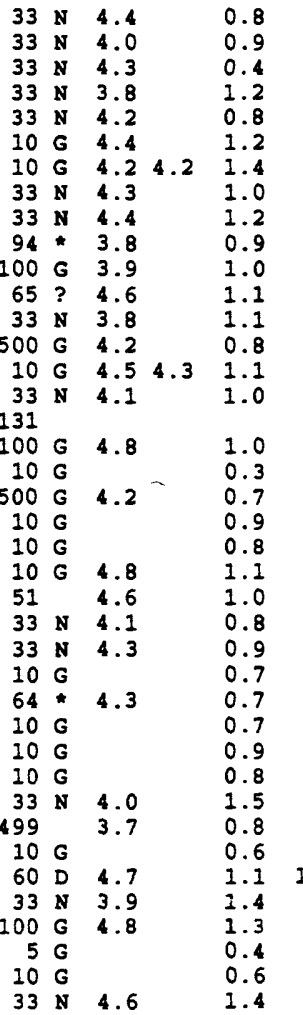

NT. USED
REGION, CONTRIBUTED MAGNITUDES AND COMMENTS

4 OFF EAST COAST OF KAYCHATKA

3 SAKHALIN ISIAND. Felt (III) at Okha. Also felt (III) at Nikolayevsk-na-Amure.

0.716 OFF EAST COAST OF KAMCHATKA

1.121 NEAR NORTH COAST OF IRIAN JAYA

1.239 GREENLAND SEA

0.910 OFF BAST COAST O! KAMCHATRA

52 SOUTHERN ALASKA. 〈AEIC>

0.812 FLORES REGION, INDONESIA

0.57 BANDA SEA

55 CENTRAI CALIFORNIA. 〈GM-P〉. YD 3.1 (GM). ML 3.4 (BRK). Felt throughout the San Erancisco Bay area.

25 OFF EAST COAST OF KAYCHATKA

80 SAMAR, PHILIPPINE ISLANDS. MW 5.5 (ERV).

Centroid, Moment Tensor (HRV): Centroid origin time $04: 52: 13.3$; Lat $12.56 \mathrm{~N}$; Lon $125.78 \mathrm{E}$; Dep $15.0 \mathrm{Bdy}$; Halfduration 1.2 sec; Principal axes (scale 10**17 Nm): (T) Val $=2.14, P 1 g=63, A 2 m=265$; (N) Val $=-0.15, P 1 g=5, A 2 m=164$ (P) Val=-1.98, P1g=26, Azm=72; Best double couple: No-2.1*10**17 Nm; NP1: Strike-150, Dip-20, S1ip-75; NP2: Strike=346, Dip=71, S1ip=95.

MINDORO, PHILIPPINE ISLANDS

OFF EAST COAST OF KAYCHATKA

NORTH ISLAND, NEW ZEALAND NEW IRELAND REGION, P.N.G. OFF EAST COAST OF KAMCHATKA AUCKLAND ISLANDS REGION CENTRAL BAST PACIFIC RISE SOUTHERN IRAN SOUTH OF MARIANA ISLANDS CENTRAL PERO

NEW GUINEA, PAPUA NEW GOINEA MINDANAO, PHILIPPINE ISILANDS OFF EAST COAST OF KAYCHATRA FIJI ISLANDS REGION JAN MAYEN ISLAND REGION IRIAN JAYA REGION, INDONESIA SOUTHERN ALASKA. <AEIC>. HALYAHERA, INDONESIA SPAIN. mbLg 2.2 (MDD). SOUTH OF FIJI ISIANDS SPAIN, mbLg 2.4 (MDD) STRAIT OF GIBRALTAR. mbLg 2.5 (MDD). SOUTHEAST INDIAN RIDGE EASTERN NEW GUINEA REG., P.N.G. OFE EAST COAST OF HONSHO, JAPAN KERMADEC ISLANDS REGION SPAIN. mbLg 3.0 (YDD). MARIANA ISLANDS SPAIN. mbLg 2.5 (MDD). SPAIN. mbLg 2.8 (MDD).

STRAIT OF GIBRALTAR. mbLg 2.5 (MDD). MINAHASSA PENINSOLA, SULAWESI MARIANA ISLANDS SPAIN. mbLg 2.6 (MDD). ALASKA PENINSULA. ML 4.9 (PMR). Felt (III) at Chignik. IRIAN JAYA, INDONESIA

VANUATU ISLANDS

CHILE-ARGENTINA BORDER REGION. WD 3.3 (SAN).

SPAIN. mbLg 2.5 (MDD).

SOUTH SANDWICH ISLANDS REGION 
$\begin{array}{lllllll}01 & 21 & 22 & 03.78 & 37 & 041\end{array}$

01

01 $\begin{array}{llll}57 & 59.87 & 24.47 \mathrm{~s}\end{array}$ $\begin{array}{lllll}0 & 03 & 59.3 ? & 32.12 & s\end{array}$ $0100 \quad 44.38 \quad 37.051$ 35
58.98
07.069 $024531.6 * 49.414$ $031155.1 * 51.587$ $31355.5 * 20.369 \mathrm{~s}$ 4.8 .12 .494 $\begin{array}{llll}07 & 05 & 06.0 & 26.966 \mathrm{~N}\end{array}$ $074209.2 * 26.933 \mathrm{~N}$ $\begin{array}{llll}07 & 45 & 37.7 & 42.133\end{array}$ $085902.5 * 11.840 \mathrm{~N}$ $090425.5 * 32.096 \mathrm{~s}$ $09 \quad 18 \quad 38.8 \& \quad 57.977$ $094122.7 * 13.503 \mathrm{~s}$ $\begin{array}{lll}0 & 31 & 12.1 \text { ? } 31.60 \mathrm{~s}\end{array}$ $\begin{array}{llll}1 & 49 & 42.5 ? & 34.53\end{array}$ $\begin{array}{llll}12 & 13 & 56.5 & 51.085\end{array}$ $124204.4 * 43.069 \mathrm{~N}$

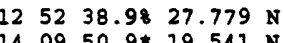
$40950.9 * 19.541 \mathrm{~N}$ $144108.58 \quad 37.022$ $1446 \quad 34.38 \quad 37.069$ $\begin{array}{llll}16 & 03 & 32.4 & 60.894 \mathrm{~s}\end{array}$ $\begin{array}{lllllllll}01 & 21 & 24 & 55.6 & 53.436 & \mathrm{~N} & 163.529 & \mathrm{~W} \\ 01 & 21 & 46 & 11.68 & 60.085 & \mathrm{~N} & 141.698 \mathrm{~W}\end{array}$

$215623.8 * 51.617 \mathrm{~N} 160.308 \mathrm{E}$ $\begin{array}{lllllll}21 & 59 & 59.6 & 4.704 \mathrm{~S} & 152.969 \mathrm{E}\end{array}$ $221912.2837 .396 \mathrm{~N} 139.552 \mathrm{E}$ $223732.6 ? 14.49$ S 166.39 E 224857.1 ? 51.90 N $159.90 \quad \mathrm{E}$ $\begin{array}{lllllll}56 & 08.8 ? & 36.02 & \text { N } & 140.61 & \mathrm{E}\end{array}$ $232948.1 * 8.319 \mathrm{~s} 107.739 \mathrm{E}$ $144412.38 \quad 36.999$ N

$4.985 \mathrm{~W}$

$4.991 \mathrm{~W}$

$156.470 \mathrm{E}$

$16.315 \mathrm{E}$

178.546

$147.192 \mathrm{E}$

$100.241 \mathrm{E}$

100.322

$7.784 \mathrm{~W}$

86.241 W

$70.470 \mathrm{~K}$

154.992

$111.204 \mathrm{~W}$

$69.51 \mathrm{~W}$

$70.43 \mathrm{~W}$

$177.380 \mathrm{~W}$

$126.626 \mathrm{~W}$

$130.133 \mathrm{E}$

$77.798 \mathrm{~W}$

$4.919 \mathrm{~W}$

$4.906 \mathrm{~W}$

$5.003 \mathrm{~W}$

21.217 พ
$33 \mathrm{~N} 3.8$ 5 $\begin{array}{lll}10 \mathrm{G} & & 0.6 \\ 33 \mathrm{~N} & 4.3 & 1.0\end{array}$ 0

$33 \mathrm{~N} 4.2$

$76 * 5.0$

$10 \mathrm{G}$

$33 \mathrm{~N} 4.2$

$33 \mathrm{~N} 4.1$

$33 \mathrm{~N}$

$33 \mathrm{~N} 4.1$

179.53 E $600 \mathrm{G} 3.8$

$71.36 \mathrm{~W} 33 \mathrm{~N}$

71.42 W $33 \mathrm{~N}$

$5 \mathrm{G} \quad 1.3$

$600 \mathrm{G} \quad 4.2 \quad 0.8$

$33 \mathrm{~N} 5.01 .1$

$33 \mathrm{~N} 5.04 .5 \quad 0.9$

$\begin{array}{lll}33 \mathrm{~N} & 4.3 & 1.5\end{array}$

$33 \mathrm{~N} 4.2 \quad 1.3$

83

$10 \mathrm{G} \quad 4.6$

$170 \mathrm{G}$

$600 \mathrm{G} \quad 4.5$

$5 \mathrm{G}$

$33 \mathrm{~N} 4.0$

$10 \mathrm{G}$

$33 \mathrm{~N}$

$10 \mathrm{G}$

$\begin{array}{ll}10 & \mathrm{G} \\ 10 \mathrm{G}\end{array}$

$\begin{array}{ll}10 & G \\ 10 & G\end{array}$

$33 \mathrm{~N} 5.0 \quad 1.1$
0.78 IRIAN JAYA, INDONESIA

49 CALIFORNIA-NEVADA BORDER REGION. SGM-P>. MD 3.2 (GM). ML 3.1 (GS).

$6 \quad 13$ SPAIN mbLg 2.8 (MDD)

34 UNIMAK ISLAND REGION. ML 4.1 (PMR).

45 SOUTHEASTERN ALASKA. CAEIC>. ML 3.3 (AEIC), 3.7 (PMR). Felt (IV) at Icy Bay Logging Camp.

1.021 OFF EAST COAST OF KAMCHATKA

0.959 NEW BRITAIN REGION, P.N.G.

0.65 EASTERN HONSHU, JAPAN

0.612 VANUATU ISLANDS

0.79 OFF EAST COAST OF KAMCHATKA

0.15 NEAR EAST COAST OF HONSHO, JAPAN

1.217 JAWA, INDONESIA

0.711 SOUTH OF FIJI ISLANDS

0.312 NEAR COAST OF CENTRAL CHILE. MD 4.2 (SAN).

0.312 NEAR COAST OF CENTRAL CHILE. MD 4.3 (SAN)

$150.9 \quad 9$ SPAIN. mbLg 2.3 (MDD)

$10 \mathrm{G} \quad 1.0 \quad 6$ SPAIN. mbIg 2.4 (MDD)

$33 \mathrm{~N} \quad 3.9 \quad 1.2 \quad 17$ KURIL ISLANDS

$10 \mathrm{G} \quad 0.8$

17 POLAND. MI 3.2 (GRF), 3.1 (VIE), 2.9 (MOX).

28 FIJI ISLANDS REGION

49 SAMAR, PHILIPPINE ISLANDS

33 KORIL ISLANDS

74 YUNNAN, CHINA

15 YUNNAN, CHINA

9 SPAIN. mbLg 3.7 (NDD).

20 NEAR COAST OF NICARAGUA

15 CHILE-ARGENTINA BORDER REGION. MD 4.4 (SAN).

43 KODIAK ISLAND REGION. LAEIC>.

30 CENTRAL EAST PACIFIC RISE

0.49 SAN JUAN PROVINCE, ARGENTINA

0.821 FIJI ISLANDS REGION

9 CHILE-ARGENTINA BORDER REGION. MD 3.3 (SAN).

26 ANDREANOF ISLANDS, ALEUTIAN IS. ML 4.4 (PMR).

38 OFF COAST OF OREGON

5 RYUKYU ISLANDS

34 CUBA REGION

9 SPAIN. mbLg 2.4 (MDD).

11 STRAIT OF GIBRALTAR. mbLg 2.7 (MDD).

8 SPAIN. MbLg 2.4 (MDD).

50 SOUTHWESTERN ATLANTIC OCEAN. MW 5.2 (HRV).

Centroid, Moment Tensor (HRV): Centroid origin time

$16: 03: 31.3$; Lat $61.20 \mathrm{~s}$; Lon $21.66 \mathrm{w}$; Dep $15.0 \mathrm{Fix}$; Half duration $1.0 \mathrm{sec}$; Principal axes (scale 10**16 Nm): (T) Val-6.61, Plg-19, Azm=172; (N) Val=-0.44, Plg-24, Azm=73; (P) Val=-6.18, Plg-58, Azm=296; Best double couple: Mo-6. 4*10*16 Nm; NP1: Strike-296, Dip-34, slip--42; NP2: strike-62, Dip=68, S1ip=-116.

$33 \mathrm{~N} 4.1$

$33 \mathrm{~N} 4.2$

600 G 3.8

$33 \mathrm{~N} 4.0$

$10 \mathrm{G}$

$150 \mathrm{G}$

13

500 G 4.1

$10 \mathrm{G} 4.0$

$5 \mathrm{G}$

$10 \mathrm{G} 4.2$

$25 \mathrm{D} \quad 4.6$

$33 \mathrm{~N} 4.2$

$6 \mathrm{G}$

B7 D 4.3

$33 \times 4.4$

$100 \mathrm{G} \quad 4.3$

$53 \mathrm{D} \quad 4.5$

$33 \mathrm{~N}$

$33 \mathrm{~N} 4.44 .11$

$200 \mathrm{G} \quad 4.2 \quad 1.1$

$33 \mathrm{~N} 4.6$

33 i 3.9

$5 \mathrm{G}$

$33 \times 5.65 .4$
1.6

1.0
0.7

1.5

0.6

1.5

0.6

0.1

1.0

0.9

1.0

1.1

0.9

1.3

.0

1.1

1.7

0.8

9 NEAR NORTH COAST OF IRIAN JAYA

18 EAST OF KURIL ISLANDS

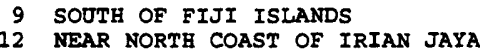

5 NEAR EAST COAST OF HONSHO, JAPAN

6 LEEWARD ISLANDS. ND 3.4 (TRN).

46 CENTRAL AIAASKA. CAEIC>. ML 2.5 (AEIC), 3.0 (PMR).

15 FIJI ISLANDS REGION

12 CHAGOS ARCHIPELAGO REGIO

6 CHILE-ARGENTINA BORDER REGION

54 CENTRAL CALIFORNIA. LGM-P>. MD 3.6 (GM), 3.3 (PAS). ML 3.7 (BRK).

9 WEST CHILE RISE

29 SAMAR, PHILIPPINE ISLANDS

20 SAMAR, PHILIPPINE ISLANDS

5 SOUTHERN CALIFORNIA. 〈PAS-P>. MD 2.1 (PAS). Felt in the Glendale area.

28 HOKKAIDO, JAPAN REGION

26 SAMAR, PHILIPPINE ISLANDS

14 SOUTH SANDWICH ISLANDS REGION

27 NORTHERN COLOMBIA

7 STRAIT OF GIBRALTAR. mbLg 2.9 (MDD).

21 NEAR COAST OF PERU

26 TONGA ISLANDS

16 NORTHERN MOLUCCA SEA

9 OFF COAST OF NORTHERN CHILE

21 CALIFORNIA-NEVADA BORDER REGION. ML 3.0 (GS). MD 3.0 (GM).

221 XIZANG. NW 5.7 (GS), 5.7 (HRV). Me 5.2 (GS).

Broadband source Parameters (GS): Dep 7; NP1: Strike-167,

Dip=33, slip=-114; NP2: Strike-15, Dip-60, slip=-75;

Radiated energy $1.6 * 10 * * 12 \mathrm{Nm}$.

Moment Tensor (GS): Dep 5; Principal axes (scale 10**17 Nm): (T) Val-3.54, Plg=2, Azm=84; (N) Val=-0.01, Plg=13, Azm-175; (P) val=-3.53, P1g-76, Azm-344; Best double couple: Mo-3.5*10*\$17 Nm; NPI: Strike-161, Dip=44, Slip=-109; NP2: Strike-7, Dip-49, Slip=-72.

Centroid, Moment Tensor (HRV): Centroid origin time $06: 44: 52.3$; Lat $29.77 \mathrm{~N}$; Lon $88.32 \mathrm{E}$; Dep $15.0 \mathrm{Bdy}$; Half duration $1.7 \mathrm{sec}$; Principal axes (scale 10**17 Nm): (T) Val-3.72, Plg=1, Azm=90; (N) Val=-0.37, Plg=8, Azm=180; (P) val=-3.36, Plg-82, Azm-355; Best double couple: Mo-3.5*10**17 Nm; NP1: Strike-172, Dip=45, Slip--102; NP2: Strike $=8$, Dip $=46$, slip $=-78$. 
$0307 \quad 10 \quad 15.9 * 30.468 \mathrm{~N}$ $0307 \quad 14 \quad 39.08 \quad 44.679 \mathrm{~N}$

$0307 \quad 15 \quad 42.9 * 29.993 \mathrm{~N}$

$\begin{array}{llllll}03 & 07 & 38 & 06.08 & 33.500 \mathrm{~s}\end{array}$

$\begin{array}{lllllll}03 & 07 & 42 & 35.2 \& & 35.752 & \mathrm{~N}\end{array}$

$\begin{array}{llllll}03 & 07 & 55 & 25.58 & 33.806 & \mathrm{~s}\end{array}$ $\begin{array}{lllll}03 & 08 & 06 & 51.9 & 36.280 \mathrm{~N}\end{array}$ $030847 \quad 34.9 * 13.090 \mathrm{~N}$ 03091519.9 ? 3.99 S $\begin{array}{lllllll}03 & 09 & 49 & 53.28 & 44.705 & N\end{array}$ $\begin{array}{llllll}03 & 10 & 10 & 33.8 & 29.923 & \mathrm{~N}\end{array}$
$88.187 \mathrm{E}$ $7.195 \mathrm{E}$ $87.944 \mathrm{E}$ 70.686 W $121.262 \mathrm{~W}$ 71.119 म $138.982 \mathrm{E}$ 143.346 $127.09 \mathrm{E}$ $7.666 \mathrm{E}$ $88.187 \mathrm{E}$
$33 \mathrm{~N} 4.1$

$10 \mathrm{G}$

$33 \mathrm{~N} 4.0$

$80 \mathrm{G}$

3

$60 \mathrm{G}$

$167 \mathrm{D} \quad 3.9$

$216 ? 4.0$

$33 \mathrm{~N} 4.4$

$10 \mathrm{G}$

$\begin{array}{lllll}33 \mathrm{~N} & 5.0 & 4.7 & 0.9\end{array}$
$03101942.9 * 30.017 \mathrm{~N}$

$03104950.5 * 29.873 \mathrm{~N}$

$\begin{array}{llllllll}03 & 11 & 37 & 16.5 ? & 11.66 & \mathrm{~s}\end{array}$

$\begin{array}{llllll}03 & 11 & 38 & 28.0 & 42.158 \mathrm{~N}\end{array}$

$03121322.9 * 21.427 \mathrm{~s}$

$03124357.1 * 35.522 \mathrm{~N}$

$\begin{array}{lllllll}03 & 12 & 53 & 19.6 ? & 59.54 \quad S\end{array}$

$\begin{array}{lllllll}03 & 12 & 59 & 06.9 ? & 30.10 & \mathrm{~N}\end{array}$

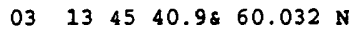

$\begin{array}{lllllll}03 & 13 & 57 & 05.28 & 34.376 & \mathrm{~s}\end{array}$

$\begin{array}{lllllll}03 & 16 & 09 & 02.4 ? & 34.19 & \mathrm{~S}\end{array}$

$\begin{array}{lllllll}03 & 16 & 11 & 09.5 ? & 34.22 & \mathrm{~S}\end{array}$

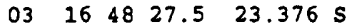

$88.214 \mathrm{E} \quad 33 \mathrm{~N} 4.3$

$87.846 \mathrm{E} 33 \mathrm{~N} 4.1$

$\begin{array}{lllll}166.73 & \mathrm{E} & 198 \mathrm{D} & 4.2\end{array}$

$0.271 \mathrm{E}$

$69.963 \mathrm{~W}$

$140.936 \mathrm{E}$

26.11 W

$151.588 \mathrm{~W}$

$70.666 \mathrm{~W}$

$72.12 \mathrm{~W}$

72.20

$70.405 \mathrm{~W}$
$5 \mathrm{G}$

$55 \mathrm{D} \quad 4.4$

$33 \mathrm{~N} 3.8$

$111 ? 4.5$

$33 \mathrm{~N} 3.8$

70

$100 \mathrm{G}$

$10 \mathrm{G}$

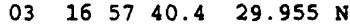
$\begin{array}{llllllllllllll}03 & 17 & 07 & 19.6 ? & 30.48 \quad \mathrm{~N}\end{array}$ $03175224.4 * 12.688 \mathrm{~N}$ $\begin{array}{lllll}03 & 18 & 02 & 43.9 * 30.091 \mathrm{~N}\end{array}$ $03 \quad 18 \quad 34 \quad 59.18 \quad 33.318 \mathrm{~s}$

$\begin{array}{llllll}03 & 18 & 59 & 26.4 & 40.683 & \mathrm{~N}\end{array}$
$88.061 \mathrm{E}$ $88.68 \mathrm{E}$ $125.183 \mathrm{E}$ $87.731 \mathrm{E}$ $70.652 \mathrm{~W}$ $142.570 \mathrm{E}$
$33 \mathrm{~N} 4.2$

$33 \mathrm{~N} 3.8$

$33 \mathrm{~N} 4.6$

$33 \mathrm{~N} 3.9$

$60 \mathrm{G}$

49

1.3
0.8
1.2
0.8

0.3

0.9

0.7

1. 3

15 XIZANG

8 NORTHERN ITALY. ML 2.3 (GEN).

13 XIZANG

10 CHILE-ARGENTINA BORDER REGION

68 CENTRAL CALIFORNIA. LGM-P>. MD 3.7 (GM), 3.7 (PAS). ML 4.0 (BRK).

9 NEAR COAST OF CENTRAL CHILE

30 EASTERN HONSBU, JAPAN

16 SOUTH OF MARIANA ISLANDS

11 SERAM, INDONESIA

6 NORTHERN ITALY. ML 2.3 (GEN).

63 XIZANG. MW 5.0 (HRV).

Centroid, Moment Tensor (HRV): Centroid origin time

10:10:41.6; Lat $29.92 \mathrm{~N} \mathrm{Fix;} \mathrm{Lon} 88.19 \mathrm{EF}$ Fix; Dep $33.0 \mathrm{Fix}$; Half-duration $1.0 \mathrm{sec}$; Principal axes (scale 10**16 $\mathrm{Nm}$ ): (T) Val=3.45, Plg=18, Azm-80; (N) Val=0.55, Plg=3, Azm=349; (P) Val- -4.00, Plg-72, Azm=249; Best double couple: Mo-3.7*10*16 Nm; NP1: Strike-175, Dip=27, Slip=-83; NP2: Strike-347, Dip=63, Slip=-94.

$\begin{array}{lll}1.2 & 24 & \text { XIZANG } \\ 0.8 & 11 & \text { XIZANG }\end{array}$

1.218 SANTA CRUZ ISLANDS

0.914 PYRENEES. MI 2.9 (STR)

1.429 NORTHERN CBILE

1.112 NEAR EAST COAST OF BONSEO, JAPAN

1.329 SOUTE SANDWICH ISLANDS REGION

0.15 XIZANG

93 KENAI PENINSULA, ALASKA. <AEIC>. ML 3.0 (AEIC), 3.3 (PMR)

0.110 CBILE-ARGENTINA BORDER REGION

10 NEAR COAST OF CENTRAL CEILE. MD 3.6 (SAN).

7 NEAR COAST OF NORTHERN CEILE. NW 5.9 (HRV), 5.8 (GS). Moment Tensor (GS): Dep 29; Principal axes (scale 10**17 $\mathrm{Nm}$ : (T) Val-6.61, Plg-62, Azm=54; (N) Val--0.05, Plg=18, Azm-182; (P) Val=-6.55, Plg=21, Azm-279; Best double couple: $M 0-6.6 \star 10 \star \star 17 \mathrm{Nm}$; NP1: Strike-38, Dip=29, Slip=130; NP2: Strike-174, Dip-68, Slip-70.

Centroid, Moment Tensor (HRV): Centroid origin time $16: 48: 33.9$; Lat $23.24 \mathrm{~s}$; Ion $71.22 \mathrm{~W}$; Dep 15.0 Fix; Balfduration $2.2 \mathrm{sec}$; Principal axes (scale 10**17 $\mathrm{km}$ ): (T) Val-7.51, Plg-63, Azm-53; (N) Val-0.28, Plg-13, Azm=171; (P) Val=-7.79, Plg-23, Azm-266; Best double couple: Mo-7.7*10*17 Nm; NP1: Strike-20, Dip-25, Slip=122; NP2 : Strike-165, Dip-69, Slip=76.

$0.7 \quad 18$ XIZANG

0.98 XIZANG

22 SAMAR,

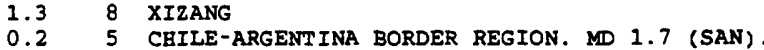

108 NEAR EAST COAST OF BONSHO, JAPAN. NW 5.2 (HRV) Centroid, Moment Tensor (HRV): Centroid origin time $18: 59: 27.5$; Lat $40.73 \mathrm{~N}$; Lon $143.00 \mathrm{E}$; Dep 15.8; Balf duration 1.0 sec; Principal axes (scale 10*\$17 $\mathrm{Nm}$ ): (T) Val-1.01, Plg-57, Azm-339; (N) Val=-0.39, Plg-32, Azm-144; (P) Val--0.62, Plg-7, Azm-238; Best double couple: Mo-8.1*10*16 km; NP1: Strike-359, Dip-47, Slip=136; NP2 : Strike-122, Dip=59, Siip=52.

$032005 \quad 29.4 * 13.210 \mathrm{~N} 124.647 \mathrm{E}$

$33 \mathrm{~N} 4.3$

1.214 LU2ON, PEILIPPINE ISLANDS

$\begin{array}{llllllll}03 & 20 & 35 & 28.9 ? & 30.33 & \mathrm{~N} & 87.98 & \mathrm{E}\end{array}$

$\begin{array}{llllllll}03 & 20 & 35 & 54.28 & 63.045 & N & 150.619 & N \\ 03 & 20 & 47 & 15.48 & 44.262 & N & 7.205 & \end{array}$

$032100 \quad 59.5 \& 64.581 \mathrm{~N} \quad 152.199 \mathrm{~W}$

$\begin{array}{lllllll}03 & 21 & 13 & 33.98 & 37.032 & \mathrm{~N} & 4.953 \mathrm{~W}\end{array}$

$\begin{array}{lllllll}03 & 21 & 38 & 32.28 & 36.977 & \mathrm{~N} & 4.940 \mathrm{~W}\end{array}$

$\begin{array}{lllllllll}03 & 21 & 44 & 42.08 & 37.027 & \mathrm{~N} & 4.926 \mathrm{~W}\end{array}$

$\begin{array}{lllllll}03 & 21 & 59 & 08.78 & 37.086 \mathrm{~N} & 4.970 \mathrm{~W}\end{array}$

$032204 \quad 43.5 \& 47.773 \mathrm{~N} \quad 121.885 \mathrm{~W}$

$04003248.5 * 14.632 \mathrm{~S} \quad 176.418 \mathrm{~W}$

$04015247.5 \& 61.600 \mathrm{~N} 149.759 \mathrm{~W}$

$0402 \quad 2250.0 ? \quad 34.45$ S 70.39 W

$\begin{array}{llllllll}04 & 02 & 28 & 06.0 ? & 21.14 & \mathrm{~S} & 168.57 & \mathrm{E}\end{array}$

$\begin{array}{llllllll}04 & 02 & 56 & 44.2 & 53.631 & \mathrm{~N} & 160.475 \mathrm{E}\end{array}$

$04.03 \quad 38 \quad 23.6 \quad 30.069 \mathrm{~N} \quad 88.219 \mathrm{E}$

$040505 \quad 46.7 * 8.842 \mathrm{~S} \quad 124.162 \mathrm{E}$

$04 \quad 0709 \quad 36.6 ? \quad 7.04 \quad S \quad 154.74 \quad E$

$\begin{array}{lllllllll}04 & 08 & 16 & 59.2 \& & 33.995 & \mathrm{~N} & 116.298 \mathrm{~W}\end{array}$

$04081905.5 \& 63.296 \mathrm{~N} \quad 151.053 \mathrm{~W}$

$\begin{array}{llllllll}04 & 09 & 04 & 58.58 & 32.992 \mathrm{~s} & 70.207 \mathrm{~W}\end{array}$

$041004 \quad 05.6 * 8.929 \mathrm{~S} \quad 124.077$

3.9

$12 \quad 3.3$

$10 \mathrm{G}$

15 *

$15 \star$

$10 \mathrm{G}$

3

30064.1

39

$33 \mathrm{~N} \quad 4.2$

$33 \mathrm{~N} 4.4$

$33 \mathrm{~N} 4.3$

$33 \mathrm{~N} 4.4$

$33 \times 4.0$

5

13

$10 \mathrm{G}$

$04113939.8 \& 61.852 \mathrm{~N} 150.830 \mathrm{~W} \quad 55 \quad 5.6$

1.798 CENTRAL ALASKA. <AEIC>.

0.28 NORTBERN ITALY. ML 2.2 (GEN).

34 CENTRAL ALASKA. CAEIC>. MIL 2.9 (AEIC), 3.2 (PMR)

0.812 SPAIN. mbLg 2.5 (MDD).

0.611 STRAIT OF GIBRALTAR. mbLg 2.5 (MDD)

0.612 SPAIN. mbLg 2.6 (MDD)

0.77 SPAIN. mbIg 2.0 (MDD)

35 WASHINGTON. $\langle$ SEA-P>. MD 3.0 (SEA). Felt.

0.617 FIJI ISLANDS REGION

46 SOUTHERN ALASKA. CAEIC>. ML 2.7 (AEIC).

0.16 CEILE-ARGENTINA BORDER REGION

1.420 LOYALTY ISLANDS

1.140 NEAR EAST COAST OF KAMCHATKA

0.518 XIZANG

1.317 TIMOR REGION, INDONESIA

0.98 SOLONON ISLANDS

32 SOUTHERN CALIFORNIA. <PAS-P>. MD 3.4 (PAS). ML 3.2 (GS).

56 CENTRAL ALASKA. CAEIC>. ML 2.7 (AEIC), 3.0 (PMR).

0.210 CHILE-ARGENTINA BORDER REGION. MD 2.5 (SAN)

1.013 TIMOR REGION, INDONESIA

324 SOUTHERN ALASKA. CAEIC>. MW 5.4 (GS), 5.4 (HRV). ML 5.4 (AEIC), 5.7 (PMR). Felt (IV) at Anchorage, Palmer, wasilla and Willow.

Moment Tensor (GS): Dep 60; Principal axes (scale 10**17 Nim): (T) Val-1.10, Plg-23, Azm-264; (N) Val-0.17, Plg=23, Azm=4; (P) val=-1.26, P1g-56, Azm-133; Best double couple:

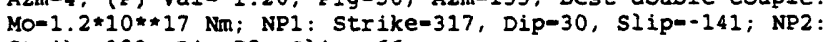
Strike-192, Dip-72, Slip--66.

Centroid, Moment Tensor (HRV): Centroid origin time $11: 39: 40.4$; Lat $61.91 \mathrm{~N}$; Lon 151.21 w; Dep 58.7 ; Balfduration $1.2 \mathrm{sec}$; Principal axes (scale 10*17 $\mathrm{Nm}$ ): (T) Val=1.40, Plg-33, Azm=279; (N) Val=-0.01, Plg-8, Azm-183; (P) Val-1.39, Plg-56, Azm-81; Best double couple:

Mo=1.4*10*17 Nm; NP1: Strike-37, Dip=15, S1ip=-55; NP2: 
Strike-181, Dip-78, slip=-99.

04

$\begin{array}{llllll}12 & 34 & 38.98 & 61.853 \mathrm{~N} \\ 04 & 13 & 20 & 41.3 * & 40.896 & \mathrm{~N}\end{array}$

$04135647.1 * 72.441 \mathrm{~N}$

$04 \quad 1406 \quad 45.48 \quad 15.734 \mathrm{~N}$

04

$144913.6 * 53.316 \mathrm{~N} 159.950 \mathrm{E} \quad 55 \mathrm{D} \quad 3.9$ $153750.67 .148 \mathrm{~S} \quad 122.395 \mathrm{E} 600 \mathrm{G} \quad 5.3$
$041440 \quad 38.1842 .103 \mathrm{~N}$
127.24 E 400 G 4.1

150.851 $159.277 \mathrm{E}$ $141.469 \mathrm{E}$ $147.537 \mathrm{E}$ $3.324 \mathrm{E}$ 61.092 $0.216 \mathrm{E}$ 54 G 4.1 $33 \times 3.7$ 51 $97 * 3.7$ $33 \mathrm{~N} 4.6$ $10 \mathrm{G} 3.6$ $100 \mathrm{G}$

$10 \mathrm{G}$ 150.879 W

0.8 B BANDA SEA

78 SOUTHERN ALASKA, <AEIC>. ML 3.1 (AEIC).

1.412 OFF EAST COAST OF KAMCHATKA

67 SOUTHERN ALASKA. CAEIC>. ML 2.6 (AEIC).

1.212 NEAR EAST COAST OF HONSHU, JAPAN

1.145 MARIANA ISLANDS REGION

1.512 NORWEGIAN SEA

0.210 LEEWARD ISLANDS. MD 3.5 (TRN).

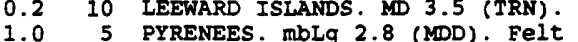
Spain.

1.013 NEAR EAST COAST OF RAMCHATKA

0.8118 FLORES SEA. MW 5.4 (HRV).

Centroid, Noment Tensor (HRV): Centroid origin time

$15: 37: 49.4$; Lat $7.62 \mathrm{~S}$; Lon $122.83 \mathrm{E}$; Dep 614.2 ; Half

duration $1.2 \mathrm{sec}$; Principal axes (scale 10*17 Nm): (T)

Val-1.48, Plg=26, Azm=342; (N) Val-0.15, Plg-21, Azm=83

(P) Val=-1.62, Plg=55, Azm-206; Best double couple:

No=1.5*10**17 Nm; NP1: Strike-33, Dip-26, S1ip=-143; NP2:

Strike-269, Dip-75, Sl1p=-68.

$\begin{array}{lllllllllllll}04 & 15 & 50 & 38.7 & 8.489 & \mathrm{~N} & 141.564 \mathrm{E} & 33 & \mathrm{~N} & 5.1 & 5.4 & 1.3 & 103\end{array}$

Noment Tensor (GS): Dep 9; Principal axes (scale 10**17 Nm): (T) Val-6.24, Plg-37, Azm-349; (N) Val-0.10, Plg-45, Azm-210; (P) val--6.34, Plg-22, Azm-96; Best double couple: No-6.3*10*17 Nm; NP1: Strike-138, Dip-47, Slip-13; NP2: Strike-39, Dip-81, Slip-136.

Centroid, Noment Tensor (HRV): Centroid origin time

$15: 50: 38.8$; Lat 8.09 N; Lon $141.63 \mathrm{E}$; Dep $16.0 \mathrm{Bdy}$; Halfduration 2.1 sec: Principal axes (scale 10**17 Nm): (T) Val-6.55, Plg-21, Azm-348; (N) Vai=0.31, P1g-67, Azm=197; (P) val--6.86, Plg-10, Azm-82; Best double couple: No-6.7*10*17 $\mathrm{Nm}$; NP1: Strike-126, Dip 68 , S1ip-8; NP2: strike-34, Dip=B3, Slip-158.

$04155350.5 * 8.868 \mathrm{~N} 141.495 \mathrm{E}$

04

$04 \quad 165416.1863 .139 \mathrm{~N}$

$04170759.4 * 6.128 \mathrm{~s}$

$04172520.9 * 28.070 \mathrm{~s}$

$\begin{array}{llllll}04 & 18 & 11 & 04.6 & 30.009 & \mathrm{~N}\end{array}$

$\begin{array}{llllll}04 & 18 & 41 & 42.2 & 5.227 & \mathrm{~s}\end{array}$

$\begin{array}{lllll}04 & 19 & 42 & 09.2 ? \quad 4.72 \mathrm{~s}\end{array}$

$04201109.0 * 56.090 \mathrm{~N}$

$\begin{array}{lllllll}04 & 21 & 53 & 09.78 & 32.984 & \mathrm{~s}\end{array}$

$\begin{array}{llllll}04 & 21 & 57 & 20.0 & 38.191 & N\end{array}$

$\begin{array}{lllllll}04 & 22 & 14 & 54.28 & 32.983 & \mathrm{~s}\end{array}$

$0422 \quad 2526.5$ ? 39.34 N

$04224046.8 * 11.825 \mathrm{~N}$

$\begin{array}{llllll}22 & 47 & 37.8 ? & 23.99 \quad \mathrm{~S}\end{array}$

$\begin{array}{llllll}04 & 23 & 26 & 08.8 & 42.346 & \mathrm{~N}\end{array}$

$\begin{array}{lllllll}04 & 23 & 30 & 41.8 ? & 17.84 \mathrm{~S}\end{array}$

$\begin{array}{lllllll}04 & 23 & 46 & 38.1 & 23.808 & \mathrm{~S}\end{array}$

$\begin{array}{llllll}0 & 00 & 07 & 41.68 & 62.952 & \mathrm{~N}\end{array}$

$\begin{array}{llllll}00 & 4722.08 & 34.082 & \mathrm{~s}\end{array}$

$501 \quad 1237.1 * 50.186 \mathrm{~N}$

$011918.9 * 4.729 \mathrm{~s}$

$\begin{array}{lllll}02 & 03 & 55.88 & 32.989 \mathrm{~s}\end{array}$

$030029.0641 .710 \mathrm{~N}$

$\begin{array}{lllll}05 & 15 & 05.5 & 51.474 \mathrm{~N}\end{array}$

$0525 \quad 48.6 ? 11.18 \mathrm{~N}$

$063810.0 * 5.286 \mathrm{~N}$

$081521.8 * 51.850 \mathrm{~N}$

$\begin{array}{lllll}09 & 16 & 43.08 & 63.031 & \mathrm{~N}\end{array}$

$\begin{array}{lllllll} & 09 & 17 & 12.68 & 44.282 & \mathrm{~N}\end{array}$

$\begin{array}{llll}09 & 19 & 20.28 & 60.922 \mathrm{~N}\end{array}$

093644.8 ? $32.34 \mathrm{~s}$

$\begin{array}{lllll}09 & 54 & 53.68 & 44.397 & \mathrm{~N}\end{array}$

$\begin{array}{lllll}10 & 02 & 29.5 * 17.790 \mathrm{~N}\end{array}$

$\begin{array}{lllll}10 & 37 & 17.2 * 51.768 \mathrm{~N}\end{array}$

$\begin{array}{llllll}5 & 11 & 23 & 52.3 & 51.769 \mathrm{~N}\end{array}$

$\begin{array}{lllll}05 & 11 & 52 & 57.0 * 51.814 \mathrm{~N}\end{array}$

$5 \quad 120052.7 * 8.934 \mathrm{~s}$

$\begin{array}{lllll}12 & 17 & 52.0863 .063 \mathrm{~N}\end{array}$

$5130956.1 * 30.060 \mathrm{~N}$

$5132121.4 * 19.329 \mathrm{~s}$

$140148.0 * 21.817 \mathrm{~N}$

$\begin{array}{llllll}15 & 04 & 07.8 & 1.136 & \mathrm{~s}\end{array}$

$\begin{array}{llllll}16 & 38 & 26.38 & 59.236 & N\end{array}$

$\begin{array}{llllll}5 & 17 & 43 & 28.2 & 28.696 & \mathrm{~N}\end{array}$

$\begin{array}{llllll}5 & 18 & 34 & 42.0 & 10.112 & \mathrm{~S}\end{array}$

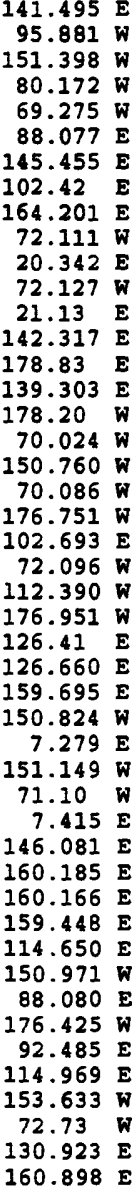

33 N 5.2

$10 \mathrm{G} 4.2$

8

$74 ? 4.1$

$106 \mathrm{D} \quad 4.1$

$33 \times 4.9$

$56 \star 4.4$

$33 \mathrm{~N} 4.0$

$33 \mathrm{~N} 4.1$

$15 \mathrm{G}$

$35 * 4.4$

$15 \mathrm{G}$

$33 \mathrm{~N} 3.8$

$60 ? 4.2$

600 G 4.4

$25 \mathrm{D} 4.8$

600 G 3.9

$43 \mathrm{D} 4.4$

110

$10 \mathrm{G}$

33 N 4.0

$33 \mathrm{~N} 4.3$

$10 \mathrm{G}$

2

$33 \mathrm{~N} 4.5$

$33 \mathrm{~N} 4.1$

$\begin{array}{lll}33 & \mathrm{~N} & 4.1 \\ 3 & \mathrm{~N} & 4.7\end{array}$

33 i

121

$10 \mathrm{G}$

63

$60 \mathrm{G}$

$10 \mathrm{G}$

$200 \mathrm{G} 3.4$

$33 \mathrm{~N} 4.4$

$33 \mathrm{~N} 4.0$

$33 \mathrm{~N} 4.74 .1$

$100 \mathrm{G} \quad 4.8$

130

$33 \mathrm{~N} 4.2$

$250 \mathrm{G} \quad 4.1$

$33 \mathrm{~N} 4.1$

$33 \mathrm{~N} 4.9$

107

$10 \mathrm{G}$

$33 \mathrm{~N} 4.1$

$83 * 5.5 \quad 5.2 \quad 1.0 \quad 110$

0.2

1.4

0.8

15
1.155 WESTERN CAROLINE ISLANDS

0.99 SOOTHERN PACIFIC OCEAN

10 CENTRAL ALASKA. <AEIC>. MC 2.4 (AEIC), 2.8 (PMR).

1.117 NEAR COAST OF NORTHERN PERO

1.219 CHILE-ARGENTINA BORDER REGION

1.057 XIZANG

0.718 EASTERN NEW GUINEA REG., P.N.G.

1.09 SOUTHERN SUMATERA, INDONESIA

1.014 KOMANDORSKY ISLANDS REGION

0.311 OFF COAST OF CENTRAL CHILE. MD 4.0 (SAN).

1.152 GREECE

0.411 OFF COAST OF CENTRAL CHILE. MD 4.0 (SAN).

1.415 GREECE

1.018 SOOTH OF MARIANA ISLANDS

0.8 17. SOOTH OF FIJI ISLANDS

0.988 HOKKAIDO, JAPAN REGION

0.913 FIJI ISLANDS REGION

1.027 NEAR COAST OF NORTHERN CHILE

CENTRAL ALASKA. 〈AEIC>

CHILE-ARGENTINA BORDER REGION. MD 3.8 (SAN).

SOUTHERN SUMATERA, INDONESIA

OFF COAST OF CENTRAL CHILE. ND 4.0 (SAN).

OTAH. 〈SLC-P>. MD 3.8 (SLC). ML 3.5 (GS).

ANDREANOF ISLANDS, ALEOTIAN IS. ML 4.6 (PMR).

PHILIPPINE ISLANDS REGION

MINDANAO, PHILIPPINE ISLANDS

OFF EAST COAST OF KAMCHATKA

CENTRAL ALASKa. 〈AEIC>.

NORTHERN ITALY MI 2.1 (GEN)

KENAI PENINSULA, ALASKA. CAEIC>. NI 3.1 (AEIC).

NEAR COAST OF CENTRAL CHILE. ND 3.8 (SAN)

NORTHERN ITALY. ML 1.9 (GEN).

MARIANA ISLANDS

OFF EAST COAST OF KAMCHATKA

OFF EAST COAST OF KAMCHATKA

OFF EAST COAST OF KAMCHATKA

BAII REGION, INDONESIA

CENTRAL ALASKA. 〈AEIC>.

XIZANG

FIJI ISLANDS REGION

MYANMAR-BANGLADESH BORDER REGION

BORNEO

SOUTHERN ALASKA. <AEIC>

OFF COAST OF CENTRAL CHILE. MD 3.7 (SAN).

RYOKYU ISLANDS

(III) at HODIATA.

Broadband Source Parameters (GS): Dep 36; NP1: Strike-285,

Dip-50, S1ip-45; NP2: Strike-162, Dip=57, Slip-130;

Radiated energy $1.9 * 10 * 12 \mathrm{Nm}$.

Moment Tensor (GS): Dep 36; Principal axes (scale 10*17

Nin) : (T) Val-3.01, Plg-59, Azm-133; (N) Val=0.19, Plg-30

Azm-297; (P) Val--3.20, Plg=7, Azm-31; Best double couple:

No-3.1*10*17 Nm; NP1: Strike-150, Dip-46, s1ip-133; NP2:

strike-277, Dip-58, Silp-54.

Centroid, Moment Tensor (HRV): Centroid origin time

18:34:39.7; Lat $10.25 \mathrm{~S}$; Lon $161.13 \mathrm{E}$; Dep 33.0; Half-

duration $1.5 \mathrm{sec}$; Principal axes (scale 10*17 $\mathrm{Nm}$ ): (T)

val-2.70, Plg-64, Azm=113; (N) Val=-0.33, Plg-26, Azm=301; 
$\begin{array}{lllllllll}05 & 19 & 06 & 04.4 & 48.248 & \mathrm{~N} & 155.684 \mathrm{E}\end{array}$ $05 \quad 20 \quad 3621.4 * 34.177$ N $05 \quad 213044.4858 .662 \mathrm{~N}$

$05 \quad 2205 \quad 55.8 ? \quad 17.30 \mathrm{~s}$ $\begin{array}{lllllll}05 & 23 & 30 & 05.2 & 43.517\end{array}$ $33150.08 \quad 36.613$ N $00 \quad 4157.48 \quad 44.369 \mathrm{~N}$ $0053 \quad 05.4$ * $6.589 \mathrm{~s}$ $35.081 \mathrm{E}$ $152.843 \mathrm{~W}$ $84.000 \mathrm{w}$

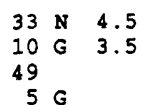

$171.56 \mathrm{~W}$ $110.312 \mathrm{~W}$ $139.452 \mathrm{E}$ $7.233 \mathrm{E}$ $129.062 \mathrm{E}$
$33 \times 4.5$

$5 \mathrm{G}$

$10 \mathrm{G}$

$5 \mathrm{G}$ $222 ? 4.9$

(P) Val--2.37, Plg-3, Azm-209; Best double couple: Mo-2.5*10**17 Nm; NP1: Strike-274, Dip-48, Slip-53; NP2: Strike-142, Dip-53, Slip-123.

1.152 KURIL ISLANDS

0.99 NEAR $S$. COAST OF WESTERN HONSHO

65 KODIAK ISIAND REGION. 〈AEIC>. MI 3.4 (AEIC).

TENNESSEE. <MACRO>. mbLg 2.8 (GS). Felt in cherokee county, North Carolina.

TONGA ISLANDS REGION

WYOMING. ML 3.1 (BUT).

5 EASTERN HONSHU, JAPAN

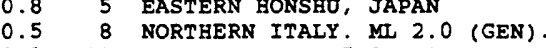

0.933 BANDA SEA. MW 5.2 (HRV).

Centroid, Moment Tensor (BRV): Centroid origin time

00:53:15.9; Lat $6.59 \mathrm{~S}$ Fix; Lon 129.06 E Fix; Dep 240.9;

Half-duration $1.0 \mathrm{sec}$; Principal axes (scale $10 * 16 \mathrm{Nm}$ )

(T) Val=5.74, Plg-44, Azm-2; (N) Val=0.90, Plg=30, Azm-238; (P) Val=-6.64, Plg-31, Azm-128; Best double couple: Mo-6.2*10*16 Nm; NP1: Strike-166, Dip-31, Slip-15; NP2: Strike-63, Dip-82, Slip-120.

$06012706.1 * 22.361 \mathrm{~N}$ $0602 \quad 22 \quad 15.7 * 43.476 \mathrm{~N}$ $06025042.9 * 48.443 \mathrm{~N}$ $\begin{array}{llllll}06 & 04 & 23 & 05.28 & 44.040\end{array}$ $06 \quad 044145.0 \quad 51.621 \mathrm{~N}$ $\begin{array}{llllll}06 & 05 & 02 & 25.9 & 3.641 \mathrm{~S}\end{array}$ $\begin{array}{llllll}06 & 05 & 03 & 33.5 & 2.973\end{array}$
$120.737 \mathrm{E}$ 110.523 $155.721 \mathrm{E}$ $6.952 \mathrm{E}$ $16.271 \mathrm{E}$ 139.951 $128.354 \mathrm{E}$
$33 \times 4.0$

$5 \mathrm{G}$

$10 \mathrm{G}$

$5 \mathrm{G}$

$\begin{array}{llll}33 \mathrm{~N} & 4.9 & & 1.0 \\ 33 \mathrm{~N} & 5.4 & 5.2 & 1.0\end{array}$
$33 \mathrm{~N} 3.9$ $\begin{array}{llllll}06 & 05 & 11 & 16.68 & 44.461\end{array}$ $06 \quad 052422.7 * 31.128$ $06 \quad 052906.8844 .549 \mathrm{~N}$ $06 \quad 05 \quad 58 \quad 37.1 * 32.953 N$ $\begin{array}{lllllllll}06 & 08 & 12 & 39.4 ? & 29.96 & \mathrm{~N}\end{array}$ $06 \quad 08 \quad 45 \quad 32.5 * 52.342 \mathrm{~N}$ $06 \quad 0856 \quad 55.08 \quad 47.160 \mathrm{~N}$ $06 \quad 1008 \quad 52.1 * 10.188 \mathrm{~N}$ $\begin{array}{lllllllll}06 & 10 & 09 & 26.4 & 43.288 & \mathrm{~N}\end{array}$ $\begin{array}{llllll}06 & 11 & 06 & 43.8 \& 60.439 \mathrm{~N}\end{array}$ $\begin{array}{llllll}06 & 11 & 23 & 35.38 & 37.114 & N\end{array}$ $06 \quad 1146 \quad 48.9$ ? $11.41 \mathrm{~s}$ $\begin{array}{llllll}06 & 11 & 56 & 44.8 & 15.514 & \mathrm{~N}\end{array}$
$7.328 \mathrm{E}$ 129.393 $6.739 \mathrm{E}$ $140.782 \mathrm{E}$ $87.89 \mathrm{E}$ 158.824 $150.786 \mathrm{E}$ $120.876 \mathrm{E}$ $147.408 \mathrm{E}$ $152.345 \mathrm{~W}$ $1.801 \mathrm{~W}$ $165.92 \mathrm{E}$ $147.657 \mathrm{E}$
$10 \mathrm{G}$

$91 ? 4.1$

$5 \mathrm{G}$

$65 * 4.0$

$33 \times 3.8$

$33 \mathrm{~N} 3.9$

$100 \mathrm{G} 3.8$

$33 \mathrm{~N} 4.4$

$33 \mathrm{~N} 4.3$

92

$10 \mathrm{c}$

$33 \mathrm{~N} 4$.

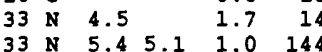

\section{TAIWAN}

$\begin{array}{rrl}1.3 & 16 & \text { TATWAN } \\ 0.8 & 8 & \text { WYOMING. MI } 3.2 \text { (BOT) }\end{array}$

$\begin{array}{rrl}0.8 & 8 & \text { WYOMING. ML } \\ 1.0 & 18 & \text { KORIL ISLANDS } \\ 0.2 & 6 & \text { FRANCE }\end{array}$

0.26 FRANCE. ML 2.8 (GEN).

$\begin{array}{lll}0.3 & 11 & \text { POLAND. MI } 2.0 \text { (MOX). } \\ 1.0 & 33 & \text { IRIAN JAYA, INDONESIA }\end{array}$

84 HAIMAHERA, INDONESIA. MW 5.6 (GS), 5.5 (HRV).

Moment Tensor (GS): Dep 33; Principal axes (scale 10**17

$\mathrm{Nm}$ ): (T) Val-3.27, Plg-75, Azm-149; (N) Val-0.00, Plg-13, Azm-297; (P) Val--3.27, Plg-8, Azm-29; Best double couple: Mo-3.3*10*17 Nm; NP1: Strike-133, Dip-39, Slip=110; NP2: Strike-288, Dip-54, Slip-74

Centroid, Moment Tensor (HRV): Centroid origin time

$05: 03: 38.1$; Lat $3.22 \mathrm{~N}$; Ion $128.24 \mathrm{E}$; Dep $40.0 \mathrm{Bdy}$; Halfduration $1.4 \mathrm{sec}$; Principal axes (scale $10 * 17 \mathrm{Nm}$ ): (T) Val-2.41, Plg-79, Azm-57; (N) Val--0.24, Plg-10, Azm-259; (P) Val--2.17, Plg-4, Azm-168; Best double couple: Mo-2.3*10**17 Nm; NP1: Strike-248, Dip-42, Slip-75; NP2: Strike-88, Dip-50, Slip-103.

NORTHERN ITALY. ML 1.9 (GEN).

$\begin{array}{rrl}0.1 & 6 & \text { NORTHERN ITALY } \\ 0.8 & 16 & \text { KYOSHO, JAPAN }\end{array}$

0.35 FRANCE. ML 2.7 (GEN)

1.017 SOUTH OF HONSHO, JAPAN

$1.5 \quad 11$ XIZANG

0.811 NEAR EAST COAST OF KAMCHATKA

1.38 KURIL ISLANDS

$1.3 \quad 15$ SULU SEA

1.030 KURIL ISIANDS

SOUTHERN ALASKA. LAEIC>.

SPAIN. mbLg 3.3 (MDD). Felt (III) in the carboneras area. SANTA CRUZ ISLANDS

MARIANA ISLANDS REGION. MW 5.6 (GS), 5.6 (HRV)

Moment Tensor (GS): Dep 9; Principal axes (scale 10**17 $\mathrm{Nm}$ ) (T) Val-3.04, Plg-28, A2m-319; (N) Val-0.01, Plg-38, Azm=204; (P) Val=-3.04, Plg-39, Azm-74; Best double couple: Mo-3.0*10*17 Nm; NP1: Strike-100, Dip=39, S1ip=-11; NP2: Strike-199, Dip-83, Slip--129.

Centroid, Moment Tensor (BRV): Centroid origin time $11: 56: 45.6$; Lat $15.54 \mathrm{~N}$; Lon $148.35 \mathrm{E}$; Dep $15.0 \mathrm{Fix}$; Halfduration $1.5 \mathrm{sec}$; Principal axes (scale 10**17 $\mathrm{Nm}$ ): (T) val-2.97, Plg-30, Azm-290; (N) Val-0.01, Plg-4, Azm-198; (P) Val--2.96, Plg-60, Azm-101; Best double couple: Mo-3.0*10*17 Nm; NP1: Strike-31, Dip-16, Slip--76; NP2 : Strike=197, Dip=75, Slip=-94.

scalar Moment (PPT): Mo-1.6*10**18 Nm.

0.9244 MARIANA ISIANDS REgION. MW 6.2 (GS) 6.2 (BRV). Me 5.9 (GS) mb 5.8 (BRK).

Broadband Source Parameters (GS): NP1: Strike-315, Dip=55, Slip=140; NP2: Strike=71, Dip=58, Slip=42; Radiated energy $1.7 * 10 * 13 \mathrm{Nm}$

Moment Tensor (GS): Dep 238; Principal axes (scale 10**18 $\mathrm{Nm}$ ): (T) Val-2.47, Plg-51, A2m-284; (N) Val=-0.25, Plg=39, $A z m=103$; (P) Val=-2.22, Plg=1, Azm=193; Best double couplé Mo-2.3*10**18 Nm; NP1: Strike=316, Dip=56, Slip-140; NP2: Strike=71, Dip-57, Slip=41.

Centroid, Moment Tensor (HRV): Centroid origin time $21: 36: 34.8$; Lat $22.02 \mathrm{~N}$; Lon $142.99 \mathrm{E}$; Dep 252.5; Halfduration $3.2 \mathrm{sec}$; Principal axes (scale 10**18 N(m): (T) Val=2.66, Plg=53, Azm=274; (N) Val=-0.06, Plg=37, Azm-95; (P) Val=-2.59, Plg-0, Azm=5; Best double couple: Mo $=2.6 * 10 * 18 \mathrm{Nm}$; NP1: Strike=64, Dip=55, Slip=43; NP2 : Strike=306, Dip=56, Slip=137. 
0. $232234.1836 .950 \mathrm{~N}$ $0 . \quad 23 \quad 32 \quad 38.3 * 22.285 \mathrm{~N}$ $0700 \quad 37 \quad 17.2844 .571 \mathrm{~N}$ $07 \quad 0143 \quad 46.5 \quad 46.684 \mathrm{~N}$ $\begin{array}{llllll}07 & 01 & 54 & 04.5 & 39.775 & N\end{array}$ $\begin{array}{lllllll}07 & 02 & 02 & 08.28 & 37.122 & \mathrm{~N}\end{array}$ $07 \quad 0252 \quad 10.0866 .461 \mathrm{~N}$ $\begin{array}{lllllll}07 & 03 & 45 & 57.1 ? & 15.78 & \mathrm{~N}\end{array}$ $7041329.6 * 18.173 \mathrm{~N}$ $7 \begin{array}{llllll}04 & 16 & 34.3 & 15.555 & \mathrm{~N}\end{array}$ $7041932.2 * 53.180 \mathrm{~N}$ $\begin{array}{lllllll}7 & 04 & 22 & 35.88 & 45.098 \mathrm{~N}\end{array}$ 7 O5 $07 \quad 30.2844 .420 \mathrm{~N}$ $706 \quad 0501.5 * 60.040 \mathrm{~s}$ $\begin{array}{llllll}7 & 06 & 11 & 35.3 & 0.088 & \mathrm{~s}\end{array}$ $\begin{array}{llllll}07 & 06 & 27 & 08.0 & 31.761 & \mathrm{~N}\end{array}$ $\begin{array}{lllllll}07 & 07 & 39 & 03.4 * 36.509 \mathrm{~N}\end{array}$ $\begin{array}{llllll}07 & 07 & 53 & 53.5 ? & 62.02 & \mathrm{~S}\end{array}$

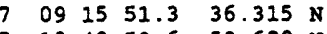
$\begin{array}{llllll}07 & 10 & 49 & 59.6 & 58.620 & \mathrm{~N}\end{array}$
$1.604 \mathrm{~W}$ $45.058 \mathrm{~W}$ $7.182 \mathrm{E}$ $152.563 \mathrm{E}$ $14.850 \mathrm{E}$ $3.882 \mathrm{~W}$ $148.612 \mathrm{~W}$ $148.03 \mathrm{E}$ $68.189 \mathrm{~W}$ $147.730 \mathrm{E}$ $166.114 \mathrm{~W}$ $7.309 \mathrm{E}$ $7.344 \mathrm{E}$ 27.581 W $123.842 \mathrm{E}$ $115.708 \mathrm{~W}$ $70.711 \mathrm{E}$ 56.34 W $70.850 \mathrm{E}$ $157.752 \mathrm{E}$
$10 \mathrm{G}$

$10 \mathrm{G} \quad 3.8$

$10 \mathrm{G}$

$33 \mathrm{~N} 4.0$

343

$10 \mathrm{G}$

$20 \mathrm{G}$

$33 \mathrm{~N} 3.9$

$93 \quad 3.7$

$33 \mathrm{~N} 4.4$

$33 \mathrm{~N} 4.4$

$10 \mathrm{G}$

$10 \mathrm{G}$

$100 \mathrm{G} \quad 4.3$

$65 ? 4.8$

5 G 3.9

200 G 3.8

$33 \mathrm{~N} 4.3$

10 G $5.6 \quad 5.6 \quad 1.9 \quad 20$

Scalar Moment (PPT) : Mo-1.0*10**18 Nm.

0.512 WESTERN MEDITERRANEAN SEA. MbLg 3.0 (MDD)

1.111 NORTHERN MID-ATLANTIC RIDGE

0.58 NORTHERN ITALY. MU 1.9 (GEN).

0.834 KORIL ISLANDS

1.178 TYRRHENIAN SEA

0.46 SPAIN. mbLg 2.2 (MDD)

13 NORTHERN ALASKA. <AIIC). ML 2.6 (AEIC).

1.515 MARIANA ISLANDS REGION

1.121 MONA PASSAGE

1.233 MARIANA ISLANDS REGION

1.336 FOX ISLANDS, ALEUTIAN ISLANDS, ML 4.6 (PMR).

0.99 NORTHERN ITALY. MI 2.3 (GEN).

0.47 NORTHERN ITALY. MI 1.9 (GEN).

1.317 SOUTH SANDWICH ISLANDS REGION

1.149 MINAHASSA PENINSULA, SULAKESI

1.140 BAJA CALIFORNIA, MEXICO

1.313 HINDO KUSH REGION, AFGHANISTAN

20 HINDU KUSH REGION, AFGHANISTAN

KAMCHATKA. MW 6.4 (OBN), 5.8 (GS), 5.8 (HRV). Me 5.7 (GS). MS 5.5 (BRK). Felt (IV) at Magadan and (III) in the palana area.

Broadband Source Parameters (GS): Dep 6; NP1: Strike-215, Dip-40, Slip-140； NP2: Strike-338, Dip=66, Slip=57;

Radiated energy $8,2 * 10 * 12 \mathrm{Nm}$

Moment Tensor (GS): Dep 7; Principal axes (scale 10*17 Nm): (T) Val=5.17, Plg=60, Azm=197; (N) Val=-0.22, Plg=27, Azm=351; (P) Val--4.95, Plg-11, Azm-87; Best double couple: Mo-5.1*10**17 Nin; NP1: Strike=207, Dip-42, Slip-134; NP2: Strike=335, Dip $=61$, Slip=58.

Centroid, Moment Tensor (HRV): Centroid origin time $10: 50: 05.3$; Lat $58.47 \mathrm{~N}$; Lon $157.85 \mathrm{E}$; Dep $15.0 \mathrm{Bdy}$; Half duration $1.8 \mathrm{sec}$; Principal axes (scale 10*17 $\mathrm{Nm}$ ): (T)

Val=4.07, Plg=44, Azm=185; (N) Val=1.60, Plg=46, $A z m=4 ;$ (P)

Val $=-5.67, P I g=1, A z m=95$; Best double couple: Mo-4.9*10*17 Nm; NP1: Strike-221, Dip=60, Slip=146; NP2: Strike-329, Dip $=61$, slip -35 .

scalar Moment $(O B N)$ : MO-4.9*10**18 Nm.

$071055 \quad 23.1 * 58.642 \mathrm{~N}$

$07112829.6 * 41.976 \mathrm{~s}$

$07 \quad 1135 \quad 30.3$ ? $18.60 \mathrm{~s}$

$07 \quad 130523.6844 .357 \mathrm{~N}$

$\begin{array}{llllll}07 & 13 & 35 & 46.2 & 54.785 & \mathrm{~N}\end{array}$

$\begin{array}{lllllll}07 & 14 & 05 & 44.6 & 37.278 & \mathrm{~N}\end{array}$

$\begin{array}{lllllll}07 & 14 & 07 & 44.08 & 38.027 & \mathrm{~N}\end{array}$

$07 \quad 14 \quad 23 \quad 38.3$ ? $63.65 \quad \mathrm{~N}$

$\begin{array}{llllll}07 & 14 & 34 & 46.4 & 15.691 & \mathrm{~N}\end{array}$

$\begin{array}{lllll}07 & 15 & 12 & 11.1 ? \quad 62.50 \mathrm{~s}\end{array}$

$07 \quad 153154.0 * 31.701 \mathrm{~N}$

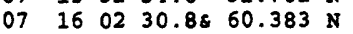

$07 \quad 1603 \quad 15.1 * 38.844 \mathrm{~N}$

$07 \quad 16 \quad 43 \quad 32.7 ? \quad 1.80 \mathrm{~N}$

$\begin{array}{llllll}07 & 16 & 47 & 43.46 & 60.016 & N\end{array}$

$\begin{array}{lllll}07 & 18 & 06 & 56.6 & 35.480\end{array}$

$\begin{array}{lllllll}07 & 19 & 24 & 17.5 ? & 18.50 \quad \mathrm{~S}\end{array}$

$0721 \quad 1731.9$ ? $6.49 \mathrm{~s}$

$07215304.6 * 51.602 \mathrm{~N}$

$\begin{array}{llllll}07 & 22 & 03 & 58.3 & 15.540 \mathrm{~N}\end{array}$

$07 \quad 22 \quad 53 \quad 57.2$ ? $1.92 \mathrm{~S}$

$072323 \quad 47.5 * 58.680 \mathrm{~N}$

$07234649.0 * 13.037 \mathrm{~N}$

$\begin{array}{llllllll}08 & 00 & 18 & 36.2 & 30.050 \mathrm{~N}\end{array}$

$08 \quad 0053 \quad 59.9 ? 34.39 \mathrm{~S}$

$08012307.5 * 51.177 \mathrm{~N}$

08 01 32 35.5 $23.763 \mathrm{~N}$

$08014600.0 * 42.656 \mathrm{~s}$

$08023256.0 ? 50.81 \mathrm{~N}$

$08031602.8 * 43.104 \mathrm{~N}$

$\begin{array}{llllll}08 & 04 & 15 & 35.6 & 37.976 \mathrm{~N}\end{array}$

$\begin{array}{lllllll}08 & 04 & 35 & 20.1 ? \quad 43.12 & \mathrm{~s}\end{array}$

$\begin{array}{llllll}08 & 04 & 42 & 53.38 & 39.570 \mathrm{~N}\end{array}$

$080503 \quad 23.2 * 23.443 \mathrm{~s}$

$080552 \quad 31.4 * 57.762 \mathrm{~N}$

$\begin{array}{llllllll}08 & 06 & 07 & 43.08 & 59.400 & \mathrm{~N}\end{array}$

$08061826.5 * 51.045 \mathrm{~N}$

$08 \quad 0730 \quad 27.3$ ? 14.81 s

$\begin{array}{llllll}08 & 07 & 49 & 31.28 & 60.370 \mathrm{~N}\end{array}$

$08093055.1 * 41.931 \mathrm{~N}$

$08093056.6 * 15.165 \mathrm{~s}$

$\begin{array}{llllll}08 & 10 & 22 & 29.4 & 16.325 & \mathrm{~N}\end{array}$

$081106 \quad 16.5 * 36.860 \mathrm{~N}$

$\begin{array}{llllll}08 & 11 & 33 & 50.1 & 20.701 & \mathrm{~s}\end{array}$

$\begin{array}{llllll}08 & 11 & 40 & 38.2 & 21.509 & \mathrm{~N}\end{array}$

$08132950.5 * 58.785 \mathrm{~N}$

$\begin{array}{lllllll}08 & 14 & 03 & 46.8 \text { ? } & 6.51\end{array}$

$\begin{array}{lllllll}08 & 16 & 03 & 33.7 ? & 37.57 & N\end{array}$

$08165603.1 * 7.972 \mathrm{~s}$

$\begin{array}{lllllll}08 & 17 & 32 & 19.58 & 33.107 \mathrm{~s}\end{array}$

$081855 \quad 50.5$ ? $20.08 \mathrm{~S}$

$\begin{array}{llllll}08 & 19 & 53 & 50.1 & 42.996\end{array}$

$\begin{array}{lllllll}08 & 20 & 06 & 44.58 & 34.042 & \mathrm{~N} \\ 08 & 20 & 08 & 55.88 & 65.130 & \mathrm{~N}\end{array}$
$157.229 \mathrm{E}$ $84.445 \mathrm{E}$ 72.23 $6.268 \mathrm{E}$

$162.591 \mathrm{E}$ $139.876 \mathrm{E}$ $4.008 \mathrm{~W}$ 159.54 พ $147.693 \mathrm{E}$ 55.50 W $115.684 \mathrm{~W}$ $152.966 \mathrm{~W}$ $94.142 \mathrm{E}$ 127.02 $\begin{array}{r}22.755 \\ \hline\end{array}$ 175.48 $147.71 \mathrm{E}$ $16.506 \mathrm{E}$ $147.688 \mathrm{E}$ 128.08 E $157.899 \mathrm{E}$ $88.275 \mathrm{~W}$ $88.155 \mathrm{E}$ $71.16 \mathrm{~W}$ $177.473 \mathrm{~W}$ $122.889 \mathrm{E}$ $42.257 \mathrm{E}$ $178.99 \mathrm{~W}$ $47.174 \mathrm{E}$ $140.130 \mathrm{E}$ 122.31 $66.456 \mathrm{~W}$ $156.360 \mathrm{~W}$ $152.890 \mathrm{~W}$ $177.682 \mathrm{~W}$ $268.28 \mathrm{E}$ $147.250 \mathrm{~W}$ $142.517 \mathrm{E}$ $167.369 \mathrm{E}$ 61.545 71.503 $94.853 \mathrm{E}$
$157.730 \mathrm{E}$ $157.730 \mathrm{E}$ $150.27 \mathrm{E}$ 8.51 W $127.802 \mathrm{E}$ $70.301 \mathrm{~K}$ 177.85 W $14.289 \mathrm{E}$ $139.318 \mathrm{E}$
$148.590 \mathrm{~W}$
$10 \mathrm{G} 4.4$ $10 \mathrm{G} 4.6$ $33 \mathrm{~N} 4.4$ 5 G $33 \mathrm{~N} 4.0$ $10 \mathrm{G}$ $10 \mathrm{G}$ $10 \mathrm{G}$ $33 \mathrm{~N} 4.2$ $10 \mathrm{G} 4.2$ 5 G 137 $33 \mathrm{~N} 4.2$ $100 \mathrm{G} 4.3$ $138 \quad 3.6$ $33 \mathrm{~N} 4.3$ $250 \mathrm{G} 4.2$ $63 * 4.1$

$5 \mathrm{G}$ $33 \times 4.7$ $33 \mathrm{~N} 4.6$ $10 \mathrm{G} 4.1$ $33 \mathrm{~N} 4.3$

$33 \times 4.5$ 706

$33 \mathrm{~N} 4.0$ $33 \mathrm{~N} 4.3$ $10 \mathrm{G} 4.0$

$33 \mathrm{~N} 4.0$ $33 \mathrm{~N} 3.7$ $138 \quad 4.1$ $10 \mathrm{G} \quad 4.3$ 23

$200 \mathrm{G} 3.6$

$33 \mathrm{~N} 3.4$

$33 \mathrm{~N} 3.9$

$33 \mathrm{~N} 4.4$

143.2

$57 * 3.6$

$200 \mathrm{G} \quad 4.5$

$33 \mathrm{~N}$
147.3 .8

500 G 4.2

$102 \mathrm{D} 5.1$

$10 \mathrm{G} 4.0$

$33 \mathrm{~N} 3.9$

$10 \mathrm{G}$

$33 \mathrm{~N} 4.7$

$110 \mathrm{G}$

500 G 4.0

$10 \mathrm{G}$

$10 \mathrm{G}$ 13

1.0

1.2 21

1.1

0.4

0.915

0.3

0.8

0.6

0.9

0.8

1.0

1.013

$.2 \quad 32$

$1.3 \quad 13$

$1.4 \quad 11$

$1.4 \quad 10$

$\begin{array}{ll}1.2 & 51 \\ 1.8 & 23\end{array}$

0.7

1.123

1.0

0.2

0.9

1.1

1.0

1.1

1.2
1.5

1.0

1.2

1.2 


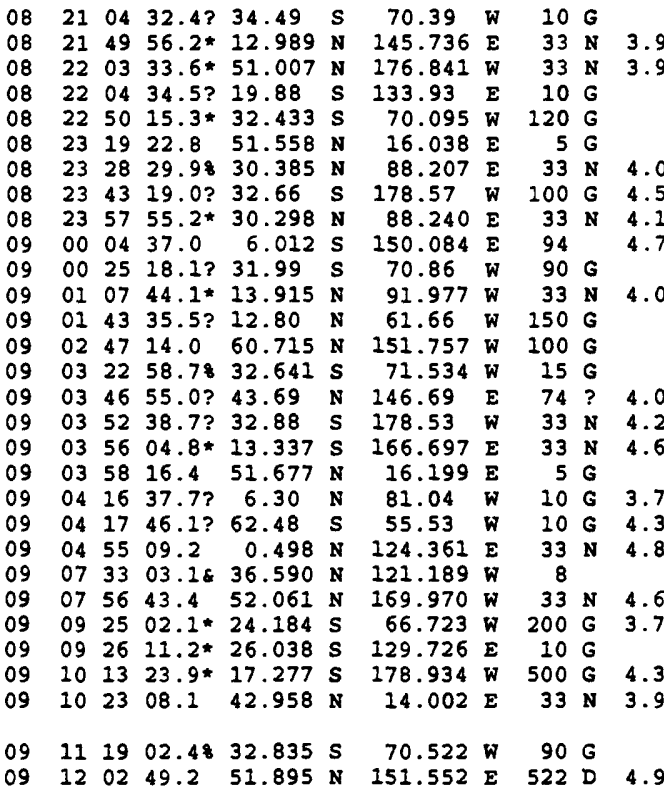

$\begin{array}{lllllll}12 & 29 & 10.1 * & 51.050 & \mathrm{~N} & 176.788 & \mathrm{~W} \\ 12 & 36 & 03.5 * & 36.328 & \mathrm{~N} & 1.811 & \mathrm{E} \\ 12 & 57 & 57.0 * & 50.967 & \mathrm{~N} & 176.718 & \mathrm{~W} \\ 13 & 04 & 23.9 ? & 33.16 & \mathrm{~S} & 179.88 & \mathrm{E} \\ 14 & 41 & 59.6 ? & 79.42 & \mathrm{~N} & 5.49 & \mathrm{E} \\ 15 & 24 & 59.4 ? & 19.34 & \mathrm{~S} & 178.00 & \mathrm{~W} \\ 16 & 40 & 23.3 ? & 13.86 & \mathrm{~N} & 92.24 & \mathrm{~W} \\ 17 & 44 & 38.4 * & 29.552 & \mathrm{~N} & 141.196 & \mathrm{E} \\ 18 & 35 & 30.4 & 34.123 & \mathrm{~N} & 138.529 & \mathrm{E} \\ 19 & 22 & 28.2 * & 6.436 & \mathrm{~S} & 154.970 & \mathrm{E} \\ 20 & 10 & 16.5 * & 51.174 & \mathrm{~N} & 177.307 & \mathrm{~W} \\ 21 & 29 & 31.08 & 37.211 & \mathrm{~N} & 3.739 & \mathrm{~W} \\ 21 & 33 & 29.6 ? & 29.94 & \mathrm{~S} & 179.88 & \mathrm{E} \\ 21 & 36 & 23.9 ? & 41.88 & \mathrm{~S} & 172.49 & \mathrm{E} \\ 21 & 58 & 30.0 * & 62.210 & \mathrm{~N} & 150.860 & \mathrm{~W} \\ 22 & 19 & 12.37 & 4.61 & \mathrm{~S} & 134.21 & \mathrm{E} \\ 22 & 35 & 53.2 & 51.630 & \mathrm{~N} & 16.374 & \mathrm{E} \\ 22 & 49 & 59.8 * & 11.939 & \mathrm{~S} & 166.456 & \mathrm{E} \\ 23 & 06 & 42.6 * & 17.682 & \mathrm{~S} & 178.702 & \mathrm{~W} \\ 00 & 04 & 19.7 * & 51.184 & \mathrm{~N} & 177.040 & \mathrm{~W} \\ 00 & 14 & 34.0 * & 32.030 & \mathrm{~S} & 71.449 & \mathrm{~W} \\ 00 & 32 & 45.68 & 33.636 & \mathrm{~S} & 71.607 & \mathrm{~W} \\ 00 & 49 & 26.4 & 4.267 & \mathrm{~S} & 102.819 & \mathrm{E}\end{array}$

$33 \mathrm{~N} 4.0$

$10 \mathrm{G} \quad 3.7$

$33 \mathrm{~N} 3.8$

$200 \mathrm{G} 4.1$

$10 \mathrm{G} \quad 3.5$

$600 \mathrm{G} 4.3$

$33 \mathrm{~N} 4.5$

$33 \mathrm{~N} 4.3$

$242 \quad 4.2$

$33 \mathrm{~N} 4.1$

$33 \mathrm{~N} 3.6$

$10 \mathrm{G}$

$500 \mathrm{G} \quad 4.1$

$100 \mathrm{G} \quad 3.8$

70

$33 \mathrm{~N} 4.3$

56

$158 \mathrm{D} \quad 4.4$

$600 \mathrm{G} \quad 4.3$

$33 \mathrm{~N} 4.2$

$33 \mathrm{~N}$

$33 \mathrm{~N}$

$109 ? 5.1$
6 CHILE-ARGENTINA BORDER REGION. MD 3.0 (SAN).

0.78 SOUTH OF MARIANA ISLANDS

1.214 ANDREANOF ISLANDS, ALEUTIAN IS.

0.96 NORTHERN TERRITORY, AUSTRALIA

0.413 CHILE-ARGENTINA BORDER REGION. MD 3.8 (SAN).

0.612 POLAND. ML 2.9 (MOX).

1.512 XIZANG

1.415 SOUTH OF KERMADEC ISLANDS

1.316 XIZANG

1.241 NEW BRITAIN REGION, P.N.G.

0.410 CHILE-ARGENTINA BORDER REGION. MD 3.1 (SAN).

1.118 NEAR COAST OF GUATEMALA

0.16 WINDWARD ISLANDS. MD 3.6 (TRN).

0.611 KENAI PENINSOLA, ALASKA

0.611 NEAR COAST OF CENTRAL CHILE. MD 4.2 (SAN).

1.312 KURIL ISIAANDS

1.38 SOUTH OF KERMADEC ISLANDS

1.125 VANOATO ISLANDS

1.021 POLAND. MI 3.5 (VIE), 3.3 (MOX).

1.211 SOOTH OF PANAYA

$1.3 \quad 12$ SOUTHWESTERN ATLANTIC OCEAN

1.031 MINAHASSA PENINSOLA, SULAKESI

27 CENTRAL CALIFORNIA. 〈GM-P>. MD 2.9 (GM). MI 2.8 (GS).

1.138 FOX ISLANDS, ALEUTIAN ISLANDS

1.014 SALTA PROVINCE, ARGENTINA

1.36 SOUTH AUSTRALIA

1.024 FIJI ISLANDS REGION

1.375 CENTRAL ITALY. YI 4.5 (VIE). Felt (VI) in the epicentral area.

0.310 CHILE-ARGENTINA BORDER REGION. MD 2.8 (SAN)

0.8174 SEA OF OKHOTSK. MW 5.1 (HRV).

Centroid, Noment Tensor (HRV): Centroid origin time

$12: 02: 51.5$; Lat $51.59 \mathrm{~N}$; Lon $151.58 \mathrm{E}$; Dep 516.9 ; Halfduration $1.0 \mathrm{sec}$; Principal axes (scale $10 * 16 \mathrm{Nm}$ ): (T) Val-4.36, Plg=15, Azm=212; (N) Val=0.89, Plg=12, Azm=305; (P) Val=-5.25, Plg=71, Azm-74; Best double couple: Mo-4.8*10*16 $\mathrm{Nm}$; NP1: Strike-285, Dip-32, Slip=-114; NP2: Strike-132, Dip-61, S1ip=-76.

1.325 ANDREANOF ISLANDS, ALEOTIAN IS.

1.225 NORTHERN ALGERIA. mbLg 3.4 (MDD).

0.85 ANDREANOF ISLANDS, ALEUTIAN IS.

1.318 SOUTH OF KERMADEC ISLANDS

1.710 SVALBARD REGION

0.714 FIJI ISLANDS REGION

1.07 OFF COAST OF CHIAPAS, MEXICO

1.017 SOUTH OF HONSHO, JAPAN

1.129 NEAR S. COAST OF HONSHO, JAPAN

0.913 SOLOYON ISLANDS

1.16 ANDREANOF ISLANDS, ALEUTIAN IS.

$0.8 \quad 8$ SPAIN. MbLg 2.3 (YDD).

$1.0 \quad 15$ KERMADEC ISLANDS REGION

1.68 SOUTH ISLAND, NEW ZEALAND

83 CENTRAL ALASKA. <AEIC>

1.610 IRIAN JAYA REGION, INDONESIA

$0.8 \quad 18$ POLAND. ML 3.5 (VIE), 3.0 (MOX).

0.928 SANTA CRDZ ISLANDS

0.939 FIJI ISLANDS REGION

1.129 ANDREANOF ISLANDS, ALEOTIAN IS. ML 4.6 (PMR)

0.412 NEAR COAST OF CENTRAL CHILE. MD 4.5 (SAN).

0.410 NEAR COAST OF CENTRAL CHILE. MD 3.0 (SAN).

1.161 SOUTHERN SUMATERA, INDONESIA. NW 5.1 (HRV).

Centroid, Moment Tensor (HRV): Centroid origin time

$00: 49: 30.1$; Lat $4.48 \mathrm{~s}$; Lon $102.82 \mathrm{E}$; Dep 66.1 ; Half-

duration 1.0 sec; Principal axes (scale $10 * 16 \mathrm{Nm}$ ): (T) Val=4.90, Plg=69, Azm=42; (N) Val=-0.51, Plg=6, Azm=295; (P) Val=-4.39, Plg=20, Azm=202; Best double couple:

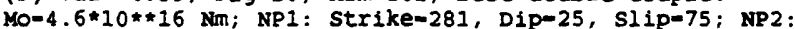
Strike-118, Dip $=65$, Slip $=97$.

0.616 ANDREANOF ISLANDS, ALEUTIAN IS.

1.420 VOLCANO ISLANDS REGION

0.921 NORTHERN MOLUCCA SEA

0.210 CHILE-ARGENTINA BORDER REGION

1.171 ANDREANOF ISLANDS, ALEUTIAN IS. MIL 4.7 (PMR).

0.913 IRIAN JAYA REGION, INDONESIA

1.034 MINDANAO, PHILIPPINE ISLANDS

0.74 EASTERN HONSEU, JAPAN

0.45 EASTERN HONSEU, JAPAN

$0.8 \quad 6$ KYOSHO, JAPAN

1.036 GREECE-ALBANIA BORDER REGION. Felt (V) in the Leskovik area, Albania.

0.726 NEAR EAST COAST OF KAMCHATKA

FOX ISLANDS, ALEUTIAN ISLIANDS. MW 5.9 (HRV). ML 5.9 (PMR). Centroid, Moment Tensor (HRV): Centroid origin time

05:48:24.9; Lat 52.09 N; Lon $171.01 \mathrm{~W}$; Dep 32.0 Bdy; Halfduration $2.1 \mathrm{sec}$; Principal axes (scale 10**17 Nm): (T)

$\mathrm{Val}=6.63, \mathrm{Plg}=71, \mathrm{Azm}=322$; (N) Val=0.82, Plg=4, Azm=63; (P)

Val=-7.46, PIg-19, Azm=154; Best double couple:

Mo-7.1*10*17 Nm; NP1: Strike-250, Dip-26, Slip-98; NP2

Strike-61, Dip=64, Slip-86.

0.99 NEAR COAST OF CENTRAL CEILE

1.19 FOX ISLANDS, ALEUTIAN ISLANDS

0.3 IO NEAR COAST OF CENTRAL CHILE $\begin{array}{lll}0.3 & 10 & \text { NEAR COAST OF CEN } \\ 0.8 & 75 & \text { SEA OF OKHOTSK }\end{array}$ $\begin{array}{lllllllllll}10 & 05 & 48 & 20.9 ? & 33.90 & \mathrm{~S} & 71.71 & \mathrm{~W} & 40 & \mathrm{G} & \\ 10 & 06 & 00 & 32.7 * & 52.285 & \mathrm{~N} & 171.073 & \mathrm{~W} & 33 & \mathrm{~N} & 3.3 \\ 10 & 06 & 17 & 51.88 & 33.668 & \mathrm{~S} & 71.425 & \mathrm{~W} & 40 & \mathrm{G} & \end{array}$

$10 \quad 06 \begin{array}{lllllllll} & 53.9 & 51.906 & \mathrm{~N} & 151.479 & \mathrm{E} & 507 & \mathrm{D} & 4.4\end{array}$ 
24 FOX ISLANDS, ALEUTIAN ISLANDS

38 KAMCHATKA

10 DODECANESE ISLANDS

27 NORTHERN MOLUCCA SEA

42 FOX ISLANDS, ALEUTIAN ISLAANDS

7 SOUTHERN MOLUCCA SEA

29 KORIL ISLANDS

8 FOX ISLANDS, ALEUTIAN ISLANDS

8 GERMANY

7 JUJUY PROVINCE, ARGENTINA

13 NEW BRITAIN REGION, P.N.G.

11 POLAND. MI 3.3 (VIE), 2.9 (MOX).

32 NEAR S. COAST OF HONSHO, JAPAN

12 FOX ISLANDS, ALEUTIAN ISLANDS

9 NEPAL

16 NORTHERN MOLUCCA SEA

10 SEA OF OKHOTSK

23 NEAR NORTH COAST OF IRIAN JAYA

5 STRAIT OF GIBRALTAR. mbLg 3.3 (YDD).

12 AFGHANISTAN-TAJIKISTAN BORD REG.

9 YELLOWSTONE REGION, WYOMING. ML 3.1 (GS).

10 CHILE-ARGENTINA BORDER REGION

103 SOUTHERN ALASKA. <AEIC>.

14 KORIL ISLANDS

2 EAST CENTRAL PACIFIC OCEAN

22 KORIL ISLANDS

13 SALTA PROVINCE, ARGENTINA

14 CHILE-BOLIVIA BORDER REGION

19 KORIL ISLANDS

23 NEAR COAST OF NORTHERN CAIIF. $\langle G M-P\rangle$. ND 3.0 (GM).

SOUTH OF JAWA, INDONESIA

8 BANDA SEA

42 ALASKA PENINSULA. <AEIC>.

21 OFF COAST OF CENTRAL AMERICA

12 NEW IRELAND REGION, P.N.G.

15 KURIL ISLANDS

11 NEAR COAST OF PERU

19 NORTHWESTERN CADCASUS. Felt (IV) at Myskhako and

Novorossiysk; (III) at Abrau-Dyurso and Verkhnebakanskiy; (II) at Anapa.

19 MINAHASSA PENINSULA, SULAKESI

19 SAKBALIN ISLAND

18 NEAR S. COAST OF HONSHO, JAPAN

45 NORTHERN ITALY

5 HALMAHERA, INDONESIA

10 MINDANAO, PHILIPPINE ISLANDS

10 WEST OF MACQUARIE ISLAND

28 KURIL ISLANDS

49 CENTRAI CAIIFORNIA. 〈GM-P>. MD 3.2 (GM). MI 3.0 (GS).

ANDREANOF ISLANDS, ALEUTIAN IS.

18 OFF EAST COAST OF HONSHO, JAPAN

20 XIZANG

28 TONGA ISLANDS

11 XIZANG

23 NEAR EAST COAST OF HONSHU, JAPAN

12 XIZANG

15 ANDREANOF ISLANDS, ALEUTIAN IS.

14 NEW BRITAIN REGION, P.N.G.

17 JUJUY PROVINCE, ARGENTINA

103 KURIL ISLANDS. MW 5.2 (HRV).

Centroid, Moment Tensor (HRV): Centroid origin time 08:50:05.0; Lat $45.36 \mathrm{~N}$; Lon $151.18 \mathrm{E}$; Dep 49.5; Halfduration $1.0 \mathrm{sec}$; Principal axes (scale $10 \star \star 16 \mathrm{Nm}$ ): (T) Val-5.71, Plg=66, Azm=257; (N) Val=0.79, $1 \mathrm{~g}=19$, Azm-39. (P) Val-6.50, Plg-14, Azm-134; Best double couple: Mo-6.1*10*16 Nm; NP1: Strike-249, Dip-35, S1ip-125; NP2: Strike-28, Dip-62, Slip=68.

\begin{abstract}
092030.3 ? $19.78 \mathrm{~s}$ $\begin{array}{rrrrrr}10 & 10 & 35.5 * 26.735 \mathrm{~N} \\ 10 & 17 & 34.7 & 8.984 \mathrm{~N}\end{array}$ $1154 \quad 31.5 \quad 30.066 \mathrm{~N}$ $123455.88 \quad 32.810 \mathrm{~N}$ $123838.2 * 27.007 \mathrm{~N}$ $\begin{array}{llllll}13 & 01 & 45.47 & 28.12 & N \\ 13 & 06 & 21.9 & 35.936 & N\end{array}$ $133110.0 * 22.034 \mathrm{~s}$ $142356.930 .389 \mathrm{~N}$ $\begin{array}{lllll}15 & 19 & 23.58 & 33.009 & 5\end{array}$ $\begin{array}{rrrr}15 & 43 & 44.6 * 4.686 \mathrm{~N} \\ 17 & 33 & 07.8 * 25.986 \mathrm{~s}\end{array}$

$175310.6 * 24.491 \mathrm{~s}$ $\begin{array}{llll}18 & 49 & 04.3 & 31.82\end{array}$ $\begin{array}{llllll}19 & 03 & 37.0 & 63.262 & \mathrm{~N}\end{array}$ $\begin{array}{llll}19 & 27 & 00.9 & 31.781 \mathrm{~S}\end{array}$ $195228.3 * 14.399 \mathrm{~N}$ $\begin{array}{llllll}20 & 03 & 50.9 ? & 20.91 \mathrm{~S}\end{array}$ $\begin{array}{ccccc}20 & 06 & 30.6 ? & 17.75 & \mathrm{~S} \\ 20 & 42 & 03.3 * & 2.832 & \mathrm{~N}\end{array}$ $204808.8 * 31.806 \mathrm{~s}$ $222353.5 * 21.1335$ $\begin{array}{llll}22 & 29 & 32.7 ? & 32.41 \mathrm{~s}\end{array}$
\end{abstract}

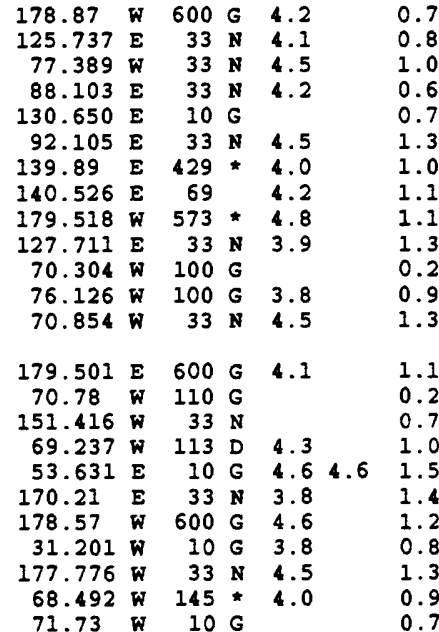

12 FIJI ISLANDS REGION

10 NORTHEAST OF TAIWAN

31 PANAMA-COLOMBIA BORDER REGION

23 XIZANG

5 KYUSHU, JAPAN

25 EASTERN XIZANG-INDIA BORDER REG

16 BONIN ISLANDS REGION

31 NEAR EAST COAST OF HONSHO, JAPAN

44 SOUTH OF FIJI ISLANDS

16 NORTHWEST OF RYOKYO ISLANDS

10 CHILE-ARGENTINA BORDER REGION. MD 2.2 (SAN).

13 COLOMBIA

24 NEAR COAST OF NORTHERN CHILE. Felt (III) at Chanaral and Copiapo: (II) at Diego de Almagro and Tierra Amarilla.

23 SOUTH OF FIJI ISLANDS

11 CEILE-ARGENTINA BORDER REGION. MD 4.0 (SAN).

10 CENTRAL ALASKA. ML 3.1 (PMR).

40 SAN JUAN PROVINCE, ARGENTINA. MD 4.6 (SAN).

48 ARABIAN SEA

10 VANUATO ISLANDS

21 FIJI ISLANDS REGION

11 CENTRAL MID-ATLANTIC RIDGE

26 KERMADEC ISLANDS REGION

16 CHILE-BOLIVIA BORDER REGION

11 NEAR COAST OF CENTRAL CHILE. MD 3.8 (SAN). 


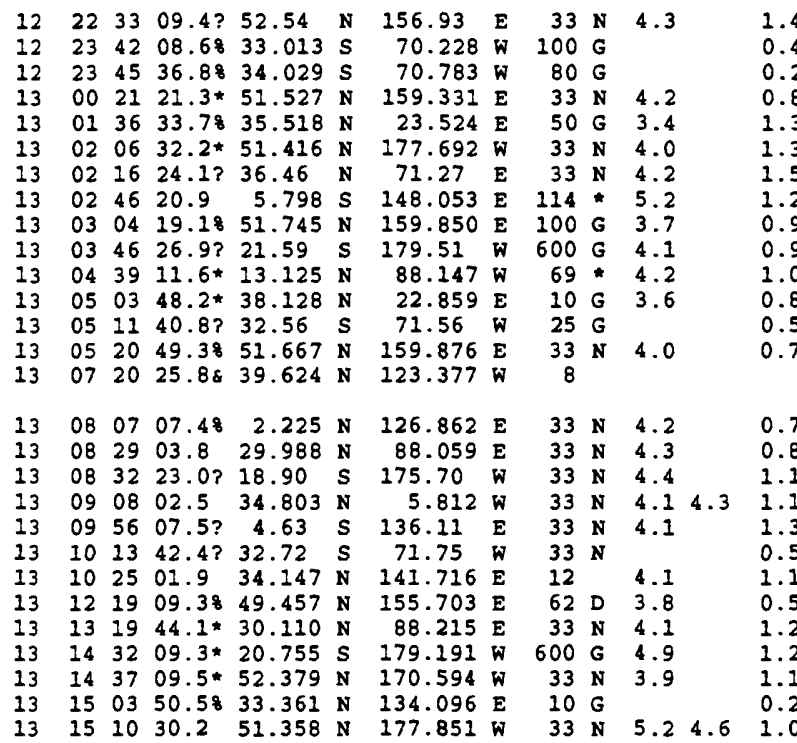

$\begin{array}{lllll}13 & 15 & 32 & 01.5 * 6.966 \mathrm{~s}\end{array}$

$\begin{array}{llllll}13 & 15 & 57 & 07.3 ? & 5.01 & \mathrm{~s}\end{array}$ $\begin{array}{lllllll}3 & 17 & 36 & 18.5 ? & 31.61 & \mathrm{~S}\end{array}$ $3 \quad 1903 \quad 43.6 * 7.231 \mathrm{~N}$ 3 I9 $0932.9 * 55.997 \mathrm{~N}$ $200505.5 * 7.146 \mathrm{~N}$ $200950.4 * 51.181 \mathrm{~N}$ $\begin{array}{llll}20 & 29 & 25.3 ? & 14.08 \mathrm{~N}\end{array}$ $\begin{array}{rrrrr}21 & 55 & 32.1 & 0.083 \mathrm{~N} \\ 22 & 04 & 38.5 * & 36.468\end{array}$ $\begin{array}{lllll}22 & 15 & 42.48 & 32.991 \mathrm{~S}\end{array}$ $\begin{array}{lllll}22 & 34 & 15.9 & 64.600 \mathrm{~N}\end{array}$ $\begin{array}{lllll}00 & 40 & 06.1 & 32.627 & \mathrm{~N}\end{array}$ $\begin{array}{llllll}02 & 03 & 53.6 ? & 32.56 \quad \mathrm{~S}\end{array}$ $\begin{array}{llllll}03 & 03 & 05.5 \& & 40.490 \mathrm{~N}\end{array}$ $045952.88 \quad 34.576 \mathrm{~s}$ $\begin{array}{llllll}05 & 03 & 38.78 & 34.300 \mathrm{~s}\end{array}$ $\begin{array}{lll}05 & 15 & 01.4 \text { ? } 35.24 \mathrm{~N}\end{array}$ $052126.1 * 36.796 \mathrm{~N}$ $\begin{array}{llllll}06 & 10 & 29.88 & 24.329 & \mathrm{~N}\end{array}$ $070021.0 ? 31.07 \mathrm{~s}$ $070940.6 ? 33.44$ N $072738.6 * 29.207 \mathrm{~N}$ $\begin{array}{llllll}08 & 59 & 16.6 ? & 3.87 & \mathrm{~N}\end{array}$ $\begin{array}{llll}09 & 01 & 53.3 & 3.613 \mathrm{~N}\end{array}$ $112539.6 * 55.312 \mathrm{~S}$ $132138.8 * 15.175 \mathrm{~S}$ $\begin{array}{lllll}13 & 29 & 58.9 ? & 23.62 & \mathrm{~S}\end{array}$ $\begin{array}{llllll}14 & 54 & 41.7 ? & 12.97 \mathrm{~N}\end{array}$ $\begin{array}{llllll}15 & 00 & 15.38 & 34.306 \mathrm{~S}\end{array}$ $\begin{array}{llllll}15 & 01 & 16.0 ? & 11.99 \mathrm{~N}\end{array}$ $\begin{array}{llll}15 & 02 & 06.2 * 11.840 \mathrm{~N}\end{array}$ $\begin{array}{lllll}15 & 15 & 14.38 & 40.259 \mathrm{~N}\end{array}$ $\begin{array}{llll}15 & 17 & 51.48 & 34.271 \mathrm{~s}\end{array}$ $\begin{array}{llllll}15 & 43 & 49.2 & 36.342 & \mathrm{~N}\end{array}$ $\begin{array}{llll}17 & 02 & 32.2 ? & 50.96 \mathrm{~N}\end{array}$ $\begin{array}{lllll}17 & 07 & 13.9 & 51.093 \mathrm{~N}\end{array}$
$13191221.5 * 56.071 \mathrm{~N}$

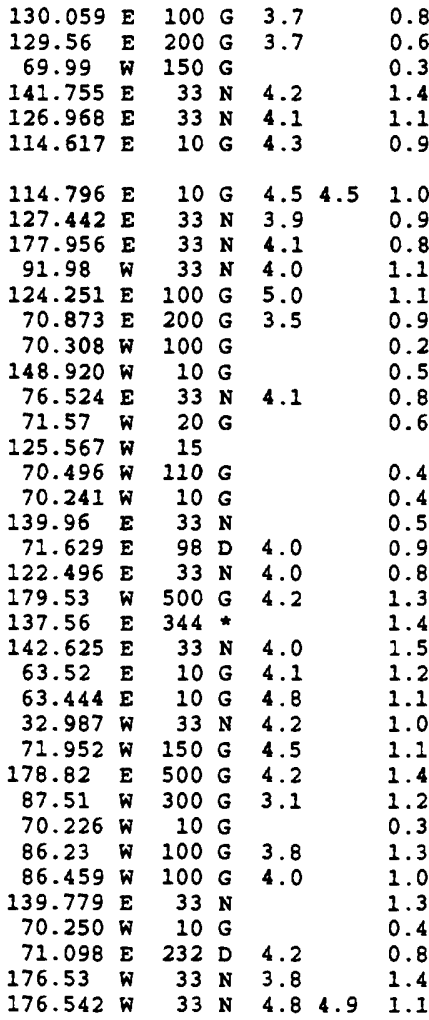

13 KAMCHATKA

0.4 10 CEILE-ARGENTINA BORDER REGION. MD 2.1 (SAN).

211 CHTLE-ARGENTINA BORDER REGION. MD 3.3 (SAN)

0.818 OFF EAST COAST OF KAMCHATKA

1.3 6 CRETE

117 ANDREANOF ISLANDS, ALEUTIAN IS.

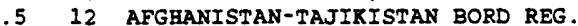

232 NEW BRITAIN REGION, P.N.G

8 OFF EAST COAST OF KAMCHATKA

0.911 FIJI ISLANDS REGION

1.020 EL SALVADOR. Felt (II) at San Salvador.

0.810 GREECE

.510 NEAR COAST OF CENTRAL CHILE. MD 3.6 (SAN).

9 OFF EAST COAST OF KAMCHATKA

25 NEAR COAST OF NORTHERN CALIF. 〈GM-P>. MD 3.0 (GM). MU 3.1 (GS), 3.0 (BRK)

710 NORTHERN MOLUCCA SEA

26 XIZANG

18 TONGA ISLANDS

43 MOROCCO. mbLg 3.8 (MDD).

6 IRIAN JAYA REGION, INDONESIA

NEAR COAST OF CENTRAL CHILE. MD 3.3 (SAN).

22 OFF EAST COAST OF HONSHO, JAPAN

13 KURIL ISLANDS. Felt (II) at Severo-Kurilsk.

18 XIZANG

21 FIJI ISLANDS REGION

17 FOX ISLANDS, ALEUTIAN ISLANDS

7 SHIKOKU, JAPAN

125 ANDREANOF ISLANDS, ALEUTIAN IS. MW 5.2 (HRV). ML 5.3 (PMR). Centroid, Moment Tensor (HRV): Centroid origin time

$15: 10: 32.8$; Lat $51.49 \mathrm{~N}$; Lon $177.67 \mathrm{~W}$; Dep 40.8 ; Halfduration $1.0 \mathrm{sec}$; Principal axes (scale 10*16 Nm): (T) Val=5.09, Plg=61, Azm=243; (N) Val-1.81, Plg=25, Azm=31; (P) Val=-6.90, Plg-13, Azm-127; Best double couple: Mo-6.0*10**16 Nm; NP1: Strike-247, Dip-38, Slip=133; NP2: Strike=17, Dip=63, Slip-62.

BANDA SEA

11 SAN JUAN PROVINCE, ARGENTINA. MD 3.9 (SAN).

15 SOUTH OF HONSEO, JAPAN

MINDANAO, PHILIPPINE ISLANDS

20 EAST OF LARE BAYKAL, RUSSIA. Felt (V) at severomuysk and raksimo; (IV) at Tonnelnyy; (III) at Bodaybo.

22 EAST OF LAKE BAYKAL, ROSSIA

9 PHILIPPINE ISIANDS REGION

10 RAT ISLANDS, ALEUTIAN ISLANDS

12 GUATEMALA

40 MINARASSA PENINSULA, SULANESI

11 HINDU KOSH REGION, AFGHANISTAN

11 CHILE-ARGENTINA BORDER REGION. MD 3.4 (SAN).

CENTRAL ALASKA. ML 3.0 (PMR).

12 KASHMIR-INDIA BORDER REGION

10 NEAR COAST OF CENTRAL CHILE

OFF COAST OF NORTHERN CAIIFORNIA. 〈GM-P>. MD 2.9 (GM).

CEILE-ARGENTINA BORDER REGION

1 CHILE-ARGENTINA BORDER REGION

NEAR S. COAST OF HONSHU, JAPAN

AFGEANISTAN-TAJIKISTAN BORD REG.

TAIWAN REGION

KERMADEC ISILANDS REGION

NEAR S. COAST OF HONSHU, JAPAN

SOUTH OF HONSHO, JAPAN

CARLSBERG RIDGE

CARLSBERG RIDGE

SOUTH GEORGIA ISLAND REGION

SOUTHERN PERU

SOUTH OF FIJI ISLANDS

NEAR COAST OF NICARAGUA

11 CHILE-ARGENTINA BORDER REGION. MD 4.0 (SAN).

12 NEAR COAST OF NICARAGUA

2 NEAR COAST OF NICARAGUA

5 NEAR WEST COAST OF HONSHU, JAPAN

11 CHILE-ARGENTINA BORDER REGION. MD 3.5 (SAN).

34 AFGHANISTAN-TAJIKISTAN BORD REG.

7 ANDREANOF ISLANDS, ALEUTIAN IS.

88 ANDREANOF ISLANDS, ALEUTIAN IS. MW 5.1 (HRV)

Centroid, Moment Tensor (HRV): Centroid origin time

17:07:11.9; Lat $50.92 \mathrm{~N}$; Lon $176.18 \mathrm{~W}$; Dep 26.1; Halfduration $1.0 \mathrm{sec}$; Principal axes (scale 10*16 Nm): (T) Val-5.56, Plg-57, Azm-308; (N) Val--0.15, Plg-10, Azm-53; (P) Val--5.41, Plg=31, Azm-149; Best double couple:

Mo-5.5*10**16 Nm; NP1: Strike-270, Dip=16, Slip-128; NP2:

Strike-5I, Dip-77, Slip-80.

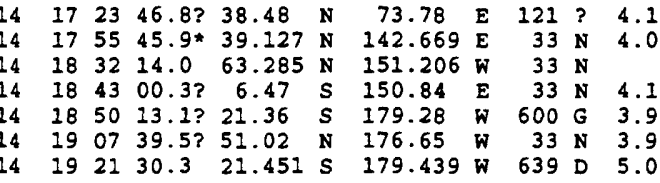

1.110 TAJIKISTAN-XINJIANG BORDER REG

1.216 NEAR EAST COAST OF HONSHO, JAPAN

0.911 CENTRAL ALASKA. ML 3.5 (PMR), 3.2 (AEIC).

1.410 NEW BRITAIN REGION, P.N.G.

1.219 FIJI ISLANDS REGION

1.38 ANDREANOF ISLANDS, ALEUTIAN IS

0.9105 FIJI ISLANDS REGION. NW 5.4 (HRV).

Centroid, Moment Tensor (HRV): Centroid origin time

$19: 21: 33.9$; Lat $21.58 \mathrm{~s}$; Lon 179.22 W; Dep 637.2 ; Half-

duration $1.1 \mathrm{sec}$; Principal axes (scale 10**17 Nm): (T) 
$14 \quad 1950 \quad 01.0 ? 11.60 \mathrm{~s}$ $\begin{array}{lllllll}14 & 22 & 06 & 38.98 & 36.256 & \mathrm{~N}\end{array}$ $14224011.9 * 10.306 \mathrm{~N}$ $15001328.6 \quad 46.015 \mathrm{~N}$

$052801.6 ? \quad 21.89 \mathrm{~N}$ $052859.6 * 3.580 \mathrm{~S}$ $\begin{array}{lllll}06 & 01 & 11.7 & 3.502 \mathrm{~S}\end{array}$ $\begin{array}{lllllll}05 & 46 & 12.7 & 46.052 \mathrm{~N}\end{array}$
$99.22 \mathrm{E}$ 11.873 $6.034 \mathrm{E}$ $12.143 \mathrm{~W}$
$33 \mathrm{~N} 4.3$

$10 \mathrm{G} \quad 4.3$

$5 \mathrm{G}$ $10 G 4.55 .10 .8$
Val-1.29, Plg=72, A2m=125; (N) Val=-0.13, Plg-7, Azm-11 (P) Val--1.16, Plg-16, A2m-279; Best double couple: Mo-1.2*10**17 Nm; NP1: Strike-358, Dip-30, Slip-75; NP2 : Strike-195, Dip-61, S1ip=98.

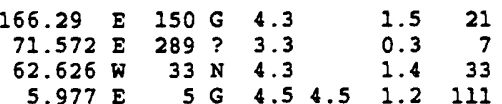

SANTA CRUZ ISLANDS

AFGHANISTAN-TAJIKISTAN BORD REG.

NEAR COAST OF VENEZUELA

FRANCE. mbLg 4.3 (MDD). One person sightly injured and minor damage (VI) at Cruseilles. Felt strongly at Annecy. Also felt at Lyon. Widely felt in the French Alps and southwestern Switzerland.

$33 \mathrm{~N} 4.5 \quad 1.4 \quad 8$ SOUTH OF RERMADEC ISLANDS

$54 \mathrm{D} \quad 3.8 \quad 1.0 \quad 23$ NEAR EAST COAST OF HONSHO, JAPAN

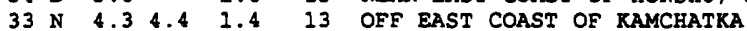

$10 \mathrm{G} \quad 4.6 \quad 4.8 \quad 1.0 \quad 33$ NORTH OF ASCENSION ISLAND. NW 5.2 (HRV).

Centroid, Moment Tensor (HRV): Centroid origin time

05:01:52.5; Lat $3.55 \mathrm{~S}$; Lon $12.05 \mathrm{~W}$; Dep $15.0 \mathrm{Fix}$; Halfduration $1.0 \mathrm{sec}$; Principal axes (scale 10**16 $\mathrm{Nm}$ ): (T) Val-7.63, Plg=6, Azm-79; (N) Val--0.68, Plg-28, Azm-346 (P) Val--6.95, Plg-61, Azm-179; Best double couple:

Mo-7.3*10**16 Nm; NP1: Strike-196, Dip=46, Slip-49; NP2: Strike-325, Dip-57, Slip--124.

MYANMAR-CHINA BORDER REGION

NORTH OF ASCENSION ISLAND

SWITZERLAND. ML 3.3 (VIE)

NORTH OF ASCENSION ISLAND. NW 5.2 (HRV).

Centroid, Moment Tensor (HRV): Centroid origin time

$06: 01: 21.2$; Lat $3.65 \mathrm{~S}$; Lon $12.34 \mathrm{~W}$; Dep $15.0 \mathrm{Fix}$; Halfduration $1.0 \mathrm{sec}$; Principal axes (scale 10**16 Nm): (T) Val-9.31, Plg-19, A2m-81; (N) Val--2.17, Plg-10, A2m-348;

(P) Val=-7.13, Plg-69, Azm=231; Best double couple: Mo-8.2*10*\#16 Nm; NP1: Strike-187, Dip-28, Slip--68; NP2: Strike-343, Dip-64, Slipm-101.

$\begin{array}{llllllllrll}07 & 54 & 00.5 ? & 33.20 & \mathrm{~S} & 178.64 & \mathrm{~W} & 33 & \mathrm{~N} & 4.6 \\ 09 & 12 & 21.98 & 23.039 & \mathrm{~N} & 121.596 & \mathrm{E} & 33 & \mathrm{~N} & 3.8 \\ 10 & 41 & 02.78 & 31.361 & \mathrm{~N} & 132.180 & \mathrm{E} & 33 & \mathrm{~N} & \\ 10 & 46 & 30.9 ? & 1.86 & \mathrm{~N} & 126.70 & \mathrm{E} & 33 & \mathrm{~N} & 4.3 \\ 11 & 25 & 00.68 & 62.574 & \mathrm{~N} & 149.590 & \mathrm{~W} & 73 & & \\ 12 & 52 & 52.3 & 36.364 & \mathrm{~N} & 70.690 & \mathrm{E} & 217 & \mathrm{D} & 4.8 \\ 13 & 01 & 24.68 & 52.730 & \mathrm{~N} & 142.526 & \mathrm{E} & 100 & \mathrm{G} & 3.9 \\ 14 & 10 & 59.4 * & 51.186 & \mathrm{~N} & 178.852 & \mathrm{~W} & 33 & \mathrm{~N} & 4.3 \\ 14 & 15 & 33.3 * & 23.873 & \mathrm{~S} & 115.357 & \mathrm{~W} & 10 & \mathrm{G} & 4.2 \\ 14 & 47 & 57.28 & 39.113 & \mathrm{~N} & 70.277 & \mathrm{E} & 33 & \mathrm{~N} & 4.0 \\ 14 & 50 & 35.7 ? & 43.85 & \mathrm{~N} & 151.91 & \mathrm{E} & 45 & \mathrm{D} & 3.5 \\ 15 & 05 & 49.68 & 36.137 & \mathrm{~N} & 139.830 & \mathrm{E} & 10 & \mathrm{G} & \\ 15 & 39 & 15.3 * & 15.752 & \mathrm{~N} & 93.479 & \mathrm{~W} & 100 & \mathrm{G} & 4.4 \\ 15 & 45 & 50.27 & 23.63 & \mathrm{~S} & 115.41 & \mathrm{~W} & 10 & \mathrm{G} & 4.0 \\ 16 & 06 & 26.1 & 51.670 & \mathrm{~N} & 16.230 & \mathrm{E} & 10 & \mathrm{G} & \\ 16 & 09 & 56.0 * & 3.753 & \mathrm{~S} & 140.066 & \mathrm{E} & 78 & \mathrm{D} & 4.1 \\ 16 & 51 & 22.0 & 18.726 & \mathrm{~N} & 145.628 & \mathrm{E} & 177 & \mathrm{D} & 5.9\end{array}$

0.910 SOUTH OF RERMADEC ISLANDS

1.09 TAIWAN

0.78 SOUTHEAST OF SHIKOKU, JAPAN
0.9

1.0

1.3

1.3

0.7

0.7

1.1

1.4

0.8

1.2

1.1

1.1

1.1

NORTHERN MOLOCCA SEA

HINDU KOSH REGION, AFGHANISTAN

SAKRALIN ISLAND

ANDREANOF ISLANDS, ALEUTIAN IS

SOUTHERN EAST PACIFIC RISE

TAJIKISTAN

EAST OF KURIL ISLANDS

EASTERN HONSHU, JAPAN

NEAR COAST OF CHIAPAS, MEXICO

SOUTHERN EAST PACIFIC RISE

POLAND. MC 3.1 (MOX)

IRIAN JAYA, INDONESIA

MARIANA ISLANDS. NW 6.3 (GS), 6.3 (HRV), Me 6.3 (GS). mb 5.9 (BRK).

Broadband Source parameters (GS): NP1: Strike-220, Dip=35 Slip-150; NP2: Strike-335, Dip-73, Slip-59; Radiated energy $6.0 * 10 * 13 \mathrm{Nm}$.

Moment Tensor (GS): Dep 176; Principal axes (scale 10**18 $\mathrm{Nm}$ ): (T) Val-2.25, Plg-51, Azm-197; (N) Val=0.81, Plg-25, Azm-324; (P) val--3.06, Plg-27, Azm-67; Best double couple: Mo-2.7*10**18 Nm; NP1: Strike-202, Dip-29, Slip-151; NP2 Strike-317, Dip-76, Slip-64.

Centroid, Moment Tensor (HRV): Centroid origin time

$16: 51: 28.4$; Lat $18.76 \mathrm{~N}$; Lon $145.82 \mathrm{E}$; Dep 189.0; Halfduration $3.3 \mathrm{sec}$; Principal axes (scale 10**18 $\mathrm{Nm}$ ): (T) Val-2.78, Plg-48, Azm-210; (N) Val-0.02, Plg-25, Azm-331: (P) Val--2.80, Plg-31, Azm-77; Best double couple: Mo-2.8*10**18 Nm; NP1: Strike-218, Dip-26, Slip-159; NP2: Strike-327, Dip-81, Slip-65. $180128.7 * 51.285 \mathrm{~N}$ $190156.2 * 23.913$ $193145.5 * 33.063 \mathrm{~N}$ $\begin{array}{llll}19 & 58 & 37.9 & 33.804 \mathrm{~N}\end{array}$ $141.862 \mathrm{E}$ 159.262 67.518 W $49.840 \mathrm{E}$ $135.508 \mathrm{E}$ $2123 \quad 34.0 \quad 17.600 \mathrm{~N} 100.965 \mathrm{~W}$
$33 \mathrm{~N} 4.0$ $33 \mathrm{~N} 4.0$ $138 * 4.6$ $33 \mathrm{~N} 4.4$ $55 \star 4.1$ 18 G $5.76 .5 \quad 1.2 \quad 165$
$126.270 \mathrm{E}$ $121.869 \mathrm{~W}$ 178.71 W $12.348 \mathrm{E}$

$\begin{array}{rll}33 & N & 4.5 \\ 21 & & \\ 600 & G & 4.1 \\ 10 & G & \end{array}$

1. 4 19
0.38 VOLCANO ISLANDS REGION

0.614 OFF EAST COAST OF KAMCHATKA

1.246 CHILE-ARGENTINA BORDER REGION CHILE-ARGENT

WESTERN IRAN

GOERRERO, MEXICO. NW 6.8 (GS), 6.6 (HRV). Me 6.1 (GS). MS 6.4 (BRK). Felt strongly at Acapulco, Ixtapa and Mexico City. Also felt in Oaxaca.

Broadband Source parameters (GS): Dep 18; Radiated energy $3.3 * 10 * * 13 \mathrm{Nm}$

Moment Tensor (GS): Dep 9; Principal axes (scale 10**19 Nm): (T) Val-1.77, Plg-60, A2m-30; (N) Val--0.06, Plg-9, Azm-285; (P) Val--1.70, Plg-28, Azm-191; Best double couple: MO-1.7*10**19 Nm; NP1: Strike-258, Dip=18, Slip=62; NP2: Strike-108, Dip-74, Slip-99.

Centroid, Moment Tensor (HRV): Centroid origin time $21: 23: 42.2$; Lat $17.50 \mathrm{~N}$; Lon $101.12 \mathrm{~W}$; Dep 22.4 ; Halfduration $5.1 \mathrm{sec}$; Principal axes (scale 10**19 $\mathrm{Nm}$ ): (T) Val-1.01, Plg-66, A2m-21; (N) Val--0.03, Plg-1, A2m-114; (P) Val--0.98, Plg-24, A2m-204; Best double couple: Mo-9.9*10**18 Nm; NP1: Strike-297, Dip-21, Slip-93; NP2 : Strike-113, Dip-69, Slip-89.

Strike-113, Dip-69, SIIP
PHILIPPINE ISLANDS REGION

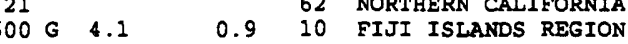

0.621 AUSTRIA. ML 3.4 (FUR), 3.1 (VIE). Felt (IV) at Kitzbuhel and Sankt Johann in Tirol. 
$33 \mathrm{~N} 4.1$

$33 \mathrm{~N} 4.7$

$154 \star 4.5$

$10 \mathrm{G}$

$33 \mathrm{~N} 4.3$

650 G 4.3

$33 \mathrm{~N} 4.1$

$\begin{array}{lllll}33 & \mathrm{~N} & 4.1 & & 0.3 \\ 33 & \mathrm{~N} & 5.8 & 6.4 & 1.2\end{array}$

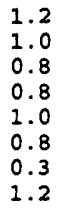

1.2

1.0
0.8

.8

205

21 GUERRERO, MEXICO

5 NEAR COAST OF GUERRERO, MEXICO

50 KURIL ISLANDS

7 NORTHWESTERN BALKAN REGION

8 VANUATU ISLANDS

3 SOUTH OF FIJI ISLANDS

KURIL ISLANDS

KOMANDORSKY ISLANDS REGION. NW 6.6 (OBN), 6.5 (GS), 6.5 (HRV). Me 6.8 (GS).

Broadband Source Parameters (GS): NPI: Strike-24, Dip-89, S1ip-3; NP2: Strike-294, Dip-87, Slip-179; Radiated energy $3.2 * 10 * * 14 \mathrm{Nm}$

Moment Tensor (GS): Dep 21; Principal axes (scale 10**18 $\mathrm{Nm}$ ): (T) Val=5.02, Plg=1, $\mathrm{Azm}=267$; (N) Val-0.79, Plg=81,

Azm-168; (P) Val=-5.81, Plg=9, Azm=357; Best double couple: Mo-5. $4 * 10 * \star 18 \mathrm{Nm} ;$ NP1: Strike-42, Dip-83, Slip=-5; NP2: Strike-133, Dip-85, Slip--173.

Centroid, Moment Tensor (HRV): Centroid origin time $03: 48: 34.0$; Lat $56.16 \mathrm{~N}$; Lon $164.98 \mathrm{E}$; Dep 15.0 Bdy; Halfduration $4.4 \mathrm{sec}$; Principal axes (scale 10**18 Nm): (T) Val-7.46, Plg-10, Azm=259; (N) Val=-0.55, Plg=78, Azm=111; (P) Val=-6.91, Plg-6, Azm-350; Best double couple: MO-7.2*10**18 Nm; NP1: Strike-35, Dip-79, Slip-3; NP2: Strike-304, Dip-87, Slip-169.

Scalar Moment $(O B N): M O=9.3 * 10 * * 18 \mathrm{Nm}$.

Scalar Moment (PPT): Mo-1.2*10**19 $\mathrm{km}$.

$\begin{array}{llllllll}16 & 04 & 08 & 11.0 & 56.222 & \mathrm{~N} & 164.485 \mathrm{E}\end{array}$
$33 \times 4.5$

42

$5 G$

$33 \mathrm{~N} 4.1$

$33 \mathrm{~N} 4.1$

$33 \mathrm{~N} 4.1$

$33 \mathrm{~N} 4.0$

$33 \mathrm{~N} 4.7$

$33 \mathrm{~N} 4.64 .6$

$33 \mathrm{~N} 4.7$

$33 \mathrm{~N} 5.0$

$33 \mathrm{~N}$

$33 \mathrm{~N}$

$33 \mathrm{~N} 4.1$

$\begin{array}{lll}33 \mathrm{~N} & 4.7 & 3.9\end{array}$

$33 \mathrm{~N} 3.7$

$33 \mathrm{~N} 4.1$

$\begin{array}{llll}33 \mathrm{~N} & 6.0 & 6.4 & 1.0\end{array}$

0.7

0.8

1.0

1.1

0.1

0.7

0.8

0.3

0.9

0.5

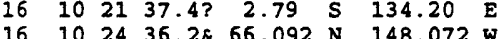

. $148.072 \mathrm{~W}$

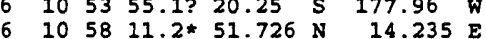

$\begin{array}{llllll}11 & 39 & 51.1 & 17.267 & \mathrm{~N} & 101.036 \mathrm{~W}\end{array}$

$121617.3 * 3.829 \mathrm{~S} 131.293 \mathrm{E}$

$6121918.5 \% 35.098 \mathrm{~N} 117.006 \mathrm{~W}$

$\begin{array}{llllllll}16 & 13 & 25 & 21.08 & 1.144 & \mathrm{~N} & 120.193 \mathrm{E}\end{array}$

$\begin{array}{llllllll}16 & 13 & 27 & 23.58 & 1.191 & \mathrm{~N} & 120.300 \mathrm{E}\end{array}$

$\begin{array}{lllllllll}16 & 13 & 40 & 45.9 & 34.589 & \mathrm{~N} & 140.489 & \mathrm{E}\end{array}$

$\begin{array}{lllllll}16 & 15 & 24 & 19.78 & 26.569 \mathrm{~N} & 126.667 \mathrm{E}\end{array}$

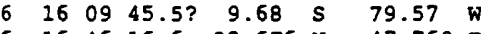

$\begin{array}{llllllll}16 & 16 & 46 & 16.6 & 32.676 \mathrm{~N} & 47.769 \mathrm{E}\end{array}$

$\begin{array}{lllllll}16 & 17 & 02 & 17.5 & 21.092 & \mathrm{~S} & 177.260 \mathrm{w}\end{array}$

$\begin{array}{lllllll}6 & 17 & 10 & 28.2 \& & 60.187 \mathrm{~N} & 153.536 \mathrm{~W}\end{array}$

$\begin{array}{llllll}16 & 18 & 08 & 00.38 & 34.282 \mathrm{~N} & 118.377 \mathrm{~W}\end{array}$

$\begin{array}{llllllll}16 & 18 & 17 & 22.3 & 13.572 & \mathrm{~N} & 91.281 & \mathrm{w}\end{array}$

$16 \quad 183021.3 * 17.754 \mathrm{~s} \quad 178.305 \mathrm{k}$

$16191317.6 * 39.641 \mathrm{~N} r 3.725 \mathrm{E}$

$\begin{array}{llllllll}16 & 21 & 31 & 48.0 & 40.454 & \mathrm{~N} & 127.396 \mathrm{~W}\end{array}$

$16 \quad 223735.8 \% 38.940 \mathrm{~N} 123.008 \mathrm{~W}$

$16225520.8 * 2.830 \mathrm{~N} 126.959 \mathrm{E}$

17003930.0 ? 5.64 S 103.21 E

$\begin{array}{lllllll}17 & 00 & 50 & 06.8 & 47.195 & \mathrm{~N} & 11.424 \mathrm{E}\end{array}$

17

$00 \quad 54 \quad 12.5 \quad 47.175 \mathrm{~N}$

$11.519 \mathrm{E}$ $\begin{array}{lllllll}16 & 13 & 04 & 25.4 & 51.699 & \mathrm{~N} & 16.035 \mathrm{E}\end{array}$

$1700 \quad 05 \quad 35.3 \quad 27.928 \mathrm{~N} \quad 130.329$
$33 \mathrm{~N} 4.8$

32

500 G 3.8

$10 \mathrm{G}$

$33 \mathrm{~N} 4.4$

$33 \mathrm{~N} \quad 4.4$

$10 \mathrm{G} \quad 3.3$

$33 \mathrm{~N} 4.4$

$33 \mathrm{~N} 4.2$

$33 \mathrm{~N} 4.1$

$33 \mathrm{~N} 4.1$

$\begin{array}{llllll}33 & \mathrm{~N} & 3.9 & 3.8 & 1.2\end{array}$

$33 \mathrm{~N} 4.2 \quad 0.9$

$282 \mathrm{D} 4.8$

147
6

$33 \mathrm{~N} 4.84 .21 .0$

$600 \mathrm{G} 4.5$

$33 \mathrm{~N} 4.3$

$10 \mathrm{G} 4.5$

5

$33 \mathrm{~N} 4.7$

$27 \quad 4.1$

$33 \mathrm{~N} 4.1$

$10 \mathrm{G}$

$10 \mathrm{G}$
0.738 KOMANDORSKY ISLANDS REGION

31 SOUTEERN ALASKA. <AEIC>. ML 2.6 (AEIC).

16 KOMANDORSKY ISIANDS REGION

18 KOMANDORSKY ISLANDS REGION

17 KOMANDORSKY ISLANDS REGION

12 ANDREANOF ISLANDS, ALEUTIAN IS.

33 SOUTHEAST OF SHIROKO, JAPAN

50 KOMANDORSKY ISLANDS REGION

57 KOMANDORSKY ISLANDS REGION

84 KOMANDORSKY ISLANDS REGION

7 SHIKOKU, JAPAN

8 EASTERN HONSHO, JAPAN

19 IRAN-IRAQ BORDER REGION

52 KOMANDORSKY ISLANDS REGION

6 IRIAN JAYA REGION, INDONESIA
9 YELLOWSTONE REGION, WYOMING. ML 3.0 (GS).

5 KURIL ISLANDS

145 MINAHASSA PENINSULA, SULAWESI. WW 6.6 (GS), 6.6 (HRV). Me 6.4 (GS). Ms 6.4 (BRK). Some damage in the rolitoli area. Felt widely in northwestern Sulawesi.

Broadband Source Parameters (GS): Dep 18; NP1: Strike-250, Dip-85, Slip=60; NP2: Strike-151, Dip=30, Slip=170 Radiated energy $1.0 * 10 * * 14 \mathrm{Nm}$.

Moment Tensor (GS): Dep 5; Principal axes (scale 10**18 Nm): (T) Val=7.82, Plg-52, Azm=161; (N) Val=2.17, Plg=8, Azm-262; (P) Val=-9.99, Plg-37, Azm=358; Best double couple: Mo-8.9*10**18 Nm; NP1: Strike-128, Dip-11, S1ip-136; NP2: Strike-261, Dip-82, Slip-82.

Centroid, Moment Tensor (BRV): Centroid origin time $10: 07: 42.4$; Lat $1.27 \mathrm{~N}$; Lon $120.35 \mathrm{E}$; Dep 21.0 Bdy; Half duration $4.5 \mathrm{sec}$; Principal axes (scale 10*18 $\mathrm{km}$ ): (T) Val=8.25, Plg=58, Azm=178; (N) Val=0.29, Plg=5, Azm-81; (P) Val=-8.54, Plg-31, Azm-348; Best double couple:

MO=8.4*10**18 Nin; NP1: Strike-63, Dip-14, Slip-71; NP2: Strike-262, Dip-76, Slip-95.

1.46 IRIAN JAYA REGION, INDONESIA

14 NORTHERN ALASKA. LAEIC>. ML 2.8 (AEIC)

1.19 FIJI ISLANDS REGION

1.45 GERMANY

36 NEAR COAST OF GUERRERO, MEXICO

8 IRIAN. JAYA REGION, INDONESIA

24 CENTRAL CALIFORNIA. <PAS-P>. MD 3.1 (PAS). ME 3.0 (GS). Felt at Barstow.

1.517 POLAND. ML 3.6 (VIE)

0.78 MINAHASSA PENINSULA, SULAWESI

0.58 MINAHASSA PENINSULA, SULAWESI

13 NEAR EAST COAST OF HONSHU, JAPAN

10 RYUKYO ISLANDS

9 OFF COAST OF NORTHERN PERU

17 IRAN-IRAQ BORDER REGION

69 FIJI ISLANDS REGION

41 SOUTHERN ALASKA. CAEIC>.

5 SOUTHERN CALIFORNIA. 〈PAS-P>. MD 2.6 (PAS). Felt in the Lake view Terrace area.

55 NEAR COAST OF GUATEMALA

$\begin{array}{lll}1.2 & 24 & \text { FIJI ISLANDS REGION } \\ 1.4 & 21 & \text { TAJIKISTAN-XINJIANG BORDER REG. }\end{array}$

65 OFF COAST OF NORTHERN CALIFORNIA. MW 4.8 (BRK). ML 4.4 (BRK). Scalar Moment (BRK): MO-2.1*10**16 Nm.

26 NEAR COAST OF NORTHERN CALIF, 〈GM-P>. MD 3.1 (GM). ML 3.1 (GS).

1.517 NORTHERN MOLUCCA SEA

1.116 RYUKYU ISLANDS

0.58 SOUTHERN SUMATERA, INDONESIA

1.243 AUSTRIA. ML 3.6 (VIE), 3.5 (FUR), 3.4 (IDG). Felt (IV) at Innsbruck.

1.265 AUSTRIA. ML 4.1 (VIE), 3.9 (FUR), 3.8 (IDG). Felt (IV) at 

$0128 \quad 40.3847 .600 \mathrm{~N}$ $013333.6 * 14.105 \mathrm{~s}$ $\begin{array}{llll}02 & 15 & 47.5 & 47.141 \mathrm{~N}\end{array}$ $0218 \quad 40.1 * 79.688 \mathrm{~N}$ $\begin{array}{llll}02 & 31 & 30.8 & 51.094 \mathrm{~N}\end{array}$ $\begin{array}{lllll}02 & 46 & 41.0 & 51.638 & \mathrm{~N}\end{array}$ $\begin{array}{lllll}03 & 25 & 15.8 & 56.105 \mathrm{~N}\end{array}$ $\begin{array}{lllll}03 & 35 & 13.6 & 34.302 \mathrm{~N}\end{array}$ $\begin{array}{llll}03 & 37 & 10.6 & 34.251\end{array}$

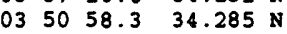
$\begin{array}{llll}04 & 21 & 12.7 & 34.311 \\ \mathrm{~N}\end{array}$ $\begin{array}{llll}04 & 52 & 51.1 & 47.156 \mathrm{~N}\end{array}$ $045900.5 ? \quad 34.23 \quad \mathrm{~N}$ $052347.6 * 26.039 \mathrm{~N}$ $065606.9 * 31.391 \mathrm{~N}$ $070054.6 * 44.537 \mathrm{~N}$ $071737.9 * 47.162 \mathrm{~N}$ $075932.8061 .459 \mathrm{~N} 147$

$\begin{array}{lllll}09 & 05 & 05.6 & 40.754\end{array}$

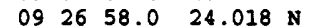
$103512.3 * 41.943 \mathrm{~N}$ $110314.4 * 51.576 \mathrm{~N}$ $120146.3 * 51.613 \mathrm{~N}$ $122556.9 * 22.027 \mathrm{~s}$ $\begin{array}{lll}13 & 24 & 39.7 \& 62.956 \mathrm{~N}\end{array}$ $135101.3 * 21.074 \mathrm{~s}$ $\begin{array}{llllll}14 & 05 & 03.28 & 17.294 \mathrm{~N}\end{array}$ $141900.1 * 36.586 \mathrm{~s}$ $\begin{array}{llllll}15 & 10 & 48.08 & 45.008 \mathrm{~N}\end{array}$ $\begin{array}{llll}15 & 18 & 37.6 & 25.575\end{array}$ $\begin{array}{llll}15 & 35 & 19.78 & 35.070 \mathrm{~N}\end{array}$ $\begin{array}{lllll}16 & 04 & 40.9 ? & 13.07 \mathrm{~N}\end{array}$ $\begin{array}{llll}16 & 32 & 26.1 & 51.670 \mathrm{~N}\end{array}$ $1653 \quad 32.0818 .900 \mathrm{~N}$ $\begin{array}{lllll}17 & 13 & 47.38 & 60.144 \mathrm{~N}\end{array}$ $\begin{array}{lllll}17 & 22 & 48.8 & 25.464 & N\end{array}$ $\begin{array}{llllll}18 & 01 & 41.2 ? & 46.31 \mathrm{~N}\end{array}$ $1822 \quad 14.0863 .258 \mathrm{~N}$ $\begin{array}{lll}18 & 26 & 08.5 * 44.666 \mathrm{~N}\end{array}$ $\begin{array}{llllll}18 & 51 & 57.4 ? & 54.04 \mathrm{~s}\end{array}$ $\begin{array}{llll}18 & 54 & 01.28 & 34.005 \mathrm{~N}\end{array}$ $1908 \quad 36.0 * 23.859 \mathrm{~s}$ $\begin{array}{lllll}21 & 15 & 31.0 & 7.820 \mathrm{~N}\end{array}$ $21 \quad 1548.1859 .933 \mathrm{~N}$ $213425.5 * 20.144 \mathrm{~S}$

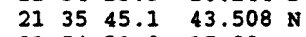
215432.8 ? $17.00 \mathrm{~s}$ $221217.8 * 37.513 \mathrm{~N}$ $223615.4 * 43.468 \mathrm{~N}$

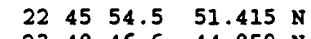
$\begin{array}{llll}23 & 48 & 46.6 & 44.859 \mathrm{~N}\end{array}$ $\begin{array}{lllllll}01 & 30 & 36.08 & 46.057 \mathrm{~N}\end{array}$ $013944.0 * 11.754 \mathrm{~N}$ $\begin{array}{llll}03 & 39 & 44.7 & 21.856 \mathrm{~s}\end{array}$ $035257.7 * 52.093 \mathrm{~N}$ $044414.0 * 35.096 \mathrm{~N}$ $\begin{array}{llll}04 & 47 & 53.7 ? \quad 34.92 \mathrm{~S}\end{array}$ $052149.3 * 12.970 \mathrm{~N}$ $\begin{array}{llll}08 & 16 & 43.8 & 17.600 \mathrm{~N}\end{array}$

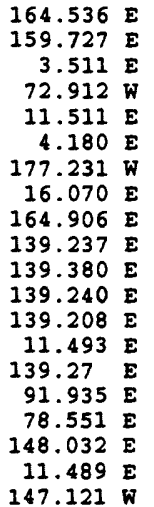

$15.312 \mathrm{E}$ $122.598 \mathrm{E}$ $120.510 \mathrm{E}$ $14.463 \mathrm{E}$ $14.490 \mathrm{E}$ $68.135 \mathrm{~W}$ $150.664 \mathrm{~W}$ $68.370 \mathrm{~W}$ $145.040 \mathrm{E}$

$71.608 \mathrm{~W}$ $6.626 \mathrm{E}$ 109.526 $27.447 \mathrm{E}$ 88.68 W

$16.204 \mathrm{E}$ $155.250 \mathrm{~W}$ $153.443 \mathrm{~W}$ $109.672 \mathrm{~W}$ $12.43 \mathrm{E}$ $151.131 \mathrm{~W}$ $142.443 \mathrm{E}$ 134.43 $135.232 \mathrm{E}$ $69.957 \mathrm{~W}$ $72.710 \mathrm{~W}$ $152.406 \mathrm{~W}$ $133.880 \mathrm{E}$ $146.789 \mathrm{E}$ 172.90 W $49.838 \mathrm{E}$ $126.450 \mathrm{~F}$ $159.402 \mathrm{E}$ $10.418 \mathrm{E}$ $6.496 \mathrm{E}$ 125.485 68.449 W 30.181 W $135.727 \mathrm{E}$

$71.14 \mathrm{~N}$ 88.538 h $100.963 \mathrm{~W}$

$\begin{array}{rll}33 & \mathrm{~N} & 4.4 \\ 33 & \mathrm{~N} & 4.1 \\ 10 & \mathrm{G} & \\ 95 & \star & 3.8 \\ 7 & & \\ 10 & \mathrm{G} & 4.0 \\ 33 & \mathrm{~N} & 4.4 \\ 10 & \mathrm{G} & \\ 33 & \mathrm{~N} & 4.3 \\ 10 & \mathrm{G} & 4.3 \\ 10 & \mathrm{G} & 4.1 \\ 10 & \mathrm{G} & 4.1 \\ 10 & \mathrm{G} & 4.0 \\ 10 & \mathrm{G} & \\ 10 & \mathrm{G} & \\ 33 & \mathrm{~N} & 4.3 \\ 33 & \mathrm{~N} & 4.6 \\ 33 & \mathrm{~N} & 4.0 \\ 10 & \mathrm{G} & \\ 20 & & 4.0\end{array}$

$\begin{array}{lll}10 \mathrm{G} & 3.7 & 0.9\end{array}$

$33 \mathrm{~N} 4.6$

$10 \mathrm{G} 2.5$

$10 \mathrm{G}$

$141 * 3.0$

111

$139 * 4.3$

$33 \mathrm{~N} 3.8$

$100 \mathrm{G} \quad 3.5$

$10 \mathrm{G}$

$\begin{array}{lll}10 & \mathrm{G} & 4.1\end{array}$

$33 \mathrm{~N} 3.9$

$50 \mathrm{G} \quad 4.3$

$10 \mathrm{G}$

$10 \quad 4.2$

10

10

9

$234 * 4.1$

$10 \mathrm{G} \quad 4.4$

$33 \mathrm{~N}$

$82 * 4.1$

$33 \mathrm{~N} 4.5$

95

10 G 4.9

$33 \mathrm{~N} 4.0$

$33 \mathrm{~N} 4.2$

$33 \times 4.3$

$10 \mathrm{G} \quad 3.6$

$33 \mathrm{~N} 4.8$

$10 \mathrm{G}$

$10 \mathrm{G}$

$50 \mathrm{G} \quad 4.5$

$122 \mathrm{D} \quad 4.6$

$10 \mathrm{G} \quad 3.5$

$10 \mathrm{G} \quad 3.9$

$100 \mathrm{G}$

$33 \mathrm{~N} 4.4$

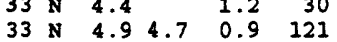

38

1.0

1.0

0.8

0.9

1.2

0.8

1.2

1.2

0.2

0.5

1.4

1.3

0.45

1.3

50

Innsbruck

DERKY ISLANDS REGION

1.016 OFF EAST COAST OF KAMCHATKA

0.86 FRANCE

1.313 CENTRAL PERO

AUSTRIA

15 GREENLLAND SEA

57 ANDREANOF ISLANDS, ALEUTIAN IS.

12 POLAND. MII 3.3 (MOX).

1 KOMANDORSKY ISLANDS REGION

30 NEAR S. COAST OF HONSHO, JAPAN

18 NEAR S. COAST OF HONSHO, JAPAN

29 NEAR S. COAST OF HONSHO, JAPAN

26 NEAR S. COAST OF HONSHU, JAPAN

AUSTRIA. ML 1.9 (VIE).

5 NEAR $S$. COAST OF HONSHU, JAPAN

10 NORTHEASTERN INDIA

9 XIZANG-INDIA BORDER REGION

KORIL ISLANDS

AOSTRIA. ML 1.7 (VIE)

SOUTHERN ALASKA. 〈AEIC>. MU 4.5 (AEIC), 4.5 (PMR). Felt (III) at Anchorage and (II) at Eagle River, Palmer and valdez. Also felt at Pump station $\$ 12$ of the Trans-Alaska Pipeline.

18 SOUTHERN ITALY

42 TAIWAN REGION

NORTHEASTERN CHINA

GERYANY. MIL 2.7 (MOX).

GERMANY

10 NORTHERN CHILE

44 CENTRAL ALASKA. $\angle A E I C>$

10 CHILE-BOLIVIA BORDER REGION

1.110 MARIANA ISLANDS

0.99 CENTRAI CHILE

1.411 FRANCE. MI 2.6 (LDG).

0.626 GULF OF CALIFORNIA

1.08 DODECANESE ISLANDS

1.510 EL SALVADOR

0.810 POLAND. ML 2.8 (MOX).

9 HAWAII. <SPEC>.

46 SOUTHERN ALASKA. <AEIC>.

0 GULF OF CALIFORNIA

0.941 EOKKAIDO, JAPAN REGION

1.413 PACIFIC-ANTARCTIC RIDGE

1.17 NEAR S. COAST OF WESTERN EONSHO

1.515 NORTHERN CHILE

0.755 NORTEERN COLOMBIA

73 SOUTHERN ALASKA. <AEIC>

1.15 NORTHERN TERRITORY, AUSTRALIA

1.126 KORIL ISLANDS

1.58 TONGA ISLANDS REGION

1.59 CASPIAN SEA

1.313 OFF COAST OF OREGON

1.074 OFE EAST COAST OF KAMCHATKA

1.216 NORTHERN ITALY. ML 2.6 (IDG), 2.5 (VIE).

1.112 SWITZERIAND. MI 2.6 (IDG)

0.9 Il SAMAR, PHILIPPINE ISLANDS

1.240 CHILE-BOLIVIA BORDER REGION

1.29 NORTEERN MID-ATLANTIC RIDGE

1.413 WESTERN EONSHO, JAPAN

0.311 NEAR COAST OF CENTRAL CEILE. ND 3.9 (SAN)

OFF COAST OF CENTRAL AMERICA

GUERRERO, MEXICO. WW 5.4 (KRV). Felt at Mexico City.

Centroid, Moment Tensor (HRV): Centroid origin time

08:16:47.1; Lat $17.35 \mathrm{~N}$; Lon $101.02 \mathrm{w}$; Dep 26.2; Half-

duration $1.2 \mathrm{sec}$; Principal axes (scale 10**17 Nm): (T)

val=1.54, Plg-64, Azm-29; (N) Val-0.10, Plg-3, Azm-293; (P)

Val=-1.64, Plg-26, Azm-202; Best double couple:

Mo-1.6*10**17 Nin; NP1: Strike-285, Dip-19, Slip-81; NP2:

Strike-114, Dip=71, Slip=93.

0.610 FRANCE. ML 2.6 (IDG).

$6.164 \mathrm{E}$

$10 \mathrm{G}$

77 KENAI PENINSULA, ALASKA. <AEIC>. ML 3.0 (AEIC).

$\begin{array}{lllllllll}18 & 08 & 42 & 07.38 & 60.047 & \mathrm{~N} & 151.789 \mathrm{~W}\end{array}$

$\begin{array}{lllllll}08 & 55 & 20.5 & 51.139 & \mathrm{~N} & 176.482 & \mathrm{~W} \\ 09 & 02 & 33.28 & 33.598 & \mathrm{~S} & 70.028 \mathrm{~W}\end{array}$

$091809.3862 .278 \mathrm{~N} 151.367 \mathrm{~W}$

092127.2 ? $51.57 \mathrm{~N} 160.03 \mathrm{E}$

$092553.68 \quad 9.634 \mathrm{~N} \quad 126.282 \mathrm{E}$

$092654.4 \quad 52.071 \mathrm{~N}$

$179.598 \mathrm{E}$

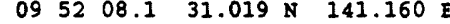

$\begin{array}{llll}10 & 13 & 47.0860 .170 \mathrm{~N}\end{array}$

$\begin{array}{llllll}10 & 19 & 32.1 ? & 35.62 \mathrm{~N}\end{array}$

$153.609 \mathrm{p}$

$141.02 \mathrm{E}$

$88.173 \mathrm{E}$

70.696

$16.114 \mathrm{E}$

$\begin{array}{llll}12 & 5522.78 & 34.347 \mathrm{~s}\end{array}$

$\begin{array}{llllll}13 & 01 & 45.4 & 51.645 \mathrm{~N}\end{array}$

$1333 \quad 39.4816 .352 \mathrm{~N}$

$134240.0 * 31.090 \mathrm{~N} 141.547 \mathrm{E}$

$\begin{array}{rrrrrr}14 & 22 & 41.28 & 60.056 \mathrm{~N} & 153.385 \mathrm{~W} \\ 14 & 27 & 57.78 & 31.256 & \mathrm{~N} & 55.590\end{array}$

$142757.78 \quad 31.256 \mathrm{~N}$

$33 \times 4.3$

$10 \mathrm{G}$

ANDREANOF ISLANDS, ALEUTIAN IS. ML 4.0 (PMR).

0.310 CEILE-ARGENTINA BORDER REGION

9279 CENTRAL ALASKA. <AEIC>.

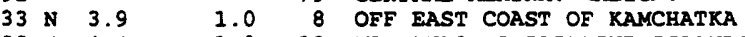

$33 \mathrm{~N} \quad 4.4$

$150 \mathrm{G} \quad 4.6$

$49 \mathrm{D} 4.6$

$150 \quad 4.1$

$10 \mathrm{G}$

$33 \mathrm{~N} \quad 4.6$

$100 \mathrm{G}$

$\begin{array}{lll}10 & G & \\ 33 & N & 3.8\end{array}$

$10 \mathrm{G} 3.9$

137

$33 \mathrm{~N} 4.0$

1.012 MINDANAO, PEILIPPINE ISLANDS

1.246 RAT ISIANDS, ALEUTIAN ISLANDS

1.136 SOUTH OF HONSHO, JAPAN

$\begin{array}{lrl}0.8 & 135 & \text { SOUTHERN ALASRA. CAEIC>. } \\ 0 & 5 & \text { NEAR EAST COAST OF HONSHO, JAPAN }\end{array}$

$0.7 \quad 43$ XIZANG

0.110 CHILE-ARGENTINA BORDER REGION. MD 2.6 (SAN).

1.113 POLAND. ML 3.1 (MOX)

0.366 LOZON, PHILIPPINE ISLANDS

1.510 SOUTH OF HONSHO, JAPAN

49 SOUTHERN ALASKA. <AEIC> 


$\begin{array}{lllllllll}18 & 18 & 30 & 42.18 & 40.423 & \mathrm{~N} & 125.493 & \mathrm{~W} \\ 18 & 19 & 00 & 21.87 & 34.36 & \mathrm{~S} & 72.17 & \mathrm{~W} \\ 18 & 20 & 36 & 22.98 & 45.997 & \mathrm{~N} & 6.080 & \mathrm{E} \\ 18 & 20 & 52 & 46.3 * & 4.638 & \mathrm{~N} & 125.680 & \mathrm{E} \\ 18 & 21 & 03 & 36.38 & 46.378 & \mathrm{~N} & 7.161 & \mathrm{E} \\ 18 & 21 & 09 & 26.38 & 60.423 & \mathrm{~S} & 25.113 & \mathrm{~W} \\ 18 & 21 & 11 & 33.88 & 32.565 & \mathrm{~S} & 70.569 & \mathrm{~W} \\ 18 & 21 & 17 & 23.27 & 10.55 & \mathrm{~N} & 62.12 & \mathrm{~W} \\ 18 & 21 & 28 & 06.7 ? & 34.29 & \mathrm{~S} & 72.19 & \mathrm{~W} \\ 18 & 22 & 31 & 04.18 & 33.763 & \mathrm{~S} & 70.128 & \mathrm{~W} \\ 18 & 22 & 55 & 03.4 & 51.494 & \mathrm{~N} & 159.442 & \mathrm{E}\end{array}$

26

$5 \mathrm{G}$

$10 \mathrm{G}$

$150 \mathrm{G} \quad 4.6$

$10 \mathrm{G}$

$33 \mathrm{~N} 4.5$

$90 \mathrm{G}$

$10 \mathrm{G}$

$5 \mathrm{G}$

$10 \mathrm{G}-0.3$

$\begin{array}{lllll}33 \mathrm{~N} & 5.5 & 5.2 & 0.9\end{array}$

\begin{abstract}
$225937.3 * 51.488 \mathrm{~N} 158.951 \mathrm{E}$

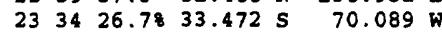
$\begin{array}{lllllll}00 & 49 & 19.98 & 33.377 & \mathrm{~S} & 69.856 \mathrm{~W}\end{array}$ 011207.7 ? 51.51 N 174.98 W OI $5048.7 * 25.948 \mathrm{~s} 179.740 \mathrm{E}$ $\begin{array}{llllll}02 & 28 & 49.3 & 39.821 & \mathrm{~N} & 54.085 \mathrm{E}\end{array}$ $\begin{array}{llllll}02 & 40 & 55.47 & 18.43 & S & 177.94\end{array}$ 032052.5 ? $51.83 \mathrm{~N} \quad 6.89 \mathrm{E}$ $\begin{array}{llllll}03 & 41 & 28.9 & 5.877 \mathrm{~N} & 95.334 \mathrm{E}\end{array}$ $0400 \quad 59.5856 .231 \mathrm{~N} \quad 164.573 \mathrm{E}$ $\begin{array}{lllllll}04 & 23 & 13.4 ? & 0.80 & \mathrm{~S} & 136.71 \mathrm{E}\end{array}$ $042728.9860 .049 \mathrm{~N} 253.574$ W $050439.0 ? \quad 9.56 \mathrm{~N} 141.75 \mathrm{E}$ $\begin{array}{llllll}05 & 34 & 24.4 & 5.821 & \mathrm{~N} & 95.327 \mathrm{E}\end{array}$ $060655.5 * 51.718 \mathrm{~N} 159.194 \mathrm{E}$ $061228.8 * 15.604 \mathrm{~S} 167.806 \mathrm{E}$

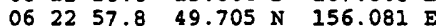
$071326.9 * 5.689 \mathrm{~N} \quad 95.280 \mathrm{E}$ 082856.951 .521 N $177.665 \mathrm{~W}$
\end{abstract}

$33 \mathrm{~N} \quad 4.7$

$10 \mathrm{G}$

$10 \mathrm{G}$

$33 \mathrm{~N} 3.8$

$500 \mathrm{G} 4.3$

$33 \mathrm{~N} 5.0$

$550 \mathrm{G} 4.2$

$10 \mathrm{G}$

$33 \mathrm{~N} 4.7$

$33 \mathrm{~N} 3.8$

$33 \times 4.4$

160

$33 \mathrm{~N} 4.1$

$33 \mathrm{~N} 4.6$

$33 N 4.1$

$100 \mathrm{G} 4.9$

$33 \mathrm{~N} 4.2$

$33 \mathrm{~N} 4.3$

$33 \mathrm{~N} 4.94 .20$.
$100.123 \mathrm{~W}$ $149.400 \mathrm{E}$ $14.607 \mathrm{E}$ $14.510 \mathrm{E}$ 140.96 $128.867 \mathrm{~W}$

$71.493 \mathrm{w}$ $56.342 \mathrm{~W}$ 152.901 W $152.462 \mathrm{~W}$ $173.764 \mathrm{~W}$ $179.73 \mathrm{E}$ $135.583 \mathrm{E}$ 80.82 W $139.938 \mathrm{E}$ 152.772 ผ $141.747 \mathrm{E}$ $142.653 \mathrm{~W}$ $178.831 \mathrm{~W}$ $152.924 \mathrm{E}$ $149.525 \mathrm{E}$ $149.589 \mathrm{E}$ $159.784 \mathrm{E}$ $145.50 \mathrm{E}$ 28.175 พ $6.235 \mathrm{E}$ $70.329 \mathrm{~W}$ 70.03 W $31.623 \mathrm{E}$ $31.642 \mathrm{E}$ $70.639 \mathrm{~W}$ $27.103 \mathrm{E}$ $\begin{array}{llllll}33 & N & 4.8 & 3.9 & 1.0 & 118\end{array}$ $33 \mathrm{~N} 4.1$ $300 \mathrm{G} \quad 4.0$ $10 \mathrm{G}$ $33 \mathrm{~N}$

$40 \mathrm{G}$ $\begin{array}{ll}10 \mathrm{G} & 4.1 \\ 108 & \end{array}$ $33 \mathrm{~N} 4.8$ $600 \mathrm{G} \quad 3.7$ $33 \mathrm{~N} 4.0$ $10 \mathrm{G} \quad 3.3$ $33 \mathrm{~N}$ $33 \mathrm{~N}$ 86 200 G 4.0 $33 \mathrm{~N} 4.2$ $10 \mathrm{G}$ $33 \times 4.6$ $33 \mathrm{~N} 4.7$ $33 \mathrm{~N} 4.5$ $33 \mathrm{~N} 4.3$ $100 \mathrm{G} 4.5$ $33 \mathrm{~N} 4.1$ $33 \mathrm{~N} 3.8$ 10 100 $150 \mathrm{G}$ $10 \mathrm{G}$ $10 \mathrm{G}$ $33 \times 5.7 \quad 6.2 \quad 1.0$

1.5
1.2
0.2 throughout the island of Hawait.

26 OFF COAST OF NORTHERN CALIFORNIA. (BRK>. MI 3.1 (BRK), 2.8 (GS). MD 2.8 (GM)

$0.8 \quad 8$ NEAR COAST OF CENTRAL CHILE

1.112 FRANCE. ML 2.7 (LDG).

0.722 TALAUD ISLANDS, INDONESIA

6 SWITZERIAND. ML 2.2 (IDG)

11 SOUTH SANDWICH ISLANDS REGION

10 CHILE-ARGENTINA BORDER REGION. MD 2.9 (SAN).

5 NEAR COAST OF VENEZUELA. MD 3.2 (TRN).

8 NEAR COAST OF CENTRAI CHILE

8 CHILE-ARGENTINA BORDER REGION

187 OFF EAST COAST OF KAMCHATRA. NW 6.0 (OBN), 5.8 (HRV). Felt (III) at Petropavlovsk-Kamchatskiy.

Centroid, Moment Tensor (HRV): Centroid origin time $22: 55: 10.7$; Lat $51.44 \mathrm{~N}$; Lon $159.83 \mathrm{E}$; Dep 22.6; Halfduration $2.0 \mathrm{sec}$; Princlpal axes (scale $10 * * 17 \mathrm{Nm}$ ): (T) Val=5.34, $P 1 g=76, A z m=310$; (N) Val=1.06, Plg=2, Azm=214; (P) Val=-6.40, Plg-14, Azm-124; Best double couple: Mo-5.9*10**17 Nm; NP1: Strike-211, Dip=31, slip=87; NP2: Strike=35, Dip=59, slip=92.

Scalar Moment (OBN): MO=1.2*10*18 $\mathrm{Nm}$.

1.320 NEAR EAST COAST OF KAMCHATKA

$\begin{array}{rrl}1.3 & 20 & \text { NEAR EAST COAST OF KAMCHATKA } \\ 0.8 & 8 & \text { CHILE-ARGENTINA BORDER REGION. MD } 3.3 \text { (SAN). }\end{array}$

0.29 CHIIE-ARGENTINA BORDER REGION. MD 3.2 (SAN).

0.44 ANDREANOF ISLANDS, ALEUTIAN IS.

0.819 SOUTH OF FIJI ISLANDS

$0.8 \quad 113$ TURKMENISTAN

1.4 II FIJI ISLANDS REGION

1.011 GERMANY. ML 2.7 (LDG), 2.2 (UCC).

1.064 NORTHERN SUMATERA, INDONESIA

0.26 KOMANDORSKY ISLANDS REGION

0.99 IRIAN JAYA REGION, INDONESIA

28 SOUTHERN ALASKA. CAEIC>.

8 KESTERN CAROIINE ISLANDS

33 NORTHERN SUMATERA, INDONESIA

21 OFF EAST COAST OF KAMCHATKA

62 VANUATU ISLANDS

34 KURII ISLANDS

12 NORTHERN SUMATERA, INDONESIA

143 ANDREANOF ISLANDS, AIEUTIAN IS. NW 5.1 (HRV). ML 4.8 (PMR). Felt (III) on Adak.

Centroid, yoment Tensor (BRV): Centroid origin time 08:29:00.0: Lat $51.46 \mathrm{~N}$; Lon $177.71 \mathrm{w}$; Dep 53.1; Halfduration $1.0 \mathrm{sec}$; Principal axes (scale 10**16 (min): (T) Val=4.98, Plg=54, Azm=287; (N) Val=-1.26, Plg=12, Azm=33; (P) Val=-3.71, Plg-33, Azm-131; Best double couple: Mo=4.3*10**16 Nm; NPI: Str1ke=260, Dip=16, Slip=138; NP2: Strike-31, D1p=79, S11p=78.

GUERRERO, MEICO. Felt at Acapulco and Mexico city.

5 KURII ISLANDS

52 SICIIY

GERMANY

NEAR EAST COAST OF HONSHO, JAPAN

35 CALIFORNIA-NEVADA BORDER REGION. 〈GM-P>. MD 3.1 (GM). ML 3.0 (BRK), 3.0 (GS).

NEAR COAST OF CENTRAL CHILE

13 SOUTH SHETLAND ISLANDS

20 SOUTHERN ALASKA. 〈AEIC>.

28 SOUTHERN ALASKA. 〈AEIC>.

1.355 TONGA ISLANDS

1.199 SOUTH OF FIJI ISLANDS

0.76 BONIN ISLANDS REGION

1.57 SOUTH AUSTRALIA

NEAR COAST OF NORTHERN PERO

26 SOUTERN BONSHD, JAPAN

26 SOUTHERN ALASKA. 〈AEIC>

1.113 VOLCANO ISLANDS REGION

0.920 OFF EAST COAST OF RAMCHATKA

0.965 GULF OF ALASKA. ML 3.6 (AEIC)

24 SOUTH OF KERMADEC ISLANDS. Feit on RaOul.

24 SOUTH OF KERMADEC ISLANDS.

16 KORIL ISLANDS

14 KURIL ISLANDS

27 KERMADEC ISLANDS, NEW ZEALAND. Felt (I) On RaOUl.

15 OFF EAST COAST OF RAMCHATKA

7 MARIANA ISLANDS REGION

31 NORTHERN MID-ATLANTIC RIDGE

8 FRANCE. ML 2.1 (LDG).

10 CHILE-ARGENTINA BORDER REGION. MD 2.1 (SAN).

10 CHILE-ARGENTINA BORDER REGION. MD 3.4 (SAN).

SOUTH OF AFRICA

4 SOUTH OF AFRICA

9 CHILE-ARGENTINA BORDER REGION. MD 2.5 (SAN).

279 DODECANESE ISLAANDS. MW 6.2 (HRV), 6.0 (GS). Me 5.9 (GS). MS 6.2 (BRK).

Broadband Source parameters (GS): Dep 7; NPI: Strike-195. Dip=45, Slip--50; NP2: Strike-325, D1p-57, Slip=-123; Radiated energy $2.5 * 10 * * 13 \mathrm{Nm}$. Two events about 2.5 seconds apart. Depth based on first event.

Moment Tensor (GS): Dep 14; Principal axes (scale 10**17

$\mathrm{Nin}$ ): (T) Val-9.96, Plg=1, Azm=101; (N) Val=0.01, Plg=4,

Azm=11; (P) Val=-9.97, Pig=86, Azm=207; Best double couple: 
$002133.08 \quad 48.973 \mathrm{~N}$ $\begin{array}{lllllll}00 & 41 & 45.5 ? & 36.03 & \mathrm{~N}\end{array}$ $\begin{array}{lllllll}01 & 04 & 16.9 ? & 35.84 \mathrm{~N}\end{array}$ $010545.1 * 21.612 \mathrm{~N}$ $\begin{array}{lllllll}01 & 10 & 07.4 ? & 36.06 \mathrm{~N}\end{array}$ $\begin{array}{lllll}01 & 16 & 06.3 & 36.115 & \mathrm{~N}\end{array}$ $0117 \quad 10.8845 .950 \mathrm{~N}$ $\begin{array}{lllll}01 & 29 & 00.88 & 36.206 & N\end{array}$ $\begin{array}{llll}01 & 34 & 43.6 & 36.220 \mathrm{~N}\end{array}$

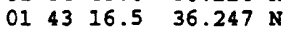
$\begin{array}{lllll}02 & 25 & 52.7 & 52.563 & \mathrm{~N}\end{array}$ $\begin{array}{lllll}02 & 4729.17 & 36.15\end{array}$ $\begin{array}{lllll}03 & 04 & 29.48 & 36.071 & N\end{array}$ $031253.4 * 2.129 \mathrm{~s}$ $033445.6 * 58.587 \mathrm{~N}$ $034722.3 * 36.133$ $\begin{array}{llll}04 & 14 & 15.8 & 20.554 \\ \mathrm{~S}\end{array}$ $\begin{array}{lllll}04 & 41 & 43.9 & 36.157 & N\end{array}$ $054618.3 * 13.539 \mathrm{~N}$ $055930.3 * 12.138 \mathrm{~N}$ $063506.6 * 5.135$ $070616.7860 .197 \mathrm{~N}$

$\begin{array}{llll}07 & 17 & 03.6 & 11.852\end{array}$ $\begin{array}{lllll}07 & 18 & 45.3 & 36.148\end{array}$ 073230.9 ? 20.40 $\begin{array}{lllll}07 & 41 & 15.2 & 19.820 \mathrm{~s}\end{array}$ $014634.8 * 36.046 \mathrm{~N}$ $\begin{array}{lllllll}02 & 20 & 37.7 & 58.066 & N\end{array}$ $035659.7 \quad 36.118 \mathrm{~N}$ $\begin{array}{llll}04 & 17 & 33.9 & 20.993 \mathrm{~S}\end{array}$
$1.906 \mathrm{E}$ 27.45 $27.09 \mathrm{E}$ $143.059 \mathrm{E}$ $27.67 \mathrm{E}$ $27.190 \mathrm{E}$

$6.244 \mathrm{E}$ $26.973 \mathrm{E}$ $27.210 \mathrm{E}$ $27.203 \mathrm{E}$ $27.035 \mathrm{E}$ 142.697 31.742 พ $27.25 \quad \mathrm{E}$ $27.212 \mathrm{E}$ $133.772 \mathrm{E}$ 142.669 $27.310 \mathrm{E}$ $27.163 \mathrm{E}$ $69.032 \mathrm{~W}$ $68.591 \mathrm{~W}$ $27.066 \mathrm{E}$ $91.620 \mathrm{~W}$ $123.244 \mathrm{E}$ 82.523 153.468 W

\section{$142.202 \mathrm{E}$} $27.302 \mathrm{E}$ $67.77 \mathrm{E}$

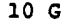

$33 \mathrm{~N} 4.0$

$33 \mathrm{~N} 3.6$

$300 \mathrm{G} 3.6$

$33 \mathrm{~N} 3.4$

$33 \mathrm{~N} 3.8$

$10 \mathrm{G}$

$33 \mathrm{~N} 3.5$

$33 \mathrm{~N} 4 . \mathrm{I}$

$33 \mathrm{~N} 4.1$

$33 \mathrm{~N} 3.9$

$10 \mathrm{G}$

$10 \mathrm{G} 4.3$

$33 \mathrm{~N} 3.8$

$33 \mathrm{~N} 3.4$

$33 \mathrm{~N} 4.5$

$10 \mathrm{G}$

$33 \times 4.0$

$33 \mathrm{~N} 4.5$

$86 \mathrm{D} 4.7$

$130 \mathrm{G} 4.1$

$33 \mathrm{~N} 3.8$

$33 \mathrm{~N} 4.3$

$33 \mathrm{~N} 4.4$

$10 \mathrm{G} \quad 4.6$
$146 \quad 3.8$

$33 N 4.4$

$33 \mathrm{~N} 4.0$

$10 \mathrm{G} 4.3$

\begin{tabular}{l}
$357 \mathrm{D} 5.7$ \\
\hline
\end{tabular} $085130.3 * 35.983 \mathrm{~N} 27.099 \mathrm{E}$ $085555.0 * 3.091 \mathrm{~N} 127.134 \mathrm{E}$ $\begin{array}{lllllll}09 & 09 & 47.8 & 36.094 & N & 27.180 & E\end{array}$ $\begin{array}{lllllll}09 & 14 & 04.1 & 13.856 \mathrm{~N} & 120.671 \mathrm{E}\end{array}$
$33 \mathrm{~N} 3.7$ $33 \mathrm{~N} 3.8$ $33 \mathrm{~N} 4.2$ $33 \mathrm{~N} 3.6$ $33 \mathrm{~N} 4.8$ $110 \mathrm{D} 5.5$ $33 \mathrm{~N} 4.6$

Mo-1.0*10**18 Nm; NP1: Strike-195, Dip -44 , Slip -85 ; NP2 Strike-8, Dip-46, S1ip- -95

Centroid, Moment Tensor (HRV): Centroid origin time

$00: 00: 47.1$; Lat $36.07 \mathrm{~N}$; Lon $26.92 \mathrm{E}$; Dep $15.0 \mathrm{Bdy}$; Halfduration $3.0 \mathrm{sec}$; Principal axes (Scale 10*18 Nm): (T) Val-2.37, P1g-8, Azm-114; (N) Val-0.01, Plg-7, Azm-205; (P) Val=-2.38, Plg-79, Azm-338; Best double couple:

No-2.4*10**18 Nm; NP1: Strike-196, Dip-38, S1ip--102; NP2: Strike-30, Dip=53, Slip=-81. 5.9 (BRK).

Broadband Source Parameters (GS): NPI: Strike=285, Dip=75 Slip=75; NP2: Strike-151, Dip-21, Slip-134; Radiated energy $1.5 * 10 * 13 \mathrm{Nm}$

Moment Tensor (GS): Dep 364; Principal axes (scale 10**17 $\mathrm{Nm}$ ): (T) Val-6.71, Plg-50, $\mathrm{Azm}=238$; (N) Val-1.17, Plg-25 Azm-113; (P) Val=-7.88, Plg=28, Azm=8; Best double couple: Mo=7. 3*10**17 Nm; NP1: Strike-52, Dip-28, Slip-26; NP2: Strike=299, Dip=78, Slip=116.

Centroid, Moment Tensor (HRV): Centroid origin time $07: 41: 21.2$; Lat $19.72 \mathrm{~S}$; Lon $177.46 \mathrm{~W}$; Dep 373.1 ; Halfduration $2.1 \mathrm{sec}$; Principal axes (scale 10*17 Nm): (T) Val-6.43, Plg-56, Azm-220; (N) Val=0.91, Plg-15, Azm-106; (P) Val--7.34, Plg-30, Azm-7; Best double couple: Mo-6.9*10**17 Nm; NP1: Strike-60, Dip-21, Slip-42; NP2: Strike-290, Dip-76, Slip-106.

1.017 DODECANESE ISLANDS

$\begin{array}{lrl}0.8 & 5 & \text { DODECANESE ISLANDS } \\ 0.9 & 11 & \text { NEW IRELAND REGION, P.N.G. }\end{array}$

$\begin{array}{lrl}0.6 & 9 & \text { DODECANESE ISLANDS } \\ 1.0 & 23 & \text { TALAUD ISLANDS, INDONESIA }\end{array}$

1.197 DODECANESE ISLANDS

1.0180 MINDORO, PHILIPPINE ISLANDS. NW 5.9 (GS), 5.9 (HRV). Felt (IV RF) at Tagaytay, Luzon. Felt (III RF) at puerto Galera. Moment Tensor (GS): Dep 108; Principal axes (scale 10**17 Nin): (T) Val-8.87, Plg-46, Azm-6; (N) Val=-0.93, Plg=37 Azm-148; (P) Val=-7.93, Plg=20, Azm-254; Best double couple: Mo-8.4*10**17 Nm; NP1: Strike-27, Dip-42, S1ip-156 NP2: Strike-136, Dip-74, Slip=51.

Centroid, Noment Tensor (HRV): Centroid origin time $09: 14: 07.4$; Lat $13.87 \mathrm{~N}$; Lon $120.48 \mathrm{E}$; Dep 119.4; Halfduration $2.2 \mathrm{sec}$; Principal axes (scale 10*17 $\mathrm{Nm}$ ): (T) Val-8.69, Plg-51, Azm-10; (N) Val=-0.89, Plg-21, Azm-128; (P) Val--7.80, Plg-32, Azm-231; Best double couple: Mo-8.2*10**17 Nm; NP1: Strike-10, Dip-23, Slip-154; NP2: Strike-124, Dip $=80$, Slip=69.

$\begin{array}{lllllll}20 & 09 & 19 & 47.08 \quad 45.935 \mathrm{~N} & 6.085 \mathrm{E}\end{array}$ $10 \mathrm{G}$ $093557.1 ? 36.12 \mathrm{~N} \quad 26.33 \mathrm{E} \quad 33 \mathrm{~N} 3.7$

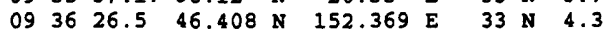
095748.4 ? 36.18 N 27.37 E 33 N 3.8 $105124.3 * 15.072 \mathrm{~S} 167.392 \mathrm{E} 100 \mathrm{G} \quad 4.7$

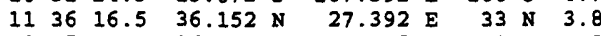

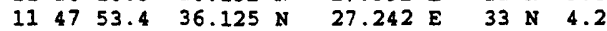
$\begin{array}{llllll}12 & 02 & 57.0 & 43.983 & \mathrm{~N} & 83.596 \mathrm{E}\end{array}$ $\begin{array}{llllllll}12 & 18 & 27.4 ? & 36.21 & N & 27.17 & E\end{array}$

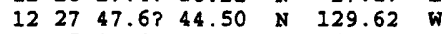
123757.0 ? 11.81 S 66.18 E $\begin{array}{rllllll}13 & 07 & 15.1 * 35.530 & \mathrm{~N} & 27.279 & \mathrm{E} \\ 14 & 55 & 52.8 & 10.529 & \mathrm{~S} & 165.315 & \mathrm{E}\end{array}$

DODECANESE ISLANDS

45 VANUATO ISLANDS

16 DODECANESE ISLANDS

68 DODECANESE ISLANDS

37 NORTHERN XINJIANG, CHINA

5 DODECANESE ISLANDS

29 OFF COAST OF OREGON

6 NID-INDIAN RIDGE

31 DODECANESE ISIANDS

33 N 5.05 .1
SANTA CRUZ ISLANDS. MW 5.2 (ERV).

Centroid, Moment Tensor (HRV): Centroid origin time $14: 56: 00.1$; Lat $10.08 \mathrm{~S}$; Lon $165.10 \mathrm{E}$; Dep 39.3 ; Half duration $1.0 \mathrm{sec}$; Principal axes (scale $10 * \star 16 \mathrm{Nm}$ ): (T) Val-7.36, Plg-59, Azm=211; (N) Val-2.11, Plg-25, Azm=353; 
$\begin{array}{llll}15 & 56 & 56.3 & 36.239\end{array}$ $\begin{array}{llll}16 & 45 & 39.5 ? & 25.31 \mathrm{~S}\end{array}$ $\begin{array}{llll}16 & 59 & 19.9 & 9.929\end{array}$ $\begin{array}{llll}17 & 12 & 36.6 & 35.217\end{array}$

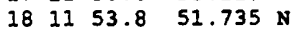
$\begin{array}{lll}18 & 37 & 40.8 * 36.184 \mathrm{~N}\end{array}$ $\begin{array}{lllll}18 & 59 & 44.1 & 10.185 \mathrm{~N}\end{array}$ $\begin{array}{llll}19 & 14 & 53.28 & 45.033\end{array}$ $\begin{array}{llll}19 & 25 & 07.5 & 36.161\end{array}$ $2048 \quad 17.6 * 35.915 \mathrm{~N}$ $\begin{array}{lllll}21 & 40 & 58.8 & 36.124 & \mathrm{~N}\end{array}$ 214453.1 ? $36.04 \quad N$ $\begin{array}{lllll}22 & 04 & 33.2 & 45.981 & N\end{array}$ $220617.5 \& 59.200 \mathrm{~N}$ $221209.9 * 21.614 \mathrm{~N}$ $\begin{array}{lllll}22 & 28 & 27.4 & 35.97 & N\end{array}$ $\begin{array}{llll}22 & 38 & 29.7 & 36.076\end{array}$ $2248 \quad 25.1 * 35.956 \mathrm{~N}$ $\begin{array}{lllll}22 & 48 & 57.78 & 45.942 & N\end{array}$ $\begin{array}{lllll}23 & 44 & 34.8 & 8.655 & s\end{array}$ $00 \quad 0937.4 \quad 51.212$
$27.214 \mathrm{E}$ $\begin{array}{lllll}.72 & W & 100 \mathrm{G} & 4.1\end{array}$ $2.953 \mathrm{E}$ $27.977 \mathrm{E}$ $16.093 \mathrm{E}$ $27.146 \mathrm{E}$ $121.528 \mathrm{E}$ $6.616 \mathrm{E}$ $27.266 \mathrm{E}$ $27.162 \mathrm{E}$ $27.190 \mathrm{E}$ $27.39 \mathrm{E}$ $6.051 \mathrm{E}$ $147.431 \mathrm{~W}$ $144.446 \mathrm{E}$ $27.46 \mathrm{E}$ $27.153 \mathrm{E}$ $27.101 \mathrm{E}$ $6.244 \mathrm{E}$ $114.675 \mathrm{E}$ $6.746 \mathrm{E}$ $169.037 \mathrm{~W}$
$33 \mathrm{~N} 4.6$

$33 \mathrm{~N} 3.8$

$\begin{array}{lll}10 \mathrm{G} & 3.7\end{array}$

$33 \mathrm{~N} 3.7$

$33 \mathrm{~N} 4.1$

$10 \mathrm{G}$

$33 \mathrm{~N} 4.2$

33 N 3.9

$33 \mathrm{~N} 3.9$

$33 \times 3.6$

$10 \mathrm{G} \quad 3.7$

35

$100 \mathrm{G} 3.5$

$33 \mathrm{~N} 3.5$

$33 \mathrm{~N} 3.7$

$33 \mathrm{~N} 3.8$

$10 \mathrm{G}$

$33 \mathrm{~N} 5.1$

$10 \mathrm{G}$

5.34 .5

(P) Val--9.47, Plg-17, Azm-91; Best double couple: Mo-8.4*10**16 Nm; NP1: Strike-214, Dip=36, Slip-137; NP2: Strike-341, Dip=66, Slip-62.

26 DODECANESE ISLANDS

1.210 SOUTH OF FIJI ISLANDS

1.137 NICOBAR ISLANDS, INDI

1.321 POLAND. ML 3.8 (VIE), 3.5 ( $\mathrm{HOX}$ ).

1.311 DODECANESE ISLANDS

1.218 PANAY, PHILIPPINE ISLANDS

1.28 FRANCE. ML 1.8 (LDG)

1.367 DODECANESE ISLANDS

1.527 DODECANESE ISLANDS

1.251 DODECANESE ISLANDS

1.513 DODECANESE ISLANDS

1.137 FRANCE. ML 3.4 (LDG).

70 GULF OF ALASKA. CAEIC>. MIL 2.9 (AEIC).

1.110 MARIANA ISLANDS REGION

1.511 DODECANESE ISLANDS

1.124 DODECANESE ISLANDS

1.428 DODECANESE ISLANDS

1.16 FRANCE. ML $1.9^{\circ}$ (LDG).

1.1 S1 BALI REGION, INDONESIA

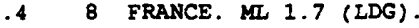

212 FOX ISLANDS, ALEUTIAN ISLANDS. 5.2 (HRV). Centroid, Moment Tensor (BRV): Centroid origin time

00:09:38.1; Lat 51.24 N; Lon $169.28 \mathrm{~W}$; Dep 15.0 Bdy; Halfduration $1.0 \mathrm{sec}$; Principal axes (scale $10 * \star 16 \mathrm{Nm}$ ): (T) Val-8.04, Plg=5, Azm=162; (N) Val=-0.66, Plg-5, Azm-71; (P) Val=-7.38, Plg-83, Azm-294; Best double couple: Mo-7.7*10**16 Nm; NP1: Strike-258, Dip-40, Slipm-82; NP2: strike-67, Dip-50, slip=-97.

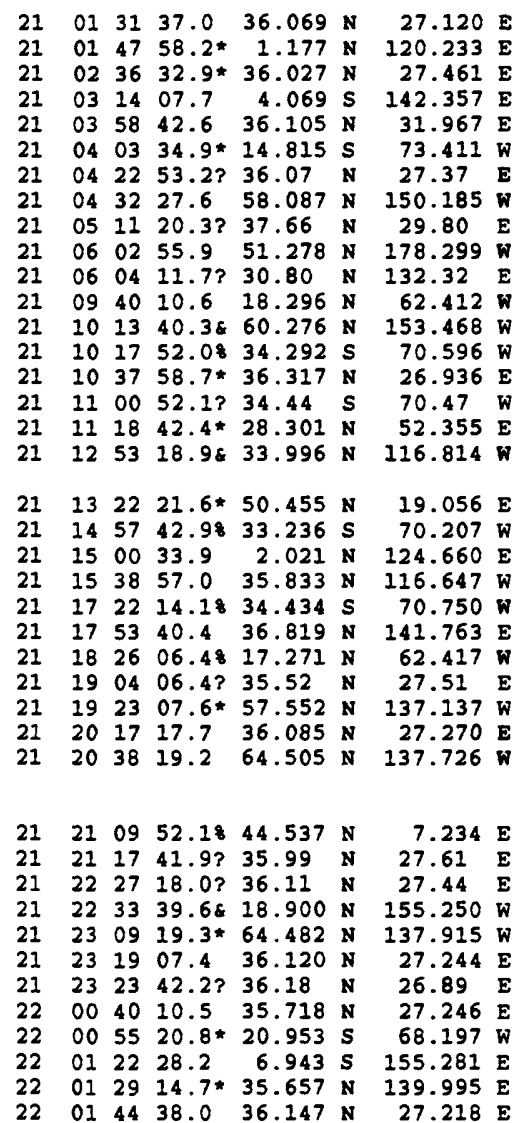

$\begin{array}{rrrrr}22 & ? & 3.8 & 0.9 & 47 \\ 33 & \text { N } & 4.4 & 0.8 & 11 \\ 100 & ? & 3.4 & 0.8 & 14 \\ 127 & \text { * } & 4.9 & 0.9 & 77 \\ 120 & ? & 3.9 & 0.9 & 50 \\ 124 & ? & 4.4 & 1.0 & 31 \\ 115 & ? & 3.7 & 1.2 & 26 \\ 10 & G & & 0.7 & 53 \\ 10 & G & 3.9 & 1.0 & 11 \\ 33 & \text { N } & 4.7 & 0.9 & 96 \\ 33 & \text { N } & & 1.0 & 4 \\ 61 & & 4.5 & 0.6 & 78 \\ 169 & & & & 13 \\ 100 & G & & 0.3 & 9 \\ 127 & ? & 3.8 & 1.0 & 30 \\ 120 & G & & 0.1 & 8 \\ 33 & \text { N } & 4.6 & 0.6 & 19 \\ 13 & & & & 31 \\ & & & & \\ 10 & G & & 0.7 & 7 \\ 104 & ? & & 0.3 & 11 \\ 229 & & 5.0 & 0.9 & 55 \\ 5 & G & & 0.8 & 29 \\ 100 & G & & 0.2 & 10 \\ 33 & \text { N } & 4.5 & 1.0 & 48 \\ 50 & G & & 0.7 & 5 \\ 33 & \text { N } & 3.8 & 1.5 & 6 \\ 10 & G & & 1.1 & 16 \\ 33 & \text { N } & 4.0 & 0.9 & 15 \\ 10 & G & 4.9 & 0.7 & 185\end{array}$

47 DODECANESE ISLANDS

1 MINARASSA PENINSULA, SULAKESI

4 DODECANESE ISIANDS

77 NEW GUINEA, PAPUA NEW GUINEA

TO TUREY

1 CENTRAL PERO

26 DODECANESE ISLANDS

3 GULF OF ALASKA. ML 2.8 (AEIC).

11 TURKEY

96 ANDREANOF ISLANDS, ALEUTIAN IS. MIL 4.6 (PMR).

4 SOUTHEAST OF SHIKOKD, JAPAN

8 LEEWARD ISLANDS

13 SOUTHERN ALASRA, <AEIC>.

9 CHILE-ARGENTINA BORDER REGION. MD 2.8 (SAN).

DODECANESE ISLANDS

CHILE-ARGENTINA BORDER REGION. ID 2.1 (SAN).

31 SOUTHERN CAIIFORNIA. LPAS-P>. 30.4 (PAS). $2[1.3$ (GS). Felt.

7 POLAND

11 CHILE-ARGENTINA BORDER REGION. MD 3.1 (SAN).

55 CETEBES SEA

29 CENTRAL CALIFORNIA. ML 3.4 (GS).

10 CHILE-ARGENTINA BORDER REGION. ID 2.7 (SAN).

48 NEAR EAST COAST OF HONSHU, JAPAN

5 LEEHARD ISLANDS. MD 3.1 (TRN).

6 DODECANESE ISLANDS

6 OFF COAST OF SOUTHEASTERN ALASKA. ML 3.4 (AEIC).

DODECANESE ISLANDS

SOUTEERN YUKON TERRITORY, CANADA. ML 5.0 (PGC), 4.9 (PMR). Felt strongly in the Flat Creek area. Also felt slightly at Dawson City.

$10 \mathrm{G}$

$33 \mathrm{~N} 3.7$

0.2

NORTBERN ITALY. MI 1.5 (IDG).

$10 \mathrm{G} \quad 4.3$

$10 \mathrm{G}$

$33 * 4.4$

$33 \mathrm{~N} .8$

$114 \mathrm{D} 4.0$

$163 * 4.3$

$71 \quad 4.7$

3.8

1.56 DODECANESE ISLANDS

1.416 DODECANESE ISLANDS

5 HAWAII. <SPEC>.

1.015 SOUTEERN YUKON TERRITORY, CANADA. ML 3.3 (AEIC).

1.293 DODECANESE ISLANDS

1.336 DODECANESE ISLANDS

1.166 DODECANESE ISLANDS

1.029 CHILE-BOLIVIA BORDER REGION

0.937 SOLOMON ISLANDS

1.2 17 NEAR S. COAST OF HONSHO, JAPAN

$33 \times 4.74 .7 \quad 1.1164$ DODECANESE ISLANDS. NW 5.1 (ERV).

Centroid, Moment Tensor (HRV): Centroid origin time

$01: 44: 42.7$; Lat $36.11 \mathrm{~N}$; Ion $26.91 \mathrm{E}$; Dep $15.0 \mathrm{Fix}$; Halfduration $1.0 \mathrm{sec}$; Principal axes (scale 10*16 Nm): (T) Val-4.55, Plg=9, Azm=125; (N) Val-0.05, Plg=7, Azm=34; (P) Val=-4.61, Plg=78, Azm=267; Best double couple: Mo-4.6*10*16 Nm; NP1: Strike-223, Dip=36, Slip=-78; NP2 : Strike=29, Dip=55, Slip=-99.

$\begin{array}{lllllllcl}22 & 02 & 20 & 35.9 ? & 1.81 & \mathrm{~N} & 127.42 & \mathrm{E} \\ 22 & 02 & 41 & 05.2 ? & 9.98 & \mathrm{~N} & 61.67 & \mathrm{~W} \\ 22 & 03 & 21 & 53.7 * & 39.250 & \mathrm{~N} & 140.756 & \mathrm{E} \\ 22 & 04 & 03 & 56.9 ? & 35.62 & \mathrm{~N} & 27.63 & \mathrm{E} \\ 22 & 04 & 04 & 49.48 & 18.907 & \mathrm{~N} & 155.265 & \mathrm{~W} \\ 22 & 04 & 05 & 16.38 & 18.900 & \mathrm{~N} & 155.250 & \mathrm{~W} \\ 22 & 04 & 14 & 01.6 ? & 37.03 & \mathrm{~N} & 141.24 & \mathrm{E} \\ 22 & 04 & 17 & 58.7 ? & 35.45 & \mathrm{~N} & 27.53 & \mathrm{E} \\ 22 & 04 & 28 & 24.9 ? & 36.04 & \mathrm{~N} & 27.31 & \mathrm{E} \\ 22 & 04 & 54 & 03.3 ? & 5.93 & \mathrm{~S} & 150.66 & \mathrm{E}\end{array}$

$33 \times 4.1$ $10 \mathrm{G}$

$123 * 3.5$

$33 \times 3.6$

14

$10 \mathrm{G} \quad 3.9$

$10 \mathrm{G}$

$33 \mathrm{~N} 3.5$

$33 \times 3.7$

$33 \mathrm{~N} 4.1$
0.9

1.0

1.1

B HALWAHERA, INDONESIA

4 NEAR COAST OF VENEZUELA. MD 3.5 (TRN).

1 EASTERN HONSHO, JAPAN

16 DODECANESE ISLANDS

5 HAWAII. 〈HVO-P>. MD 4.0 (HVO).

4 HAWAII. <SPEC>.

0.35 NEAR EAST COAST OF HONSHO, JAPAN

1.47 DODECANESE ISLANDS

1.419 DODECANESE ISLANDS

1.3 8 NEW BRITAIN REGION, P.N.G. 


$\begin{array}{rrrlrrrrr}22 & 05 & 02 & 11.1 & 36.152 & \mathrm{~N} & 27.181 & \mathrm{E} \\ 22 & 05 & 10 & 39.6 & 36.167 & \mathrm{~N} & 27.343 & \mathrm{E} \\ 22 & 05 & 31 & 03.6 * & 51.761 & \mathrm{~N} & 179.268 & \mathrm{~W} \\ 22 & 05 & 38 & 32.8 * & 51.821 & \mathrm{~N} & 177.962 & \mathrm{~W} \\ 22 & 05 & 44 & 36.0 & 5.075 & \mathrm{~S} & 151.805 & \mathrm{E}\end{array}$

$33 \mathrm{~N} 4.1$

$33 \mathrm{~N} 4.0$

$33 \mathrm{~N} 3.8$

$33 \mathrm{~N} 4.2$

$46 \mathrm{D} 5.0$

0.963 DODECANESE ISLANDS

1.543 DODECANESE ISLANDS

1.199 ANDREANOF ISLANDS, ALEUTIAN IS.

1.217 ANDREANOF ISLANDS, ALEUTIAN IS

1.068 NEW BRITAIN REGION, P.N.G. NW 5.4 (HRV)

Centroid, koment Tensor (HRV): Centroid origin time

05:44:42.6; Lat 5.29 S; Lon 152.08 E; Dep 43.1; Half-

duration $1.2 \mathrm{sec}$; Principal axes (scale 10*17 $\mathrm{Nm}$ ): (T)

Val=1.19, Plg=67, Azm=11; (N) Val=0.00, Plg=15, Azm=241;

(P) Val-1.20, Plg-17, Azm=147; Best double couple: Mo-1.2*10*17 Nm; NP1: Strike-215, Dip=31, slip=60; NP2 :

Strike=69, Dip=63, Slip=107.
1.420 DODECANESE ISLANDS
1.4

$\begin{array}{lllllllllll}22 & 06 & 00 & 27.4 & 36.192 & \mathrm{~N} & 27.340 & \mathrm{E} & 33 & \mathrm{~N} & 3.9 \\ 22 & 06 & 41 & 09.3 & 51.38 & \mathrm{~N} & 179.51 & \mathrm{~W} & 33 & \mathrm{~N} & 3.7 \\ 22 & 07 & 41 & 32.0 * & 20.316 & \mathrm{~N} & 122.076 & \mathrm{E} & 193 & ? & 4.1 \\ 22 & 07 & 41 & 32.98 & 18.900 & \mathrm{~N} & 155.250 & \mathrm{~W} & 10 & \mathrm{G} & \\ 22 & 08 & 22 & 02.28 & 18.836 & \mathrm{~N} & 155.233 & \mathrm{~W} & 12 & & 4.2 \\ 22 & 08 & 23 & 41.28 & 18.900 & \mathrm{~N} & 155.250 & \mathrm{~W} & 10 & \mathrm{G} & \\ 22 & 08 & 29 & 07.8 & 17.487 & \mathrm{~S} & 178.943 & \mathrm{~W} & 539 & ? & 4.6\end{array}$

0.919 PHILIPPINE ISLANDS REGION

2 HAWAII, <SPEC>.

HAWAII. <HVO-P>. MO 3.7 (HVO).

4 HAWAII. <SPEC>

1.116 FIJI ISLANDS REGION

1.1249 EL SALVADOR. NW 5.6 (GS), 5.6 (HRV). Felt (V) at San Salvador.

Moment Tensor (GS): Dep 35; Principal axes (scale 10* $\$ 17$ $\mathrm{Nm}$ ): (T) Val-2.44, Plg=10, $\mathrm{Azm}=38$; (N) Val=-0.06, Plg=5,

Azm=129; (P) Val=-2.38, Plg=79, Azm=247; Best double couple: Mo-2.4*10*17 Nm; NPI: Strike-122, Dip=36, S1ip=-99; NP2: Strike-313, Dip-55, S1ip=-84.

Centroid, Moment Tensor (HRV): Centroid origin time $08: 30: 24.1$; Lat $12.85 \mathrm{~N}$; Lon $89.24 \mathrm{~W}$; Dep 65.5 ; Halfduration $1.6 \mathrm{sec}$; Principal axes (scale 10*17 $\mathrm{Nm}$ ): (T) Val=3.07, Plg=14, Azm=33; (N) Val=0.01, Plg=1, Azm=303; (P) Val=-3.09, Plg=76, Azm-209; Best double couple: Mo=3.1*10*17 Nm; NP1: Strike=125, Dip=31, Slip=-88; NP2: Strike=302, Dip=59, Slip=-91.

$22083100.7 * 35.132 \mathrm{~S} \quad 71.030 \mathrm{~W}$ $22091154.7 * 1.031 \mathrm{~N} 120.366 \mathrm{E}$ $220952 \quad 36.28 \quad 43.797 \mathrm{~N} \quad 8.292 \mathrm{E}$ $221006 \quad 14.98 \quad 34.204 \mathrm{~N} 105.711 \mathrm{~W}$

$\begin{array}{llllllll}22 & 10 & 09 & 22.5 & 31.002 & \mathrm{~s} & 138.797 & \mathrm{E}\end{array}$ $2210 \quad 15 \quad 47.1 \% 38.755 \mathrm{~N} 122.725 \mathrm{~W}$

$22103055.4 * 24.119 \mathrm{~s}$ $221032 \quad 49.4 * 36.601$ * $2210 \quad 3348.5 \& 18.863 \mathrm{~N}$ $221038 \quad 16.6 * 51.592 \mathrm{~N}$ $22111653.6 \% 18.884 \mathrm{~N}$ $\begin{array}{llllll}11 & 20 & 36.8 & 12.501 \mathrm{~S}\end{array}$ $1136 \quad 42.8 * 24.583$ $\begin{array}{llllll}22 & 12 & 31 & 23.78 & 18.903 \mathrm{~N}\end{array}$ $22 \quad 123435.6 * 15.584 \mathrm{~N}$ $22 \quad 124302.5 ? \quad 34.98$ S $\begin{array}{llllllll}22 & 12 & 52 & 07.8 \& & 65.156 & \mathrm{~N}\end{array}$ $\begin{array}{llllll}22 & 13 & 23 & 51.68 & 60.965 \mathrm{~N} & 1\end{array}$ $\begin{array}{rrrlrrr}22 & 14 & 09 & 48.98 & 18.911 & \mathrm{~N} & 155.198 \\ 22 & 14 & 19 & 35.7 & 1.000 & \mathrm{~N} & 120.450\end{array}$
90 G 3.9

33 N 5.1

$10 \mathrm{G}$

10

$10 \mathrm{G} \quad 3.3$

2

$66.890 \mathrm{~W} 198 ? 3.3$

$71.445 \mathrm{E}$

$155.238 \mathrm{~W}$

$14.482 \mathrm{E}$

$155.247 \mathrm{~W}$

$166.922 \mathrm{E}$

155.264

$145.499 \mathrm{E}$

$71.13 \mathrm{~W}$

$148.438 \mathrm{~W}$

$151.519 \mathrm{~W}$

$120.450 \mathrm{E}$
$150 \mathrm{G} 3.3$

12

$10 \mathrm{G} \quad 4.0$

$216 \mathrm{D} 4.5$

$33 \times 4.3$

14

2284.0

$90 \mathrm{G}$

20

334.2

$\begin{array}{llll}33 & \times & 4.2 & 5 \\ 6.0 & 6.9 & 1.2 & 214\end{array}$

1.0
0.5

$\begin{array}{lll}0.8 & 16 & \text { CENTRAL CHILE. MD } 4.3 \text { (SAN). } \\ 1.0 & 30 & \text { MINAEASSA PENINSULA, SULANESI }\end{array}$

$\begin{array}{rrr}1.0 & 30 & \text { MINAEASSA PENINSULA, } \\ 0.3 & 8 & \text { CORSICA. ML } 2.1 \text { (GEN) }\end{array}$

25 NEW MEXICO. 〈SNM-PS. MD 3.4 (SNM). mbLg 3.5 (GS). Felt in the Corona area.

1.013 SOUTH AOSTRALIA

63 MORTHERN CALIFORNIA. LGM-P>. MD 3.2 (GM). MIL 3.3 (BRK), 3.2 (GS).

1.112 SALTA PROVINCE, ARGENTINA

0.78 AFGHANISTAN-TAJIKISTAN BORD REG

HAWAII. <HVO-P>. MD 3.7 (HVO)

1.27 GERYANY

98 SANTA CRUZ ISLANDS

1.225 VOLCANO ISLANDS REGION

HAWAII. 〈HVO-P>. MD 3.7 (HVO).

MARIANA ISLANDS

10 NEAR COAST OF CENTRAL CHILE. MD 3.2 (SAN).

25 NORTHERN ALASKA. CAEIC>. NI 2.8 (AEIC).

6 KENAI PENINSUIA, ALASKA. <AEIC>.

HAWAII. <HVO-P>. MD 3.9 (HVO).

MINAEASSA PENINSULA, SULAWESI. NW 7.0 (GS), 7.0 (HRV). Me 6.6 (GS). MS 6.9 (BRR). Some damage (VIII) in the Tolitoli area. Felt (V) in the Palu area.

Broadband Source Parameters (GS): Radiated energy $1.6 * 10 * * 14$ $\mathrm{Nm}$. Complex earthquake, with at least two events about 6 seconds apart.

Moment Tensor (GS): Dep 36; Principal axes (scale 10*\#19 Nm): (T) Val-3.01, Plg=41, Azm=194; (N) Val=0.32, Plg=33, Azm=70; (P) Val=-3.33, Plg-31, Azm-316; Best double couple: Mo $-3.2 * 10 * 19 \mathrm{Nm}$; NP1: Strike-351, Dip=34, Slip=10; NP2: Strike-253, Dip-85, Slip=124.

Centroid, Moment Tensor (HRV): Centroid origin time $14: 19: 48.1$; Lat $1.34 \mathrm{~N}$; Lon $120.65 \mathrm{E}$; Dep 28.2 ; Halfduration $7.1 \mathrm{sec}$; Principal axes (scale 10**19 $\mathrm{Nm}$ ): (T) Val=3.54, Plg=57, Azm=183; (N) Val-0.13, Plg=6, Azm-83; (P) Val $=-3.67, P l g=32, A z m=349$; Best double couple:

Mo-3.6*10**19 Nm; NP1: Strike-57, Dip=14, Slip-63; NP2 : Strike $=264$, Dip $=77$, Slip $=96$.

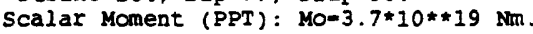

$33 \mathrm{~N} \quad 5.6$

$33 \mathrm{~N} 4.0$

134.3

$33 \times 5.0$

$13 \quad 3.8$

$33 \mathrm{~N} 5.0$

$380 \mathrm{G} \quad 4.3$

$33 \mathrm{~N} \quad 4.5$

$33 \mathrm{~N} 4.1$

$33 \mathrm{~N} 4.4$

$12 \quad 3.9$

24

$12 \times 4.0$

13

70 G 4.3

$12 \quad 3.9$

1845.0

$33 \mathrm{~N} 4.1$
1.180 MINAHASSA PENINSULA, SULAWESI

1.410 SERAM, INDONESIA

HAWAII. 〈HVO-P>. MD 4.0 (HVO).

0.951 TONGA ISLANDS

HAWAII. <HVO-P>. MD 4.1 (HVO).

11 CENTRAL ALASKA. <AEIC>. ML 2.4 (AEIC), 2.9 (PMR).

1.363 NORTHERN SUMATERA， INDONESIA

0.912 BANDA SEA

0.837 XIZANG

0.819 KURIL ISLANDS

1.037 OFF EAST COAST OF KAMCHATKA

HAWAII. <HVO-P>. MD 4.1 (HVO).

HAWAII. 〈HVO-P>. MD 3.8 (HVO).

HAWAII. 〈HVO-P>. MD 3.8 (EVO)

1.319 ANDREANOF ISLANDS, ALEUTIAN IS

HAWAII. <HVO-P>. MD 3.7 (HVO).

1.19 VANUATU ISLANDS

7 HAWAII. <HVO-P>. MD 4.3 (HVO).

0.874 NEW BRITAIN REGION, P.N.G.

1.210 LOZON, PHILIPPINE ISLANDS 
$33 \mathrm{~N} 3.5$

$10 \mathrm{G}$

$33 \mathrm{~N} 4.3$

$14 \quad 4.3$

$33 \mathrm{~N} 4.2$

134.3

$33 \mathrm{~N} 4.3$

$33 \mathrm{~N} 4.1$

$113 ? 3.7$

$10 \mathrm{G} \quad 4.0$

$10 \mathrm{G}$

13

$33 \mathrm{~N} 4.0$

28
$5 \mathrm{G}$

$33 \mathrm{~N} 4.3$

$5 \mathrm{G}$

$33 \mathrm{~N} 4.3$

124.1

$33 \mathrm{~N} 4.3$

$\begin{array}{lllll}33 & 5.8 & 6.4 & 1.1 & 11\end{array}$

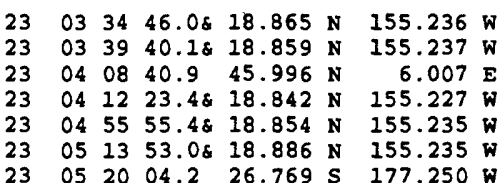
$\begin{array}{lll}38 & 15.6 & 26.647\end{array}$

$\begin{array}{lll}1.1 & 6 & \text { SAMOA ISLANDS REGION } \\ 1.2 & 8 & \text { POLAND. ML } 2.4 \text { (CLL) }\end{array}$

1.426 POLAND. MIL 2.4 (CLL).

6 HAWAII. 〈HVO-P>. MD 4.1 (HVO).

1.012 SAMAR, PHILIPPINE ISLANDS

28 HAWAII. <HVO-P>. MD 4.5 (HVO)

0.814 TONGA ISLANDS REGION

0.915 LUZON, PHILIPPINE ISLANDS

1.313 GUERRERO, MEXICO

19 HAWAII. <SPEC>.

0.913 POLAND. ML 3.2 (VIE).

1 HAWAII. 〈HVO-P>. MD 3.7 (HVO).

4 HAWAII. <HVO-P>. MD 3.9 (HVO).

1.224 KOMANDORSKY ISIANDS REGION

0.99 HAWAII. <HVO-P>. MD 4.2 (HVO).

19 NEW YORX. mbLg 2.6 (GS), 2.8 (OTT). Felt strongly at Vermontville. Also felt at Bloomingdale and Saranac Lake.

1.116 SOUTH OF EIJI ISLANDS

1.067 FRANCE. ML 3.1 (IDG), 3.1 (STR).

0.818 NORTHERN SUMATERA, INDONESIA

16 HAWAII. <HVO-P>. MD 4.4 (HVO)

NORTHERN SUMATERA, INDONESIA

SOUTH OF FIJI ISLANDS. MW 6.5 (HRV), 6.3 (GS). Me 6.7 (GS). Ms 6.4 (BRR). Felt (III) on Raoul, Kermadec Islands.

Broadband Source parameters (GS): Radiated energy $2.6 * 10 * * 14$ $\mathrm{Nm}$.

Moment Tensor (GS): Dep 29; Principal axes (scale 10**18

$\mathrm{Nm}$ ): (T) Val=3.17, Plg=16, Azm=165; (N) Val=-0.32, Plg=64, Azm=40; (P) Val=-2.85, Plg=20, Azm=261; Best double couple: Mo=3.0*10*18 Nm; NP1: Strike-303, Dip-64, Slip--3; NP2: Strike=34, Dip=87, Slip=-154.

Centroid, Moment Tensor (HRV): Centroid origin time $03: 32: 17.0$; Lat $26.97 \mathrm{~s}$; Lon $176.60 \mathrm{w}$; Dep $15.0 \mathrm{Fix}$; Halfduration $4.1 \mathrm{sec}$; Principal axes (scale $10 * \star 18 \mathrm{Nm}$ ): (T) val-6.98, Plg-3, Azm-167; (N) val--2.10, Plg-67, Azm=70 (P) Val=-4.87, Plg=23, Azm=259; Best double couple: Mo=5.9*10**18 Nm; NP1: Strike-301, Dip=72, Slip=-15; NP2 : Strike=35, Dip=76, Slip=-161.

Scalar Moment (PPT): $10-1.1 * 10 * * 19 \mathrm{~km}$.

HAWAII. 〈HVO-P>. YD 4.2 (HVO).

$13 \quad 4.1$

12

$10 \mathrm{G} \quad 4.4$

12

12
13

$13 \quad 3.9$

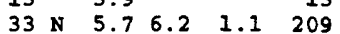

2 HAWAII. <HVO-P>. MD 3.9 (HVO)

1 FRANCE. M 4.2 (IDG), 4.0 (VIE)

HAWAII. <HVO-P>. MD 3.7 (HVO).

HAWAII. <HVO-P>. MD 3.8 (HVO).

SOUTH OF FIJI ISLANDS. MW 6.2 (BRV). MS 6.1 (BRK). Felt (III) on Raoul, Kermadec Islands.

Centroid, Moment Tensor (HRV): Centroid origin time $05: 20: 06.3$; Lat $26.87 \mathrm{~s}$; Lon $176.88 \mathrm{w}$; Dep 15.0 ; Halfduration $3.1 \mathrm{sec}$; Principal axes (scale 10**18 Nm): (T) Val-2.30, Plg-3, Azm-356; (N) Val-0.00, Plg=74, Azm=96; (P) val=-2.31, Plg=16, Azm=265; Best double couple:

MO=2.3*10*18 Nm; NP1: Strike=42, Dip=77, SIip=-171; NP2: Strike-309, Dip-81, Slip--13.

Scalar Moment (PPT) : Mo-5.1*10*\#18 Nm.

27.023 W 100 G 4.6 17.162

$\begin{array}{ll}1.0 & 14 \\ 1.1 & 81\end{array}$

SOUTH OF FIJI ISLANDS. MW 5.9 (HRV). Felt (III) on Raoul, Kermadec Islands.

Centroid, Noment Tensor (BRV): Centroid origin time

$06: 38: 16.6$; Lat $26.95 \mathrm{~S}$; Lon 176.84 พ; Dep 15.0 Fix; Halfduration 2.0 sec; Principal axes (scale $10 * 17 \mathrm{Nm}$ ): (T) Val=6.20, Plg=14, Azm=153; (N) Val=1.16, Plg=59, Azm=266; (P) val=-7.37, Plg=27, Azm=56; Best double couple: Mo=6.8*10**17 Nm; NP1: Strike-197, Dip=61, Slip=-170; NP2: Strike=102, Dip=81, Slip=-30.

8 NORTHERN ITALY. ML 2.1 (GEN)

.55 AUSTRIA. ML 2.1 (VIE).

6 PYRENEES. ML 2.3 (LDG).

7 HAWAII. <HVO-P>. MD 4.1 (HVO).

9 SOUTH OF FIJI ISLANDS

20 HAWAII. <HVO-P>. MD 4.1 (HVO)

8 HAWAII. <HVO-P>. MD 4.0 (HVO).

8 NEAR SOUTH COAST OF FRANCE

4 TRINIDAD. MD 2.6 (TRN).

18 BANDA SEA

2 HAWAII. <HVO-P>. MD 4.2 (HVO).

13 HAWAII. <HVO-P>. ID 4.1 (HVO).

59 HAWAII. <HVO-P>. MD 4.8 (HVO)

28 CENTRAI CALIFORNIA. 〈PAS-P>. MD 2.3 (PAS). Precursor.

2 CENTRAL CALIFORNIA. <PAS-P>. MD 3.2 (PAS). ML 3.2 (GS).

.9 10 BANDA SEA

. 14 NEAR SOUTH COAST OF FRANCE. ML 2.2 (GEN), 1.8 (IDG).

36 PERO-ECOADOR BORDER REGION. Felt throughout southern Ecuador.

56 DODECANESE ISIAANDS

5 AUSTRIA. ML 1.4 (VIE).

7 CORSICA. ML 2.1 (IDG).

6 ERANCE. ML 2.3 (LDG).

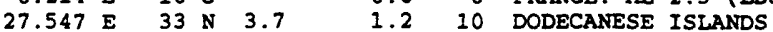




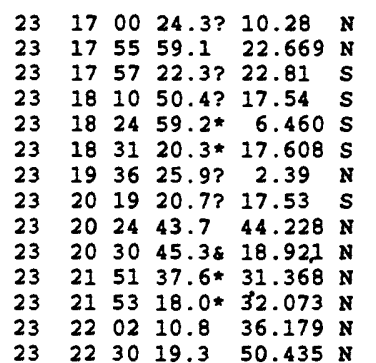

$61.29 \mathrm{~W}$ $142.832 \mathrm{E}$
$114.31 \mathrm{~W}$ $177.51 \mathrm{ผ}$ $146.030 \mathrm{E}$ $166.947 \mathrm{E}$ $129.24 \mathrm{E}$ $178.97 \mathrm{~W}$ $10.817 \mathrm{E}$ 155.247 ผ $87.788 \mathrm{E}$ $88.319 \mathrm{E}$ $27.270 \mathrm{E}$

$5.746 \mathrm{E}$

\section{0}

$\begin{array}{rll}139 & \mathrm{D} & 4.4 \\ 33 & \mathrm{~N} & 3.8\end{array}$

400 G 3.9

$104 * 3.4$

$33 \times 4.4$

$33 \mathrm{~N} 4.2$

550 G 4.3

$10 \mathrm{G}$

16

$33 \mathrm{~N} 4.1$

$33 \times 4.0$

0.8
1.1
1.0
0.6
0.7
1.1
1.4
0.6
1.2
1.0
1.0
1.2
0.9

0.2115 DODECANESE ISLANDS

VANUATU ISLANDS

16 XIZANG

8 XIZANG $\begin{array}{lllllllll}23 & 23 & 04 & 01.5 ? & 33.94 & \mathrm{~S} & 71.95 & \mathrm{~W} & 33 \mathrm{~N}\end{array}$ $\begin{array}{lllllllllll}23 & 23 & 09 & 34.3 ? & 26.03 & \mathrm{~S} & 177.38 & \mathrm{~W} & 33 & \mathrm{~N} & 4.5\end{array}$ $2323 \quad 43 \quad 39.8 * 19.295$ S 169.028 E 119 D 5.3
4 TRINIDAD. MD 2.4 (TRN).

VOLCANO ISLANDS REGION

8 EASTER ISLAND REGION

FIJI ISLANDS REGION

EASTERN NEW GUINEA REG., P.N.G

9 HALMAHERA, INDONESIA

10 FIJI ISLANDS REGION

5 NORTHERN ITALY. MI 2.8 (LDG).

HAWAII. 〈HVO-P>. MD 3.8 (HVO).

5 BELGIOM. MU 3.9 (UCC), 3.9 (VIE), 3.6 (CLL). Felt (IV) at Spa. Felt in the Aachen and Bonn areas, Germany. Also felt in the southwestern part of The Netherlands.

0.57 NEAR COAST OF CENTRAL CHILE

1.314 SOUTH OF FIJI ISLANDS

1.247 VANOATO ISLANDS. Mw 5.2 (HRV). Felt at Port-Vila.

Centroid, Moment Tensor (HRV): Centroid origin time

23:43:39.0; Lat $19.89 \mathrm{~s}$; Lon 168.74 $\mathrm{E}$; Dep 127.3; Halfduration $1.0 \mathrm{sec}$; Principal axes (scale 10*\$16 $\mathrm{Nm}$ ): (T) Val=6.93, Plg=9, Azm=330; (N) Val=0.96, Plg=53, Azm=72; Val=-7.89, Plg-36, Azm=234; Best double couple: Mo*7.4*10*16 km; NP1: Strike-18, Dip=59, Slip=-159; NP2: Strike-277, Dip=72, Slip=-33.

$\begin{array}{lllllll}23 & 23 & 47 & 31.6 \& & 18.912 & \mathrm{~N} & 155.259 \mathrm{~W}\end{array}$ $30 \quad 4.0$ $\begin{array}{lllllllll}24 & 00 & 19 & 23.8 & 17.835 \mathrm{~S} & 173.670 \mathrm{~N} & 33 & \mathrm{~N} & 4.5\end{array}$ $24002520.0 ? 41.10$ S $88.12 \quad \mathrm{~W} \quad 33 \mathrm{~N} \quad 4.3$ $24004322.0 \quad 36.600 \mathrm{~N} 27.318 \mathrm{~B}$ $2400 \begin{array}{llllll}50 & 13.3 & 34.099 & \mathrm{~N} & 137.318 \mathrm{E}\end{array}$ $240056 \quad 38.6 ? \quad 16.93 \quad N \quad 102.14 \quad \mathrm{~N}$ $2401 \quad 18 \quad 42.4 \& 18.887$ N 155.242 W 24 OI $5217.5 * 64.263 \mathrm{~N} 17.993$ ต $\begin{array}{llllllll}24 & 02 & 52 & 22.6 & 49.268 & \mathrm{~S} & 116.756 \mathrm{E}\end{array}$

HAWAII. 〈HVO-P>. MD 3.8 (HVO)

0.933 TONGA ISLANDS

1.110 WEST CHILE RISE

1.083 DODECANESE ISLANDS

1.0110 NEAR $S$. COAST OF HONSHO, JAPAN

1.58 OFF COAST OF GUERRERO, MEXICO

9 HAWAII. 〈HVO-P〉. MD 4.0 (HVO).

18 LUZON, PHILIPPINE ISLANDS

11 ICEIAND

61 SOUTH OF AUSTRAIIA. NW 5.2 (HRV). $24015037.9 * 12.590$ N $123.205 \mathrm{E}$ $\begin{array}{ll}328 & 4.6\end{array}$

$33 \mathrm{~N}$

134.0

$33 \times 4.2$

$10 \mathrm{G} \quad 3.7$

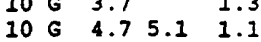

Centroid, Moment Tensor (BRV): Centroid origin time $02: 52: 28.2$; Lat $49.33 \mathrm{~s}$; Lon $116.95 \mathrm{E}$; Dep $15.0 \mathrm{Fix}$; Half duration $1.1 \mathrm{sec}$; Principal axes (scale $10 * 16 \mathrm{Nm}$ ): (T) val-6.52, Plg=9, Azm-31; (N) Val-3.17, Plg-23, Azm-125; (P) Val--9.69, Plg-65, Azm-281; Best double couple:

Mo-8.1*10*16 Nm; NP1: Strike-96, Dip-41, Slip--126; NP2: Strike-320, Dip-58, Slip=-63.

$\begin{array}{lllllllrlll}24 & 02 & 57 & 38.68 & 18.879 & \mathrm{~N} & 155.234 & \mathrm{~N} & 13 & 3.9 \\ 24 & 03 & 21 & 17.3 & 33.065 & \mathrm{~S} & 70.976 & \mathrm{~N} & 80 & 4.5\end{array}$

HAWAII. <HVO-P>. MD 3.8 (HVO)

$\begin{array}{llllllll}24 & 03 & 21 & 17.3 & 33.065 \mathrm{~s} & 70.976 \mathrm{~W}\end{array}$

$\begin{array}{rrlllllll}24 & 03 & 24 & 32.3 & 31.104 & \mathrm{~N} & 68.580 & \mathrm{E} \\ 24 & 03 & 33 & 04.7 & 1.325 & \mathrm{~N} & 120.465 & \mathrm{E}\end{array}$

$\begin{array}{llllllll}24 & 03 & 33 & 04.7 * & 1.325 & \mathrm{~N} & 120.465 & \mathrm{E} \\ 24 & 03 & 45 & 05.2 ? & 32.28 & \mathrm{~S} & 71.31 & \mathrm{~W}\end{array}$

24035955.0864 .638 N 146.908 W

$\begin{array}{lllllllll}24 & 04 & 09 & 26.2 & 26.795 & \mathrm{~S} & 177.086 & \mathrm{~W}\end{array}$ $24041131.2 * 51.070 \mathrm{~N} \quad 15.871 \mathrm{E}$

$24050220.1 \% 61.709$ N $149.731 \mathrm{~W}$

$24050410.5818 .900 \mathrm{~N} 155.250 \mathrm{~W}$

$24 \quad 05 \quad 26 \quad 34.3844 .349 \mathrm{~N} \quad 7.275 \mathrm{E}$

$\begin{array}{lllllll}24 & 05 & 48 & 37.38 & 18.905 \mathrm{~N} & 155.259 \mathrm{~W}\end{array}$

24055313.0 ? 6.10 S 104.09 E

$\begin{array}{lllllll}24 & 05 & 55 & 10.4 & 36.265 \mathrm{~N} & 27.347 \mathrm{E}\end{array}$

$\begin{array}{llllllll}24 & 06 & 11 & 36.1 & 51.241 & \mathrm{~N} & 178.053 \mathrm{~W}\end{array}$

$24061222.9818 .873 \mathrm{~N} 155.238 \mathrm{~W}$

$\begin{array}{llllllll}24 & 06 & 24 & 39.4 & 45.888 & \mathrm{~N} & 6.039 & \mathrm{E}\end{array}$

24062525.3 ? 52.52 N 168.67 W

$24072747.6833 .914 \mathrm{~N} 116.342 \mathrm{~W}$

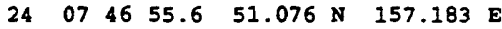

$\begin{array}{llllllll}24 & 08 & 02 & 52.2 & 36.574 & \mathrm{~N} & 27.250 \mathrm{E}\end{array}$

$\begin{array}{lllllll}24 & 08 & 03 & 12.3 \& & 36.014 & \mathrm{~N} & 117.814\end{array}$

$24085153.1818 .900 \mathrm{~N} \quad 155.250 \mathrm{~N}$

$240852 \quad 33.7 * 51.452 \times 16.267 \mathrm{E}$

24102417.4835 .684 N 117.602 W

$24 \quad 1054 \quad 04.98 \quad 18.838 \mathrm{~N} \quad 155.233 \mathrm{~W}$

$24105421.4818 .900 \mathrm{~N} 155.250 \mathrm{~W}$

$24105534.3818 .900 \mathrm{~N} 155.250 \mathrm{~W}$

$24 \quad 113418.3$ ? 14.66 S 172.95 N

$24 \quad 124534.6 * 34.055 \mathrm{~N} 139.450 \mathrm{E}$

$24 \quad 1247 \quad 41.9 \quad 34.325$ N $139.174 \mathrm{E}$

$24133925.8 * 31.322 \mathrm{~N} 87.608 \mathrm{E}$

$24 \quad 1351 \quad 02.8 \% 18.884 \mathrm{~N} 155.226 \mathrm{~N}$

$\begin{array}{lllllll}24 & 16 & 11 & 55.4 & 4.527 & \mathrm{~S} & 101.883\end{array}$

$241647 \quad 16.26 \quad 61.492 \times 150.677$

$24 \quad 1652 \quad 48.7 \& 18.871 \mathrm{~N} 155.233 \mathrm{~W}$ $24172356.335 .998 \times 68.760 \mathrm{E}$

$\begin{array}{rrrrrr}10 & G & 4.8 & 4.8 & 1.0 \\ 33 & N & 4.3 & & 0.8 \\ 70 & G & & & 0.4 \\ 7 & & & & \end{array}$

$33 \times 4.84 .40 .9$

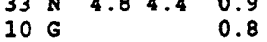

41

$10 \mathrm{G}$

15

$33 \times 4.2$

$33 \mathrm{~N} 4.2$

$33 \times 4.5$

12

$10 \mathrm{G}$

$33 \mathrm{~N} 4.0$

$33 \times 4.5$

$33 \times 4.5$

2

$10 \mathrm{G} 4.0$

$10 \mathrm{G}$

124.1

$10 \mathrm{G} \quad 4.4$

$10 \mathrm{G} 4.3$

$33 N \quad 4.7$

$10 \mathrm{G}$

$\begin{array}{lll}33 & N & 3.5 \\ 33 & N & 4.2\end{array}$

164.3

$58 \quad 4.2$

134.1 CHILE-ARGENTINA BORDER REGION. Felt (V) at La Ligua, LOS Andes and Quillota; (III) at santiago and valparaiso, chile.

76 PAKISTAN

14 MINAHASSA PENINSULA, SULAWESI

10 NEAR COAST OF CENTRAL CHILE. MD 2.6 (SAN).
51 CENTRAL ALASKA. CAEIC). MIL 3.8 (AEIC), 4.2 (PMR). Felt at Eielson AFB, North Pole and Salcha.

53 SOUTH OF FIJI ISLANDS

7 POLAND. MG 2.8 (WAR).

75 SOUTEERN ALASKA. CAEIC>. ML 2.9 (AEIC), 3.1 (PMR).

4 HAMAII. KSPEC>.

0.57 NORTHERN ITALY. ML 2.2 (GEN)

8 BAWAIT. 〈HVO-P>. MD 4.0 (HVO).

SUNDA STRAIT

$\begin{array}{rrl}0.1 & 8 & \text { SUNDA STRAIT } \\ 1.3 & 22 & \text { DODECANESE ISLANDS }\end{array}$

1.344 ANDREANOF ISLANDS, ALEUTIAN IS. ML 4.2 (PMR).

4 HAWAII. <HVO-P>. MD 4.3 (EVO).

0.910 ERANCE. MLC 2.5 (STR).

1.412 FOX ISLANDS, ALEUTIAN ISLANDS

21 SOUTHERN CALIFORNIA. LPAS-P>. MD 2.9 (PAS).

0.736 NEAR EAST COAST OF KAMCHATKA

1.258 DODECANESE ISLANDS

9 CALIFORNIA-NEVADA BORDER REGION, LPAS-P>. MO 2.9 (PAS).

6 HAWAII. $\langle S P E C\rangle$.

0.56 POLAND. MG 3.0 (WAR).

6 CENTRAL CALIFORNIA. <PAS-P>. MD 2.8 (PAS).

10 HAWAII. 〈HVO-P>. Precursor.

19 HAWAII. 〈SPEC>.

12 HAWAII. <SPEC>

0.514 SAMOA ISLANDS

0.57 NEAR S. COAST OF HONSHO, JAPAN

1.016 NEAR S. COAST OF HONSHO, JAPAN

16 NEAR S

5 HAWAII. 〈EVO-P〉. MD 4.1 (EVO)

37 SOUTHERN SUMATERA, INDONESIA

135 SOUTHERN ALASKA. CAEIC>. ML 4.3 (AEIC), 4.4 (PMR). Felt (IV) at Anchorage and Eagle River; (III) at Butte and Palmer.

7 HAWAII. <HVO-P>. MD 4.0 (EVO)

127 HINDU KUSH REGION, AFGHANISTAN. MW 5.0 (HRV).

Centroid, Moment Tensor (HRV): Centroid origin time $17: 23: 58.5$; Lat $36.08 \mathrm{~N}$; Lon $68.19 \mathrm{E}$; Dep 35.8 ; Halfduration $1.0 \mathrm{sec}$; Principal axes (scale 10*\$16 $\mathrm{Nm}$ ): (T) Val=4.31, Plg=81, Azm-187; (N) Val=-0.88, Plg-8, Azm-36; (P) Val=-3.44, Plg=4, Azm-305; Best double couple: Mo-3.9*10*16 Nm; NP1: Strike-27, Dip-41, slip-78; NP2: Strike-223, Dip-50, Slip-101.

$24 \quad 173849.4 \& 18.861 \times 155.246 \mathrm{~N} \quad 13 \quad 4.6$ $24 \quad 173923.5$ ? 5.36 S 146.08 E $134 * 4.3$ $2418 \quad 0558.9860 .786 \times 152.311 \mathrm{~W} 101$

$\begin{array}{lll}5.3 & 57 & \text { HAWAII. <HVO-P>. MD } 4.8 \text { (HVO). } \\ 16 & \text { EASTERN NEW GUINEA REG., P.N.G. }\end{array}$

49 SOUTHERN ALASKA. 〈AEIC>. 
$\begin{array}{lllll} & 113.202 & W & 10 \mathrm{G} & 4.2\end{array}$

$\begin{array}{llllll}S & 179.97 & E & 480 & G & 3.9\end{array}$

$24 \begin{array}{llllllllll}19 & 21 & 15.8 ? & 7.06 & \mathrm{~S} & 129.30 & \mathrm{E} & 173 & \text { ? } & 4.1\end{array}$

$242015 \begin{array}{llllllllllll} & 4.7 & 41.784 & \mathrm{~N} & 125.911 & \mathrm{~W} & 10 & \mathrm{G} & 5.4 & 5.4 & 1.2\end{array}$

1.0 10 BAJA CALIFORNIA, MEXICO

1.012 SOUTH OF FIJI ISLANDS

0.510 MARIANA ISLANDS REGION

1.414 BANDA SEA

175 OFF COAST OF NORTHERN CALIFORNIA. MW 5.9 (GS), 5.7 (HRV), 5.6 (BRK). Me 6.2 (GS). ML 5.3 (BRK). MD 4.9 (GM) Broadband Source Parameters (GS): Dep 6; NP1: Strike-330, Dip-88, Slip=-168; NP2: Strike-240, Dip-78, Slip=-2 Radiated energy $4.0 * 10 * 13 \mathrm{Nm}$.

Moment Tensor (GS): Dep 26; Principal axes (scale $10 * \star 17$ $\mathrm{Nm}$ ): (T) Va1-7.86, Plg-6, Azm=95; (N) Va1=0.51, Plg-83, Azm=258; (P) Val=-8.37, Plg-2, Azm=4; Best double couple: Mo-8.1*10*17 Nm; NP1: Strike-139, Dip=84, Slip-177; NP2: Strike=230, Dip-87, Slip=6.

Centroid, Moment Tensor (HRV): Centroid origin time

$20: 15: 47.6$; Lat $41.81 \mathrm{~N}$; Lon $126.03 \mathrm{~W}$; Dep $15.0 \mathrm{Fix}$; Half duration $1.8 \mathrm{sec}$; Principal axes (scale 10**17 Non): (T) Val=3.98, Plg=9, Azm=281; (N) Val=0.20, Plg-75, Azm-49; (P) Val--4.18, Plg-11, Azm=189; Best double couple:

Mo-4.1*10*17 Nm; NP1: Strike=325, Dip=75, Slip=-179; NP2 Strike-234, Dip-89, Slip=-15.

Scalar Moment (BRK): MO-2.8*10**17 Nm.

Scalar Moment (PPT): MO-6.8*10**17 Nm.

1.118 NORTHERN SUMATERA, INDONESIA

$4210033.4 \quad 15.141 \mathrm{~S} \quad 173.493 \mathrm{~W} \quad 33 \mathrm{~N} \quad 5.35 .2 \quad 0.9 \quad 124$

TONGA ISI

Centroid, Moment Tensor (HRV): Centroid origin time

$21: 00: 39.3$; Lat $15.26 \mathrm{~s}$; Lon $173.11 \mathrm{~W}$; Dep 63.5 ; Halfduration $1.1 \mathrm{sec}$; Principal axes (scale 10*17 Nm): (T)

Val-1.22, Plg-41, Azm=183; (N) Val=-0.34, Plg=32, Azm-306; (P) Val=-0.88, Plg=33, Azm-60; Best double couple: MO=1.1*10*17 Nm; NP1: Strike=206, Dip=33, Slip=172; NP2 : Strike-303, Dip $=85$, Slip=57.

$24 \quad 21 \quad 27 \quad 07.5 \& \quad 19.003$ N 155.310 W 13

$24214142.1 \quad 41.890 \mathrm{~N} \quad 126.044$ พ

$214309.2 ? 41.97$ N 126.10 พ $214411.7 * 25.984 \mathrm{~N} \quad 99.093 \mathrm{E}$ $\begin{array}{llllll}22 & 33 & 10.1 & 37.388 \mathrm{~N} & 2.300 \mathrm{~W}\end{array}$ $\begin{array}{lllllll}23 & 16 & 47.0 & 51.653 \mathrm{~N} & 16.182 \mathrm{E}\end{array}$ $234306.6 ? 2.36$ S $140.19 \mathrm{E}$ $\begin{array}{lllll}23 & 5322.4 & 51.701 \mathrm{~N} & 177.124 & \mathrm{~W}\end{array}$ $2338 \quad 16.8859 .905 \mathrm{~N} 153.400 \mathrm{~W}$
$10 \mathrm{G} \quad 4.1$

$10 \mathrm{G} \quad 3.8$

$33 \mathrm{~N} \quad 4.2$

$10 \mathrm{G}$

$10 \mathrm{G}$

125

$33 \times 4.1$

$55 \mathrm{D} \quad 5.1$
1 HAWAII. <HVO-P>. MO 3.7 (EVO).

1.152 OFF COAST OF NORTHERN CALIFORNIA. ML 4.0 (BRK), 3.5 (GS). MD 3.9 (GM).

0.87 OFF COAST OF NORTHERN CALIFORNIA

$1.1 \quad 17$ YONNAN, CHINA

1.023 SPAIN. mbig 3.7 (MDD). Felt (III) in the olula del Rio area.

0.513 POLAND. ML 2.6 (CLL)

76 SOUTHERN ALASKA. CAEIC>.

1.3 I3 NEAR NORTH COAST OF IRIAN JAYA

0.9107 ANDREANOF ISLANDS, ALEUTIAN IS. NW 5.1 (HRV). ML 5.0 (PMR). Felt (IV) on Adak.

Centroid, Moment Tensor (HRV): Centroid origin time

$23: 53: 21.4$; Lat $51.40 \mathrm{~N}$; Ion $176.56 \mathrm{w}$; Dep 60.4 ; Halfduration $1.0 \mathrm{sec}$; Príncipal axes (scale 10**16 $\mathrm{Nm}$ ): (T) Val=4.32, Plg=69, Azm=326; (N) Val=0.60, Plg=5, Azm=224; (P) Val=-4.92, Plg=21, Azm-132; Best double couple: Mo $=4.6 * 10 * 16 \mathrm{Nm} ; \mathrm{NP1}$ : Strike=213, Dip=25, Slip=79; NP2: Strike=46, Dip-66, Slip=95.

$\begin{array}{lllll}01 & 26 & 44.1 & 36.318 & N\end{array}$ 012645.1217 .52 S $\begin{array}{lllll}01 & 27 & 55.58 & 45.069 & \mathrm{~N}\end{array}$ 014655.6818 .909 024326.7 ? $0.07 \mathrm{~S}$ $0245 \quad 20.6 \& \quad 18.876 \mathrm{~N}$ $\begin{array}{llll}02 & 58 & 08.8 \& \quad 18.903\end{array}$ $\begin{array}{lllll}03 & 12 & 33.5 & 51.867 \mathrm{~N}\end{array}$ $03 \quad 30 \quad 49.3 \quad 51.872 \mathrm{~N} \quad 150.775 \mathrm{E}$

$33 \mathrm{~N} 3$. 8.243 $580 \mathrm{G} \quad 4.6$ $10 \mathrm{G}$ 143.9 121.92 E 273 D 4.5 $155.248 \mathrm{~W}$ $155.278 \mathrm{~W}$ $160.010 \mathrm{E}$
26

$\begin{array}{llllll}38 & D & 4.6 & 4.1 & 0.9 & 50\end{array}$ $\begin{array}{llllll}41 \mathrm{D} & 5.1 & 5.0 & 1.0 & 167\end{array}$

$\begin{array}{lllllrl}04 & 13 & 48.48 & 18.960 & \mathrm{~N} & 155.270 & \mathrm{~W} \\ 04 & 14 & 03.98 & 18.900 & \mathrm{~N} & 155.250 & \mathrm{~W} \\ 04 & 57 & 51.4 & 22.421 & \mathrm{~S} & 68.406 & \mathrm{~W} \\ 05 & 33 & 56.3 * & 17.469 & \mathrm{~S} & 178.850 & \mathrm{~W} \\ 07 & 04 & 37.9 * & 5.452 & \mathrm{~S} & 147.055 & \mathrm{E} \\ 07 & 53 & 01.1 * & 51.839 & \mathrm{~N} & 159.906 & \mathrm{E} \\ 09 & 30 & 18.5 & 50.465 & \mathrm{~N} & 177.247 & \mathrm{E} \\ 09 & 46 & 09.9 * & 16.080 & \mathrm{~S} & 173.658 & \mathrm{~W} \\ 10 & 45 & 13.1 * & 41.907 & \mathrm{~N} & 142.274 & \mathrm{E} \\ 10 & 58 & 33.38 & 16.824 & \mathrm{~N} & 98.221 & \mathrm{~W} \\ 12 & 03 & 58.0 & 44.370 & \mathrm{~N} & 7.373 & \mathrm{E} \\ 12 & 49 & 37.0 * & 50.149 & \mathrm{~S} & 113.538 & \mathrm{~W}\end{array}$

22

$10 \mathrm{G} \quad 4.2$

$85 * 4.4$

$514 ? 4.5$

$200 \quad 4.4$

$33 \mathrm{~N} 4.2$

$33 \mathrm{~N} \quad 4.4$

$33 \mathrm{~N} 4.4$

$33 \mathrm{~N} \quad 3.9$

$33 \mathrm{~N}$

$13 \%$

$\begin{array}{llll}10 \mathrm{G} & 5.1 & 5.7 & 1.2\end{array}$
0.720 DODECANESE ISIANDS

0.812 FIJI ISLANDS REGION

$\begin{array}{lrlll}0.8 & 12 & \text { FIJI ISLANDS REGION } \\ 1.1 & 9 & \text { NORTHERN ITALY. ML } 2.1 \text { (GEN) }\end{array}$

HAWAII. <HVO-P>. MD 3.8 (HVO)

6 MINAHASSA PENINSULA, SULAWESI

HAWAII. 〈HVO-P>. MD 4.1 (HVO)

HAWAII. <HVO-P>. MD 3.8 (HVO)

OFF EAST COAST OF KAMCHATKA

OFF EAST COAST OF KAMCHATKA. NW 5.4 (HRV).

Centroid, Moment Tensor (HRV): Centroid origin time

$03: 30: 50.5$. Lat $51.73 \mathrm{~N}$; Lon $160.46 \mathrm{E}$; Dep 26.1 ; Halfduration $1.1 \mathrm{sec}$; Principal axes (scale $10 \star 17 \mathrm{Nm}$ ): (T) Val-1.34, Plg=61, Azm=342; (N) Val=0.02, Plg-14, Azm=225; (P) Val=-1.36, Plg=25, Azm-128; Best double couple: Mo-1.4*10**17 Nm; NP1: Strike-190, Dip=24, Slip-53; NP2 : Strike=50, Dip-71, Slip-105.

HAWAII: <HVO-P>. MD 3.9 (HVO).

2 HAWAII. <SPEC>.

$0.6 \quad 47$ FIJI ISLANDS REGION

1.3 13 EASTERN NEW GUINEA REG., P.N.G.

0.820 OFF EAST COAST OF KAMCEATKA

0.839 RAT ISLANDS, ALEUTIAN ISLANDS

0.511 TONGA ISLANDS

1.48 HOKKAIDO, JAPAN REGION

1.28 NEAR COAST OF GUERRERO, MEXICO

9 NORTHERN ITALY. MI 2.3 (GEN)

41 SOUTHERN EAST PACIFIC RISE. 6.1 (HRV), 6.0 (GS). MS 5.9 (BRK).

Moment Tensor (GS): Dep 14; Principal axes (scale 10*18 Non): (T) Val=1.09, Plg-9, Azm=144; (N) Val-0.00, Plg=76, Azm-276; (P) Val-1.09, Plg-10, Azm-53; Best double couple Mo-1.1*10*18 Nm; NP1: Strike-189, Dip-76, Slip=-179; NP2: Strike-99, Dip=89, Slip=-14.

Centroid, Moment Tensor (HRV): Centroid origin time

$12: 49: 47.4$; Lat $50.11 \mathrm{~s}$; Lon $114.17 \mathrm{~W}$; Dep $15.0 \mathrm{Fix}$; Half duration $2.5 \mathrm{sec}$; Principal axes (scale 10**18 Nm): (T) Val=1.40, Plg-5, Azm=324; (N) Val=-0.04, PIg=83, Azm=197; (P) Val=-1.36, P1g=6, Azm-55; Best double couple: Mo-1.4*10**18 Nm; NP1: Strike-99, Dip=83, slip=-1; NP2 Strike-189, Dip=89, Slip=-173.

scalar Moment (PPT): Mo $=3.3 * 10 * 18 \mathrm{Nm}$. 


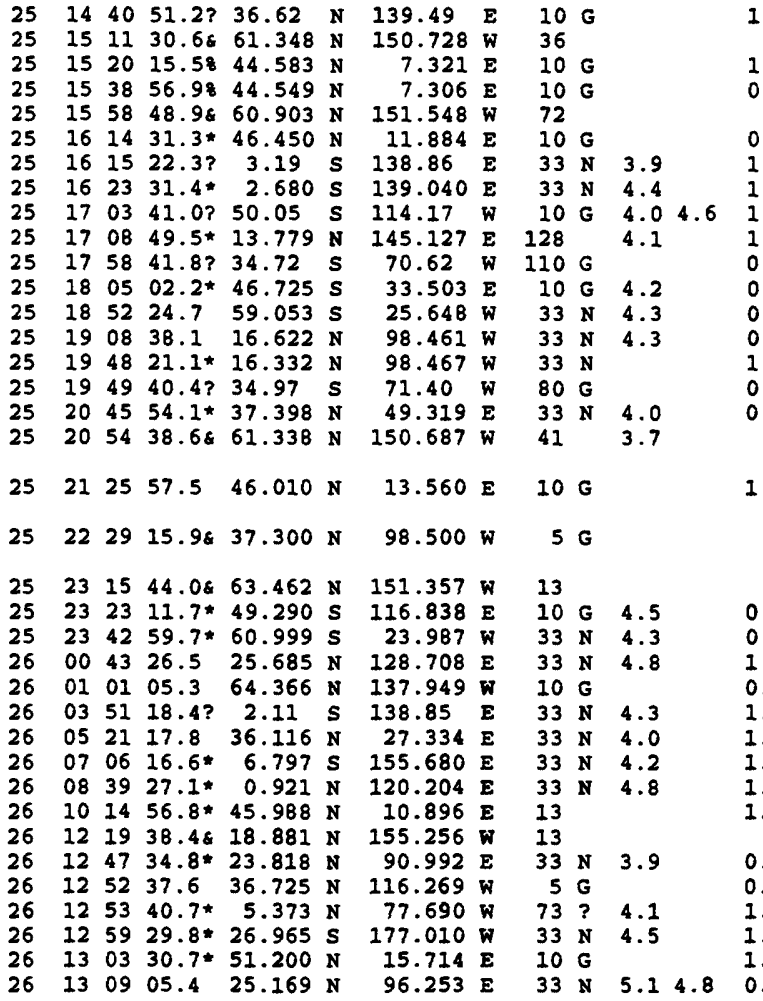

1.24 EASTERN BONSHU, JAPAN

372 SOUTHERN ALASKA. $\angle A E I C>$. ML 3.2 (AEIC), 3.5 (PMR).

12 NORTHERN ITALY. ML 2.5 (GEN).

76 KFNAI

0.66 NORTHERN ITALY. ML 2.1 (VIE).

1.16 IRIAN JAYA, INDONESIA

1.315 NEAR NORTH COAST OF IRIAN JAYA

1.29 SOUTHERN EAST PACIFIC RISE

25 MARIANA ISLANDS. Felt (IV) in the Agat-Santa Rita area, Guam.

10 CHILE-ARGENTINA BORDER REGION. MD 2.3 (SAN).

$\begin{array}{rrl}0.9 & 10 & \text { CEILE-ARGENTINA BORDER REGION } \\ 0.9 & 7 & \text { PRINCE EDWARD ISLANDS REGION }\end{array}$

0.714 SOUTE SANDWICE ISLANDS REGION

.92 21 NEAR COAST OF GUERRERO, MEXICO

1.37 NEAR COAST OF GUERRERO, MEXICO

0.410 NEAR COAST OF CENTRAL CHILE. MD 2.9 (SAN).

0.715 CASPIAN SEA

94 SOUTHERN ALASKA. 〈AEIC>. ML 3.8 (AEIC), 3.8 (PMR). Felt (III) at Anchorage and Palmer.

1.118 AUSTRIA. MU 3.5 (VIE), 3.4 (GRF), 3.3 (FUR). Felt (V) at Anhovo and Dobrovo, Slovenia.

4 RANSAS. <MACRO>. mbLg 2.2 (GS), 2.2 (TUL). Felt in the Medicine Lodge area.

63 CENTRAL ALASKA. LAEIC>. ML 3.4 (AEIC), 3.7 (PMR).

0.824 SOUTH OF AUSTRALIA

0.512 SOUTH SANDWICH ISLANDS REGION

1.139 RYUKYO ISLANDS

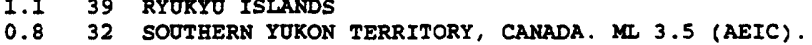

1.39 IRIAN JAYA, INDONESIA

1.229 DODECANESE ISLANDS

1.216 SOLOMON ISLANDS

1.025 MINAHASSA PENINSULA, SULAWESI

$\begin{array}{lrl}1.0 & 25 & \text { MINAHASSA PENINSULA, SULAWESI } \\ 1.0 & 8 & \text { NORTHERN ITALY. MI } 2.9 \text { (VIE) }\end{array}$

8 NORTHERN ITALY. MI 2.9 (VIE).
I HAWAII. <HVO-P>. MD 3.7 (HVO).

7 BANGIADESE

0.511 CALIFORNIA-NEVADA BORDER REGION. ML 3.5 (GS).

1.215 NEAR WEST COAST OF COLOMBIA

$1.2 \quad 22$ SOUTH OF FIJI ISLANDS

1.412 POLAND. ML 3.1 (CLL), 3.1 ( $\mathrm{KOX}$ ).

8120 MYAMNAR. NW 5.4 (HRV).

Centroid, Moment Tensor (HRV): Centroid origin time

$13: 09: 09.6$; Lat $24.68 \mathrm{~N}$; Lon $96.53 \mathrm{E}$; Dep 67.6 ; Halfduration $1.1 \mathrm{sec}$; Principal axes (scale 10*17 Nm): (T) Val=1.22, PIg=22, Azm-315; (N) Val=-0.04, Plg=67, Azm=154; (P) Val=-1.19, PIg=7, Azm=48; Best double couple:

Mo-1.2*10**17 Nm; NP1: Strike=93, Dip-70, Slip-11; NP2:

Strike-359, Dip-80, Slip=159.

$26 \quad 1324 \quad 48.3 \& 18.886$ \& 155.260 \%

$26 \quad 14 \quad 14 \quad 09.9 * 27.695 \mathrm{~N} \quad 66.982 \mathrm{E}$ $26 \quad 142950.73 \quad 5.94$ N 77.07 W $144041.9 * 36.146 \mathrm{~N} 139.886 \mathrm{E}$ $144751.5 * 24.099 \mathrm{~S} 179.732 \mathrm{~W}$

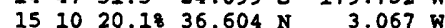
$\begin{array}{lllllll}15 & 11 & 34.88 & 36.380 \mathrm{~N} & 3.149 \mathrm{~W}\end{array}$ $\begin{array}{lllll}15 & 14 & 06.8 * & 6.566 \mathrm{~S} & 29.421 \mathrm{E}\end{array}$ 174408.7 ? 50.01 S 115.84 E $174552.1 * 17.991 \mathrm{~N} 67.729 \mathrm{~W}$ $180229.0860 .771 \mathrm{~N} 147.272 \mathrm{~W}$

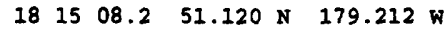

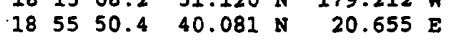

$13 \quad 4.2$

$\begin{array}{lll}33 & \mathrm{~N} & 4.0 \\ 33 & \mathrm{~N} & 3.9\end{array}$

$33 \mathrm{~N}$

$500 \mathrm{G} \quad 4.4$

$10 \mathrm{G}$

25

$10 \mathrm{G} \quad 4.2$

$10 \mathrm{G} \quad 4.3$

$33 \mathrm{~N}$

15

$33 \mathrm{~N} 4.5$

$11 \quad 5.2 \quad 5.2 \quad 1.2$
11

1.1

1.4
0.7

1.0

1.0

0.9
0.8

1.1

1.0

0.5 2199

\begin{abstract}
$192912.4 * 7.642 \mathrm{~S} \quad 119.462 \mathrm{E}$ $193407.5 \quad 0.981 \mathrm{~N} \quad 120.586 \mathrm{E}$ $202756.7 * 51.397 \mathrm{~N} 176.324 \mathrm{~W}$ $213125.1 * 1.059 \mathrm{~N} 120.121 \mathrm{E}$ $213815.0 * 0.513 \mathrm{~N} 120.527 \mathrm{E}$

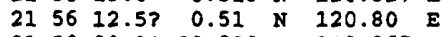
$215929.3 * 52.832 \mathrm{~N} 142.867 \mathrm{E}$ $220033.0 * 47.627 \mathrm{~N} 154.113 \mathrm{E}$ $220231.8818 .881 \mathrm{~N} 155.256 \mathrm{~W}$

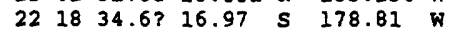
$\begin{array}{lllllll}22 & 23 & 58.2 ? & 49.35 & N & 153.99 & E\end{array}$ $224603.1 * 51.075 \mathrm{~N} \quad 176.183 \mathrm{~W}$ $\begin{array}{lllllll}23 & 01 & 17.68 & 36.531 \mathrm{~N} & 3.084 \mathrm{~W}\end{array}$

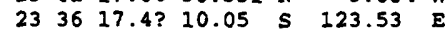
$\begin{array}{llllllll}23 & 59 & 47.5 * & 18.940 & \mathrm{~N} & 155.269 & \mathrm{~W} \\ 00 & 26 & 41.9 * & 24.660 \mathrm{~N} & 121.995 & \mathrm{E}\end{array}$
\end{abstract}

$00 \quad 27 \quad 40.8 \quad 35.790 \mathrm{~N}$ $003657.1 * 5.925 \mathrm{~N}$ $\begin{array}{llll}00 & 52 & 00.7 & 35.929\end{array}$ $\begin{array}{llll}02 & 49 & 49.6 & 32.066 \mathrm{~N}\end{array}$ $\begin{array}{llll}02 & 5622.436 .059 \mathrm{~N} \\ 03 & 01 & 03.1 * 33.511\end{array}$

$27.619 \mathrm{E}$ 77.801 พ $27.275 \mathrm{E}$ $131.536 \mathrm{E}$ $27.215 \mathrm{E}$ $69.980 \mathrm{~W}$
$341 ? 4.1$ $33 \mathrm{~N} 4.9$ $33 \mathrm{~N} 4.1$ $33 \mathrm{~N} 4.7$ $33 \mathrm{~N} 4.2$ $33 \mathrm{~N} 4.1$ 33 N 3.7

$33 \times 4.54 .2 \quad 1.1$ 13

400 G 3.9

$33 \mathrm{~N} 3.6$

$33 \mathrm{~N} 4.2$ $10 \mathrm{G}$

$120 \mathrm{G} 4.1$

$24 \quad 4.0$

$48 ? 4.3$

$33 \mathrm{~N} 3.2$ $10 \mathrm{G} 4.74 .2$

$33 \mathrm{~N} 3.7 \quad 1.0$

694.1 $33 \mathrm{~N} 3.3$

$10 \mathrm{G}$
PAKISTAN

TEST COAST OF COLOMBIA

EASTERA HONSHO, JAPAN

SOUTH OF FIJI ISLANDS

10 STRAIT OF GIBRALTAR. mbLg 3.0 (MDD).

10 STRAIT OF GIBRAITAR. mbLg 2.8 (MDD).

14 LAKE TANGANYIKA REGION

\section{SOUTH OF AUSTRALIA}

MONA PASSAGE

1 SOUTHERN ALASKA. <AEIC>, ML 3.0 (AEIC).

ANDREANOF ISLANDS, ALEUTIAN IS

GREECE-ALBANIA BORDER REGION. MW 5.3 (GRV). ML 4.8 (ROM). Felt in the Ioannina area, Greece. Also felt (IV) at Bitola, Ohrid and Resen, former Yugoslav Republic of Macedonia.

Centroid, Moment Tensor (HRV): Centroid origin time $18: 55: 55.8$; Lat $39.92 \mathrm{~N}$; Lon $20.77 \mathrm{E}$; Dep $15.0 \mathrm{Fix}$; Halfduration $1.1 \mathrm{sec}$; Principal axes (scale $10 * 17 \mathrm{Nm}$ ): (T) Val=0.91, Plg-9, Azm=117; (N) Val-0.14, Plg=6, Azm-26; (P) Val=-1.05, Plg=79, Azm=262; Best double couple:

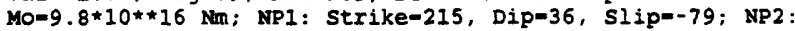
Strike-22, Dip-54, Slip=-98.

\section{FLORES SEA}

40 MINAHASSA PENINSULA, SULAWESI

10 ANDREANOF ISLANDS, ALEUTIAN IS

22 MINAFASSA PENINSULA, SULAWESI

9 MINAHASSA PENINSULA, SULAKESI

11 MINAHASSA PENINSULA, SULAWESI

8 SAKHALIN ISLAND

28 KURIL ISLANDS

1 HAWAII. 〈HVO-P>. MD 4.0 (HVO).

10 FIJI ISLANDS REGION

KURIL ISLANDS

20 ANDREANOF ISLANDS, ALEUTIAN IS.

STRAIT OF GIBRALTAR. mbLg 3.2 (MDD).

TIMOR REGION, INDONESIA

9 HAWAII. <HVO-P>. MD 4.3 (HVO)

TAIWAN. Felt (III JMA) at Nan-ao and (II JMA) at I-lan, Suao and Taipei.

16 DODECANESE ISLANDS

36 NEAR NEST COAST OF COLOMBIA

18 DODECANESE ISLANDS

28 KYUSHO, JAPAN

11 DODECANESE ISLANDS

11 CHILE-ARGENTINA BORDER REGION. MD 3.5 (SAN). 
$\begin{array}{llllllll}27 & 03 & 03 & 58.3 & 50.483 & \mathrm{~N} & 18.966 \mathrm{E}\end{array}$ $2703 \quad 3322.8 \& 18.846 \mathrm{~N} \quad 155.242 \mathrm{~W}$ $270405 \quad 41.6 \& 18.888 \mathrm{~N} \quad 155.265 \mathrm{~W}$ $270427 \quad 37.6 \& 18.903 \mathrm{~N} \quad 155.257 \mathrm{~W}$ $\begin{array}{lllllll}27 & 05 & 26 & 12.9 ? & 23.92 & S & 179.95\end{array}$ $27055030.0818 .889 \mathrm{~N} 155.249 \mathrm{~W}$ $27071048.4 * 11.004 \mathrm{~S} 162.340 \mathrm{E}$ $2708 \quad 0538.4818 .874 \mathrm{~N} 155.248 \mathrm{~W}$ $\begin{array}{llllllll}27 & 08 & 16 & 46.8 & 18.208 & \mathrm{~N} & 87.433 \mathrm{~F}\end{array}$ $27 \quad 082451.1 * 12.432 \mathrm{~N} 124.854 \mathrm{E}$ $27 \quad 08 \quad 45 \quad 18.4 \quad 21.307 \quad \mathrm{~N} \quad 94.804 \mathrm{E}$

$\begin{array}{rll}10 \mathrm{G} & \\ 12 & & \\ 13 & & 4.3 \\ 14 & & 4.1 \\ 500 \mathrm{G} & 4.1 \\ 13 & & \\ 79 & \mathrm{D} & 4.4 \\ 13 & & 4.1 \\ 33 & \mathrm{~N} & 4.7 \\ 33 & \mathrm{~N} & 4.3 \\ 110 & \mathrm{D} & 5.0\end{array}$

1.110 POLAND. MG 3.0 (WAR).

2 HAWAII. <HVO-P>. YD 3.7 (HVO).

21 HANAII. <HVO-P>. YD 4.3 (HVO).

HANAII <HVO-P>. YD 3.9 (HVO)

0.99 SOUTH OF FIJI ISLANDS

2 HAWAII. <HVO-P>. MD 3.9 (HVO).

30 SOLOMON ISLANDS

17 HAWAII. <HVO-P>. MD 4.2 (HVO)

1.231 BAY OF BENGAI

0.820 SAMAR, PHILIPPINE ISLANDS

0.8158 MYANMAR. MW 5.2 (BRV).

Centroid, Moment Tensor (HRV): Centroid origin time

$08: 45: 19.9$; Lat $20.80 \mathrm{~N}$; Lon $94.85 \mathrm{E}$; Dep 128.9; Half-

duration 10 sec; Principal axes (scale 10**16 $\mathrm{Nm}$ ): (T)

Val=7.67, Plg=43, Azm-59; (N) Val-0.26, Plg=47, Azm-241; (P) Val=-7.93, Plg=1, Azm=150; Best double couple: MO-7.8*10**16 Nm; NPI: Strike=204, Dip=60, S1ip=33; NP2: Strike-96, Dip=62, Slip-146.

$270848 \quad 01.78 \quad 61.740 \mathrm{~N} \quad 151.680 \mathrm{~N}$ $27 \quad 094100.48 \quad 36.481 \mathrm{~N} \quad 2.870 \mathrm{~W}$ $27095454.2 * 20.982 \mathrm{~N} \quad 122.515 \mathrm{E}$ $27 \quad 10 \quad 0925.9818 .848 \mathrm{~N} 155.223 \mathrm{~W}$ $27 \quad 10 \quad 1050.9 \quad 38.300 \mathrm{~N} \quad 22.303 \mathrm{E}$ $27 \quad 1025 \quad 46.4 \& \quad 18.871$ N $155.229 \mathrm{~N}$ $27 \quad 11 \quad 1757.3861 .450 \mathrm{~N} 149.990 \mathrm{~W}$ $\begin{array}{llllllll}27 & 11 & 40 & 02.8 & 28.655 & S & 177.465 & \mathrm{~W}\end{array}$

93

$10 \mathrm{G}$ $200 \mathrm{G} \quad 4.0$ $12 \quad 4.1$

$27 * 4.1$ $13 \quad 3.8$

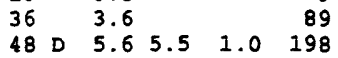

91 $\begin{array}{llllll}27 & 11 & 52 & 58.1 ? & 36.19\end{array}$

$27115904.9818 .900 \mathrm{~N} \quad 155.250 \mathrm{~N}$

$27 \quad 121744.3 \& 18.852 \mathrm{~N} 155.239$ ผ

$\begin{array}{lllllll}27 & 12 & 39 & 51.58 & 36.559 \mathrm{~N} & 2.806 \mathrm{~W}\end{array}$

$\begin{array}{llllll}27 & 12 & 59 & 17.78 & 36.541 \mathrm{~N} & 2.821\end{array}$

$27 \quad 125919.3844 .412 \mathrm{~N} \quad 7.317 \mathrm{~F}$

$27131029.8 * 18.271$ S 175.130 .

$\begin{array}{lllllll}27 & 14 & 03 & 17.5 & 30.600 \mathrm{~N} & 137.659 \mathrm{E}\end{array}$

$\begin{array}{lllllll}27 & 14 & 40 & 52.48 & 36.545 \mathrm{~N} & 2.840 \mathrm{~N}\end{array}$

$27 \quad 1548 \quad 54.7 * 41.181 \mathrm{~N} \quad 72.628 \mathrm{E}$

27 I5 $5003.8 * 27.794 \mathrm{~s} \quad 176.428 \mathrm{~W}$

$\begin{array}{lllllllll}27 & 16 & 23 & 14.7 ? & 44.60 & \mathrm{~N} & 147.39 \quad \mathrm{E}\end{array}$

$27 \quad 16 \quad 31 \quad 54.98 \quad 63.340 \mathrm{~N} \quad 151.260 \mathrm{~W}$

$27163622.5 \& 63.330 \mathrm{~N} 151.270 \mathrm{~N}$

$27 \quad 16 \quad 4134.6 * 36.098 \mathrm{~N} \quad 27.407 \mathrm{E}$

$271654 \quad 51.6 \& 63.316 \mathrm{~N} \quad 151.265 \mathrm{~N}$

$\begin{array}{llllllll}7 & 17 & 20 & 21.9 ? & 1.18 & S & 127.55 & E\end{array}$

$\begin{array}{llllllll}7 & 18 & 10 & 16.38 & 44.009 \mathrm{~N} & 7.205 \mathrm{E}\end{array}$

$\begin{array}{llllll}18 & 35 & 34.1 & 36.00 & \mathrm{~N} & 27.79\end{array}$

$191352.3861 .710 \mathrm{~N} 149.660 \mathrm{~W}$

$193301.0861 .010 \mathrm{~N} 150.150 \mathrm{~N}$

$200424.6 * 1.247 \mathrm{~N} 120.477 \mathrm{E}$

$2016 \quad 01.3859 .980 \mathrm{~N} \quad 152.510 \mathrm{~W}$

$2018 \quad 39.2 * 36.315 \mathrm{~N} \quad 71.059 \mathrm{E}$

$\begin{array}{lllllll}20 & 29 & 47.78 & 18.937 \mathrm{~N} & 155.257 \mathrm{~W}\end{array}$

$2051 \quad 42.8 ? 44.58 \mathrm{~N}$

$\begin{array}{llllllll}21 & 08 & 27.2 * & 5.276 \mathrm{~N} & 126.004\end{array}$

$\begin{array}{llllllll}77 & 21 & 33 & 42.9 \& 59.882 & \mathrm{~N} & 153.195 \mathrm{~W}\end{array}$

$27213640.2 \& 18.875 \mathrm{~N} 155.249 \mathrm{~W}$

$272202 \quad 12.8 \& 18.858 \mathrm{~N} \quad 155.258 \mathrm{~N}$

$72210 \quad 05.6818 .848 \mathrm{~N} \quad 155.235 \mathrm{H}$

$\begin{array}{lllllll}22 & 18 & 26.48 & 18.875 & \mathrm{~N} & 155.236\end{array}$

$27222505.3 * 27.018$ S 177.088 K

$\begin{array}{llllllll}27 & 22 & 29 & 35.4 & 51.687 & \mathrm{~N} & 16.105 & \mathrm{E}\end{array}$
$27 \begin{array}{llllll}12 & 03 & 26.18 & 18.924 & \mathrm{~N} & 155.279\end{array}$

$27 \quad 13 \quad 3536.48 \quad 35.654 \mathrm{~N} 119.585 \mathrm{~N}$

$27161219.5 * 53.111 \mathrm{~N} 142.686 \mathrm{E}$

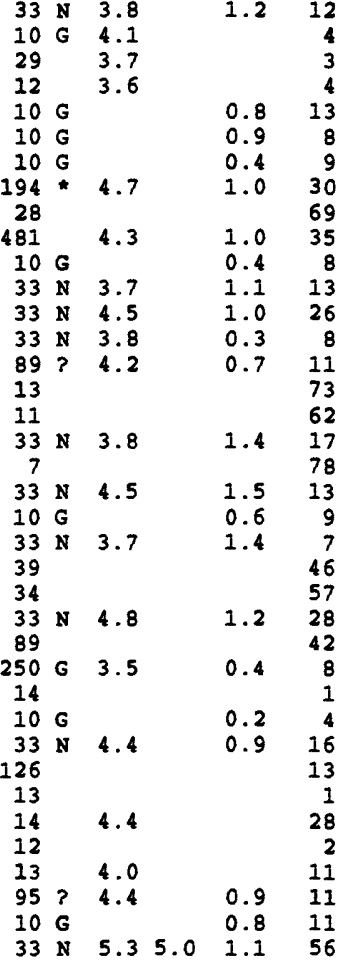

$\begin{array}{llllllll}27 & 23 & 02 & 57.2 ? & 35.08 & 5\end{array}$

2723 (16.31

$\begin{array}{llllllll}27 & 23 & 16 & 17.0 & 37.428 & \mathrm{~N} & 141.650 \\ 27 & 23 & 30 & 07 & 9 ? & 37.72 & \mathrm{~N} & 139.59\end{array}$

$27 \quad 235005.3 * 37.489 \mathrm{~N} r 20.912$

$27235911.0818 .866 \mathrm{~N} 155.222 \mathrm{~N}$

$280000 \quad 10.5859 .930 \mathrm{~N} \quad 153.000 \mathrm{~W}$

$\begin{array}{llllll}28 & 00 & 12 & 38.68 & 33.228 \mathrm{~S} & 71.488 \mathrm{~W}\end{array}$

$\begin{array}{lllllll}8 & 00 & 40 & 24.8 & 21.227 \mathrm{~N} & 120.123 \mathrm{E}\end{array}$

$\begin{array}{llllllll}8 & 01 & 02 & 09.7 & 36.083 & \mathrm{~N} & 27.276 \mathrm{E}\end{array}$

$28011038.5 \& 18.920 \mathrm{~N} 155.263 \mathrm{~K}$

$\begin{array}{llllllll}28 & 01 & 12 & 03.8 & 39.978 & \mathrm{~N} & 20.731 & \mathrm{E}\end{array}$

$\begin{array}{llllllr}28 & 01 & 14 & 39.9 & 43.101 & \mathrm{~N} & 0.717 \\ & 01 & 44 & 27.28 & 18.870 & \mathrm{~N} & 155.251\end{array}$

$\begin{array}{rrrrr}100 & \mathrm{G} & & 0.3 & 10 \\ 33 & \mathrm{~N} & & 0.7 & 6 \\ 145 \mathrm{P} & 3.5 & 0.9 & 10 \\ 10 \mathrm{G} & 3.8 & 1.1 & 22 \\ 12 & & & & 4 \\ 107 & & & & 57 \\ 40 & \mathrm{G} & & 0.4 & 10 \\ 33 & \mathrm{~N} & 4.2 & 0.8 & 21 \\ 33 \mathrm{~N} & 4.1 & 1.3 & 63 \\ 17 & & & & 2 \\ 10 & \mathrm{G} & 4.0 & 1.3 & 35 \\ 10 & \mathrm{G} & & 0.6 & 17 \\ 13 & & & & 1\end{array}$

0.310 PHILIPPINE ISLANDS REGION

11 HAWAII. <HVO-P>. MD 4.0 (HVO)

O GREECE

HAWAII. <HVO-P>. AD 4.4 (HVO)

SOUTAERN ALASKA. <AEIC>. ML 3.5 (AEIC) KERMADEC ISLANDS

Centroid, Moment Tensor (HRV): Centroid origin time

$11: 40: 07.6$; Lat $28.58 \mathrm{~s}$; Lon $177.04 \mathrm{w}$; Dep 50.9; Halfduration $2.1 \mathrm{sec}$; Principal axes (scale $10 * \star 17 \mathrm{Nm}$ ): (T) Val=6.88, Plg=76, Azm=294; (N) Val-1.80, Plg=3, Azm=191; (P) Va1=-8.68, Plg-13, Azm=100; Best double couple: Mo-7.8*10*17 $\mathrm{Nm}$; NP1: Strike=186, Dip=32, Slip=84; NP2: strike=13, Dip-58, Slip=94.

Scalar Moment (PPT): MO-7.9*10*17 km.

\section{NORTHWESTERN IRAN}

HAWAII. <SPEC>

HAWAII. <HVO-P>. MD 3.9 (HVO).

HANAII. <HVO-P>. MD 3.9 (HVO)

STRAIT OF GIBRALTAR. MbLg 3.3 (MDD).

STRAIT OF GIBRALTAR, mbLg 2.7 (MDD).

NORTHERN ITALY. ML 2.2 (GEN).

\section{TONGA ISLANDS}

CENTRAL CALIFORNIA. 〈GM-P>. MD 3.4 (GM), 3.2 (PAS).

SOUTH OF HONSHO, JAPAN

STRAIT OF GIBRALTAR. mbLg 2.5 (MDD).

KYRGYZSTAN

KERMADEC ISLANDS REGION

SAKHALIN ISLAND

RORIL ISLANDS

CENTRAL ALASKA. LAEIC). ML 3.5 (AEIC).

CENTRAL ALASKA. <AEIC>. MI 3.1 (AEIC).

DODECANESE ISLANDS

CENTRAL ALASKA. LAEIC>. MU 3.5 (AEIC).

HALMAFERA, INDONESIA

NORTHERN ITALY. MI 2.4 (GEN).

DODECANESE ISLANDS

SOOTHERN ALASKA. CAEIC>. MI 2.6 (AEIC).

SOUTHERN ALASKA. <AEIC>. MI 2.9 (AEIC).

MINAHASSA PENINSULA, SULAWESI

SOUTHERN ALASKA. <AEIC>

AFGHANISTAN-TAJIKISTAN BORD REG.

HAWAII. <HVO-P>. YD 3.8 (HVO)

NORTHERN ITALY. ML 1.8 (GEN).

MINDANAO, PHILIPPINE ISLANDS

SOUTHERN ALASKA. <AEIC>

HAWAII. <HVO-P>. ID 3.7 (HVO).

HAWAII. <HVO-P>. AD 4.5 (HVO)

BAWAII. <HVO-P>. MD 4.3 (HVO)

HAWAII. <HVO-P>. MD 4.0 (HVO)

KERMADEC ISLANDS REGION

POLAND. ML 2.7 (MOX), 2.5 (CIL).

MINAHASSA PENINSULA, SULAWESI. MW 5.4 (HRV)

Centroid, Moment Tensor (BRV): Centroid origin time

$22: 34: 12.3$; Lat $1.59 \mathrm{~N}$; Lon $120.55 \mathrm{E}$; Dep 42.7 ; Half-

duration $1.2 \mathrm{sec}$; Principal axes (scale $10 * * 17 \mathrm{Nm}$ ): (T)

Val=1.28, Plg=66, Azm-202; (N) Val=0.34, P1g-9, Azm=91; (P)

Val=-1.63, Plg=22, Azm-357; Best double couple:

Mo-1.5*10**17 Nm; NP1: Strike-70, Dip=25, Slip-68; NP2:

Strike-274, Dip=67, S1ip=100.

CENTRAL CHILE. MD 3.4 (SAN).

NEAR EAST COAST OF HONSHO, JAPAN

EASTERN HONSHO, JAPAN

IONIAN SEA

HAWAII. <HVO-P>. MD 3.7 (HVO).

SOUTHERN ALASKA. <AEIC>.

NEAR COAST OF CENTRAL CHILE. MD 3.7 (SAN).

TAIWAN REGION

DODECANESE ISLANDS

HAWAII. <HVO-P>. UD 3.8 (HVO)

GREECE-ALBANIA BORDER REGION. MI 3.8 (ROM).

PYRENEES. mbLg 3.3 (MDD).

HAWAII. <HVO-P>. ND 4.1 (HVO). 


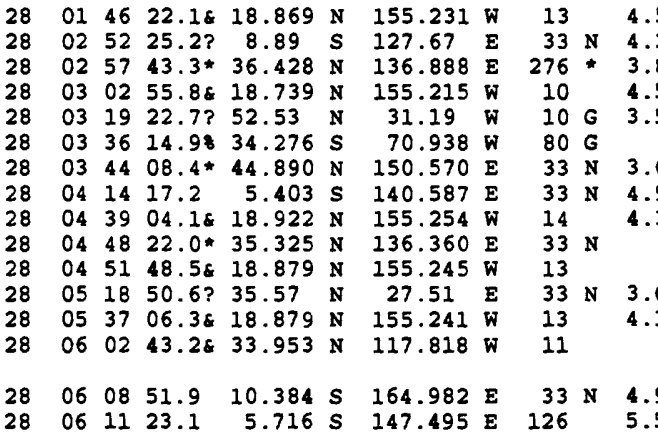

21 HAWAII. 〈HVO-P>. MD 4.4 (HVO).

1.114 TIMOR REGION, INDONESIA

0.77 NEAR WEST COAST OF HONSHU, JAPAN

26 HAWAII. <HVO-P>. MD 4.6 (HVO).

1.58 NORTHERN MID-ATLANTIC RIDGE

0.1 10 CHILE-ARGENTINA BORDER REGION. MD 2.3 (SAN)

1.310 EAST OF KURIL ISLANDS

0.943 IRIAN JAYA, INDONESIA

6 HAWAII. 〈HVO-P>. MD 3.9 (HVO).

0.45 WESTERN HONSHU, JAPAN

6 HAWAIT. <HVO-P>. MD 4.3 (HVO).

8 DODECANESE ISLANDS

4 HAWAII. 〈HVO-P>. MD 3.7 (HVO).

24 SOUTHERN CALIFORNIA. 〈PAS-P>. MD 2.8 (PAS). ML 2.7 (GS). Felt.

0.950 SANTA CRUZ ISLANDS REGION

1.1151 EASTERN NEW GUINEA REG., P.N.G. MW 5.4 (HRV).

Centroid, Moment Tensor (HRV): Centroid origin time

$06: 11: 24.8$; Lat $5.92 \mathrm{~S}$; Lon $147.67 \mathrm{E}$; Dep 133.0; Halfduration 1.1 sec; Principal axes (scale 10*\#17 Nm): (T) Val=1.46, $\mathrm{Plg}=58, \mathrm{Azm}=11$; (N) Val=0.09, PIg-28, Azm=160; (P) Val-1.56, Plg-14, Azm=257; Best double couple: Mo-1.5*10*\#17 Nm; NP1: Strike-20, Dip-39, S1ip-137; NP2 : Strike=145, Dip=65, Slip=59.

$\begin{array}{lllllll}06 & 25 & 13.9 * & 49.714 & \mathrm{~N} & 156.458 & \mathrm{E} \\ 07 & 08 & 11.5 & 23.653 & \mathrm{~N} & 94.140 & \mathrm{E} \\ 07 & 42 & 01.3 \& & 18.854 & \mathrm{~N} & 155.228 & \mathrm{~W} \\ 07 & 44 & 13.48 & 18.902 & \mathrm{~N} & 155.241 & \mathrm{~W} \\ 08 & 00 & 05.8 & 36.246 & \mathrm{~N} & 27.344 & \mathrm{E} \\ 09 & 16 & 57.6 \& & 18.890 & \mathrm{~N} & 155.249 & \mathrm{~W} \\ 09 & 30 & 21.3 \& & 18.891 & \mathrm{~N} & 155.247 & \mathrm{~W} \\ 09 & 46 & 13.5 \& & 33.992 & \mathrm{~N} & 116.274 & \mathrm{~W} \\ 09 & 53 & 54.16 & 37.644 & \mathrm{~N} & 119.029 & \mathrm{~W} \\ 10 & 12 & 57.6 & 39.571 & \mathrm{~N} & 15.687 & \mathrm{E} \\ 10 & 27 & 25.2 & 1.045 & \mathrm{~N} & 120.112 & \mathrm{E} \\ 10 & 35 & 00.98 & 62.739 & \mathrm{~N} & 150.810 & \mathrm{~W} \\ 10 & 40 & 43.6 & 1.006 & \mathrm{~N} & 120.196 & \mathrm{E}\end{array}$

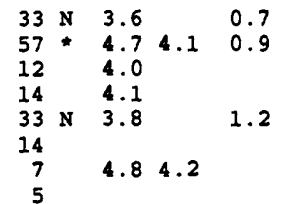

4 261
33
99 99 $\begin{array}{llll}33 & \mathrm{~N} \quad 5.5 & 5.5 \quad 1.1 & 18\end{array}$

8 KORII ISLANDS

MYAMMAR-INDIA BORDER REGION

6 HAWAII. 〈HVO-P>. MD 4.3 (HVO).

5 HAWAIT <HVO-P>. MD 4.3 (HVO).

DODECANESE ISLANDS

4 HAWAII. <HVO-P>. MD 3.7 (HVO).
91 HAWAII. 〈HVO-P>. MD 4.9 (HVO). SOUTHERN CALIFORNIA. <PAS-P>. MD 3.4 (PAS). ML 3.2 (GS) Felt.

7 CENTRAL CALIFORNIA. 〈GM-P>. MD 2.8 (GM).

1 SOUTHERN ITALY

2 MINAHASSA PENINSULA, SULAWESI

67 CENTRAL ALASKA. CAEIC>.

MINAHASSA PENINSULA, SULAWESI. MW 6.0 (GS), 6.0 (HRV).

Moment Tensor (GS): Dep 5; Principal axes (scale 10**18 Nm) (T) Val=0.96, Plg=49, Azm=167; (N) Val=0.19, Plg-5, Azm=71; (P) Val=-1.15, Plg=40, Azm=336; Best double couple:

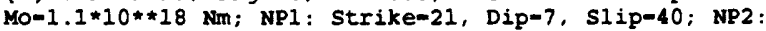
Strike-251, Dip-86, Slip-95.

Centroid, Moment Tensor (HRV): Centroid origin time

$10: 40: 49.3$; Lat $1.36 \mathrm{~N}$; Lon 120.35 E; Dep 20.0 Bdy; Halfduration 2.2 sec; Principal axes (scale 10**17 Nm): (T) Val=9.55, Plg=60, Azm=174; (N) Val=0.23, Plg=5, Azm=75; (P) Val=-9.78, Plg=30,Azm=342; Best double couple:

Mo-9.7*10*17 Nm; NP1: Strike-57, Dip-16, Slip-71; NP2: Strike=256, Dip=75, Slip=95.

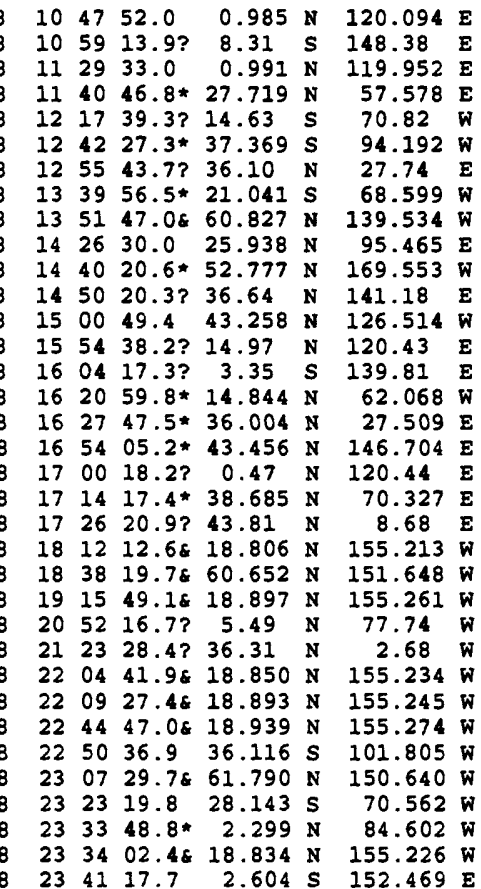

$33 \times 5.0$

$33 \mathrm{~N} 3.7$

$33 \mathrm{~N} 4.7$

$33 \mathrm{~N} 3.8$

$182 ? 3.7$

$10 \mathrm{G} \quad 4.4$

$33 \mathrm{~N} 3.8$

$126 ? 3.8$

15

$91 \mathrm{D} 4.5$

$33 \mathrm{~N} 3.9$

$33 \mathrm{~N}$

$10 \mathrm{G} \quad 3.1$

$33 \mathrm{~N} 3.8$

$33 \mathrm{~N} 4.0$

32 *

$33 \times 3.7$

$77 \cdot 4.4$

$33 \mathrm{~N} 4.3$

$33 \mathrm{~N} 3.6$

5 G

12

60

26

$10 \mathrm{G} \quad 3.9 \quad 1.2$

$10 \mathrm{G}$

12

13

15

10 G 4.5

46

$62 \mathrm{D} \quad 4.6$

$33 \mathrm{~N} 4.4$

12

$33 \times 5.15 .01 .0$

0.5

1.4

1.1

1.1

0.3
0.951 MINAHASSA PENINSULA, SULANES

1.26 EASTERN NEW GUINEA REG., P.N.G.

0.932 MINAHASSA PENINSULA, SULAWESI

0.810 SOUTHERN IRAN

1.213 CENTRAI PERU

0.822 WEST CHILE RISE

1.410 DODECANESE ISLANDS

1.015 CHILE-BOLIVIA BORDER REGION

SOUTHEASTERN ALASKA. <AEIC>. ML 3.3 (AEIC).

42 MYANMAR-INDIA BORDER REGION

0.98 FOX ISLANDS, ALEUTIAN ISILANDS

1.49 NEAR EAST COAST OF HONSHO, JAPAN

0.745 OFF COAST OF OREGON

1.47 LUZON, PHILIPPINE ISLANDS

1.58 IRIAN JAYA, INDONESIA

8

WINDWARD ISLANDS. MD 3.7 (TRN). Felt (II) on Martinique. DODECANESE ISLANDS

37 KURIL ISLANDS

10 MINAHASSA PENINSULA, SULAWESI

AFGHANISTAN-TAJIKISTAN BORD REG.

CORSICA. MI 2.2 (GEN)

3 HAWAII. <HVO-P>. MD 4.3 (HVO)

74 KENAI PENINSULA, ALASKA. <AEIC>. ML 3.4 (AEIC), 3.2 (PMR).

2 HAWAII. 〈HVO-P>. MD 3.8 (HVO)

NEAR WEST COAST OF COLOMBIA

STRAIT OF GIBRALTAR. MbLg 3.0 (MDD).

HAWAII. <HVO-P>. MD 4.0 (HVO).

3 HAWAII. 〈HVO-P>. MD 4.3 (HVO).

2 HAWAII. <HVO-P>. ND 4.1 (HVO).

23 SOUTHERN PACIFIC OCEAN

66 SOUTHERN ALASKA. CAEIC>. MI 3.1 (AEIC).

39 CENTRAL CHILE

20 OFF COAST OF CENTRAL AMERICA

2 HAWAII. <HVO-P>. MD 3.8 (HVO)

59 NEW IRELAND REGION, P.N.G. MW 5.4 (HRV).

Centroid, Moment Tensor (HRV): Centroid origin time

23:41:19.5; Lat $2.83 \mathrm{~s}$; Lon 152.90 E; Dep 29.5; Half-

duration 1.1 sec; Principal axes (scale 10**17 Nm): (T)

Val-1.31, Plg=56, Azm=285; (N) Val=-0.05, Plg-24, Azm=154;

(P) Val--1.26, Plg-23, Azm=54; Best double couple:

NO-1.3*10*\#17 Nm; NPI: Strike-106, Dip-31, S1ip=37; NP2:

Strike=343, Dip=72, Slip=115. 
$\begin{array}{llllll}29 & 00 & 28 & 13.2 ? & 1.93\end{array}$ $2900 \quad 29 \quad 26.38 \quad 44.481 \mathrm{~N}$ $\begin{array}{llllll}9 & 00 & 47 & 37.5 & 54.028 \mathrm{~N}\end{array}$ $\begin{array}{lllll}29 & 01 & 48 & 57.8 & 41.824\end{array}$

$\begin{array}{llllll}29 & 01 & 52 & 11.3 ? & 21.28 \quad \mathrm{~s}\end{array}$ $\begin{array}{llllll}29 & 02 & 13 & 39.0 ? & 1.28 \quad \mathrm{~N}\end{array}$ $\begin{array}{lllllll}29 & 03 & 18 & 22.38 & 18.750 & \mathrm{~N}\end{array}$ $\begin{array}{lllllll}29 & 03 & 19 & 58.98 & 18.866 & \mathrm{~N}\end{array}$ $903 \quad 3410.2818 .952 \mathrm{~N}$ $042513.8 * 37.181 \mathrm{~N}$ $\begin{array}{lllllll} & 04 & 38 & 38.7 ? & 6.06 & \mathrm{~S}\end{array}$ $\begin{array}{lllllll}9 & 04 & 49 & 40.38 & 18.867 \mathrm{~N}\end{array}$ $9 \quad 04 \quad 58 \quad 39.6 \& 59.775 \mathrm{~N}$ $\begin{array}{lllll}05 & 58 & 49.4 & 44.344 & N\end{array}$ $\begin{array}{lllll}06 & 08 & 03.15 & 18.820 \mathrm{~N}\end{array}$ $\begin{array}{llll}06 & 32 & 22.28 & 18.856 \mathrm{~N}\end{array}$ $0655 \quad 55.8 \& 18.900 \mathrm{~N}$ $\begin{array}{llllll}07 & 18 & 49.5 \& & 63.251 & \mathrm{~N}\end{array}$ $\begin{array}{llllll}07 & 24 & 04.08 & 18.914 & \mathrm{~N}\end{array}$ $080525.5 * 60.405 \mathrm{~s}$ $\begin{array}{lllll}08 & 20 & 53.48 & 18.893 \mathrm{~N}\end{array}$ $\begin{array}{lll}08 & 31 & 03.58 \\ 0 & 18.857 \mathrm{~N}\end{array}$ 083206.1 * $12.901 \mathrm{~N}$

$\begin{array}{llll}09 & 03 & 29.8 \& \quad 18.896 \mathrm{~N}\end{array}$ $094722.0 * 60.017 \mathrm{~s}$ $095120.7 ? 50.09$ N $\begin{array}{lll}10 & 56 & 09.9 * 55.780 \mathrm{~s}\end{array}$ $110032.5 \% 18.891 \mathrm{~N}$ $\begin{array}{lllll}11 & 13 & 07.98 & 18.936 & \mathrm{~N}\end{array}$ $113653.9 * 32.761 \mathrm{~s}$ $132550.0 * 18.056 \mathrm{~N}$ $\begin{array}{lllll}13 & 32 & 29.4 ? & 34.81 \mathrm{~S}\end{array}$ $\begin{array}{llll}13 & 46 & 45.96 & 18.842 \mathrm{~N}\end{array}$ $\begin{array}{lllll}14 & 28 & 35.56 & 18.872 \mathrm{~N}\end{array}$ $\begin{array}{llll}14 & 44 & 51.0 & 24.382\end{array}$ 151210.7 * $31.419 \mathrm{~S}$ $\begin{array}{llll}15 & 34 & 30.7 & 46.018\end{array}$ $\begin{array}{llll}16 & 05 & 11.9 * 13.896 \mathrm{~N}\end{array}$ $161432.4 * 48.374 \mathrm{~s}$ $\begin{array}{lllll}16 & 53 & 21.8 ? & 10.27 \mathrm{~S}\end{array}$ $\begin{array}{llll}17 & 27 & 58.5 & 7.475\end{array}$ $\begin{array}{lllll}17 & 33 & 42.6 & 21.863 \mathrm{~s}\end{array}$ $175716.5 * 31.530 \mathrm{~s}$ $\begin{array}{llll}19 & 1722.6 ? \quad 2.70 \quad \mathrm{~N}\end{array}$

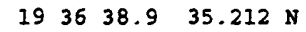
$200005.3 * 51.520 \mathrm{~N}$ $\begin{array}{lllll}20 & 03 & 37.8 & 50.863 \mathrm{~N}\end{array}$ $\begin{array}{lllll}20 & 12 & 32.38 & 18.906 & \mathrm{~N}\end{array}$ $\begin{array}{llll}20 & 20 & 52.9 & 24.602\end{array}$
80.63 $7.260 \mathrm{E}$ $109.227 \mathrm{~B}$ $88.420 \mathrm{E}$

178.72 $125.73 \mathrm{~B}$ $155.205 \mathrm{~h}$ $155.237 \mathrm{~W}$ 155.285 $72.157 \mathrm{E}$ 154.86 155.244 W $153.476 \mathrm{~W}$ $9.800 \mathrm{E}$ $155.215 \mathrm{~W}$

$155.242 \mathrm{~W}$ $155.265 \mathrm{~W}$ $149.752 \mathrm{~W}$ 155.254 27.973 h 155.244 พ $155.233 \mathrm{~W}$ $88.824 W$

155.247 พ $159.789 \mathrm{E}$ 176.40 W $27.924 \mathrm{~W}$ $155.259 \mathrm{~W}$ 155.284 W $71.685 \mathrm{~W}$ $145.667 \mathrm{E}$ $71.11 \mathrm{~W}$ $155.228 \mathrm{~W}$ $155.239 \mathrm{~W}$ $127.403 \mathrm{E}$ $69.546 \mathrm{~W}$ $6.016 \mathrm{E}$ $145.150 \mathrm{E}$ $31.120 \mathrm{E}$ 151.63 $126.858 \mathrm{E}$ 178.283 ผ 68.893 W 126.56 E $136.400 \mathrm{E}$ 176.494 $175.980 \mathrm{E}$ $155.264 \mathrm{~W}$ $122.282 \mathrm{E}$
$33 \times 4.0$

14 .

33 N 4.1

0 G 4.9

$613 ? 4.0$

$33 \mathrm{~N} 3.9$

10

133.8

15

$200 \mathrm{G} 3.6$

$33 \mathrm{~N} 4.0$

13

$27 \quad 3.8$

$5 \mathrm{G}$

13

14

100

12
33

$33 \mathrm{~N} 4.0$

13

$\begin{array}{ll}13 & 3.8\end{array}$

13

$10 \mathrm{G} 4.5$

$33 \mathrm{~N} 3.4$

$33 \mathrm{~N} 4.6$

134.4

15

$50 \mathrm{G}$

204 D 4.1

$90 \mathrm{G}$

12

13

$33 \mathrm{~N}$
$180 \mathrm{G}$

$10 \mathrm{G}$

$142 \quad 4.0$

$10 \mathrm{G} 4.5$

$33 \mathrm{~N} 3.7$

$33 \mathrm{~N} 4.5$

$331 \star 4.7$

$135 \star 4.2$

$33 \times 4.0$

$33 \mathrm{~N} 3.5$

$33 \mathrm{~N} 4.1$

$33 \mathrm{~N} 3.9$

14

$74 \mathrm{D} 5.2$

Province. Also felt (III) at Mendoza.

1.315 OFF COAST OF ECUADOR

0.210 NORTHERN ITALY. ML 2.3 (GEN).

0.715 LAKE BAYKAL REGION, ROSSIA

0.797 SOUTHERN XINJIANG, CHINA. Probable underground nuclear explosion.

0.614 FIJI ISLANDS REGION

1.16 NORTHERN MOLUCCA SEA

1 HAWAII, <HVO-P>. YD 3.7 (HVO).

10 HAWAII. <HVO-P>. MD 4.3 (HVO).

1 HAWAII. <HVO-P>. ND 3.9 (HVO).

0.98 TAJIKISTAN

0.98 SOLOMON ISLANDS

1 HAWAIT. 〈HVO-P>. MD 3.9 (HVO)

108

NOUTHERN ALASKA. CAEIC> (GEN).

2 HAWAII. <HVO-P>. MD 3.8 (HVO)

HAWAII. 〈HVO-P>. MD 3.9 (HVO)

HAWAII. 〈HVO-P>. MD 3.8 (HVO)

6 CENTRAL ALASKA. LAEIC>.

1 HAWAII. 〈HVO-P>. MD 3.8 (HVO)

0.87 SOUTH SANDWICH ISLANDS REGION

1 HAWAII. 〈HVO-P>. MD 3.8 (HVO).

4 HAWAII. <HVO-P>. MD 4.3 (HVO)

0.916 OFF COAST OF CENTRAL AMERICA. MD 3.6 (SSS). Felt (II) at San Salvador, El Salvador.

1 HAWAII. <HVO-P>. MD 3.9 (HVO)

0.94 ANDREANOF ISLANDS, ALEUTIAN IS

0.813 SOUTH SANDWICH ISLANDS REGION

25 HAWAII. 〈HVO-P>. MD 4.5 (HVO)

1 HAWAII. 〈HVO-P>. MD 3.9 (EVO).

0.411 NEAR COAST OF CENTRAL CHILE. MD 4.0 (SAN)

0.918 MARIANA ISLANDS

0.211 NEAR COAST OF CENTRAL CHILE. MD 2.6 (SAN).

HAWAII. <HVO-P>. MD 4.3 (HVO).

2 HAWAII. <HVO-P>. MD 4.0 (HVO)

0.822 SOUTHEAST OF RYUKYU ISLANDS

0.411 SAN JOAN PROVINCE, ARGENTINA. MD 4.1 (SAN).

$0.8 \quad 17$ SWITZERLAND. MI 2.8 (STR).

0.916 MARIANA ISLANDS

1.199 SOUTH OF AFRICA

1.48 D'ENTRECASTEAUX ISLANDS REGION

0.827 MINDANAO, PHILIPPINE ISLANDS

0.982 FIJI ISLANDS REGION

0.919 SAN JOAN PROVINCE, ARGENTINA. MD 4.3 (SAN)

0.810 NORTHERN MOLUCCA SEA

1.313 WESTERN HONSHU, JAPAN

1.211 RAT ISLANDS, ALEUTIAN ISLANDS

0.618 RAT ISLANDS, ALEUTIAN ISLANDS

$1.1 \quad 153$

TAIWAN REGION, 5.5 (HRV). Felt on Taiwan.

Centroid, Moment Tensor (HRV): Centroid origin time 20:20:55.6; Lat $24.44 \mathrm{~N}$; Lon $122.22 \mathrm{E}$; Dep 67.9 ; Half-

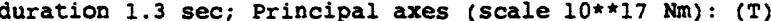
Val=1.77, Plg=57, $A z m=21$; (N) Val $=0.00, P l g=32, A z m=207$ (P) Val=-1.77, Plgm, Azm-116; Best double couple:

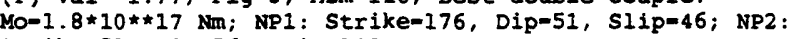
Strike-53, Dip=56, Slip-130.

$21 \quad 1731.8659 .498 \mathrm{~N}$ $\begin{array}{lllll}23 & 15 & 04.68 & 18.925 \mathrm{~N}\end{array}$ $001512.9 * 0.997 \mathrm{~N}$ $\begin{array}{llllll}00 & 21 & 03.1 ? & 11.53 & \mathrm{~N} \\ 00 & 22 & 52.4 * & 7.115 & \mathrm{~S}\end{array}$ $\begin{array}{llll}0055 & 06.2 & 6.792 \mathrm{~S}\end{array}$ $010402.6 ? \quad 34.56$ s $01 \quad 1744.0 * 13.422 \mathrm{~S}$ $012505.8836 .192 \mathrm{~N}$ $015917.7 \& 18.933 \mathrm{~N}$ $0208 \quad 18.98 \quad 36.353 \mathrm{~N}$ $\begin{array}{lllll}02 & 14 & 38.58 & 18.878 \mathrm{~N}\end{array}$ $\begin{array}{lllll}02 & 43 & 54.5 & 6.891 \mathrm{~S}\end{array}$ $\begin{array}{lllll}02 & 57 & 47.78 & 36.114 \mathrm{~N}\end{array}$ $030111.9 * 41.945 \mathrm{~N}$ $\begin{array}{llll}03 & 07 & 05.8 * 43.657 \mathrm{~N}\end{array}$ $\begin{array}{llll}03 & 45 & 22.5 \& \quad 18.891 \mathrm{~N}\end{array}$ $\begin{array}{lllll}04 & 09 & 01.2 & 39.450 \mathrm{~N}\end{array}$ $\begin{array}{llllll}04 & 28 & 04.88 & 44.422 & \mathrm{~N}\end{array}$ $\begin{array}{llllll}04 & 28 & 55.5 & 43.817 & \mathrm{~N}\end{array}$ $\begin{array}{lll}06 & 37 & 08.0 ? \quad 32.46\end{array}$

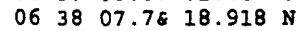
$065021.0861 .514 \mathrm{~N}$ $\begin{array}{llllll}06 & 58 & 12.1 \text { ? } & 34.97 & \mathrm{~S} \\ 07 & 45 & 56.08 & 36.233\end{array}$ $\begin{array}{lllll}08 & 32 & 13.38 & 36.247 & \mathrm{~N}\end{array}$ 0932 21.0? $48.99 \mathrm{~N}$ $094353.8 * 17.703 \mathrm{~s}$ $\begin{array}{llllll}10 & 14 & 32.7 ? 31.76 & s\end{array}$ $\begin{array}{lllll}11 & 04 & 02.2 * 51.921 & \mathrm{~N}\end{array}$ $114819.6 * 3.876 \mathrm{~s}$ $114831.8 * 51.697 \mathrm{~N}$ $\begin{array}{llllll}11 & 59 & 55.6 ? & 12.55 \mathrm{~N} \\ 13 & 05 & 43.28 & 62.400 \mathrm{~N}\end{array}$
152.575 ผ 155.258 $120.144 \mathrm{E}$ 86.04 W

$310 ? 4.7$ $70.33 \mathrm{~W}$ $167.298 \mathrm{E}$ $27.225 \mathrm{E}$ $155.275 \mathrm{~W}$ $27.196 \mathrm{E}$ $155.254 \mathrm{~W}$ $129.527 \mathrm{E}$ $27.280 \mathrm{E}$ $146.128 \mathrm{E}$ $85.145 \mathrm{E}$ $155.255 \mathrm{~W}$ $25.769 \mathrm{E}$ $8.620 \mathrm{E}$ $84.636 \mathrm{E}$ $71.96 \mathrm{~W}$ $155.255 \mathrm{~W}$ $147.874 \mathrm{~W}$ 71.19 $27.194 \mathrm{E}$ 27.194
155.85 $178.848 \mathrm{~W} 530 \star 4.4$ $69.63 \mathrm{~W} 170 \mathrm{G}$ $14.254 \mathrm{E} \quad 10 \mathrm{G}$ $141.404 \mathrm{E} 113 \star 4.4$ $14.677 \mathrm{E} \quad 10 \mathrm{G}$ 87.16 W $33 \mathrm{~N} 4.1$ $151.390 \mathrm{~W}$

77

$14 \quad 3.5$

$33 \mathrm{~N} 4.7$

$10 \mathrm{G}$

$10 \mathrm{G}$

145

13

$120 ?$
5 G

$33 \mathrm{~N} 4.1$

$33 \mathrm{~N} 4.0$ 
$\begin{array}{llllllll}30 & 13 & 29 & 53.28 & 18.836 \mathrm{~N} & 155.226 \mathrm{~W}\end{array}$ $30 \quad 135555.4618 .900 \mathrm{~N} 155.250 \mathrm{~W}$ 30142516.4 ? 48.17 N 128.98 W $30 \quad 14 \quad 3959.98 \quad 44.077 \mathrm{~N}$ $30 \quad 16 \quad 05 \quad 25.3 * 28.477$ $30 \quad 1605 \quad 45.5 \quad 46.032$ $30 \quad 17 \quad 10 \quad 25.6 ? \quad 6.36 \quad \mathrm{~s}$ $\begin{array}{lllll}30 & 17 & 38 & 30.7 & 14.509 \mathrm{~N}\end{array}$ $\begin{array}{cc}128.98 & \mathrm{~W} \\ 7.090 \mathrm{E}\end{array}$

$113.134 \mathrm{~V}$

$10.818 \mathrm{E}$

76.96 W

$119.954 \mathrm{E}$
124.3

$10 \mathrm{G} 4.3$

$10 \mathrm{G} \quad 3.3$

$10 \mathrm{G}$

$10 \mathrm{G} \quad 4.0$

$10 \mathrm{G}$

$\begin{array}{lll}153 & ? & 3.7\end{array}$

$33 \times 6.15 .7 \quad 1.3 \quad 13$

4

4 HAWAII <HVO-P) MD 3.8 (HVO).

$\begin{array}{lrl} & 24 & \text { HAWAII. CSPEC>. MD } 4.6 \text { (HVO) } \\ 0.6 & 7 & \text { VANCOUVER ISLAND REGION }\end{array}$

0.15 NORTHERN ITALY. ML 2.2 (GEN).

0.1 21 BATA CALIFORNIA MTXICO

NORTHERN ITALY. ML 2.5 (VIE).

NORTHERN ITALY
NORTHERN PERO

LUZON, PHILIPPINE ISLANDS. WW 6.1 (GS), 6.0 (HRV). Me 6.1 (GS). MS 5.6 (BRK). Some damage in the epicentral area.

Felt at Angeles, Baguio, Manila, Olongapo and Tagaytay.

Broadband Source Parameters (GS): Dep 32; NP1: Strike-230, Dip-40, Slip=140; NP2: Strike-353, Dip=66, Silp=57;

Radiated energy $2.8 * 10 * \pi 13 \mathrm{Nm}$. Two events about 5 seconds apart. Depths 32 and $28 \mathrm{~km}$, respectively.

Moment Tensor (GS): Dep 36; Principal axes (scale 10*\#18

$\mathrm{Nm}$ ): (T) Val=1.25, Plg=70, Azm=270; (N) Val=0.21, Plg=0, Azm-1; (P) Val=-1.46, P1g=20, Azm=91; Best double couple: Mo-1.4*10**18 Nm; NP1: Strike-182, Dip=25, SIip-91; NP2: Strike-1, Dip $=65$, slip $=90$.

Centroid, Moment Tensor (HRV): Centroid origin time

$17: 38: 34.3$; Lat $14.56 \mathrm{~N}$; Lon $119.87 \mathrm{E}$; Dep $43.0 \mathrm{Bdy}$; Half duration $2.4 \mathrm{sec}$; principal axes (scale 10*\#18 Nm): (T) Val-1.26, Plg=76, Azm-294; (N) Val=-0.05, Plg=5, Azm-185; (P) Val-1.21, Plg-13, Azm-94; Best double couple:

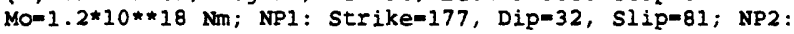
Strike=8, Dip-58, Slip=96.

Scalar Moment (PPT): Mo-1.5*10*\#18 Nm.

$\begin{array}{llllll}30 & 1800 & 26.48 & 34.131 \mathrm{~S}\end{array}$

$30 \quad 18 \quad 17 \quad 07.2 * 8.926 \mathrm{~s}$

$\begin{array}{lllllll}30 & 18 & 23 & 17.5 ? & 23.99 & \mathrm{~N}\end{array}$

$\begin{array}{lllllll}30 & 19 & 10 & 36.6 ? & 10.83 & \mathrm{~N}\end{array}$

$\begin{array}{llllll}30 & 19 & 14 & 02.6 & 39.248 \mathrm{~N}\end{array}$

$\begin{array}{llllllllll}30 & 19 & 43 & 07.4 ? & 36.30 & \mathrm{~N}\end{array}$

$\begin{array}{lllllll}30 & 19 & 54 & 28.58 & 10.938 & N\end{array}$

$30201422.8 * 25.785 \mathrm{~N}$

$\begin{array}{lllllll}30 & 21 & 39 & 11.9 & 34.74 \mathrm{~N}\end{array}$

$\begin{array}{llllllll}30 & 21 & 46 & 09.4 & 38.819 & \mathrm{~N}\end{array}$

$\begin{array}{lllllll}30 & 21 & 59 & 32.4 \& \quad 18.781\end{array}$

$30 \quad 215954.7$ ? $14.65 \mathrm{~s}$

$\begin{array}{lllllll}30 & 22 & 08 & 01.68 & 36.213 & \mathrm{~N}\end{array}$

$\begin{array}{lllllll}30 & 22 & 41 & 45.6 ? & 59.48 \quad \mathrm{~N}\end{array}$

$\begin{array}{lllllll}30 & 22 & 43 & 37.5 * 59.918 & N\end{array}$

$\begin{array}{llllll}30 & 22 & 58 & 19.88 & 44.720 & \mathrm{~N}\end{array}$

$30 \quad 234151.5 * 29.071 \mathrm{~s}$

$\begin{array}{lllllll}31 & 00 & 06 & 27.88 & 18.898 & \mathrm{~N}\end{array}$

$\begin{array}{llllllll}31 & 00 & 07 & 44.08 & 36.239 & \mathrm{~N}\end{array}$

$\begin{array}{lllllll}31 & 00 & 15 & 01.3 & 51.582 & \mathrm{~N}\end{array}$

$\begin{array}{llllll}31 & 00 & 32 & 15.9 & 37.631 & \mathrm{~N}\end{array}$

$\begin{array}{llllll}31 & 02 & 19 & 17.0 \quad 3.820 \mathrm{~s}\end{array}$ $\begin{array}{lllllll}30 & 21 & 10 & 59.08 & 33.142 & \mathrm{~S}\end{array}$

$\begin{array}{llllll}30 & 23 & 30 & 00.3 ? & 36.12 \mathrm{~N}\end{array}$

$310113 \quad 31.7 * 41.958$
$70.993 \mathrm{~W}$ 109.358 $121.98 \mathrm{E}$ 62.15 W $22.065 \mathrm{E}$ $27.15 \mathrm{E}$ $61.665 \mathrm{~W}$ $124.225 \mathrm{E}$ 70.295 $22.96 \mathrm{E}$ $20.705 \mathrm{E}$ $155.226 \mathrm{~W}$ $167.90 \mathrm{E}$ $27.199 \mathrm{E}$ 30.42 W $30.332 \mathrm{~W}$ $6.595 \mathrm{E}$ $27.13 \mathrm{E}$ $13.010 \mathrm{~W}$ $155.255 \mathrm{~W}$ $27.337 \mathrm{E}$ $16.157 \mathrm{E}$ $143.022 \mathrm{~B}$ $125.928 \mathrm{~W}$ $151.305 \mathrm{E}$
$80 \mathrm{G}$

$10 \mathrm{G} 4$.

$89 ? 4.0$

$66 ?$

$10 \mathrm{G}$

$5 \mathrm{G}$

33

$205 ? \quad 3.7$

10

$5 \mathrm{G}$

$10 \mathrm{G} \quad 4.0$

11

$33 \mathrm{~N} 4.1$

$5 \mathrm{G}$

$10 \mathrm{G} \quad 3.8$

$10 \mathrm{G} \quad 3.8$

11

$10 \mathrm{G}$

10

$33 \mathrm{~N}$

$10 \mathrm{G} 3$.

$33 \mathrm{~N} 4.1$

10 G 3.2

$\begin{array}{llll}10 \mathrm{G} & 3.2 & & 1.3 \\ 33 \mathrm{~N} & 5.1 & 5.2 & 1.1\end{array}$
$3102 \quad 2735.0 * \quad 3.735$ s $150.995 \mathrm{E}$ $\begin{array}{lllllll}31 & 02 & 34 & 38.9 & 39.568 \mathrm{~N} & 23.537 \mathrm{E}\end{array}$ $\begin{array}{lllllll}31 & 03 & 20 & 51.9 & 41.954 & \mathrm{~N} & 23.194 \mathrm{E}\end{array}$ $310347 \quad 31.98 \quad 60.449$ N 151.997 $31040613.1 * 3.783 \mathrm{~s} 151.507 \mathrm{E}$

$\begin{array}{rll}33 & N & 4.4 \\ 5 & G & \\ 10 & G & \\ 85 & & \end{array}$

33 N 4.9
0.6

1.1

1.5

0.3

0.7

0.6

0.4

0.7

0.5

1.4

1.0

0.6

0.4

1.5

0.2

1.5

0.3

0.7

0.7
9 CHILE-ARGENTINA BORDER REGION

CENTRAI EAST PACIFIC RISE

7 TAIWAN

NEAR COAST OF VENEZUELA

9 GREECE. MD 3.0 (ATH)

4 DODECANESE ISLANDS. MD 3.6 (ATH).

5 TRINIDAD

13 NORTHEAST OF TAIWAN

CHILE-ARGENTINA BORDER REGION. MD 3.0 (SAN).

4 CENTRAL MEDITERRANEAN SEA. MD 3.6 (ATH)

40 GREECE. ML 3.9 (ATH)

1 HAWAII. <HVO-P>. MD 3.7 (HVO).

7 VANOATU ISLANDS

5 DODECANESE ISLANDS. MD 3.6 (ATH).

5 NORTH ATLANTIC OCBAN

8 NORTH ATLANTIC OCEAN

11 FRANCE. ML 2.4 (GEN)

4 DODECANESE ISLANDS. MD 3.6 (ATH).

34 SOUTHERN MID-ATLANTIC RIDGE

HAWAII. 〈HVO-P>. MD 3.8 (HVO)

5 DODECANESE ISLANDS. MD 3.8 (ATH)

29 POLAND. MIL 4.0 (GRF), 3.9 (FUR), 3.8 (MOX).

20 OFF EAST COAST OF HONSHO, JAPAN

10 OFF COAST OF NORTHERN CALIFORNIA

42 NEW IRELAND REGION, P.N.G. NW 5.5 (HRV)

Centroid, Moment Tensor (HRV): Centroid origin time

$02: 19: 18.8$; Lat $3.72 \mathrm{~S}$; Lon $151.69 \mathrm{E}$; Dep 15.0 Fix; Halfduration 1.4 sec; Principal axes (scale 10**17 Nin): (T) Val=2.55, $P 1 g=21, A 2 m=337$; (N) Val--0.49, $P 1 g=69, A z m=155$; (P) Val-2.06, Plg=1, Azm-247; Best double couple: Mo=2.3*10*17 Nm; NP1: Strike-20, Dip=75, S1ip=165; NP2: Strike=114, Dip=76, Slip=16.

19 NEW IRELAND REGION, P.N.G.

19 NEW IRELAN REA ML 3.3 (ATH).

10 GREECE-BULGARIA BORDER REGION. ML 2.7 (SKO).

54 KENAI PENINSULA, ALASKA. <AEIC>.

28 NEW IRELAND REGION, P.N.G. MW 5.3 (HRV).

Centroid, Moment Tensor (BRV): Centroid origin time

$04: 06: 15.4$; Lat 4.50 S; Lon $151.19 \mathrm{E}$; Dep 15.0 Fix; Halfduration 1.0 sec; Principal axes (scale 10**17 Nm): (T) Val=1.12, Plg=35, Azm=317; (N) Val=-0.29, Plg=20, Azm=62 (P) Val=-0.84, Plg=48, Azm-175; Best double couple: MO $=9.8 * 10 * * 16 \mathrm{Nm}$; NP1: Strike-353, Dip=21, Slip=-160; NP2: Strike=244, Dip=83, Slip=-70.

$31041411.6 ? \quad 1.78 \quad \mathrm{~N}$ $\begin{array}{llllll}31 & 04 & 29 & 27.2 & 40.035 \mathrm{~N}\end{array}$ $\begin{array}{llllllll}31 & 04 & 36 & 42.8 \text { ? } 10.91 \mathrm{~N}\end{array}$ $\begin{array}{llllll}31 & 05 & 31 & 52.0 & 41.302 & \mathrm{~N}\end{array}$ $\begin{array}{llllll}31 & 05 & 38 & 55.78 & 18.922 & N\end{array}$ $\begin{array}{lllllll}31 & 05 & 44 & 31.18 & 18.899 & \mathrm{~N}\end{array}$ $\begin{array}{lllllll}31 & 05 & 48 & 24.0 & 3.839 & \mathrm{~N}\end{array}$ $3106 \quad 1202.3 * 30.471 \mathrm{~N}$ $3106 \quad 3959.2 * \quad 4.856 \mathrm{~s}$ $\begin{array}{llllll}31 & 06 & 57 & 31.7 ? \quad 44.47 \quad \mathrm{~N}\end{array}$ $\begin{array}{llllll}31 & 07 & 14 & 49.9 ? & 18.34 & S\end{array}$ 31074301.5 ? $10.55 \mathrm{~N}$ $\begin{array}{llllll}31 & 08 & 00 & 27.5 & 30.174 & \mathrm{~N}\end{array}$
99.59 E $149 ? 4.0$ $20.670 \mathrm{E}$ $84.91 \mathrm{~W}$ $14.381 \mathrm{E}$ $155.272 \mathrm{~F}$ $155.260 \mathrm{~W}$ $31.533 \mathrm{~W}$ $140.775 \mathrm{E}$ $151.537 \mathrm{E}$ $7.32 \mathrm{E}$ 174.65
61.76 $88.178 \mathrm{E}$

149
$5 \mathrm{G}$
$100 \mathrm{G}$
25
14
14
$10 \mathrm{G}$
93
$166 ?$
$10 \mathrm{G}$
$33 \mathrm{G}$
$10 \mathrm{G}$
33

4.2

4.5

4.3 $\begin{array}{lllllll}31 & 08 & 02 & 52.7 & 30.111 & \mathrm{~N}\end{array}$ $31 \quad 08 \quad 20 \quad 11.48 \quad 47.804 \quad \mathrm{~N}$
$88.143 \mathrm{E}$ $8.024 \mathrm{E}$
4.0

0.6

0.8

1.2

7 GREECE-ALBANIA BORDER REGION. MD 3.2 (ATH)

13 COSTA RICA

41 SOUTHERN ITALY. ML 3.2 (ROM)

HAWAII. 〈HVO-P>. MD 3.8 (HVO)

3 HAWAII. 〈HVO-P>. MD 4.0 (HVO)

$\begin{array}{llll}4 & 4.6 & 4.3 & 0.9\end{array}$

0.8

1.0

0.1

52 CENTRAL MID-ATLANTIC RIDGE

10 SOUTH OF HONSHO, JAPAN

20 NEW BRITAIN REGION, P.N.G.

4 NORTHERN ITALY. MI 1.8 (GEN).

12 TONGA ISLANDS

4 TRINIDAD
Centroid, Moment Tensor (HRV): Centroid origin time

08:00:34.0; Lat $29.74 \mathrm{~N}$; Lon $88.67 \mathrm{E}$; Dep $15.0 \mathrm{Bdy}$; Half duration $1.0 \mathrm{sec}$; Principal axes (scale 10*17 Nm): (T) val=1.78, Plg=21, Azm-258; (N) val=0.02, $P 1 g=23, A z m=159$; (P) Val=-1.80, Plg-58, Azm=26; Best double couple: Mo-1.8*10**17 Nm; NP1: Strike-23, Dip-32, slip=-41; NP2: Strike=150, Dip=70, Slip=-115.

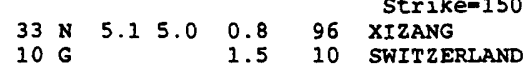


$31 \quad 09 \quad 18 \quad 30.98 \quad 36.252$ $\begin{array}{lllll}09 & 23 & 41.0 & 36.194\end{array}$ $0936 \quad 08.58 \quad 36.298$ $\begin{array}{lllll}09 & 42 & 00.58 & 36.275\end{array}$ $\begin{array}{llll}10 & 14 & 14.58 & 36.176 \mathrm{~N}\end{array}$ $\begin{array}{lllll}10 & 19 & 33.1 & 2.731 \mathrm{~N}\end{array}$

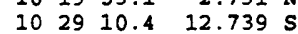

$27.146 \mathrm{E} \quad 5 \mathrm{G}$ $27.225 \mathrm{E} \quad 33$ $27.180 \mathrm{E} \quad 5 \mathrm{G}$ $27.159 \mathrm{E} \quad 33 \mathrm{~N}$ $27.163 \mathrm{E} \quad 5 \mathrm{G}$ $128.616 \mathrm{E} 250 \mathrm{G}$ $66.307 \mathrm{E}$
0.1

1. 3

0.1

1.0

0.8 $250 \mathrm{G} \quad 4.5$ $\begin{array}{ll}4.5 & \\ 5.1 & 4.9\end{array}$

5 DODECANESE ISLANDS. MD 3.9 (ATH)

54 DODECANESE ISLANDS. ML 4.5 (ATH)

5 DODECANESE ISLANDS. MD 3.7 (ATH).

6 DODECANESE ISLANDS. ND 3.9 (ATH).

5 DODECANESE ISLANDS. MD 3.7 (ATH)

27 HALMAHERA, INDONESIA

164 MID-INDIAN RIDGE. NW 5.2 (HRV).

Centroid, Moment Tensor (HRV): Centroid origin time

$10: 29: 15.4$; Lat $12.86 \mathrm{~S}$; Lon $66.10 \mathrm{E}$; Dep $15.0 \mathrm{Fix}$; Halfduration 1.1 sec; Principal axes (scale 10**16 Nm): (T) Val-7.56, Plg-0, Azm-216; (N) Val-0.35, Plg=0, Azm-126 (P) Val--7.21, Plg-90, Azm-180; Best double couple: MO-7.4*10**16 Nm; NP1: Strike-306, Dip-45, slip--90; NP2: Strike-126, Dip-45, Slip--90.

$152.855 \mathrm{~W}$ $142.688 \mathrm{E}$

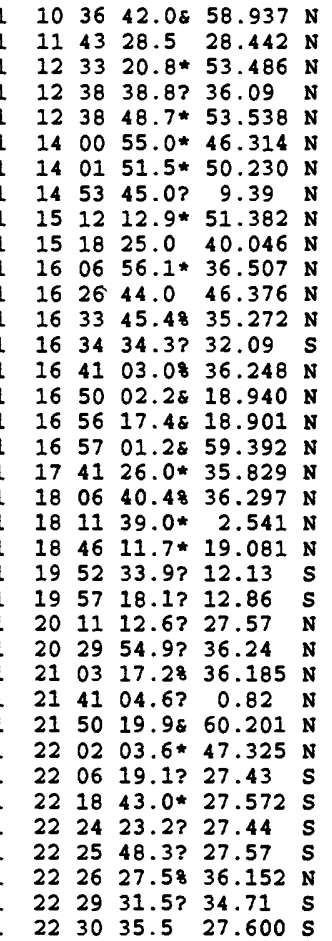
$165.718 \mathrm{~W}$ $141.56 \mathrm{E}$ $165.722 \mathrm{~W}$ $153.063 \mathrm{E}$ $18.985 \mathrm{E}$ $141.33 \mathrm{E}$ $16.173 \mathrm{E}$ $20.720 \mathrm{E}$ $71.029 \mathrm{E}$ $7.389 \mathrm{E}$ $26.978 \mathrm{E}$ $71.49 \mathrm{~W}$ $27.226 \mathrm{E}$ $155.267 \mathrm{~W}$ 155.256 W $152.948 \mathrm{~W}$ $70.707 \mathrm{E}$ $27.198 \mathrm{E}$ $126.953 \mathrm{E}$ $39.160 \mathrm{E}$ $66.11 \mathrm{E}$ $66.27 \quad \mathrm{E}$ 140.58 $27.14 \quad \mathrm{E}$ 27.089 $16.21 \mathrm{~W}$ 153.483 พ $152.179 \mathrm{E}$ $65.50 \mathrm{E}$ $65.447 \mathrm{E}$ $65.49 \quad \mathrm{~B}$ 65.88 27.129 70.48 K $\begin{array}{ll}68 & 3.0\end{array}$

$33 \mathrm{~N} 4.2$

$33 \mathrm{~N} 3.8$

$10 \mathrm{G}$

$33 \mathrm{~N} 4.2$

$33 \mathrm{~N}$
$10 \mathrm{G}$

$10 \mathrm{G}$

$33 \mathrm{~N}$

$10 \mathrm{G}$
$211 ?$

$211 ?$
$5 \mathrm{G}$

$5 \mathrm{G}$

10

5

24

14

99

$110 \mathrm{D}$
$5 \mathrm{G}$

$\begin{array}{rr}5 & G \\ 91 & D\end{array}$

$\begin{array}{lll}91 & \mathrm{D} & 4.4 \\ 10 & \mathrm{G} & 4.1\end{array}$

$10 \mathrm{G} 3.8$

10 G 4.0

$300 \mathrm{G} 3.4$

$5 \mathrm{G}$

$10 \mathrm{G}$

$10 \mathrm{G} \quad 3.7$

137

$33 \mathrm{~N} 3.8$

$10 \mathrm{G} 4.3$

$10 \mathrm{G} 4.1$

$10 \mathrm{G} \quad 4.3$

$10 \mathrm{G} 4.3$

$5 \mathrm{G}$

$115 \mathrm{G}$

0.6

.21 .2

$\begin{array}{lll}74.2 & 1.1\end{array}$

$.3 \quad 0.3$
$4 \quad 0.8$

0.5

1.3

1.3

0.8
0.9

0.9
0.5

0.4

1.3

4.1

15 FOX ISLANDS, ALEUTIAN ISLANDS

5 NEAR EAST COAST OF HONSHU, JAPAN

6 FOX ISLANDS, ALEUTIAN ISLANDS

55 KORIL ISLANDS

POLAND. MG 2.8, (WAR).

WESTERN CAROLINE ISLANDS

POLAND. ML 2.6 (MOX), 2.4 (CLL)

GREECE-ALBANIA BORDER REGION. MD 3.1 (ATH).

2 AFGHANISTAN-TAJIKISTAN BORD REG.

39 SWITZERLAND. ML 3.0 (IDG).

5 CRETE. MD 3.9 (ATH).

10 NEAR COAST OF CENTRAL CHILE. MD 3.8 (SAN).

6 DODECANESE ISLANDS. MD 3.9 (ATH) .

HAWAII. <HVO-P>. MD 3.7 (EVO).

HAWAII. <HVO-P>. MD 4.3 (EVO).

41 SOUTHERN ALASKA. <AEIC>.

20 HINDU KUSH REGION, AFGHANISTAN

6 DODECANESE ISLANDS. MD 4.0 (ATH).

23 NORTHERN MOLUCCA SEA

20 RED SEA

7 MID-INDIAN RIDGE

14 MID-INDIAN RIDGE

BONIN ISLANDS REGION

4 DODECANESE ISLANDS. MD 4.0 (ATH).

7 DODECANESE ISLANDS. MD 3.9 (ATH).

5 NORTH OF ASCENSION ISLAND

67 SOUTHERN ALASKA. 〈AEIC>.

8 KURIL ISLANDS

9 SOUTH INDIAN OCEAN

10 SOUTH INDIAN OCEAN

SOUTH INDIAN OCEAN

SOUTH INDIAN OCEAN

6 DODECANESE ISLANDS. MD 4.0 (ATH).

6 CHILE-ARGENTINA BORDER REGION

232 SOUTH INDIAN OCEAN. NW 5.6 (HRV), 5.4 (GS).

Moment Tensor (GS): Dep 14: Principal axes (scale 10**17 $\mathrm{Nm}$ ): (T) Val=1.57, Plg=4, Azm=169; (N) Val-0.04, Plg=16, Azm-260; (P) Val--1.61, Plg=73, Azm-66; Best double couple: Mo=1.6*10*\$17 Nm; NP1: Strike=242, Dip-43, Slip--114; NP2: Strike=94, Dip-51, S1ip=-69.

Centroid, Moment Tensor (HRV): Centroid origin time

$22: 30: 42.7$; Lat $27.53 \mathrm{~s}$; Lon $65.34 \mathrm{E}$; Dep 15.0 Bdy; Half-

duration $1.5 \mathrm{sec}$; Principal axes (scale 10**17 $\mathrm{Nm}$ ): (T)

val=2.88, Plg=0, Azm-160; (N) Val=-0.18, Plg-8, Azm=250;

(P) Val--2.69, P1g=82, Azm=70; Best double couple:

Mo-2.8*10**17 Nm; NP1: Strike=242, Dip=46, Slip=-101; NP2: Strike-78, Dip-46, Slip- -79 .

$\begin{array}{lllll}31 & 22 & 34 & 49.1 \quad 51.609\end{array}$

$31 \quad 223923.8 * 21.337 \mathrm{~s}$

$\begin{array}{lllllll}31 & 22 & 54 & 37.5 ? & 27.72 \quad S\end{array}$

$\begin{array}{lllll}31 & 23 & 03 & 07.7 * 20.315 & \mathrm{~N}\end{array}$

$\begin{array}{llllllll}31 & 23 & 13 & 04.9 ? & 27.58 & \mathrm{~S}\end{array}$

$\begin{array}{llllll}31 & 23 & 19 & 45.88 & 37.533 & \mathrm{~N}\end{array}$

$\begin{array}{lllll}23 & 32 & 07.3 & 20.306 \mathrm{~N}\end{array}$

$31235939.9 * 27.634 \mathrm{~s}$

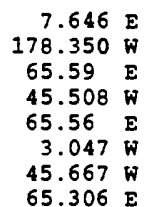

$10 \mathrm{G}$

$500 \mathrm{G} \quad 4.5$

$10 \mathrm{G} \quad 4.0$

$10 \mathrm{G} \quad 4.1$

$10 \mathrm{G} \quad 4.3$

$10 \mathrm{G}$

$10 \mathrm{G} \quad 4.6 \quad 4.4 \quad 0.8$

1.012 GERMANY. ML 2.3 (DBN).

0.827 FIJI ISLANDS REGION

0.7

1.416 SOUTH INDIAN OCEAN

1.26 SPAIN. mbLg 2.2 (MDD).

16 SOUTH INDIAN OCEAN
49 NORTHERN MID-ATLANTIC RIDGE

Compiled by Francis w. Baldwin, Pamela J. Benfield, Don L. Blakeman, George L. Choy, Stuart K. Koyanagi,

John H. Minsch, Waverly J. Person, Stuart A. Sipkin and Madeleine D. Zirbes. 
Earthquake Focal Mechanisms for July 1996

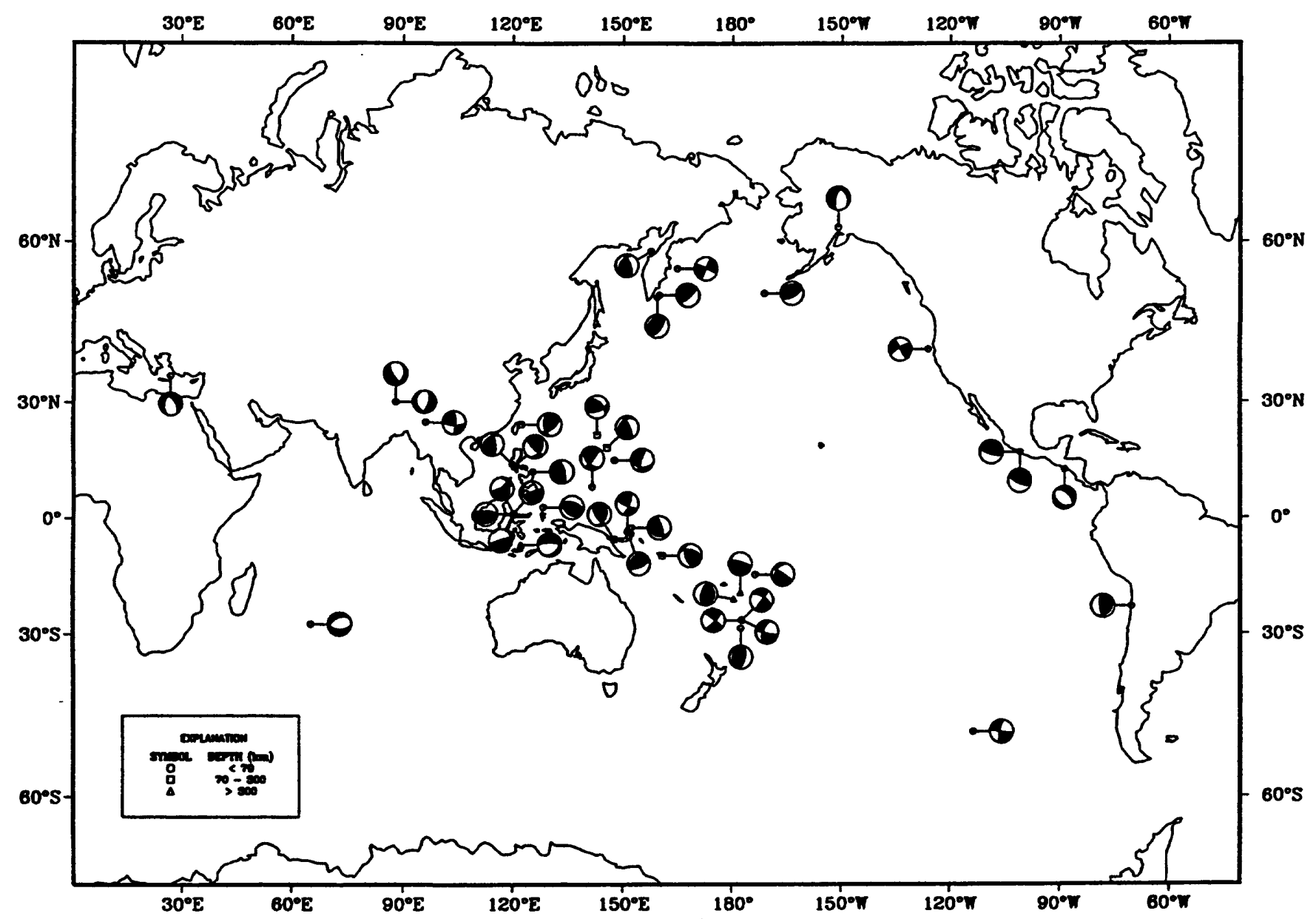

Earthquake epicenters in Alaska and adjacent regions for July 1996

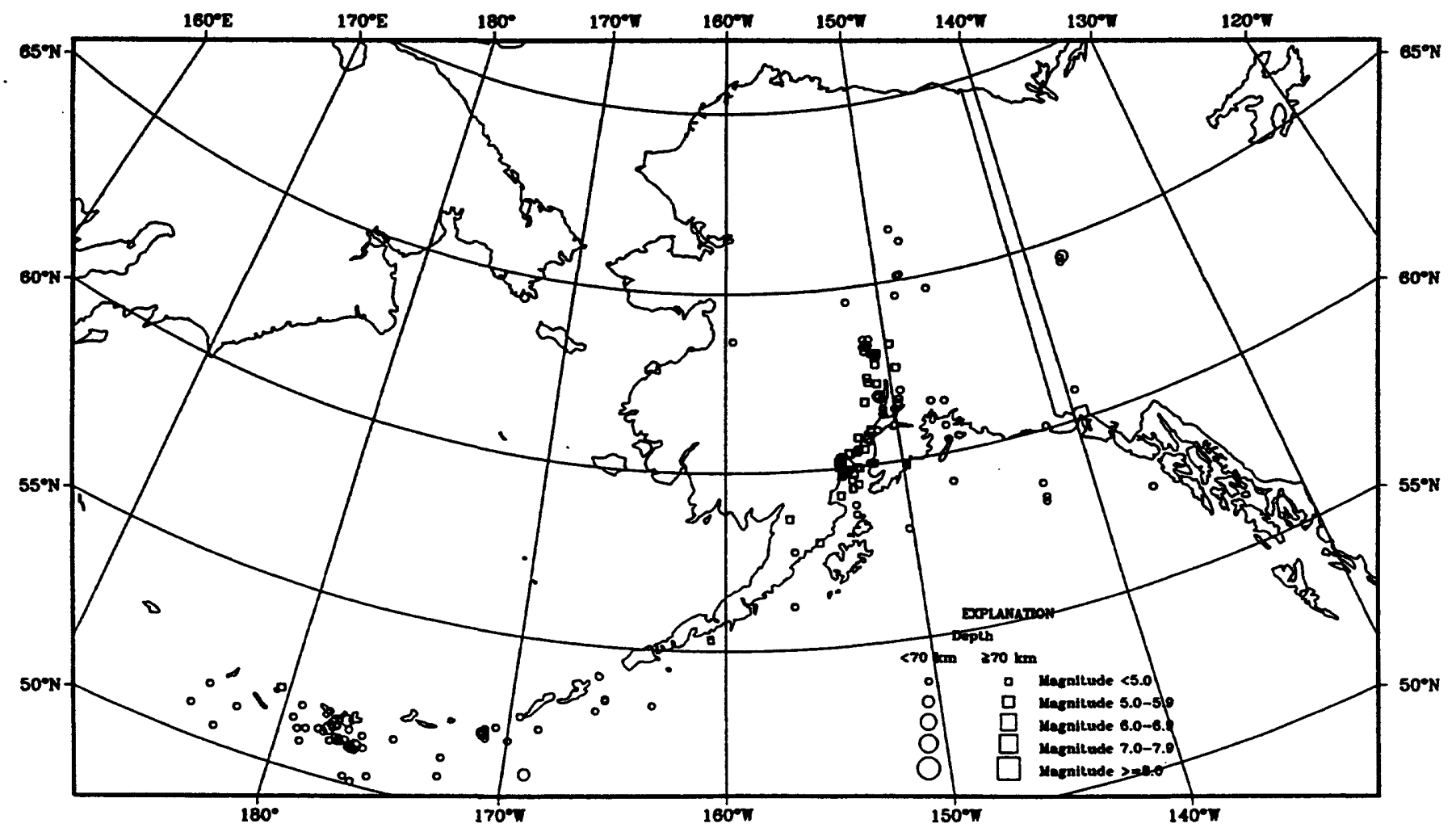


PAGE 27

Earthquake epicenters in the conterminous United States and adjacent regions for July 1996

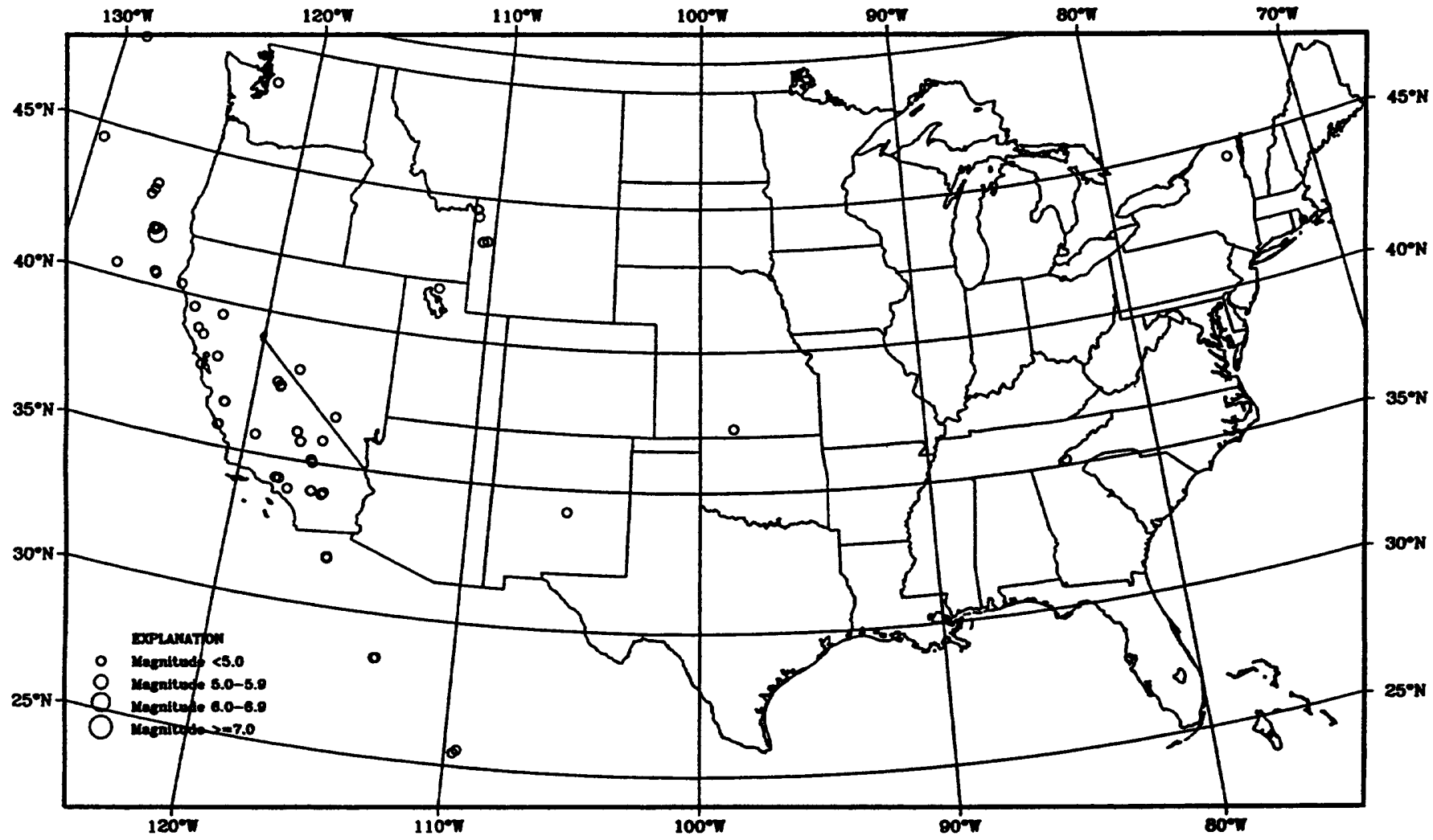

Earthquakes located worldwide in July 1996

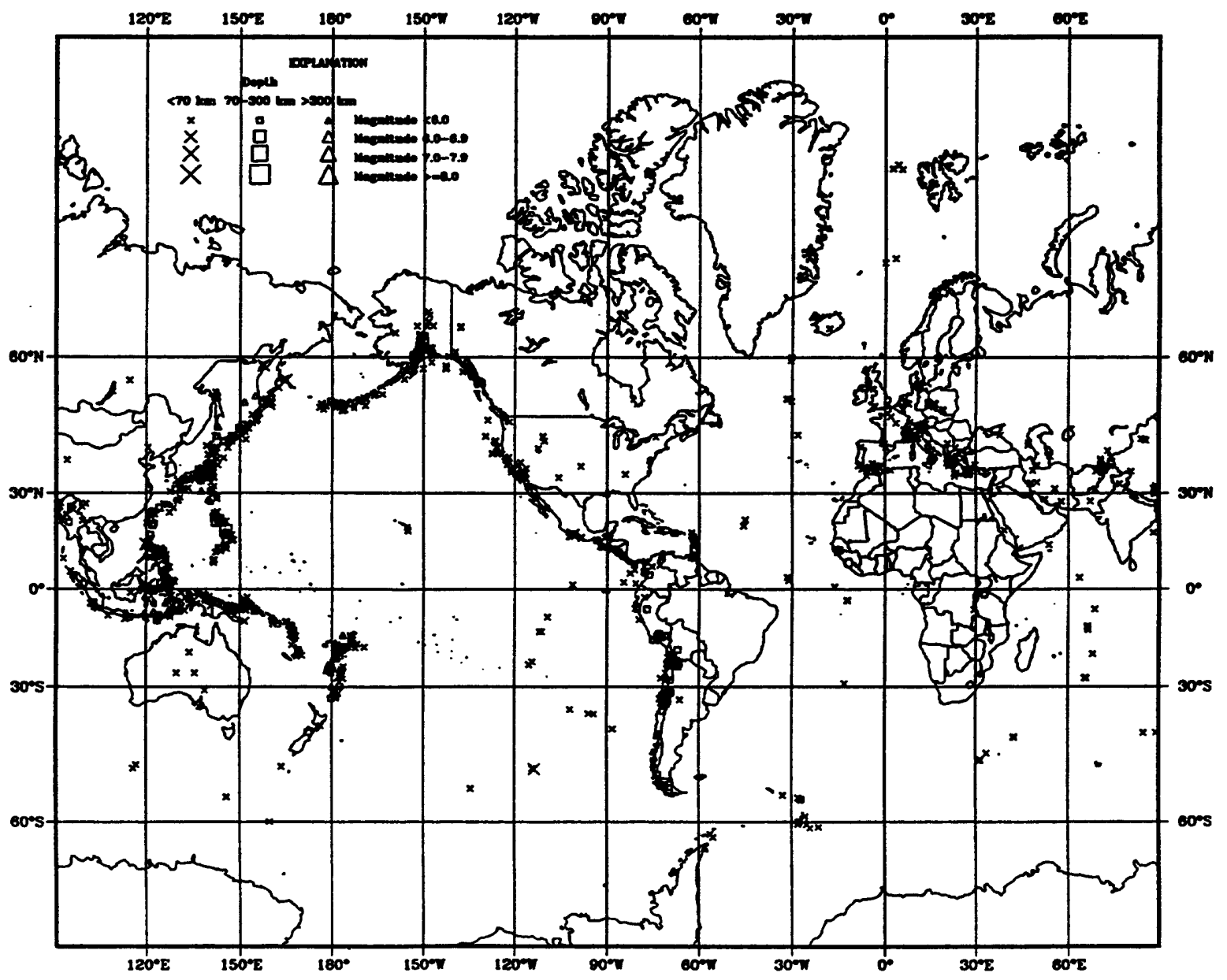


MB - Body wave magnitudes.

Msz - Vertical surface wave magnitudes.

OTC - Coordinated Universal Time. HR iN SEC - Hour, minute, second.

SD - Standard Deviation from the arithmetic mean of residuals.

No. Sta. - Number of stations reporting P or PKP phases used in computation

KEY - (Printed vertically). An "a" in this column indicates additional source parameters are published for this event in a separate section following the list of hypocenters.

Symbols and Abbreviations osed in Comments

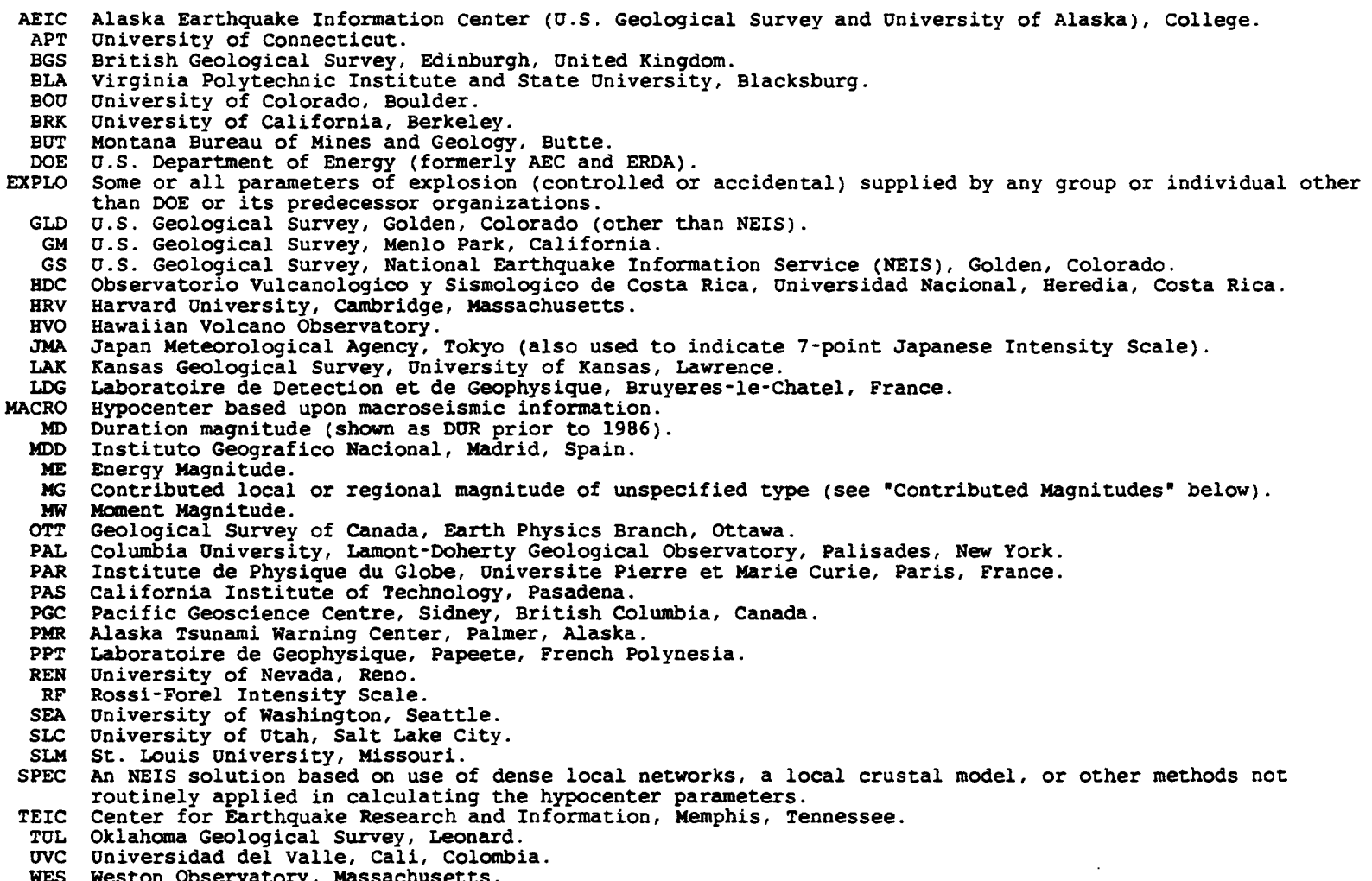

Roman Used to indicate intensity (when not followed by RF or JMA they refer to the Modified Mercalli Scale or Numerals any 12-point intensity scale closely related to it).

- Geographic degrees, minutes, seconds.

-p Supplied hypocenter is a preliminary computation.

Any additional 3 to 5 letter codes enclosed in parentheses or angle brackets refer to individual station codes. These codes may be found in Geological Survey Open File Report 85-714, Seismograph Station codes and coordinates (1985). Addenda to OF 85-714 are printed at the end of the Earthquake Data Report for this month.

Symbols Following Depth

N Indicates the depth was restrained at $33 \mathrm{~km}$ for earthquakes whose character on seismograms indicates a shallow focus but whose depth is not satisfactorily determined by the data.

D Indicates the depth was restrained by the computer program based on 2 or more compatible pp phases and/or unidentified secondary arrivals used as pP.

$G$ Indicates the depth was restrained by a geophysicist.

* Indicates a less well-constrained free depth. The 908 marginal confidence interval on depth is greater than 8.5 $\mathrm{km}$ and less than or equal to $16.0 \mathrm{~km}$.

? Indicates a poorly-constrained free depth. The 908 marginal confidence interval on depth is greater than 16.0 km.

The lack of any symbol indicates that the 908 marginal confidence interval on depth is less than or equal to 8.5 $\mathrm{km}$, or that a contributed hypocenter was computed with a free depth, regardless of the size of the confidence interval. 
- Indicates that parameters of the hypocenter were supplied or determined by a computational procedure not normally used by the National Earthquake Information Service (NEIS). The source or nature of the determination is indicated by a 2 to 5 letter code enclosed by angle brackets and appearing in the first line of comments. A " $P$ " appended to the code indicates that the computation is preliminary. These codes are included with the list of abbreviations above.

\& Indicates a single network solution. A non-furnished hypocenter has been computed using data reported by single network of stations for which the date and/or origin time cannot be confirmed from seismograms available to a NEIS analyst. The geometric mean of the semi-major and semi-minor axes of the horizontal 908 confidence ellipse is less than or equal to $16.0 \mathrm{~km}$.

* Indicates a less reliable solution. In general, the geometric mean of the semi-major and semi-minor axes of the horizontal 908 confidence ellipse is greater than $8.5 \mathrm{~km}$ and less than or equal to $16.0 \mathrm{~km}$.

? Indicates a poor solution, published for completeness of the catalog. In general, the geometric mean of the semi-major and semi-minor axes of the horizontal $90 \%$ confidence ellipse is greater than $16.0 \mathrm{~km}$. This includes a poor solution computed using data reported by a single network.

The lack of any symbol indicates that the geometric mean of the semi-major and semi-minor axes of the horizontal 908 confidence ellipse is less than or equal to $8.5 \mathrm{~km}$.

APPROXIMATE CORRELATION OF GRADES FOR INTENSITY SCALES REPORTED IN PRELIMINARY DETERMINATION OF EPICENTERS

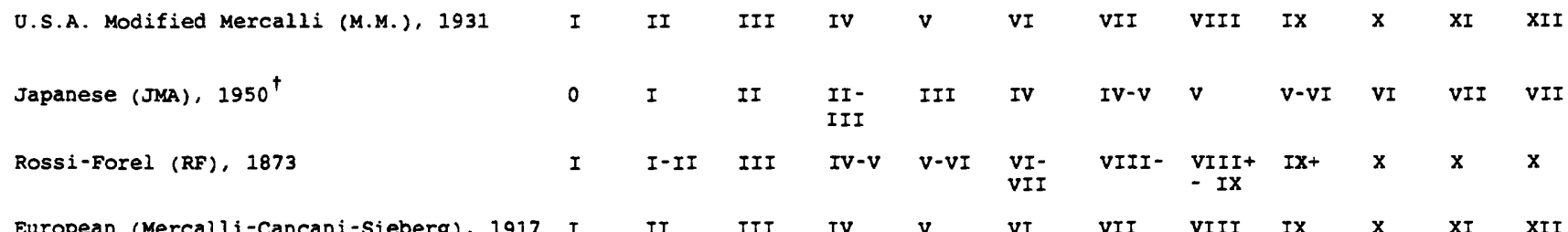

† Beginning 1993, Japan Meteorological Agency (JMA) intensities for earthquakes felt in Japan may be instrumentally determined.

\section{TRAVEL-TIME TABLES}

In general, all hypocenters have been computed based on the 1940 Jeffreys-Bullen $P$ and 1968 Bolt PKP travel-time tables. Some other earth model or computational procedure may have been used for those hypocenters which have been indicated by an ampersand ( $\delta$ ) following the origin time.

\section{MACROSEISMIC INFORMATION}

Macroseismic information is compiled from various sources, including newspaper articles, Foreign Broadcast Information Service messages, 0.s. Geological survey Earthquake Reports and seismological station reports. Macroseismic information for southwestern France is contributed by Dr. Pierre stahl, Pau. Sources of information for particular events can be supplied on request from: 0.S. Geological Survey, National Earthquake Information Center, Stop 967 , Box 25046, Denver Federal Center, Denver, Co 80225 , O.S.A.

\section{GEOGRAPHIC REGIONS}

The regions shown in the comments column are from the seismic and geographical regionalization of Flinn, Engdahl and Hill (1974), with occasional name changes which have been given in various issues of the Monthly Listing. The boundaries of these regions are defined at one degree intervals and differ slightly from irregular political boundaries.

\section{DEPTHS FROM BROADBAND DISPLACEMENT SEISMOGRAMS}

The NEIS routinely interprets broadband data from the GDSN, USNSN and other global digital seismograph networks for events with $\mathrm{MB} \geq 5.8$. Records that are flat to displacement between approximately 0.01 and 5 . 0 Hz are obtained using methods described by Harvey and Choy (1982). The notation that a depth is obtained from broadband seismograms indicates that a depth was obtained by inversion of differential travel times that are clearly identifiable at several stations using methods described by Choy and Engdahl (1987). Depths of selected events may also be constrained by modelling broadband $P$ and transversely polarized $S$ waves using methods described by Choy and Dewey (1988).

Choy, G. L. and Dewey, J. W., 1988, Rupture process of an extended earthquake sequence: Teleseismic analysis of the Chilean earthquake of March 3, 1985: Journal of Geophysical Research, v. 93, p. 1103-1118.

Choy, G. L. and Engdahl, E. R., 1987, Analysis of broadband seismograms from selected IAspeI events: Physics of the Earth and planetary Interiors, v. 47, p. 80-92.

Harvey, D. and Choy, G. L., 1982, Broadband deconvolution of GDSN data: Geophysical Journal of the Royal Astronomical Society, v. 69, p. $659-668$.

\section{BROADBAND FAULT PLANE SOLUTIONS}

A fault plane solution is determined when possible for any earthquake having a magnitude $\geq 5.8$. Beginning January 1996 , the fault plane solution is determined primarily from least-squares fitting of synthetic waveforms and broadband body waves that are flat to displacement between approximately 0.01 to 5.0 Hz. The fault plane solution derived from broadband data is sensitive to the dynamic or high frequency part of the earthquake. For complex earthquakes, the fault plane solution corresponds to the largest subevent unless otherwise stated. Prior to January 1996 , fault plane solutions were constrained primarily by using first motions from P, PP and PRP waves. Polarities were also obtained by using broadband displacement records of surface-reflected body waves (e.g., pP and sP), Hilbert-transformed body waves of certain secondary arrivals (e.g., PP), and transversely polarized $s$ waves. 


\section{FOCAI MECHANISM MAPS}

Best double couple focal mechanisms are plotted as lower-hemisphere, equal-area projections for earthquakes having a seismic moment greater than $1 * 10 * \star 17 \mathrm{Nm}$. The shaded quadrants represent compressional first motions. For each event, the mechanism shown is selected from either the Fault Plane solution, Moment rensor solution or centroid, Moment Tensor Solution. All these solutions are given in the Additional Source parameters section of the Monthly Listing.

\section{NEIS MAGNITUDES}

All magnitudes are NEIS magnitudes unless otherwise indicated. Beginning with August, 1983, average magnitudes are computed by a 258 trimmed mean as described by Rosenberger, J. L. and Gasko, M., 1983, "Comparing location estimators: trimmed means, medians, and trimean" in Understanding Robust and Exploratory Data Analysis, ed. Hoaglin, D.C.,

Mosteller, F., and Tukey, J. W., John Wiley, New York.

Mw These moment magnitudes are computed from the scalar moment of the moment tensor using the Kanamori (1977) formula:

$$
\text { Mw }=(2 / 3) \log \text { Mo }-10.7
$$

where Mo is the scalar moment of the best double couple in dyne-cm. Mo, computed from low frequency seismic data, is a measure of the area ruptured by an earthquake. Beginning with January, 1993 , a moment magnitude 1s computed routinely from the usGS moment tensor and Harvard centroid moment tensor solutions.

Me These energy magnitudes are computed from the radiated energy using the Choy and Boatwright (1995) formula (eq. 6):

$$
\text { Me }-(2 / 3) \log E s-2.9
$$

where Es is the radiated seismic energy in Newton-meters. Me, computed from high frequency seismic data, is a measure of seismic potential for damage. Beginning from July 1995, an energy magnitude is computed routinely from the usGS radiated energy.

These surface wave magnitudes are computed from the IASPEI formula:

$$
\text { MS }=\log (A / T)+1.66 \log D+3.3
$$

where:

A is the maximun ground amplitude in micrometers (microns) of the vertical component of the surface wave within the period range $18 \leq T \leq 22$.

$T$ is the period in seconds.

$D$ is the distance in geocentric degrees (station to epicenter) and $20^{\circ} \leq D \leq 160^{\circ}$.

No depth corrections are applied, and us magnitudes are not generally computed for depths greater than $50 \mathrm{~km}$. The Ms value published is the average of the individual station magnitudes from reported $T$ and $A$ data.

If the uncertainty of the computed depth is considered great enough that the depth could be less than $50 \mathrm{~km}$, an Ms value may still be published, computed by the IAspeI formula and not corrected for depth.

In general, the Ms magnitude is more reliable than the MB magnitude as a means of yielding the relative "size" of a shallow-focus earthquake.

These compressional body wave (P-wave) magnitudes are computed according to the formula:

$$
M B=\log (A / T)+Q(D, h)
$$

defined by Gutenberg and Richter (1956) except that $T$, the period in seconds, is restricted to $0.1 \leq T \leq 3.0$ and $A$, the ground amplitude in micrometers, is not necessarily the maximum in the $P$ group. $Q$ is $a$ function of distance (D) and depth (h) where $D \geq 5^{\circ}$.

These Ig body wave magnitudes are computed according to the formula:

$$
\begin{aligned}
& \operatorname{mbLg}-3.75+0.90 \log D+\log (A / T) \text { for } 0.5^{\circ} \leq D \leq 4^{\circ} \\
& \operatorname{mbLg}=3.30+1.66 \log D+\log (A / T) \text { for } 4^{\circ} \leq D \leq 30^{\circ}
\end{aligned}
$$

as proposed by Nuttli (1973) where $A$ is the ground amplitude in micrometers and $T$ is the period in seconds calculated from the vertical component 1 -second $\mathrm{Lg}$ waves. $\mathrm{D}$ is the distance in geocentric degrees.

ML

These local magnitudes are computed according to the formula:

$$
\text { MIL - } \log A-\operatorname{LOg} A O
$$

defined by Richter (1935) where $A$ is the maximum trace amplitude in micrometers recorded on a standard shortperiod torsion seismometer and $\log A O$ is a standard value as a function of distance where distance $\leq 600$ km.

\section{CONTRIBUTED MAGNITUDES}

Magnitudes appearing in the comments which have been contributed by organizations operating a network of stations may have been calculated from any one station in the network or may be an average magnitude from a number of stations from the network.

Beginning with January, 1986, a contributed magnitude of unspecified type may be quoted (using the designator MG) for events which have no other magnitudes given or computed. These MG magnitudes either have been reported by the contributor without 1isting the type (such as "Mag 3.5") or have been computed using procedures which are not defined by the magnitude types routinely reported in this bulletin. Direct inquiries should be made to the contributor (shown in parentheses after the magnitude) concerning the specific details of the computational procedures used to determine these values. 
Choy, G. L., and Boatwright, J. L., 1995, Global patterns of radiated seismic energy and apparent stress: Journal of Geophysical Research, v. 100, p. 18205-18228.

Gutenberg, B., and Richter, C. F., 1956, Magnitude and energy of earthquakes: Annali di Geofisica, v. 9, no. 1, p. 1-15. Kanamori, H., 1977, The energy release in great earthquakes: Journal of Geophysical Research, v. 82, p. $2981-2987$. Nuttli, O. W., 1973, Seismic wave attenuation and magnitude relations for eastern North Anerica: Journal of Geophysical Research, v. 78, no. 5, p. 876-885.

Richter, C. F., 1935, An instrumental earthquake scale: Bulletin of the Seismological Society of America, v. 25, p. $1-32$.

\section{USGS RADIATED ENERGY}

The energy radiated by an earthquake is estimated from the energy spectral density of the broadband $P$ waves, using the method described by Boatwright and Choy (1986), where the energy flux in the $P$ waves is integrated directly. No correction for source directivity or frequency-dependent interference of the depth phases is incorporated into these estimates of radiated energy. Data used are either direct $P$ waves (for deep earthquakes) or the $P$ wave group consisting of P, PP and SP (for shallow earthquakes) from GDSN and other stations that contribute digital data to the NEIC within two months of the occurrence of an event. The data are processed using the method of Harvey and Choy (1982) so that they are flat to velocity from low frequencies (generally $0.01 \mathrm{~Hz}$ ) to at least $2.0 \mathrm{~Hz}$. The effect of attenuation is they are flat to velocity from low frequencies (generally 0.01 Hz) to at least 2.0 Hz. The effect of attenuation is
corrected with the frequency-dependent $t$ * of choy and cormier (1986). The focal mechanism used is either the $P$-wave first-motion solution (F), the USGS moment tensor solution (M) or the Harvard centroid solution (C).

Boatwright, J. and Choy, G. L., 1986, Teleseismic estimates of the energy radiated by shallow earthquakes: Journal of Geophysical Research, v. 91, p. 2095-2112.

Choy, G. L. and Cormier, V. F., 1986, Direct measurement of the mantle attenuation operator from broadband $P$ and $S$ waveforms: Journal of Geophysical Research, v. 91, p. 7326-7342.

Harvey, D. and Choy, G. L., 1982, Broadband deconvolution of GDSN data: Geophysical Journal of the Royal Astronomical Society, v. 69, p. $659-668$

EXPLANATION OF THE ENTRIES "MOMENT TENSOR SOLUTION" (USGS)

These solutions have been determined using the body-wave moment tensor inversion method described by sipkin (1982).

1. NUMBER OF STATIONS: Number of GDSN stations with distances between approximately 30 and 95 degrees found to have suitable $P$ waveforms. Only long-period vertical components are used.

2. DEPTH: The source depth which gives the smallest normalized mean-squared-error. This is the only hypocentral parameter determined since the inversion procedure is insensitive to small errors in both epicenter and origin time.

3. SCALE

4. PRINCIPAL AXES

5. BEST DOUBLE COUPLE See "Centroid, Noment Tensor (HRV)"

S. A. Sipkin, J.s. Geological Survey, Mail Stop 967, Box 25046, Denver Federal Center, Denver, Co 80225 0SA

Sipkin, S. A., 1982, Estimation of earthquake source parameters by the inversion of waveform data: synthetic seismograns: Physics of the Earth and Planetary Interiors, v. 30, no. 2-3, p. 242-259.

\section{EXPLANATION OF THE ENTRIES "GEOSCOPE MOMENT TENSOR (PAR)"}

These solutions have been obtained from very long period Rayleigh wave data in the period range $180-310$ seconds ( 1 and R2 trains) using a two step moment tensor inversion method as described in Romanowicz and Guillemant (1984) and Romanowicz

and Monfret (1986). Parameters solved for are centroid time, seismic moment, depth and moment tensor. origin time and epicentral coordinates are kept fixed as given in the USGS Quick Epicenter Determinations (QED) or PDE. For shallow earthquakes the precision on depth is in general no greater than $\pm 10 \mathrm{~km}$.

The data used presently come from GEOSCOPE teletransmitted stations (usually 8 - 10 stations) and are available within a week after the event. The solutions are computed by the Institute de Physique du Globe, Universite Pierre et Marie Curie, Paris, France.

Romanowicz, B. and Guillemant, P., 1984, An experiment in the retrieval of depth and source mechanism of large earthquakes using very long-period Rayleigh wave data: Bulletin of the Seismological Society of America, v. 74, no. 2 , p. $417-437$.

Romanowicz, B. and Monfret, T., 1986, Source process times and depths of large earthquakes by moment tensor inversion of mantle wave data and the effect of lateral heterogeneity: Annales de Geophysique, v. B4, no. 3 , p. $271-282$.

EXPLANATION OF THE ENTRIES "CENTROID, MOMENT TENSOR (HRV)"

These solutions have been determined using the long period body and mantle wave moment tensor inversion method described by Dziewonski, et.al. (1981) considering corrections due to an aspherical earth structure of model sH8/04L8 (Dziewonski and Woodward, 1991).

1. DATA USED: currently GDSN, GSN and IDA/IRIS data are used. The numbers following the entries L.P.B. and M.W. indicate the number of stations (S) and total number of records (C) for the long-period body waves and mantle waves, respectively. Mantle waves are routinely used in inversion for sources with moments greater than $5 \star 10 \star * 18$ Newton-meters (Nm). 
2. CENTROID LOCATION: hypocentral parameters obtained by adding perturbations resulting from inversion to the parameters reported in the PDE; standard errors follow the individual entries. If a given parameter is not perturbed in inversion, this is indicated by the letters FIX. If the depth is fixed to be consistent with waveform matching of reconstructed broad-band body waves (Ekstrom, 1989), this is indicated by the letters BDY. The default depth for shallow earthquakes is increased to $15 \mathrm{~km}$. in order to improve the stability of solutions; it was $10 \mathrm{~km}$. in $1981-1985$.

3. PRINCIPAI AXES: rotation of the moment tensor, constrained to have zero trace, into the principal axes system. Most of the solutions are predominantly of the double couple type: the largest positive eigenvalue corresponds to the tension axis (T): the usually small, intermediate eigenvalue is associated with the null axis (N); the smallest negative eigenvalue is 1dentified with the compression axis (P). PLG are the plunges and AzM the azimuths of the axes.

4. BEST DOUBLE COUPLE: If the eigenvalue $(T)$ is $\sigma_{1}$ and $(P)$ is $-\sigma_{2}$, then the scalar seismic moment is defined as Mo $-1 / 2\left(\sigma_{1}+\sigma_{2}\right)$. The strike, dip and slip of the first (NP1) and second (NP2) nodal planes are calculated from the directions of the $P, T$, and $N$ axes. The remainder is a linear-vector dipole; in most cases the magnitude of LVD is small. Although all such decompositions are highly non-unique, this particular one is the best in estimating the starting solution for the non-linear, constrained double couple inverse problem. The strike, dip, and slip angles are defined using the convention of Aki and Richards (1980, $p$. 106) and are the angles designated there as $\phi_{s}, \delta, \lambda$, respectively.

A. M. Dziewonski, G. Ekstrom and M. P. Salganik, Department of Earth and Planetary Sciences, Harvard University, Cambridge, MA 02138

Aki, K. and Richards, P. G., Quantitative Seismology, Volume 1, W. H. Freeman, San Francisco, 1980 , 557 Pp.

Dziewonski, A. M., Chou, T. A., and woodhouse, J. H., 1981, Determination of earthquake source parameters from waveform data for studies of global and regional seismicity: Journal of Geophysical Research, v. $86, \mathrm{p}$. $2825-2852$.

Dziewonski, A. M. and woodward, R.L., 1991, Acoustic imaging at the planetary scale, in Acoustical Imaging, vol. I9, E. Ermert and H.-P. Harjes, eds., Plenum Press (in press).

Ekstrom, G., 1989, A very broad band inversion method for the recovery of earthquake source parameters: Tectonophysics, v. 166, p. $73-100$.

\section{OTHER SEISMIC MOMENTS}

1. The seismic moment (MO) contributed by the University of California, Berkeley (BRK), is given for regional earthquakes based on Wood-Anderson torsion seismograms recorded within $300 \mathrm{~km}$ of the epicenter with peak-to-peak ampli. tudes of at least $3 \mathrm{~mm}$. This seismic moment (MO) in dyne-cm is defined by Log Mo $=16.74+1.22 \mathrm{LOg}$ (CDA), where C is the maximum peak-to-peak amplitude in $\mathrm{mm}, \mathrm{D}$ is whe duration in seconds from the time of the $S$-wave onset to the last time that the peak-to-peak amplitude exceeds $c / 3$, and $\Delta$ is the epicentral distance in km. Seismic moments quoted in "Preliminary Determination of Epicenters" are converted to Newton-meters ( 1 Newton-meter - $10 \star * 7$ dyne-cm).

Bolt, B.A. and Berraiz, M. 1983, Simplified estimation of seismic moment from seismograms: Bulletin of the Seismological Society of America, $v .73, \mathrm{p} .735-748$.

2. Beginning with November, 1988, seismic moments for selected events have been contributed by the Laboratoire de Geophysique, Papeete, French Polynesia (PPT). These moments are computed from mantle Rayleigh and Love waves using the method of Talandier, Reymond and Okal (1987 and 1990).

Talandier, J., Reymond, D. and Okal, E.A. 1987, Use of a variable period mantle magnitude for the rapid one-station estimatión of seismic moments: Geophysical Research Letters, v. 14, no. 8, p.840-843.

Okal, E.A., and Talandier, J. 1990, Mm: Extension to Love Waves of the Concept of a Variable-Period Mantle Magnitude: Pure and Applied Geophysics, v. 134, p. 355-384. 


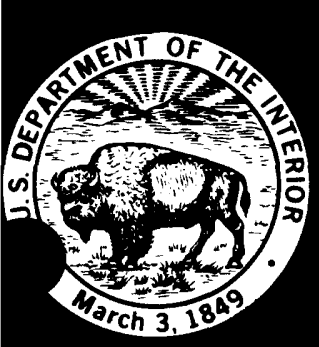

ORIGIN TIME UTC

DAY ER MN SEC GEOGRAPHIC COORDINATES

$0100 \quad 05 \quad 30.8 * 32.409 \mathrm{~s}$ $\begin{array}{lllllllll}01 & 00 & 13 & 02.9 & 45.287 & \mathrm{~N}\end{array}$ $01 \quad 0015 \quad 44.8 ? 27.59 \quad S$ $\begin{array}{lllllll}01 & 01 & 11 & 42.9 & 3.071 & \mathrm{~s}\end{array}$ $\begin{array}{llllll}01 & 01 & 40 & 48.0 & 36.412 \mathrm{~N}\end{array}$ $\begin{array}{llllll}01 & 02 & 06 & 09.4 & 3.453 & \mathrm{~N}\end{array}$ $\begin{array}{llllll}01 & 03 & 36 & 06.0 & 45.587 & \mathrm{~N}\end{array}$
70.372 ผ $6.245 \mathrm{E}$ $65.29 \mathrm{E}$ $129.656 \mathrm{E}$ $70.285 \mathrm{E}$ $96.349 \mathrm{E}$ $97.312 \mathrm{E}$
AUGUST

DEPTH MAGNITUDE SD NO. GS

$100 G$

$5 \mathrm{G}$

$10 \mathrm{G} \quad 4.4$

$33 \mathrm{~N} 4.9$

$204 \mathrm{D} 4.4$

$82 * 4.8$

1.2111

\section{$\begin{array}{llllllllll}01 & 04 & 08 & 23.2 & 0.018 & \mathrm{~S} & 122.939 \mathrm{E} & 149 \mathrm{D} & 5.5\end{array}$}

$010436 \quad 22.08 \quad 61.039$ N $\quad 252.728$ W 171

$\begin{array}{lllllll}01 & 04 & 49 & 18.4 & 27.594 \mathrm{~S} & 65.712 \mathrm{E} & 10\end{array}$

0.018 S 122.939 E $149 \mathrm{D} \quad 5.5$

$0.8 \quad 197$

\title{
1996
}

\author{
REGION, CONTRIBUTED MAGNITUDES AND COMNENTS \\ CHILE-ARGENTINA BORDER REGION. MD 3.1 (SAN). \\ FRANCE. MIL 2.7 (IDG), 2.6 (STR). \\ SOUTH INDIAN OCEAN \\ SERAK, INDONESIA \\ HINDU KDSE REGION, AFGHANISTAN \\ NORTHERN SUMATERA, INDONESIA \\ MONGOLIA. MW 5.1 (HRV).
}

Centroid, Moment Tensor (HRV): Centroid origin time 03:36:07.8; Lat 45.19 N; Lon 96.97 E; Dep 15.0 Fix; Halfduration $1.0 \mathrm{sec}$; Principal axes (scale 10*\$16 $\mathrm{Nm}$ ): (T) Val-4.68, Plg-2, Azm=126; (N) Val-1.59, Plg-63, Azm-221; (P) Val--6.27, Plg-26, Azm-35; Best double couple: Mo-5.5*10*16 Nm; NP1: Strike-174, Dip-70, Slip--162; NP2: Strike-78, Dip=73, slip--21.

MINAHASSA PENINSULA, SULAWESI. MW 5.6 (GS), 5.6 (HRV). Felt in the Gorontalo-Tolitoli area.

Moment Tensor (GS): Dep 140; Principal axes (scale 10*\#17 $\mathrm{Nm}$ ): (T) Val-2.72, Plg-73, Azm-289; (N) Val-0.11, Plg-16, Azm-84; (P) Val--2.84, Plg-7, Azm-176; Best double couple: Mo-2.8*10**17 Nm; NP1: Strike-284, Dip-41, Slip-115; NP2: Strike-72, Dip-54, Slip-70.

Centroid, Moment Tensor (HRV): Centroid origin time $04: 08: 26.0$; Lat $0.07 \mathrm{~N}$; Lon $123.17 \mathrm{E}$; Dep 133.3 ; Half duration $1.4 \mathrm{sec}$; Principal axes (scale 10**17 $\mathrm{Nin}$ ): (T) Val-2.25, Plg-55, Azm-260; (N) Val-0.51, Plg-35, Azm-79; (P) Val--2.75, Plg-1, Azm-169; Best double couple: MO-2.5*10**17 Nm; NP1: Strike-289, Dip-54, Slip-135; NP2: Strike-50, Dip-55, Slip-46.

17 SOUTHERN AIASKA. <AEIC>

SOUTH INDIAN OCEAN. NW 5.1 (HRV).

Centroid, Moment Tensor (HRV): Centroid origin time 04:49:25.8; Lat $27.59 \mathrm{~S} \mathrm{Fix;} \mathrm{Lon} 65.71$ E Fix; Dep 15.0 Bdy; Half-duration $1.0 \mathrm{sec}$; Principal axes (scale 10**16 Nm): (T) Val-4.05, PIg-0, Azm-152; (N) Val-1.66, Plg-0, Azm-62; (P) Val- -5.70, Plg-90, Azm-180; Best double couple: MO-4.9*10*16 Nm; NP1: Strike-242, Dip-45, S1ip--90; NP2 Strike-62, Dip-45, Slip--90.

$65.497 \mathrm{E}$ $104.247 \mathrm{~W}$ $71.263 \mathrm{~W}$ $104.196 \mathrm{~W}$ $6.035 \mathrm{E}$ $65.83 \mathrm{E}$ $143.92 \mathrm{E}$ $155.266 \mathrm{~W}$ 178.68 พ $147.910 \mathrm{E}$ 68.81 $169.034 \mathrm{E}$ $153.167 \mathrm{~W}$ $152.859 \mathrm{E}$ $125.875 \mathrm{E}$ $171.385 \mathrm{~W}$ $157.989 \mathrm{E}$ $65.594 \mathrm{E}$ $155.280 \mathrm{~W}$ $155.263 \mathrm{~W}$ $151.376 \mathrm{~W}$ $65.436 \mathrm{E}$ $66.911 \mathrm{E}$ $65.49 \mathrm{E}$

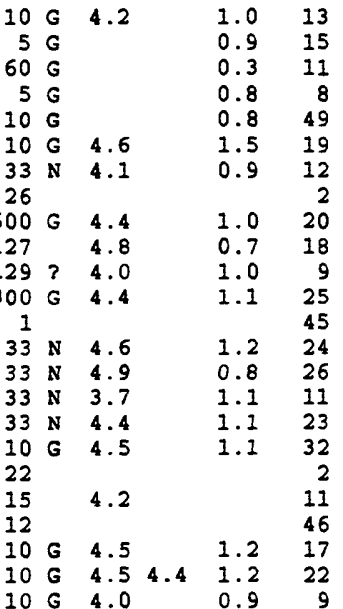
SOTTE INDIAN OCEAN

COLORADO. mbLg 3.8 (GS). Felt at rrinidad.

NEAR COAST OF CENTRAL CEILE. MD 2.8 (SAN).

COLORADO. mbLg 3.2 (GS).

FRANCE. ML 3.0 (IDG)， 2.9 (STR).

SOUTH INDIAN OCEAN

VOLCANO ISLANDS REGION

HAWAII. <HVO-P>. MD 4.0 (HVO)

FIJI ISLLANDS REGION

EASTERN NEW GUINEA REG., P.N.G.

CHILE-BOLIVIA BORDER REGION

VANUATU ISLANDS

SOUTHERN ALASKA. <AEIC>, ML 2.7 (AEIC)

D'ENTRECASTEAUX ISLANDS REGION

SAMAR, PHILIPPINE ISLANDS

FOX ISLANDS, ALEUTIAN ISLANDS

KAMCHATKA

SOUTH INDIAN OCEAN

HAWAII. 〈HVO-P>. MD 4.0 (HVO).

HAWAII. 〈HVO-P>. MD 4.3 (HNO)

CENTRAL ALASKA. CAEIC>. ML 2.9 (AEIC).

SOUTH INDIAN OCEAN

MID-INDIAN RIDGE

SOUTH INDIAN OCEAN 
$01 \quad 18 \quad 15 \quad 05.1 ? \quad 36.58$ N $139.43 \quad \mathrm{E} 10$ $01 \quad 183819.582 .61$. $139.43 \mathrm{~B}$ 1019.532 .61 S $139.56 \mathrm{E}$ $01 \quad 191049.5 \quad 56.111 \mathrm{~N} \quad 164.373 \mathrm{E}$ $\begin{array}{llllllllll}01 & 19 & 14 & 45.7 ? & 20.96 & S & 177.99 & \text { W }\end{array}$ $01195329.2 * 49.014 \mathrm{~N} 8.621 \mathrm{E}$

$\begin{array}{lllllll}01 & 20 & 55 & 01.1 & 55.124 \mathrm{~N} & 4.686 \mathrm{E}\end{array}$ $\begin{array}{llllll}01 & 21 & 51 & 47.8 & 17.810 & \mathrm{~s}\end{array}$ $70.623 \mathrm{~W} \quad 10 \mathrm{G}$
$10 \mathrm{G}$

33 N 4.2 33 N 4.2 500 G 4.0

5 G

$74 \mathrm{D} 5.0$
1.1

1.2

0.9

1.1

1.4

1.3
1.0

4 EASTERN HONSHO, JAPAN

9 NEAR NORTH COAST OF IRIAN JAYA

22 KOMANDORSKY ISLANDS REGION

11 EIJI ISLANDS REGION

10 GERMANY. MIL 2.6 (LDG).

NORTH SEA

NEAR COAST OF PERO. MW 5.2 (HRV). Felt (IV) at Locumba

(III) at Arequipa, Candarave, Ilo, Tacna and Tarata; (II) at vollendo and Yoquegua.

Centroid, Moment Tensor (HRV): Centroid origin time

$21: 51: 50.3$; Lat $18.22 \mathrm{~s}$; Lon $70.84 \mathrm{~W}$; Dep 97.5 ; Half-

duration $1.0 \mathrm{sec}$; Principal axes (scale 10*\$16 $\mathrm{Nm}$ ): (T)

Val=5.79, Plg=23, $A z m=98$; (N) Val=0.94, Plg=30, Azm=353;

(P) Val=-6.73, Plg-50, Azm=219; Best double couple:

Mo=6.3*10*16 Nm; NP1: Strike=230, Dip=35, Slip=-28; NP2 : Strike-344, Dip=74, Slip=-122.

$01 \quad 2156 \quad 20.7 ? 31.06 \quad s$

$012200 \quad 42.2 * 13.885 \mathrm{~N}$

$\begin{array}{llllll}01 & 22 & 13 & 36.8 & 51.909 & \mathrm{~N}\end{array}$

$02001706.1 * 6.816 \mathrm{~s} \quad 155.630 \mathrm{E}$

$02003438.9 * 17.383 \mathrm{~s}$

$02.00 .58 \cdot 39.0 * 31.820 \mathrm{~s}$

$020141 \quad 10.2$ ? $34.54 \mathrm{~s}$

$\begin{array}{lllllll}02 & 02 & 04 & 17.98 & 46.076 & \mathrm{~N}\end{array}$

$\begin{array}{llllll}02 & 02 & 23 & 32.98 & 18.877 & \mathrm{~N}\end{array}$

$\begin{array}{lllllll}02 & 03 & 35 & 44.5 & 33.141 & \mathrm{~N}\end{array}$

$\begin{array}{llllll}02 & 03 & 39 & 29.0 * & 11.995 \\ 02 & 05 & 38 & 15.5 \% & 60.970\end{array}$

$\begin{array}{lllllll}02 & 05 & 38 & 15.5 \% & 60.970 & \mathrm{~N} \\ 02 & 06 & 49 & 26.98 & 65.197 & \mathrm{~N}\end{array}$

$\begin{array}{llllllll}02 & 06 & 51 & 08.1 ? & 7.57 & \mathrm{~N}\end{array}$

$\begin{array}{llllll}02 & 07 & 00 & 05.08 & 45.492 & N\end{array}$

$\begin{array}{lllll}02 & 07 & 05 & 09.9 * 12.050 \mathrm{~N}\end{array}$

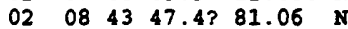

$02094916.5 * 8.559 \mathrm{~s}$

$\begin{array}{llllll}02 & 09 & 56 & 36.18 & 44.389 & \mathrm{~N}\end{array}$

$02 \quad 1001 \quad 10.98 \quad 36.770 \mathrm{~N}$

$\begin{array}{llllll}02 & 10 & 18 & 56.7 & 1.163 & \mathrm{~N}\end{array}$

$\begin{array}{lllllll}02 & 10 & 33 & 15.88 & 34.231 & \mathrm{~S}\end{array}$

$\begin{array}{llllll}02 & 11 & 36 & 12.3 & 29.398 & \mathrm{~N}\end{array}$

$168.763 \mathrm{~B}$

$70.083 \mathrm{~W}$

70.39 W

$2.905 \mathrm{E}$

155.254 W

$137.003 \mathrm{E}$

$149.680 \mathrm{H}$

$148.516 \mathrm{~W}$

77.41 พ

$97.544 \mathrm{E}$

$125.700 \mathrm{E}$

$120.53 \mathrm{E}$

$33.239 \mathrm{E}$

$7.385 \mathrm{E}$

$3.203 \mathrm{~W}$

$121.566 \mathrm{E}$

$70.744 \mathrm{~W}$

$67.695 \mathrm{E}$

$426 ? 4.0$

$33 \mathrm{~N} 4.2$

$33 \mathrm{~N} 4.3$

$86 * 4.0$

$112 ? 4.7$

200 G 4.7

$120 \mathrm{G}$

$10 \mathrm{G}$

$10 \mathrm{G}$

13
391

33

37
11

$33 \mathrm{~N} 3$.

$33 \mathrm{~N} 3.9$

$33 \mathrm{~N} 4.8$

$10 \mathrm{G} 3.8$

$10 \mathrm{G} 4.1$

$10 \mathrm{G}$

$10 \mathrm{G}$

$33 N 4.8$

$100 \mathrm{G}$

$33 \times 4.9$
1.1

1.5

0.4

1.1

1.1

0.1

1.0

1.0

0.9

1.2

1.1

1.6

1.3

0.4

1.0

0.4 $\begin{array}{lllllllll}02 & 11 & 36 & 21.68 & 37.853 & \mathrm{~N} & 3.797 & \mathrm{~N} & 10 \mathrm{G}\end{array}$

$02114619.0844 .980 \mathrm{~N} 122.650 \mathrm{~W} 27$

$\begin{array}{lllllllllll}02 & 12 & 07 & 54.2 ? & 22.28 & \mathrm{~S} & 171.25 & \mathrm{E} & 75 & ? & 5.1\end{array}$

32 KERMADEC ISLANDS REGION

25 OFE COAST OF CHIAPAS, MEXICO

48 OFF EAST COAST OF KAMCHATKA

EASTERN NEW GUINEA -REG., P.N.G

40 SOLONON ISIANDS

30 VANUATO ISLANDS

CHILE-ARGENTINA BORDER REGION. MD 3.5 (SAN).

CHILE-ARGENTINA BORDER REGION

FRANCE. MIL 1.5 (LDG)

2 HAWAII. 〈HVO-P>. MD 4.0 (HVO).

88 NEAR S. COAST OF HONSHO, JAPAN

6 SAMAR, PHILIPPINE ISLANDS

74 KENAI PENINSULA, AIASKA. 〈AEIC>. MI 3.0 (AEIC)

20 NORTHERN ALASKA. 〈AEIC>. ML 2.6 (AEIC).

9 PANAMA-COLOMBIA BORDER REGION

15 MONGOLIA

SAMAR, PHILIPPINE ISLANDS

7 EAST OF SEVERNAYA ZEMUYA, ROSSIA

6 TANZANIA

5 NORTHERN ITALY. ML 1.6 (GEN).

5 STRAIT OF GIBRALTAR. IBLI 2.3 (MDD).

32 MINAHASSA PENINSULA, SULAKESI

10 CHILE-ARGENTINA BORDER REGION. MD 2.2 (SAN).

77 PAKISTAN. NW 5.2 (HRV).

Centroid, Moment Tensor (HRV): Centroid origin time

$11: 36: 16.2$; Lat $29.29 \mathrm{~N}$; Lon $67.63 \mathrm{E}$; Dep $33.0 \mathrm{Fix}$; Halfduration $1.0 \mathrm{sec}$; Principal axes (scale 10*16 Nm): (T) Val=7.34, Plg=60, Azm=273; (N) Val=-1.14, Plg=15, Azm=31; (P) Val=-6.20, Plg=25, Azm=128; Best double couple: Mo=6.8*10**16 Nm; NP1: Strike=247, Dip=24, Slip=129; NP2 : Strike=25, Dip=72, Slip=74.

0.65 SPAIN. MbLg 2.6 (MDD)

47 OREGON. 〈SEA-P>. MD 3.3 (SEA). Felt at Estacada, Lebanon, Sandy and Silverton.

1.048 LOYALTY ISLANDS REGION. NW 5.4 (HRV).

Centroid, Moment Tensor (HRV): Centroid origin time

$12: 07: 57.4$; Lat $22.52 \mathrm{~s}$; Lon $171.23 \mathrm{E}$; Dep 76.1 ; Half-

duration $1.2 \mathrm{sec}$; Principal axes (scale 10*\$17 Nm): (T) Val=1.42, Plg=45, Azm=308; (N) Val=-0.22, Plg=35, Azm=173; (P) Val=-1.20, Plg=25, Azm-65; Best double couple: vo=1.3*10**17 Nm; NP1: Strike=107, Dip=38, Slip=19; NP2: Strike-2, Dip=78, Slip=126.

$02 \quad 124325.0 * \quad 5.341 \mathrm{~S} \quad 147.119 \mathrm{E} \quad 280 \quad 4.2 \quad 0.8 \quad 12$ EASTERN NEW GUINEA REG., P.N.G.

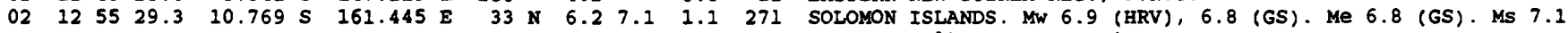
(BRK). Felt (IV) at Honiara.

Broadband Source Parameters (GS): Dep 30; NP1: Strike-125 Dip=50, Slip=130; NP2: Strike-252, Dip=54, slip=53; Radiated energy $4.1 * 10 * 14 \mathrm{Nm}$. Complex earthquake with at least one larger event occurring about 6 seconds after the onset.

Moment Tensor (GS): Dep 35; Principal axes (scale 10**19

$\mathrm{Nm}$ ): (T) Val=1.61, Plg=60, Azm=108; (N) Val=-0.03, Plg=27, Azm=260; (P) Val=-1.57, Plg=12, Azm=356; Best double

couple: Mo=1.6*10**19 Nm; NPI: Strike-117, Dip=40, Slip=134; NP2: Strike-245, Dip=62, Slip=59.

Centroid, koment Tensor (HRV): Centroid origin time 12:55:40.3; Lat $10.78 \mathrm{~s}$; Lon $161.46 \mathrm{E}$; Dep 47.4; Halfduration $7.5 \mathrm{sec}$; Principal axes (scale 10*19 Nm): (T) Val=2.60, $P l g=54, A z m=125$; (N) $\operatorname{Val}=0.50, \quad P l g=36, A z m=282$; (P) Val=-3.10, Plg=6, Azm=17; Best double couple: Mo=2.8*10**19 Nm; NP1: Strike=139, Dip=50, Slip=139; NP2 Strike-258, Dip=60, Slip=48.

Scalar Moment (PPT): MO-4.3*10**19 Nm.

$021346 \quad 27.1 * 10.580 \mathrm{~s}$

$\begin{array}{llllllll}02 & 15 & 29 & 03.58 & 48.514 \mathrm{~N} & 0.575 \mathrm{~W}\end{array}$

$02 \quad 1544 \quad 40.7833 .656 \mathrm{~s} \quad 71.763 \mathrm{~W}$

$\begin{array}{llllllll}02 & 16 & 23 & 36.6 & 26.923 \mathrm{~S} & 177.171 \mathrm{~W}\end{array}$
$33 \times 4.4$

$86 ? 3.8$

$10 \mathrm{G}$

$10 \mathrm{G}$

1.0
0.9
0.4
0.4
0.2
1.4
23 SOLOMON ISLANDS

10 KURIL ISIAANDS

5 NEAR EAST COAST OF HONSHO, JAPAN

5 FRANCE. ML 2.4 (LDG).

11 NEAR COAST OF CENTRAL CHILE. MD 4.0 (SAN).

91 SOUTH OF FIJI ISLANDS. MW 5.9 (HRV)

Centroid, Moment Tensor (HRV): Centroid origin time $16: 23: 38.5$; Lat $26.80 \mathrm{~s}$; Lon $177.14 \mathrm{~W}$; Dep $33.0 \mathrm{Fix}$; Half duration $2.0 \mathrm{sec}$; Principal axes (scale 10**17 $\mathrm{Nm}$ ): (T) Val=9.72, Plg=2, Azm=4; (N) Val=-1.04, Plg=88, Azm=190; (P)

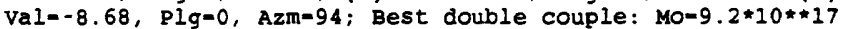
Nm; NPI: Strike-139, Dip-88, Slip-2; NP2: Strike-49, Dip=88, Slip $=178$. 


\begin{tabular}{|c|c|c|c|c|c|c|c|c|c|c|c|c|}
\hline $\begin{array}{l}02 \\
02 \\
02 \\
02 \\
02 \\
02 \\
02 \\
02\end{array}$ & $\begin{array}{l}17 \\
17 \\
17 \\
18 \\
18 \\
18 \\
20 \\
20 \\
20 \\
20 \\
20 \\
21 \\
21 \\
22 \\
23 \\
23 \\
23 \\
00 \\
01 \\
01 \\
02\end{array}$ & $\begin{array}{l}44 \\
50 \\
52 \\
12 \\
13 \\
40 \\
01 \\
09 \\
25 \\
36 \\
53 \\
07 \\
20 \\
55 \\
00 \\
18 \\
59 \\
14 \\
07 \\
41 \\
53\end{array}$ & $\begin{array}{l}51.1 * \\
21.4 ? \\
54.48 \\
16.6 \star \\
13.1 \\
23.08 \\
40.58 \\
11.68 \\
38.8 * \\
37.38 \\
10.2 \star \\
04.0 ? \\
51.0 \\
35.2 * \\
09.5 \\
29.5 ? \\
56.3 * \\
18.7 \\
49.3 * \\
05.68 \\
27.6\end{array}$ & $\begin{array}{c}34.554 \\
23.65 \\
33.689 \\
21.141 \\
51.519 \\
29.050 \\
18.881 \\
18.895 \\
18.994 \\
32.003 \\
73.248 \\
21.49 \\
4.337 \\
7.575 \\
73.085 \\
35.31 \\
20.755 \\
23.891 \\
18.216 \\
64.900 \\
40.431\end{array}$ & $\begin{array}{l}N \\
S \\
S \\
S \\
N \\
N \\
N \\
N \\
N \\
N \\
N \\
N \\
S \\
N \\
N \\
N \\
N \\
N \\
N \\
N \\
N\end{array}$ & $\begin{array}{c}83.504 \\
179.74 \\
72.008 \\
68.396 \\
16.310 \\
130.217 \\
155.256 \\
155.253 \\
145.456 \\
129.826 \\
6.795 \\
94.48 \\
134.798 \\
127.277 \\
6.412 \\
136.36 \\
121.050 \\
142.657 \\
111.197 \\
149.607 \\
1.407\end{array}$ & $\begin{array}{l}E \\
E \\
W \\
W \\
E \\
E \\
W \\
W \\
E \\
E \\
E \\
E \\
E \\
E \\
E \\
E \\
E \\
E \\
W \\
W \\
W\end{array}$ & $\begin{array}{r}33 \\
600 \\
10 \\
121 \\
5 \\
33 \\
14 \\
13 \\
244 \\
10 \\
10 \\
100 \\
33 \\
33 \\
10 \\
33 \\
33 \\
33 \\
10 \\
17 \\
10\end{array}$ & $\begin{array}{l}\mathbf{D} \\
\mathbf{G} \\
\mathbf{G} \\
\mathbf{G} \\
\mathbf{N} \\
\mathbf{N} \\
\mathbf{G} \\
\mathbf{N} \\
\mathbf{N} \\
\mathbf{N} \\
\mathbf{G}\end{array}$ & $\begin{array}{l}3.8 \\
3.5 \\
4.8 \\
4.4 \\
3.9 \\
4.3 \\
5.0 \\
4.4\end{array}$ & $\begin{array}{l}4.5 \\
4.1\end{array}$ & $\begin{array}{l}1.3 \\
0.3 \\
1.6 \\
0.4 \\
1.3 \\
1.5 \\
0.8 \\
0.3 \\
1.1 \\
0.9 \\
1.0\end{array}$ \\
\hline $\begin{array}{l}03 \\
03 \\
03 \\
03 \\
03 \\
03 \\
03 \\
03\end{array}$ & $\begin{array}{l}03 \\
03 \\
04 \\
05 \\
05 \\
05 \\
05 \\
05 \\
06\end{array}$ & $\begin{array}{l}35 \\
42 \\
45 \\
02 \\
09 \\
22 \\
24 \\
40 \\
15\end{array}$ & $\begin{array}{l}42.5^{\star} \\
10.7^{\star} \\
53.6 \\
57.28 \\
10.9 \star \\
05.8 ? \\
34.9 \star \\
03.58 \\
16.4\end{array}$ & $\begin{array}{r}8.352 \\
52.132 \\
73.184 \\
73.060 \\
24.085 \\
30.86 \\
73.370 \\
37.786 \\
44.517\end{array}$ & $\begin{array}{l}S \\
N \\
N \\
N \\
S \\
S \\
N \\
N \\
N\end{array}$ & $\begin{array}{r}113.729 \\
158.243 \\
6.356 \\
6.509 \\
66.761 \\
176.54 \\
6.473 \\
139.200 \\
114.144\end{array}$ & $\begin{array}{l}\mathbf{E} \\
\mathbf{E} \\
\mathbf{E} \\
\mathbf{E} \\
W \\
W \\
\mathbf{E} \\
\mathbf{E} \\
W\end{array}$ & $\begin{array}{r}33 \\
33 \\
10 \\
10 \\
187 \\
33 \\
10 \\
10 \\
5\end{array}$ & $\begin{array}{l}\mathbf{N} \\
\mathbf{N} \\
\mathbf{G} \\
\mathbf{G} \\
\mathbf{k} \\
\mathbf{N} \\
\mathbf{G} \\
\mathbf{G} \\
\mathbf{G}\end{array}$ & $\begin{array}{l}4.4 \\
4.2 \\
4.6 \\
3.5 \\
4.1 \\
4.4 \\
4.4 \\
4.0\end{array}$ & 4.3 & $\begin{array}{l}1.1 \\
0.8 \\
1.2 \\
0.6 \\
1.0 \\
1.4 \\
1.5 \\
0.6 \\
0.9\end{array}$ \\
\hline $\begin{array}{l}03 \\
03 \\
03 \\
03 \\
03 \\
03 \\
03 \\
03\end{array}$ & $\begin{array}{l}07 \\
07 \\
07 \\
09 \\
09 \\
09 \\
09 \\
10\end{array}$ & $\begin{array}{l}12 \\
30 \\
40 \\
01 \\
04 \\
32 \\
51 \\
06\end{array}$ & $\begin{array}{l}06.48 \\
16.9 ? \\
42.6 * \\
28.5 * \\
20.88 \\
27.9 ? \\
38.8 \\
26.9\end{array}$ & $\begin{array}{c}30.076 \\
3.87 \\
18.567 \\
54.800 \\
18.908 \\
40.30 \\
27.530 \\
40.466\end{array}$ & $\begin{array}{l}N \\
S \\
S \\
N \\
N \\
N \\
S \\
N\end{array}$ & $\begin{array}{c}88.255 \\
103.96 \\
175.706 \\
163.080 \\
155.258 \\
1.57 \\
65.627 \\
1.475\end{array}$ & $\begin{array}{l}E \\
W \\
W \\
W \\
W \\
W \\
E \\
W\end{array}$ & $\begin{array}{r}33 \\
10 \\
200 \\
33 \\
14 \\
10 \\
10 \\
10\end{array}$ & $\begin{array}{l}\mathbf{N} \\
\mathbf{G} \\
\mathbf{G} \\
\mathbf{N}\end{array}$ & $\begin{array}{l}4.1 \\
4.3 \\
4.0 \\
3.3\end{array}$ & 3.9 & $\begin{array}{l}0.5 \\
0.8 \\
0.9 \\
0.5 \\
0.4 \\
1.0 \\
1.1\end{array}$ \\
\hline $\begin{array}{l}03 \\
03 \\
03 \\
03 \\
03 \\
03 \\
03 \\
03 \\
03 \\
03 \\
03 \\
03 \\
03 \\
03 \\
03 \\
03 \\
03 \\
03 \\
03 \\
03 \\
03 \\
03\end{array}$ & $\begin{array}{l}10 \\
10 \\
10 \\
10 \\
11 \\
11 \\
11 \\
11 \\
11 \\
11 \\
11 \\
12 \\
12 \\
12 \\
12 \\
13 \\
13 \\
13 \\
14 \\
14 \\
15 \\
15 \\
15\end{array}$ & $\begin{array}{l}22 \\
48 \\
51 \\
56 \\
13 \\
35 \\
37 \\
37 \\
54 \\
55 \\
59 \\
11 \\
28 \\
32 \\
50 \\
02 \\
09 \\
33 \\
06 \\
54 \\
20 \\
21 \\
35\end{array}$ & $\begin{array}{l}39.4 ? \\
28.3 \\
26.6 * \\
56.1 * \\
56.7 \\
41.48 \\
50.08 \\
59.88 \\
49.38 \\
21.3 ? \\
00.08 \\
25.0 \\
56.6 \star \\
47.7 * \\
13.98 \\
37.5 ? \\
08.1 ? \\
24.2 * \\
00.5 \\
59.9 ? \\
45.9 \\
07.2 * \\
41.9\end{array}$ & $\begin{array}{c}17.62 \\
46.363 \\
24.980 \\
27.567 \\
54.128 \\
40.381 \\
37.199 \\
40.351 \\
45.962 \\
40.40 \\
40.381 \\
4.158 \\
2.483 \\
58.747 \\
62.826 \\
17.27 \\
58.53 \\
30.761 \\
48.478 \\
18.27 \\
38.045 \\
20.614 \\
44.959\end{array}$ & $\begin{array}{l}S \\
N \\
N \\
S \\
N \\
N \\
N \\
N \\
N \\
N \\
N \\
S \\
N \\
N \\
N \\
N \\
N \\
N \\
N \\
S \\
N \\
S \\
N\end{array}$ & $\begin{array}{r}178.79 \\
7.469 \\
125.782 \\
65.658 \\
163.115 \\
1.389 \\
3.712 \\
1.455 \\
6.066 \\
1.40 \\
1.456 \\
143.562 \\
126.746 \\
157.999 \\
148.861 \\
60.80 \\
157.85 \\
79.029 \\
153.715 \\
175.35 \\
139.496 \\
178.802 \\
11.318\end{array}$ & $\begin{array}{l}W \\
E \\
E \\
E \\
W \\
W \\
W \\
W \\
E \\
W \\
W \\
E \\
E \\
E \\
W \\
W \\
E \\
E \\
E \\
W \\
E \\
W \\
E\end{array}$ & $\begin{array}{r}553 \\
10 \\
51 \\
10 \\
33 \\
10 \\
10 \\
10 \\
10 \\
10 \\
10 \\
150 \\
33 \\
10 \\
61 \\
33 \\
10 \\
33 \\
111 \\
200 \\
10 \\
600 \\
20\end{array}$ & $\begin{array}{l}\text { ? } \\
\mathbf{G} \\
\mathbf{G} \\
\mathbf{G} \\
\mathbf{N} \\
\mathbf{G} \\
\mathbf{G} \\
\mathbf{G} \\
\mathbf{G} \\
\mathbf{G} \\
\mathbf{G}\end{array}$ & $\begin{array}{l}4.1 \\
4.4 \\
3.8 \\
4.1 \\
3.9 \\
3.9 \\
4.4 \\
4.2 \\
3.5 \\
4.2\end{array}$ & & $\begin{array}{l}0.9 \\
1.0 \\
1.1 \\
0.9 \\
0.9 \\
1.1 \\
0.5 \\
0.2 \\
1.5 \\
0.1 \\
1.1 \\
0.8 \\
0.9 \\
0.5 \\
0.5 \\
1.3 \\
1.0 \\
0.7 \\
0.9 \\
0.7 \\
0.9 \\
1.2\end{array}$ \\
\hline $\begin{array}{l}03 \\
03 \\
03 \\
03 \\
03 \\
03 \\
03 \\
03 \\
03 \\
03 \\
04 \\
04 \\
04 \\
04 \\
04 \\
04 \\
04\end{array}$ & $\begin{array}{l}19 \\
19 \\
20 \\
22 \\
22 \\
22 \\
23 \\
23 \\
01 \\
02 \\
02 \\
03 \\
04 \\
05 \\
06\end{array}$ & $\begin{array}{l}21 \\
05 \\
56 \\
55 \\
04 \\
20 \\
43 \\
14 \\
27 \\
43 \\
07 \\
52 \\
54 \\
20 \\
22 \\
04 \\
40\end{array}$ & $\begin{array}{l}32.3 * \\
38.28 \\
36.0 * \\
14.4 \\
14.4 \\
26.88 \\
54.5 \\
32.2 ? \\
14.9 * \\
43.1 * \\
00.2 \star \\
48.88 \\
43.5 \\
26.9 ? \\
15.7 \\
50.28 \\
32.9\end{array}$ & $\begin{array}{r}46.384 \\
60.823 \\
34.782 \\
34.317 \\
8.766 \\
31.599 \\
40.320 \\
36.74 \\
8.905 \\
6.043 \\
51.122 \\
59.991 \\
9.291 \\
8.62 \\
2.941 \\
44.039 \\
7.758\end{array}$ & $\begin{array}{l}N \\
N \\
N \\
N \\
S \\
N \\
N \\
N \\
S \\
N \\
N \\
N \\
N \\
S \\
N \\
N \\
S\end{array}$ & $\begin{array}{r}2.889 \\
151.623 \\
70.772 \\
28.265 \\
125.292 \\
131.862 \\
1.484 \\
71.07 \\
123.088 \\
124.100 \\
178.161 \\
141.391 \\
126.348 \\
110.20 \\
126.436 \\
9.775 \\
128.671\end{array}$ & $\begin{array}{l}W \\
W \\
E \\
E \\
E \\
E \\
W \\
E \\
E \\
E \\
E \\
W \\
E \\
E \\
E \\
E\end{array}$ & $\begin{array}{r}10 \\
77 \\
46 \\
33 \\
33 \\
10 \\
10 \\
200 \\
33 \\
33 \\
33 \\
3 \\
33 \\
100 \\
33 \\
5\end{array}$ & $\begin{array}{l}* \\
N \\
N \\
G \\
G \\
G \\
N \\
N \\
N\end{array}$ & $\begin{array}{l}3.7 \\
4.0 \\
4.5 \\
4.1\end{array}$ & 4.3 & $\begin{array}{l}0.8 \\
0.9 \\
1.1 \\
0.6 \\
1.3 \\
1.8 \\
1.2 \\
1.0 \\
0.8\end{array}$ \\
\hline
\end{tabular}

28 XIZANG

9 SOUTH OF FIJI ISLANDS

10 OFF COAST OF CENTRAL CEILE. MD 3.4 (SAN).

28 CBILE-BOLIVIA BORDER REGION

9 POLAND. ML 2.4 (CLI), 2.3 ( (NOX).

6 RYUKYU ISLANDS

8 HAWAII. 〈HVO-P>. MD 4.3 (HVO).

1 HAWAII. <HVO-P>. MD 3.8 (HVO).

31 MARIANA ISLANDS

5 KYUSHO, JAPAN

10 GREENLAND SEA

6 MYANMAR

34 IRIAN JAYA REGION, INDONESIA

18 PHILIPPINE ISLANDS REGION

9 GREENTAND SEA

4 WESTERN HONSHO, JAPAN

10 PHILIPPINE ISLANDS REGION

90 VOLCANO ISLANDS REGION

33 REVILLA GIGEDO ISLANDS REGION

17 CENTRAL ALASKA. CAEIC). ML 2.9 (AEIC).

70 SPAIN. ML 3.7 (LDG), 3.6 (STR). mbLg 3.6 (MDD). Felt (III) in the Albarracin area.

24 JAWA, INDONESIA

9 NEAR EAST COAST OF KAMCHATY

89 GREENLAND $S=$

5 GREENLAND SEA

24 SALTA PROVINCE, ARGENTINA

9 KERMADEC ISLANDS REGION

21 GREENLAND SEA

5 EASTERN BONSHO, JAPAN

77 WESTERN IDABO. MC 4.3 (GS). MD 4.3 (SEA). Felt at Challis and Salmon.

12 CENTRAI EAST PACIFIC RISE

TONGA ISLANDS

6 UNIMAK ISLAND REGION

1 HAWAII. <HVO-P>. MD 3.8 (HVO).

4 SPAIN. mbLg 2.6 (MDD).

6 SOUTH INDIAN OCBAN

104 SPAIN. MC 4.4 (LDG). mbLg 4.1 (MDD). Felt (IV) in the Albarracin area.

30 FIJI ISLANDS REGION

7 SWITZERLAND. ML 3.1 (LDG), 3.1 (STR).

14 SOUTEWESTERN RYUKYU ISLANDS

22 SOUTB INDIAN OCEAN

51 UNIMAR ISLAND REGION

5 SPAIN. mbLg 2.9 (YDD).

7 SPAIN. mbLg 2.5 (YDD).

6 SPAIN. WLg 3.2 (MDD).

9 FRANCE. ML 2.0 (LDG).

4 SPAIN. mbLg 2.4 (MDD).

SPAIN. mbLg 2.9 (MDD)

11 NEW GUINEA, PAPUA NEW GUINEA

4 NORTHERN MOLUCCA SEA

6 RAMCBATKA

3 CENTRAL ALASKA. CAEIC>. ML 2.9 (AEIC), 3.2 (PMR).

LEEWARD ISLANDS

7 KAMCHATKA

XIZANG-INDIA BORDER REGION

50 KORIL ISLANDS

8 TONGA ISLANDS

1 NEAR WEST COAST OF HONSHO, JAPAN

19 FIJI ISLANDS REGION

89 NORTHERN ITALY. ML 4.1 (GRF), 3.9 (STR), 3.9 (VIE), 3.6 (LDG).

(LDG) OF BISCAY. ML 3.0 (LDG)

KENAI PENINSULA, ALASKA. <AEIC>.

AFGHANISTAN

4 EASTERN MEDITERRANEAN SEA

8 TIMOR REGION, INDONESIA

KYUSHO, JAPAN

SPAIN. mbLg 2.7 (MDD). MC 2.5 (LDG).

AFGHANISTAN-TAJIKISTAN BORD REG.

FLORES REGION, INDONESIA

MINDANAO, PHILIPPINE ISLANDS

RAT ISLANDS, ALECTIAN ISLANDS

SOUTHEASTERN ALASKA. <AEIC>. ML 3.0 (AEIC).

7 MINDANAO, PHILIPPINE ISLANDS

JAWA, INDONESIA

NORTHERN MOLUCCA SEA

N NORTHERN ITALY. ML 2.5 (LDG).

94 BANDA SEA. MW 5.1 (HRV)

Centroid, Moment Tensor (HRV): Centroid origin time

06:48:41.1; Lat 7.76 S Fix; Lon 128.67 E Fix; Dep 33.0 Fix; Half-duration $1.0 \mathrm{sec}$; Principal axes (scale 10**16 Nm):

(T) Val-5.55, P1g-7, Azm-270; (N) Val-0.66, Plg-0, Azm-180; (P) Val--6.21, Plg-83, Azm-90; Best double couple:

Mo-5.9*10**16 Nm; NP1: Strike-0, Dip-38, S1ip--90; NP2: Strike-180, Dip-52, Silp--90.

$04070244.1 * 31.563 \mathrm{~N} 131.907 \mathrm{E}$

$04081242.6 * 22.087 \mathrm{~N} 144.242 \mathrm{E}$

$04 \quad 094104.7836 .874 \mathrm{~N} \quad 3.581 \mathrm{~W}$

$33 \mathrm{~N}$

$68 ?+.0$

0.9

22

FIJI ISLANDS REGION

0.55 KYUSHO, JAPAN

0.722 VOLCANO ISLANDS REGION

$10 \mathrm{G}$

0.65 STRAIT OF GIBRALTAR. mbLg 2.5 (MDD). 
$\begin{array}{lllll}04 & 09 & 51 & 57.18 & 27.480 \mathrm{~s}\end{array}$

$04 \quad 1008 \quad 47.1840 .008 \mathrm{~N}$

$04103510.2 * 18.054 \mathrm{~S}$

$04 \quad 10 \quad 44 \quad 18.4 ? \quad 33.35 \quad s$

$04111434.2 * 22.385 \mathrm{~s}$

$04 \quad 124709.1 ? \quad 18.44 \mathrm{~s}$

$04 \quad 125824.1 * 35.050 \mathrm{~N}$

$04135128.326 .419 \mathrm{~N}$

$04 \quad 14 \quad 37 \quad 33.2$ ? $2.36 \quad \mathrm{~s}$

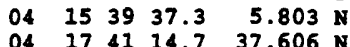

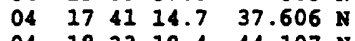

$04 \quad 183318.4 \quad 44.107 \mathrm{~N}$

$\begin{array}{llllll}04 & 19 & 02 & 02.0 & 51.582 & \mathrm{~N}\end{array}$

$\begin{array}{lllllll}04 & 19 & 10 & 32.48 & 62.490 & \mathrm{~N} \\ 04 & 19 & 59 & \mathbf{5 8 . 3} & 27.471 & \mathrm{~S}\end{array}$

$\begin{array}{lllllll}04 & 19 & 59 & 58.3 * & 27.471 & \text { s } \\ 04 & 21 & 42 & 57.9 ? & 34.85 & \text { s }\end{array}$

$215155.0 ? 16.01$ N 79.85

$04221920.5 * 52.185 \times 171.228$ \%

$04225758.8 * 22.816 \mathrm{~s} 68.368 \mathrm{~W}$

$\begin{array}{llllllll}04 & 23 & 47 & 49.9 ? & 25.16 & \text { s } & 179.73 & \text { E }\end{array}$

05 00 $40 . \ldots 28.2 \quad 40.062 \mathrm{~N} 143.540 \mathrm{E}$
$65.884 \mathrm{E} \quad 10 \mathrm{G} \quad 4.0$

$\begin{array}{llll}10.775 & \text { E } & 10 \mathrm{G} & 3.7\end{array}$

$136 \mathrm{D} \quad 4.2$

$68.803 * \quad 95 \mathrm{D} \quad 4.6$

$\begin{array}{rrr}95 & D & 4.6\end{array}$

$\begin{array}{rrr}300 & G & 3.9 \\ 33 & \times & 3.5\end{array}$

$33 \times 4.3$

$33 \times 3.8$

$116 ? 4.8$

$33 \times 4.2$

$10 \mathrm{G}$

$33 \times 4.0$

92

$501 ? 4.4$

$10 \mathrm{G} \quad 4.4$

$10 \mathrm{G} \quad 4.1$

$33 \times 4.0$

$100 \mathrm{G} \quad 4.2$

$600 \mathrm{G} \quad 4.1$

$\begin{array}{llll}33 & N & 5.2 & 4.6\end{array}$
0.811 SOUTH INDIAN OCEAN

1.017 GRGECE-ALBANIA BORDER REGION

0.915 NORTHERN CEITE

0.610 OFF COAST OF CENTRAL CHILE

1.242 NORTHERN CEILE. Felt (III) at Calama and Chuquicamata.

1.421 VANUATO ISLANDS

i.0 10 CRETE

0.927 NORTEIFASTERN INDIA

1.4 8 NEAR NORTE COAST OF IRIAN JAYA

1.265 MINDANAO, PHILIPPINE ISLANDS

1.249 SOUTHERW GREECE

0.610 NORTHERN ITALY. MIL 1.9 (LDG), 1.8 (GEN).

0.618 OFF EAST COAST OF RAMCEATRA

85 CENTRAL ALASRA. LAEIC>.

$0.9 \$ 5$ KERMADEC ISLANDS REGION

1.011 SOUTHERN EAST PACIFIC RISE

0.88 SOUTHERN INDIA. Felt at Chilakalurupet, Nekarikallu and Vinukonda.

1.010 FOX ISLANDS, ALEUTIAN ISLANDS

1.17 NORTHERN CHILE

0.714 SOUTH OF FIJI ISLANDS

0.8133 OFF EAST COAST OF HONSHO, JAPAN

0.8238 $\begin{array}{lllllllllll}05 & 02 & 07 & 16.2 ? & 20.31 & \mathrm{~s} & 175.21\end{array}$ $\begin{array}{lllllll}05 & 02 & 08 & 58.2 & 15.267 \mathrm{~S} & 173.126 & \mathrm{w}\end{array}$
$33 \times 3.6$

$215 \mathrm{D} \quad 3.9$

$10 \mathrm{G} \quad 4.4$

$33 \times 4.4$

11 G $6.06 .7 \quad 1.4$
1.5

$1.1 \quad 15$

$\begin{array}{lr}1.1 & 15 \\ 0.8 & 7\end{array}$

0.87

4319
$052423.0 * 15.231 \mathrm{~S} \quad 173.286$ म

$\begin{array}{lllllllll}05 & 03 & 45 & 06.8 ? & 36.84 & \text { S } & 179.39\end{array}$

$\begin{array}{llllllll}05 & 05 & 13 & 34.0 ? & 37.83 & \text { N } & 20.16 & \text { E }\end{array}$

$\begin{array}{llllllll}05 & 05 & 32 & 04.7 ? & 34.45 & \mathrm{~s} & 71.25 & \mathrm{~W}\end{array}$

$\begin{array}{lllll}05 & 053 & 48.9 * 33.823 & \text { N } 137.393 \mathrm{E}\end{array}$

$\begin{array}{lllllllll}05 & 06 & 16 & 13.0 & 56.224 & \mathrm{~N} & 160.376 \mathrm{~N}\end{array}$

$\begin{array}{llllll}06 & 20 & 15.3 ? & 23.77 & \mathrm{~s}\end{array}$

$\begin{array}{lllll}06 & 37 & 28.6 ? & 36.66 \quad \mathrm{~N}\end{array}$

$\begin{array}{llllll}0726 & 26.78 & 33.830 \mathrm{~N}\end{array}$

$\begin{array}{lllll}08 & 20 & 21.1 ? & 14.59 \mathrm{~N}\end{array}$

$\begin{array}{llll}08 & 42 & 59.8 ? & 14.56\end{array}$

$091050.3 * 36.930 \mathrm{~N}$

$\begin{array}{lllll}09 & 24 & 56.0 ? & 1.50 \mathrm{~s}\end{array}$

$0948 \quad 43.1867 .571 \mathrm{~N}$

$\begin{array}{lllll}10 & 00 \quad 54.48 & 34.627 \mathrm{~N}\end{array}$

05

05111224.0 ? 16.90 N

$\begin{array}{llllll}13 & 40 & 28.3 * 5.726 \mathrm{~S}\end{array}$

$\begin{array}{llllll}05 & 13 & 51 & 58.28 & 38.652 \mathrm{~N}\end{array}$

$051454 \quad 55.5 \quad 53.510 \mathrm{~N}$

$\begin{array}{lllllllll}05 & 15 & 04 & 58.1 \text { ? } & 38.91 & \mathrm{~s}\end{array}$

$05 \quad 1534 \quad 40.3 * 34.581 \mathrm{~N}$

$\begin{array}{ccccc}05 & 16 & 52 & 07.7 & 0.126 \\ 05 & 16 & 59 & 46.4 ? & 34.53\end{array}$

$05170105.2 * 15.293 \mathrm{~s}$ 7.8c $63.278 \mathrm{~N}$

$05 \quad 132613.2 * 5.755 \mathrm{~S}$
100 G 3.8

66.89 W 217 ? 3.2

$141.49 \mathrm{E}$

$116.199 \mathrm{~W}$

$45.10 \mathrm{~W}$

$45.00 \mathrm{~W}$

32.919

80.43 W

$149.171 \mathrm{~h}$

$151.443 \mathrm{~W}$

$164.161 \mathrm{~B}$

105.09

$150.417 \mathrm{E}$

$0.527 \mathrm{E}$

$0.733 \mathrm{E}$

$157.785 \mathrm{E}$

$92.18 \mathrm{~W}$
135.662

$123.757 \mathrm{E}$

70.35

173.332

$33 \mathrm{~N}$

$10 \mathrm{G} 4.2$

$10 \mathrm{G} \quad 4.5$

36

5

8

$124 ? 4.2$

$33 \mathrm{~N} 4.1$

$10 \mathrm{G}$

$143 ? 4.6$

$10 \mathrm{G} 4.1$

$391 \quad 3.7$

$147 ? 4.3$

$5 \mathrm{G}$

33
$33 \mathrm{~N} 4.7$

854.0

0.9

1.0

$33 \mathrm{~N} 4.6$

$\begin{array}{lll}33 & \mathrm{~N} & 4.6 \\ & \mathrm{~N} & 3.8\end{array}$

$60 \mathrm{G}$

$10 \mathrm{G} \quad 4.1 \quad 4.1 \quad 0.8$

$\begin{array}{lll}33 & \mathbf{4} .8 & 0.7\end{array}$

$33 \mathrm{~N} 3.73 .6 \quad 1.0$
$33 \mathrm{~N} \quad 4.2 \quad 0.9$

chenter

NBAR EAST COAST OF RAMCHATKA. MW 5.4 (BRV)

Centroid, woment Tensor (HRV): Centroid origin time

on 3 ; Lat $54.36 \mathrm{~N}$; Lon $162.17 \mathrm{E}$; Dep 41.7; Half-

dur. $\quad 2 \mathrm{sec}$; Principal axes (scale 10**17 $\mathrm{km}$ ): (T)

Val-1... Plg-80, Azm-325; (N) Val-0.15, Plg-4, Azm-214;

(P) Val=-1.43, Plg=9, Azm=123; Best double couple:

vo-1.4*10*17 km; NP1: Strike-208, Dip-36, Slip-84; NP2 : Strike-36, Dip-54, Slip-95.

NEW IRETAND REGION, P.N.G.

HINDD KUSB REGION, AFGBANISTAN

SOUTH OF AOSTRALIA

TONGA ISLANDS

TONGA ISLANDS. WW 6.7 (HRV), 6.6 (GS). We 6.0 (GS). MS 6.8

(BRR). Felt (III) at Apia, Western Samoa.

Broadband Source Parameters (GS): Dep 42; NP1: Strike=130,

Dip-73, Slip--60; NP2: Strike-247, Dip-34, S1ip--149;

Radiated energy $2.3 * 10 * \approx 13 \mathrm{Nm}$.

Moment Tensor (GS): Dep 47; Principal axes (scale 10*\#19

$\mathrm{Nan}$ : : (T) Val=0.99, Plg-18, Azm=237; (N) Val=0.02, Plg=2,

Azm-146; (P) Val--1.01, P1g-72, Azm-49; Best double couple:

vo-1.0*10*19 $\mathrm{km}$; NP1: Strike-330, Dip-28, s1ip=-85; NP2:

Strike-145, Dip-63, Slip=-93.

Centroid, Moment Tensor (BRV): Centroid origin time

02:09:07.7; Lat $15.27 \mathrm{~s}$; Lon $172.76 \mathrm{k}$; Dep 53.8 ; Half-

duration $5.3 \mathrm{sec}$; Principal axes (scale 10*19 $\mathrm{km}$ ): (T)

val-1.14, Plg-16, Azm-199; (N) Val--0.04, Plg-19, Azm-295;

(P) Val=-1.10, Plg=65, Azm=71; Best double couple:

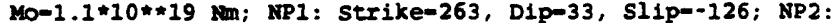

Strike-124, Dip-64, Slip=-69.

scalar Noment (PPT): $10=1.2 * 10 \star \star 19 \mathrm{~km}$.

SCalar Noment

MEAR COAST OF PERO. Felt (III) at

1.420 EAST OF NORTH ISLAND, N.Z.

1.58 IONIAN SEA

0.27 MEAR COAST OF CENTRAL CHIL

0.710 NEAR $S$. COAST OF HONSHU, JAPAN

0.825 ALASKA PENINSULA

1.35 JUJOY PROVINCE, ARGENTINA

.75 NEAR EAST COAST OF HONSHU, JAPAN

SOUTHERN CALIFORNIA. <PAS-P>. MD 2.8 (PAS).

NORTHERN MID-ATLANTIC RIDGE

8 NORTHERN MID-ATIAANTIC RIDGE

10 AZORES ISLANDS REGION

9 NEAR COAST OF ECUADOR

32 NORTHERN ALASKA. CAEIO. MI 3.3 (AEIC).

52 SOUTHERN CALIFORNIA. 〈PAS-P>. MD 3.0 (PAS). ML 3.0 (GS). Felt.

27 SOUTHERN CALIFORNIA. <PAS-P>. MD 2.7 (PAS). ML 2.8 (GS). Felt.

14 CENTRAL ALASKA. CAEIC>. YIC 2.6 (AEIC)

93 KOMANDORSKY ISLANDS REGION

9 OFF COAST OF MICHOACAN, MEXICO

16 NEW BRITAIN REGION, P.N.G.

9 NEW IRELAND REGION, P.N.G.

5 SPAIN. ImbLg 3.0 (MDD).

6 KAMCHATKA

5 WEST CHILE RISE

7 NEAR S. COAST OF WESTERN HONSHO

28 MINAHASSA PENINSULA, SULANESI

8 CEILE-ARGENTINA BORDER REGION. MD 3.1 (SAN)

44 TONGA ISLANDS. NW 5.4 ( $\mathrm{ERV}$ ).

Centrold, Moment Tensor (BRV): Centrold origin time

17:01:07.6; Lat 15.52 s; Lon 172.50 w; Dep 15.0 Fix; Half-

duration $1.4 \mathrm{sec}$; Principal axes (scale 10**17 $\mathrm{Nm}$ ): (T)

Val=1.48, Plg=45, Azm=340; (N) Val=0.16, Plg=37, Azm=200

(P) Val=-1.63, Plg-21, Azm-93; Best double couple:

Mo-1.6*10*17 Nm; NP1: Strike-138, Dip-40, Slip-22; NP2 :

Strike=31, Dip=76, slip-128.

$05175551.1 * 37.113 \times 135.532 \mathrm{E} 369 * 3.4 \quad 0.6$

12 SEA OF JAPAN

TAIWAN REGION 
$10 \mathrm{G}$

$10 \mathrm{G} 3.8$

$33 \mathrm{~N} 3.6$

$33 \mathrm{~N} 4.6$

$10 \mathrm{G}$

$10 \mathrm{G} \quad 4.2$

2

00 G 3.6

$10 \mathrm{G} \quad 4.6 \quad 4.9 \quad 1.4$ $\begin{array}{lllllll}20 & 32 & 06.9 & 29.068 & N & 139.524\end{array}$

$\begin{array}{lllllll}21 & 17 & 00.28 & 9.364 & \mathrm{~S} & 153.970 \mathrm{E}\end{array}$

$211813.8 \% 65.186 \mathrm{~N} 149.450 \mathrm{~W}$

4064.5 $33 \mathrm{~N} 4.2$

$3139.2 \quad 1.996$

\section{1}

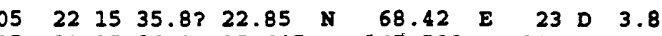
$\begin{array}{llllllllllll}05 & 22 & 35 & 06.8 & 15.647 & \mathrm{~S} & 167.733 & \mathrm{E} & 133 \mathrm{D} & 5.0\end{array}$ $05223822.0 \cdot 20.690 \mathrm{~S} 178.310 \% 550 \mathrm{D} \quad 6.4$
0.16 CHILE-ARGENTINA BORDER REGION

0.710 NORTE OF SVALBARD

0.68 LUZON, PHILIPPINE ISLANDS

1.126 TONGA ISLANDS REGION

0.59 SPAIN. mbLg 2.4 (MDD).

7 SVALBARD REGION

9 CALIFORNIA-NEVADA BORDER REGION. <GM-P>. MD 2.8 (GM).

8 FLORES SEA

26 CENTRAI EAST PACIFIC RISE. NW 5.3 (BRV).

Centroid, voment Tensor (HRV): Centroid origin time

20:27:42.2; Lat $6.07 \mathrm{~s}$; Lon $107.16 \mathrm{w}$; Dep $15.0 \mathrm{Fix}$; Half duration 1.2 sec; Principal axes (scale 10*17 $\mathrm{Nm}$ ): (T) Val=1.13, Plg-7, Azm-140; (N) Val--0.08, Plg-77, Azm-264; (P) Val--1.05, Plg-10, Azm-48; Best double couple:

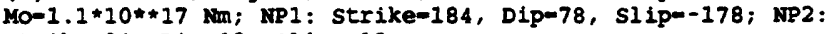
Strike-94, Dip=88, S11p=-12.

68 SOUTH OF HONSHO, JAPAN

4 D'ENTRECASTEAOX ISLANDS REGION

7 NORTHERN ALASKA. CAEIC>. ML 3.0 (AEIC), 3.3 (PMR). Felt at Goldstream Valley.

OFF COAST OF.ECUADOR. M 6.3 (GS) 6.3 (BRV). Me 6.0 (GS) Ms 5.7 (BRK). Felt in Guayas and Manabi Provinces.

Broadband Source Parameters (GS): Dep 16; Radiated energy $1.9 * 10 * 13 \mathrm{~km}$.

Moment Tensor (GS): Dep 7: Principal axes (scale 10**18 $\mathrm{Nm}$ ): (T) Val-2.97, Plg-55, Azm-70; (N) Val--0.02, Plg-5, Azm-168; (P) val-2.95, Plg-35, Azm-261; Best double couple: Mo=3.0\#10*18 $\mathrm{Nm}$; NP1: Strike-16, Dip=11, Slip-119; NP2: Strike-167, Dip $=80$, Slip $=84$.

Centroid, Moment Tensor (BRV): Centrold origin time

$21: 39: 19.3$; Lat $2.10 \mathrm{~s}$; Lon $81.08 \mathrm{w}$; Dep $16.0 \mathrm{Bdy}$; Halfduration $3.5 \mathrm{sec}$; principal axes (scale 10*18 Nm): (T) Val=3.31, Plg-61, Azm=86; (N) Val=0.06, Plg-1, Azm-355; (P) Val=-3.37, PIg-29, Azm-265; Best double couple:

Mo-3.3*10**18 Nm; NP1: Strike-353, Dip-16, Slip-88; NP2: Strike-175, Dip $=74$, Slip -91.

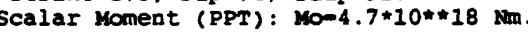

1.18 SOUTHERN INDIA

1.0130 VANUATO ISLANDS. Felt on Santo.

0.9508 FIJI ISLANDS REGION. WW 7.4 (ERV), 7.3 (GS). Ne 7.0 (GS). mb 6.8 (BRK). Felt (II) on Raoul, Kermadec Islands.

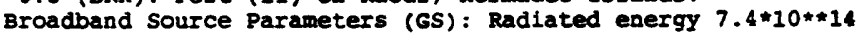
Nin.

Woment Tensor (GS): Dep 545; Principal axes (scale 10 $\$ 20$ $\mathrm{Nm}$ ): (T) Val=0.88, Plg=22, $\mathrm{Azm}=126$; (N) Val=0.18, Plg=5. Azm=218; (P) Val--1.06, PIg-67, Azm-320; Best double couple: No-9.7*10*19 Nm; NP1: Strike-206, Dip=23, S1ip=-103; NP2: Strike-40, D1p-68, Slip=-85.

Centroid, Moment Tensor (kRV): Centroid origin time $22: 38.31 .2$; Lat $20.72 \mathrm{~s}$; Ion $178.16 \mathrm{w}$; Dep 555.0 ; Balf duration $11.4 \mathrm{sec}$; Principal axes (scale 10*\$20 $\mathrm{km}$ ): (T) Val-1.30, Plg-25, Azm=131; (N) Val-0.12, Plg=3, Azm=222: (P) Val--1.42, Plg-64, Azm-319; Best double couple:

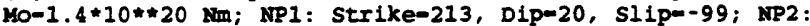
Strike-43, Dip-70, slip--87.

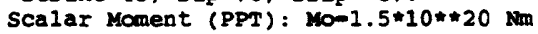
$\begin{array}{lllll}23 & 28 & 34.08 & 46.587 & N\end{array}$ $\begin{array}{llllll}23 & 37 & 15.38 & 12.251 & \mathrm{~N} \\ 23 & 49 & 15.5 & 40.341 & \mathrm{~N}\end{array}$ $1.212 \mathrm{E}$

$1.430 \mathrm{~W}$ $\begin{array}{lllll}23 & 58 & 40.8 ? \quad 39.60 \quad \mathrm{~N}\end{array}$ $000121.6 * 25.178 \mathrm{~S}$ $002902.1 * 20.697 \mathrm{~s}$ $003545.3 \% 30.790 \mathrm{~N}$ $\begin{array}{lllll}00 & 49 & 12.8 ? & 19.90 \mathrm{~s}\end{array}$ $005943.3 * 16.984 \mathrm{~s}$ $\begin{array}{llll}02 & 07 & 41.27 \quad 36.83\end{array}$ 023257.3 ? $20.65 \mathrm{~s}$ $\begin{array}{llllll}03 & 04 & 32.1 & 24.898 & N\end{array}$ $033353.2 * 13.429 \mathrm{~N}$ $034859.0 \& 59.177 \mathrm{~N}$ $0403 \quad 38.7859 .167 \mathrm{~N}$ $041436.0 * 6.765 \mathrm{~s}$ $052056.5 ? 20.66 \mathrm{~s}$ $062104.0 * 25.350 \mathrm{~N}$ $\begin{array}{llll}06 & 29 & 32.4 & 38.794\end{array}$ $\begin{array}{llll}10 & 13 & 14.3 & 21.356 \mathrm{~s}\end{array}$
$20.49 \mathrm{E}$ $177.780 \mathrm{~W}$ $178.434 \mathrm{~W}$ $139.940 \mathrm{E}$ 178.53
70.083 $6.93 \mathrm{~W}$ 178.25 พ $95.279 \mathrm{E}$ $146.108 \mathrm{E}$ $153.120 \mathrm{~W}$ $153.075 \mathrm{~W}$ $147.441 \mathrm{E}$ $178.32 \mathrm{~W}$ $95.540 \mathrm{E}$ $175.461 \%$ $118.706 \mathrm{~W}$
73

$10 \mathrm{G}$

$5 \mathrm{G}$

$33 \mathrm{~N} 3.8$

$10 \mathrm{G}$

$10 \mathrm{G} \quad 3.2$

$205 \mathrm{D} \quad 4.0$

$160 * 3.7$

500 G 3.9

$146 * 3.9$

$10 \mathrm{G}$

500 G 4.1

$125 \mathrm{D} 4.5$

$66 ? 4.3$

14

13

$73 * 4.4$

$500 \mathrm{G} \quad 4.1$

100 G 3.9

$5 \mathrm{G}$

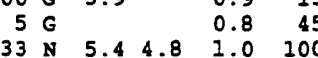

13

0.84

0.910

1.114

0.98

$\begin{array}{ll}1.1 & 22 \\ 1.1 & 56\end{array}$

1.210

1.210

1.211

0.3

0.7

1.0

28

$0.8 \quad 17$

1.213

00

GREECE-ALBANIA BORDER REGION.
buildings at Konitsa, Greece.

KODIAR ISIAAND REGION. <AEIC>

SPAIN. mbLg 2.6 (MDD).

FRANCE. ML 2.3 (LDG).

SAMAR, PHILIPPINE ISLANDS

Albarracin area.

GREECE-ALBANIA BORDER REGION

GREECE-ALBANIA BORDER
SOUTH OF FIJI ISLANDS

FIJI ISLANDS REGION

SOUTH OF HONSEU, JAPAN

FIJI ISLANDS REGION

SOUTEERN PERU

STRAIT OF GIBRALTAR. mbLg 2.5 (MDD).

FIJI ISLANDS REGION

MYANMAR

SOUTH OF MARIANA ISLANDS

SOUTHERN ALASKA. CAEIC>. MI 2.6 (AEIC).

SOUTHERN ALASKA, 〈AEIC>. MI 2.7 (AEIC).

EASTERN NEW GUINEA REG., P.N.G.

FIJI ISLANDS REGION

MYANMAR-INDIA BORDER REGION

CALIFORNIA-NEVADA BORDER REGION. ML 3.5 (GS), 3.5 (BRK).

TONGA ISLANDS. MW 5.6 (HRV). $\begin{array}{llll}10 & 26 & 30.6 & 35.095 \mathrm{~N}\end{array}$ $\begin{array}{llllll}10 & 28 & 37.0 ? & 36.00 & \mathrm{~N} \\ 10 & 32 & 23.6 ? & 20.55 & \mathrm{~S}\end{array}$ $115211.5 \% 34.079 \mathrm{~N}$
$27.783 \mathrm{E}$

$69.92 ? 3.9$

178.32 \% 500 G 3.8 $116.782 \% 13$
1.4

0.9

Centroid, Moment Tensor (HRV): Centroid origin time

$10: 13: 22.0$; Lat $21.20 \mathrm{~s}$; Ion 174.94 \%; Dep 43.8; Half-

duration $1.5 \mathrm{sec}$; Principal axes (scale 10 $\star 17 \mathrm{Nm}$ ): (T)

val-3.32, Plg-51, Azm-87; (N) val--0.44, Plg-4, Azm-182

(P) Val--2.87, Plg-39, Azm=275; Best double couple:

Mo-3.1*10*\#17 Nm; NP1: Strike-33, Dip-7, Slip-121; NP2:

Strike=182, Dip=84, slip $=86$.

DODECANESE ISLANDS

EINDU KUSH REGION, AFGHANISTAN

FIJI ISILANDS REGION

36 SOUTHERN CALIFORNIA. <PAS-P>. MD 3.4 (PAS). ML 3.3 (GS). Felt in the Banning area. 
$\begin{array}{llll}06 & 15 & 25 & 04.4 * 21.631\end{array}$

$\begin{array}{lllllllll}06 & 16 & 25 & 37.6 ? & 32.07 & \mathrm{~s}\end{array}$

06165639.1 *1.342

$\begin{array}{lllll}06 & 17 & 01 & 35.7 & 7.171\end{array}$

$06 \quad 195918.4833 .783 \mathrm{~s}$

$\begin{array}{llllr}06 & 20 & 10 & 39.3 & 6.119 \\ 06 & 20 & 27 & 19.6 & 27.676\end{array}$

$\begin{array}{lllllll}06 & 20 & 38 & 47.0 ? & 33.13 & \mathrm{~s}\end{array}$

$06205427.5 * 27.672$ N

$06 \quad 205829.2 * \quad 5.519 \mathrm{~s}$

$06213517.1 * 6.816 \mathrm{~N}$

$06224124.7 * 20.586$

$07005052.4 \quad 14.898 \mathrm{~s}$

$\begin{array}{lllllll}07 & 01 & 03 & 00.7 ? & 36.74\end{array}$

07012130.2 * 07.207 औ

$07 \quad 0130 \quad 47.08 \quad 62.028 \mathrm{~N}$

$\begin{array}{lllllll}07 & 02 & 02 & 09.18 & 18.889 & \mathrm{~N}\end{array}$

$07 \quad 0212 \quad 08.4860 .091$ N

$07 \quad 02 \quad 39 \quad 45.3 * 23.695 \mathrm{~s}$

070355 i4.6? 24.60 s

$\begin{array}{llllll}07 & 07 & 06 & 21.2 & 12.202 & \mathrm{~N}\end{array}$

$\begin{array}{llllll}07 & 07 & 21 & 01.7 ? & 1.29 & \mathrm{H}\end{array}$

$\begin{array}{llllll}07 & 08 & 03 & 44.4 * 30.139 & \mathrm{~N}\end{array}$

$07082431.8 * 18.123 \mathrm{~s}$

$\begin{array}{lllllll}07 & 08 & 57 & 43.8 & 53.196 & \mathrm{~N}\end{array}$

$07 \quad 100013.1 * 8.995 \mathrm{~N}$

$\begin{array}{lllll}07 & 12 & 21 & 50.36 & 35.743 \mathrm{~N}\end{array}$

$\begin{array}{lllll}07 & 12 & 59 & 02.0 * & 3.916 \\ \mathrm{~N}\end{array}$

$07 \quad 132901.1 * 51.474 \mathrm{~N}$

$\begin{array}{llllll}07 & 14 & 36 & 33.6 & 23.907 & \mathrm{~N}\end{array}$

$07 \quad 1439 \quad 56.6 \quad 5.968 \mathrm{~s}$

$\begin{array}{llllll}07 & 15 & 13 & 47.6 ? & 25.07 & \mathrm{~S}\end{array}$

$\begin{array}{lllll}07 & 15 & 29 & 02.7 * 18.943 \mathrm{~s}\end{array}$

$\begin{array}{lllllllll}07 & 15 & 47 & 48.78 & 61.855 & \mathrm{~N}\end{array}$

$\begin{array}{llllll}07 & 15 & 57 & 04.08 & 42.843 & \mathrm{~N} \\ 07 & 16 & 11 & 24.8 & 9.795 & \mathrm{~N}\end{array}$

$\begin{array}{lllllll}07 & 16 & 31 & 27.38 & 33.078\end{array}$

$\begin{array}{lllllll}07 & 16 & 38 & 42.4 & 24.465 & s\end{array}$

$07165513.6844 .799 \mathrm{~N}$

$07 \quad 171811.4 \quad 39.254 \mathrm{~N}$

$\begin{array}{llllll}07 & 17 & 50 & 24.78 & 42.805\end{array}$

$07185110.3 * 58.665 \mathrm{x}$

$07185156.0 * 58.694$ H

$\begin{array}{llllll}07 & 19 & 29 & 28.88 & 59.648 \text { H }\end{array}$

$07202655.2 ? 27.47$ s

$07211410.6 * 52.244 \mathrm{H}$

$\begin{array}{lllllll}07 & 21 & 17 & 26.7 ? & 5.27 & 5\end{array}$

$07212613.7 * 28.023 \mathrm{~N}$

$\begin{array}{llllll}07 & 22 & 08 & 03.1 ? & 33.88\end{array}$

$072221 \quad 13.1833 .174$

$\begin{array}{lllll}07 \quad 22 & 54 & 13.98 & 37.346 & \mathrm{~N}\end{array}$

$\begin{array}{lllllll}07 & 23 & 33 & 17.7 & 20.887 & \mathrm{~s} \\ 07 & 23 & 34 & 24.37 & 1.79 & \end{array}$

$08 \quad 0052 \quad 34.4$ ? $9.47 \quad s$

$08011934.7 * 22.135 \mathrm{~s}$

$\begin{array}{llllll}08 & 01 & 52 & 04.6 & 36.309 & \mathrm{~N}\end{array}$

$08020939.8 * 37.275 \mathrm{~N}$

$080215 \quad 36.7 * 2.026 \mathrm{~s}$

$\begin{array}{lllllll}08 & 02 & 34 & 50.3 \& & 61.107 \mathrm{~N}\end{array}$

$\begin{array}{llllllll}08 & 03 & 24 & 48.1 ? & 18.97 \mathrm{~s}\end{array}$

$\begin{array}{llllllll}08 & 05 & 15 & 11.68 & 59.734 \text { H }\end{array}$

$08 \quad 06 \quad 22 \quad 49.5 * 40.352 \mathrm{~N}$

$08091217.0 * 28.417 \mathrm{~s} 136.169 \mathrm{E}$

$08091906.3 \% 40.360 \mathrm{~N} \quad 124.735 \mathrm{~W}$

$\begin{array}{lllllllll}08 & 10 & 04 & 04.0 ? & 0.88 & \mathrm{~s} & 136.70 \quad \mathrm{E}\end{array}$ $\begin{array}{llllllllll}08 & 10 & 23 & 36.2 \& & 18.903 & \mathrm{~N} & 155.259 & \mathrm{H}\end{array}$ $08 \quad 1028 \quad 48.1 \quad 12.010 \mathrm{~N} \quad 125.733 \mathrm{E}$ 65.57 .
$67.535 \mathrm{~W}$

$143.167 \mathrm{E}$ 70.59 $75.258 \mathrm{~B}$ $71.067 \mathrm{~F}$ $149.180 \mathrm{E}$ $53.012 \mathrm{E}$ $53.066 \mathrm{E}$ $129.300 \mathrm{E}$ $73.156 \mathrm{n}$ 78.643 พ

179.012 4.66 W

151.336 ต 155.250 พั $153.089 \mathrm{~W}$ 70.47 म $126.427 \mathrm{E}$ $19.72 \mathrm{E}$ $175.235 \mathrm{H}$ $159.400 \mathrm{E}$ $117.626 \mathrm{~N}$ $28.099 \mathrm{E}$ $16.372 \mathrm{E}$
$96.772 \mathrm{E}$ $46.232 \mathrm{E}$ $179.65 \mathrm{E}$ 173.519 $7.092 \mathrm{~W}$ $69.609 \mathrm{H}$

$31.325 \mathrm{E}$ $6.648 \mathrm{E}$ $143.112 \mathrm{E}$ $157.735 \mathrm{E}$ $157.682 \mathrm{E}$ 88.114 E $159.385 \mathrm{E}$ $\begin{array}{ll}136.12 & \mathrm{E} \\ 108.390 & \mathrm{E}\end{array}$ $135.33 \mathrm{E}$ $115.601 \mathrm{w}$

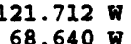
$126.28 \mathrm{E}$ $118.28 \mathrm{E}$ $63.719 \mathrm{~W}$ 49.873 $79.218 \mathrm{n}$ $150.812 \mathrm{\%}$ 173.59 ต $150.471 \mathrm{~W}$ $124.637 \mathrm{~W}$ $23 G \quad 4.5$

$33 \times 4.3$ 13 $33 \mathrm{~N} \quad 5.04 .5 \quad 1.0$

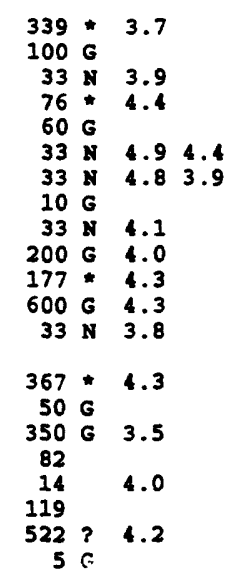

0.2

1.3

0.8

1.3

0.7

0.4

0.9

0.4
0.7

500 .

$33 \times 4.6$

$33 \times 4.3$

$33 \mathrm{~N} 3.9$

$200 G 4.3$

$101 \mathrm{D} 4.3$

$61 ? 4.6$

$137 ? 4.7$

$5 \mathrm{G}$
85

424.84 .31 .0

$\begin{array}{lll}33 & 6.1 & 1.1 \\ 34 & 4.7 & 1.0\end{array}$

34

$20 \mathrm{G}$

$33 \times 3.9$

$33 \mathrm{~N}$

$53 \mathrm{D} \quad 4.3$

$10 \mathrm{G}$

$10 \mathrm{G} \quad 3.6$

$20 \mathrm{G}$

$10 \mathrm{G} 4.0$

$10 \mathrm{G} \quad 4.1$

$10 \mathrm{G} \quad 4.3$

33 N 3.8

33 N 3.7

33 N 4.0

$33 \times 4.0$$$
5
$$

8

$112 \mathrm{D} 4.2$

$33 \times 4.3$

$33 \times 4.3$

$331 \star 3.9$

33 N 3.5

$119 \star 4.5$

$162 ? 4.1$

49

$100 ? 4.5$

14 MARIANA ISLANDS REGION

0.310 CHILE-ARGENTINA BORDER RBGION. MD 2.9 (SAN).

0.812 EASTERN CAOCASUS

1.130 NORTHERN COLOMBIA

$0.7 \quad 114$

0.410

0.7

$\begin{array}{ll}1.1 & 35 \\ 0.8 & 9\end{array}$

NORTEERN COLOMBIA

vaAR coAst of central chils

NEW BRITAIN REGION, P.N.G.

SOUTHERN IRAN

OFF COAST OF CENTRAL CHILE. ND 3.4 (SAN)

7 SOUTHERN IRAN

8 BANDA SEA

WORTHERN COLOMBIA

FIJI ISIANDS REGION

2 GREECE. MC 3.5 (ROM). Felt in Akhaia and Aitolia kai Akarnania.

1 FIJI ISLANDS REGION

STRAIT OF GIBRALTAR

9 SEA OF JAPAN

77 CENTRAL ALASKA. <AEIC>.

3 BAWAII. <EVO-P>. MD 4.3 (EVO).

SOUTE OF FIJI ISIANTDS

0.599 SOUTH OF FIJI ISIANDS

1.331 PHILIPPINE ISLANDS REGION

1.18 CELEBES SEA

1.213 XIZANG

0.817 TOMGA ISLANDS

1.044 NTAR EAST COAST OF KAMCEATKA

1.230 PAILIPPINE ISLANDS REGION

10 CENTRAL CALIFORNIA. 〈PAS-P>. MD 2.8 (PAS).

129 NORTH OF HALMAHERA, IKDONESIA

1.76 POLAND. MG 2.9 (WAR).

22 MAAMMAR

37 EASTERN NEW GOINEA REG., P.N.G. MC 5.0 (PMG).

12 SOUTH OF FIJI ISTANDS

26 TONGA ISLANDS

65 SOUTHERN ALASKA. <AEIC). MC 2.8 (AEIC), 2.9 (PMR).

0.76 SPAIN. mbLg 3.0 (MDD). Felt (III) in the Becerrea area.

0.610 VENEZUEMA

1.35 KYOSBO, JAPAM

0.824 NORTHERN CEILE. Felt (III) in the Cerro paranal area.

0.56 FRANCE. MU 1.8 (GEN)

0.714 OFF EAST COAST OE HOLSHO, JAPAN

0.96 SPAIN. mbIg 2.9 (MDD). Felt (III) in the Becerrea area.

0.715 KAMCHATKA

$0.8 \quad 17$ KAYCHATKA

SOUTHER ALASKa. <AEIC>.

0.97 SOUTH INDIAN OCEAN

5 XIZANG

1.010 OFF EAST COAST OF KAYCAATKA

1.110 IRIAN JAYA REGION, IKDONESIA

0.816 SICHOAN, CEINA

1.210 NEAR S. COAST OF WESTERN EONSHO

SOUTHERN CALIFORNIA. <PAS-P>. MD 2.8 (PAS).

5 CENTRAL CALIFORNIA. $\langle G M-P\rangle$. ID 2.8 (GM).

1.329 CHILE-BOLIVIA BORDER REGION

1.210 NORTHERN MOLUCCA SEA

1.712 SUMBAWA REGION, INDONESIA

0.79 SALTA PROVINCE, ARGENTINA

1.019 DODECANESE ISLANDS

0.68 CASPIAN SEA. Felt in the Rasht area, Iran.

1.244 NEAR COAST OF ECUADOR

52 SOUTEBRN ALASKA. <AEIC). MI 2.5 (AEIC).

1.020 TONGA ISIANDS

68 RENAI PENINSULA, ALASKA. CAEIC). ML 2.6 (AEIC).

1.246 KORIL ISLANDS

47 NEAR COAST OE NORTHERN CALIE. SGM-P>. MD 3.5 (GM). YIL 3.6 (GS), 3.4 (BRK).

1.36 SOUTE AOSTRAITA

167 NEAR COAST OF NORTHERN CALIF. ¿BRK>. MW 4.7 (BRK). MIL 4.4 (BRK). MD 4.2 (GM). Felt in the Eureka-Petrolia area. Scalar Noment (BRK): Ko-1.4*10**16 $\mathrm{Nm}$.

IRIAN JAYA REGION, INDONESIA

2 HAWAII. 〈HVO-P>. MD 3.8 (HVO).

50 SAMAR, PHILIPPINE ISLANDS. NW 5.3 (BRV). Felt (II RF) at Palo, Leyte.

Centroid, Moment Tensor (HRV): Centroid origin time

$10: 28: 49.1$; Lat $12.39 \mathrm{~N}$; Lon $125.80 \mathrm{E}$; Dep $15.0 \mathrm{Bdy}$; Halfduration $1.0 \mathrm{sec}$; Principal axes (scale $10 * 16 \mathrm{Nm}$ ): (T) val-9.76, Plg-54, Azm-304; (N) Val=-1.86, Plg-19, Azm-187; (P) val--7.89, Plg-30, Azm-86; Best double couple: Mo-8.8*10*16 Nm; NP1: Strike-133, Dip-23, Slip-34; NP2: Strike-11, Dip-77, SIip-109.

$08103442.8 ? 36.46$ N $140.99 \quad \mathrm{E}$ $08105630.3 * 12.033 \mathrm{~N} 125.627 \mathrm{E}$ $08 \quad 112733.1 * 12.029$ N $125.986 \mathrm{E}$ $08 \quad 113333.2 * 54.635 \mathrm{~N} 161.442 \mathrm{~g}$ 08 11 $44 \begin{array}{lllllll} & 38.5 & 1.124 & \mathrm{~N} & 127.858 & \mathrm{E}\end{array}$ $08121432.2811 .997 \mathrm{~N} 125.705 \mathrm{E}$ $\begin{array}{llllllllll}08 & 13 & 38 & 48.1 & 28.461 & \mathrm{~S} & 69.358 \mathrm{w}\end{array}$ 08 $141736.8 \& 65.466 \times 144.878 \mathrm{~W}$

$33 \mathrm{~N}$

$33 \mathrm{~N} 4$.

$33 \mathrm{~N} 4.3$

$33 \mathrm{~N} 4.1$

$33 \mathrm{~N} 4.84$

$33 \mathrm{~N} 4.0$

16
0.3

1.

0.8

0.9

1.0

0.7

5 NEAR EAST COAST OF EONSHO, JAPAN

22 SAMAR, PHILIPPINE ISLANDS 
$\begin{array}{lllllllll}08 & 14 & 29 & 13.1 & 57.274 & \mathrm{~N} & 142.932 & \mathrm{~W}\end{array}$ $\begin{array}{llllllllll}08 & 14 & 30 & 21.6 ? & 0.39 & N & 122.85 & E\end{array}$ $\begin{array}{lllllllll}08 & 14 & 58 & 21.6 & 33.981 & \mathrm{~N} & 72.876 & \mathrm{E}\end{array}$ $08 \quad 155624.0 * \quad 6.849 \mathrm{~S} \quad 146.619 \mathrm{E}$ $08 \quad 161453.6860 .055 \mathrm{~N} 153.668 \mathrm{~W}$ $08 \quad 165257.9 * 35.846 \mathrm{~N} 68.169 \mathrm{E}$ $\begin{array}{lllllllll}08 & 17 & 09 & 39.9 & 58.636 \mathrm{~N} & 157.560 \mathrm{E}\end{array}$ $08 \quad 171052.753 .061 \mathrm{~N} \quad 167.094 \mathrm{~W}$
$10 \mathrm{G}$

$33 \mathrm{~N} 3$.

$33 \mathrm{~N} 4.84 .3 \quad 1.0$

$33 \mathrm{~N} 3.7$ 148

$37 ? 4.0$

3355.01 .4

$44 \quad 5.74 .9 \quad 0.9$

Springs.

0.422 GULF OF ALASKA. UC 2.8 (AEIC).

14 MINABASSA PENINSULA, SULAKESI

.0 94 PAKISTAN

9 EASTERN NEW GOINEA REG., P.N.G.

SOUTHERN ALASKA. 〈AEIC>

17 HINDO KOSH REGION, AFGBANISTAN

83 KAMCEATKA

380 FOX ISLANDS, ALEUTIAN ISLANDS. 5.5 (HRV). MI 5.3 (AEIC). Felt (IV) at Dutch Harbor and Dnalaska.

Centroid, Moment Tensor (BRV): Centroid origin time

$17: 10: 54.9$; Lat $52.86 \mathrm{~N}$; Lon $166.88 \mathrm{w}$; Dep 44.0 Bdy; Halfduration $1.4 \mathrm{sec}$; Principal axes (scale 10*17 $\mathrm{Nm}$ ): (T) Val-2.03, Plg-71, Azm=337; (N) Val-0.16, Plg=7, Azm=227; (P) val=-2.19, Plg-17, Azm-135; Best double couple: Mo-2.1*10\#\#17 km; NP1: Strike-214, Dip=28, S1ip-76; NP2: Strike-50, Dip -63 , Slip $=98$.

$\begin{array}{lllllllll}08 & 18 & 16 & 06.8 & 35.427 & \mathrm{~N} & 138.934 \mathrm{E}\end{array}$ $08 \quad 18 \quad 22 \quad 11.7 * 45.717$ N $150.955 \mathrm{E}$ $08182356.3 * 5.455 \mathrm{~S} \quad 146.998 \mathrm{E}$ $08185150.7844 .483 \times 7.316 \mathrm{E}$ $\begin{array}{lllllllll}08 & 20 & 27 & 01.82 & 23.29 & \mathrm{~S} & 179.13 & \mathrm{~W}\end{array}$ $\begin{array}{llllllll}08 & 22 & 14 & 32.2 & 51.674 & \mathrm{~N} & 16.180 \mathrm{E}\end{array}$ $08222511.0 ? 22.11$ N 80.18 W

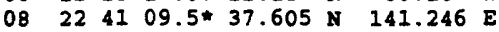
$\begin{array}{lllllllll}08 & 23 & 12 & 35.1 & 11.977 & \mathrm{~N} & 125.677 \mathrm{E}\end{array}$

$47 \quad 4.54 .2 \quad 1.0$

$33 \times 4.5$ $200 \mathrm{G} 5.0$

$5 \mathrm{G}$

$500 \mathrm{G} 3.9$

$5 \mathrm{G}$

$10 \mathrm{G} 3.8$

$89 * 3.8$ $\begin{array}{llllllllll}09 & 00 & 20 & 59.0 & 41.098 & \mathrm{~N} & 144.815 \mathrm{E}\end{array}$

$\begin{array}{lllllll}09 & 00 & 27 & 05.9 & 2.017 \mathrm{~S} & 99.675 \mathrm{E}\end{array}$
$33 \mathrm{~N} 4.8$ $33 \times 5.45 .51 .1$

EASTERN HONSHU, JAPAN

KURIL ISLANDS

26 EASTERN NEW GUINEA REG., P.N.G

NORTHERN ITALY. MI 1.8 (GEN)

10 SOUTH OF FIJI ISLANDS

20 POLAND. ME 3.5 (VIE), 3.4 iGK, T.A (YOX).

10 POLAND. ME

13 NEAR EAST COAST OF HONSHO, JAPAN

130 SAMAR, PHILIPPINE ISLANDS. NW 5.8 (HRV). Felt (IV RF) at Palo, Leyte.

Centrold, voment Tensor (BRV): Centroid origin time

$23: 12: 37.3$; Lat $11.86 \mathrm{~N}$; Lon $126.09 \mathrm{E}$; Dep $15.0 \mathrm{~F} 1 \mathrm{x}$; Halfduration $2.0 \mathrm{sec}$; Principal axes (scale 10**17 $\mathrm{km}$ ): (T) Val-5.52, Plg=63, Azm=260; (N) Val-0.28, Plg=3, Azm=355; (P) Val--5.80, Plg-27, Azm-87; Best double couple:

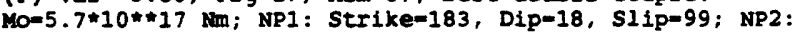
Strike-354, Dip=72, S1ip=87.

.0 82 HOKKAIDO, JAPAN RBGION

SOOTHERN SUNATERA, INDONESIA. 5.8 (BRV), 5.7 (GS). He 5.8

(GS). Felt in the southern part of siberut Island.

Broadband Source parameters (GS): Dep 23; Radiated energy $1.2 * 10 * 13 \mathrm{Nm}$

Moment Tensor (GS): Dep 22; Principal axes (scale 10"t17 $\mathrm{Nm}$ ): (T) Val-4.00, Plg-58, $\mathrm{Azm}=41$; (N) Val=-0.52, Plg=3, Azm-306; (P) Val--3.48, P1g=32, Azm=214; Best double couple: Mo-3.7*10*17 Nm; NPI: Strike-294, Dip-13, S1ip=77; NP2: Strike=127, Dip=77, S1ip=93.

Centroid, Moment Tensor (HRV): Centroid origin time $00: 27: 09.3$; Lat $2.26 \mathrm{~S}$; Lon $99.32 \mathrm{E}$; Dep $15.0 \mathrm{Fix}$; Halfduration $2.0 \mathrm{sec}$; Principal axes (scale 10*17 $\mathrm{Nm}$ ): (T) val -5.07, Plg=56, $A z m-33$; (N) Val=0.58, Plg=2, $A z m=300$; (P) Val=-5.64, Plg-34, Azm-209; Best double couple:

Mo-5.3*10*\#17 Nm; NP1 : Strike=289, Dip-11, Slip-79; IP2: Strike-121, Dip=79, Slip=92. $09010416.8 * 11.931 \mathrm{~N} 125.714 \mathrm{E}$ $09021016.2 \& 60.647 \mathrm{~N} 151.112 \mathrm{~K}$
$09005653.7 \quad 1.942 \mathrm{~s} \quad 99.690 \mathrm{E}$ $\begin{array}{llllllll}09 & 01 & 27 & 03.3 * 41.336 \mathrm{~N} & 1.364 \mathrm{E}\end{array}$ $09014900.3 * 12.011 \times 125.724 \mathrm{E}$ $09023913.6 \& 35.101 \mathrm{~N} 117.495 \mathrm{~W}$ $09023953.1 * 11.958 \mathrm{~N} 125.628 \mathrm{E}$ $09025931.5 ? \quad 1.95$ S $99.81 \quad \mathrm{E}$ $09 \quad 03 \quad 01 \quad 41.2 * 23.425 \mathrm{~S} \quad 179.845 \mathrm{E}$ $09 \quad 03 \quad 20 \quad 48.7 ? \quad 17.86$ S $168.05 \quad \mathrm{E}$ $0903 \quad 3756.1 \% 33.260 \mathrm{~N} 116.287 \mathrm{~W}$ $\begin{array}{lllllllll}09 & 03 & 43 & 35.1 ? & 12.03 & \mathrm{~N} & 125.50 & \mathrm{E}\end{array}$ $09043618.4 ? \quad 11.95 \quad \mathrm{~N} 125.37 \mathrm{E}$ $\begin{array}{llllllll}09 & 04 & 54 & 29.6 ? & 9.52 & \mathrm{~S} & 116.09 & \mathrm{E}\end{array}$ $\begin{array}{lllllllll}09 & 04 & 57 & 16.37 & 3.61 & \mathrm{~N} & 126.59 & \mathrm{E}\end{array}$ $\begin{array}{llllllll}09 & 05 & 24 & 47.13 & 7.16 & \mathrm{~s} & 129.10 & \mathrm{E}\end{array}$ $\begin{array}{lllllllll}09 & 05 & 29 & 04.1 & 36.154 & \mathrm{~N} & 27.249 & \mathrm{E}\end{array}$ $\begin{array}{lllllllll}09 & 06 & 22 & 08.4 & 50.112 & \mathrm{~N} & 178.303 \mathrm{E}\end{array}$ $0906 \quad 36 \quad 42.9 \& 36.762 \mathrm{~N} \quad 121.439 \mathrm{~N}$

$33 \mathrm{~N} 4.6$ $10 \mathrm{G}$ $33 \times 4.2$ 50 6 $33 \mathrm{~N} 4.5$ $33 \mathrm{~N} 3.9$ $600 \mathrm{G} 4.3$ 250 G 4.5 9 $33 \mathrm{~N} 4.2$ $33 \mathrm{~N} 4.3$ $33 \mathrm{~N} 4.4$ $33 \mathrm{~N} 4.3$ 150 G 4.3 $33 \mathrm{~N} 3.5$ $33 \mathrm{~N} 4.5$ 10

$33 \mathrm{~N} 3.8$ $99 ? 4.1$ $\begin{array}{llllllll}09 & 08 & 08 & 06.4 & 13.647 \mathrm{~N} \quad 120.861 \mathrm{E}\end{array}$ $33 \mathrm{~N} 4.4$ $\begin{array}{rllllll}09 & 07 & 41 & 36.8 ? & 23.15 & \mathrm{~N} & 144.04 \\ 09 & 46 & 46.3 * & 15.675 & \mathrm{~N} & 93.286\end{array}$

0.731 SOUTHERN SUMATERA, INDONESIA

0.615 SAYAR, PHILIPPINE ISLANDS

0.48 SPAIN. mbLg 3.0 (MDD). KC 2.8 (STR).

0.510 SAMAR, PHILIPPINE ISIANDS

61 KENAI PENINSULA, ALASKA. CAEIC>. MC 2.6 (AEIC)

28 CENTRAL CAIIFORNIA. <PAS-P>. MD 2.9 (PAS). MI 2.7 (GS).

1.019 SAMAR, PHILIPPINE ISLANDS

0.39 SOUTHERN SULATERA, INDONESIA

0.613 SOUTH OF FIJI ISLANDS

1.019 VANDATU ISLANDS

25 SOUTHERN CALIFORNIA. LPAS-P>. YD 3.3 (PAS). NC 3.3 (GS)

0.899 SAMAR, PHILIPPINE ISLANDS

1.09 SAMAR, PHILIPPINE ISLANDS

1.211 SUMBAWA REGION, INDONESIA

1.213 TALAUD ISLANDS, INDONESIA

1.6 9 BANDA SEA

1.426 DODECANESE ISLANDS

1.373 RAT ISIANDS, ALEUTIAN ISLANDS, YC 4.7 (PMR)

64 CENTRAL CALIFORNIA. $\langle G M-P\rangle$. MD 3.1 (GK). ML 3.2 (BRK), 3.1 (GS). Felt at salinas.

I.5 6 VOLCANO ISLANDS REGION

1.022 NEAR COAST OF CHIAPAS, MEXICO

0.9143 MINDORO, PHILIPPINE ISLANDS. NW 5.4 (GS) 5.4 (HRV)

Moment Tensor (GS): Dep 118; Principal axes (scale 10**17 $\mathrm{Nm}$ ): (T) Val-1.30, Plg-66, Azm=346; (N) Val=-0.01, Plg=24, Azm-167; (P) Val=-1.28, Plg=0, Azm-77; Best double couple: Ho=1.3*10**17 Vin; NPI: Strike-145, Dip=49, Slip=58; NP2 : Strike-9, Dip-50, Slip-122.

Centroid, Moment Tensor (HRv) : Centroid origin time 08:08:06.9; Lat $13.61 \mathrm{~N}$; Lon $120.69 \mathrm{E}$; Dep 134.0; Halfduration 1.2 sec; Principal axes (scale 10*17 $\mathrm{Nm}$ ): (T) val-1.40, Plg=67, Azm-339; (N) Val=-0.35, Plg-23, Azm-158 (P) val-1.06, Plg=0, $A z m=248$; Best double couple: Mo-1.2*10*17 $\mathrm{Nm} ;$ NPI: Strike-0, Dip=49, S1ip-121; NP2 Strike-137, Dip-50, Slip-59.

2 HAWAII. 〈HVO-P>. MD 4.0 (HVO). Felt.

$09 \quad 082454.7 \& 19.357 \mathrm{~N} \quad 155.064 \mathrm{~K}$ $09092444.9 * 1.951 \mathrm{~S} 99.682 \mathrm{E}$ $090952 \quad 46.2 * 27.706 \mathrm{~N} 129.940 \mathrm{E}$ $09 \quad 100639.3$ ? 5.08 N 127.56 E $09101726.6 * 10.516 \mathrm{~S} 117.354 \mathrm{E}$ $09102928.7 * 35.847 \mathrm{~N} 141.170 \mathrm{E}$ $09105035.9840 .380 \mathrm{~N} \quad 1.459 \mathrm{~W}$
4

$33 \times 4.2$

$33 \mathrm{~N} 4.5$

$33 \mathrm{~N} 4.0$

$33 \mathrm{~N} 4.3$

$10 \mathrm{G} \quad 3.3$

$10 \mathrm{G}$
0.7

0.9

1.4

1.4

1.4
21 SOUTHERN SUMATERA, INDONESIA

6 RYUKYO ISIANDS

9 PHILIPPINE ISLANDS REGION

18 SOUTH OF SUMBAWA, INDONESIA

5 NEAR EAST COAST OF HONSHO, JAPAN

10 SPAIN. mbLg 3.0 (MDD). Felt (III) in the Albarracin area. 
$09 \quad 110938.7 ? 36.73 \quad \mathrm{~N} \quad 7.56 \quad \mathrm{H}$ $09112459.0 * 51.474 N 278.636$ ผ $09115413.9 * 43.959$ \& 147.931 $09120538.4 * 35.634 N 80.959 \mathrm{E}$ $09131232.0 * 51.551$ \& $178.672 \mathrm{~N}$ 09140424.5 ? 33.03 \& $141.76 \mathrm{E}$ $9 \begin{array}{lllll}15 & 07 & 03.7 * 1.809 \mathrm{~S} & 99.933 \mathrm{E}\end{array}$ $09151022.6 * 35.079$ N $141.406 \mathrm{E}$ $09151625.9 * 2.105 \mathrm{~S} 99.563 \mathrm{E}$ $09 \quad 1534 \quad 49.5 \quad 49.256 \mathrm{~N} \quad 155.630 \mathrm{E}$
$20 \mathrm{G}$

$33 \mathrm{~N} 3.7$

$33 \mathrm{~N} 3.9$

$33 \times 4.0$

$33 \times 4.0$

$33 \mathrm{~N} 4.2$

$33 \mathrm{~N} 4.1$

$10 \mathrm{G} 3.6$

$\begin{array}{llll}33 \times 5.4 & 4.8 & 1.1 & 32 \\ 0.9 & 272\end{array}$

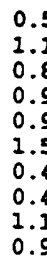

0.5
1.1
0.8
0.9
0.9
1.5
0.4
0.4
1.1
0.9
12

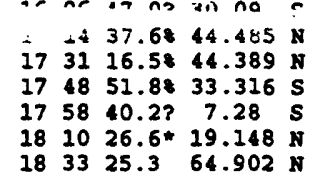

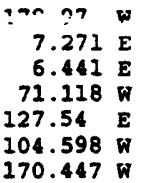

$\begin{array}{rll}\operatorname{san} & = & 4.2 \\ 10 & G & \\ 5 & G & \\ 60 & G & \\ 100 & G & 3.7 \\ 33 & N & 4.4 \\ 10 & G & 4.3\end{array}$

9 ANDREAMOE ISLANDS, ALEUTIAN IS.

11 KORIL ISLANDS

15 KASEIIR-XIZANG BORDER REGIOH

15 ANDREANOF ISLANDS, ALEUTIAN IS

11 OFF EAST COAST OE MONSEO, JAPAN

12 SOUTHERN SUNATERA, INDONESIA

6 NEAR EAST COAST OF MONSBO, JAPAN

32 SOUTHERN SUMATERA, INDONESIA

KURIL ISLANDS. MW 5.5 (GS), 5.5 (日RV).

Moment Tensor (GS): Dep 34: Principal axes (scale 10**17 N(n) : (T) Val-1.75, Plg-74, Azm-259; (N) Val--0.14, Plg-14, Azm-54; (P) val--1.61, Plg-6, Azm-146; Best double couple: Mo-1.7*10*\#17 Nm; NP1: Strike-251, Dip-41, Slip-112; NP2: Strike-43, Dip -53, Silp $=72$

Centroid, koment Tensor (HRV): Centroid origin time $15: 34: 54.2$; Lat $49.13 \mathrm{~N}$; Lon $156.23 \mathrm{E}$; Dep 50.6 ; Halfduration $1.4 \mathrm{sec}$; Principal axes (scale $10 * 17 \mathrm{Nm}$ ): (T) Val-1.99, Plg-76, $A z m=253$; (N) Val-0.50, Plg-10, Azm-31; (P) Val=-2.48, Plg-9, Azm-123; Best double couple: No-2.2*10*17 Nm; NP1: Strike-225, Dip-37, slip-107; NP2: Strike-24, Dip-55, Slip-77.

TERIADEC ISTANDS, NET ZEATAND

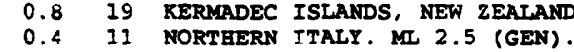

MANCE. NL 2.6 , SE:

NEAR COAST OF CENTRAL CEILE. MO 3.1 (SAN).

9 BANDA SEA

36 NEAR COAST OF JALISCO, MEXICO

65 BERIMG STRAIT. 5.0 (HRV). Felt (III) at savoonga, st. Lawrence Island.

Centroid, Moment Tensor (ERV): Centroid origin time

18:33:30.1: Lat 64.90 N Fix; Ion $170.45 \mathrm{~W} \mathrm{Fix;} \mathrm{Dep} 15.0$ F1x; Half-duration $1.0 \mathrm{sec;}$ Principal axes (scale $10 * * 16$ $\mathrm{Nm}$ ): (T) val-3.01, Plg-48, $A 2 m-208$; (N) Val-0.11, Plg-41, Azm-47; (P) val--3.13, Plg-10, Azm-308; Best double couple: vo-3.1*10*16 Nm; NP1: Strike-1, Dip-50, Slip-32; NP2:

Strike-249, Dip-66, Slip-135.

$09184055.1 * 44.418$ N $6.564 \mathrm{E}$ $09184538.6843 .087 \times \quad 8.384$ W $091845 \quad 43.4 \quad 64.994 \mathrm{~N} 170.416$ N

5 G

$10 \mathrm{G}$

0.9

15

FRANCE. ML 2.9 (GBN)

BERING STRATT. 5.2 (HRV). Felt (III) at Port clarence,

Alaska .

Centroid, Moment Tensor (HRV): Centroid origin time

$18: 45: 46.2$; Lat $64.56 \mathrm{~N}$; Lon 170.97 v; Dep 19.8; Half-

duration $1.0 \mathrm{sec}$; Principal axes (scale $10 * 16 \mathrm{~km}$ ): (T)

Val=7.27, Plg=25, Azm=7; (N) Val=0.20, Plg=58, $A z m=230$; (P)

Val=-7.37, Plg-19, Azm=106; Best double couple:

Yo-7.3*10**16 Nin; NP1: Strike-148, Dip-58, SIip-4; NP2 :

Strike-55, Dip-86, Slip-148.

$09 \quad 1905 \quad 56.9 \quad 64.796$ N 170.239 \%

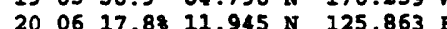
204937.1012 .3585 $.205854 .5 ? \quad 17.55 \mathrm{~s}$ $215432.8861 .743 \mathrm{~N}$ $\begin{array}{llllll}22 & 22 & 39.17 & 34.75 & N\end{array}$ $\begin{array}{lll}22 & 2728.4844 .417 \mathrm{~N}\end{array}$ $231141.4 ? 44.04 \mathrm{~N}$ $\begin{array}{lll}23 & 48 & 12.2 * 51.546 \mathrm{~N}\end{array}$ $33 \mathrm{G} 4.4$ 166.906 E 215 D 4.4 168.26 E $100 \mathrm{G} \quad 4.5$ $150.369 \mathrm{~W}$ $83.31 \mathrm{E}$ $7.317 \mathrm{E}$ $147.05 \mathrm{E}$ $001027.1 * 3.706 \mathrm{~S} 151.269 \mathrm{E}$

1.3

0.4

1.1

1.5

$33 \times 3.6$

5 G

$33 \mathrm{~N} 4.5$

5 G

$33 \mathrm{~N}$

0.8

6 SAMAR, PEILIPPINE ISLANDS

43 SANTA CRUZ ISLANDS

18 VANOATO ISLANDS

64 SOUTHERN ALASKA. CAEIC). IC 3.1 (AEIC), 3.2 (PMR).

1 NORTBERN ITALY. MI 2.4 (GEN).

KURIL ISLANDS

6 POLAND. YL 2.5 (MOX).

NEW IRELAND REGION, P.N.G. MW 5.4 (ERV).

Centroid, Moment Tensor (HRV): Centroid origin time 00:10:30.6; Lat 3.71 S Fix; Ion 151:27 E Fix; Dep 15.0 Fix; galf-duration 1.0 sec; Principal axes (scale $10 * 17 \mathrm{Nm}$ ): (T) Val-1.18, Plg-29, Azm-336; (N) Val=0.03, Plg=61, Azm-150; (P) Val--1.20, Plg-3, Azm-244; Best double couple: Ko=1.2*10*17 Nm; NP1: Strike-16, Dip=68, Slip=160; NP2: Strike-114, Dip=72, Slip-23.

NEW IRELAND REGION, P.N.G. NW 5.7 (HRV).

Centroid, Moment Tensor (HRV): Centroid origin time

00:20:53.5; Lat 3.83 S Eix; Lon 151.16 E Fix; Dep 42.1;

Half-duration $1.3 \mathrm{sec}$; Principal axes (scale 10*\$17 $\mathrm{Nm}$ ):

(T) Val-3.59, Plg-25, Azm-338; (N) Val=0.84, Plg-65,

Azm-152; (P) val--4.43, Plg-2, Azm-247; Best double couple: Mo-4.0*10*17 $\mathrm{km}$; NP1: Strike-20, Dip-71, S1ip-164; NP2:

Strike-115, Dip-75, Slip-20.

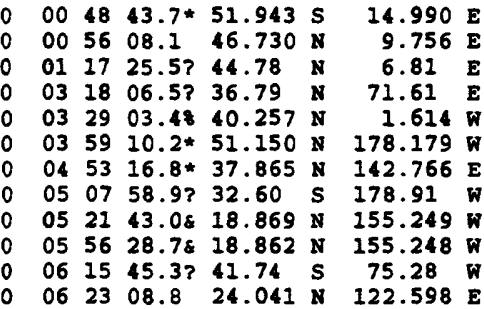

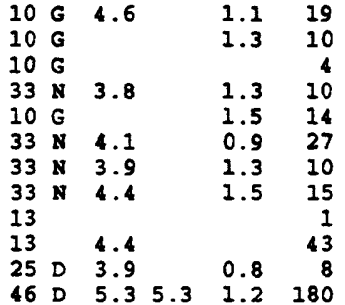

SOUTHFSST OF AFRICA

SWITZERLAND. ML 2.0 (VIE).

FRANCE. MI 1.5 (GEN).

AFGHANISTAN-TAJIKISTAN BORD REG.

SPAIN. mbLg 3.0 (MDD). Felt (III) in the Albarracin area.

ANDREANOF ISLANDS, ALEUTIAN IS. ML 4.3 (PMR).

OFF EAST COAST OF HONSHO, JAPAN

SOUTH OF KERMADEC ISLANDS

BAWAII. 〈HVO-P>. MD 3.8 (HVO).

HAWAII. <HVO-P>. MD 4.6 (HVO)

OFE COAST OF SOUTHERN CHILE

TAIWAN REGION. MW 5.7 (HRV). Felt in eastern and northern Taiwan.

Centroid, Moment Tensor (ARV): Centroid origin time

$06: 23: 09.9$; Lat $23.81 \mathrm{~N}$; Lon $122.62 \mathrm{E}$; Dep 33.3 ; Half-

duration $1.7 \mathrm{sec}$; Principal axes (scale 10*\#17 $\mathrm{Nm}$ ): (T)

Val-3.11, Plg=52, Azm-282; (N) Val=0.63, Plg=25, Azm=48;

(P) Val--3.74, Plg-27, Azm-152; Best double couple:

Mo-3.4*10*17 Nm; NP1: Strike-286, Dip-29, Slip-151; NP2:

Strike-42, Dip-76, Slip=64. 
$\begin{array}{rlllllllllllll}10 & 06 & 33 & 53.9 & 64.881 & \text { N } & 169.986 & \text { W } & 10 & G & 4.1 & 0.9 & 40 \\ 10 & 07 & 23 & 18.9 & 12.514 & \text { S } & 166.977 & \text { E } & 209 & \text { D } & 4.5 & 1.1 & 69 \\ 10 & 07 & 38 & 43.87 & 24.27 & \text { S } & 179.75 & \text { E } & 550 & \text { G } & 3.5 & 0.9 & 5 \\ 10 & 09 & 34 & 26.0 & 43.722 & \text { N } & 127.805 & \text { W } & 10 & G & 3.5 & & 0.7 & 78 \\ 10 & 11 & 20 & 19.7 & 4.938 & \text { S } & 152.131 & \text { E } & 33 & N & 5.6 & 6.0 & 1.0 & 235\end{array}$

BERING STRAIT

SANTA CRDZ ISLANDS

SOOTH OF FIJI ISLANDS

OFF COAST OF OREGON

IEW BRITAIN REGION, P.N.G. 6.2 (HRV), 6.1 (GS), Me 5.6 (GS). MS 6.0 (BRK).

Broadband Source Parameters (GS): Dep 38; NP1: Strike-80, Dip-60, Slip-110; NP2: Strike-224, Dip-36, slip-59;

Radiated energy $5.7 * 10 * 12 \mathrm{~km}$.

Moment Tensor (GS): Dep 35; Principal axes (scale 10*18 Nim): (T) Val-1.49, P1g=78, Azm=329; (N) Val=-0.04, Plg=12, Azm-132; (P) Val--1.45, Plg-4, Azm-223; Best double couple Mo-1.5*10*18 km; NP1: Strike-325, Dip-43, S1ip-108; NP2: Strike-122, Dip -50 , Slip $=74$.

Centroid, Moment Tensor (HRV): Centroid origin time $11: 20: 29.2$; Lat 5.19 S; Lon 152.29 E; Dep 46.7; Halfduration $3.2 \mathrm{sec}$; Principal axes (scale $10 * * 18 \mathrm{Nm}$ ): $(T)$ Val-2.21, Plg-76, Azm-5; (N) Val-0.21, Plg-6, Azm=252; (P) val--2.42, Plg-13, Azm=160; Best double couple: Mo-2.3*10**18 $\mathrm{km} ; \mathrm{NP1}$ : Strike-243, Dip-32, Slip-79; NP2 Strike=75, Dip-58, slip-97.

$10 \quad 113204.9837 .405 \mathrm{~N}$ $10 \quad 120017.8 * 21.980 \mathrm{~S}$ $\begin{array}{lllllll}12 & 09 & 19.0 * & 38.396 & N & 142.394 & E \\ 12 & 35 & 10.0 ? & 32.23 & N & 141.68 & E \\ 12 & 48 & 23.6 ? & 11.15 & N & 126.49 & E \\ 12 & 48 & 53.68 & 61.676 & N & 151.787 & W \\ 13 & 05 & 28.2 * & 8.489 & N & 122.837 & E \\ 13 & 33 & 14.47 & 36.85 & N & 72.02 & E \\ 13 & 57 & 47.7 & 47.679 & N & 8.638 & E \\ 14 & 25 & 32.3 * & 44.715 & N & 149.333 & E \\ 15 & 08 & 05.7 * & 19.749 & S & 177.965 & \text { W } \\ 15 & 11 & 41.2 & 19.540 & S & 178.081 & \text { W } \\ 15 & 38 & 08.1 ? & 40.35 & N & 78.39 & E \\ 15 & 51 & 39.7 * & 51.649 & N & 159.535 & E \\ 18 & 12 & 17.3 & 38.909 & N & 140.530 & E\end{array}$
$2.211 \mathrm{~W} 10 \mathrm{G}$

68.770 W 101 D 4.4

$33 \times 4.0$

$33 \mathrm{~N} \quad 3.7$

$33 \times 3.9$

82

$33 \times 4.3$

$33 \mathrm{~N} 3.8$

$10 \mathrm{G}$

33 N 3.8

500 G 5.0

550 G 4.5

$33 \times 3.6$

$33 \times 3.8$

10 G $6.05 .7 \quad 0.9 \quad 369$
$140.636 \mathrm{E}$ $140.673 \mathrm{E}$ 140.539 $140.706 \mathrm{E}$ $136.808 \mathrm{E}$ $140.719 \mathrm{E}$ $140.651 \mathrm{E}$ $140.556 \mathrm{E}$
$10 \mathrm{G} \quad 3.9$

$10 \mathrm{G} \quad 3.8$

$10 \mathrm{G} \quad 4.5$

$10 \mathrm{G} \quad 3.8$

$33 \mathrm{~N} 4.4$

10 G. 3.8

$10 \mathrm{G} \quad 5.75 .1 \quad 0.9$
$10 \mathrm{G} \quad 4.4$

1.37 SPAIN. mbLg 2.5 (LDD).

1.023 CHILE-BOLIVIA BORDER REGION. Felt (IV) at Calama and Chuquicamata, Chile.

1.316 NEAR EAST COAST OF HONSHO, JAPAN

1.077 SOUTH OF HONSHO, JAPAN

1.07 PHILIPPINE ISLANDS REGION

51 SOUTHERA ALASKA. CAEIC).

1.014 MINDANAO, PHILIPPINE ISLANDS

1.3 11 AFGHANISTAN-TAJIKISTAN BORD REG.

1.532 SWITZERLAND. ML 3.2 (STR), 3.1 (GRF), 3.0 (VIE), 2.9 (FUR).

1.215 KORIL ISLANDS

1.694 FIJI ISLANDS REGION

$\begin{array}{rrll}1.1 & 64 & \text { FIJI ISLANDS REGION } \\ 0.5 & 8 & \text { SOUTHERN XINJIANG, CHINA }\end{array}$

0.917 OFF EAST COAST OF RAMCHATKA

EASTERN HONSEO, JAPAN. WW 6.0 (GS), 6.0 (BRV). Me 5.9 (GS). MS 5.5 (BRK). Six people injured in northeastern Yamagata Prefecture. Four people injured and fifteen houses damaged in Myagi Prefecture. Felt ( $V$ JNA) in the Kurikona area, (IV JNA) at Shinjo and (III JMA) at Ichinoseki and oga.

Broadband Source Parameters (GS): Dep 9; IP1: Strike-190, Dip-50, slip=135; NP2: Strike-313, Dip-57, slip=50; Radiated energy $1.7 * 10 * 13 \mathrm{Nm}$.

Moment Tensor (GS): Dep 4; Principal axes (scale 10**18 Na) : (T) Val-1.02, Plg=77, Azm=280; (N) Val=-0.03, Plg=1,

Azm-188; (P) Val $=-0.98, P l g=13$, Azm-98; Best double couple: MO-1.0*10*18 Nm; NP1: Strike-187, Dip-32, Slip=89; NP2: Strike-8, Dip-58, slip-91.

Centroid, Moment Tensor (BRV): Centroid origin time

$18: 12: 22.7$; Lat $38.95 \mathrm{~N}$; Lon $140.53 \mathrm{E}$; Dep $15.0 \mathrm{Fix}$; Balfduration $2.4 \mathrm{sec}$; Principal axes (scale 10**18 $\mathrm{km}$ ): (T) Val=0.88, Plg=76, $\mathrm{AzIm}=145$; (N) Val=0.14, Plg=12, Azm=0; (P) Val=-1.02, Plg-8, Azm=268; Best double couple: Mo*9.5*10*17 $\mathrm{km}$; NPI: Strike-345, Dip-38, s1ip-71; NP2 Strike-189, Dip=54, Slip-105.

$\begin{array}{lll}1.5 & 11 & \text { EASTERN HONSHO, JAPAN } \\ 1.2 & 16 & \text { EASTERN HONSHO, JAPAN }\end{array}$

$\begin{array}{lll}1.2 & 16 & \text { EASTERN HONSHU, JAPAN } \\ 1.2 & 43 & \text { EASTERN HONSHO, JAPAN }\end{array}$

1.210 EASTERN HONSHU, JAPAN

1.020 IRIAN JAYA REGION, INDONESIA

1.011 EASTERN HONSHO, JAPAN

1.130 EASTERN HONSEO, JAPAN

EASTERN HONSHU, JAPAN. 5.5 (HRV). Additional damage (V JMA) in the Kurikome area.

Centroid, Moment Tensor (HRV): Centroid origin time

$18: 54: 17.5$; Lat $39.27 \mathrm{~N}$; Lon $140.55 \mathrm{E}$; Dep $15.0 \mathrm{Fix}$; Halfduration $1.4 \mathrm{sec}$ : Principal axes (scale 10**17 $\mathrm{Nm}$ ): (T) Val-1.76, Plg=69, Azm=136; (N) Val-0.11, Plg-14, Azm-3; (P) Val=-1.88, Plg-14, Azm=269; Best double couple:

Mo-1.8*10*17 Nm; NP1: Strike=340, Dip-33, Slip-63; NP2 : Strike=191, Dip-61, Slip=107.

$101903 \quad 34.3862 .097 \mathrm{~N} 151.125 \mathrm{~W}$ $10 \quad 1908$ 44.3? 38.97 N 140.99 E $10192028.9 * 38.976$ N $140.669 \mathrm{E}$ $10192540.4 * 39.040 \times 140.696 \mathrm{E}$ $10193344.7 \& 33.740 \mathrm{~N} 116.166 \mathrm{~W}$

$\begin{array}{llllllll}10 & 19 & 44 & 17.6 & 38.936 \mathrm{~N} & 140.764 \mathrm{E}\end{array}$ $10 \quad 1951 \quad 17.5 * 38.932 \mathrm{~N} 140.734 \mathrm{E}$ $10195631.5 * 38.866$ N $140.847 \mathrm{E}$ $10195850.7 * 39.000 \mathrm{~N} 140.739 \mathrm{E}$ $10201458.5 * 38.934 \mathrm{~N} 140.788 \mathrm{E}$ $10201710.6 * 58.749 \mathrm{~S} 26.566 \mathrm{~W}$ $\begin{array}{llllllll}10 & 20 & 26 & 03.6 & 38.894 & N & 140.661 & E\end{array}$

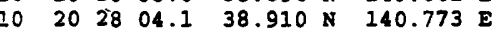
$10203147.6 ? 7.54$ N 126.53 E

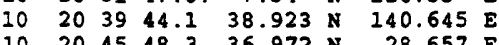
\begin{tabular}{lllllll}
10 & 20 & 45 & 48.3 & 36.972 & $N$ & 28.657 \\
\hline
\end{tabular} $\begin{array}{llllllll}10 & 20 & 59 & 25.7 & 3.070 & \mathrm{~S} & 139.167\end{array}$ $102210 \quad 00.3 * 12.737$ N 87.880 W $1022 \quad 2715.0 * 2.088 \mathrm{~s} \quad 99.600 \mathrm{E}$ $\begin{array}{lllllll}10 & 22 & 35 & 48.8 & 1.956 \mathrm{~s} & 99.622 \mathrm{E}\end{array}$

$\begin{array}{rrrrr}82 & & & & \\ 10 & G & & & 0.3 \\ 10 & G & 3.6 & & 1.0 \\ 10 & G & 3.9 & & 1.5 \\ I & & & & \\ & & & & \\ 10 & G & 3.7 & & 1.3 \\ 10 & G & 3.8 & & 1.3 \\ 10 & G & 3.6 & & 1.5 \\ 10 & G & 3.7 & & 1.2 \\ 10 & G & 3.5 & & 1.3 \\ 100 & G & 4.7 & & 1.0 \\ 10 & G & 4.4 & & 1.3 \\ 10 & G & 4.1 & & 0.9 \\ 33 & N & 4.0 & & 1.0 \\ 10 & G & 4.4 & & 1.3 \\ 33 & N & 3.6 & & 1.3 \\ 33 & N & 4.8 & 4.3 & 1.0 \\ 33 & N & 4.5 & & 1.3 \\ 33 & N & 4.3 & & 0.8 \\ 33 & N & 5.3 & 5.5 & 0.8\end{array}$

A. $\angle A E I C>$.

TERN HONSHO, JAPAN

EASTERN HONSHO, JAPAN

14 EASTERN HONSHO, JAPAN

23 SOUTHERN CALIFORNIA. <PAS-P>. MD 2.9 (PAS). MI 2.8 (GS) Felt in the Indio area.

1.316 EASTERN HONSHD, JAPAN

1.315 EASTERN HONSHO, JAPAN

9 EASTERN HONSHO, JAPAN

11 EASTERN HONSHO, JAPAN

9 EASTERN HONSHO, JAPAN

37 EASTERN HONSHO, JAPAN

12 EASTERN HONSHO, JAPAN

7 MINDANAO, PHILIPPINE ISLANDS

20 DODECANESE ISLANDS

36 IRIAN JAYA, INDONESIA

52 NEAR COAST OF NICARAGUA

52 NEAR COAST OF NICARAGUA

100 SOUTHERN SUMATERA, INDONESIA. NW 5.7 (HRV). Felt in the
26 SOUTH SANDWICH ISLANDS REGION

49 EASTERN HONSHO, JAPAN 


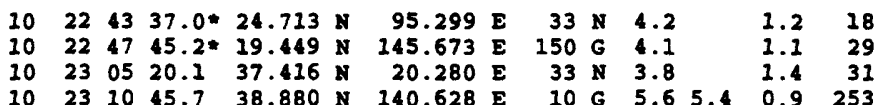
$233609.1 * 38.868 \times 140.904 \mathrm{E}$ $4335.1 ? 9.82$ S $99.92 \mathrm{E}$ $235706.8 * 54.854-136.135$ พ $001505.1 * 38.909 \mathrm{~N} 140.683 \mathrm{E}$ $003542.9 * 1.048 \times 120.019 \mathrm{E}$ $005953.7 ? \quad 28.35$ S 177.79 $\begin{array}{lllllll}01 & 31 & 16.7 & 13.396 \mathrm{~s} & 166.692 \mathrm{E}\end{array}$
$10 \mathrm{G} \quad 3.7$

$33 \mathrm{~N} \quad 4.2$

$10 \mathrm{G} \quad 4.8$

$10 \mathrm{G} \quad 3.7$

$33 \mathrm{~N} 4.8$ 100
1.4

$\begin{array}{lll}5.6 & 5.8 & 1.3\end{array}$ southern part of Siberut Island.

Centroid, yoment Tensor (HTV): Centroid origin time

$22: 35: 53.3$; Lat $2.28 \mathrm{~s}$; Lon $99.30 \mathrm{E}$; Dep 15.0 Fix; Half-

duration $1.6 \mathrm{sec}$; Principal axes (scale 10**17 $\mathrm{km}$ ): (T)

Val-3.13, Plg-64, Azm-28; (H) Val-0.74, Plg=0, Azm-297; (P)

Val--3.87, Plg-26, Azm-207; Best double couple:

No-3.5*10*\$17 Nan; IP1: Strike-296, Dip-19, Slip-89; NP2 :

Strike-117, Dip-71, S1ip-90.

MANDAR

IONIAN SEA. ML 4.0 (ROM).

EASTERN BONSEO, JAPAN. NW 5.7 (HRV).

Centroid, Moment Tensor (HRV): Centroid origin time

$23: 10: 51.0$; Lat $39.04 \mathrm{~N}$; Lon $140.81 \mathrm{E}$; Dep $15.0 \mathrm{Fix}$; Half -

duration 1.9 sec; Principal axes (scale $10 \% 17 \mathrm{Nm})$ : (T)

Val-3.32, Plg-25, Azm-177; (N) Val-1.05, Plg-60, Azm=35

(P) Val--4.37, Plg-16, Azm-275; Best double couple:

No-3.8*10*17 $\mathrm{Nm}$; NP1: Strike-318, Dip-61, S11p-6; NP2:

Strike=225, Dip-84, Slip=150.

1.29 EASTERN HONSEU

1.014 SOUTHERN SUMATERA, INDONESIA

1.423 PACIFIC-ANTARCTIC RIDGE

1.012 EASTERN HONSHO, JAPAN

1.028 MINARASSA PENINSULA, SULAWESI

CBRIADEC ISIANDS REGION. Felt (I) on Raoul.

VANUATU ISLANDS. NW 6.1 (GS), 6.0 (ERV). Me 5.8 (GS).

Broadband Source Parameters (GS): Dep 38; NP1: Strike-135, Dip $=40$, S11p $=75$; NP2: Strike-334, Dip-52, S11p-102;

Radiated energy $1.2 * 10 * 13 \mathrm{Nm}$.

Moment Tensor (GS): Dep 38; Principal axes (scale 10*18

$\mathrm{Nm}$ ): (T) Val-1.48, Plg-83, A2m=252; (N) Val=0.00, Plg=0

Azm-159; (P) Val--1.48, Plg-7, Azm-69; Best double couple: Mo-1.5*10*18 Nm; NP1: Strike=158, Dip-38, S11p=89; NP2:

Strike-339, Dip-52, slip -90 .

Centroid, Moment Tensor (HRV): Centroid orfgin time

$01: 31: 16.5$; Lat $13.40 \mathrm{~s}$; Lon $166.56 \mathrm{E}$; Dep 58.6 ; Half -

duration 2.4 sec; Principal axes (scale 10*t18 $\mathrm{km}$ ): (T)

Val-1.13, Plg=80, Azm=255; (N) Val-0.00, Plg-1, Azm-353;

(P) Val=-1.13, Plg=10, Azm-84; Best double couple:

Mo-1.1*10**18 Nm; NP1: Strike-176, Dip-35, S11p-93; NP2:

Strike-352, Dip-55, Silp 88 .

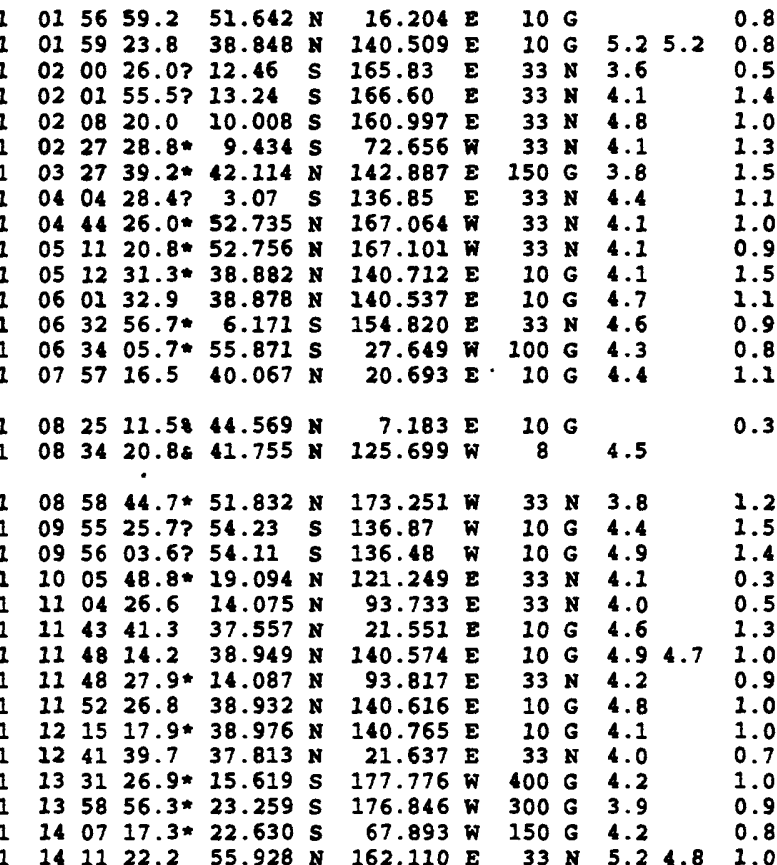

0.811 POLAND. MC 2.6 (MOX)

GAST BRN BONSEO, JAPAN

SANTA CROZ ISLANDS

VANUATO ISLANDS

SOLONON ISLANDS

PERD-BRAZIL BORDER REGION

BORKAIDO, JAPAN REGION

IRIAN JAYA, INDONESIA

FOX ISLANDS, ALEUTIAN ISLANDS

20 FOX ISLANDS, ALEOTIAN ISLANDS

19 EASTERN KONSHO, JAPAN

72 BASTERN BONSHO, JAPAN

SOLOMON ISLANDS

SOLONON ISLANDS
SOUTE SANDWICE ISLANDS REGION

60 GREECE-ALBANIA BOROER REGION. ML 4.2 (ROM). Additional minor damage to bufldings at Konitsa, Greece.

11 NORTEIRN ITALY. MU 2.4 (GEN)

143 OFE COAST OF NORTEERN CALIFORNIA. SGM-P>. MD 4.2 (GM). ML 4.4 (BRR)

1.213 ANDREANOF ISLANDS, ALEOTIAN IS.

1.514 PACIFIC-ANTARCTIC RIDGE

1.416 PACIFIC-ANTARCTIC RIDGE

0.39 PEILIPPINE ISLANDS REGION

0.512 ANDAMAN ISIANDS, INDIA

90 SOUTEERN GREECE

99 EASTERN BONSEO, JAPAN

10 AKDAMAN ISLANDS, INDIA

72 EASTERN HONSHO, JAPAN

14 EASTERN BONSHO, JAPAN

16 SOUTHERN GREECE

37 FIJI ISIANDS REGION

24 SOUTH OF FIJI ISLANDS

11 CHILE-BOLIVIA BORDER REGION

172 NEAR EAST COAST OF KAMCHATRA. MW 5.3 (ERV)

Centroid, Moment Tensor (HRV): Centroid origin time

$14: 11: 23.9$; Lat $56.12 \mathrm{~N}$; Lon $162.50 \mathrm{E}$; Dep 31.1 ; Half -

duration 1.0 sec; Principal axes (scale $10 * 17 \mathrm{Nm}$ ): (T)

Val-1.05, Plg-27, Azm-145; (N) Val--0.10, Plg-55, Azm-8

(P) Val=-0.94, Plg-21, Azm-246; Best double couple:

No=9.9*10**16 Nin; NP1: Strike-287, Dip=55, Slip=5; NP2 :

Strike-194, Dip-86, Slip-145.

$\begin{array}{lllllllllll}11 & 15 & 01 & 08.1 & 6.782 & \mathrm{~S} & 126.625 \mathrm{E} & 350 \mathrm{G} & 4.8\end{array}$ 
95.62 E $100 \mathrm{G} \quad 3.8$ $174.552 \mathrm{~W} 33 \mathrm{~N} 4.5$ 4.5 $0.423 \mathrm{~W}$ $55.635 \mathrm{E}$ $60.285 \mathrm{E}$ $105.774 \mathrm{E}$ $63.767 \mathrm{~W}$ 70.60 ฟ 142.997 $139.06 \mathrm{E}$ $153.364 \mathrm{~W}$ $176.352 \mathrm{~W}$ $152.70 \mathrm{E}$ $169.311 \mathrm{~W}$ $170.890 \mathrm{E}$ 121.33 W $71.351 \mathrm{~W}$ $7.509 \mathrm{E}$ $151.995 \mathrm{~W}$ $140.573 \mathrm{E}$ $140.513 \mathrm{E}$ $169.81 \mathrm{E}$ $119.456 \mathrm{E}$ $160.893 \mathrm{E}$ $56.903 \mathrm{E}$ $120.891 \mathrm{E}$ $10 \mathrm{G}$ $33 \times 3.8$ $33 \mathrm{~N} 3.9$ $53 \mathrm{D} \quad 4.7$ $100 \mathrm{G}$

$33 \mathrm{~N} 4.1$ $89 ? 3.8$ 29

$33 \times 4.0$

$33 \mathrm{~N} 4.2$

$33 \mathrm{~N} 4.0$

$100 \mathrm{G} 4.2$

$10 \mathrm{G} 4.4$

$100 \mathrm{G} 3.9$ $5 \mathrm{G}$

56

$10 \mathrm{G} 3.6$

$10 \mathrm{G}$

$100 \mathrm{G} 4.3$

$33 \mathrm{~N} 3.6$

$200 \mathrm{G} \quad 3.7$

$10 \mathrm{G} 4.5$ $33 \mathrm{~N} 4.9$ $33 \times 4.0$

1.411 NORTEFRN SUMATERA, IMDONESIA

1.115 TONGA ISLANDS

$1.1 \quad 17$ BANDA SEA

0.89 SPAIN. mbLg 3.0 (MDD). Felt (III) in the Torrevieja area.

0.89 SOUTBERN IRAN

1.27 NORTEWESTERN OZBERISTAN

1.410 WESTERN NEI MONGOL, CBINA

1.255 CENTRAL BOLIVIA. Felt at Samaipata and Santa Cruz.

0.3 il CEILE-ARGENTINA BORDER REGION. MD 3.7 (SAN).

0.812 NEAR EAST COAST OF HONSHO, JAPAN

1.211 NEAR NORTH COAST OF IRIAN JAYA

56 SOUTHERN ALASKA. CAEIC>

1.37 ANDREANOE ISLANDS, ALEUTIAN IS

1.09 NEW BRITAIN REGION, P.H.G.

1.225 FOX ISLANDS, ALEUTIAN ISLANDS

1.026 LOYALTY ISLANDS REGION

1.611 SOUTHERN BAST PACIFIC RISE

0.826 CENTRAL CHILE. YD 3.8 (SAN). Felt (III) at Curico add Talca.

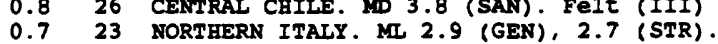

65 KEMAI PENINSULA, ALASKA. CAEIC>. ML 3.4 (AEIC), 3.3 (PMR).

1.29 EASTERN HONSHO, JAPAN

1.410 EASTERN HONSHO, JAPAN

1.016 VANOATO ISLANDS

1.09 LUZON, PAILIPPINE ISLANDS

1.016 RAYCHATKA

1.118 SOUTHWEST INDIAN RIDGE

1.143 MINAHASSA PENINSULA, SULAWESI. NW 5.1 (BRV).

Centroid, Monent Tensor (HRV): Centroid origin time

15:44:52.3; Lat $1.79 \mathrm{~N}$; LOD $120.97 \mathrm{E}$; Dep $33.0 \mathrm{Fix}$; Half duration $1.0 \mathrm{sec;}$ Pribcipal axes (scale $10 * 16 \mathrm{sm}$ ): (T)

val-5.18, Plg-55, Azm=216; (N) Val=0.04, Plg-19, Azm=96; (P) Val--5.22, Plg-28, Azm-356; Best double couple:

Ho=5.2*10*16 vin; NP1: Strike-47, Dip=24, S1ip=38; IP2: Strike-281, Dip $=75$, Slip=110.

\section{$57 * 4.0 \quad 1.2 \quad 39$ DODECANESE ISLALDS}

$27.720 \mathrm{E}$

$10 \mathrm{G}$

$\begin{array}{rr}1.2 & 39 \\ 0.4 & 8\end{array}$

$\begin{array}{lllll}16 & 35 & 48.8 ? & 43.74\end{array}$

$\begin{array}{cc}7.50 & E \\ 125.094 & E\end{array}$ $5.7 \quad 5.5 \quad 1.2$

NEAR SOUTE COAST OF FRANCE. ML 2.3 (GEN).

SOUTHERN MOLUCCA SEA. MW 6.0 (GS), 6.0 (ERV). Me 5.5 (GS). Ms 5.3 (BRK).

Broadband Source Parameters (GS): Dep 36; MP1: Strike-120 Dip=50, Slip-30; IP2: Strike-10, Dip=67, Slip=136; Radiated energy $4.1 * 10 * 12 \mathrm{~km}$.

voment Tensor (GS): Dep 35; Principal axes (scale 10*18 Nim): (T) Val-1.05, Plg-51, Azm-318; (N) Val=-0.12, Plg-35, Azm-167; (P) Val--0.93, Plg-14, Azm-67; Best double couple: Mo=9.9*10*17 Nm; NP1: Strike-119, Dipm44, Slip-33; IP2: Strike-4, Dip-68, Slip-129.

Centroid, koment Tensor (घRV): Centroid origin time

$17: 00: 34.4$; Lat $0.18 \mathrm{~S}$; Lon $125.52 \mathrm{E}$; Dep 37.0 Bdy; Half -

duration $2.4 \mathrm{sec}$; Prícipal axes (scale 10**18 $\mathrm{Nm}$ ): (T)

Val-1.25, Plg=68, Azm=280; (ki) Val=-0.04, Plg=10, Azm=35

(P) Val-1.22, Plg=19, Azm=129; Best double couple:

No-1.2*10*18 $\mathrm{km}$; NP1: Strike-235, Dip=27, slip-112; NP2:

Strike-31, Dip=65, slip-79.

$11 \quad 4.4$

$100 \mathrm{G} \quad 4.2$

$6 \mathrm{G}$

$254 \quad 4.4$

$33 \mathrm{~N} 4.2$

$600 \mathrm{G} \quad 4.2$

$33 \mathrm{~N} 4.2$

$82 \mathrm{D} 4.0$

11

500 G 4.8

10

$292 * 3.7$

$25 \mathrm{D} \quad 4.4$

6

$33 \mathrm{~N} 4.0$

$178 \mathrm{D} \quad 4.4$

$33 \mathrm{~N}$

$33 \mathrm{~N} 4.2$

5

$33 \mathrm{~N} 4.4$

$5 \mathrm{G}$

$10 \mathrm{G}$
1.2

1.1

1.1
1.0
0.7

1.0

1. 1

1.0

0.8

1.0

1.0

1.2
1.2
0.7
1.1

1.1

2.2

0.6
17 IOYALTY ISLAIDS REGION

4 SOUTHERN CALIFORNIA. <PAS-P>. MD 2.9 (PAS).

33 JUJOY PROVINCE, ARGENTINA

1 ANDREANOF ISLANDS, ALEUTIAN IS.

14 SOUTH OF FIJI ISLANDS

22 SOUTHERN MOLUCCA SEA

18 NEW BRITAIN REGION, P.N.G

30 SOUTHERN CALIFORNIA. <PAS-P>. YD 2.9 (PAS). Felt in the west Los Angeles area.

88 FIJI ISLANDS REGION

3 SOUTHERN CAIIFORNIA. <PAS-P>. MD 2.2 (PAS). Felt in the west Los Angeles area.

16 TONGA ISLANDS

28 NORTHERN PERU

29 SOUTHERN CALIFORNIA. 〈PAS-P>, MD 2.9 (PAS).

14 NEAR EAST COAST OF RAYCHATKA

25 SOUTAERA PERO

14 STRAIT OF GIBRALTAR, mbLg 2.7 (2DD).

13 SOUTHERN IRAN

2 OFF COAST OF NORTAERN CALIFORNIA. (GM-P>. MD 3.0 (GM).

27 SOUTHERN MOLUCCA SEA

18 POLAND. ML 3.5 (GRF).

EASTERN BONSEO, JAPAN. NW 5.3 (HRV).

Centroid, Moment Tensor (BRV): Centroid origin time

$02: 13: 08.9$; Lat $38.85 \mathrm{~N}$; Lon $140.45 \mathrm{E}$; Dep $15.0 \mathrm{Fix}$; Balfduration 1.0 sec; Principal axes (scale 10w17 Nm): (T)

Val-1.10, Plg-43, Azm-35; (N) Val=-0.05, Plg=27, Azm=154;

(P) val=-1.05, Plg-35, Azm=265; Best double couple: Mo-1.1*10*17 Nm; NP1: Strike-53, Dip-28, Slip-170; NP2: Strike=152, Dip=85, Siip-62.

$\begin{array}{lllll}13 & 02 & 25 & 06.7 * 38.802 \mathrm{~N} & 140.914 \mathrm{E}\end{array}$ $043011.5 \quad 30.040 \mathrm{~S} \quad 143.560 \mathrm{E}$ $054431.8 ? 29.96$ s $143.60 \mathrm{E}$ $\begin{array}{llllll}06 & 15 & 21.7 & 39.543 \mathrm{~N} & 75.326 \mathrm{E}\end{array}$ $\begin{array}{llllll}06 & 38 & 17.2 & 0.239 \mathrm{~s} & 125.017 & \mathrm{E}\end{array}$
$10 \mathrm{G} \quad 3.6$

$10 \mathrm{G} \quad 5.0$

$10 \mathrm{G} \quad 4.3$

28 D $5.75 .3 \quad 1.4$
$53 \star 4.5$

1.27 EASTERN BONSHO, JAPAN
1.045 NEW SOUTH WALES, AOSTRALIA

4 NEW SOUTH WALES, AUSTRALIA

SOUTEERN XINJIANG, CEINA

SOUTHERN MOLUCCA SEA. NW 5.7 (GS), 5.7 (HRV). MS 5.2 (BRK).

Moment Tensor (GS): Dep 36; Principal axes (scale 10*17

$\mathrm{Nm}$ ): (T) Val-4.88, PIg=0, Azm-75; (N) Val=-0.63, PIg=38

Azm=344; (P) Val=-4.25, Plg-52, Azm=165; Best double

couple: MO=4.6*10*17 Nm; NP1: Strike-197, Dip=56, 


$\begin{array}{lllllrlrll}07 & 04 & 59.5 * & 0.164 & \mathrm{~S} & 124.973 & \mathrm{E} & 33 & \mathrm{~N} & 4.7 \\ 07 & 18 & 50.2 * & 38.541 & \mathrm{~N} & 73.817 & \mathrm{E} & 150 \mathrm{G} & 4.1 \\ 07 & 24 & 42.8 * & 1.752 & \mathrm{~N} & 126.216 & \mathrm{E} & 33 & \mathrm{~N} & 4.5 \\ 07 & 49 & 48.6 * & 12.137 & \mathrm{~N} & 125.626 & \mathrm{E} & 33 & \mathrm{~N} & 4.6 \\ 09 & 09 & 21.2 * & 18.489 & \mathrm{~S} & 177.863 & \mathrm{~N} & 600 & \mathrm{G} & 4.0 \\ 09 & 12 & 51.7 & 34.434 & \mathrm{~N} & 25.596 & \mathrm{E} & 33 & \mathrm{~N} & 3.5 \\ 09 & 11 & 21.3 & 21.601 & \mathrm{~S} & 170.351 & \mathrm{E} & 110 & \mathrm{D} & 5.3\end{array}$

$\begin{array}{rr}1.2 & 32 \\ 1.2 & 13 \\ 0.9 & 20 \\ 0.7 & 20 \\ 1.2 & 25 \\ 1.4 & 32 \\ 1.1 & 167\end{array}$

$1.1 \quad 167$
$094143.7 * 34.506 \mathrm{~s}$ $\begin{array}{llllllllll}0 & 55 & 32.3 ? & 8.57 & S & 118.40 & E & 126 & ? & 4.5\end{array}$ 179.156 E $200 \mathrm{G} \quad 5.3$ $127.397 \mathrm{E} 75$ $\begin{array}{ll}1.2 & 40 \\ 0.9 & 15\end{array}$ $1.0 \quad 125$

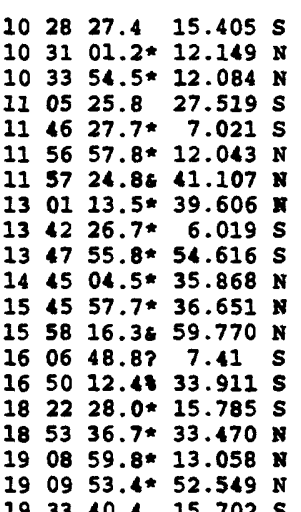

$193340.415 .702 \mathrm{~S}$

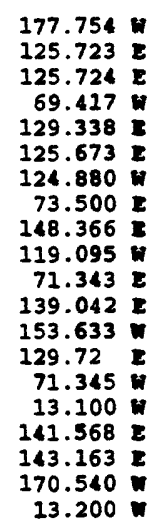

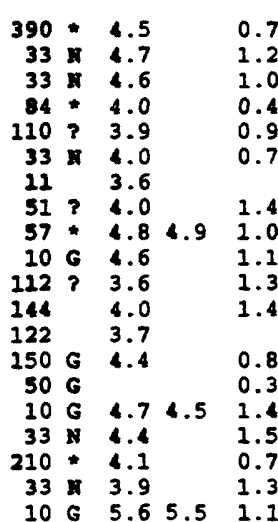

Slip=-42; JP2: Strike-313, Dip-57, Slip=-138.

Centroid, Moment Tensor (BRV): Centrold origin time 06:38:23.0; Lat $0.02 \mathrm{~S}$; Lon $124.43 \mathrm{E}$; Dep 57.1; Halfduration $1.8 \mathrm{sec}$; Prícipal axes (scale 10*17 $\mathrm{Nm}$ ): (T) val-2.80, Plg=3, Azm=26; (N) Val-2.19, Plg=31, Azm=294; (P) val=-4.99, Plg-59, Azm-122; Best double couple: Mo-3.9*10**17 Nm; NP1: Strike=145, Dip=50, Slip=-47; NP2: Strike-270, Dip=56, Slip=-129.

SOUTHERN MOLUCCA SEA

TAJIKISTAN-XINJIANG BORDER REG.

NORTHERN MOLUCCA SEA

SAMAR, PAILIPPINE ISLANDS

EIJI ISLANDS REGION

CRETE

LOYALTY ISLANDS REGION. NW 5.7 (HRV)

Centroid, Moment Tensor (HRV): Centroid origin time

09:41:26.6; Lat $21.76 \mathrm{~s}$; Lon 170.17 E; Dep 108.8; Balfduration $1.8 \mathrm{sec}$; Principal axes (scale 10*17 Nm): (T) Val=4.59, Plg=39, Azm=344; (N) Val=-0.40, Plg=34, Azm=106; (P) Val=-4.19, Plg-33, Azm=223; Best double couple: Mo-4.4*10**17 Nim; NP1: Strike-10, Dip-34, Slip-174; NP2: Strike-104, Dip-87, Slip-56.

SOUTA OF KERMADEC ISLANDS

SUMBAKA REGION, INDONESIA

TALAUD ISLANDS, INDONESIA. NW 5.7 (BRV).

Centroid, Moment Tensor (BRV): Centroid origin time

$10: 07: 11.2$; Lat $4.87 \mathrm{~N}$; Lon $127.34 \mathrm{E}$; Dep 82.5; Halfduration $1.7 \mathrm{sec;}$ Principal axes (scale $10 * 17 \mathrm{Nm}$ ): (T) val-4.01, Plg=57, Azm=19; (N) Val=-1.07, Plg-19, Azm=141; (P) Val=-2.94, PIg=26, Azm-241; Best double couple:

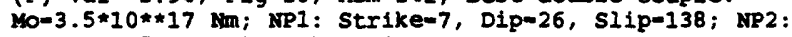
strike-135, Dip-73, silp-70.

\section{FIJI ISLANDS RBGION}

21 SAMAR, PAILIPPINE ISLANDS

1.018 SAMAR, PAILIPPINE ISLANDS

0.412 NORTHERN CHILE

0.911 BANDA SBA

9.79 SAMAR, PAILIPPINE ISLANDS

45 MEAR COAST OF NORTHERN CALIF. <BRK>. MI 3.7 (BRK), 3.8 (GS). 15 TAJIKISTAN-XINJIANG BORDER REG.

TAJIKISTAN-XINJIANG BORDER
NEN BRITAIN REGION, P.N.G.

6 SOUTHERN EAST PACIFIC RISE

13 PAXISTAN

11 BASTERN BONSHO, JAPAN

141 SOUTBER ALASKA. CAEIC>. Felt (II) at pedro Bay.

BANDA SEA

NGAR COAST of CENTRAL CHILE

44 SOUTHERN MID-ATLANTIC RIDGE

10 OFF EAST COAST OF HONSHO, JAPAN

20 SOUTH OF MARIANA ISLANDS

10 FOX ISIANDS, ALEUTIAN ISLANDS

181 SOUTHERN MID-ATLANTIC RIDGE. MW 5.6 (GS), 5.6 (HRV)

Moment Tensor (GS): Dep 7; principal axes (scale 10**17 Nm):

(T) val-3.18, Plg-2, Azm-279; (N) val=-0.27, Plg=7, Azm=10; (P) Val=-2.91, PIg-83, Azm-175; Best double couple: No-3.0*10*17 km; MPI: Strike-2, Dip=44, Slip=-100; NP2: Strike-196, Dip-47, Slip- -80 .

Centroid, Moment Tensor (HRV): Centroid origin time

19:33:45.8; Lat $15.80 \mathrm{~s}$; Lon $13.31 \mathrm{~W}$; Dep 15.0 Fix; Halfduration $1.6 \mathrm{sec;}$ Principal axes (scale $10 * 17 \mathrm{~km}$ ): (T) val=3.09, Plg=10, Azm=72; (N) val-0.38, Plg=4, Azm-342; (P) val=-3.47, Plg=79, Azm=228; Best double couple:

Mo-3.3*10**17 $\mathrm{km}$; NP1: Str1ke-168, Dip-35, S11p--82; NP2: strike-338, D1p $=55$, Slip $=-95$.

$13195120.2 * 9.911 \mathrm{~N}$ $\begin{array}{llll}20 & 09 & 28.9 & 15.635\end{array}$ $204207.3 * 51.395 \mathrm{~N}$ $2042 \quad 22.6834 .041 \mathrm{~S}$ 215117.2 ? 38.28 N $215120.0659 .618 \mathrm{~N}$ $2157 \quad 48.1$ ? $3.74 \mathrm{~S}$ $221159.8 ? \quad 34.42 \mathrm{~S}$ $224827.4 \quad 42.211 N$
$N$

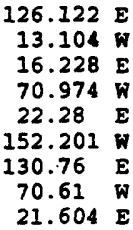

$33 \times 4.6$ $10 \mathrm{G} \quad 4.8$ $5 \mathrm{G}$ $70 \mathrm{G}$ $\begin{array}{lll}70 & G & \\ 10 & G & 3.7\end{array}$ 65 $33 \times 4.0$ $310 \mathrm{G}$ $10 \mathrm{G} 3.2$

\subsection{MINDANAO, PAILIPPINE ISLANDS}

0.947 SOUTHERN MID-ATLANTIC RIDGE

0.45 POLAND. MC 2.4 ( $\mathrm{NOX}$ )

CHILE-ARGENTINA BORDER REGION CRILE-ARC

45 SOUTHERN ALASKa. CAEIC>. MI 2.7 (AEIC).

$1.1 \quad 11$ SERAY, INDONESIA

0.4
1.3 23 NORTEWESTERN BALXAN REGION. MI 3.5 (ROM). Felt (VI) 10 the NORTHWESTERN BALXAN REGION. MI 3.5 (ROM). Felt (VI)
Skopje area. Also felt in other parts of the former Yugoslav Republic of Macedonia. $\begin{array}{lllllllllll}13 & 22 & 52 & 18.4 & 38.814 & \mathrm{~N} & 140.514 & \mathrm{E} & 10 \mathrm{G} & 4.4 \\ 14 & 00 & 09 & 19.0 ? & 17.91 & \mathrm{~S} & 178.12 & \mathrm{~W} & 600 & \mathrm{G} & 3.7\end{array}$ 001827.3 ? 17.80 S 178.09 W $600 \mathrm{G} 3.8$ $011102.8 * 52.404$ N 168.192 พ 33 N 3.7

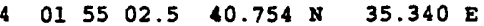

$\begin{array}{lllll}10 \mathrm{G} & 5.3 & 5.6 & 1.1 & 11 \\ 116\end{array}$
EASTERN HONSHU, JAPAN. Felt (III JMA) in the Kurikoma area.

1.226 EASTERN BONSHO, JAPAN

$\begin{array}{llll}1.5 & 12 & \text { EIJI ISLANDS REGION } \\ 0.7 & 13 & \text { FIJI ISIANDS REGION }\end{array}$

$\begin{array}{lll}0.7 & 13 & \text { FIJI ISLANDS REGION } \\ 1.1 & 11 & \text { FOX ISLANDS, ALEUTIAN ISLANDS }\end{array}$

TURKEY. MW 5.8 (GS), 5.7 (HRV). MS 5.6 (BRK). Nine people injured by a landslide at Oymaagac. Some damage in Amasya. Felt strongly in Cankir1, Kastamonu, Samsun, Sivas and Tokat.

Moment Tensor (GS): Dep 17; Principal axes (scale 10**17 $\mathrm{Nm}$ ): (T) Val-6.42, PIg=9, Azm-81; (N) val-0.32, Plg=79, Azm-223; (P) Val--6.74, Plg-6, Azm-350; Best double couple:

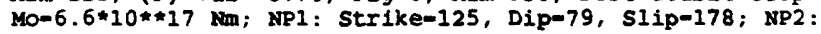
Strike-215, Dip-89, Si1p-11.

Centroid, Moment Tensor (HRV): Centroid origin time 01:55:08.3; Lat $40.52 \mathrm{~N}$; Lon $35.02 \mathrm{E}$; Dep 15.0 Fix; Halfduration $1.8 \mathrm{sec}$; Principal axes (scale 10**17 $\mathrm{Nm}$ ): (T) Val-5.18, PIg=16, Azm=74; (N) Val=-1.21, Plg=70, Azm=217; (P) val=-3.97, PIg=11, Azm-340; Best double couple: 
$14021229.2 ? 31.51$ S 178.83 W $14022558.9 \quad 40.910 \mathrm{~N} \quad 35.373 \mathrm{E}$ $14023902.0 * 0.792 \mathrm{~s} 12.805 \mathrm{~W}$ $14024250.6 * 28.566 \mathrm{~N} 142.689 \mathrm{E}$ $140257 \quad 45.6 * 5.558$ S $154.886 \mathrm{E}$ $14025941.1 \quad 40.801 \mathrm{~N} 35.370 \mathrm{E}$
$33 \times 4.5$

$10 \mathrm{G} \quad 4.0$

$10 \mathrm{G} \quad 4.3$

$33 \mathrm{~N} 4.1$

70 D 4.7

Mo-4.6*10**17 Nin; NP1: Strike-116, Dip-70, S1ip-176; NP2: strike-208, Dip-87, Slip-20.

1.517 RERMADEC ISLANDS REGION

1.022 TURKEY

1.318 NORTH OF ASCENSION ISLAND

0.811 BONIN ISLANDS REGION

1.221 SOLOMON ISLANDS

267 TURKEY. Ww 5.7 (HRV). Additional damage in Amasya. Centroid, Moment Tensor (HRV): Centroid origin time

$02: 59: 47.7$; Lat $40.86 \mathrm{~N}$; Lon $35.18 \mathrm{E}$; Dep 15.0 Bdy; Halfduration $1.6 \mathrm{sec}$; Principal axes (scale 10*17 $\mathrm{km}$ ): (T) Val-2.79, Plg-12, Azm=61; (N) Val-1.49, Plg-69, Azm-297; (P) Val=-4.28, Plg-17, Azm-155; Best double couple: Mo-3.5*10*17 Nm; NP1: Strike-197, Dip-69, Slipm-4; NP2: Strike-288, Dip-87, Slip=-159.

$1403 \quad 05 \quad 27.48 \quad 34.599 \mathrm{~N} 116.280 \mathrm{~N}$

$1403 \quad 16 \quad 50.1 \quad 30.068 \mathrm{~S} \quad 143.435 \mathrm{E}$

$140356 \quad 06.0 \quad 25.033$ N $145.299 \mathrm{E}$

$\begin{array}{llllll}14 & 04 & 37 & 45.9 & 46.376 & \mathrm{~N}\end{array}$

$14043852.8 * 14.294 \mathrm{~N}$

$14043937.9846 .339 \mathrm{~N}$

$14055124.0 * 20.205 \mathrm{~s}$

$140558 \quad 27.3 * 17.508 \mathrm{~N}$

$\begin{array}{lllll}14 & 07 & 02 & 57.18 & 61.956 \mathrm{~N}\end{array}$

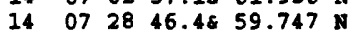

$\begin{array}{llllll}14 & 08 & 39 & 57.88 & 11.171 & \mathrm{~N}\end{array}$

$\begin{array}{llllll}14 & 09 & 05 & 37.6 ? & 4.58 & N \\ 14 & 09 & 29 & 06.9 & 30.003 & \text { S }\end{array}$

14094644.3 ? $5.80 \mathrm{~s}$

141045 58.9* $0.463 \mathrm{~s}$

$14 \quad 105158.3$ ? $30.65 \mathrm{~s}$

$14105750.2 * 17.448 \mathrm{~s}$

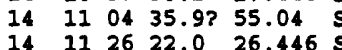

$14 \quad 112622.0 \quad 26.446 \mathrm{~s}$

$14 \quad 1218 \quad 40.9 * 60.138 \mathrm{~N}$

$14123502.1 * 38.446 \mathrm{~N}$

$141236 \quad 05.8 * 42.025 \mathrm{~N}$

$\begin{array}{llllll}14 & 12 & 36 & 10.9 & 45.966 & \mathrm{~N}\end{array}$

$\begin{array}{llllll}14 & 12 & 37 & 21.7 & 22.506 & \mathrm{~s}\end{array}$

$14 \quad 125632.8 * 22.348 \mathrm{~s}$

$\begin{array}{lllllll}14 & 13 & 12 & 39.1 ? & 36.18 & \mathrm{~N}\end{array}$

$\begin{array}{lllll}14 & 13 & 36 & 41.9 * 36.257 & \mathrm{~N}\end{array}$

$14143313.4 * 36.310 \mathrm{~N}$

$\begin{array}{llllll}14 & 15 & 39 & 32.5 & 23.857 & \mathrm{~s}\end{array}$

$\begin{array}{llllll}14 & 16 & 06 & 50.1 & 37.766 & \mathrm{~N}\end{array}$

$\begin{array}{lllll}14 & 16 & 17 & 04.8 & 37.877\end{array}$

$14163501.0 * 9.188 \mathrm{~s}$

$\begin{array}{llllll}14 & 17 & 26 & 00.8 & 37.781 & \mathrm{~N}\end{array}$

$\begin{array}{lllll}14 & 17 & 30 & 30.2 & 37.301\end{array}$

$\begin{array}{lllllll}14 & 17 & 34 & 34.58 & 60.184 & \mathrm{~N}\end{array}$

$\begin{array}{lllllll}14 & 17 & 39 & 53.08 & 62.029 & \mathrm{~N} \\ 14 & 17 & 55 & 28.98 & 45.901\end{array}$

$\begin{array}{lllllll}14 & 17 & 55 & 28.98 & 45.901 & \mathrm{~N}\end{array}$

$14 \quad 1828 \quad 43.3 \quad 38.874$ N

$141849 \quad 36.3 * 32.990 \mathrm{~s}$

14

14

$\begin{array}{lll}45 & 01.5 & 22.457\end{array}$

$\begin{array}{lllllll}14 & 20 & 02 & 32.38 & 33.162 & 5\end{array}$

$14201829.1 * 16.481 \mathrm{~S}$

$14202802.9 * 50.496 \mathrm{~N}$

$142123 \quad 47.4 \quad 42.547 \mathrm{~s}$

$126.71 \mathrm{E}$

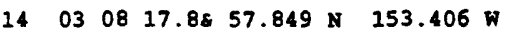

$140903 \quad 31.1 * 23.986 \mathrm{~s}$

$4 \quad 172737.98 \quad 35.749 \mathrm{~N}$
$119.479 \quad 8$

$7.490 \mathrm{E}$

68.502 ผ

$94.210 \mathrm{~W}$

$151.434 \mathrm{~W}$

152.865 ผ

61.612

$124.77 \mathrm{E}$

143.434

$132.419 \mathrm{E}$

178.82 H

173.688 \%

132.71

$68.006 \mathrm{E}$

143.097

$25.369 \mathrm{E}$

$179.030 \mathrm{E}$

$78.897 \mathrm{~B}$

$4.54 \mathrm{~W}$

$26.980 \mathrm{E}$

$70.751 \mathrm{E}$

$179.701 \mathrm{E}$

$14.945 \mathrm{E}$

$14.846 \mathrm{E}$

$125.261 \mathrm{E}$

$14.974 \mathrm{E}$

$71.474 \mathrm{~W}$

153.301 พ

$150.000 \mathrm{~W}$

$6.160 \mathrm{E}$

$152.484 \mathrm{~W}$

$140.363 \mathrm{E}$

68.936 W

$140.232 \mathrm{E}$

45.181 พ

$70.744 \mathrm{~V}$

$69.311 \mathrm{~W}$

$18.879 \mathrm{E}$
73.587
$26.627 \mathrm{E}$
10
6 $\quad 4.2$

1.3

52

$10 \mathrm{G} 4.6$

$38 \mathrm{D} 4.4$

$10 \mathrm{G}$

$33 \mathrm{~N} 4.0$

$10 \mathrm{G}$

$146 * 4.0$

$50 \mathrm{G} \quad 3.9$

80

$87 \quad 4.0$

33 N 3.9

$10 \mathrm{G} 4.3$

$450 \mathrm{G} 3.8$

$33 \mathrm{~N} 4.8$

$33 \mathrm{~N} 4.6$

$33 \mathrm{~N} 4.2$

$10 \mathrm{G} 4.4$

$10 \mathrm{G} \quad 4.8$

$33 \mathrm{~N} 3.6$

$10 \mathrm{G} 4.1$

$129 \mathrm{D} 4.0$

$595 \mathrm{D} \quad 4.8$

600 G 4.1

$\begin{array}{rr}33 & \mathrm{~N} \\ 150 \mathrm{G} & 3.8\end{array}$

$89 ? 4.1$

$500 \mathrm{G} \quad 4.4$

$10 \mathrm{G}$

$20 \mathrm{G} \quad 3.6$

$33 \mathrm{~N} 4.6$

$10 \mathrm{G}$

10

$132 \mathrm{D} 4.4$

124

47
10

163

$49 \quad 4.1$

$20 \mathrm{G}$

$33 \mathrm{~N} 3.9$

$10 \mathrm{G} 4.3$

$75 \mathrm{G}$

197

$10 \mathrm{G}$

$33 N 4.95 .0 \quad 0.8$

1.0

1.5
$200 \mathrm{G} \quad 3.8$

44 TURKEY

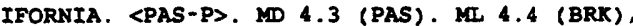

30 KODIAK ISLAND REGION. CAEIC>. MC 2.8 (AEIC).

NEW SOUTH WALES, AUSTRALIA

NORTE PACIEIC OCEAN

SWITZERLAND. ML 3.6 (STR), 3.5 (VIE), 3.4 (GRF), 3.3 (LDG). 3.3 (FUR).

1.213 LUZON, PHILIPPINE ISLALDS

0.577 SWITZERLAND

0.69 CHILE-BOLIVIA BORDER REGION

0.925 CHIAPAS, MEXICO

60 SOUTHERN ALASKA. $\angle A E I C>$.

128 SOUTHERN ALASKA. CAEIC>.

0.47 WINDWARD ISLANDS. JD 3.8 (TRN)

0.816 JUJUY PROVINCE, ARGENTINA

1.311 CELEBES SEA

1.525 NEN SOUTH WALES, AUSTRALIA

$1.1 \quad 12$ BANDA SEA

1.323 IRIAN JAYA REGION, IKDONESIA

1.211 CERMADEC ISLANDS, NEW ZEALAND

0.914 TONGA ISLANDS

1.09 PACIFIC-ANTARCTIC RIDGE

1.134 SOUTH INDIAN OCEAN

53 SOUTHERN ALASKA, CAETC>.

0.910 OFF EAST COAST OF HONSHO, JAPAN

1.67 BULGARIA

1.538 ROMANIA

0.882 SOUTH OF FIJI ISLANDS

0.921 SOUTH OF FIJI ISIANDS

0.77 STRAIT OF GIBRALTAR. mbLg 3.1 (MDD).

0.98 DODECANESE ISLANDS

0.910 HINDU KUSH REGION, AFGEANISTAN

1.160 SOUTH OF FIJI ISLANDS

0.69 SICILY. 3.2 (ROM)

1.516 SICILY. MD 3.3 (ROM).

1.530 TIMOR REGION, INDONESIA

0.67 SICILY. MD 3.4 (ROM)

31 CENTRAL CALIFORNIA. CPAS-P>. ID 3.3 (PAS). ML 3.0 (GS).

AFGBANTSTAN-TAJIKISTAN BORD REG.

43 SOUTHERN ALASKA. <AEIC>.

89 CENTRAL ALASKA. LAEIC>. ML 3.2 (AEIC), 3.3 (PMR).

0.8 TO SOUERN ALASKA. CAEICO.

20 GASTERN HONSEU, JAPAN

12 MENDOZA PROVINCE, ARGENTINA. MD 4.0 (SAN). Felt (III) by people in tall buildings at Mendoza.

$1.1 \quad 17$ WESTERN CAROLINE ISLANDS

0.829 NORTEERN MID-ATLANTIC RIDGE

0.38 CHILE-ARGENTINA BORDER REGION. MD 1.7 (SAN)

17 PERU-BOLIVIA BORDER REGION

6 POLAND

34 SOUTHERN CHILE. MW 5.2 (HRV). Felt (IV) at Ancud and Castro. Centroid, Moment Tensor (BRV): Centrold origin time $21: 23: 49.0$; Lat $42.36 \mathrm{~s}$; Lon $72.81 \mathrm{~W}$; Dep $33.0 \mathrm{Fix}$; Halfduration $1.0 \mathrm{sec}$; Principal axes (scale 10*16 $\mathrm{km}$ ): (T) Val-8.74, Plg=33, Azm=69; (N) Val=-3.01, Plg=29, Azm=317; (P) Val--5.73, Plg=43, Azm-196; Best double couple: Mo-7.2*10*16 Nm; NP1: Strike-215, Dip-29, S1ip=-11; NP2: Strike-314, Dip=85, Slip -119

152.613 E 200 G 3.7

0.7

12 NORTHWEST OF KORIL ISLANDS

$75.171 \mathrm{E}$

$122.91 \mathrm{~B}$

170.210

$14.948 \mathrm{E}$

$35.112 \mathrm{E}$

$24.75 \mathrm{E}$

174.994

$75.245 \mathrm{E}$

$5.628 \mathrm{E}$

$128.15 \mathrm{E}$

$10 \mathrm{G}$

40.972 W 10 G $3.94 .2 \quad 0.5$

$71.143 \mathrm{H}$
$33 \mathrm{~N} 4.5$

0.9

$33 \mathrm{~N} 3.9$

0.8

$\begin{array}{lllll}10 \mathrm{G} & 3.8 & 4.1 & 0.8\end{array}$

$\begin{array}{llll}10 \mathrm{G} & 4.0 & 0.7\end{array}$

$10 \mathrm{G} 3$.

$33 \times 3.8$

1.2

$33 \mathrm{~N} \quad 4.94 .7 \quad 1.2$

$79 * 4.44 .7 \quad 0.9$

$400 \mathrm{G} \quad 4.0$

0.9

61 TAJIXISTAN-XINJIANG BORDER REG

6 TAIWAN REGION

25 BERING STRAIT

11 SICILY. MD 3.5 (ROM).

16 CRETE

54 TONGA ISLANDS REGION

50 TAJIKISTAN-XINJIANG BORDER REG.

7 FRANCE

10 BANDA SEA

10 NORTHERN MID-ATLANTIC RIDGE

$5805^{5.1} \quad 1.0 \quad 150$
15 TURKEY
NEAR COAST OF PERO. 5.4 (ERV). Felt (V) at Arica, Chile and (IV) at Arequipa.

Centroid, Moment Tensor (HRV) : Centroid origin time

$06: 25: 36.8$; Lat $17.45 \mathrm{~S}$; Lon $71.25 \mathrm{~W}$; Dep 72.0 ; Half-

duration $1.2 \mathrm{sec}$; Principal axes (scale $10 *+17 \mathrm{Nm}$ ): (T) 
Val-1.33, Plg=3I, Azm=114; (N) Val=-0.19, Plg=39, Azm=232; (P) Val--1.15, Plg-36, Azm-358; Best double couple: Mo-1.2*10*17 $\mathrm{km}$; NPI: Strike-149, Dip-39, Slip-174; NP2: Strike-55, Dip -87, slip -51 .

(BRK) Broadband Source parameters (GS): Dep 39; NP1: Strike-333, Dip=57, Slip=50; IR2: Strike-210, Dip=50, Slip-135; Radiated energy $3.5 * 10 * 13 \mathrm{~km}$. Two events about 2.5 seconds apart. Depth based on first event.

Moment Tensor (GS): Dep 33; Principal axes (scale 10**18 $\mathrm{Nm}$ ): (T) Val=1.80, Plg-81, A2m-264; (N) Val=0.01, Plg=0 Azm=171; (P) Val--1.81, Plg-9, Azm-81; Best double couple:

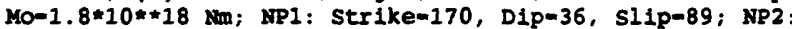
Strike-351, Dip-54, slipm-9I.

Centroid, Moment Tensor (HRV): Centroid origin time $07: 33: 59.9$; Lat $13.30 \mathrm{~s}$; Ion $166.50 \mathrm{E}$; Dep 48.5; Halfduration $3.1 \mathrm{sec}$; Principal axes (scale $10 * \pm 18 \mathrm{Nm}):(T)$ Val-2.30, Plg=81, Azm=272; (N) Val=0.08, Plg=1, Azm=175; (P) Val--2.38, Plg=9, Azm-85; Best double couple: No-2.3*10**18 Nm; NPI: Strike-173, Dip-36, Slip-88; KP2 : Strike-356, Dipm54, S1ip=91

Scalar Moment (PPT): Mo-3.9*10**18 $\mathrm{km}$

$\begin{array}{lllllllll}15 & 08 & 53 & 52.96 & 36.234 & \mathrm{~N} & 120.678 & \mathrm{~W}\end{array}$ $105506.6 * 40.583$ N $\begin{array}{llll}11 & 45 & 55.8 & 8.253\end{array}$

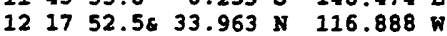

$1323 \quad 39.86 \quad 61.697 \quad N$ $135413.3 \quad 47.491 \mathrm{~N}$ $152653.7 * 43.149 \mathrm{~N}$ $155709.9 * 6.326 \mathrm{~s}$ $\begin{array}{lllll}16 & 03 & 32.5 & 36.023\end{array}$

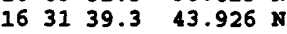
$\begin{array}{lllll}16 & 36 & 09.38 & 60.191 & \mathrm{~N}\end{array}$ $\begin{array}{lllll}16 & 36 & 37.28 & 37.157 & \mathrm{~N}\end{array}$ $\begin{array}{llll}17 & 39 & 13.7 & 42.845\end{array}$ $\begin{array}{lllll}18 & 12 & 05.98 & 60.385 & 8\end{array}$ $193627.1 * 23.831 \mathrm{~s}$ $193945.1 * 15.401 \mathrm{~N}$ 0729.9849 .076

$203503.8 * 37.274$ $210854.9 \& 59.910 \mathrm{~N}$ $211918.3 * 50.027$ H $234613.6 * 3.584 \mathrm{~S}$ $000253.0 * 32.941 \mathrm{~N}$ $\begin{array}{llll}00 & 52 & 05.8 & 44.270 \mathrm{~N}\end{array}$ $010132.9 * 38.614$ $010930.1844 .364 \mathrm{~N}$ $011402.9 \times 12.264 \mathrm{~N}$ $\begin{array}{lllll}01 & 32 & 23.98 & 49.021 & N\end{array}$ $\begin{array}{lllllll}01 & 48 & 32.0 ? & 10.66 \mathrm{~s}\end{array}$ $015123.8833 .331 \mathrm{~s}$ $025727.9 * 11.695 \mathrm{~N}$ $\begin{array}{lllll}03 & 00 & 15.5 & 19.129 & \mathrm{~N}\end{array}$ $\begin{array}{lllll}03 & 41 & 10.6 & 51.322 \mathrm{~N}\end{array}$ $0456 \quad 46.08 \quad 49.210 \mathrm{~N}$ $\begin{array}{lllll}06 & 00 & 06.6 & 16.832 \mathrm{~N}\end{array}$ $\begin{array}{llll}06 & 06 & 27.4 * 49.720 \mathrm{~s}\end{array}$ $\begin{array}{lllll}06 & 11 & 07.2 & 46.772\end{array}$ $061630.3 * 44.386 \mathrm{~N}$ $\begin{array}{lllll}07 & 28 & 17.38 & 46.451 & \mathrm{~N}\end{array}$ $080044.8 * 18.317 \mathrm{~s}$ $\begin{array}{lllll}08 & 04 & 07.9 * 14.805 \mathrm{~S}\end{array}$ $084157.958 .635 \mathrm{~s}$
150.331 พ $8.953 \mathrm{E}$ 126.389 म $153.329 \mathrm{E}$ $139.785 \mathrm{E}$ $28.642 \mathrm{~W}$ 138.314 $3.733 \mathrm{n}$ $2.570 \mathrm{E}$

$149.043 \mathrm{~N}$ $179.962 \mathrm{E}$ $60.963 \mathrm{w}$ 112.405 n

$142.589 \mathrm{E}$ $151.390 \mathrm{~W}$ $28.953 \mathrm{~W}$ $131.163 \mathrm{E}$ $140.998 \mathrm{E}$ $7.353 \mathrm{E}$ 7.812 ผ $7.318 \mathrm{E}$ $144.017 \mathrm{E}$ $1.825 \%$ $165.98 \mathrm{E}$ $72.295 \mathrm{~W}$ $125.940 \mathrm{E}$ $49.015 \mathrm{~W}$ $130.410 \mathrm{k}$ 82.920 i $61.456 \mathrm{~N}$

$114.193 \mathrm{E}$ 8.705 E $8.752 \mathrm{E}$ $6.161 \mathrm{E}$ $87.361 \mathrm{E}$ 176.582 W 25.026 ผ
10

$33 \mathrm{~N} 4.5$

$10 \mathrm{G} 3.9$

$86-4.1$

18

47

$10 \mathrm{G}$

$33 \mathrm{~N}$

$55 \mathrm{D} 4$.

$10 \mathrm{G} 4.74$

8

$10 \mathrm{G}$

$10 \mathrm{G}$

27

$500 \mathrm{G} 4.6$

5 G

$33 \times 3.9 \quad 1.0$

67

10 G 3.93 .4

$33 \mathrm{~N} 4.7$

$33 \mathrm{~N} 4.3$

$10 \mathrm{G}$

$10 \mathrm{G}$

$10 \mathrm{G}$

$43 *$

$10 \mathrm{G}$

$33 \mathrm{~N}$

$5 \mathrm{G}$

$33 \mathrm{~N} 4.4$

10 G 3.93 .81 .1

$10 G 4.5461 .6$

$18 \mathrm{G} 3.5$

$55 \quad 4.43 .9$

$10 \mathrm{G} 4.3$

$10 \mathrm{G}$

$10 \mathrm{G}$

$5 \mathrm{G}$

$10 \mathrm{G} 4.2$

$33 \mathrm{~N} 4.6$

$33 \mathrm{~N} 5.0$

0.9

1.0

0.9

0.3

50

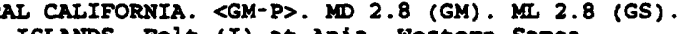

TORKEY

5 EASTERN NEW GUINEA REG., P.N.G.

31 SOUTHERN CALIFORNIA. <PAS-P>. MD 3.3 (PAS). ML 3.1 (GS). Felt.

52 SOUTHERN ALASKA. LAEIC>. YL 2.5 (AEIC)

15 SKITZERLAND. ML 2.7 (STR), 2.6 (IDG).

41 OFF COAST OF OREGON

4 NEN BRITAIN REGION, P.N.G.

56 BASTERN HONSHO, JAPAN

105 NORTAERN MID-ATLANTIC RIDGE

20 SOUTAERN YUKON TERRITORY, CANADA. CAEIC). MI 2.7 (AEIC).

SPAIN. mbIg 2.3 (YDD).

53 PYRENEES. MIL 3.4 (STR), 3.4 (IDG). mbLg 3.2 (YDD).

72 KENAI PENINSULA, ALASKA. CAEIC). MI 2.9 (AEIC).

41 SOUTH OF FIJI ISLANDS

ALBERTA, CANADA. <PGC-P>. mbLg 3.6 (PGC). ML 3.4 (GS). Felt at Lethbridge.

9 OFF EAST COAST OF HONSHO, JAPAN

69 KENAI PENINSULA, ALASKA. CAEIC>. 2IL 2.7 (AEIC).

18 HORTHERN MID-ATLANTIC RIDGE

30 IRIAN JAYA REGION, INDONTESIA

17 SOUTH OF HONSHO, JAPAN

13 NORTHERN ITALY. ML 1.9 (LDG), 1.8 (GEN).

13 PORTUGAL. mbLg 2.9 (IDD). Felt (III) in the Evora area.

7 NORTAERN ITALY. ML 1.8 (GEN).

30 SOUTH OF MARIANA ISLANDS

FRAMCE. MU 1.6 (IDG).

9 SANTA CRUZ ISLANDS

10 OFF COAST OF CEMTRAL CAILE. MD 3.7 (SAN).

15 SAMPR, PHILIPPINE ISLANDS

16 NORTH ATLANTIC OCEAN

114 QUEEN CHARLOTTE ISLANDS RBGION. MU 4.5 (PGC)

17 ONTARIO, CANADA. 〈OTT-P>. mbLg 3.6 (OTT).

50 LEDWARD ISLANDS. ND 4.6 (TRN).

16 SOUTH OF AUSTRAIIA

9 SWITZERLAND. IIL 2.6 (VIE)

16 NORTEERN ITALY. ML 2.6 (IDG), 2.4 (GEN).

13 SWITZERLAND. ML 2.3 (IDG).

21 SOUTH INDIAN OCEAN

55 FIJI ISLANDS REGION

81 SOOTH SANDWICE ISLANDS REGION. NW 5.5 (HRV)

Centroid, Moment Tensor (HRV): Centroid origin time

$08: 42: 03.8$; Lat $59.08 \mathrm{~s}$; Lon $25.04 \mathrm{~W}$; Dep $15.0 \mathrm{Fix}$; Half duration $1.3 \mathrm{sec}$; Principal axes (scale 10**17 Nm): (T)

Val=2.37, P1g=62, Azm=309; (N) val=-0.21, Plg=6, Azm=207 (P) Val-2.16, Plg=27, Azm-114; Best double couple: Mo-2.3*10*17 Nm; NPI: Strike-189, Dip-19, Slip-71: NP2: Strike-29, Dipm72, slip=96.

$\begin{array}{llllllll}16 & 09 & 04 & 52.0 & 49.476 \mathrm{~N} & 155.793 \mathrm{E}\end{array}$ $16092400.5 * 14.792 \mathrm{~S} \quad 176.484 \mathrm{~W}$ $16092702.6 * 14.594 \mathrm{~S} 176.611 \mathrm{~W}$ $\begin{array}{llllll}16 & 09 & 31 & 58.8 & 14.707 \mathrm{~S} & 176.286 \mathrm{~W}\end{array}$ $\begin{array}{llllll}33 & N & 5.2 & 4.9 & 0.9 & 257\end{array}$

$33 \times 4.6$

$33 \mathrm{~N} 4.8$

$33 \mathrm{~N} 5.05 .4$ 1.350 1.482 112

KURIL ISLANDS. Felt (IV) at Severo-Kurilsk.

FIJI ISLANDS REGION

FIJI ISLANDS REGION

FIJI ISLANDS REGION. WW 5.6 (HRV).

Centroid, Moment Tensor (HRV) : Centroid origin time

09:32:01.0; Lat 14.59 S; Lon $175.95 \mathrm{~W}$; Dep 15.0 Fix; Halfduration $1.6 \mathrm{sec}$; Principal axes (scale $10 * 17 \mathrm{vm}$ ): (T) Val=3.15, Plg=6, Azm=142; (N) Val=-0.09, Plg-84, Azm-293; (P) Val--3.07, Plg=3, Azm=52; Best double couple:

Mo-3.1*10*17 Nm; NPI: Strike-187, Dip-84, Slip=178; NP2:

Strike-277, Dip-88, Slip-6.

$\begin{array}{llllll}16 & 09 & 54 & 40.9 & 51.305 \mathrm{~N} & 130.343 \mathrm{~W}\end{array}$ $16095815.2 * 0.160 \mathrm{~N} 123.930 \mathrm{E}$ $\begin{array}{llllllll}16 & 10 & 41 & 19.6 & 39.358 \mathrm{~N} & 116.649 \mathrm{~W}\end{array}$ $112820.0 * 21.338$ S $177.486 \mathrm{~W}$ $115844.2 * 11.910 \mathrm{~S} \quad 127.215 \mathrm{E}$ $125111.6 * 43.345 \mathrm{~N} 147.734 \mathrm{E}$

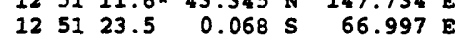

10 G 4.3

$33 \mathrm{~N} 4.2$

5 G

$400 \mathrm{G} \quad 4.2$

$33 \mathrm{~N} 4.1$

$33 \times 4.2$

$\begin{array}{llll}33 \mathrm{~N} & 4.2 & & 1.4 \\ 10 \mathrm{G} & 4.9 & 4.6 & 1.2\end{array}$
1.3

1.3

0.6

1.1 
$12: 51: 26.8$; Lat $0.61 \mathrm{~s}$; Ion $67.36 \mathrm{E}$; Dep $15.0 \mathrm{Bdy}$; Half duration $1.1 \mathrm{sec}$; Principal axes (scale 10**16 $\mathrm{Nm}$ ): (T) Val-8.71, Plg=0, Azm=228; (N) Val=-2.84, Plg=0, Azm=138 (P) Val $=-5.87$, Plg $=90$, Azm-180; Best double couple: (P) Val=-5.87, Plg=90, Azm-180; Best double couple:
Mo-7.3*10*16 Nm; NP1: Strike-318, Dip-45, Slip--90; NP2: Strike-138, Dip=45, slip=-90.

$\begin{array}{rrrlrrrr}16 & 12 & 56 & 32.4 & 0.032 & \mathrm{~S} & 66.997 & \mathrm{E} \\ 16 & 13 & 27 & 17.7 & 34.596 & \mathrm{~N} & 116.274 & \mathrm{~W} \\ 16 & 13 & 27 & 42.88 & 34.606 & \mathrm{~N} & 116.284 & \mathrm{~W} \\ 16 & 13 & 36 & 34.7 & 4.340 & \mathrm{~S} & 155.039 & \mathrm{E} \\ 16 & 14 & 22 & 21.7 * & 0.838 & \mathrm{~N} & 126.218 & \mathrm{E} \\ 16 & 14 & 34 & 10.5 ? & 9.61 & \mathrm{~S} & 107.61 & \mathrm{E} \\ 16 & 14 & 48 & 22.3 * & 16.540 & \mathrm{~N} & 98.825 & \mathrm{~W} \\ 16 & 14 & 57 & 05.38 & 37.201 & \mathrm{~N} & 3.011 & \mathrm{~W} \\ 16 & 15 & 16 & 46.5 * & 15.568 & \mathrm{~S} & 176.001 & \mathrm{~W}\end{array}$

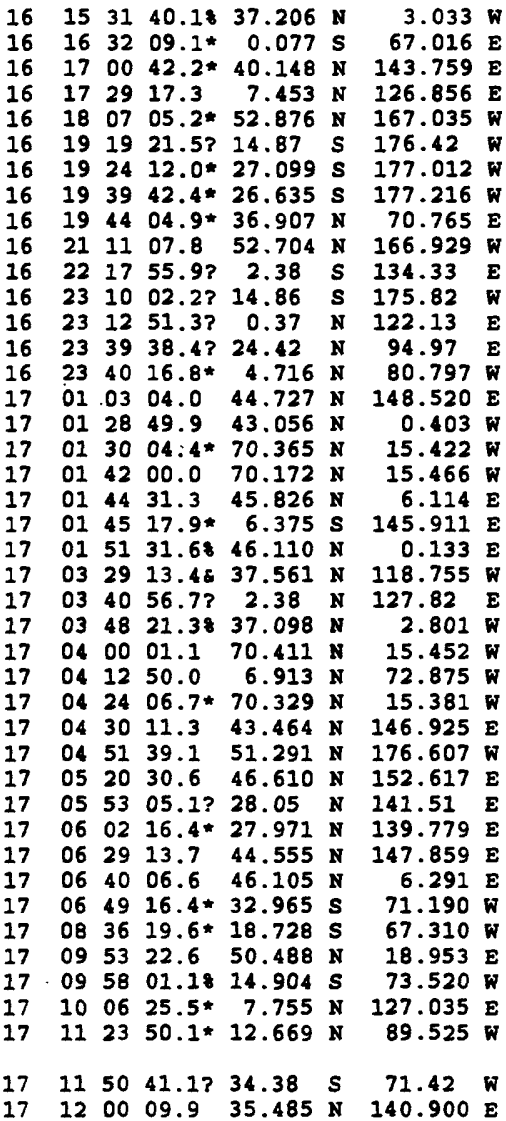

$\begin{array}{rllll}10 & \mathrm{G} & 4.9 & & 1.0 \\ 6 & & & \\ 6 & & & \\ 495 & \mathrm{D} & 4.8 & & 1.0 \\ 33 & \mathrm{~N} & 4.3 & & 1.2 \\ 33 & \mathrm{~N} & 3.3 & & 0.6 \\ 33 & \mathrm{~N} & 4.6 & & 0.9 \\ 10 & \mathrm{G} & & & 0.4 \\ 33 & \mathrm{~N} & 4.6 & 4.8 & 1.1\end{array}$

$10 \mathrm{G}$

$10 \mathrm{G} 4$

$33 \times 4.2$

$33 \mathrm{~N} 4.94 .9$

33 N 3.9

$33 \times 4.3$

$33 \mathrm{~N} 4.3$

$33 \times 4.4$

$33 \times 4.0$

$33 \times 4.4$

$33 \mathrm{~N} 3.8$

33 N 4.0

$33 \times 4.3$

$33 \times 4.1$

$33 \mathrm{~N} 4.0$

$33 \mathrm{~N} 4.3$

$10 \mathrm{G}$

$10 \mathrm{G} \quad 3.8$

$10 \mathrm{G} \quad 4.54 .3$

$10 \mathrm{G}$

33

10

$33 N 4.2$

$10 \mathrm{G}$

$10 \mathrm{G}$

$150 \mathrm{G} 4.1$

10 G 3.93 .7

$33 \mathrm{~N} 4.6$

$33 \mathrm{~N} 4.3$

$33 \mathrm{~N} 4.44 .0$

$33 \times 4.4$

$423 * 4.0$

$33 \mathrm{~N} 5.1$

$5 \mathrm{G}$

$50 \mathrm{G}$

$241 * 4.1$

10 G 2.8

$33 \mathrm{~N} 3.9$

$33 \mathrm{~N} 4.1$

$33 \mathrm{~N} 3.5$

$\begin{array}{lllllll}10 & \mathrm{G} & & & & 0.2 & 7 \\ 33 & \mathrm{~N} & 5.1 & 4.9 & 0.9 & 146\end{array}$
1.0

1.1

0.8

1.5

1.2

0.9

1.1

0.6

1.6

0.9

1.2

1.5

1.2

0.5

1.3

1.1

0.4

1.0

0.9

0.9

0.4

0.8

1.0

1.5

1.3

1.0

0.5

1.0

1.0

0.9
2

\begin{tabular}{|c|c|c|c|c|}
\hline $\begin{array}{ll}4 & 05 \\
5 & 47 \\
5 & 54\end{array}$ & $\begin{array}{l}06.98 \\
59.18 \\
07.6\end{array}$ & $\begin{array}{l}63.254 \\
33.592 \\
43.969\end{array}$ & $\begin{array}{l}\mathbf{N} \\
\mathbf{N}\end{array}$ & $\begin{array}{r}151.097 \\
75.730 \\
15.410\end{array}$ \\
\hline $\begin{array}{l}07 \\
30 \\
32 \\
43 \\
49 \\
30 \\
29 \\
52 \\
05 \\
51 \\
35 \\
27 \\
11 \\
27 \\
18 \\
36\end{array}$ & $\begin{array}{l}56.3 \star \\
53.4 ? \\
38.6 \star \\
09.1 \star \\
55.8 \star \\
19.08 \\
06.3 \\
41.9 \\
18.6 \\
19.58 \\
23.0 ? \\
09.6 ? \\
05.3 ? \\
18.4 \star \\
18.28 \\
31.68\end{array}$ & $\begin{array}{c}55.246 \\
32.44 \\
6.025 \\
6.040 \\
5.427 \\
36.976 \\
44.387 \\
44.364 \\
44.374 \\
45.997 \\
53.97 \\
26.90 \\
36.04 \\
44.691 \\
36.798 \\
47.830\end{array}$ & $\begin{array}{l}N \\
S \\
S \\
S \\
S \\
N \\
N \\
N \\
N \\
N \\
S \\
S \\
N \\
N \\
N \\
N\end{array}$ & $\begin{array}{c}162.378 \\
70.02 \\
103.841 \\
154.184 \\
146.326 \\
3.841 \\
7.356 \\
7.307 \\
7.282 \\
6.326 \\
133.30 \\
176.97 \\
22.03 \\
148.206 \\
5.565 \\
7.478\end{array}$ \\
\hline
\end{tabular}

80 CARLSBERG RIDGE

7 SOUTHERN CAIIFORNIA. <PAS-P>. M 2.7 (PAS).

7 SOUTHERN CALIFORNIA. 〈PAS-P>. YD 2.9 (PAS).

84 SOLOKON ISLANDS

18 NORTEERN MOLUCCA SEA

8 SOUTE OF JANA, INDONESIA

46 NEAR COAST OF GUERRERO, MEXICO

8 SPAIN. mbLg 2.6 (NDD).

34 FIJI ISLANDS REGION. MW 5.2 (HRV).

Centroid, Monent Tensor (BRv): Centroid origin time

$15: 16: 51.4$; Lat $14.40 \mathrm{~s}$; Lon 176.38 \%; Dep $15.0 \mathrm{Fix}$; Halfduration $1.0 \mathrm{sec}$; Principal axes (scale $10 * * 16 \mathrm{Nm}$ ): (T)

Val=8.40, Plg=33, Azm=141; (N) Val=-3.99, Plg=57, Azm=321; (P) Val=-4.41, Plg=0, Azm-51; Best double couple:

Yo-6.4*10**16 km; NP1: Strike-181, Dip-67, Slip-155; NP2 :

Strike-281, Dip=67, Slip=25.

SPAIN. mbLg 2.1 (MDD).

22 CARISBERG RIDGE

12 OFF EAST COAST OF BONSEU, JAPAN

65 MINDANAO, PHILIPPINE ISLANDS

2 FOX ISIANDS, ALEUTIAN ISIAANDS

FIJI ISLAKDS REGION

1 RERMADEC ISLANDS REGION

6 SOUTH OF FIJI ISLANDS

9 HINDU KOSB REGION, AFGEANISTAN

4 FOX ISLANDS, ALEUTIAN ISIANDS

8 IRIAN JAYA REGION, INDONESIA

22 SAMOA ISLANDS REGION

MINARASSA PENINSULA, SULAFES

MTANAAR-IKDIA BORDER REGION

SOUTH OF PARAYA

KURIL ISLANDS

8 PYRENTES. ML 2.8 (LDG). mbLg 2.8 (MDD).

JAN MAYEN ISIAND REGION

JAN YATEN ISTANO BEGTON

FRANCE. MI 2.0 (LDG).

NEW GUINEA, PAPOA NEN GUINEA

FRANCE. ML 2.1 (LDG).

CALIFORNIA-NEVADA BORDER REGION, SGM-P>. ID 3.1 (GM).

NORTHERN MOLOCCA SEA

SPAIN. mbLg 2.3 (2DDD).

14 JAN MAYEN ISTAND REGION

1 NORTHERN COLOIBIA

JAN MAYEN ISEAND REGION

TURIL ISLANDS

ANDREANOF ISLANDS, ALEUTIAN IS. ML 4.4 (PMR).

RIL ISLANDS

BONIN ISLANDS REGION

BONIN ISLAKDS REGION

KURIL ISLANDS

SWITZERIAND. UL 2.6 (IDG), 2.6 (STR).

CENTRAL BOLIVIA

POIAND

CENTRAL PERU

ILIPPINE ISLANDS REGION

Salvador OF CENTRAL A

NEAR COAST OF CENTRAL CHILE. ND 3.1 (SAN).

NEAR EAST COAST OF HONSHO, JAPAN. MW 5.2 (HRV)

Centroid, Moment Tensor (HRV): Centroid origin time

12:00:17.7; Lat $35.93 \mathrm{~N}$; Lon $141.48 \mathrm{E}$; Dep $15.0 \mathrm{Fix}$; Halfduration $1.1 \mathrm{sec}$; Principal axes (scale 10**16 $\mathrm{Nm}$ ): (T) Val=6.39, Plg=67, Azm=177; (N) Val=0.48, Plg=22, Azm=19; (P) Val--6.88, Plg-8, Azm-286; Best double couple: Mo-6.6*10*16 Nm; NP1: Strike-353, Dip=42, Slip=56; NP2: Strike=215, Dip=56, Slip=117.

$73 \times 3.4 \quad 53$

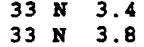

$33 \mathrm{~N} 4.1$

$120 \mathrm{G}$

$33 \mathrm{~N} 4.4$

$33 \mathrm{~N} 4.2$

$33 \mathrm{~N} \quad 3.7$

$10 \mathrm{G}$

$10 \mathrm{G}$

$10 \mathrm{G}$

$10 \mathrm{G}$

$10 \mathrm{G}$

$10 \mathrm{G} 4.4$

$33 \mathrm{~N} 3.9$

$10 \mathrm{G} \quad 3.7$

$33 \mathrm{~N} 3.7$

$10 \mathrm{G}$

$10 \mathrm{G}$ $\begin{array}{lr}1.5 & 6 \\ 1.2 & 116\end{array}$

$1.4 \quad 12$

0.410

$\begin{array}{ll}1.1 & 21 \\ 1.0 & 22\end{array}$

$1.3 \quad 16$

1.012

0.838

$0.4 \quad 14$

$1.5 \quad 12$

1. 414

0.7

0.7

1.1

0.2
CENTRAI AIASKa. 〈AEIC> MT 2.6 (AEIC), 2.9 (PMR).

EASTERN KASHMIR

ADRIATIC SEA. MU 4.3 (IDG), 3.8 (ROM). Felt (VI) in the Vodice, Croatia area.

NEAR EAST COAST OF KAMCHATKA

CHILE-ARGENTINA BORDER REGION. MD 2.8 (SAN).

SOUTHWEST OF SUMATERA, INDONESIA

SOLOMON ISLANDS

EASTERN NEW GUINEA REG., P.N.G.

STRAIT OF GIBRALTAR. MbLg 2.6 (MDD) .

NORTBERN ITALY. ML 2.7 (GEN), 2.5 (STR), 2.5 (IDG).

NORTHERN ITALY. MI 2.3 (GEN).

NORTGERN ITALY. NC 2.5 (GEN), 2.3 (LDG).

FRANCE. NC 2.0 (LDG).

PACIFIC-ANTARCTIC RIDGE

SOUTH OF FIJI ISLANDS

SOUTHERN GREECE

11 KURIL ISLANDS

STRAIT OF GIBRALTAR. mbLg 2.8 (MDD).

SWITZERLAND. ML 1.1 (IDG) 
$\begin{array}{lllll}18 & 01 & 47 & 37.8 & 44.148\end{array}$ $\begin{array}{lllll}18 & 02 & 48 & 00.9 & 25.875\end{array}$ $\begin{array}{llllll}18 & 03 & 30 & 09.18 & 47.951\end{array}$ $\begin{array}{llllll}18 & 04 & 03 & 42.3 & 43.946 & \mathrm{~N}\end{array}$ $\begin{array}{lllll}18 & 04 & 16 & 57.8 \% 38.815 & N\end{array}$ $\begin{array}{llllllll}18 & 04 & 33 & 43.4 ? & 32.54 & 5\end{array}$ $\begin{array}{llllll}18 & 05 & 31 & 57.7 & 39.024 & N\end{array}$ $\begin{array}{lllll}18 & 06 & 52 & 51.0 * 32.829 & s\end{array}$ $\begin{array}{lllll}18 & 07 & 16 & 10.8 & 0.696\end{array}$ $\begin{array}{llllll}18 & 04 & 07 & 32.98 & 36.463\end{array}$ $\begin{array}{llllll}18 & 05 & 04 & 59.0 ? & 12.38 \mathrm{~s}\end{array}$
$10.081 \mathrm{E}$ $90.156 \mathrm{E}$ $7.042 \mathrm{E}$ $15.412 \mathrm{E}$ 2.645 W $73.361 \mathrm{E}$ 71.76 พ $172.36 \mathrm{E}$ $78.962 \mathrm{E}$ 71.821 w 30.021 พ

$\begin{array}{rrrrr}10 \mathrm{G} & & 1.4 & 13 \\ 18 & & 4.4 & 1.0 & 32 \\ 5 \mathrm{G} & & 0.6 & 5 \\ 10 \mathrm{G} & 3.8 & 1.4 & 75 \\ 10 \mathrm{G} & & 0.7 & 13 \\ 33 \mathrm{~N} & 4.1 & 1.1 & 6 \\ 20 \mathrm{G} & & 0.4 & 11 \\ 33 \mathrm{~N} & 4.3 & 1.3 & 31 \\ 33 \mathrm{~N} & 4.1 & 1.0 & 28 \\ 10 \mathrm{G} & & 0.8 & 12 \\ 10 \mathrm{G} & 5.05 .2 & 1.4 & 171\end{array}$

$10 \mathrm{G} \quad 5.05 .2 \quad 1.4 \quad 179$ $\begin{array}{lllll}18 & 07 & 50 & 05.3 * 36.007 \mathrm{~N}\end{array}$

$\begin{array}{llllll}18 & 08 & 21 & 04.58 & 47.353 \mathrm{~N}\end{array}$

$\begin{array}{llllll}18 & 08 & 43 & 42.3 & 35.873 \mathrm{~s}\end{array}$

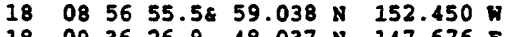

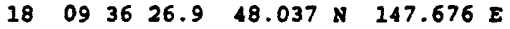

$\begin{array}{lllllll}18 & 10 & 22 & 35.1 & 6.542 & S & 123.163 \\ E\end{array}$

$181058 \quad 46.0 * 13.387 \mathrm{~S} 111.204 \mathrm{w}$

$\begin{array}{llllll}18 & 12 & 28 & 13.88 & 60.025 \mathrm{~N} & 149.380\end{array}$

$18 \quad 323606.0 * 19.026 \times 145.523 \mathrm{E}$

$18131906.0 * 34.334 \mathrm{~N} 140.430 \mathrm{E}$

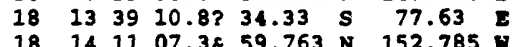

$18141851.0 \quad 35.604 \mathrm{~N} 84.951 \mathrm{E}$

$\begin{array}{llllll}18 & 15 & 02 & 12.9 & 36.245 \mathrm{~N} & 27.285 \mathrm{E}\end{array}$

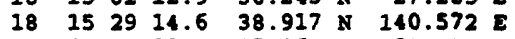

$\begin{array}{llllllll}18 & 15 & 31 & 22.1 ? & 37.86 & \mathrm{~S} & 78.05 & \mathrm{E}\end{array}$

$18 \quad 1550 \quad 03.6863 .432 \mathrm{~N} 151.879$ N

$\begin{array}{lllllllll}18 & 16 & 07 & 46.9 ? & 2.97 & S & 128.74 & \text { E }\end{array}$

$18161743.5866 .570 \mathrm{~N} 151.216 \mathrm{w}$

$\begin{array}{llllll}18 & 16 & 41 & 26.3 & 1.063 \mathrm{~N} & 120.448 \mathrm{E}\end{array}$

$18164900.0844 .454 \mathrm{~N} 7.298 \mathrm{E}$

$18172643.9 * 37.049 \mathrm{~s} \quad 78.071$ :

$\begin{array}{llllllllll}18 & 18 & 01 & 07.6 ? & 21.64 & S & 170.44\end{array}$

$18180222.8 * 10.264$ N 124.967 w

$\begin{array}{lllllll}18 & 18 & 11 & 28.9 ? & 17.44 & S & 178.62\end{array}$

$18182216.3 * 51.572 \times 159.591 \mathrm{E}$

$\begin{array}{lllllll}18 & 18 & 25 & 48.3 ? & 40.71 & \mathrm{~N} & 34.19\end{array}$

$18 \quad 183135.8862 .561 \mathrm{~N} 149.104$ w

$\begin{array}{llllllll}18 & 18 & 35 & 28.73 & 11.19 & \mathrm{~N} & 125.56 & \mathrm{E}\end{array}$

18 19 $3028.4 * 27.644$ S 68.884 .

$18 \quad 195223.8861 .967 \mathrm{~N} 151.003$ w

$\begin{array}{rrrrrrrrr}18 & 20 & 49 & 29.16 & 59.982 & \mathrm{~N} & 140.638 & \mathrm{~W} \\ 18 & 21 & 20 & 10.2 * & 13.059 & \mathrm{~N} & 57.938 & \mathrm{E}\end{array}$

$\begin{array}{lllll}18 & 21 & 38 & 08.3 & 43.180\end{array}$

$\begin{array}{lllll}18 & 22 & 36 & 19.2 * 31.713 \mathrm{~S}\end{array}$

$\begin{array}{lllllll}18 & 23 & 30 & 58.13 & 27.15 & S\end{array}$

$\begin{array}{lllll}18 & 23 & 42 & 23.7 & 14.339 \mathrm{~N}\end{array}$

$190051 \quad 12.88 \quad 45.940$

$\begin{array}{llllll}19 & 01 & 05 & 38.1 & 39.341 & \mathrm{~N}\end{array}$ $19025602.9660 .038 \times 153.148$ W $\begin{array}{lllllll}19 & 03 & 06 & 42.9 ? & 28.06 & \text { N } 139.16 \quad \mathrm{E}\end{array}$ $\begin{array}{llllllll}19 & 03 & 08 & 46.0 & 37.754 & \mathrm{~N} & 20.817 \mathrm{E}\end{array}$ $\begin{array}{llllll}19 & 03 & 13 & 16.1 & 48.088 \mathrm{~N} & 147.699 \mathrm{E}\end{array}$

19041916.151 .451 N 178.367 w
$18105436.4861 .289 \mathrm{~N} 151.158 \mathrm{w}$

$182005 \quad 41.3$ ? 41.13 N 79.14 E

$\begin{array}{lllll}18 & 22 & 55 & 26.3 & 10.920\end{array}$

$19024023.454 .622 \times 161.020 \mathrm{w}$
$1.054 \mathrm{w}$

$62.320 \mathrm{~W}$

177.54 W

$60.724 \mathrm{~W}$

$6.174 \mathrm{E}$

$25.985 \mathrm{~B}$

$\begin{array}{rlll}126 * & 4.0 & 1.0 & 13 \\ 5 G & & 1.5 & 14 \\ 91 D & 4.9 & 1.0 & 78\end{array}$

79

400 G 4.7

550 G 5.0

56

$10 \mathrm{G} \quad 4.64 .6$

24.3 .6

71 D 4.3

$10 \mathrm{G} \quad 3.9$

$8 B$

$33 \times 4.1$

$33 \times 4.4$

$33 \mathrm{~N} 4.4$

1064.3

$33 \times 4.2$

77

$33 \times 4.94 .5$

$10 \mathrm{G}$

$10 \mathrm{G} 4.8$

$33 \times \quad 4.5$

$10 \mathrm{G}$

$500 \mathrm{G} 3$.

5

$33 \times 4$

$106 \quad 3.7$

58

33 N 4.3

$113 \quad 3.9$

100 G 3.4

63

$33 \times 3.7$

7
25

25 D 4.1

$10 \mathrm{G}$

$170 \mathrm{G}$

$100 \mathrm{D} 4.2$

$200 \mathrm{G} 4.1$

100

5
33
$\mathrm{G}$

$33 \times 4.4$

1

400 G 3.5

33 N 3.8

300 G 3.7

$33 \times 5.75 .6 \quad 0.9$
3 NORTHERN ITALY. ML 2.1 (LDG).

INDIA-BANGLADESB BORDER REGION

SWITZERLAND. MI 1.6 (LDG).

ADRIATIC SEA. ML 4.0 (LDG), 3.5 (ROM)

STRAIT OF GIBRALTAR. mbLg 2.9 (MDD).

TAJIKISTAN-XINJIANG BORDER REG.

NEAR COAST OF CENTRAL CHILE. MD 4.0 (SAN).

SANTA CRUZ ISLANDS REGION

SOUTHERN XINJIANG, CHINA

NEAR COAST OF CENTRAL CBILE. MD 4.3 (SAN).

CENTRAL MID-ATLANTIC RIDGE. 5.8 (HRV).

Centroid, Moment Tensor (HRV): Centroid origin time

$07: 16: 20.8$; Lat $0.81 \mathrm{~N}$; Lon $30.12 \mathrm{~W}$; Dep 15.0 Fix; Half -

duration $2.0 \mathrm{sec}$; Principal axes (scale $10 * 17 \mathrm{Nm}$ ): (T)

Val-6.39, Plg-12, Azm-221; (N) Val=0.14, Plg=75, Azm=5; (P)

val--6.54, Plg=9, Azm-129; Best double couple:

Mo-6.5*10**17 Nm; NP1: Strike-265, Dip =75, Slip-177; NP2 :

Strike-355, Dip-88, Slip-15.

BINDO KDSH REGION, AFGHANISTAN

(ERANCE. YL 2.7 (LDG)

CENTRAL CHILE. MD 4.8 (SAN). Felt (IV) at ralca and (III) at Cauquenes, Chillan, Constitucion, Curico, Iloca, Licanten, Linares and San Carlos.

68 SOUTHERN ALASKA. LAEIC

$0.7 \quad 158$ SEA OF OKHOTSK

0.764 BANDA SEA

59 SOUTHERN ALASKA. LAEIC). ML 2.6 (AEIC).

32 CEATRAL EAST PACIFIC RISE

KENAI PENINSULA, ALASKA. CAEIC>. ML 2.8 (AEIC).

MARIANA ISLANDS

1.7217 NEAR EAST COAST OF HONSHU, JAPAN

$\begin{array}{lrl}1.2 & 17 & \text { NEAR EAST COAST } \\ 0.9 & 6 & \text { MID-INDIAN RIDGE }\end{array}$

101 SOUTHERN ALASRA. <AEIC>.

1.018 XIZANG

1.3110 DODECANESE ISLANDS

0.828 EASTERN HONSHU, JAPAN

1.410 MID-INDIAN RIDGE

35 CENTRAL ALASKA. CAEIC>. MC 2.4 (AEIC), 2.6 (PMR).

11 CERAM SEA

12 MORTEERN ALASKA. CAETC>.

1.243 MINAHASSA PENINSULA, SULAWESI

0.25 NORTEERN ITALY. MLI 1.9 (GEN).

1.429 MID-INDIAN RIDGE

1.421 LOYALTY ISLANDS REGION

0.913 NEAR COAST OF NORTHERN CALIF. ML 2.9 (GS).

1.123 FIJI ISLANDS REGION

52 SOUTHERN ALASKA. CAEIC). IL 2.4 (AEIC), 2.9 (PMR).

1.126 OFF EAST COAST OF RAYCBATKA

1.46 TURREY

51 CEMTRAL ALASKA. CAEIC). ME 2.5 (AEIC).

1.515 SAMAR, PHILIPPINE ISLANDS

0.918 NEAR $S$. COAST OF BONSHO, JAPAN

1.512 CBILE-ARGENTINA BORDER REGION

94 SOUTHERN ALASKA. CAEIC. MC 3.1 (AEIC), 3.2 (PMR).

0.88 KYRGYZSTAN-XINJIANG BORDER REG.

22 SOUTHEASTERN ALASKA. <AEIC>. MC 2.6 (AEIC).

1.414 ARABIAN SEA

0.97 PYRENEES. mbLg 2.6 (YDD). ML 2.4 (LDG).

0.713 SAN JUAN PROVINCE, ARGENTINA. YD 2.6 (SAN).

1.131 NEAR COAST OF VENEZUELA

1.413 KERYADEC ISLANDS REGION

0.926 WINDWARD ISLANDS

0.911 FRANCE. MC 2.0 (LDG).

1.1152 AEGEAN SEA. Felt at Ayvacik, Bayramic, Ezine and Izmir, rurkey.

1.076 ALASKA PENINSULA. YL 4.1 (PMR), 3.6 (AEIC)

76 SOUTEERN ALASKA. CAEIC>. ML 3.4 (AEIC), 3.6 (PMR).

0.27 BONIN ISLANDS REGION

1.117 IONIAN SEA

9 SEA OF OKHOTSK

391 ANDREANOF ISLANDS, ALEUTIAN IS. MW 6.0 (RRV), 5.9 (GS). Me 5.8 (GS). ML 5.7 (PMR). MS 5.4 (BRK). Felt (III) on Adak. Broadband Source Parameters (GS): Dep 28; NPI: Strike-50, Dip-65, Slip-75; NP2: Strike-262, Dip-29, Slip-119; Radiated energy $1.2 * 10 * * 13 \mathrm{~km}$.

Moment Tensor (GS) : Dep 24; Principal axes (scale 10**17 Nin): (T) Val=9.09, Plg-61, Azm=290; (N) Val-0.18, Plg-22, Azm-67; (P) val--9.27, Plg-18, Azm-165; Best double couple Mo-9.2*10*17 Nm; NP1: Strike-285, Dip-34, Slip-133; NP2: Strike-57, Dip $=66$, Slip $=66$.

Centroid, Moment Tensor (HRV): Centroid origin time

$04: 19: 19.7$; Lat $51.43 \mathrm{~N}$; Lon $178.02 \mathrm{w}$; Dep 42.1; Halfduration $2.6 \mathrm{sec}$; Principal axes (scale 10**18 $\mathrm{Nm}$ ): (T) val-1.08, P1g=66, Azm-307; (N) val=0.06, P1g-10, Azm-61; (P) val--1.14, Plg=21, Azm-155; Best double couple: Mo-1.1*10*18 Nm; NP1: Strike-263, Dip-25, Slip-114; NP2: Strike-56, Dip-67, Slip-79.

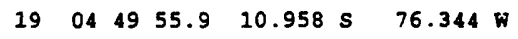

$33 \times 4.5$

$1.0 \quad 14$ CENTRAI

$914.8 \quad 1.0 \quad 40$ MINDANAO, PHILIPPINE ISLANDS

50 G $4.0 \quad 0.9$

$10 \mathrm{G} \quad 5.45 .9 \quad 1.4 \quad 74$ MID-INDIAN RIDGE. MW 6.1 (GS), 5.9 (HRV).

Moment Tensor (GS): Dep 11; Principal axes (scale 10**18
$19060230.4 * 36.627$ \& $141.110 \mathrm{E}$

$19062411.0 \quad 41.523 \mathrm{~s} \quad 80.416 \mathrm{E}$ 


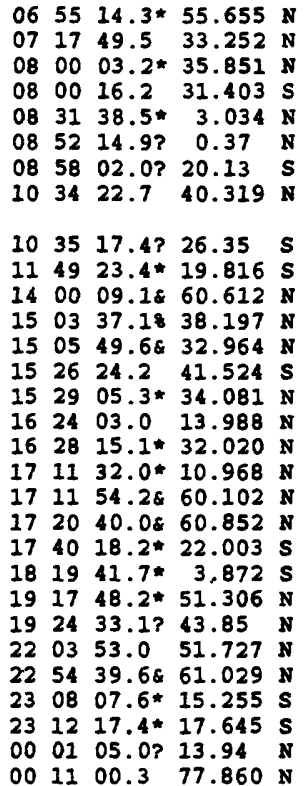

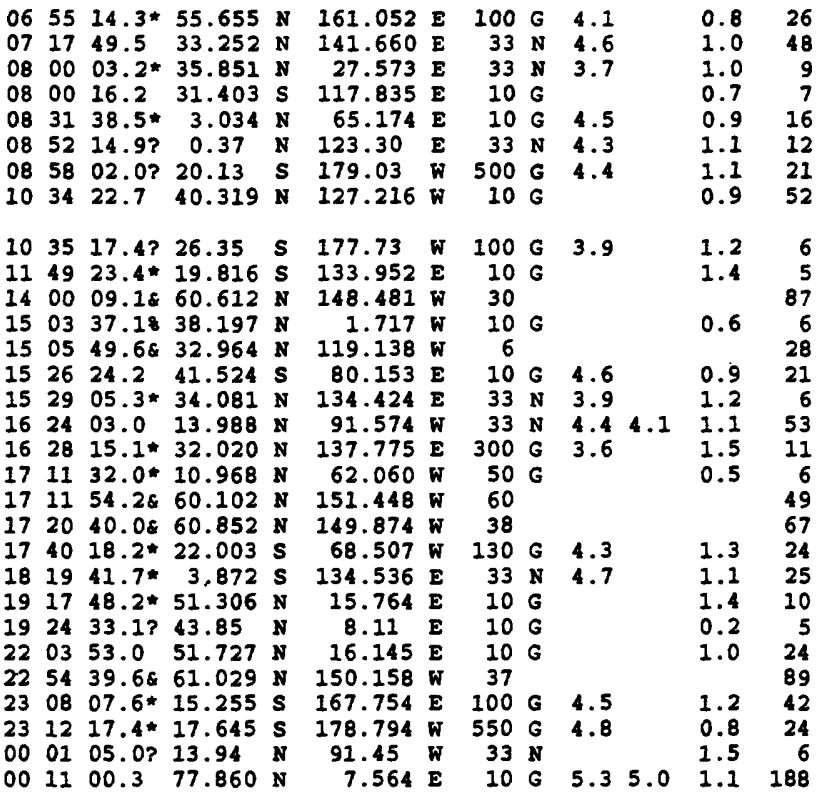

N(m): (T) Val=1.81, Plg-11, Azm=351; (N) Val=-0.21, Plg=79, Axm-174; (P) Val--1.60, Plg-1, Azm-81; Best double couple: Ho-1.7*10*18 Ni NPI: Strike-127, Dip-82, S11p-7; NP2: Strike-36, Dip-83, Slip-172.

Centroid, voment Tensor (BRV): Centroid origin time $06: 24: 16.8$; Lat $41.53 \mathrm{~S}$; Lon $80.07 \mathrm{E}$; Dep 15.0 F1x; Halfduration $2.2 \mathrm{sec}$; Principal axes (scale 10*17 $\mathrm{Nm}$ ): (T) Val-8.87, Plg=13, Azm=1; (N) Val=-0.54, Plg-67, Azm=238; (P) Val=-8.33, Plg=19, Azm-95; Best double couple: Mo-8.6*10*17 $\mathrm{km}$; NP1: Str1ke-137, Dip-68, S11p-4; IP2: Strike-229, Dip-86, Slip=-158.

NEAR EAST COAST OF KAMCHATKa

OFE EAST COAST OF BONSHU, JAPAN

DODECANESE ISLANDS

WESTERN AUSTRALIA

16 CARLSBERG RIDGE

MINAHASSA PENINSULA, SULAFESI

1 FIJI ISLANDS REGION

2 OFE COAST OF NORTHERN CALIFORNIA. MW 3.8 (BRK). MII 4.0 (BRK). Scalar Moment (BRK): MO=5.7*10*14 Nm.

SOUTE OF FIJI ISLANDS

NORTEERN TERRITORY, AOSTRALIA

8 KENAI PENINSULA, ALASKA. CAEIC). ML 3.6 (AEIC), 3.3 (PMR).

SPAIN. mbLg 2.6 (MDD).

OFF COAST OF CALIFORNIA. <PAS-P). MD 3.3 (PAS). MLC 3.5 (GS). MID-INDIAN RIDGE

NEAR S. COAST OF WESTERN HONSHD

NEAR COAST OF GOATEMALA

SOUTE OF BONSHO, JAPAN

NEAR COAST OF VENEZUELA

KENAI PENINSULA, ALASRA. <AEIC>. NI 2.5 (AEIC).

KENAI PENINSULA, ALASKa. CAEIC>. MI 2.9 (AEIC).

NORTHERN CAILE

IRIAN JAYA REGION, INDONESIA

POLAND

CORSICA. YLI 2.0 (IDG).

POLAND. ML 4.2 (GRF), 3.8 (VIE), 3.6 (MOX).

SOUTHERN ALASKA. CAEIC>. MC 3.3 (AEIC), 3.1 (PMR).

VANOATU ISLANDS

FIJI ISLANDS REGION

NEAR COAST OE GUATDMAIA

SVATBARD REGION. WW 5.2 (GRV), 5.1 (GS).

Moment Tensor (GS): Dep 6; Principal axes (scale 10*16 (n):

(T) Val=5.65, Plg-3, Azm-103; (N) Val=0.11, Plg=4, Azm=13;

(P) val- -5.77, Plg-84, Azm-229; Best double couple:

Mo-5.7*10*16 $\mathrm{km}$; MP1: Strike-198, Dip=42, S11p--83; IP2:

Strike-9, Dip=48, Slip--96.

Centroid, Moment Tensor (HRV): Centrold origin time

00:11:04.9; Lat 78.07 N; Lon 6.95 E; Dep 15.0 Fix; Half-

duration 1.0 sec; Principal axes (scale $10 * 16 \mathrm{Nm}$ ): (T)

Val-8.39, Plg-3, Azm=102; (N) Val-1.40, Plg=10, Azm=11;

(P) val- -6.98, Plg-80, Azm-207; Best double couple:

ko-7.7*10*16 Nm; NP1: Strike-202, Dip=43, S1ip-75; NP2:

Strike-2, DIp-48, Slip--103.

$\begin{array}{llllll}20 & 01 & 26 & 50.6 & 40.036\end{array}$
$20.629 \mathrm{E}$

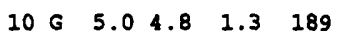

$\begin{array}{lllll}03 & 10 & 05.0 ? & 42.69 & 1\end{array}$

7.68 W $10 \mathrm{G}$

$032140.7 * 6.549 \mathrm{~s} \quad 147.121 \mathrm{E} \quad 100 \mathrm{G} 4.3$

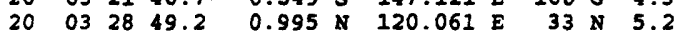

GREECE-ALBANIA BORDER REGION. mbLg 5.0 (MDD). ML 4.8 (ROM).

some damage to houses at Ronitsa, Greece.

0.44 SPAIN. mbLg 2.8 (2DD).

0.812 EASTERN NEW GUINEA REG., P.N.G.

1.049 MTNAHASSA PENINSOLA, SOLAWESI. 5.3 (BRV).

Centrold, Moment Tensor (BRV): Centrold origin time

$03: 28: 56.5$; Lat $1.69 \mathrm{~N}$; Ion $120.26 \mathrm{E}$; Dep 43.4 ; Half

duration $1.1 \mathrm{sec}$; Principal axes (scale 10*\#17 Nuil):(T)

Val-0.82, Plg-59, Azm-191; (N) Val-0.30, Plg-13, Azm=78;

(P) Val=-1.12, Plg=27, Azm=341; Best double couple:

No-9.7*10*16 im; NP1: Strike-40, Dip-21, Slip-50; NP2:

Strike-262, Dip=74, slip-104.

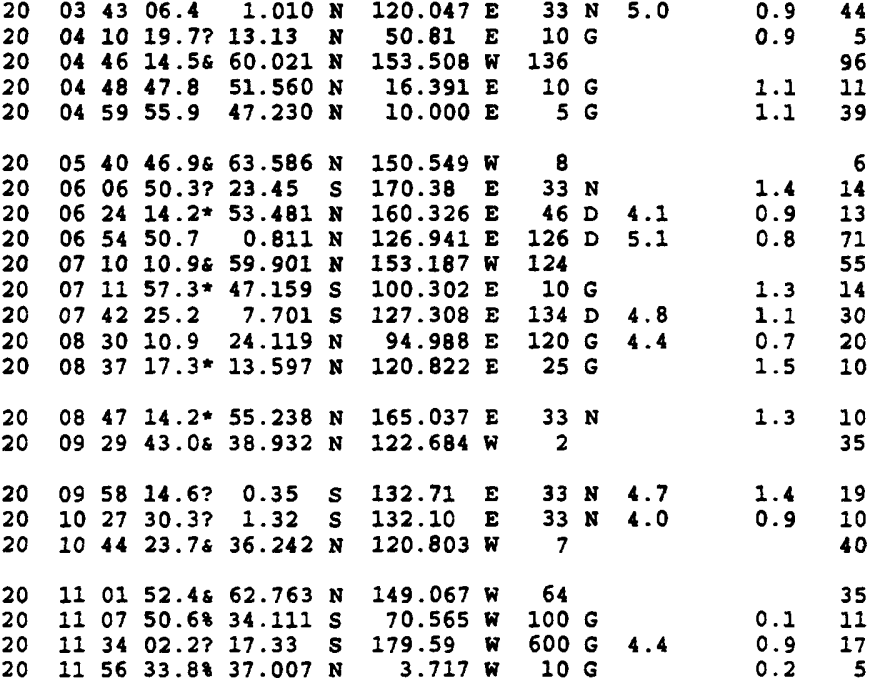

MINAHASSA PENINSULA,

6 SOUTHERN ALASKA. LAEIC>

POLAND. AL 3.2 (GRF), 3.1 (MOX).

AUSTRIA. YC 3.5 (STR), 3.4 (VIE), 3.1 (GRF), 3.0 (IDG), 2.9

(FUR). Felt (IV) at Rlosterle.

6 CENTRAL ALASRA. CAEIC>. ML 2.4 (AEIC), 2.8 (PMR).

LOXALTY ISLANDS REGION

NEAR EAST COAST OF RAMCHATKA

NORTHERN MOLUCCA SEA

SOUTHERN ALASKA. LAEIC>.

SOUTBEAST INDIAN RIDGE

BANDA SEA

MYANMAR-INDIA BORDER REGION

MINDORO, PHILIPPINE ISLANDS. Felt (II RF) at puerto Galera.

Felt (I RF) at Tagaytay, Luzon.

10 KOMANDORSKY ISLANDS REGION at Lower Lake.

IRIAN JAYA REGION, INDONESIA

IRIAN JAYA REGION, INDONESIA

CENTRAL CAIIFORNIA. 〈GM-P〉. MD 3.0 (GM), 2.8 (PAS). ML 3.1 (BRK).

CENTRAL ALASKA. LAEIC>. ML 2.6 (AEIC).

11 CHILE-ARGENTINA BORDER REGION. MD 3.8 (SAN).

0.917 FIJI ISLANDS REGION 
$20120658.4 * 56.063 \mathrm{~s}$ $\begin{array}{llllll}20 & 12 & 08 & 11.2 & 23.548 & \mathrm{~s}\end{array}$ $\begin{array}{llllllll}20 & 15 & 27 & 05.18 & 32.728 & 5\end{array}$ $20154429.5 * 36.498$ \& $\begin{array}{lllll}20 & 16 & 10 & 36.7 & 36.244\end{array}$ $20163944.5 * 44.128$ N $20164637.1 * 56.139 \mathrm{~s}$ $20 \quad 171956.7 \quad 5.369$
28.567 ค 250 G 4.1 179.744 ค $500 \mathrm{G} 5.1$ $71.553 \mathrm{~W} 20 \mathrm{G}$ 26.935 E $130 \mathrm{G} \quad 3.6$ 27.261 E 33 N 4.1 $14.972 \mathrm{E} \quad 10 \mathrm{G}$ $27.012 \mathrm{~N} \mathrm{~N} 5.0$ $82.637 \%$
$10 \mathrm{G} 5.25 .1$

ANDREAMOF ISLANDS, ALEUTIAN IS. MW 5.3 (ERV). MC 5.4 (PMR). Felt on Adak.

Centroid, Moment Tensor (HRV): Centroid origin time

$12: 02: 40.0$; Lat $51.42 \mathrm{k}$; Lon $178.18 \mathrm{w}$; Dep 38.3 ; Balfduration $1.0 \mathrm{sec}$; Principal axes (scale 10*17 $\mathrm{kn}$ ): (T) Val-1.09, Plg-58, Azm=293; (K) Val=-0.18, Plg-15, Azm=50; (P) Val=-0.90, Plg-27, Azm-148; Best double couple: Mo-9.9*10*16 Nm; NP1: Strike-271, Dip-23, slip-133; NP2: Strike-45, Dip=73, Slip=74.

SOUTR SANDWICR ISLANDS REGION

$\begin{array}{lll}0.6 & 11 & \text { SOUTR SANDWICR ISLANDS } \\ 0.9 & 67 & \text { SOUTR OF FIJI ISLANDS }\end{array}$

0.59 NEAR COAST OF CENTRAL CBILE. MD 3.5 (SAN).

0.712 DODECANESE ISLANDS

1.352 DODECANESE IST.ANDS

$\begin{array}{lll}1.3 & 52 & \text { DODECANESE ISLANDS } \\ 0.6 & 14 & \text { ADRIATIC SEA. 2II } 3.5 \text { (LDG) }\end{array}$

1.427 SOUTH SANDWICH ISLANDS REGION

135 SOUTB OF PANAMA. NW 5.9 (BRV).

Centroid, Moment Tensor (BRV): Centroid origin time

$17: 20: 03.6$; Lat $5.29 \mathrm{*}$; Lon 82.51 w; Dep 15.0 Bdy; Halfduration $2.1 \mathrm{sec}$; Principal axes (scale 10*17 $\mathrm{Nm}$ ): (T) Val-7.72, Plg-20, Azm-315; (N) Val=-0.87, Plg-70, Azm-142; (P) Val--6.85, Plg=2, Azm-46; Best double couple: Mo-7.3*10*17 $\mathrm{Nm} ;$ IP1: Strike-92, Dip=75, Slip-13; NP2. Strike-359, Dip=78, slip=164.

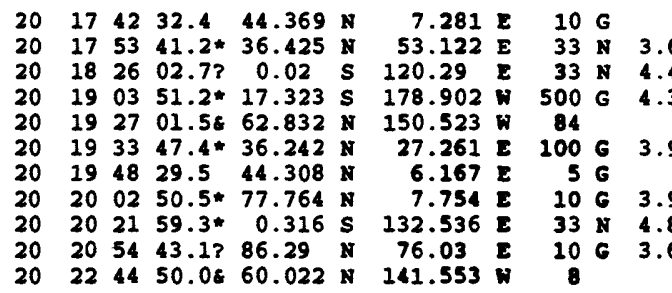

$\begin{array}{llllll}20 & 23 & 00 & 01.5 & 45.495 & \mathrm{~N}\end{array}$ $\begin{array}{llllll}20 & 23 & 13 & 50.5 & 53.433 & \mathrm{~N}\end{array}$ $20232532.1 * 40.653$ : $20 \quad 23 \quad 2858.6 \quad 44.356$ N $\begin{array}{llllll}20 & 23 & 42 & 52.3 & 13.234 & \%\end{array}$ $20235553.8 \% 46.730 \quad N$ $21005714.1 * 20.104 \mathrm{~s}$ $21 \quad 013521.1 \quad 0.987 \mathrm{~N}$ $21015408.7 * 12.404 \mathrm{~N}$ $\begin{array}{lllllll}21 & 02 & 17 & 58.2 & 40.746 & N\end{array}$ $\begin{array}{lllllll}21 & 03 & 08 & 55.07 & 16.09 & \mathrm{~N}\end{array}$ $21033835.0 * 0.910 \mathrm{~s}$ $21 \quad 03 \quad 41 \quad 42.88 \quad 33.762 \mathrm{~s}$ $210404 \quad 33.9 * 50.760 \mathrm{~N}$ $\begin{array}{llllll}21 & 04 & 31 & 28.37 & 2.31 & N\end{array}$ $\begin{array}{llllll}21 & 04 & 37 & 22.3 & 44.357 & \mathrm{~N}\end{array}$ $2104 \quad 4701.68 \quad 58.391 \mathrm{~N}$ $21055147.4 * 15.905 \mathrm{~N}$ 21072138.9858 .170

$6.668 \mathrm{E}$ 160.357 $35.497=$ $7.269 \mathrm{E}$ 87.414 w 122.145 177.837 120.053 $144.092=$ $35.420 \mathrm{E}$ 120.35 97.084 70.713 16.4415 126.53 $7.313 \mathrm{E}$ 154.834 98.216 พ $151.759 \%$ $71.352 \mathrm{n}$

\section{$10 \mathrm{G}$}

$\begin{array}{lll}33 & \mathrm{~N} & 4.4 \\ 10 & \mathrm{G} & 3.9\end{array}$

56

$212 \mathrm{D} \quad 4.3$

18

$480 ? 4.5$

$45 ? 4.6$

33 \% 4.1

$86 ? 4.9$

33 \& 4.4

$80 \mathrm{G}$

28 *

$130 ? 4.3$

14

1
$34 \mathrm{D} 5.14 .8 \quad 1.0$

$33 \times 4.1$

0.5

1.0

0.6

1.0

1.5

0.9

1.3

1.4

1.5

\section{3}

0.8

1.4
0.6

0.6

8.8

0.8

1.2

1.1

1.4

0.9

0.3

1.3

0.4

1.3

1.1

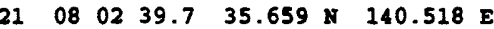
$210850 \quad 55.9 * 35.987$ \& 140.845 E $\begin{array}{llllllll}21 & 08 & 52 & 25.87 & 28.19 & \text { N } & 139.07 & E \\ 21 & 08 & 54 & 04.9 & 51.367 & \text { N } & 178.268 & \end{array}$ $\begin{array}{lll}39 & 0 & 4.7\end{array}$ $33 \mathrm{~N}$ 520 * $33 \times 4.9$
73 SOUTE OF SEVERMAYA ZEYIYA

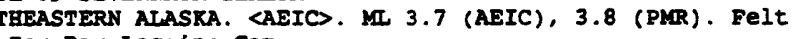
at ICy Bay Logging Camp.

26 FRANCE. MI 2.3 (LDG)

26 MEAR EAST COAST OF RAMCHATKA

18 TORKEY

10 NORTHERN ITALY. MI 1.9 (GEN), 1.5 (IDG).

63 MASBINGTON. 〈SEA-P〉. MD 2.6 (SEA). Felt.

20 FIJI ISLANDS REGION

50 MINAEASSA PENINSULA, SULAKESI

24 SOUTE OF MARIANA ISLANDS

24 TORKEY

9 LUZON, PHILIPPINE ISLANDS

22 SOUTHWEST OF SUMATERA, INDONESIA

11 CHILE-ARGENTINA BORDER REGION. YD 2.6 (SAN).

6 POLAND. MIL 3.1 (GRF), 2.8 ( $M O X)$.

9 NORTHERN MOLUCCA SEA

19 NORTHERN ITALY. MC 2.5 (GEN), 2.3 (LDG).

43 ALASKA PENINSULA. CAEIC). MI 3.7 (AEIC), 3.7 (PMR).

16 OFF COAST OF GUERRERO, MEXICO

40 KODIAK ISLAND REGION. ¿AEIC>. ML 2.8 (AEIC)

15 VERMONT-NEW HAMPSHIRE REGION. mbLg 3.8 (GS), 3.6 (OTT). Felt (IV) at Bethlehem, (III) at South Tamworth and (II) at vilan, New Bampshire. Also felt at Twin Nountain, New Hampshire.

1.260 NEAR EAST COAST OF HOMSBO, JAPAN

1.510 NEAR EAST COAST OF HONSHO, JAPAN

$0.4 \quad 8$ BONIN ISLANDS REGION

ANDREANOF ISLANDS, ALEUTIAN IS. NW 5.2 (HRV). MC 4.7 (PMR). Centroid, Moment Tensor (HRV): Centrold origin time 08:54:04.2; Lat $51.33 \mathrm{~N}$; Lon $177.72 \mathrm{w}$; Dep 25.8; Halfduration $1.0 \mathrm{sec}$; Principal axes (scale 10**16 $\mathrm{Nm}$ ): (T) val=7.56, Plg=60, Azm-309; (N) Val-0.31, Plg=5, Azm-48; (P) val=-7.86, Plg-30, Azm-141; Best double couple:

Mo-7.7*10*16 Nm; NP1: Strike-246, Dip-16, Slip-109; NP2 : Strike-46, Dip-75, Slip-85.

$21090130.0 * 47.649 \mathrm{~s} 99.698 \% \quad 10 \mathrm{G} 4.9 \quad 1.1 \quad 32$ SOUTHERN PACIFIC OCEAN. NW 5.0 (BRV).

Centroid, Noment Tensor (HRV): Centroid origin time 09:01:34.5; Lat 48.05 $\mathrm{S}$; Lon $99.34 \mathrm{~W}$; Dep 15.0 Fix; Halfduration $1.0 \mathrm{sec}$; Principal axes (scale 10*16 $\mathrm{Nm}$ ): (T) Val-3.70, Plg-0, Azm-230; (N) Val-0.93, Plg-90, Azm-180; (P) Val-4.63, PIg-0, Azm-140; Best double couple: Mo-4.2*10**16 Nm; NP1: Strike=275, Dip-90, Slip--180; NP2 : Strike-5, Dip-90, slip=0.
$21 \quad 110915.4 ? 50.96 \mathrm{~N}$

$21 \quad 111755.4736 .08 \mathrm{~N}$

21 11 $3327.5 * 11.345 \mathrm{~N}$

B6.662 พ 33

0.7

$155.489 \mathrm{E}$
$33 \mathrm{~N} 5.14 .8 \quad 1.0$
6 POLAND YG 3.2 (WAR)

8 HINDD KOSH REGION, AFGHANISTAN

18 NEAR COAST OF NICARAGOA

64 D'ENTRECASTEAUX ISLANDS REGION. NW 5.2 (HRV). Centroid, Moment Tensor (HRV): Centroid origin time $12: 17: 46.9$; Lat $9.87 \mathrm{~S}$; Lon $155.65 \mathrm{E}$; Dep $15.0 \mathrm{Bdy}$; Halfduration $1.0 \mathrm{sec}$; Principal axes (scale 10**16 $\mathrm{Nm}$ ): (T) val-8.21, Plg-7, Azm-4; (N) Val=-2.96, Plg-6, Azm-94; (P) val--5.25, PIg-81, Azm-221; Best double couple: Mo-6.7*10**16 Nm; NP1: Strike-87, Dipm38, S1ipm-99; NP2 : Strike-279, Dip-53, Slip=-83.

$21122812.4 * 56.225 * 163.981 \mathrm{E} 33 \mathrm{~N} 4.2$

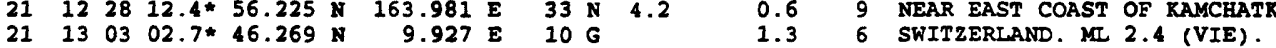

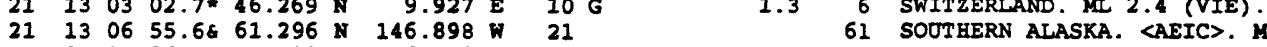
$21 \quad 140856.8 * 46.705 \times 146.240 \mathrm{E} 304 ? 4.1 \quad 0.9 \quad 17$ NORTHWEST OF KORIL ISLANDS 
$\begin{array}{lllllllllll}21 & 14 & 27 & 39 & 57 & 9 & 36 & 5 & 155.59 & 5\end{array}$

$21 \quad 14 \quad 4053.8 \quad 45.746 \mathrm{~N}$ $161300.8 * 5.665$ N

$\begin{array}{lllll}17 & 26 & 58.6 ? & 9.48 & \end{array}$
$33 \times 4.1$
$125.816 \mathrm{E} \quad 33 \mathrm{~N} 4.5$

$33 \times 5.15 .01 .1$
$49.4 \quad 5.462 \mathrm{~s}$
$25.45 \mathrm{E}$

$147.153 \mathrm{E}$ 179.50 $155.315 \mathrm{E}$
$33 \mathrm{~N}$

$223 \quad 4.9$

$\begin{array}{lll}652 ? & 4.2\end{array}$ $33 \times 5.05 .0 \quad 0.6$ $\begin{array}{lllll}21 & 19 & 11 & 57.8 ? & 52.60\end{array}$ $21 \quad 191502.9 ? \quad 44.38$ $\begin{array}{llllll}21 & 20 & 43 & 00.5 & 12.168 & \mathrm{~N}\end{array}$ $\begin{array}{llllllll}21 & 21 & 09 & 47.7 \text { ? } & 27.18 & \text { N }\end{array}$ $\begin{array}{llllll}21 & 21 & 53 & 07.6 & 19.145 \mathrm{~s}\end{array}$

$\begin{array}{rrrrr}30.36 & \text { W } & 10 & G & 3.8 \\ 7.84 & \text { E } & 10 & G & \\ 60.890 & \text { W } & 79 & & 4.5 \\ 125.62 & \text { E } & 243 & ? & 3.9\end{array}$

1.9

$0.9 \quad 173$

1.119
D'EATRECASTEAOX ISLANDS REGION

RURIL ISLANDS

\section{MINDANAO, PHILIPPINE ISLANDS}

D' BNTRECASTEAUX ISLANDS REGION

D' EATRECASTEAOX ISLANDS REGION. 5.4 (BRV)

Centroid, Moment rensor (HRV): Centroid origin time

17:27:37.4; Lat $9.90 \mathrm{~s}$; Ion $155.62 \mathrm{E}$; Dep $15.0 \mathrm{Fix}$; Halfduration $1.6 \mathrm{sec}$; Principal axes (scale 10**17 $\mathrm{sm}$ ): (T)

Val=1.46, Plg=8, $A z m=352$; (N) Val=0.13, Plg=8, $A 2 m=83$; (P) Val=-1.60, PIg=79, Azm-215; Best double couple:

Mo-1.5*10*17 Nm; NP1: Strike-73, Dip-37, Slip--102; NP2: Strike-268, Dip=54, Slip=-81.

$\begin{array}{lrl}1.3 & 5 & \text { AEGEAN SEA } \\ 0.9 & 41 & \text { EASTERN NEW GUINEA REG., P.N.G. }\end{array}$

SOUTE OE FIJI ISLANDS

D'ENTRECASTEAUX ISLANDS REGION. WW 5.5 (HRV).

Centroid, Moment Tensor (HRV): Centroid origin time

$19: 06: 55.8$; Lat $9.92 \mathrm{~S}$; Ion $155.83 \mathrm{E}$; Dep 15.4; Balfduration $1.3 \mathrm{sec}$; Principal axes' (scale 10*17 $\mathrm{km}$ ): (T) Val=1.68, Plg=11, Azm=355; (N) Val=0.11, Plg=2, Azm=264; (P) Val=-1.80, Plg=79, Azm-164; Best double couple: Mo-1.7*10*17 Nm; NP1: Strike-87, Dip-34, slip=-86; NP2: Strike-263, Dip-56, Slip--92.

NORTHERN MID-ATLANTIC RIDGE

$\begin{array}{rrl}1.2 & 6 & \text { NORTHERN MID-ATLANTIC RIDGE } \\ 0.4 & 5 & \text { NORTHERN ITALY. NC } 2.1 \text { (IDG) } \\ 1.0 & 52 & \text { WINDWARD ISIANDS. MD } 4.4 \text { (TRN) }\end{array}$

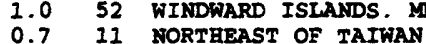

125.62 E $243 ? 3.9$

1.2182 VANUATO ISLANDS. 5.4 (HRV)

Centroid, Moment Tensor (HRV): Centroid origin time $21: 53: 14.3$; Lat $19.19 \mathrm{~s}$; Ion $169.60 \mathrm{E}$; Dep 276.2 ; Halfduration $1.2 \mathrm{sec}$; Principal axes (scale $10.17 \mathrm{Nm}$ ) : (T) Val=1.45, Plg=40, Azm=55; (N) Val=-0.02, Plg=50, $\mathrm{Azm}=228$; (P) $\mathrm{Val}=-1.43$, Plg=3, Azm=322; Best double couple: Yo-1.4*10**17 $\mathrm{Nm} ;$ NP1: Strike-91, Dip-61, Slip-152; NP2: Strike-196, Dip-66, Slip-33

$212209 \quad 49.4 ? \quad 32.44 \quad N$ $22003522.1 * 37.865 N$ $\begin{array}{llllll}00 & 46 & 00.6 & 9.495 \mathrm{~S} & 155.493 \mathrm{E}\end{array}$ $005001.0 ? \quad 9.39$ S 155.59 E $010900.2 * 36.548$ \& $012912.13 \quad 9.15 \quad 5$ $014455.6 \quad 53.292 \mathrm{~N}$ $020101.6 \quad 38.358 \mathrm{~N}$ $021055.0 \quad 10.430 \mathrm{~s}$ 71.357 $35.314 \mathrm{k}$ 21.769

$\begin{array}{rllll}43 & \mathrm{D} & & & 1.1 \\ 33 & \mathrm{~N} & & & 1.1 \\ 33 \mathrm{~N} & 4.5 & & 0.8 \\ 33 \mathrm{~N} & 4.2 & & 1.4 \\ 158 * & 4.2 & & 1.2 \\ 33 \mathrm{~N} & 4.7 & & 0.4 \\ 10 \mathrm{G} & 4.8 & 4.6 & 0.9 \\ 42 & 3.7 & & 1.0 \\ 130 & \mathrm{~F} & 5.0 & & 1.1\end{array}$

1.1

1.1
0.8

1.4

1.2 1.0
$22021431.8 * 47.832 N$ $22044706.6 \quad 5.578$ N $22051911.5 * 21.642 \mathrm{~N}$ $22052439.5 * 20.010 \mathrm{~N}$ $\begin{array}{lllll}22 & 05 & 35 & 42.0 & 7.124 \mathrm{~s}\end{array}$
152.179 $125.313 \mathrm{E}$ $143.132 \mathrm{E}$ $121.736 \mathrm{E}$ $123.275 \mathrm{E}$
160

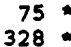
33 $596 \mathrm{D} 5.8$ $\begin{array}{lllllclcl}22 & 06 & 01 & 57.9 * & 9.519 & \text { S } & 155.630 & E \\ 22 & 06 & 02 & 39.58 & 46.842 & \text { N } & 0.836 & \text { W } \\ 22 & 06 & 06 & 18.47 & 9.48 & \text { S } & 155.71 & E \\ 22 & 06 & 09 & 08.1 & 9.517 & \text { S } & 155.355 & E\end{array}$

$\begin{array}{llll}33 & N & 4.5 & 1.2 \\ 10 & G & & 1.2 \\ 33 & N & & 0.9\end{array}$

$33 \mathrm{~N} 5.14 .9 \quad 0.9$ 065630.3 ? 24.26 $\begin{array}{lllll}07 & 14 & 44.7 & 14.625 \mathrm{~s}\end{array}$ $071616.6 * 46.181 \mathrm{~s}$ $\begin{array}{llll}07 & 56 & 33.6 & 33.831\end{array}$ $0840 \quad 43.68 \quad 32.653 \mathrm{~s}$ $090522.2 ? 26.96 \mathrm{~s}$ $\begin{array}{llcll}10 & 09 & 18.1 ? & 1.74 & \mathrm{~N} \\ 10 & 26 & 22.38 & 11.478\end{array}$ $\begin{array}{lllll}10 & 26 & 22.38 & 11.478 \mathrm{~N}\end{array}$ $\begin{array}{lllll}10 & 29 & 48.0 ? & 3.69 & 5 \\ 11 & 56 & 53.6 * & 6.147\end{array}$
$155.855 \mathrm{E} \quad 105 \mathrm{D} \quad 4.8$ $125.09 E$ $41.288 \mathrm{E}$ $0.917 \mathrm{E}$ $94.050 \mathrm{E}$ 71.596 พ $107.60 \mathrm{~W}$ $121.81 \mathrm{E}$ $61.303 \mathrm{H}$ $144.87 \quad \mathrm{E}$ $\begin{array}{lll}33 & \mathrm{~N} & 4.0 \\ 10 & \mathrm{G} & 4.6\end{array}$ $10 \mathrm{G} \quad 4.8$ 33 N 4.8 $10 \mathrm{G}$ $10 \mathrm{G} \quad 4.7$ $33 \mathrm{~N} 4.5$ $10 \mathrm{G}$
0.6

0.8

1.3
7 SOUTR OF BONSHO, JAPAN

7 AFGEANISTAN-TAJIKISTAN BORD REG.

28 D' ENTRECASTEAUX ISLANDS REGION

12 D' ENTRECASTEAUX ISLANDS REGION

27 AFGEANISTAN-TAJIKISTAN BORD REG

D'ENTRECASTEAOX ISLANDS REGION

131 NORTE ATLANTIC OCEAN

24 GREECE

83 SOLOMON ISLANDS. NW 5.3 (HRV).

Centroid, Moment Tensor (HRV): Centroid origin time 02:10:47.9; Lat 10.43 S Fix; Lon 161.13 E Fix; Dep 44.4; Half-duration $1.0 \mathrm{sec}$; Principal axes (scale 10**16 $\mathrm{Nm}$ ): (T) Val-8.98, Plg=64, Azm-9; (N) val=-0.37, Plg-24, Azm=166; (P) Val--8.61, Plg-9, Azm=260; Best double couple Mo-8.8*10*16 Mm; MP1: Strike=16, Dip=42, Slip=127; NP2: Strike-150, Dip=58, Slip $=61$.

13 KURIL ISLANDS

38 MINDANAO, PHILIPPINE ISLANDS

MARIANA ISLANDS REGION

PHILIPPINE ISLANDS RPGION. Felt (II RF) at Basco.

BANDA SEA. NW 6.0 (GS), 5.9 (BRV). We 5.7 (GS)

Broadband source parameters (GS): NPI: Strike-205, Dip-40, Slip-110; NP2: Str1ke-50, Dip-53, Silp=-74; Radiated energy $6.7 * 10 * 12 \mathrm{Nm}$.

yoment Tensor (GS): Dep 618; Principal axes (scale 10*18 $\mathrm{Nim}$ ): (T) Val $=0.98, \mathrm{Plg}=0, \mathrm{Azm}=296$; (N) Val $=0.03, \mathrm{Plg}=20$, Azm-206; (P) Val=-1.02, Plg=70, Azm-26; Best double couple: Mo-1.0:10*18 Min; NP1: strike-44, Dip-48, Slip=-63; NP2: Strike-187, Dip $=48$, Slip $=-117$.

Centroid, Moment Tensor (HRV): Centroid origin time $05: 35: 47.4$; Lat $7.14 \mathrm{~s}$; Ion $123.52 \mathrm{E}$; Dep 614.7 ; Half duration $2.3 \mathrm{sec}$; Principal axes (scale 10**17 $\mathrm{Nm}$ ): (T) Val=8.59, $\mathrm{Plg}=2, \mathrm{Azm}=300$; (N) Val-1.28, Plg-13, Azm-210; (P) val=-9.87, Plg-77, Azm-37; Best double couple: Mo-9.2*10*17 km; NP1: Strike-43, Dip-45, Slip=-71; NP2 : Strike-198, Dipe-48, Slip=-108.

20 D'ENTRECASTEAUX ISLANDS REGION

12 FRANCE. ML 2.5 (IDG).

D'ENTRECASTEAOX ISLANDS REGION

63 D'ENIRECASTEAUX ISLANDS REGION. NW 5.5 (HRV).

Centroid, Moment Tensor (HRV): Centroid origin time $06: 09: 10.3$; Lat $9.82 \mathrm{~s}$; Ion $155.59 \mathrm{E}$; Dep $15.0 \mathrm{Bdy}$; Halfduration $1.2 \mathrm{sec}$; Principal axes (scale 10*17 $\mathrm{Nm}$ ): (T) Val-1.64, Plg-7, Azm-347; (N) Val-0.19, Plg-10, Azm-78; (P) Val=-1.83, Plg-78, Azm=223; Best double couple: Mo-1.7*10*17 Nm; NP1: Strike=65, Dip-39, Slip=-106; NP2: Strike-266, Dip=53, Slipm-77.

0.8141 KORIL ISLANDS. Felt (II) at Severo-Kurilsk.

1.46 SOUTHWESTERN RYUKYU ISLANDS

$1.0 \quad 42$ MOZAMBIQUE CHANNEL

$1.1 \quad 18$ SOUTH ATLANTIC OCEAN

1.143 QINGBAI, CHINA

0.511 NEAR COAST OF CENTRAL CHILE. ND 3.7 (SAN)

1.27 EASTER ISIAND REGION

1.217 MINAHASSA PENIMSOLA, SULAWESI

0.96 WINDWARD ISIANDS. ND 2.1 (TRN).

1.17 NEAR N COAST OF NEW GUINEA, PNG.

1.1

14 NEW BRITAIN REGION, P.N.G. 


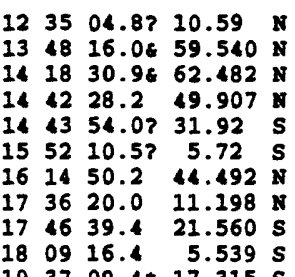

$193709.4 * 17.315 \mathrm{~s}$

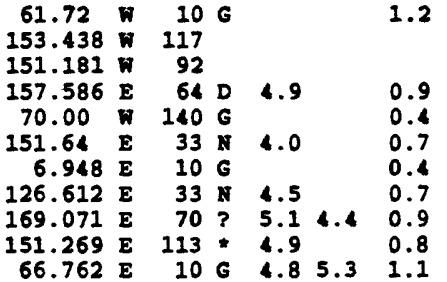

\begin{abstract}
$193838.3 * 11.173 \mathrm{~N}$ $200609.1 * 24.186 \mathrm{~S}$ $\begin{array}{lllll}20 & 28 & 59.97 & 24.27 & \text { N }\end{array}$ 2050 08.5* $11.310 \mathrm{~s}$ $\begin{array}{llll}21 & 03 & 13.7 & -0.207 \mathrm{~s} \\ 21 & 0.53 & \end{array}$ $\begin{array}{llll}21 & 27 & 54.5 & 41.837\end{array}$ $\begin{array}{llll}22 & 15 & 17.6 & 33.951 \mathrm{~s}\end{array}$ $221954.7 * 37.935 \mathrm{~N}$ $222311.9232 .66 \mathrm{~s}$ $\begin{array}{lllll}22 & 43 & 13.2 & 34.958 & \mathrm{~N}\end{array}$ $\begin{array}{llll}22 & 55 & 34.6 & 18.809 \mathrm{~N}\end{array}$ $002916.6 * 51.356 \mathrm{~N}$ 010154.3 ? $0.64 \mathrm{~s}$ $\begin{array}{llllllll}01 & 37 & 43.0 & 45.715 & \mathrm{~N}\end{array}$ $0144 \quad 34.3 \quad 13.181 \mathrm{~N}$ $022905.8=30.353 \mathrm{~s}$ $033004.5 * 11.916 \mathrm{~N}$ $034319.0 * 23.066 \mathrm{~s}$ $051129.47 \quad 7.74 \mathrm{~S}$ $\begin{array}{lll}0533 & 20.5 & 70.293 \mathrm{~N}\end{array}$ $\begin{array}{llll}05 & 46 & 37.2 & 37.065 \mathrm{~N}\end{array}$ $\begin{array}{lllll}05 & 54 & 39.0 & 44.463 \mathrm{~N}\end{array}$ $061339.9 * 24.878 \mathrm{~s}$ $061955.9 \quad 36.045 \mathrm{~N}$ $064132.2 * 38.916 \mathrm{~N}$ $072417.0720 .73 \mathrm{~S}$ $075532.7 * 6.776$ N 080033.7636 .885 N
\end{abstract}

$\begin{array}{lllll}08 & 10 & 29.27 & 37.49 \quad \mathrm{~N}\end{array}$ $\begin{array}{lllllll}08 & 53 & 33.97 & 32.58 \quad \mathrm{~S}\end{array}$ $085754.2 * 36.025 \mathrm{~N}$ $085827.8 * 43.838 \mathrm{~N}$ $\begin{array}{lllll}09 & 13 & 46.9 & 47.942 & \text { N }\end{array}$ $09 \quad 1546.1844 .472 \mathrm{~N}$ $\begin{array}{lllll}10 & 02 & 05.3 ? & 12.28 \quad \mathrm{~N}\end{array}$ 102531.9769 .89 N 1141 21.07 0.16 N $115721.3 * 14.790 \mathrm{~N}$ $\begin{array}{llll}12 & 01 & 15.98 & 61.044\end{array}$ $125821.6 ? 36.56 \mathrm{~N}$ $\begin{array}{lllll}13 & 27 & 45.9 & 46.831 & \text { N }\end{array}$ $151200.2 * 65.073 \mathrm{~N}$ $152552.2 * 38.988 \mathrm{~N}$

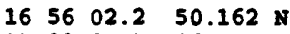
$\begin{array}{lllll}17 & 22 & 39.7 & 26.355 \mathrm{~N}\end{array}$ $173213.1827 .842 \mathrm{~N}$ $\begin{array}{llll}17 & 53 & 06.1 & 19.035 \mathrm{~s}\end{array}$ 1338 18.9* $14.084 \mathrm{~N}$

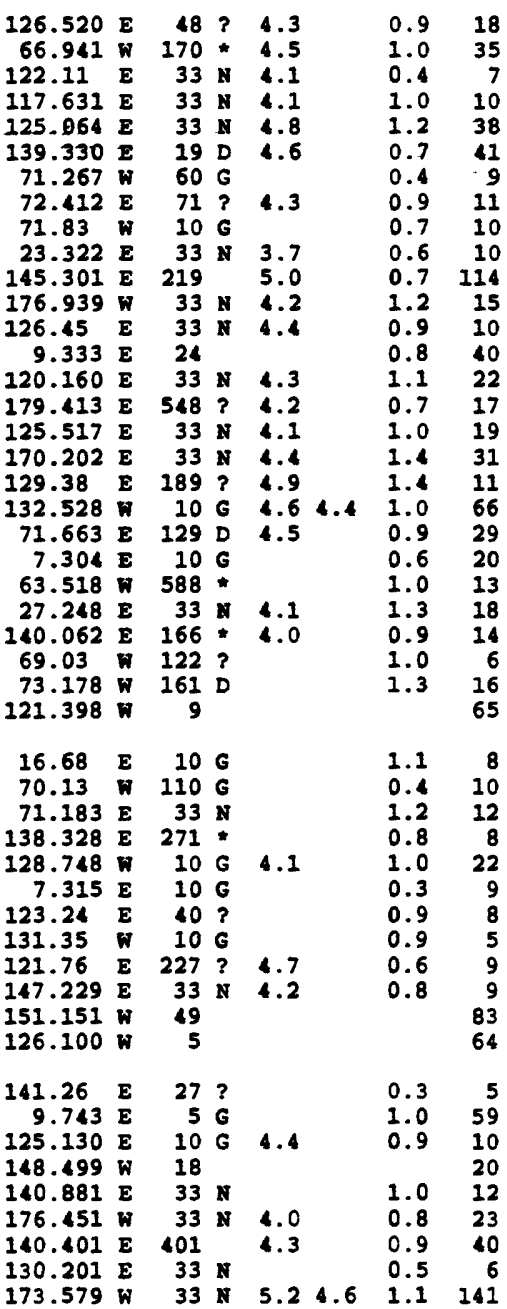

$\begin{array}{lllll}19 & 28 & 27.2 & 51.408 & 11\end{array}$ $\begin{array}{llll}19 & 35 & 38.6 & 50.266 \mathrm{~N}\end{array}$ $\begin{array}{lllll}19 & 38 & 35.7 & 50.356 & \text { N }\end{array}$ $\begin{array}{llll}19 & 53 & 47.78 & 61.819\end{array}$ $205600.2 ? 32.62 \mathrm{~s}$ $\begin{array}{llllll}20 & 57 & 58.28 & 33.726 & \mathrm{~S}\end{array}$ $\begin{array}{llllll}21 & 23 & 17.08 & 44.454 & \mathrm{~N}\end{array}$ $215606.0 \quad 4.088 \mathrm{~s}$

$151.863 \mathrm{~W}$

$70.417 \mathrm{w}$

$7.280 \mathrm{E}$

$\begin{array}{rrrrrr}450 & ? & 4.2 & & 0.7 & 28 \\ 10 G & & & 1.3 & 34 \\ 5 \text { G } & & & 0.7 & 15 \\ 103 & & & & 21 \\ 10 & G & 4.5 & & 1.0 & 8 \\ 17 & \star & & & 0.2 & 10 \\ 10 & G & & & 0.3 & 7 \\ 10 & G & 5.0 & 5.6 & 1.1 & 74\end{array}$

4 TRIKIDAD. MD 2.9 (TRH).

27 SOUTHERN ALASKA. CAEIC>.

46 CDMTRAL ALASKA. <AEIC>

40 EAST OF KURIL ISLANDS

7 NEN BRITAIA REGION, P.N.G.

19 FRAKCE. NII 2.4 (GEN), 2.3 (IDG).

26 PHILIPPINE ISLANDS REGION

52 LOYALTY ISLANDS REGION

29 NEN BRITAIN REGION, P.N.G.

22 NAURITIUS-REUNION REGION. NW 5.1 (BRV).

Centroid, koment Tensor (BRV): Centroid origin time

$19: 37: 15.8$; Lat $17.09 \mathrm{~s}$; Lon $66.51 \mathrm{E}$; Dep 15.0 F1x; Halfduration $1.0 \mathrm{sec}$; Principal axes (scale 10**16 Nm): (T) Val-5.23, Plg-12, Azm-107; (N) Val=-0.92, Plg-78, A2m-306; (P) Val=-4.32, Plg=4, Azm=197; Best double couple: Mo $4.8 * 10 * 16 \mathrm{Nm}$; NP1: Strike=243, Dip-79, S11P=6; NP2 : Strike-151, Dip=85, Silp=169.

18 PHILIPPINE ISLANDS REGION

SALTA PROVINCE, ARGENTINA

7 TAIWAN REGION

10 SOUTH OF SUMBAWA, INDONESIA

38 SOUTHERN MOLUCCA SEA

1 HOKRAIDO, - JAPAN . REGION

9 IBAR:COAST OF CENERAL CHILE

1 TAJTKISTAN

O MEAR COAST OF CENTRAL CHILE. MD 3.4 (SAN).

10 CRETE

MARIANA ISLANDS

ANDREANOF ISLANDS, ALEUTIAN IS.

SOUTHERN MOLUCCA SEA

MORTHERN ITALY. NC 3.2 (STR), 2.6 (IDG).

2 MINDORO, PHILIPPINE ISLANDS

KERLADEC ISLANDS REGION

SAMAR, PHILIPPINE ISLANDS

IOYALTY ISIANDS REGION

BANDA SEA

BEAUFORT SEA

AFGEANISTAN-TAJIKISTAN BORD REG.

NORTHERN ITALY. ML 2.9 (GEN), 2.8 (IDG).

SALTA PROVINCE, ARGENTINA

DODECANTSE ISLANDS

EASTERN HONSHU, JAPAN

NORTHERN CHILE

NORTHERN COLOYBIA

CENKRAI CALIFORNIA. 〈GM-P>. MD 3.0 (GM). ML 3.0 (BRK), 3.0 (GS). Felt in the Hollister area.

8 IONIAN SEA. MU 3.4 (ROM).

CHILE-ARGENTINA BORDER REGION, MD 3.2 (SAN).

AFGHANISTAN-TAJIKISTAN BORD REG.

EASTERN SEA OF JAPAN

OFF COAST OF MASHINGTON

WORTHIRN ITALT. MIL 2.0 (GEN).

IOZON, PHILIPPINE ISLANDS

NORTHAEST TERRITORIES, CAKADA

MINAFASSA PENINSULA, SULAWESI

MaRIANA ISLANDS REgION

3 SOUTHERN AIASKA, CAEIC), MI 3.6 (AEIC), 3.6 (PMR).

OFF COAST OF NORTHERN CALIFORNIA. <GM-P>. MD 3.2 (GM). MI 3.8 (BRK), 3.5 (GS).

NEAR BAST COAST OF HONSHU, JAPAN

SWITZERLAND. ML 3.9 (FUR), 3.5 (GRF), 3.5 (STR), 3.4 (VIE).

PHILIPPINE ISTANDS REGION

NORTHERN ALASKA. CAEIC>. ML 2.6 (AEIC).

EASTERN HONSHO, JAPAN

ANDREANOF ISLANDS, ALEUTIAN IS.

BONIN ISIANDS REGION

RYOKYO ISILANDS

TONGA ISLANDS. MW 5.2 (HRV).

Centroid, Moment Tensor (BRV): Centroid origin time

$17: 53: 06.5$; Lat $19.45 \mathrm{~S}$; Lon $172.98 \mathrm{w}$; Dep 17.3 ; Half-

duration 1.0 sec; Principal axes (scale 10*16 Nm): (T)

Val=8.45, $\mathrm{Plg}=11$, Azm=86; (N) Val=-1.23, $P 1 g=1, A z m=355$;

(P) Val=-7.23, Plg=79, Azm-263; Best double couple:

MO-7.8*10*16 Nm; NP1: Str1ke=176, Dip-34, Slip=-89; NP2:

Strike-355, Dip=56, Slip=-91.

SEA OF OKHOTSK

GERMANY. YI 3.3 (VIE), 3.0 (BNS), 3.0 (UCC)

GERMANY. ML 2.8 (LDG), 2.6 (STR), 2.4 (UCC).

SOUTHERN ALASKA. LAEIC>

SOUTHWEST INDIAN RIDGE

CAILE-ARGENTINA BORDER REGION. MD 3.3 (SAN).

NORTHERN ITALY. ML 1.8 (GEN).

CENTRAL EAST PACIFIC RISE. WW 5.9 (HRV).

Centroid, Moment Tensor (HRV): Centroid origin time

$21: 56: 13.2$; Lat $3.80 \mathrm{~s}$; Lon $104.40 \mathrm{w}$; Dep $15.0 \mathrm{Fix}$; Halfduration 2.2 sec; Principal axes (scale $10 * 17 \mathrm{Nm}$ ): (T)

Val=9.10, Plg=5, Azm=134; (N) Val=-0.60, Plg=80, Azm=15; (P) Val=-8.51, Plg=9, Azm=225; Best double couple:

Mo-8.8*10*17 Nm; NP1: Strike=270, Dip-80, Slip--3; NP2:

Strike=0, Dip=87, Slip--170.

scalar Noment (PPT): MO-1.5*10*18 Nm.

HOKKAIDO, JAPAN REGION 
$\begin{array}{lllll}0 & G & 5.0 & 5.4 & 1.1\end{array}$

$\begin{array}{lllllrl}22 & 30 & 38.2 ? & 29.74 & \mathrm{~S} & 177.10 & \mathrm{~W} \\ 22 & 33 & 58.9 ? & 33.99 & \mathrm{~S} & 71.74 & \mathrm{~W} \\ 23 & 00 & 15.6 * & 19.986 & \mathrm{~N} & 121.463 & \mathrm{E} \\ 23 & 42 & 59.7 * & 44.844 & \mathrm{~N} & 8.877 & \mathrm{E} \\ 02 & 18 & 24.96 & 66.952 & \mathrm{~N} & 147.276 & \mathrm{~W} \\ 02 & 38 & 20.5 & 47.462 & \mathrm{~N} & 9.015 & \mathrm{E} \\ 02 & 42 & 24.7 & 47.479 & \mathrm{~N} & 9.040 & \mathrm{E} \\ 03 & 54 & 44.4 * & 0.862 & \mathrm{~N} & 99.460 & \mathrm{E} \\ 04 & 15 & 38.2 & 44.373 & \mathrm{~N} & 7.327 & \mathrm{E} \\ 04 & 17 & 49.0 ? & 24.66 & \mathrm{~S} & 65.32 & \mathrm{~W} \\ 05 & 15 & 56.3 * & 31.464 & \mathrm{~N} & 51.013 & \mathrm{E} \\ 06 & 14 & 28.67 & 19.01 & \mathrm{~N} & 67.21 & \mathrm{~W} \\ 07 & 09 & 49.6 & 12.267 & \mathrm{~N} & 125.693 & \mathrm{E} \\ 08 & 09 & 04.7 * & 39.369 & \mathrm{~N} & 74.157 & \mathrm{E} \\ 08 & 51 & 15.2 & 30.752 & \mathrm{~S} & 178.235 & \mathrm{~W}\end{array}$
$24 \quad 10 \quad 0757.9659 .357 \times 151.732$ W $24 \quad 110240.5 \quad 37.762 \mathrm{~N} \quad 16.780 \mathrm{~W}$ $24120155.9 * 55.396 \mathrm{~S} \quad 128.255 \mathrm{~W}$ $24121526.2 * 39.606 \mathrm{~N} 91.569 \mathrm{E}$ $\begin{array}{llllllllll}24 & 12 & 41 & 19.5 & 44.359 N & 7.339 \mathrm{E}\end{array}$ $24 \quad 1303 \quad 46.18 \quad 44.384 \mathrm{~N} \quad 7.350 \mathrm{E}$ $24 \quad 13 \quad 38 \quad 33.1 * 17.622$ S $174.751 \mathrm{~W}$ $24140158.2 \quad 8.679 \mathrm{~N} \quad 72.709 \mathrm{~W}$ $\begin{array}{llllllll}24 & 14 & 14 & 19.3 & 55.35 & \mathrm{~S} & 128.53 & \mathrm{~N}\end{array}$ $24 \quad 1433 \quad 16.9 \quad 43.440 \mathrm{~N} \quad 1.813 \mathrm{~W}$

$24 \quad 144554.6 \quad 58.292 \mathrm{~s}$ $24154356.1 * 26.326 \mathrm{~s}$

$\begin{array}{lllll}24 & 15 & 58 & 51.0 & 37.296 \mathrm{~N}\end{array}$

$2418 \quad 30 \quad 43.3 * 26.312 \mathrm{~s}$

$24 \quad 18 \quad 32 \quad 45.0658 .383 \quad \mathrm{~N}$

$\begin{array}{llllll}24 & 19 & 14 & 12.9 & 44.369 & N\end{array}$

$\begin{array}{llllll}24 & 19 & 31 & 34.3 & 51.908 & N\end{array}$

$24200240.0 * 20.489 \mathrm{~N}$

$24204949.4 * 47.280 \mathrm{~N}$

$\begin{array}{llllll}24 & 21 & 11 & 35.0 \quad 7.392 \mathrm{~s}\end{array}$

$\begin{array}{llllllll}24 & 22 & 02 & 39.4 ? & 33.24 \mathrm{~S}\end{array}$

$\begin{array}{lllllllll}24 & 22 & 04 & 54.2 ? & 36.23 & S\end{array}$

$\begin{array}{lllllll}24 & 22 & 58 & 58.08 & 43.523 \mathrm{~N}\end{array}$

$\begin{array}{lllllll}24 & 23 & 04 & 36.68 & 36.734 & N\end{array}$

$24 \quad 233106.8 * 17.420 \mathrm{~s}$

$\begin{array}{llllll}24 & 23 & 38 & 59.5 & 47.918 & \mathrm{~N}\end{array}$

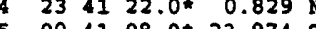

$\begin{array}{llll}5 & 004108.0 * 23.9745\end{array}$

$\begin{array}{lllll}25 & 0049 & 07.36 \quad 67.400 \mathrm{~N}\end{array}$

$250055021.8 * 55.019 \mathrm{~N}$

$25010226.5 \% 60.010 \mathrm{~N}$

$\begin{array}{llllllll}25 & 01 & 47 & 44.78 & 43.556 \mathrm{~N}\end{array}$

$\begin{array}{lllll}25 & 02 & 03 & 24.9 * 51.576\end{array}$

$\begin{array}{lllllll}25 & 02 & 28 & 20.98 & 33.062 \mathrm{~s}\end{array}$

$\begin{array}{llllll}25 & 02 & 52 & 51.6 ? & 6.48 \quad \mathrm{~s}\end{array}$

$25025959.3 * 36.467 \mathrm{~N}$

$\begin{array}{lllllll}25 & 03 & 11 & 40.2 \text { ? } & 32.37\end{array}$

$\begin{array}{lllllll}25 & 03 & 13 & 11.1 ? & 22.11 & \mathrm{~s} \\ 25 & 03 & 34 & 54 & 38 & 43.478 & \end{array}$

$03 \quad 3454.38 \quad 43.478 \mathrm{~N}$

$\begin{array}{llll}04 & 45 & 13.6 & 51.381 \\ 04 & 48 & 15.0 & 39.300\end{array}$

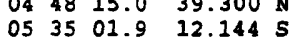

$05 \quad 3916.8$ ? $23.22 \mathrm{~N}$

$05 \quad 48 \quad 42.7 * 25.823 \mathrm{~s}$

$0606 \quad 40.8$ ? 6.39

$\begin{array}{lllllll}06 & 58 & 07.9 ? & 34.57\end{array}$

070828.8 51.779 N $175.208 \mathrm{~W}$

$\begin{array}{lllllll}08 & 27 & 21.08 & 35.097 \mathrm{~N} & 118.306 \mathrm{~W}\end{array}$

$5090752.0 \quad 51.597 \mathrm{~N} \quad 176.967 \mathrm{~W}$

$\begin{array}{rrrrr}33 & N & 4.3 & 0.9 \\ 33 & N & & 0.3 \\ 33 & N & & 0.4 \\ 33 & N & & 0.7 \\ 9 & & & \\ 20 & G & & 1.3 \\ 20 & G & & 1.2 \\ 110 & D & 5.0 & 1.2 \\ 10 & G & & 0.5 \\ 33 & N & & 0.5 \\ 33 & N & 4.4 & 1.2 \\ 33 & N & & 0.4 \\ 31 & D & 4.7 & 0.9 \\ 33 & N & 3.6 & 1.1 \\ 59 & D & 5.1 & 1.0\end{array}$

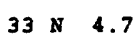

48

$10 \mathrm{G} 3.7$

$10 \mathrm{G} \quad 4.64 .4$

33

19

$10 \mathrm{G}$

$181 * 4.7$

$21 \mathrm{D} 4.4$

$10 \mathrm{G} 3.9$

27.445

$41.837 \mathrm{E}$

$70.763 \mathrm{~W}$

$7.299 \mathrm{E}$

$16.263 \mathrm{E}$

$7.935 \mathrm{E}$

4.507 ผ
45.105

69.34

16.18 พ

$1.582 \mathrm{~W}$

$3.288 \mathrm{~W}$
$67.570 \mathrm{~B}$

$7.384 \mathrm{E}$

$26.189 \mathrm{E}$

$66.694 \mathrm{~W}$

$109.458 \mathrm{E}$

$48.085 \mathrm{E}$

.100

$1.497 \mathrm{~W}$
72.537

71.108

141.96

1.709

$177.770 \mathrm{~W}$

$26.165 \mathrm{E}$
167.106

$167.106 \mathrm{E}$

70.852

$33 \mathrm{~N} 4.6$

$5 G$
$43 \quad 4$.

33

104

104
10

$33 N 4.4$

$10 \mathrm{G}$

$33 \mathrm{~N} 4.2$

$5 \mathrm{G}$

$10 \mathrm{G} \quad 4.2$

$10 \mathrm{G}$

$10 \mathrm{G}$

$10 \mathrm{G}$

$33 \mathrm{~N} 4.6$

$198 * 4.3$

$42 \quad 4.7$

$33 \times 4.4$

0

$10 \mathrm{G}$

$33 \mathrm{~N} 4.0$

$60 \mathrm{G}$

$33 \mathrm{~N} 3.9$

$243 * 4.1$

$33 \mathrm{~N}$

$33 \mathrm{~N}$

$10 \mathrm{G}$

$33 \times 4.5$

$10 \mathrm{G} 4.0$

10904.7

142 *

$33 \times 4.2$

33 N 4.1

$5 \mathrm{G}$

$33 \mathrm{~N} 4.7$

$10 \mathrm{G}$

0.7
$104.27 \mathrm{E}$

$33 \mathrm{~N} 4.6$

$33 \times 4.7$

$33 \times 4.94 .6 \quad 0.6$
59 CENTRAL EAST PACIFIC RISE. W 5.8 (BRV).

Centroid, yoment Tensor (BRV) : Centrold origin time

$22: 19: 04.0$; Lat $3.86 \mathrm{~s}$; Lon 104.25 w; Dep $15.0 \mathrm{~F} 1 \mathrm{x}$; Half duration $2.2 \mathrm{sec}$; Principal axes (scale 10**17 $\mathrm{Nm}$ ): (T)

Val $=5.80, \mathrm{Plg}=0, \mathrm{~A} 2 \mathrm{~m}=136$; (N) Val=-0.63, Plg=90, $\mathrm{Azm}=180$; (P) Val=-5.17, Plg=0, Azm=46; Best double couple:

No-5.5*10*17 Nm; NP1: Strike-181, Dip-90, Slip--180; NP2: Strike-271, Dip-90, Slip=0.

Scalar Moment (PPT): $10-1.0 * 10 * * 18 \mathrm{Nm}$.

KERMADEC ISLANDS, NEW ZEALAND

NEAR COAST OF CENTRAL CHILE

5 PEILIPPINE ISLANDS REGION

0.717 NORTHERN ITALY. ML 2.6 (GEN), 2.2 (LDG).

21 NORTEISRN ALASKA. CAEIC>. MT 3.3 (AEIC) 3.3 (PMR).

93 GERMANY. YI 4.2 (VIE), 4.1 (MOX), 4.1 (STR)

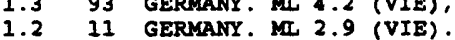

$\begin{array}{lll}1.2 & 11 & \text { GERMANY. NU } 2.9 \text { (VIE). } \\ 1.2 & 36 & \text { NORTHERN SOMATERA, INDONESI }\end{array}$

0.514 NORTHERN ITALY. MC 2.2 (GEN), 1.9 (LDG).

.55 SALTA PROVINCE, ARGENTINA

NORTHERN IRAN. Felt at Borujen.

9 MONA PASSAGE. MD 3.3 (MPR).

36 SAYAR, PHILIPPINE ISLANDS

1.112 SOUTHBRN XINJIANG, CHINA

1.068 KERMADEC ISLANDS, NEW ZEALAND. 5.2 (HRV). Felt (II) on Raoul.

Centroid, yoment Tensor (BRV): Centroid origin time

08:51:18.5; Lat $30.56 \mathrm{~s}$; Lon 177.87 w; Dep 61.9 ; Half -

duration $1.0 \mathrm{sec}$; Principal axes (scale 10**16 $\mathrm{mm}$ ): (T)

Val-6.76, $\mathrm{Plg}=71, \mathrm{Azm}=301$; (N) Val=-1.11, $\mathrm{Plg}=4, \mathrm{Azm}=201$;

(P) Val $=-5.65$, Plg=19, Azm-110; Best double couple:

No-6.2*10**16 Nm; NP1: Strike-194, Dip=27, S1ip=82; NP2: Strike-23, Dip $=64$, Slip -94

TIMOR SEA

103 KENAI PENINSULA, ALASRA. KAEIC>. ML 3.5 (AEIC).

1.034 NORTH ATLANTIC OCEAN. mbLg 3.5 (MDD).

1.324 PACIFIC-ANTARCTIC RIDGE

$1.5 \quad 11$ SOUTHERN XINJIANG, CHINA

5 NORTHERN ITALY. ML 1.8 (GEN).

$\begin{array}{rrr}0.2 & 5 & \text { NORTHERN ITALY } \\ 1.2 & 64 & \text { TONGA ISLANDS }\end{array}$

1.036 VENEZUEI.A

$0.7 \quad 10$ PACIFIC-ANTARCTIC RIDGE

1.567 PYRENEES. ML 3.9 (IDG), 3.7 (STR). mbLg 3.3 (MDD). Felt (III) in the epicentral area.

0.835 SOUTH SANDWICE ISLANDS REGION

1.57 REPUBLIC OF SOUTH AFRICA

0.830 NEAR EAST COAST OF HONSHO, JAPAN

12 NEAR COAST OF NORTHERN CHILE

32 ALASKA PENINSULA. SAEIC>.

0.312 NORTEERN ITALY. NL 2.1 (GEN), 1.8 (LDG).

0.96 POLAND. MG 2.4 (WAR).

1.219 MAAMAR

0.611 FRANCE. MU 3.5 (LDG)

1.317 NEAR $S$ COAST OF NEW GUINEA, PHG

0.810 CAILE-ARGENTINA BORDER REGION. MD 3.1 (SAN).

1.810 SOUTEERN MID-ATLANTIC RIDGE

0.913 PYRENEES. ML 2.5 (IDG).

0.59 STRAIT OF GIBRALTAR, mbLg 2.8 (MDD).

1.364 VANUATU ISLANDS

1.351 SWITZERLAND. YL 3.7 (VIE), 3.4 (IDG), 3.3 (GRF):

816 NORTEERN YOLUCCA SEA

1.029 JUJUY PROVINCE, ARGENTINA

NORTEERN ALASKA. CAEIC>. MI 4.6 (AEIC), 4.4 (PMR).

BAYKAL REGION, ROSSIA

50 SOUTHERN AIASKA. CAEIC>. ML 2.9 (AEIC), 3.1 (PMR).

0.717 PYRENEES. ML 2.5 (LDG).

0.822 ANDREANOF ISLANDS, ALEUTIAN IS.

0.311 NEAR COAST OF CENTRAL CBILE. MD 4.0 (SAN).

1.113 SUNDA STRAIT

1.114 HINDD KOSH REGION, AFGHANISTAN

0.777 SOUTH OF HONSHO, JAPAN

0.810 LOYALTY ISLANDS REGION

1.07 PYRENEES. ML 2.2 (IDG)

1.145 ANDREANOF ISLANDS, ALEUTIAN IS. YL 4.4 (PMR).

1.035 TURXEY

1.558 SANTA CRUZ ISLANDS

0.812 VOLCANO ISLANDS REGION

1.012 NEAR COAST OF NORTHERN CHILE

1.09 SOLOMON ISLANDS

0.49 CHILE-ARGENTINA BORDER REGION. MD 2.6 (SAN).

29 ANDREANOF ISLANDS, ALEUTIAN IS.

28 CENTRAI CALIFORNIA. <PAS-P>. MD 2.7 (PAS). ML 2.9 (GS).

12 PYRENEES. KCL 2.5 (LDG), 2.5 (STR).

93 ANDREANOF ISLANDS, ALEUTIAN IS. MW 5.1 (ERV). ML 4.6 (PMR). Felt on Adak.

Centroid, Moment Tensor (HRV): Centrold origin time 09:07:53.2; Lat 51.45 N; Lon 176.64 พ; Dep 52.8; Balfduration $1.0 \mathrm{sec}$; Principal axes (scale 10**16 $\mathrm{Nm}$ ): (T) $\mathrm{Val}=5.23, \mathrm{Plg}=64, \mathrm{Azm}=326$; (N) $\mathrm{Val}=0.95, \mathrm{Plg}=2, \mathrm{Azm}=60$; (P) val--6.19, Plg=26, Azm=151; Best double couple: Mo-5.7*10**16 Nm; NP1: Strike=245, Dip-19, Slip=96; NP2: strike-59, Dip=71, Slip=88. 


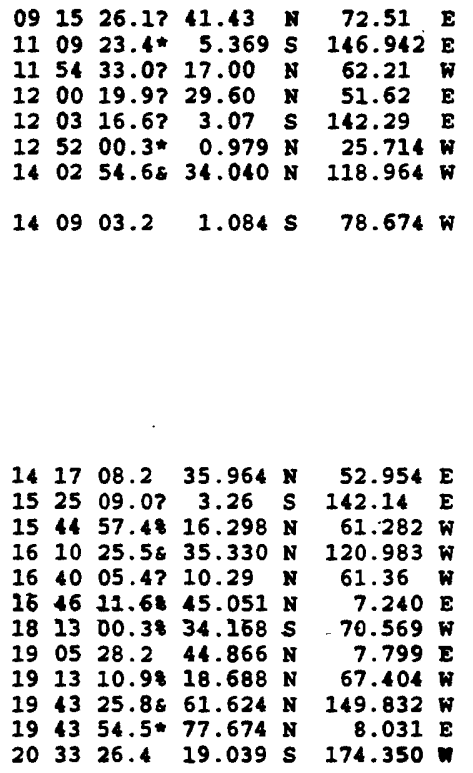

$33 \mathrm{~N}$ 182 . $10 \mathrm{G}$ $33 \mathrm{~N}$ $33 \mathrm{~N} \quad 4.1$ $10 \mathrm{G} \quad 4.7$ 15

51

$\begin{array}{rr}0.3 & 6 \\ 0.8 & 14 \\ 0.1 & 4 \\ 1.4 & 6 \\ 1.4 & 6 \\ 0.8 & 22 \\ & 30\end{array}$

6 KTREYZSTAN

14 EASTERN NIW GUINEA REG., P.N.G.

4 IEEWARD ISLANDS. MD 2.8 (TRN).

SOUTHERN IRAN

6 NEAR N COAST OF NEW GUINEA, PNG.

22 CENTRAL MID-ATLANTIC RIDGE

30 SOUTHERN CALIFORNIA. <PAS-P>. ID 3.1 (PAS). Felt at simi Valley.

5.14 .20 .8158 ECUADOR. WW 5.5 (HRV). Two people injured and several houses destroyed in the Ambato area. Also felt at Latacunga and Pelileo.

Centroid, Moment Tensor (HRV): Centroid origin time 14:09:03.7; Lat $1.12 \mathrm{~s}$; Lon $77.99 \mathrm{~W}$; Dep 15.0 Bdy; Halfduration $1.0 \mathrm{sec}$; Principal axes (scale 10 t\$17 $\mathrm{Nm}$ ): (T) Val-1.82, Plg=61, Azm=151; (N) Val=0.02, $\mathrm{Plg}=29, \mathrm{Azm}=322$; (P) Val=-1.84, Plg-4, Azm-54; Best double couple:

Mo-1.8*10*17 kn; NP1: Strike-172, Dip=48, Slip-130; NP2: Strike=300, Dip=55, Slip-54.

$33 \mathrm{~N} 4.4$

$33 \times 3.7$

$33 \mathrm{~N}$

4

$40 \mathrm{G}$

$10 \mathrm{G}$

$100 \mathrm{G}$

30

37

$10 \mathrm{G}$

$61 \mathrm{D} 5.0$
$49 * 2.9$
0.6

1.2

0.8

0.5
0.4

0.2

0.8
0.2

1.

1.1
$120.04 \mathrm{E}$

70.436

$132.183 \mathrm{z}$ $27.838 \mathrm{w}$

$7.741 \mathrm{E}$ $7.280 \mathrm{E}$

127.78 E

$174.457 \mathrm{v}$ $174.355 \mathrm{~V}$ $66.891 \mathrm{H}$ $124.511 \mathrm{z}$ 70.401 v 70.35 \% 70.38 v $93.583 \mathrm{z}$ 87.736 E 70.54 N
$10 \mathrm{G} \quad 4.3$

$100 \mathrm{G}$

$33 \mathrm{~N} 4.5$

$110 \mathrm{G}$

$193 ? 4.4$

$10 \mathrm{G}$

$10 \mathrm{G}$

$128 ? 4.4$

$53 \mathrm{D} \quad 4.9$

$54 \mathrm{D} 4.8$

23 .

$33 \times 3.4$

$5 \mathrm{G}$

$5 \mathrm{G}$

$\begin{array}{rrrr}5 & G & & \\ 10 & G & 5.2 & \end{array}$
18

18 NORTHERN IRAN

COAST OF NEN GOINEA, PNG.

64 CENTRAL CALIFORNIA. <PAS-P>. YD 2.9 (PAS).

TRINIDAD. ID 2.5 (TRN).

5 NORTHERN ITALY. YO 1.9 (GBN)

9 CHILE-ARGENTINA BORDER REGION. ND 2.1 (SAN).

19 NORTHERN ITALY

9 HONA PASSAGE. MD 3.5 (MPR).

9 SOUTHERN AIASKA. <AEIC>. ML 2.4 (AEIC).

9 SVALBARD REGION

77 TONGA ISLANDS. 5.2 (BRV)

Centroid, Moment Tensor (BRV): Centroid origin time

$20: 33: 28.5$; Lat $19.19 \mathrm{~s}$; Lon $173.65 \mathrm{w}$; Dep 62.0; Half-

duration 1.0 sec: Principal axes (scale 10*16 $\mathrm{Nm}$ ): (T)

Val=7.65, $\mathrm{Plg}=61, \mathrm{Azm}=16$; (N) Val=-0.81, $\mathrm{P} 1 \mathrm{~g}=22, \mathrm{Azm}=151$; (P) Val--6.84, Plg-19, Azm-249; Best double couple: Mo-7.2*10*16 Nm; NPI: Strike-10, Dip-33, S1ip-133; NP2: Strike-141, Dip-67, Slip=66.

0.64 IOZOH PHITIPPINE ISTANDS

0.410 CHILE-ARGENTINA BORDER REGION. MD 2.7 (SAN).

1.2 14 XIZANG

0.17 CHILE-ARGENTINA BORDER REGION

1.0

1.0

1.1
0.7

0.7

0.1

0.7

0.7

0.2

1.4

0.5

7 SOUTHEAST OF SHIROKO, JAPAN

18 SOUTH SANDWICH ISIANDS REGION

SVALBARD REGION

7 NORTHERN ITALY. YCL I.8 (LDG).

13 BANDA SEA

77 TONGA ISLANDS

54 TONGA ISLANDS

8 PUERTO RICO REGION. YD 2.8 (MPR).

6 TIMOR REGION, INDONESIA

10 CAILE-ARGENTINA BORDIR REGION. MD 3.5 (SAN).

8 CAILE-ARGENTINA BORDER REGION

8 CHILE-ARGENTINA BORDER REGION

99 SOUTH INDIAN OCEAN. NW 5.4 (BRV).

Centroid, Moment Tensor (HRV): Centroid origin time

$05: 48: 45.6$; Lat $7.76 \mathrm{~s}$; Lon $93.72 \mathrm{E}$; Dep $15.0 \mathrm{Fix}$; Halfduration $1.2 \mathrm{sec}$; Principal axes (scale 10*17 $\mathrm{Nm}$ ): (T)

Val=1.06, Plg=0, Azm-224; (N) Val-0.32, Plg=90, Azm=180; (P) Val=-1.38, PIg=0, Azm-134; Best double couple:

Mo-1.2*10*17 $\mathrm{km}$; NP1: Strike-269, Dip=90, Slip--180; NP2: Strike-359, Dipm-90, slip=0.

CENTRAL PERD

$\begin{array}{llllll}26 & 06 & 52.57 & 13.97 \text { S } 74.43 \text { \% } 100\end{array}$

06 40 05.1 * $3.458 \mathrm{~S} \quad 145.403 \mathrm{E} \quad 33 \mathrm{~N} \quad 4.7$

$071119.0 * 43.997 \mathrm{~N} 128.058 \mathrm{~W}$ 10 G 4.5

$07 \quad 1948.5834 .306$

$074659.1 * 36.068$

$080028.1666 .178 \mathrm{~N}$

$\begin{array}{llllll}08 & 37 & 44.07 & 37.15 & N\end{array}$

085032.6 * $2.432 \mathrm{~N}$

$\begin{array}{llll}09 & 36 & 03.5 & 36.275\end{array}$

$1008 \quad 50.3 * 1.661$

$\begin{array}{lllcl}11 & 58 & 55.4 ? & 4.09 & 5 \\ 12 & 00 & 12.8 * 45.697\end{array}$

70.635 พ 100

$27.255 \mathrm{E} \quad 33 \mathrm{~N} 4.0$

149.978

$141.64 \mathrm{E}$

$96.204 \mathrm{E}$

$27.314 \mathrm{E}$

$127.320 \mathrm{E} \quad 126 ? 4.7$

133.72 E $33 \mathrm{~N} \quad 3.7$

$10 \mathrm{G}$

$1303 \quad 45.7 * 42.416 \mathrm{~N}$

$\begin{array}{llllll}13 & 16 & 04.7 ? & 32.28 & 5\end{array}$

$\begin{array}{lllll}13 & 17 & 17.27 & 32.31 \mathrm{~s}\end{array}$

$\begin{array}{lllll}13 & 51 & 57.38 & 33.929 \mathrm{~s}\end{array}$

I4 $09 \quad 40.9 ? \quad 39.50 \mathrm{~s}$

$142306.5 * 3.215$ s

$\begin{array}{llll}15 & 19 & 20.5 ? & 35.20 \mathrm{~S}\end{array}$

$\begin{array}{lllll}15 & 40 & 36.9 & 38.934 & \mathrm{~N} \\ 16 & 52 & 17.5 & 51.427\end{array}$

$\begin{array}{llll}16 & 52 & 17.5 & 51.427\end{array}$

$\begin{array}{llll}17 & 29 & 10.78 & 36.898 \\ 19 & 39 & 49.8 \text { ? } & 9.70\end{array}$

$\begin{array}{lllll}19 & 39 & 49.8 \text { ? } & 9.70 & S \\ 19 & 53 & 29.2 & 9.874 & S\end{array}$

$210527.5 * 0.981 \mathrm{~s}$

$001650.1 * 40.041 \mathrm{~N}$

$0021 \quad 43.3 ? \quad 13.24 \mathrm{~N}$

$003023.9 * 52.717$

$003244.6860 .565 \mathrm{~N}$

$15.908 \mathrm{E} \quad 10 \mathrm{G}$

$\begin{array}{ll}10 & G \\ 10 & G\end{array}$

71.72 พ $10 \mathrm{G}$

$71.203 \mathrm{~N} \quad \mathrm{G}$

46.28 E $10 \mathrm{G} \quad 4.4$

103.529 E $247 ? 4.2$

71.17 พ $110 \mathrm{G}$

$140.670 \mathrm{E} \quad 10 \mathrm{G} \quad 3.6$

176.887 พ $33 \mathrm{~N} 4.5$

$2.989 \% 10 \mathrm{G}$

124.13 E 57 ? 3.8

$74.743 \mathrm{~W} 24 \mathrm{D} 5.0$

$136.696 \mathrm{E} 33 \mathrm{~N} 4.1$

$43.261 \mathrm{E} 33 \mathrm{~N} 4.1$

89.45 N $33 \times 4.6$

$32.295 \mathrm{~W} \quad 10 \mathrm{G} 4.3$

151.848 W

75

$72 \star 5.0$
1.0

1.1

1.1

0.2

1.3

0.4

1.3

0.9

0.9

1.3
0.9

0.6

0.6

0.8

1. 1

1.1
0.8
0.2

1.1

I. 3

0.9

0.5

0.7204

0.913

1.3 15

1.524

$0.8 \quad 21$

1.3
NEAR N COAST OF NEW GUINEA, PNG.

CEILE-ARGENTINA BORDER REGION. MD 3.2 (SAN).

DODECANESE ISLANDS

NORTHERN ALASKA. CAEIC>. ML 2.6 (AEIC).

NEAR EAST COAST OF HONSBU, JAPAN

NORTHERN SUMATERA, INDONESIA

DODECANESE ISLANDS

HALMAHERA, INDONESIA

IRIAN JAYA REGION, INDONESIA

NORTAWESTERN BALKAN REGION. YC 2.8 (VIE). Felt (IV) at NOvO

6 ADRIATIC SEA

11 NEAR COAST OE CENTRAL CHILE

NEAR COAST OE CENTRAL CHILE

26 SOUTHERN SUMATERA, INDONESIA

10 CENTRAL CHILE. YD 3.3 (SAN).

20 EASTERN HONSHU, JAPAN

7 ANDREANOF ISLANDS, ALEUTIAN IS. MI 4.4 (PMR).

STRAIT OF GIBRALTAR. MbLg 2.7 (MDD).

TIMOR REGION, INDONESIA

CENTRAL PERD

IRIAN JAYA REGION, INDONESIA

EI SALVADOR

KENAI PENINSULA, ALASKA. <AEIC>.

SOUTHERN MOLUCCA SEA. MW 5.2 (HRV).

Centroid, Moment Tensor (BRV): Centroid origin time
OFF COAST OF OREGON Mesto, Slovenia.

NEAR COAST OF CENTRAL CHILE

SOUTHWEST INDIAN RIDGE

GEORGIA-ARYENIA-TURKEY BORD REG.

NORTH ATIANTIC OCEAN 
$\begin{array}{lllllll}27 & 01 & 15 & 23.8 & 36.904 & \mathrm{~N}\end{array}$

$27014410.5 * 42.822 \mathrm{~N}$ $\begin{array}{llllll}27 & 01 & 33 & 03.7 & 65.204 & N\end{array}$

$27 \quad 01 \quad 4146.2 \quad 7.292 \mathrm{~s}$
$1.565 \mathrm{~W}$ $165.444 \mathrm{H}$

$143.799 \mathrm{E} 113 ? 4.6$ $91.281 \mathrm{~W}$ 107.075
$10 \mathrm{G}$ 5.05 .41 .1

$\begin{array}{lllllclrll}03 & 01 & 52.77 & 32.39 & \mathrm{~S} & 71.93 & \mathrm{~W} & 10 & \mathrm{G} & \\ 03 & 12 & 14.9 ? & 12.28 & \mathrm{~N} & 91.47 & \mathrm{~W} & 33 & \mathrm{~N} & 3.9 \\ 03 & 13 & 59.8 * & 51.257 & \mathrm{~N} & 176.618 & \mathrm{~W} & 33 & \mathrm{~N} & 4.2 \\ 03 & 56 & 20.58 & 46.615 & \mathrm{~N} & 0.484 & \mathrm{E} & \mathrm{S} & \mathrm{G} & \\ 04 & 31 & 07.47 & 24.00 & \mathrm{~S} & 179.75 & \mathrm{E} & 635 & \text { ? } & 4.4 \\ 04 & 42 & 47.8 ? & 37.11 & \mathrm{~N} & 3.60 & \mathrm{~W} & 10 & \mathrm{G} & \\ 05 & 42 & 35.98 & 33.885 & \mathrm{~S} & 71.469 & \mathrm{~W} & 50 & \mathrm{G} & \\ 06 & 19 & 31.7 & 5.779 & \mathrm{~S} & 133.720 & \mathrm{E} & 33 & \mathrm{~N} & \mathbf{5 . 1} \\ 06 & 24 & 07.9 & 22.570 & \mathrm{~S} & 179.792 & \mathrm{~W} & 575 & \mathrm{D} & \mathbf{5 . 6}\end{array}$

01:06:04.8; Lat 0.49 S; Ion $124.36 \mathrm{E}$; Dep 74.0; Halfduration $1.0 \mathrm{sec}$; principal axes (scale 10*\#16 $\mathrm{km}$ ): (T) Val-6.77, Plg=43, Azm=6; (N) Val=-1.20, Plg=47, Azm=184; (P) Val=-5.58, Plg-1, Azm-275; Best double couple: Mo-6.2*10*16 Nm; NP1: Strike-42, Dip-60, slip-147; NP2: Strike-150, Dip-62, Slip-34. $\begin{array}{rlllllllllllll}27 & 06 & 34 & 42.1 & 22.435 & \mathrm{~S} & 179.746 & \mathrm{~W} & 581 & 5.0 & & 0.9 & 155 \\ 27 & 07 & 53 & 38.7 & 36.388 & \mathrm{~N} & 139.069 & \mathrm{E} & 33 & \mathrm{~N} & & & 1.0 & 5 \\ 27 & 09 & 18 & 55.0 ? & 34.86 & \mathrm{~S} & 71.04 & \mathrm{~W} & 100 & \mathrm{G} & & & 0.1 & 9 \\ 27 & 09 & 22 & 38.8 & 3.810 & \mathrm{~N} & 126.537 & \mathrm{E} & 82 & * & 4.5 & & 0.9 & 29 \\ 27 & 10 & 50 & 49.4 & 36.883 & \mathrm{~S} & 78.486 & \mathrm{E} & 10 & \mathrm{G} & 5.3 & 5.8 & 1.2 & 70\end{array}$
0.6

0.9

0.9

0.9
14 TESTERN MEDITERRANEAN SEA mbIg 3.1 (MDD).

30 NORTHERN ALASRA. ML 4.4 (PMR).

42 JAWA, INDONESIA

14 HOKRAIDO, JAPAN REGION

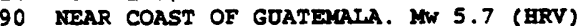

Cedtroid, Moment Tensor (HRV): Centroid origin time

$02: 12: 42.9$; Lat $13.19 \mathrm{~N}$; Lon $92.04 \mathrm{w}$; Dep 15.0 Bdy; Balfduration $1.6 \mathrm{sec}$; Principal axes (scale 10*17 $\mathrm{Nm}$ ): (T)

val-4.04, Plg-65, Azm-11; (N) Val-0.12, Plg-3, Azm-107; (P)

Val--4.16, Plg-25, Arm-199; Best double couple:

Mo-4.1*10*17 Nm; NP1: Strike-295, Dip-21, Slip-98; NP2: Strike-106, Dip-70, Slip-87.

0.310 NEAR COAST OF CENTRAL CHILE. MD 4.2 (SAN)

1.410 OFF COAST OF CBNTRAL AMIERICA

0.96 ANDREANOF ISLANDS, ALEUTIAN IS.

1.177 FRANCE. ML 2.3 (IDG).

0.418 SOUTH OF FIJI ISIANDS

0.54 SPAIN. mbIg 2.6 (KDD)

0.3

1.2

0.9

TEAR COAST OF CENTRAL CHILE. ND 3.2 (SAM).

ARO ISLANDS REGION, INDONESIA

SOUTH OF FIJI ISLANDS. WW 5.9 (GS), 5.9 (HRV). Me 5.3 (GS). mb 5.5 (BRK).

Broadband Source Parameters (GS): NP1: Strike-0, Dip-35, Slip-180; NP2: Strike-270, Dip-90, Slip--55; Radiated energy $2.3 * 10 * 12 \mathrm{Nm}$.

Moment Tensor (GS): Dep 578; Principal axes (scale 10**17 $\mathrm{Nm}$ ): (T) Val-7.45, Plg-29, Azm-339; (N) Val-0.03, Plg-16, Azm-78; (P) Val--7.47, Plg-56, Azm-193; Best double couple: Mo-7.5*10*17 Nm; MP1: Strike-30, Dip-21, Slip--140; NP2: Strike-262, Dip-76, Silip- -73 .

Centroid, Homent Tensor (HRV): Centroid origin time 06:24:15.3; Lat $22.54 \mathrm{~S}$; Lon $179.61 \mathrm{~W}$; Dep 597.5: Halfduration $2.3 \mathrm{sec}$; Principal axes (scale 10*18 $\mathrm{mm}$ ): (T) Val-1.03, Plg=26, Azm-334; (N) Val=-0.17, Plg=18, Azm=74; (P) Val--0.86, Plgm57, Azm-194; Best double couple: Mo-9.4*10*17 Nm; NP1: Strike-28, Dip-25, S1lp--138; NP2: Strike-259, Dip-74, slip--71.

SOUTH OF FIJI ISLANDS

BASTERN HONSHO, JAPAN

NEAR COAST OF CENTRAL CHILE

TALAOD ISLANDS, INDONESIA

MID-INDIAN RIDGE. WW 6.0 (GS), 5.9 (BRV)

Homent Tensor (GS): Dep 24; Principal axes (scale 10*\#18 $\mathrm{Nm}$ ): (T) Val-0.88, Plg-0, Azm-273; (N) Val-0.17, Plg-79, Azm-182; (P) Val--1.05, Plg-11, Azm-4; Best double couple: Mo-9.6*10*17 N NP1: Strike-48, Dip-82, Slip--7; NP2: Strike-139, Dip-83, Slip--172.

Centroid, Moment Tensor (HRV): Centroid origin time 10:50:55.3; Lat $36.81 \mathrm{~s}$; Lon $78.47 \mathrm{E}$; Dep 15.0 Fix; Halfduration $2.3 \mathrm{sec}$; Principal axes (scale 10*17 $\mathrm{Nm}$ ): (T) Val $=7.77, P 1 g=0, A z m-271$; (N) Val $=0.62, P 1 g=88, A z m-164$ (P) val--8.39, Plg-2, Azm-1; Best double couple:

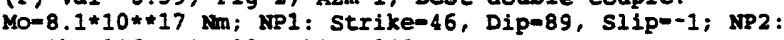
Strike-136, Dip-89, Slip--179.

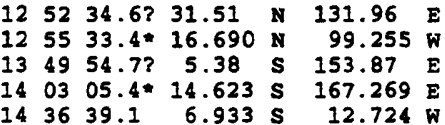

$\begin{array}{lllllcl}12 & 52 & 34.6 ? & 31.51 & \mathrm{~N} & 131.96 & \mathrm{E} \\ 12 & 55 & 33.4 * & 16.690 & \mathrm{~N} & 99.255 & \mathrm{~W} \\ 13 & 49 & 54.7 ? & 5.38 & \mathrm{~S} & 153.87 & \mathrm{E} \\ 14 & 03 & 05.4 * & 14.623 & \mathrm{~S} & 167.269 & \mathrm{E} \\ 14 & 36 & 39.1 & 6.933 & \mathrm{~S} & 12.724 & \mathrm{~W}\end{array}$

\section{$33 \mathrm{~N}$}

$33 \times 4.4$

33 N 4.5

$134 ? 4.9$

10 \& $5.25 .11 .0 \quad 34$

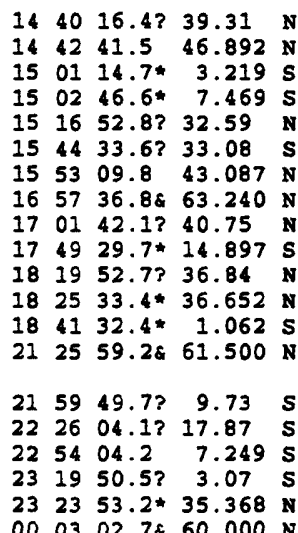

32.16 $12.900 \mathrm{E}$ $78.395 \mathrm{~W}$ $127.953 \mathrm{E}$ 133.40 72.04 $0.585 \mathrm{~W}$ $151.160 \mathrm{~W}$ $35.21 \mathrm{E}$ $166.912 \mathrm{E}$ 2.90 औ $139.522 \mathrm{E}$ $24.436 \mathrm{~W}$ $146.380 \mathrm{~W}$

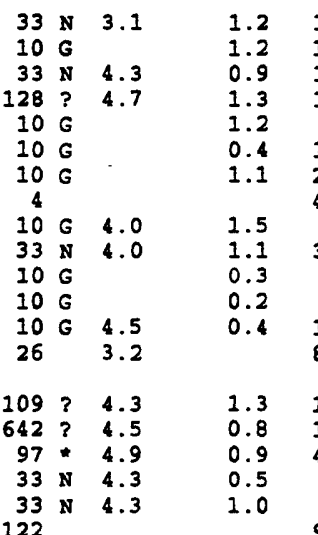

KYUSHO, JAPAN

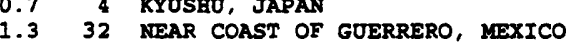

1.410 NEW IRELAND REGION, P.N.G

.0 34 VANDATU ISLANDS

ASCENSION ISLAND REGION. MW 5.6 (HRV)

Centroid, Moment Tensor (HRV): Centroid origin time $14: 36: 44.8$; Lat $6.94 \mathrm{~s}$; Lon $12.94 \mathrm{~W}$; Dep $15.0 \mathrm{Fix}$; Balfduration $1.5 \mathrm{sec}$; Principal axes (scale 10**17 $\mathrm{Nm}$ ): (T) Val-2.57, Plg=9, Azm=301; (N) Val-0.12, Plg-78, Azm=165 (P) Val--2.69, Plg-8, Azm-33; Best double couple: Mo-2.6*10*17 $\mathrm{Nm} ; \mathrm{NP1}$ : Strike-77, Dip=78, slip-0; NP2: Strike-347, Dip-90, Slip-168.

\section{TORKEY}

NORTHERN ITALY. III 3.7 (GRF).

PERO-ECUADOR BORDER REGION

BANDA SEA

SHIKOKU, JAPAN

OFF COAST OF CENTRAL CHILE. MD 3.6 (SAN)

PYRENEES. ML 3.1 (LDG), 2.8 (STR). mbLg 3.1 (MDD).

CENTRAL ALASKA. 〈AEIC>. ML 2.6 (PYR).

TURREY. Felt in the Amasya-Corum area.

VANUATU ISLANDS

STRAIT OF GIBRALTAR. mbLg 2.5 (MDD).

EASTERN HONSHO, JAPAN

CENTRAL MID-ATLANTIC RIDGE

SOUTHERN ALASKA. <AEIC>. MIL 3.7 (AEIC), 3.7 (PIR). Felt (III) at valdez.

CENTRAL PERO

FIJI ISLANDS REGION

BANDA SEA

IRIAN JAYA, INDONESIA

NEAR EAST COAST OF HONSHU, JAPAN

SOUTHERN ALASKA. 〈AEIC>. 
010613.4752 .29 \& 176.61 พ $011256.7862 .910 \mathrm{~N} 149.380 \mathrm{~W}$ $014742.6 * 77.235 \mathrm{~N} 14.855 \mathrm{E}$ $015634.0 \quad 77.806 N \quad 7.751 \mathrm{E}$ 015739.0 ? 1.70 s 136.21 $032136.5 * 7.872 \mathrm{~S} \quad 122.799 \mathrm{~B}$ $\begin{array}{llllll}03 & 39 & 17.3 & 3.627 & \mathrm{~s} & 138.921 \mathrm{E}\end{array}$ $035705.2 * 21.094 \mathrm{~N} 146.322 \mathrm{E}$ $043903.4 * 21.747 \mathrm{~N} 98.954 \mathrm{E}$ $050055.4 * 13.929 \mathrm{~N} 119.236 \mathrm{E}$ $050339.2 * 51.298 \mathrm{~N} 176.682 \mathrm{~W}$ $055408.9 ? \quad 4.20$ S $147.70 \quad E$ $061006.0 ? \quad 34.61$ s 71.07 $063049.6 \quad 0.950 N \quad 28.095$ พ

$\begin{array}{rrrr}100 \mathrm{G} & 4.2 & 0.8 \\ 87 & & 3.6 & \\ 10 \mathrm{G} & & \\ 10 \mathrm{G} & 4.5 & 0.9 \\ 33 \mathrm{~N} & 4.6 & 0.9 \\ 197 \mathrm{~T} & 4.8 & 0.8 \\ 33 \mathrm{~N} & 4.8 & 0.8 \\ 33 \mathrm{~N} & & 1.1 \\ 33 \mathrm{~N} & & 1.0 \\ 33 \mathrm{~N} & 4.6 & 0.7 \\ 33 \mathrm{~N} & 4.0 & 1.1 \\ 100 \mathrm{G} & 4.7 & 0.9 \\ 70 \mathrm{G} & & 1.4 \\ 10 \mathrm{G} & 5.14 .7 & 0.3 \\ & & & 0.6\end{array}$

$4.306 \mathrm{~W} 10 \mathrm{G}$

3.76 W $10 \mathrm{G}$

$3.579 \mathrm{~W} 10 \mathrm{G}$ $126.947 \mathrm{E}$

$70.001 \mathrm{~W}$

$177.76 \mathrm{~W}$

$45.04 \mathrm{H}$ $121.477 \mathrm{E}$

$4.975 \mathrm{~W}$

$21.419 \mathrm{E}$

$71.419 \mathrm{E}$

$71.247 \mathrm{E}$

71.136 N

$7.76 \quad E$

$4.638 \mathrm{~W}$

151.692

$151.692 \mathrm{~W}$
$15.681 \mathrm{E}$

82.514 พ

$\begin{array}{llll}16 & 0145.2 & 10.644 \mathrm{~S} & 161.345 \mathrm{E}\end{array}$

$5 \mathrm{G}$

$521 ? 4.0$

$10 \mathrm{G}$

$48 * 4.9 \quad 1.0$

$5 \mathrm{G}$

$33 \mathrm{~N}$

4.5

02 ?

$33 \mathrm{~N}$

$10 \mathrm{G}$

$10 \mathrm{G}$

$\begin{array}{llllllll}10 \mathrm{G} & & & 1.2 & 20 \\ 33 & \mathrm{~N} & 4.4 & 4.2 & 1.0 & 29\end{array}$

$\begin{array}{lllllll}33 & 0 & 5.6 & 5.3 & 1.1 & 135\end{array}$

PAGE 24

136 CEATRAL ALASKA. <AEIC>.

6 SVALBARD REGION

20 SVALBARD REGION

7 IRIAN JAYA REGION, INDONESIA

24 FLORES SEA

27 IRIAN JAYA, INDONESIA

11 MARIANA ISLANDS REGION

9 MYAMMAR

12 PHILIPPINE ISLANDS REGION

8 ANDREANOF ISLANDS, ALEUTIAN IS.

12 BISMARCK SEA

10 NEAR COAST OF CENTRAL CHILE. MD 3.0 (SAN) .

92 CENTRAL MID-ATLANTIC RIDGE. NW 5.3 (BRV)

Centroid, Moment Tensor (HRV): Centroid origin time $06: 30: 55.8$; Lat $1.08 \mathrm{~N}$; Lon $28.18 \mathrm{w}$; Dep $15.0 \mathrm{Fix}$; Halfduration $1.1 \mathrm{sec}$; Principal axes (scale 10*\$17 Nm): (T) Val-1.14, Plg=14, Azm=40; (N) Val=-0.06, Plg=74, Azm=189 (P) Val=-1.08, P1g-8, Azm-308; Best double couple: No-1.1*10*17 $\mathrm{Nm}$; NP1: Strike-83, Dip=75, s1ip=176; NP2: Strike-175, Dip-86, Slip-16.

0.412 SAN JOAN PROVINCE, ARGENTINA. YD 3.8 (SAN).

$\begin{array}{lllllll}28 & 17 & 35 & 40.9 ? & 18.23 \quad \mathrm{~N} & 63.13 & \text { W } 100 \mathrm{G}\end{array}$

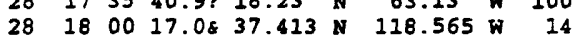

$28185123.5 * 51.211$

$28 \quad 190827.5 * 10.217 \mathrm{~N}$

$28200432.4 * 9.404 \mathrm{~s}$

$210027.0860 .116 \mathrm{~N}$

$\begin{array}{llllll}21 & 28 & 59.8 * & 0.857 & N \\ 21 & 53 & 47.3 ? & 34.44 & s\end{array}$

$8225016.2 * 1.322 \mathrm{~S}$

$\begin{array}{llllll}28 & 23 & 33 & 27.0 & 20.043 \mathrm{~N}\end{array}$

$234939.1 * 42.369 \mathrm{~N}$

$000058.9 * 24.301 \mathrm{~s}$
$15.679 \mathrm{E}$

$112.987 \mathrm{E} \quad 33 \mathrm{~N} 4.2$

$153.800 \mathrm{~W}$

$126.317 \mathrm{E}$

$70.38 \mathrm{~W}$

14.871

$145.626 \mathrm{E}$

178.903
162

$33 N 4.8$

$10 \mathrm{G}$

$10 \mathrm{G} 4.6$

$104 \mathrm{D} \quad 4.5$

$33 \mathrm{~N} 3.9$

$614 ? 4.8$
0.8

1.2

1.2

0.1

1.0

0.9

0.7
1 SPAIN mbIg 3.1 (MDD)

SPAIN

9 CORTHLRA MOLUCCA SEA -

17 FIJI ISLANDS REGION

9 NORTHERN MID-ATLANTIC RIDGE

53 PHILIPPINE ISIANDS REGION

5 SPAIN. mbLg 2.5 (YDD)

68 SOUTHERN GREECE

12 AFGEANISTAN-TAJIRISTAN BORD REG.

10 MIEAR COAST OF CENTRAL CHILE. MD 3.4 (SAN).

GERMANY

5 NORTEERN ITALY. ML 2.3 (LDG).

14 SPAIN. mbLg 3.0 (MDD)

8 KODIAK ISLAND REGION. CAEIC>. ML 2.7 (AEIC).

ADRIATIC SEA. ML 3.3 (VIE).

SOUTE OF PANAMA

SOLONON ISLANDS. NW 5.5 (ERV), 5.3 (GS). Me 5.1 (GS) Broadband Source Parameters (GS): Dep 30; Radiated energy $9.8 * 10 * 11 \mathrm{~km}$

Homent Tensor (GS): Dep 19; Principal axes (scale 10ॠ\#17 $\mathrm{Nm}$ ): (T) Val-1.03, Plg=70, Azm=335; (N) Val=-0.01, Plg=14, Azm=106; (P) Val=-1.02, Plg-15, Azm=200; Best double couple: $10-1.0 * 10 * 17 \mathrm{Nm}$; NP1: Strike-308, Dip-32, Slip-116; NP2: Strike-98, Dip-61, Slip-74.

Centroid, voment Tensor (HRV): Centrold origin time $16: 01: 48.6$; Lat $10.78 \mathrm{~s}$; Lon $161.45 \mathrm{E}$; Dep 35.6 ; Halfduration $1.3 \mathrm{sec}$; Principal axes (scale 10*17 Nm): (T) Val=1.99, Plg=77, Azm=17; (N) Val=0.08, Plg=3, Azm=121; (P) Val=-2.08, Plg=13, Azm-212; Best double couple: No-2.0*10*17 Nm; NP1: Strike-307, Dip-32, slip-96; NP2: Strike-119, Dip=58, Slip-86.

WEST OF MACQUARIE ISLAND. MW 5.9 (HRV)

Centroid, woment Tensor (HRV): Centroid origin time $16: 53: 19.0$; Lat $60.17 \mathrm{~s}$; Lon 149.94 E; Dep 15.0 Fix; Halfduration $2.0 \mathrm{sec}$; Principal axes (scale 10 $\$ 17 \mathrm{Nm}$ ): (T) Val=7.53, Plg=1, $A z m=206$; (N) Val=-0.08, Plg=89, $A z m=26$; (P) Val=-7.45, Plg=0, Azm-116; Best double couple: Mo-7.5*10**17 $\mathrm{km}$; NP1: Strike-251, Dip-89, Slip-179; NP2: strike-341, Dip=89, Slip=1.

COSTA RICA. MW 5.9 (HRV), 5.8 (GS). Me 5.3 (GS). Felt throughout Costa Rica.

Broadband Source Parameters (GS): Dep 20; NPI: Strike-300, Dip=40, Slip=120; NP2: Strike-83, Dip=56, Slip=67; Radiated energy $1.7 * 10 * * 12 \mathrm{Nm}$.

Moment Tensor (GS): Dep 19; Principal axes (scale 10*17 Nm): (T) Val=5.64, Plg=60, $A z m=9$; (N) VaI=-0.15, Plg=17, Azm=131; (P) Val=-5.49, Plg-24, Azm-229; Best double

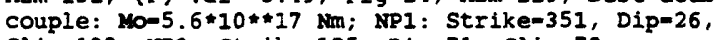
SIip=133; NP2: Strike-125, Dip=71, slip=72.

Centroid, Moment Tensor (HRV): Centroid origin time $17: 16: 22.0$; Lat $9.23 \mathrm{~N}$; Lon $84.22 \mathrm{w}$; Dep 27.9 ; Balfduration $2.3 \mathrm{sec}$; Principal axes (scale 10**17 Nm): (T) Val=7.78, Plg=68, Azm=42; (N) Val-0.65, Plg-6, Azm=296; (P) Val=-8.43, Plg=21, Azm=204; Best double couple:

Mo-8.1*10**17 Nm; NP1: Strike-283, Dip=25, slip-76; NP2: Strike=119, Dip=66, Slip=97.

LEEWARD ISLANDS. YD 3.6 (TRN) CALIFORNIA-NEVADA BORDER REGION. LGM-P>. MD 3.1 (GM). MI 3.1 (GS)

6 POIAND ML 2.5 (MOX).

4 PEILIPPINE ISLANDS REGION

74 SOUTB OF JAWA, INDONESIA
SOUTHERN ALASKA. LAEIC>

25 NORTHERN MOLUCCA SEA

6 CHILE-ARGENTINA BORDER REGION

10 NORTH OF ASCENSION ISLAND

28 MARIANA ISLANDS

9 KYRGYZSTAN

6 SOUTH OF FIJI ISLANDS 
$152.899 \mathrm{~W}$ $103.038 \mathrm{E}$ $126.15 \mathrm{E}$ $66.737 \mathrm{~W}$ 18.857 172.755
94

$33 \times 4.7$

$33 \times 4.0$

$194 * 4.2$

$10 \mathrm{G}$

$33 \mathrm{~N} 5.0$
47 SOUTEERN ALASKA. CAEIC>.

1.116 SOUTEERN SUMATERA, IMDONESIA

0.66 TALAOD ISLANDS, INDONESIA

0.929 SALTA PROVINCE, ARGEKTINA

1.06 POLAND. MG 2.9 (WAR).

1.047 SOUTE ISLAND, NEW ZEALAND. NW 5.2 (HRV). Felt strongly at Banmer Springs.

Centroid, Monent Tensor (HRV): Centroid origin time

$04: 47: 05.1$; Lat $42.41 \mathrm{~s}$; Lon $172.90 \mathrm{E}$; Dep 31.3 ; Half duration 1.1 sec; Principal axes (scale $10 \star 16 \mathrm{sm}$ ): (T) Val-8.62, Plg=3, Azm-186; (N) Val=-0.35, Plg=60, Azm-90; (P) Val=-8.27, Plg-30, Azm-277; Best double couple: No-8, 4*10*16 Nin; NP1: Strike-317, Dip -67, slip--20; NP2 : Strike-55, Dip=72, Slip=-155. $\begin{array}{llllllll}29 & 08 & 04 & 18.2 ? & 36.49 & \mathrm{~N} & 141.15 \quad \mathrm{E}\end{array}$

10 CAILE-ARGENTINA BORDER REGION

39 GREENLAND SEA

17 MINDAKAO, PAILIPPINE ISLANDS

4 NEAR EAST COAST OF HONSEU, JAPAN $\begin{array}{lllllll}29 & 05 & 09 & 05.37 & 33.16 \text { s } & 69.29\end{array}$ $\begin{array}{llllllll}29 & 06 & 22 & 07.5 & 73.307 & \mathrm{~N} & 5.417 \mathrm{E}\end{array}$ $29063310.6 * 9.448$ N $126.598 \mathrm{E}$ $2908 \begin{array}{lllllll}29 & 08.9 & 2.809 & 5 & 137.119 & E\end{array}$

$\begin{array}{rrrrrr}5 & G & & & 0.4 \\ 10 & G & 4.6 & & 1.3 \\ 33 & N & 4.4 & & 0.7 \\ 33 & N & & & 0.4 \\ 33 & N & 5.0 & 5.0 & 1.0\end{array}$

$29083825.1 * 30.089 N$ $29 \quad 085212.6 ? \quad 3.95 \mathrm{~s}$ $29092426.9 \quad 35.131 \mathrm{~N}$ $29101512.6858 .250 \mathrm{~N}$ $291156 \quad 00.8861 .548$ $29 \quad 1246 \quad 41.7 * 13.224 \mathrm{~s}$

$29 \quad 124714.7 * 21.632$ $\begin{array}{lllllllll}29 & 13 & 12 & 42.27 & 2.61 & 5 & 137.17 & E\end{array}$ $29134454.8 \quad 38.773 \times 119.561$.

$29 \quad 14 \quad 10 \quad 10.5 \quad 17.459 \mathrm{~s}$ $29 \quad 151059.2 \quad 2.9895 \quad 126.171$ E $29 \quad 152316.7$ ? 32.83 N $135.07 \mathrm{E}$ $29155001.1640 .462 \mathrm{~N} 124.383 \mathrm{~W}$

$29163605.4859 .998 \times 153.014$ พ 104 $\begin{array}{llllll}29 & 16 & 50 & 20.3 & 30.373 & 5\end{array}$ $29 \quad 172300.6 * 5.457 \mathrm{~s}$ $29175549.9 * 7.1035$ $\begin{array}{lllllll}29 & 18 & 23 & 12.37 & 31.59 & 5\end{array}$ $\begin{array}{llllll}29 & 20 & 22 & 15.9 & 1.086 \mathrm{~N}\end{array}$ $\begin{array}{rr}153.014 \text { พิ } & 104 \\ 69.080 \text { พ } & 60\end{array}$ $129.129 \mathrm{E} 141 ? 4.2$ 69.48 औ $180 \mathrm{G}$ 28.185 $\begin{array}{rlll}147.218 \mathrm{E} & 33 \times & 4.4 \\ 139.5\end{array}$
$33 \mathrm{~N} 4.7$ $33 \mathrm{~N} 4.1$ $69 \mathrm{D} \quad 4.5$ 19 39 67

$261 ? 3.7$ $33 \mathrm{~N} 4.0$

$196 \star 4.7$

$33 \times 4.3$

$34 * 5.2$

24

7
$292202 \quad 34.1865 .570 \times 144.630 \mathrm{~N}$ $292205 \quad 19.4 \quad 51.199 \times 178.121$ ผ $29 \quad 2243 \quad 09.7 * 5.604$ S 145.534 E $29224351.5 * 26.775$ s 70.548 . $29232053.47 \quad 0.61$ S $135.64 \quad \mathrm{E}$ $\begin{array}{llllll}30 & 01 & 12 & 47.8 & 42.648 \times & 105.619 \mathrm{E}\end{array}$ $30 \quad 015944.2 * 17.272 \mathrm{~S} 69.261$ $\begin{array}{llllllll}30 & 02 & 11 & 42.2 & 5.240 \mathrm{~s} & 68.588 \mathrm{E}\end{array}$
11

$33 \times 4.4$

$33 \times 4.1$

$52 *$

$33 \mathrm{~N} 4.2$

$33 \mathrm{~N} 4.5$

173 *

10 G $5.15 .0 \quad 0.9$
$57 * 3.6$ 999 $\begin{array}{rrr}5 & \mathrm{G} & \\ 575 & & 4.6\end{array}$ 575
33
33 $33 \mathrm{~N}$ $\begin{array}{rrr}33 & N & \\ 116 & ? & 4.6\end{array}$ $10 \mathrm{G}$
0.9

0.9

1.3154

0.8

0.8

0.9

0.9

1.2

5

.9

1.1

.0

0.8
58 IRIAN JAYA, INDONESIA. NW 5.2 (ARV).

Centroid, Moment Tensor (HRV): Centroid origin time

$08: 18: 48.2$; Lat $2.97 \mathrm{~S}$; Lon $137.14 \mathrm{E}$; Dep 18.1; Half-

duration $1.0 \mathrm{sec}$; Principal axes (scale 10*16 $\mathrm{mm}$ ): (T)

Val-7.69, Plg-82, Azm=255; (N) Val=-0.74, Plg-8, Azm=83.

(P) Val=-6.95, Plg-1, Azm-353; Best double couple:

Mo $=7.3 * 10 * 16 \mathrm{Nm}$; MP1: Strike-75, Dip-44, slip-78; NP2 :

Strike-271, Dip-47, Slip-101.

14 XIZANG

6 IRIAN JAYA, INDONESIA

53 KODIAK ISLAND REGION. CAEIC>. ML 3.3 (AEIC).

45 SOUTHERN ALASKA. CAEIC. MI 2.5 (AEIC).

12 NDAR COAST OF PERO. Felt (III) at Chincha Alta and San

Vicente de Canete; (II) at Cerro Azul, Pisco and Quilmana.

16 MARIANA ISLANDS REGION

60 CALIFORNIA-NEVADA BORDER REGION. MI 3.3 (GS), 3.3 (BRK). MD 3.3 (GM)

84 TONGA ISLANDS

12 IRIAN JAYA, INDONESIA

45 CERAM SEA

6 SOUTHEAST OF SHIKOKO, JAPAN

24 NEAR COAST OF MORTHERN CALIF. LGM-P>. MD 3.0 (GM). III 3.0 (GS).

65 SOUTHERN ALASKA. 〈AEIC>

37 CHILE-ARGENTIKA BORDER REGION. MD 4.5 (SAN).

19 EASTERN NEW GUIMEA REG., P.N.G.

12 BANDA SEA

10 SAN JOAN PROVINCE, ARGENTINA. ID 3.9 (SAN)

57 CENTRAI MID-ATLANTIC RIDGE. MW 5.1 (BRV).

Centroid, woment Tensor (HRV): Centroid origin time

20:22:21.7; Lat $1.04 \mathrm{~N}$; Lon $28.16 \mathrm{w}$; Dep 15.0 Fix; Halfduration $1.0 \mathrm{sec}$; Principal axes (scale $10 * 16 \mathrm{Nm}$ ): (T) Val-4.95, Plg=0, Azm=216; (N) Val=-0.02, Plg=90, Azm=180; (P) Val=-4.92, Plg=0, Azm-126; Best double couple: Mo-4.9*10*16 kn; NP1: Strike-261, Dip-90, S1ip--180; NP2: Strike-351, Dip=90, Slip-0.

28 NORTEERN ALASKA. CAEIC>. ML 3.9 (AEIC), 4.1 (PMR).

21 ANDREANOF ISLANDS, ALEUTIAN IS.

0.711 EASTERN NDW GUINEA REG., P.N.G.

0.918 NEAR COAST OF NORTHMRN CHITE

18 NGAR COAST OF NORTHARN CHILE

32 MONGOLIA

7 PERO-BOLIVIA BORDER REGION

137 CHAGOS ARCHIPELAGO REGION. NW 5.3 (BRV).

Centroid, koment Tensor (HRV): Centroid origin time $02: 11: 46.3$; Lat $5.40 \mathrm{~S}$; Lon $68.60 \mathrm{E}$; Dep $15.0 \mathrm{Fix}$; Half duration $1.0 \mathrm{sec}$; Principal axes (scale 10**16 $\mathrm{Nm}$ ): (T) Vai-9.79, Plg=9, Azm-75; (N) Val=-0.48, Plg-1, Azm-345; (P) Val=-9.31, Plg=81, Azm-251; Best double couple: Mo-9.6*10*16 Nm; NP1: Strike-166, Dip-36, Slip--89; NP2: Strike-344, Dip=54, Slip--91.

79 NEAR COAST OF NORTBERN CALIF. LGM-P>. WW 4.0 (BRK). ND 3.6 (GM). ML 3.9 (GS), 3.8 (BRK). Felt at Arcata, Eureka, Ferndale and The College of the Redwoods.

Scalar Moment (BRK): MO=9.9*10*14 Nm.

1.420 EASTERN HONSHO, JAPAN

0.817 CELEBES SEA

0.77 NORTBERN ITALY. ML 2.4 (LDG).

0.867 MARIANA ISLANDS

66 SOUTHERN ALASKA. CAEIC>. MI 2.5 (AEIC).

0.9

0.9

1.3

0.4

0.7

1.2

6 ANDAMAN ISLANDS, INDIA

18 OFF COAST OF CENTRAL CEIIE. MD 4.4 (SAN).

12 BANDA SEA

9 OFF COAST OF CENTRAL CEILE

20 ANDREANOF ISLANDS, ALEUTIAN IS.

16 TIMOR REGION, INDONESIA

13 BANDA SEA

34 CALIF. - BAJA CALIF. BORDER REGION. <PAS-P>. MD 4.0 (PAS). Felt in the Imperial valley, California.

0.927 MALAWI

0.77 EASTERN NEW GUINEA REG., P.N.G

$1.0 \quad 11$ NORTH OF ASCENSION ISLAND

1.09 SOLOMON ISLANDS 
$\begin{array}{lllllll}30 & 08 & 47 & 55.97 \quad 17.22 & \mathrm{~N} & 62.26 & \text { พ }\end{array}$

$\begin{array}{lllllll}30 & 09 & 01 & 48.07 & 52.94 \quad \mathrm{~N}\end{array}$

$\begin{array}{lllllll}30 & 09 & 16 & 04.4 ? & 2.16 & \mathrm{~s}\end{array}$

$\begin{array}{llllll}30 & 09 & 44 & 27.3 & 28.222 & \mathrm{~N}\end{array}$

$\begin{array}{llllll}30 & 10 & 56 & 31.4 & 5.507 & \mathrm{~s}\end{array}$
$135.12 \mathrm{E}$

$147.642 \mathrm{E}$

$150.716 \mathrm{E}$
139.958
$90 \mathrm{G}$

$33 \mathrm{~N}$

$33 \mathrm{~N} 3.9$

$53 ? 4.8$

1455.1
244.7

0.25 LFEAARD ISLANDS. HD 3.5 (TRN).

1.07 SOUTH OF ALASKA

0.98 IRIAN JAYA REGION, INDONESIA

1.052 BONIN ISLANDS REGION

0.924 EASTERN NEW GUINEA REG., P.N.G.

0.965 NEW BRITAIN REGION, P.N.G. NW 5.4 (BRV)

Centroid, Yoment Tensor (HRV): Centroid origin time 11:07:04.4; Lat 5.82 S; Lon $151.17 \mathrm{E}$; Dep 112.5; Halfduration $1.2 \mathrm{sec}$; Principal axes (scale 10*17 Nm): (T) Val-1.42, Plg=19, Azm=165; (N) Val=-0.17, Plg=16, Azm=261; (P) Val=-1.26, Plg-65, Azm=27; Best double couple: Mo-1.3*10*17 Nm; NP1: Strike=231, Dip=29, Slip=-123; NP2 : Strike-88, Dip=66, Slip=-73.

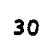

30

30

30

30

30

30

30

30

30

$215924.4 * 36.256 \mathrm{~N} 140.105 \mathrm{E}$ $225055.9840 .424 \mathrm{~N} 125.118 \mathrm{~W}$

$230030.9 \quad 3.333 \mathrm{~s}$
115.360

66.718 พ 176.704 h $146.310 \mathrm{E}$ 177.362 i $157.015 \mathrm{E}$ $70.943 \mathrm{E}$ $70.868 \mathrm{E}$ $5.17 \mathrm{E}$ 70.466 W $580 \mathrm{D} \quad 5.1$

\section{$6 G$}

-28 . 172 D 4.4 $50 \mathrm{D} \quad 4.6$ $73 ? 4.4$ $59 ?$ $33 \times 4.1$

$74 \mathrm{D} \quad 4.2$ $180139.3 * 38.952$ $2102 \quad 31.8834 .400 \mathrm{~S}$ $\begin{array}{llll}21 & 13 & 41.8 & 52.318 \mathrm{~N}\end{array}$

\section{$33 \mathrm{~N}$
7} $10 G \quad 4.9 \quad 4.8$

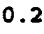

0.7
$013347.47 \quad 5.51 \quad \mathrm{~N}$ $045354.936 .910 \mathrm{~N}$ $\begin{array}{lllll}05 & 16 & 14.2 & 54.246 & N\end{array}$ $052120.8 * 5.655 \mathrm{~s}$ $\begin{array}{lllll}06 & 51 & 01.8 & 9.528 & \mathrm{~s}\end{array}$ $085535.5 * 31.277 \mathrm{~s}$ $092029.5 * 10.139 \mathrm{~N}$ $100949.8 * 18.120 \mathrm{~s}$ $\begin{array}{llllllll}10 & 19 & 27.87 & 3.67 & \mathrm{~s}\end{array}$ $\begin{array}{lllll}10 & 42 & 57.3 ? & 40.71 \quad N\end{array}$ $\begin{array}{llll}12 & 45 & 06.8 & 32.180 \mathrm{~N} \\ 12 & 59 & 12.9 * 22.905 \mathrm{~s}\end{array}$
75.30 W 22.922 E $162.501 \mathrm{~W}$ $138.462 \mathrm{E}$

$93.824 \mathrm{E}$ $175.118 \mathrm{~W}$ $145.37 \mathrm{E}$ $72.78 \mathrm{E}$ $137.355 \mathrm{E}$ $113.631 \mathrm{w}$ $147.519 \mathrm{E}$ $121.033 \mathrm{E}$
$33 \times 4.4$ $60 ? 4.0$ $33 \times 4.6$ $33 \mathrm{~N} 4.2$ $33 \mathrm{~N} 4.8$ $10 \mathrm{G}$

33 N 3.8 $189 ? 4.8$ $33 \mathrm{~N} 4.1$ $33 \mathrm{~N} 3.7$ 392 D 4.9 $10 \mathrm{G} \quad 4.8 \quad 4.8 \quad 1$.

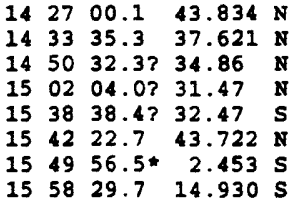

$147.218 \mathrm{E}$ $131.91 \mathrm{E}$ $\begin{array}{llll}15 & 58 & 29.7 & 14.930 \mathrm{~s}\end{array}$
120.643 E $33 \% 4.0$ $20.859 \mathrm{E}$ $141.78 \mathrm{E}$ $70.09 \mathrm{~W}$ $10.216 \mathrm{E}$

$43 D \quad 4.6$

37 ?

$33 \mathrm{~N}$

$10 \mathrm{G}$

$120 \mathrm{G}$

$33 \times 4.0$

$126 \mathrm{D} 5.2$
CENTRAI MID-ATLANTIC RIDGE. NW 5.5 (BRV).

Centroid, Moment Tensor (HRV): Centroid origin time

$11: 42: 01.0$; Lat $0.98 \mathrm{~N}$; Lon $29.02 \mathrm{w}$; Dep $15.0 \mathrm{Fix}$; Halfduration $1.2 \mathrm{sec}$; Principal axes (scale 10*17 Nm): (T) Val=1.59, Plg=2, Azm=38; (N) Va1=0.29, Plg=68, Azm=302; (P) Val-1.88, PIg=21, Azm=129; Best double couple:

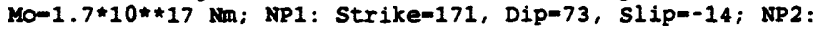
Strike=265, Dip=77, Slip=-163.

25 CALIF. -BAJA CALIF. BORDER REGION. <PAS-P>. MD 3.0 (PAS). MIL 3.1 (GS).

0.16 PUERTO RICO REGION. MD 2.6 (MPR).

0.87 ANDREANOF ISLANDS, ALEOTIAN IS

0.917 EASTERN NEW GUINEA REG., P.N.G.

1.230 SOUTH OF FIJI ISLANDS

0.951 KORIL ISLANDS

1.316 HINDU KOSH REGION, AFGEANISTAN

1.010 AFGHANISTAN-TAJIKISTAN BORD REG.

0.614 WESTERN MEDITERRANEAN SEA

0.410 CHILE-ARGENTINA BORDER REGION. ID 3.5 (SAN).

$\begin{array}{lrl}0.4 & 10 & \text { CHILE-ARGENTINA BORDER REGION. YD } 3.5 \\ 0.7 & 400 & \text { SEA OF OKHOTSK. NW } 5.5 \text { (GS), } 5.5 \text { (HRV). }\end{array}$

Moment Tensor (GS): Dep 595; Principal axes (scale 10*\#17 Nin): (T) Val=2.04, Plg=50, Azm-63; (N) Val=0.01, Plg=9,

Azm-164; (P) Val-2.05, Plg=38, Azm=261; Best double

couple: Mo-2.0*10*17 Nm; NP1: Strike=39, Dip-11, Slip-146; NP2: Strike-163, Dip=84, Slip 81 .

Centroid, Moment Tensor (BRV): Centroid origin time

$21: 13: 45.9$; Lat $52.29 \mathrm{~N}$; Lon $151.65 \mathrm{E}$; Dep 593.0 ; Halfduration $1.3 \mathrm{sec}$; Principal axes (scale 10*\#17 $\mathrm{Nm}$ ): (T) Val=1.83, Plg=51, Azm=69; (N) Val=0.12, Plg=6, Azm=167; (P) Val--1.95, Plg=39, $A z m=262$; Best double couple:

Yo=1.9*10**17 Nm; NP1: Strike=32, Dip=9, Slip-136; NP2 : Strike-166, Dip-84, Slip-84.

5 MEAR EAST COAST OF HONSHO, JAPAN

OFF COAST OF NORTHERN CALIFORNIA. <GM-P>. MD 3.2 (GM). MI 3.2 (GS), 3.1 (BRK).

94 NORTH OF ASCENSION ISLAND. NW 5.2 (HRV).

Centroid, Moment Tensor (HRV): Centroid origin time

$23: 00: 38.1$; Lat $3.21 \mathrm{~s}$; Lon $12.32 \mathrm{~W}$; Dep 15.0 Fix; Halfduration $1.0 \mathrm{sec}$; Principal axes (scale 10 $16 \mathrm{Nm}$ ): (T) Val-8.64, Plg=0, Azm=255; (N) Val=-2.26, Plg=0, Azm=165; (P) Val=-6.38, Plg=90, Azm=180; Best double couple: No-7.5*10**16 Nm; NP1: Strike=345, Dip=45, Slip=-90; NP2: Strike=165, Dip -45 , Slip $=-90$.

0.817 COLOMBIA

1.162 ALASKA PENINSULA. MU 4.8 (PMR).

$\begin{array}{rrlll}1.1 & 62 & \text { ALASRA PENINSULA. MU } 4.8 & \text { (PKR). } \\ 0.3 & 9 & \text { EASTERN NEW GUINEA REG., P.N.G }\end{array}$

0.928 SAVO SEA

1.26 SOUTB AUSTRALIA

1.18 ANDAMAN ISLANDS, INDIA

1.124 TONGA ISIANDS

1.39 NEAR N COAST OF NEW GUINEA, PNG.

1.113 KYRGYZSTAN

0.8156 SOUTH OF HONSEO, JAPAN

29 EASTER ISLAND REGION. MW 5.2 (HRV).

Centroid, Moment Tensor (HRV): Centroid origin time

12:59:22.6; Lat $22.95 \mathrm{~S}$; Lon 113.78 $\mathrm{w}$; Dep 15.0 Fix; Halfduration 1.0 sec; Principal axes (scale $10 * 16 \mathrm{Nm}):(T)$

Val=8.43, $P l g=15, A z m=278$; (N) Val=0.00, $P l g=73, A z m=67$; (P) Val=-8.43, Plg=8, Azm-186; Best double couple: Mo-8.4*10**16 Nm; NP1: Strike-321, Dip-73, S1ip-175; NP2: Strike-53, Dip=85, Slip=17.

1.144 KURIL ISLANDS

1.013 IOHTAN SEA

0.55 OFF EAST COAST OF HONSHO, JAPAN

1.05 KYUSHO, JAPAY

0.310 CHILE-ARGENTINA BORDER REGION. MD 3.4 (SAN).

0.717 CENTRAL ITALY. ML 2.9 (LDG).

1.19 SULAWESI, INDONESIA

0.9182 VANUATU ISLANDS. MW 5.6 ( $\mathrm{HRV}$ )

Centroid, Moment Tensor (HRV): Centroid origin time 15:58:34.8; Lat $14.98 \mathrm{~s}$; Lon $167.38 \mathrm{E}$; Dep 138.1; Halfduration $1.6 \mathrm{sec}$; Principal axes (scale 10\#17 $\mathrm{Nm}$ ): (T) val=3.62, Plg=63, $A z m=118$; (N) Val=-0.71, Plg-22, Azm=335; (P) Val=-2.91, Plg=15, Azm-239; Best double Couple: MO=3.3*10*17 $\mathrm{km}$; NP1: Strike=300, Dip=36, Slip=50; NP2: Strike-167, Dip-63, Slip=115.

$\begin{array}{llllllllll}31 & 16 & 03 & 25.3 ? & 34.49 & \mathrm{~S} & 70.75 & \mathrm{~W} & 100 \mathrm{G}\end{array}$ $\begin{array}{llllllllll}31 & 17 & 30 & 48.5 ? & 6.26 & \mathrm{~N} & 60.08 & \mathrm{E} & 10 \mathrm{G}\end{array}$ $31175621.1 * 51.440 \mathrm{~N} 159.692 \mathrm{E}$

SE-ARGENTINA BORDER REGION. 3.5 (SAN).

$33 N 4.1 \quad 0.7 \quad 12$ OFF EAST COAST OF KAMCHATKA 
SOUTHERN CBILE. MW 5.0 (HRV).

Centroid, Homent Tensor (BRV): Centroid origin time

18:59:20.6; Lat $53.43 \mathrm{~s}$; Lon 73.12 w; Dep 30.2 ; Halfduration $1.0 \mathrm{sec}$; Principal axes (scale $10 * * 16 \mathrm{Nm}$ ): (T) Val-3.02, Plg-12, Azm=182; (N) Val=0.23, Plg-55, Azm=74 Val-3.02, Plg-12, Azm-182; (N) Val=0.23, Plg-55, Azm
(P) Val=-3.25, Plg-32, Azm=280; Best double couple:

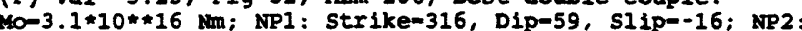
Strike-54, Dip=77, Slip=-148.

$\begin{array}{lllllllllll}31 & 19 & 02 & 50.5 & 51.643 & \mathrm{~N} & 16.194 & \mathrm{E} & 10 \mathrm{G}\end{array}$

31203506.0 ? $15.53 \mathrm{~N} \quad 98.17 \mathrm{~W} 28$.

$31204721.0 \quad 51.493 \times 178.218$ N 44 D 5.6
$0.7 \quad 16$

0.86

1.0310 DOLAND. MC 3.3 (GRF), 3.1 (MOX)

OFF COAST OF GUERRERO, MEXICO

ANDREANOF ISLANDS, ALEUTIAN IS. 5.5 (GS), 5.5 (GRV). Me 5.4 (GS). ML 6.0 (PIR). Felt (IV) on Adak.

Broadband Source Parameters (GS): Dep 40; NP1: strike=0,

Dip=25, S1ip-60; NP2: Strike-212, Dip-69, slip-103;

Radiated energy $2.8 * 10 * 12 \mathrm{~km}$.

Moment Tensor (GS): Dep 40; Principal axes (scale 10**17

$\mathrm{Nm}$ ): (T) Val=2.12, Plg=66, $A z m-146$; (N) Val=0.29, Plg-12,

Azm-29; (P) Val=-2.41, P1g=21, Azm=294; Best double couple: Mo-2.3*10**17 $\mathrm{km} ; \mathrm{NP1}$ : Strike-4, D1p-26, S1ip-63; NP2: strike-214, Dip-67, Silp-103.

Centroid, Moment Tensor (HRV): Centroid origin time

20:47:24.1; Lat $51.47 \mathrm{~N}$; Lon $178.07 \mathrm{~N}$; Dep $47.0 \mathrm{Bdy}$; Halfduration $1.4 \mathrm{sec}$; Principal axes (scale 10*\$17 $\mathrm{km}$ ): (T) Val-1.96, Plg=68, Azm=150; (N) Val=0.27, Plg=16, Azm=16; (P) Val=-2.24, Plg=15, Azm=282; Best double couple:

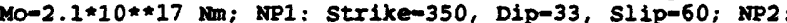
Strike-205, Dip-62, Slip-108.

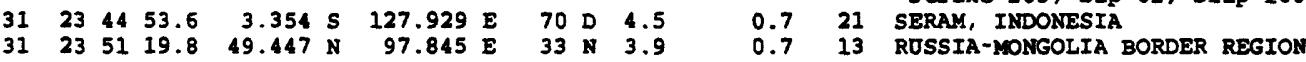

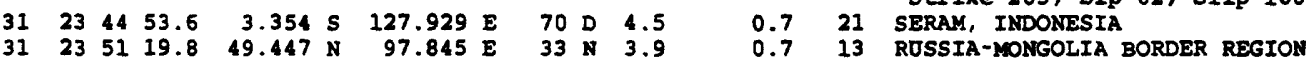

Compiled by Francis w. Baldwin, Pamela J. Benfleld, Don L. Blakeman, George L. Choy, Stuart K. Koyanagi, John H. Minsch, waverly J. Person, Stuart A. Sipkin and Madeleine D. Zirbes. 


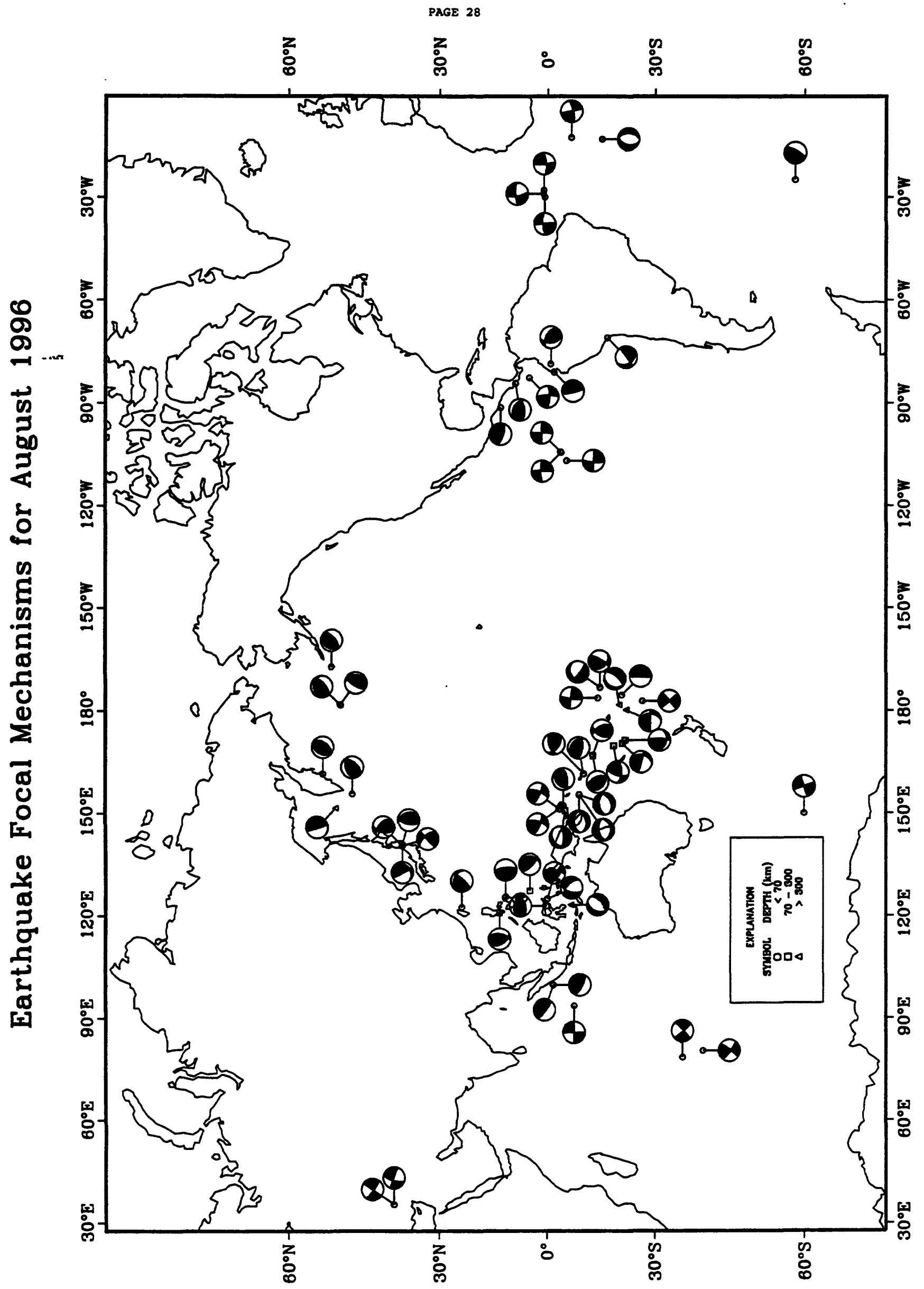


PAGE 29

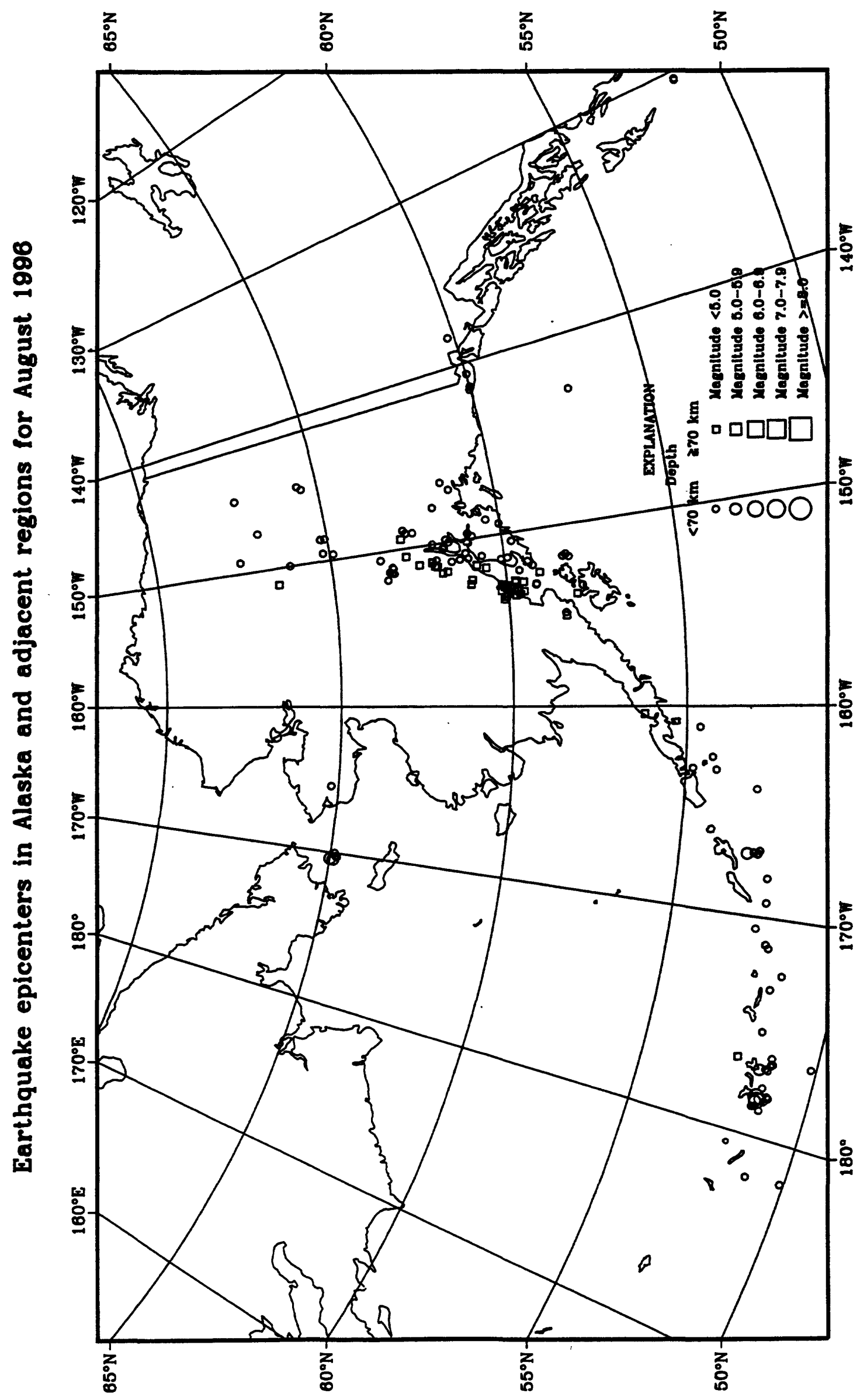


PAGE 30

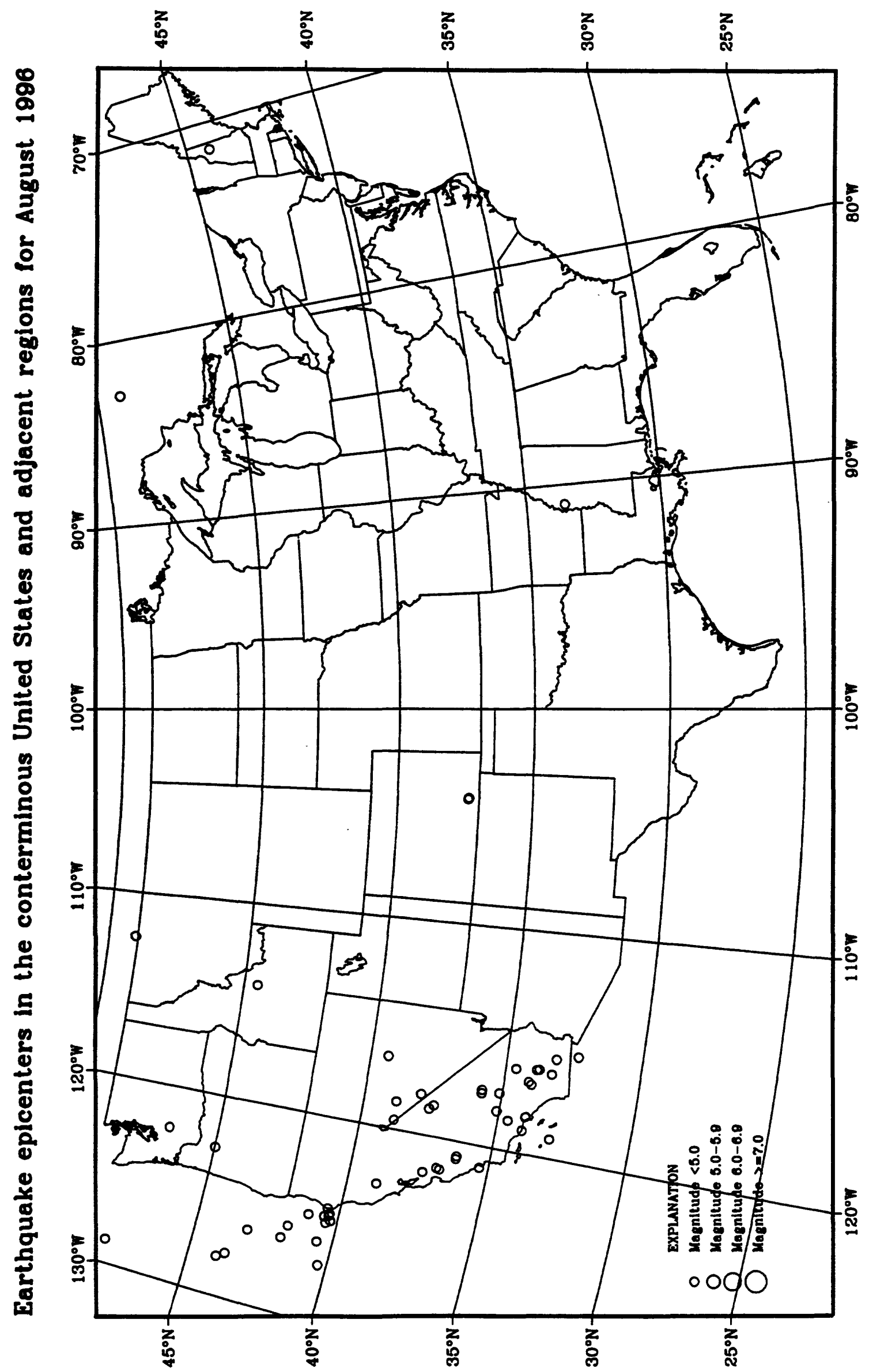




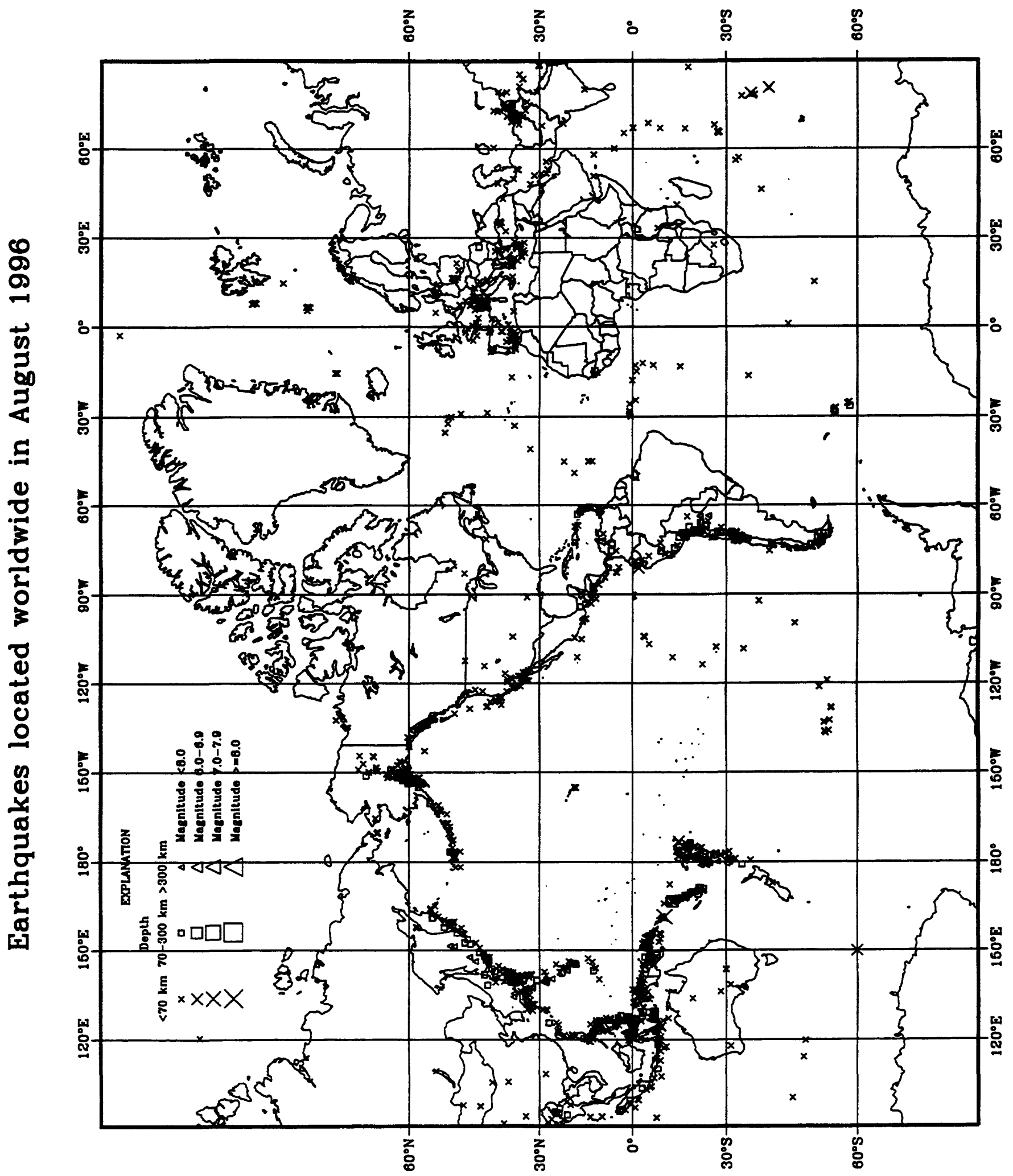


$01 \quad 20 \quad 34 \quad 11.08 \quad 35.745 \quad \mathrm{~N}$

$01 \quad 21 \quad 1525.2 * 40.055 \mathrm{~N}$

$01234557.5 * 6.942 \mathrm{~s}$

$01 \quad 23 \quad 47 \quad 29.2 * 36.363 \mathrm{~N}$

$020006 \quad 11.0 ? \quad 1.28 \mathrm{~s}$

$\begin{array}{lllllll}02 & 00 & 08 & 36.6 & 44.392 & \mathrm{~N}\end{array}$

$\begin{array}{llllll}02 & 00 & 17 & 33.7 & 44.393 & \mathrm{~N}\end{array}$

$\begin{array}{lllllll}02 & 00 & 29 & 13.6 & 1.100 & \mathrm{~N}\end{array}$

$02015720.3 * 36.048 \mathrm{~N}$

02021130.0 ? $2.40 \mathrm{~s}$

$02024523.1 * 50.630 \mathrm{~s}$

$02025904.0 * 11.623 \mathrm{~N}$

$02 \quad 030808.2 * 8.405 \mathrm{~N}$

$\begin{array}{lllllll}02 & 03 & 41 & 43.3 ? & 3.13 \mathrm{~s}\end{array}$

$\begin{array}{lllll}02 & 05 & 36 & 27.8 * 38.920 N\end{array}$

$02 \quad 0740 \quad 46.9 * 39.672 \mathrm{~N}$

$02 \quad 0743 \quad 38.6 \& 55.668 \mathrm{~N}$

$\begin{array}{llllllllll}02 & 07 & 48 & 03.4 & 44.624 & \mathrm{~N}\end{array}$

$02 \quad 0859 \quad 16.1$ ? $4.53 \mathrm{~s}$

$\begin{array}{lllllll}02 & 10 & 13 & 03.6 \& & 59.738 \mathrm{~N}\end{array}$

$\begin{array}{lllllll}02 & 12 & 10 & 15.6 & 26.231 & \mathrm{~N}\end{array}$

$\begin{array}{lllll}02 & 12 & 42 & 33.6 & 36.449 \mathrm{~N}\end{array}$

$\begin{array}{lllllll}02 & 13 & 06 & 27.27 & 11.83 & \mathrm{~N}\end{array}$

$02 \quad 140958.78 \quad 37.063 \mathrm{~N}$

$\begin{array}{lllllll}02 & 15 & 39 & 40.2 ? & 17.70 & \mathrm{~S}\end{array}$

$\begin{array}{lllllll}02 & 16 & 04 & 40.18 & 34.196 & \mathrm{~N} \\ 02 & 17 & 02 & 07.28 & 4.128 & \mathrm{~S}\end{array}$

$\begin{array}{llllrl}02 & 17 & 02 & 07.28 & 4.128 & \mathrm{~S} \\ 02 & 17 & 34 & 12.4 & 24.224 & \mathrm{~S}\end{array}$

$02 \quad 18 \quad 4512.8$ ? $3.62 \mathrm{~N}$

$\begin{array}{lllllll}02 & 18 & 56 & 22.2 ? & 3.44 & \mathrm{~N}\end{array}$

$\begin{array}{lllllll}02 & 19 & 07 & 01.5 & 37.666 \mathrm{~N}\end{array}$

02

$2204152.9 \quad 12.320 \times 143.809 \mathrm{E}$

$20.746 \mathrm{E}$

$43.673 \mathrm{E}$
$01 \quad 2011 \quad 49.3 ? \quad 6.26$ S 130.62

$\begin{array}{llllllllll}01 & 20 & 28 & 08.08 & 44.92 & \mathrm{~N} & 145.86 & \mathrm{E}\end{array}$

$\begin{array}{lllllll}01 & 20 & 31 & 12.6 & 44.411 & \mathrm{~N}\end{array}$

$02005206.5 * 39.617 \mathrm{~N}$

$0202 \quad 35 \quad 50.6 * 3.930 \mathrm{~s}$
$100 \mathrm{G} 4.3$

$10 \mathrm{G}$

117.621

$141.247 \mathrm{E}$

$136.30 \mathrm{E}$

$7.264 \mathrm{E}$

$7.256 \mathrm{E}$

$121.835 \mathrm{E}$

$143.723 \mathrm{E}$

$71.018 \mathrm{E}$

$128.10 \mathrm{E}$

$69.448 \mathrm{E}$

$62.278 \mathrm{E}$

$125.957 \mathrm{E}$

$148.19 \mathrm{E}$

$40.688 \mathrm{E}$

$158.418 \mathrm{~W}$

$149.475 \mathrm{E}$

$152.28 \mathrm{E}$

$128.816 \mathrm{E}$

$70.097 \mathrm{E}$

61.96

$139.354 \mathrm{E}$

8.96

128.936

$67.004 \mathrm{~W}$

$64.39 \mathrm{E}$

$64.13 \mathrm{E}$

$10 \mathrm{G} 4.3$

$33 \mathrm{~N} 4.1$

50 G 4.5

$33 \mathrm{~N}$

$10 \mathrm{G}$

$10 \mathrm{G}$

$33 \mathrm{~N} 4.7$

$33 \mathrm{~N} 4.5$

10704.3

$33 \mathrm{~N}$

$10 \mathrm{G} \quad 4.4$

$10 \mathrm{G}$

$33 \mathrm{~N}$

$33 \mathrm{~N}$

$33 \mathrm{~N}$

62

33
150

71

$33 \mathrm{~N} 4$

$222 \mathrm{D} 4.4$

$120 \mathrm{G}$

$10 \mathrm{G}$

$100 \mathrm{G}$

$33 \mathrm{~N}$

$33 \mathrm{~N}$

$186 \mathrm{D}$

$10 \mathrm{G}$
$33 \mathrm{~N} 3.8$

$155.116 \mathrm{E}$

$73.645 \mathrm{E}$

1.645 พ
$10 \mathrm{G} 4.3$

$\begin{array}{lllll}33 & 5.7 & 5.7 & 1.1 & 173\end{array}$

1.413
$33 \mathrm{~N} 4.5$

(GS)

1.4 8 BANDA SEA

8 HOKKAIDO, JAPAN REGION

0.732 NEAR COAST OF OREGON. MD 2.7 (SEA)

30 CENTRAL CAIIFORNIA. LGM-P>. MD 3.0 (GM). MI 3.0 (GS).

0.711 GREECE-ALBANIA BORDER REGION

1.119 SOLOMON ISIANDS

0.816 NEAR EAST COAST OF HONSBD, JAPAN

1.210 IRIAN JAYA REGION, INDONESIA

0.623 NORTHERN ITALY. ML 3.0 (LDG), 2.9 (GEN).

0.516 NORTHERN ITALY. MU 2.8 (GEN), 2.6 (IDG)

0.923 MINAHASSA PENINSULA, SULAWESI

0.612 OFE EAST COAST OF HONSBO, JAPAN

1.216 AFGEANISTAN-TAJIKISTAN BORD REG.

0.98 CERAM SEA

1.028 CEAGOS ARCHIPELAGO REGION

1.118 AUCKLAND ISLANDS REGION

1.112 ETEIOPIA

0.919 MINDANAO, PHILIPPINE ISLANDS

0.87 BISMARCK SEA

0.911 EASTERN BONSHU, JAPAN

TAJIKISTAN-XINJIANG BORDER REG

ALASKA PENINSULA. 〈AEIC>. ML 5.0 (AEIC), 5.0 (PMR). Felt

(IV) at Chignik, Ivanof Bay and Perryville.

$1.0 \quad 160 \quad$ KORTI

1.211 NEW BRITAIN REGION, P.N.G.

36 SOUTHERN ALASKA. <AEIC>

0.919 RYUKYU ISLANDS

0.9101 HINDU KUSE REGION, AFGHANISTAN

0.46 WINDWARD ISLANDS. MD 3.2 (TRN).

1.25 EASTERN HONSEO, JAPAN

0.46 FIJI ISLANDS REGION

0.810 NEAR S. COAST OF HONSEU, JAPAN

$0.7 \quad 8$ BANDA SEA

1.338 CHILE-ARGENTINA BORDER REGION

CARLSBERG RIDGE

CARISBERG RIDGE

SPAIN. mbLg 4.5 (MDD). ML 4.4 (IDG), 4.3 (STR). Felt (V) in the Mazarron area.

OF MARIANA ISLANDS. WW 5.9 (GS), 5.9 (BRV). Me 5.9

(GS). Felt (III) on Guam.

Broadband Source Parameters (GS): Dep 8; NPI: Strike-145

Dip-55, Slip=-50; NP2: Strike-269, Dip-51, Slip=-133;

Radiated evergy $1.7 * 10 * 13 \mathrm{Nm}$

Moment Tensor (GS): Dep 3: Principal axes (scale 10**17 Nm): (T) Val-6.83, Plg=8, Azm-230; (N) Val=-0.02, Plg=21, Azm-323; (P) Val--6.81, Plg-67, Azm-120; Best double couple: Mo-6.8*10*\#17 Nm; NP1: Strike=297, Dip-41,

Slip--123; NP2: Strike-158, Dip-57, Slip--64.

Centroid, Moment Tensor (BRV): Centroid origin time

20:41:52.7; Lat $12.15 \mathrm{~N}$; Lon $143.90 \mathrm{E}$; Dep 15.0 Fix; Half-

duration $2.1 \mathrm{sec}$; Principal axes (scale 10*17 Nm): (T)

Val=7.91, Plg=6, Azm=232; (N) Val-0.68, Plg=5, Azm=141

(P) Val--7.23, Plg-82, Azm-7; Best double couple:

Mo-7.6*10*17 $\mathrm{Nm}$; NPI: Strike-328, Dip-40, Slip=-81; NP2

Strike=137, Dip-51, Slip- -97

$02 \quad 22 \quad 25 \quad 48.0 * 36.704$

$71.264 \mathrm{E}$

$150 \mathrm{G}$

4.2

1.19

AFGBANISTAN-TAJIXISTAN BORD REG

$02 \quad 234157.5 \quad 45.962 \mathrm{~N} \quad 6.142 \mathrm{E} \quad 10 \mathrm{G}$

$03001131.7 \quad 7.172 \mathrm{~S} \quad 155.669 \mathrm{E}$

$\begin{array}{llllll}33 & \mathrm{~N} & 5.4 & 5.1 & 0.8 & 139\end{array}$

FRANCE. ML 2.6 (IDG).

SOLOMON ISLANDS. WW 5.5 (BRV).

Centroid, Moment Tensor (BRv): Centroid origin time

$00: 11: 33.5$; Lat $7.42 \mathrm{~S}$; Lon $155.78 \mathrm{E}$; Dep 15.0 Fix; Half duration $2.1 \mathrm{sec}$; Principal axes (scale $10 * 17 \mathrm{Nm}$ ): (T) Val-2.10, Plg-69, Azm-22; (N) Val-0.11, Plg-8, Azm-134; (P) Val=-2.21, Plg=19, Azm=227; Best double couple:

Mo-2.2*10**17 Nm; NP1: StIike=331, Dip=27, S1ip=109; NP2: Strike-130, Dip-65, Slip-81.

$03002900.8 \& 67.199 \mathrm{~N} \quad 144.817 \mathrm{~W}$

$0300 \quad 34 \quad 34.0 \quad 7.149 \mathrm{~S} \quad 155.650 \mathrm{E}$

$03015117.1 * 6.705 \mathrm{~S} \quad 72.669 \mathrm{E}$

03015357.9 ? 6.99 S 72.69 E

$\begin{array}{llllll}03 & 02 & 05 & 12.7 ? & 10.60 \mathrm{~s}\end{array}$

$03020919.0 * 7.297 \mathrm{~s}$

$03023658.3 ? 53.40 \mathrm{~N}$

$03044911.5 * 6.936 \mathrm{~s}$

$\begin{array}{lllllll}03 & 05 & 08 & 06.98 & 37.517 & \mathrm{~N}\end{array}$

$165.78 \mathrm{E}$

$155.665 \mathrm{E}$

$171.14 \mathrm{~W}$

$72.774 \mathrm{E}$

1.547 W

21

$33 \mathrm{~N} 5.0$

$10 \mathrm{G}$

$10 \mathrm{G}$

$200 \mathrm{G}$

$33 \mathrm{~N}$

200 G 3.9

$10 \mathrm{G}$

$10 \mathrm{G}$

$10 \mathrm{G} \quad 3.8$

0.8
0.9
1.0
1.
0.
1.
1.
0.

38 NORTHERN ALASRA. CAEIC>. ML 3.6 (AEIC).

$03 \quad 08 \quad 24 \quad 23.28 \quad 60.051 \mathrm{~N} \quad 151.150 \mathrm{~W}$

$0308 \quad 5951.8859 .983 \mathrm{~N} \quad 153.520 \mathrm{~W}$

$\begin{array}{llllllll}03 & 09 & 21 & 33.7 & 7.896 & \mathrm{~s} & 122.355 \mathrm{E}\end{array}$

$\begin{array}{lllllllll}03 & 09 & 44 & 52.68 & 5.975 & \mathrm{~S} & 153.078\end{array}$

40

139

250 G 5.1

$33 \mathrm{~N}$

$33 \mathrm{~N} 5.1$

$\begin{array}{lll}32 & \text { SOLOMON ISLANDS } \\ 0.9 & 18 & \text { CHAGOS ARGIPELAG }\end{array}$

6 CHAGOS ARCEIPELAGO REGION

15 SANTA CRUZ ISLANDS

16 SOLOMON ISLANDS

10 FOX ISLANDS, ALEUTIAN ISIANDS

22 CHAGOS ARCHIPELAGO REGION

5 SPAIN. mbLg 3.1 (MDD).

(AEIC).

63 RENAI PENINSULA, ALASKA. CAEIC>. ML 2.6 (AEIC).

55 SOUTHERN ALASKA. 〈AEIC>.

1.023 FLORES SEA

0.810 NEW IREIAND REGION, P.N.G.

0.8121 NEAR EAST COAST OF EONSHO, JAPAN. MW 5.1 (ERV).

Centroid, Moment Tensor (HRV): Centroid origin time 09:57:29.1; Lat $40.08 \mathrm{~N}$; Lon $142.74 \mathrm{E}_{\text {; }}$ Dep $33.0 \mathrm{Eix}$; Halfduration $1.0 \mathrm{sec}$; Principal axes (scale $10 \star * 16 \mathrm{Nm}$ ): (T) Val-6.23, Plg-59, Azm-282; (N) Val--0.60, Plg-4, Azm=185; (P) Val--5.63, Plg-31, Azm-93; Best double couple: Mo-5.9*10**16 Nm; NP1: Strike-169, Dip=14, S1ip-73; NP2: Strike=7, Dip-76, slip=94.

$03103845.5 * 18.582 \times 146.914$

$03 \quad 1058 \quad 49.4 * 23.092 \mathrm{~s}$

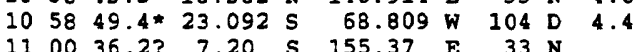

$33 \mathrm{~N} 4.0$

$03112216.2 * 14.258 \mathrm{~N} 144.039 \mathrm{E}$

33 N

0.8

11

MARIANA ISLANDS

1.334 NORTEERN CHILE

1.27 SOLOMON ISLANDS

0.69 MARIANA ISLANDS 


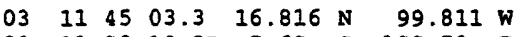

$\begin{array}{llllllllll}03 & 11 & 58 & 19.27 & 7.65 & \mathrm{~S} & 155.79 & \mathrm{E}\end{array}$ $\begin{array}{lllllll}12 & 04 & 45.7 & 14.328 \mathrm{~N} & 144.049 \mathrm{E}\end{array}$

\begin{tabular}{lllllll}
\hline & 12 & 47 & 29.1 & $33.756 \mathrm{~N}$ & $140.096 \mathrm{E}$
\end{tabular}

b3 $124934.8 * 24.636 \mathrm{~N} 96.831 \mathrm{E}$

$\begin{array}{lllllllll}03 & 13 & 25 & 42.5 ? & 7.38 & \mathrm{~S} & 155.77 & \mathrm{E}\end{array}$

$031530 \quad 25.8 \& 36.109 \mathrm{~N} 117.855 \mathrm{~W}$

$031601 \quad 53.7 * 7.219$ S $155.617 \mathrm{E}$ $\begin{array}{lllllllll}03 & 16 & 18 & 52.1 & 7.258 & \mathrm{~S} & 155.534 & \mathrm{E}\end{array}$
$150 \mathrm{G}$

$33 \mathrm{~N} 4.7$

$92 * 3.9$

$33 \mathrm{~N}$

$33 \mathrm{~N}$

$6 \mathrm{G}$

$\begin{array}{lllll}33 & \mathrm{~N} & 4.4 & & 0.9 \\ 33 & \mathrm{~N} & 4.9 & 4.5 & 1.0\end{array}$

0.968 NEAR COAST OF GUERRERO, MEXICO

$0.8 \quad 8$ SOLOMON ISLANDS

0.727 MARIANA ISLANDS

1.128 SOUTH OF HONSHU, JAPAN

0.35 MYANMAR

0.87 SOLONON ISLANDS

39 CALIFORNIA-NEVADA BORDER REGION. LPAS-P>. MD 2.8 (PAS). MI 3.2 (GS).

20 SOLOMON ISLANDS

42 SOLOMON ISLANDS. MW 5.2 (ERV).

Centroid, Moment Tensor (HRV): Centroid origin time

$16: 18: 52.0$; Lat $7.67 \mathrm{~S}$; Lon $155.75 \mathrm{E}$; Dep $15.0 \mathrm{Fix}$; Half duration $1.0 \mathrm{sec}$; Principal axes (scale 10**16 $\mathrm{Nm}$ ): (T) val-7.36, Plg-60, Azm-4; (N) Val=-0.96, Plg=12, Azm-116; (P) val- 6.40, Plg-27, Azm-212; Best double couple: Mo-6.9*10**16 Nm; NP1 : Strike-330, Dip=21, Slip=126; NP2 : Strike-112, Dip=73, Slip-77.

$031633 \quad 04.5 * 7.245$ S $155.570 \mathrm{E}$ $03163455.3 * 5.994 \mathrm{~S} 147.298 \mathrm{E}$ $\begin{array}{lllllllll}03 & 17 & 01 & 54.2 & 26.192 & \mathrm{~N} & 110.524 \mathrm{~W}\end{array}$

$33 \mathrm{~N}$

$10 \mathrm{G}$

0.7

1.2

16 SOLOMON ISLANDS

EASTERN NEW GUINEA REG., P.N.G.

146 GULF OF CALIFORNIA. MW 5.2 (HRV)

Centroid, Moment Tensor (HRV): Centroid origin time

$17: 01: 58.0$; Lat $26.60 \mathrm{~N}$; Lon $110.61 \mathrm{~W}$; Dep 15.0 Fix; Halfduration $1.0 \mathrm{sec}$; Principal axes (scale $10 * \star 16 \mathrm{Nm}$ ): (T) val-7.54, Plg-2, Azm-106: (N) Val=-1.05, Plg-52, Azm-199; (P) Val=-6.50, Plg-38, Azm-15; Best double couple: Mo-7.0*10*16 $\mathrm{Nm} ; \mathrm{NP1}$ : Strike-158, Dip-63, Slip=-153; NP2: Strike-54, Dip-65, Slip=-30.

\begin{tabular}{lllllllll}
03 & 18 & 13 & 51.38 & 64.088 & $\mathrm{~N}$ & 148.358 & $\mathrm{~W}$ \\
03 & 18 & 25 & $29.9 ?$ & 7.56 & $\mathrm{~S}$ & 156.45 & $\mathrm{E}$ \\
03 & 18 & 28 & $11.2 ?$ & 7.42 & $\mathrm{~S}$ & 72.05 & $\mathrm{E}$ \\
3 & 19 & 17 & $23.3 *$ & 18.513 & $\mathrm{~N}$ & 146.440 & $\mathrm{E}$ \\
3 & 20 & 04 & 00.0 & 12.866 & $\mathrm{~S}$ & 169.005 & $\mathrm{E}$ \\
3 & 20 & 39 & 11.58 & 65.162 & $\mathrm{~N}$ & 148.571 & $\mathrm{~W}$ \\
3 & 21 & 38 & 01.68 & 53.422 & $\mathrm{~N}$ & 165.728 & $\mathrm{~W}$ \\
3 & 23 & 11 & $01.1 *$ & 40.200 & $\mathrm{~N}$ & 49.422 & $\mathrm{E}$ \\
3 & 23 & 53 & $17.2 ?$ & 7.13 & $\mathrm{~S}$ & 155.40 & $\mathrm{E}$ \\
3 & 23 & 53 & $38.5 *$ & 38.076 & $\mathrm{~N}$ & 142.607 & $\mathrm{E}$ \\
04 & 01 & 37 & $06.0 ?$ & 20.55 & $\mathrm{~N}$ & 121.51 & $\mathrm{E}$ \\
04 & 02 & 10 & $07.4 ?$ & 16.86 & $\mathrm{~N}$ & 97.81 & $\mathrm{~W}$ \\
\hline & 03 & 37 & 53.2 & 29.966 & $\mathrm{~N}$ & 130.265 & $\mathrm{E}$
\end{tabular}

115

$33 \mathrm{~N}$

$10 \mathrm{G}$

$33 \mathrm{~N}$

$600 \mathrm{G} \quad 4.5$

18

23

$33 \mathrm{~N}$

$33 \mathrm{~N} \quad 4.6$

$33 \mathrm{~N} 3.7$

52
82 CENTRAL ALASKA. LAEIC>

1.288 SOLONON ISLANDS

0.87 CHAGOS ARCHIPELAGO REGION

0.714 MARIANA ISLANDS

1.080 SANTA CRUZ ISLANDS REGION

31 NORTHERN ALASKA. $\angle A E I C$. ML 3.0 (AEIC).

31 FOX ISLANDS, ALEUTIAN ISLANDS. CAEIC>. ML 4.2 (AEIC).

1.013 EASTERN CAUCASUS

1.112 SOLONON ISILANDS

1.217 NEAR EAST COAST OF GONSBO, JAPAN

0.877 PHILIPPINE ISLANDS REGION

0.799 OAXACA, MEXICO

$\begin{array}{lllll}5.5 & 4.7 & 1.0 & 261 & \text { RYUKYO ISIANDS. MW } 5.3 \text { (HRV) }\end{array}$

Centroid, Moment Tensor (HRV): Centroid origin time

$03: 37: 55.7$; Lat $29.49 \mathrm{~N}$; LOB $130.39 \mathrm{E}$; Dep 60.5 ; Halfduration $1.1 \mathrm{sec}$; Principal axes (scale 10**17 $\mathrm{Nm}$ ): (T) val-0.99, Plg-46, Azm=308; (N) Val=0.21, Plg=36, Azm-85 (P) val--1.20, Plg-22, Azm-193; Best double couple: Mo-1.1*10**17 Nm; NP1: Strike-328, D1P-39, Slip-158; NP2: Strike-75, Dip-76, Slip-53.

$035943.4 ? 43.14 \mathrm{~N}$ 0.33 พ $10 \mathrm{G}$ $9.353 \mathrm{~W} \quad 10 \mathrm{G}$ 0.44 PYRENEES. MI 2.7 (IDG).

$0.8 \quad 10$ $\begin{array}{llll}04 & 14 & 03.2 & 36.984\end{array}$ $\begin{array}{llllll}2.876 \text { E } & 10 \mathrm{G} & 5.3 & 5.3 & 1.2 & 316\end{array}$
PORTOGAL. MbLg 3.6 (MDD).
$0406 \quad 45 \quad 51.5 \quad 36.420 \mathrm{~N}$

04

04

04

$090030.6 \& 36.117 \mathrm{~N}$

$091818.3 * 41.791 \mathrm{~N}$

$\begin{array}{llll}10 & 18 & 26.0 & 41.915 \mathrm{~N}\end{array}$ $\begin{array}{rllllllllll}04 & 07 & 47 & 30.1 ? & 6.85 & \mathrm{~N} & 73.07 & \mathrm{~W} & 150 \mathrm{G} & 4.0 \\ 04 & 07 & 53 & 55.5 ? & 35.53 & \mathrm{~S} & 179.52 & \mathrm{E} & 33 & \mathrm{~N} & 4.6\end{array}$
$179.52 \mathrm{E} \quad 33 \mathrm{~N} \quad 4.6$

$\begin{array}{ll}0.8 & 22 \\ 0.9 & 16 \\ 1.0 & 12\end{array}$

$10 \mathrm{G} 3.6$

$126.869 \mathrm{~W}$ $126.777 \mathrm{~W}$

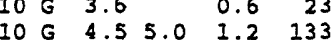
$\begin{array}{lllll}10 & 24 & 49.8 & 41.777 & \mathrm{~N}\end{array}$ $\begin{array}{lllll}10 & 32 & 57.6 \& & 35.101 & \mathrm{~N}\end{array}$ $\begin{array}{lllll}10 & 38 & 07.3 & 55.989 & \mathrm{~S}\end{array}$ $\begin{array}{lllll}10 & 47 & 15.3 & 41.739 & N\end{array}$ $\begin{array}{llllll}10 & 49 & 38.9 ? & 41.55 \quad \mathrm{~N}\end{array}$ $113055.1 * 42.188 \mathrm{~N}$ 121842.1 ? $51.20 \mathrm{~N}$ $\begin{array}{lllll}13 & 10 & 24.28 & 60.151 \mathrm{~N}\end{array}$ $\begin{array}{lllll}13 & 37 & 24.6 ? 32.15 \mathrm{~S}\end{array}$ $\begin{array}{lllll}14 & 11 & 57.9 & 20.753 & \mathrm{~S}\end{array}$ 142758.3 ? $56.92 \mathrm{~s}$ $\begin{array}{lllll}15 & 18 & 27.4 & 29.038 & \mathrm{~N}\end{array}$
$126.967 \mathrm{~W}$ $126.834 \mathrm{~W}$ $117.495 \mathrm{~W}$ $147.121 \mathrm{E}$ $126.995 \mathrm{~W}$ 126.78 W $125.664 \mathrm{w}$ $16.03 \mathrm{E}$ $152.514 \mathrm{~W}$ $70.45 \mathrm{~W}$ 178.873 W $10 \mathrm{G} 4.3$ $140.833 \mathrm{E} \quad 65 \mathrm{D} 5.4$
$10 \mathrm{G} \quad 3.7$ $\begin{array}{lll}10 G & 4.7\end{array}$

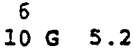
$10 \mathrm{G} \quad 3.9$ $10 \mathrm{G} 3.3$ $10 \mathrm{G} \quad 3.7$ $10 \mathrm{G}$ $100 \mathrm{G}$
NORTHERN ALGERIA. MW 5.5 (HRV). MbLg 5.1 (MDD). Felt in the Algiers area.

Centroid, Moment Tensor (HRV): Centroid origin time

$04: 14: 07.5$; Lat $37.03 \mathrm{~N}$; Ion $3.03 \mathrm{E}$; Dep $15.0 \mathrm{Fix}$; Halfduration $1.3 \mathrm{sec}$; Principal axes (scale 10*17 $\mathrm{Nm}$ ): (T) Val-1.68, Plg=7, Azm=236; (N) Val=0.46, Plg=76, Azm=118; (P) Val--2.14, Plg-12, Azm-327; Best double couple: Mo-1.9*10*17 Nm; NP1: Strike-11, Dip-76, Slip=-4; NP2: Strike=102, Dip-86, Slip=-166.

HINDU ROSB REGION, AFGHANISTAN ORTEERN COLOMBIA

ISTAND, N.2.

CALIFORNIA-NEVADA BORDER REGION. 〈PAS-P>. MD 3.5 (PAS). MLL 3.7 (GS).

OFF COAST OF NORTHERN CALIFORNIA

COAST OF NORTHERN CALIFORNIA. MW 5.4 (HRV).

Centroid, Moment Tensor (HRV): Centroid origin time

10:18:29.6; Lat 42.14 N; Lon 127.33 W; Dep 15.0 Fix; Halfduration $1.2 \mathrm{sec}$; Principal axes (scale 10*17 Nm): (T) Val=1.67, Plg-18, Azm=85; (N) Val=-0.07, Plg=4, $\mathrm{Azm}=176$; (P) val=-1.60, Plg=72, Azm=278; Best double couple: Mo-1.6*10*17 Nm; NP1: Strike-169, Dip-27, Slip=-98; NP2 : Strike=358, Dip-63, Slip=-85.

1.017 OFF COAST OF NORTHERN CALIFORNIA. ML 3.6 (GS).

$1.34 I$ OFF COAST OF NORTHERN CAIIFORNIA

29 CENTRAL CALIFORNIA. 〈PAS-P>. MD 3.2 (PAS). ML 3.1 (GS).

1.146 WEST OF MACQUARIE ISLAND

0.947 OFF COAST OE NORTHERN CALIFORNIA

1.599 OFF COAST OE NORTHERN CALIFORNIA

0.622 OFF COAST OF OREGON

0.85 POLAND. MI 2.8 (MOX).

61 SOJTHERN ALASKA. CAEIC>.

0.39 CHILE-ARGENTINA BORDER REGION. MD 2.7 (SAN).

0.852 FIJI ISLANDS REGION

0.57 SOUTHERN EAST PACIFIC RISE

0.9188 SOJTE OF HONSHO, JAPAN. MW 5.2 (HRV)

Centroid, Moment Tensor (HRV): Centroid origin time $15: 18: 28.2$; Lat $29.22 \mathrm{~N}$; LOB $141.15 \mathrm{E}$; Dep 78.7; Half duration 1.0 sec; Principal axes (scale 10**16 $\mathrm{Nm}$ ): (T) val-6.63, Plg=57, Azm=95; (N) Val-2.29, Plg=31, Azm=256 ; (P) Val--8.92, P1g=9, Azm-351; Best double couple: MO=7.8*10**16 Nm; NPI: Strike-113, Dip=46, S1ip-137; NP2: Strike-236, Dip-61, Silip-53. 
$04181601.9 \quad 31.555 \times \quad 139.931 \mathrm{E} \quad 33 \times \quad 5.45 .1 \quad 1.2 \quad 91$ and (II JMA) at Tokyo and Yokohama tsunami generated with maximum recorded wave heights (peakto-trough) of $26 \mathrm{~cm}$ on Hachijo-jima, $20 \mathrm{~cm}$ at Okada, 0shima and $16 \mathrm{~cm}$ on Miyake-jima.

moment Tensor (GS): Dep 19; Prícipal axes (scale 10**17 $\mathrm{Nm}$ ): (T) Val-4.12, Plg-81, Azm-101; (N) Val=-0.07, Plg-9, Azm=288; (P) Val=-4.05, Plg=1, Azm=198; Best double couple: Mo=4.1*10**17 Nm; NP1: Strike-279, Dip=45, Slip=77; NP2: Strike-117, Dip-47, silp-102.

Centroid, Moment Tensor (HRV): Centroid origin time $18: 16: 07.2$; Iat $31.48 \mathrm{~N}$; Lon $140.06 \mathrm{E}$; Dep 24.4; Halfduration $1.7 \mathrm{sec}$; Principal axes (scale 10*17 Nm): (T) Val=5.18, Plg=79, Azm=111; (N) Val=-1.60, Plg=3, Azm=217; (P) Val--3.58, Plg=10, Azm-307; Best double couple: Mo-4. 4*10**17 Nm; NP1: Strike=41, Dip=35, Slip=95; NP2: Strike-215, Dip=55, Slip=86.

$\begin{array}{rrrlllllllllll}04 & 18 & 42 & 09.5 ? & 20.52 & \mathrm{~S} & 179.12 & \mathrm{~W} & 600 & \mathrm{G} & 4.0 & & 1.0 & 10 \\ 04 & 18 & 55 & 34.5 * & 35.243 & \mathrm{~N} & 140.233 & \mathrm{E} & 114 & & & & 0.7 & 13 \\ 04 & 19 & 02 & 00.86 & 61.775 & \mathrm{~N} & 150.778 & \mathrm{~W} & 53 & & & & 66 \\ 04 & 19 & 06 & 49.8 & 9.365 & \mathrm{~N} & 84.266 & \mathrm{~W} & 33 & 5.8 & 5.9 & 1.0 & 321\end{array}$

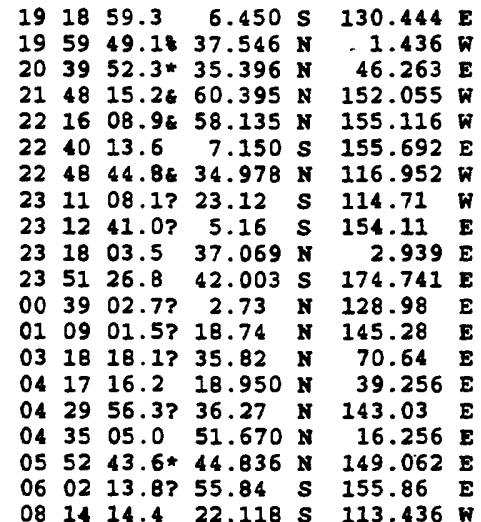

$\begin{array}{rllll}33 & \mathrm{~N} & 5.0 & & 0.8 \\ 10 \mathrm{G} & & & 0.7 \\ 33 & \mathrm{~N} & 4.4 & & 1.1 \\ 75 & & & & \\ 98 & & & & \\ 33 & \mathrm{~N} & 4.9 & 4.6 & 0.8 \\ \mathrm{G} & & & & \\ 10 \mathrm{G} & 4.5 & & 0.8 \\ 33 & \mathrm{~N} & 4.2 & & 1.4 \\ 10 \mathrm{G} & 3.4 & & 1.0 \\ 33 & \mathrm{~N} & 5.1 & & 1.1 \\ 150 \mathrm{G} & 4.1 & & 0.3 \\ 100 \mathrm{G} & & & 0.7 \\ 100 \mathrm{G} & & & 1.1 \\ 10 \mathrm{G} & 4.8 & 4.7 & 1.1 \\ 33 & \mathrm{~N} & & & 1.3 \\ 10 \mathrm{G} & & & 1.1 \\ 33 \mathrm{~N} & 3.9 & & 0.9 \\ 10 \mathrm{G} & 4.6 & & 1.2 \\ 10 \mathrm{G} & 6.2 & 7.0 & 1.1\end{array}$

$081655.4 \quad 36.729 \mathrm{~N}$ 08 20 $48.8 \quad 36.726 \quad N$ $091020.7 \quad 22.054 \mathrm{~s}$ $0922 \quad 29.7 ? \quad 7.07 \quad s$ $094659.422 .056 \mathrm{~s}$
$116.266 \mathrm{~W}$ $116.285 \mathrm{~W}$ $113.401 \mathrm{~W}$ $113.099 \mathrm{~W}$ $130.66 \mathrm{E}$ $113.083 \mathrm{~W}$
$10 \mathrm{G}$

$5 \mathrm{G}$

$10 \mathrm{G}$

$10 \mathrm{G}$ $100 \mathrm{G}$ Strike-215, Dip=55
EIJI ISIANDS REGION

SOUTHERN ALASKA. 〈AEIC>. ML 2.5 (AEIC).

COSTA RICA. NW 6.2 (GS), 6.2 (HRV). Ne 5.4 (GS).

Broadband Source Parameters (GS): Dep 18; NP1: Strike-98, Dip-67, S1ip-81; NP2: Strike-300, Dip=25, S11p-110; Radiated energy $2.9 * 10 * * 12 \mathrm{Nm}$.

Moment Tensor (GS): Dep 21; Principal axes (scale 10**18 $\mathrm{Nm}$ ): (T) Val=1.94, Plg=66, Azm=23; (N) Val=-0.01, Plg=7, Azm-129; (P) Val--1.93, Plg-23, Azm-222; Best double couple: $y=1.9 * 10 * 18 \mathrm{Nm}$; NP1: Strike-327, Dip=23, Slip=109; NP2: Strike-126, Dip=68, Slip-82.

Centroid, yoment Tensor (BRv): Centroid origin time 19:06:55.6; Lat $9.37 \mathrm{~N}$; Lon $84.36 \mathrm{~W}$; Dep $20.0 \mathrm{Fix}$; Halfduration $3.2 \mathrm{sec}$; Principal axes (scale 10**18 $\mathrm{Nm}$ ): (T) Val=2.32, Plg=65, Azm=11; (N) Val=0.05, Plg=4, Azm=110; (P) Val=-2.38, PIg=25, Azm=202; Best double couple: MO-2.3*10*18 Nm; NP1: Strike-301, Dip=21, Slip-102; NP2: Strike-109, Dip=70, Slip-86.

822 BANDA SEA

$7 \quad 12$ SPAIN mbLg 3.1 (MDD).

38 IRAN-IRAQ BORDER REGION. Felt at Marivan and Sanandaj, Iran.

66 SOUTHERN ALASKA. LAEIC>.

46 ALASKA PENINSULA. 〈AEIC>

75 SOLOMON ISLANDS

25 SOUTHERN CALIFORNIA. 〈PAS-P>. MD 3.3 (PAS). ME 3.3 (GS)

13 EASTER ISLAND REGION

6 SOLOMON ISLANDS

45 MESTERN MEDITERRANEAN SEA. mbLg 3.8 (MDD).

29 OFF E. COAST OF S. ISIAND, N.Z.

8 HALMAHERA, INDONESIA

5 MARIANA ISLANDS

5 RINDO KOSH REGION, AFGHANISTAN

34 RED SEA

6 OFF EAST COAST OF HONSHU, JAPAN

12 POLAND. MI 2.3 ( $\mathrm{MOX}$ ).

11 KORIL ISLANDS

11 MACQUARIE ISLANDS REGION

360 BASTER ISLAND REGION. NW 6.9 (HRV), 6.7 (GS). Me 6.4 (GS) Ms 7.0 (BRR). Local tsunami generated with maximum recorded wave heights (peak-to-trough) of $18 \mathrm{~cm}$ on Easter Island. Broddband Source Parameters (GS): Radiated energy $9.8 * 10 * 13$ $\mathrm{Nm}$.

Moment Tensor (GS): Dep 5; Principal axes (scale 10**19 Nm): (T) Val=1.38, Plg-66, Azm=11; (N) Val=0.11, Plg=1, Azm-279; (P) Val=-1.48, Plg-24, Azm-189; Best double couple: yo-1.4*10*19 Nm; NP1: Strike-277, Dip=21, S1ip-87; NP2 : Strike-99, Dip-69, Slipe-91.

Centrold, Moment Tensor (HRV): Centrold origin time $08: 14: 27.1$; Lat $22.32 \mathrm{~s}$; Lon $113.28 \mathrm{w}$; Dep 16.0 Fix; Halfduration $6.1 \mathrm{sec;}$ Principal axes (scale 10**19 Nm): (T) Val-1.84, Plg-75, Azm=282; (N) Val=0.65, Plg=15, Azm=109 (P) Val=-2.50, Plg=2, Azm=18; Best double couple: No-2.2*10**19 Nm; NP1: Strike-93, Dip=45, S1ip=69; NP2: Strike-303, Dip-49, S1ip=110.

Scalar Moment (PPT): MO=1.7*10**19 Nm.

32 CALIFORNIA-NEVADA BORDER REGION. NL 3.7 (GS). 0.6 11 CALIFORNIA-NEVADA BORDER REGION. ML 3.1 (GS). $4.6 \quad 0.7 \quad 23$ EASTER ISLAND REGION

$5.6 \quad 1.0 \quad 94$ EASTER ISLAND REGION

7 TANIMBAR ISLANDS REG., INDONESIA

215 EASTER ISIAND REGION. MW 6.2 (HRV). MS 6.1 (BRK).

Centroid, Moment Tensor (HRV): Centroid origin time $09: 47: 08.8$; Lat $21.94 \mathrm{~s}$; Lon $112.98 \mathrm{w}$; Dep $15.0 \mathrm{Fix}$; Half duration $3.1 \mathrm{sec;}$ Principal axes (scale $10 * 18 \mathrm{Nm}):(T)$ Val-1.59, Plg-43, Azm-301; (N) Val=0.91, Plg=38, Azm=78 (P) Val=-2.50, Plg-23, Azm=188; Best double couple: MO-2.0*10**18 Nm; NP1: Strike-324, Dip=41, Slip-162; NP2: Strike-68, Dip=78, Slip=51.

$05 \quad 0955 \quad 00.2 ? \quad 54.50$ s $148.53 \quad$ E 102511.7 ? 6.81 $105926.9 * 6.760 \mathrm{~s}$ $113323.7 * 36.376 \mathrm{~N}$ $\begin{array}{llll}12 & 52 & 03.1 & 10.330 \mathrm{~s}\end{array}$ $\begin{array}{lllll}12 & 54 & 20.1 ? & 26.02 & S\end{array}$ 76.05 W $72.810 \mathrm{E}$ $139.431 \mathrm{E}$ $161.154 \mathrm{E}$ $\begin{array}{llllll}15 & 26 & 40.8 & 0.399 & \end{array}$

$10 \mathrm{G} \quad 4.3 \quad 1.5$ $33 \mathrm{~N} 4.0$ $10 \mathrm{G} \quad 4.7$ $10 \mathrm{G}$ $33 \mathrm{~N}$ $500 \mathrm{G}$ $33 \mathrm{~N}$
1.2

0.9 5.14 .40 .7 $4.5 \quad 1.1$
WEST OF MACQUARIE ISLAND

NORTHERN COLOMBIA

29 CEAGOS ARCHIPEIAGO REGION

5 EASTERN HONSHU, JAPAN

47 SOLOMON ISLANDS. Felt at Honiara.

17 SOUTH OF FIJI ISILANDS

71 MINABASSA PENINSULA, SOLAMESI 
97 MINAHASSA PENINSULA, SULAWESI. MW 5.7 (HRV).

Centroid, Moment Tensor (BRV): Centroid origin time

$15: 36: 32.2$; Iat $0.71 \mathrm{~N}$; Lon $120.66 \mathrm{E}$; Dep 24.2; Half-

duration $1.5 \mathrm{sec}$; Principal axes (scale 10*17 $\mathrm{Nm}$ ): (T) Val-4.21, Plg-18, Azm=299; (N) Val=-0.89, Plg=11, Azm=206

(P) Val=-3.32, Plg=69, Azm=85; Best double couple: Mo-3. $* 10 \star \star 17 \mathrm{Nm}$; NP1: Strike=46, Dip $=29$, Slip $=-67 ;$ NP2 : Strike $=200$, Dip -63 , Slip $=-102$

$\begin{array}{llllcccc}05 & 15 & 43 & 37.0 & 0.338 & \mathrm{~N} & 120.228 & \mathrm{E} \\ 05 & 15 & 43 & 54.5 ? & 13.12 & \mathrm{~S} & 167.10 & \mathrm{E} \\ 05 & 16 & 21 & 25.0 ? & 0.10 & \mathrm{~N} & 120.95 & \mathrm{E} \\ 05 & 16 & 24 & 16.2 \& & 36.107 & \mathrm{~N} & 117.861 & \mathrm{~W} \\ & & & & & & & \\ 05 & 17 & 07 & 09.9 & 2.075 & \mathrm{~N} & 127.449 & \mathrm{E} \\ 05 & 17 & 16 & 56.9 ? & 36.97 & \mathrm{~N} & 141.51 & \mathrm{E} \\ 05 & 17 & 24 & 24.4 & 37.573 & \mathrm{~N} & 1.533 & \mathrm{~W} \\ 05 & 17 & 45 & 48.1 * & 0.266 & \mathrm{~N} & 120.337 & \mathrm{E} \\ 05 & 18 & 39 & 12.3 ? & 32.93 & \mathrm{~S} & 178.52 & \mathrm{~W} \\ 05 & 20 & 18 & 33.8 * & 49.333 & \mathrm{~N} & 158.514 & \mathrm{E} \\ 05 & 20 & 44 & 09.2 & 42.803 & \mathrm{~N} & 17.936 & \mathrm{E}\end{array}$

$211226.6 ? 12.65 \mathrm{~N}$ $21 \quad 1758.2 \quad 42.753 \mathrm{~N}$ $212221.0 \quad 35.999 \mathrm{~s}$

$\begin{array}{lllll}21 & 41 & 46.8 & 42.850 \mathrm{~N}\end{array}$ $\begin{array}{lllll}21 & 43 & 31.1 & 42.826 & \mathrm{~N}\end{array}$ 214409.1 ? $32.60 \mathrm{~s}$ $22 \quad 4249.8 * 25.103 \mathrm{~s}$ $2244 \quad 43.7 * 13.000 \mathrm{~N}$ $225243.1 * 13.866 \mathrm{~N}$ $233929.0 * 42.853 \mathrm{~N}$ $\begin{array}{llll}23 & 42 & 06.1 & 21.898 \mathrm{~N}\end{array}$
$143.95 \mathrm{E}$ $17.881 \mathrm{E}$

$17.983 \mathrm{E}$ $17.845 \mathrm{E}$ 178.68 W $179.371 \mathrm{E}$ $121.017 \mathrm{E}$ $120.462 \mathrm{E}$ $17.810 \mathrm{E}$ $121.498 \mathrm{E}$ $73.010 \mathrm{~W}$
$33 \mathrm{~N} 4.3$

$10 \mathrm{G}$

$20 \mathrm{G} \quad 4.6$

$10 \mathrm{G}$

$10 \mathrm{G} \quad 4.9$

$33 \mathrm{~N} 4.8$

$600 \mathrm{G} 4.3$

$33 \mathrm{~N} 5.0$

$33 \mathrm{~N} 4.6$

$10 \mathrm{G}$

$20 \mathrm{G}$
1.134

1.2

6 VANUATO ISLANDS

CALIFORNIA-NEVADA BORDER REGION. 〈PAS-P>. MD 3.4 (PAS). ML 3.5 (GS).

0.523 NORTHERN MOLUCCA SEA

1.06 NEAR EAST COAST OF HONSHO, JAPAN

0.6

1.1

1.2

0.9 1.3360
19

12 360
SPAIN. mbLg 3.4 (MDD). Felt (III) in the Mazarron area.

EAST OF RURIL ISLANDS

ADRIATIC SEA. MW 6.0 (HRV), 5.7 (GS). Me 5.7 (GS). Ms 6.1
SOUTH OF RERMADEC ISLANDS (BRR). Several people injured, 2,000 left homeless and extensive damage (VIII) in the ston-slano area, Croatia. Felt (VI) at terceg-Novi, Kotor and Tivat; (V) at Bar Budva, Niksic and Podgorica, Yugoslavia. Felt (V) in the shkoder area, Albania and (IV) at Skopje, former Yugoslav Republic of Macedonia. Felt in many parts of Bosnia and Herzegovina, Croatia and Yugoslavia.

Broadband Source Parameters (GS): Dep 12; NP1: Strike=285, Dip-55, SIip=30; NP2: Strike-177, Dip-66, Slip=141; Radiated energy $8.2 * 10 * * 12 \mathrm{Nm}$.

Moment Tensor (GS): Dep 13; Principal axes (scale 10**17 $\mathrm{N}(\mathrm{m}):$ (T) $\mathrm{VaI}=3.31, \mathrm{PIg}=68, \mathrm{Azm}=122$; (N) $\mathrm{Val}=0.13, \mathrm{Plg}=22$, Azm-308; (P) Val=-3.44, PIg-2, Azm-217; Best double couple: MO=3.4*10*17 Nm; NPI: Strike $=286$, Dip $=47$, Slip $=60$; NP2: Strike=146, Dip=51， Siip=118.

Centroid, Moment Tensor (BRV): Centroid origin time $20: 44: 17.3$; Lat $42.78 \mathrm{~N}$; Lon $17.77 \mathrm{E}$; Dep $15.0 \mathrm{Fix}$; Halfduration $2.5 \mathrm{sec}$; Principal axes (scale 10**18 $\mathrm{Nm}$ ): (T)

Val=1.23, Plg=77, $A 2 m=53$; (N) Val=-0.05, Plg-1, Azm=146; (P) Val=-1.18, Plg=13, Azm=236; Best double couple: Mo-1.2*10**18 Nm; NP1: Strike-328, Dip-32, slip=92; NP2: Strike-146, Dip=58, Slip=89.

SODTH OF MARIANA ISLANDS

$\begin{array}{rrr}1.0 & 7 & \text { SODTH OF MARIA } \\ 1.3 & 35 & \text { ADRIATIC SEA } \\ 0.7 & 19 & \text { OEF COAST OE }\end{array}$

OFF COAST OF CENTRAL CHILE. MD 4.6 (SAN). Felt (IV) at Cauquenes, Chanco, Cobquecura, Pelluhue and Quirihue.

$\begin{array}{rrl}1.0 & 17 & \text { ADRIATIC SEA } \\ 1.3 & 152 & \text { ADRIATIC SEA }\end{array}$

$1.3 \quad 152$ ADRIATIC SEA

1.314 SOOTH OF RERMADEC ISLANDS

0.820 SOUTH OF FIJI ISLANDS

1.019 MINDORO, PEILIPPINE ISIANDS

1.021 MINDORO, PAILIPPINE ISIANDS

1.210 ADRIATIC SEA

TAIWAN REGION. Iuzon, Phil

Broadband Source Parameters (GS): Dep 17; NP1: Strike=235, Dip=40, S1ip=120; NP2: Strike=18, Dip=56, S1ip=67; Radiated energy 4.9*10*14 Nm. Two events about 6 seconds apart. Depth based on second event.

Moment Tensor (GS): Dep 21; Principal axes (scale 10*19 $\mathrm{Nm}$ ): (T) Val=1.14, Plg=66, $\mathrm{Azm}=212$; (N) Val=0.01, Plg=24, Azm=43; (P) Val=-1.15, Plg-4, Azm-312; Best double couple: Mo-1.1*10*19 Nm; NP1: Strike=18, Dip=46, Slip=56; NP2: strike $=242$, Dip $=53$, siip -120 .

Centroid, Moment Tensor (HRV): Centroid origin time $23: 42: 16.6$; Lat $21.78 \mathrm{~N}$; Lon $121.45 \mathrm{E}$; Dep 30.8 ; Halfduration $6.4 \mathrm{sec}$; Principal axes (scale 10**19 $\mathrm{Nm}$ ): (T) Val=1.84, Plg=77, Azm=227; (N) Val=0.14, Plg=12, Azm=20; (P) Val=-1.98, Plg-6, Azm-111; Best double couple: Mo-1.9*10*19 Nm; NP1: Strike=214, Dip=41, Slip=108; NP2 : Strike=11, Dip $=52$, Slip=75

scalar Moment (PPT): MO=4.5*10**19 Nm

$\begin{array}{lllllll}23 & 55 & 13.8 & 21.462 & \mathrm{~N} & 121.159 & \mathrm{E} \\ 00 & 26 & 01.1 & 21.608 & \mathrm{~N} & 121.421 & \mathrm{E} \\ 00 & 31 & 15.7 & 42.796 & \mathrm{~N} & 17.811 & \mathrm{E} \\ 00 & 37 & 36.6 * & 21.631 & \mathrm{~N} & 121.430 & \mathrm{E} \\ 00 & 55 & 34.7 ? & 21.53 & \mathrm{~N} & 121.39 & \mathrm{E} \\ 00 & 57 & 50.0 * & 21.735 & \mathrm{~N} & 121.209 & \mathrm{E} \\ 01 & 13 & 19.3 * & 21.766 & \mathrm{~N} & 121.257 & \mathrm{E} \\ 01 & 14 & 18.5 & 42.654 & \mathrm{~N} & 17.872 & \mathrm{E} \\ 01 & 25 & 45.7 & 21.608 & \mathrm{~N} & 121.319 & \mathrm{E} \\ 01 & 30 & 38.8 & 42.896 & \mathrm{~N} & 17.799 & \mathrm{E} \\ 01 & 30 & 47.9 * & 6.665 & \mathrm{~N} & 126.252 & \mathrm{E} \\ 02 & 04 & 54.7 & 21.718 & \mathrm{~N} & 121.386 & \mathrm{E} \\ 02 & 11 & 28.28 & 37.574 & \mathrm{~N} & 1.556 & \mathrm{~N} \\ 02 & 20 & 29.4 & 21.638 & \mathrm{~N} & 121.397 & \mathrm{E} \\ 02 & 22 & 04.6 & 21.669 & \mathrm{~N} & 121.429 & \mathrm{E} \\ 02 & 35 & 51.3 ? & 15.44 & \mathrm{~N} & 93.17 & \mathrm{~N} \\ 02 & 47 & 39.6 ? & 6.62 & \mathrm{~N} & 123.14 & \mathrm{E} \\ 02 & 59 & 42.5 & 42.832 & \mathrm{~N} & 17.837 & \mathrm{E} \\ 03 & 31 & 52.2 & 42.812 & \mathrm{~N} & 17.853 & \mathrm{E} \\ 03 & 46 & 54.5 & 42.874 & \mathrm{~N} & 17.697 & \mathrm{E}\end{array}$

$20 \mathrm{G} \quad 4.6$

$20 \mathrm{G} \quad 5.2$

$10 \mathrm{G} \quad 4.2$

$20 \mathrm{G} \quad 4.3$

$20 \mathrm{G} \quad 4.1$

20 G 4.3

$20 \mathrm{G} \quad 4.2$

$10 \mathrm{G} \quad 4.4$

$20 \mathrm{G} \quad 5.0$

$10 \mathrm{G}$

$33 \mathrm{~N} 4.7$

$20 \mathrm{G} \quad 5.4$

$10 \mathrm{G}$

$20 \mathrm{G} 5.1$

$20 \mathrm{G} \quad 4.9$

$33 \mathrm{~N} \quad 3.8$

$600 \mathrm{G} \quad 4.7$

$10 \mathrm{G}$

$10 \mathrm{G} 4.4$

$\begin{array}{llll}03 & 46 & 54.5 & 42.874\end{array}$

$10 \mathrm{G}$

$\begin{array}{rr}0.7 & 16 \\ 0.9 & 72 \\ 1.1 & 54 \\ 1.0 & 20 \\ 1.3 & 11 \\ 0.9 & 13 \\ 0.8 & 13 \\ 1.1 & 69 \\ 1.2 & 44 \\ 1.2 & 25 \\ 0.9 & 17 \\ 0.9 & 118 \\ 0.9 & 10 \\ 1.2 & 60 \\ 1.2 & 16 \\ 1.2 & 12 \\ 1.1 & 12 \\ 1.2 & 44 \\ 1.2 & 99 \\ 1.1 & 72\end{array}$

TAIWAN REGION

TAIWAN REGION

ADRIATIC SEA. MI 3.6 (ROM)

TAIWAN REGION

TAIWAN REGION

TAIWAN REGION

TAIWAN REGION

ADRIATIC SEA

TAIWAN REGION

ADRIATIC SEA. ML 3.2 (ROM).

MINDANAO, PEILIPPINE ISIAANDS

TAIWAN REGION

SPAIN. mbLg 2.9 (MDD)

TAIWAN REGION

TAIWAN REGION

MINDANAO, PEILIPPINE ISLANDS

ADRIATIC SEA. ML 3.3 (ROM)

ADRIATIC SEA. ML 4.1 (ROM).

ADRIATIC SEA. ML 3.7 (ROM).
NEAR COAST OF CHIAPAS, MEXICO 
$06 \quad 035056.4858 .252$ N 154.283 พ $\begin{array}{llllll}06 & 04 & 02 & 08.1 & 42.916 \mathrm{~N} & 18.016 \mathrm{E}\end{array}$ $\begin{array}{lllllllll}06 & 04 & 14 & 41.7 ? & 21.73 & \text { N } 121.15 & \text { E }\end{array}$ $\begin{array}{lllllll}06 & 05 & 11 & 59.1 & 42.850 \mathrm{~N} & 17.910 \mathrm{E}\end{array}$ $06 \quad 0528 \quad 20.2 * 21.687$ N $121.319 \mathrm{E}$ $\begin{array}{llllllll}06 & 05 & 51 & 17.93 & 2.37 & N & 127.92 & E\end{array}$ $\begin{array}{lllllll}06 & 06 & 00 & 13.1 & 5.477 \mathrm{~S} & 147.986 \mathrm{E}\end{array}$ $\begin{array}{llllllll}06 & 06 & 03 & 50.38 & 45.488 & \text { N } & 26.553 \mathrm{E}\end{array}$ $\begin{array}{llllll}06 & 06 & 07 & 13.8 & 21.680 \mathrm{~N} & 121.279 \mathrm{E}\end{array}$ $\begin{array}{lllllll}06 & 06 & 17 & 35.3 \text { ? } 31.67 \text { S } 58.12 \mathrm{E}\end{array}$ $06064810.5 * 21.670 \mathrm{~N} 121.346 \mathrm{E}$ $06 \quad 06 \quad 55 \quad 24.5 * 21.590 \mathrm{~N} 121.329 \mathrm{E}$ $\begin{array}{llllll}06 & 07 & 41 & 13.78 & 44.256 \mathrm{~N} & 7.436 \mathrm{E}\end{array}$ $06075658.4 * 21.726 \times 121.463 \mathrm{E}$ $\begin{array}{llllllllll}06 & 08 & 00 & 04.7 & 42.906 & \mathrm{~N} & 17.857 \mathrm{E}\end{array}$ $\begin{array}{llllllllll}06 & 08 & 03 & 45.9 & 23.79 & S & 179.88 & E\end{array}$ $\begin{array}{lllllll}06 & 08 & 06 & 41.0 & 50.659 \mathrm{~N} & 157.104 \mathrm{E}\end{array}$ $06 \quad 0814 \quad 20.2 * 6.698$ S $147.414 \mathrm{E}$ $\begin{array}{lllllllllll}06 & 08 & 23 & 12.8 ? & 21.94 & N & 121.19 & E\end{array}$ $\begin{array}{llllllll}06 & 08 & 24 & 36.8 & 21.511 & \mathrm{~N} & 121.473 \mathrm{E}\end{array}$ $\begin{array}{llllllll}06 & 08 & 54 & 15.8 & 5.102 & \mathrm{~S} & 153.979 \mathrm{E}\end{array}$

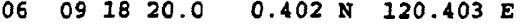
$06 \quad 095033.3844 .259 \mathrm{~N} \quad 7.457 \mathrm{E}$ $06 \quad 10 \quad 13 \quad 25.9 * 35.905 \mathrm{~N} 140.087 \mathrm{E}$ $06 \quad 105710.1 \% 33.070$ s $70.426 \mathrm{~W}$ $06 \quad 11 \quad 10 \quad 48.4 \quad 42.833 \mathrm{~N} \quad 17.915 \mathrm{E}$

$\begin{array}{lllllllll}06 & 11 & 14 & 09.98 & 44.250 \mathrm{~N} & 8.518 \mathrm{E}\end{array}$ $121.440 \mathrm{E}$ $06 \quad 04 \quad 4203.2 * 1.524 \mathrm{~N} 124.808 \mathrm{E}$ $\begin{array}{llllllll}06 & 06 & 17 & 51.3 & 21.683 & \mathrm{~N} & 121.386 & \mathrm{E}\end{array}$ $\begin{array}{llllll}06 & 07 & 27 & 11.3 & 21.681 \mathrm{~N} & 121.358 \mathrm{E}\end{array}$
75

$10 \mathrm{G}$

$20 \mathrm{G} 4$

$33 \times 4.9$

10

$20 \in 4.0$

$33 \times 4.2$

250 G 4.7

$100 \mathrm{G}$

20 G 4.5

$10 G \quad 4.2$

20 G $4.6 \quad 4.4$

$20 G \quad 4.3$

$20 \mathrm{G} \quad 4.3$

$20 G \quad 4.7$

$10 \mathrm{G}$

$20 \mathrm{G}$

100

$500 \mathrm{G} 4.4$

$33 \mathrm{~N} 4.8$

$33 \mathrm{~N} 4.3$

20 G 4.0

$20 G \quad 4.9$

$400 \mathrm{G} 4.7$

33 N 4.9

$10 \mathrm{G}$

$33 \mathrm{~N}$

$100 \mathrm{G}$

$10 \mathrm{G}$

$10 \mathrm{G}$

$20 \mathrm{G}$
PAGE 6

34 ALASTA PENTNSUTA <AEIC>.

1.422 NORTEWESTERN BALKAN REGION

1.67 TAIWAN REGION

1.226 MINABASSA PENINSULA, SULAWESI

1.341 ADRIATIC SEA. ML 3.5 (LTO)

1.08 TAIWAN REGION

1.06 NORTHERN MOLUCCA SEA

0.824 EASTERN NEW GUINEA REG., P.N.G.

0.87 ROMANIA

0.931 TAIWAN REGION

0.6

1.2

0.8

31

33 TAIWAN REGION

8 TAIWAN REGION

13 TAIWAN REGION

22 TAIWAN REGION

6 NORTHERN ITALY. ML 2.1 (GEN).

15 TAIWAN REGION

61 ADRIATIC SEA. ML 3.7 (ROM).

10 SOUTE OF FIJI ISLANDS

80 KURIL ISLANDS. Felt (III) at Severo-Kurilsk.

7 EASTERN NEW GUINEA REG., P.N.G.

TAIWAN REGION

2 TAIWAN REGION

40 NEW IRELAND REGION, P.N.G.

59 MINAHASSA PENINSULA, SUTAWESI

8 NORTHERN ITALY. MI 2.3 (GEN).

6 NEAR EAST COAST OF HONSHU, JAPAN

11 CHILE-ARGENTINA BORDER REGION. MD 3.4 (SAN).

58 ADRIATIC SEA

8 NORTHERN ITALY. ML 2.3 (GEN)

167

TAIWAN REGION. MW 5.4 (HRV).

Centroid, Moment Tensor (HRV): Centroid origin time

$11: 34: 33.3$; Lat $21.75 \mathrm{~N}$; Lon $121.38 \mathrm{E}$; Dep $15.0 \mathrm{Fix}$; Half duration $1.1 \mathrm{sec}$; Principal axes (scale 10*17 Nm): (T) Val=1.48, Plg=69, $\mathrm{Azm}=13$; (N) Val=-0.20, $\mathrm{Plg}=18, \mathrm{Azm}=160$; (P) Val=-1.27, Plg=12, Azm=254; Best double couple: mo-1 4*10*\#17 Nm; NP1: Strike=5, Dip-38, Slip-120; NP2: Strike-149, Dip-58, Slip-69.

$06 \quad 11.43 \quad 00.3 ? \quad 21.84 \quad \mathrm{~N} \quad 121.13 \quad \mathrm{E}$ $06 \quad 1200013.2 ? \quad 9.61 \quad 5 \quad 119.32 \quad E$ $06 \quad 1213 \quad 39.4$ ? 3.01 N 127.00 $06 \quad \begin{array}{llllll}12 & 36 & 56.2 & 27.809 & \mathrm{~N} & 52.417 \mathrm{E}\end{array}$ $\begin{array}{llllllll}06 & 12 & 39 & 41.4 & 7.271 & \mathrm{~S} & 155.824 & \mathrm{E}\end{array}$
$20 \mathrm{G} \quad 4.0$ $33 \mathrm{~N} \quad 4.2$ $33 \times 4.2$ $33 \times 4.5$ $33 \mathrm{~N} 5.55 .80 .8$
7 TAIWAN REGION

9 SUMBA REGION, INDONESIA

8 TALAOD ISILANDS, INDONESIA

47 SOUTHERN IRAN 208

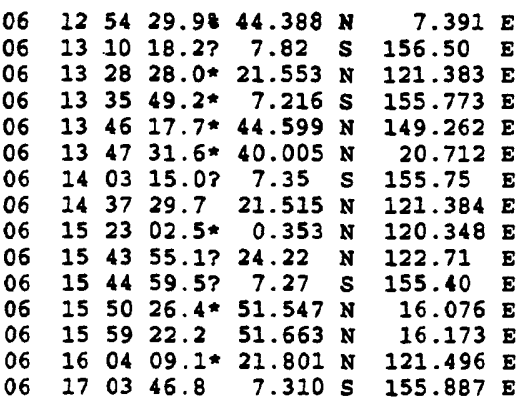

$10 \mathrm{G}$

$33 \mathrm{~N} 3.8$

$20 \mathrm{G} \quad 4.4$

$33 \mathrm{~N} 4.2$

$33 \mathrm{~N} 4.0$

$10 \mathrm{G}$

$33 \mathrm{~N} 4.3$

$20 \mathrm{G} \quad 4.6$

$33 \mathrm{~N} 4.6$

$33 \mathrm{~N}$

$33 \mathrm{~N}$

$10 \mathrm{G}$

$10 \mathrm{G}$

$20 \mathrm{G}$
0.3

0.3
0.4
1.0

1.0

0.9

1.5

0.6

1.0

1.3
0.9
0.6

0.6

0.4

0.5

1.0
SOLONON ISLANDS. MW 5.9 (HRV), 5.8 (GS). Me 5.6 (GS). Broadband Source Parameters (GS): Dep 17; NPI: Strike-150, Dip=55, SIip=75; NP2: Strike-355, Dip-38, slip=110; Radiated energy $4.8 * 10 * 12 \mathrm{Nm}$.

moment Tensor (GS): Dep 19; Principal axes (scale 10*\#17 Nim): (T) Val-5.31, Plg=60, Azm=64; (N) Val=-0.36, Plg=15, Azm-305; (P) Val=-4.95, Plg=25, Azm=208; Best double Couple: Mo-5.1*10*17 Nm; NP1: Strike-267, Dip=24, Slip=49; NP2: Strike-130, Dip-72, slip-106.

Centroid, Moment Tensor (GRV): Centroid origin time

$12: 39: 43.3$; Lat $7.37 \mathrm{~s}$; Lon $155.84 \mathrm{E}$; Dep $20.0 \mathrm{Fix}$; Halfduration $2.1 \mathrm{sec}$; Principal axes (scale 10**17 Nm): (T) val-6.80, Plg-69, Azm=13; (N) Val-0.08, Plg=8, Azm=123; (P) val=-6.88, PIg=20, Azm-216; Best double couple: Mo-6.8*10*17 Nm; NP1: Strike=319, Dip=26， Slip=108; NP2 : Strike-120, Dip-65, Slip-81.

7 NORTEERN ITALY. ML 2.0 (GEN)

6 SOLOMON ISLANDS

15 TAIWAN REGION

16 SOLOMON ISLANDS

15 KURIL ISLANDS

16 GREECE-ALBANIA BORDER REGION

6 SOLOMON ISIANDS

8 TAIWAN REGION

29 MINAEASSA PENINSULA, SULAWESI

7 TAIWAN REGION

7 SOLOMON ISLANDS

7 POLAND. ML 2.8 (MOX), 2.7 (CLL).

13 POLAND. ML 2.8 (MOX), 2.7 ( 2.7 (CLL).

4 TAIWAN REGION

(BRK)

Broadband Source Parameters (GS): Dep 18; NPI: Strike-126, Dip=71, Slip=110; NP2: Strike-258, Dip=27, S1ip=45; Radiated energy $6.8 * 10 * 12 \mathrm{Nm}$.

Monent Tensor (GS): Dep 19; Principal axes (scale 10*\#18 Nm): (T) Val-1.75, Plg=60, Azm=69; (N) Val=0.02, Plg=19, A2m-303; (P) Val=-1.76, PIg=23, A2m=204; Best double couple: Mo=1.8*10*18 Nm; NP1: Strike=262, Dip=28, Slip=46; NP2: Strike=130, Dip=70, Slip-110.

Centroid, koment Tensor (HRV): Centroid origin time $17: 03: 52.0$; Lat $7.50 \mathrm{~S}$; Lon $155.84 \mathrm{E}$; Dep 15.0 Fix; Halfduration $3.3 \mathrm{sec}$; Principal axes (scale 10**18 $\mathrm{Nm}$ ): (T) Val=2.53, Plg=71, Azm=11; (N) Val=0.12, P1g=3, Azm=111; (P) Val=-2.65, PIg=19, Azm=202; Best double couple:

MO=2.6*10**18 Nm; NPI: Strike-298, Dip=26, Slip=98; NP2: Strike-109, Dip=64, Slip=86.

Scalar Moment (PPT): MO=1.4*10**18 $\mathrm{km}$.

$\begin{array}{llllllllllllll}06 & 17 & 08 & 55.2 ? & 7.27 & 5 & 155.68 & \text { E } & 33 & \mathrm{~N}\end{array}$

$06 \quad 17 \quad 1757.8 * 7.343$ s 155.821 E . 33 N 4.8

1.18 SOLONON ISLANDS

1.142 SOLONON ISLANDS

0.210 CHILE-ARGENTINA BORDER REGION. MD 3.1 (SAN). 


$\begin{array}{llllllll}17 & 34 & 08.0 ? & 21.55 & \mathrm{~N} & 121.35 & \mathrm{E} \\ 17 & 56 & 07.9 ? & 16.94 & \mathrm{~S} & 179.34 & \mathrm{~W} \\ 18 & 04 & 40.9 * & 7.284 & \mathrm{~S} & 155.767 & \mathrm{E} \\ 18 & 22 & 28.3 & 42.800 & \mathrm{~N} & 17.923 & \mathrm{E} \\ 18 & 41 & 00.9 & 21.784 & \mathrm{~N} & 121.404 & \mathrm{E} \\ 18 & 53 & 19.7 & 0.484 & \mathrm{~N} & 120.500 & \mathrm{E} \\ 19 & 09 & 54.8 * & 21.958 & \mathrm{~N} & 121.635 & \mathrm{E} \\ 19 & 13 & 41.8 & 7.246 & \mathrm{~S} & 155.933 & \mathrm{E} \\ 19 & 14 & 55.0 ? & 7.37 & \mathrm{~S} & 156.10 & \mathrm{E} \\ 19 & 39 & 36.5 ? & 7.28 & \mathrm{~S} & 155.76 & \mathrm{E} \\ 19 & 48 & 07.0 & 42.818 & \mathrm{~N} & 17.910 & \mathrm{E} \\ 19 & 51 & 42.5 ? & 7.22 & \mathrm{~S} & 155.57 & \mathrm{E} \\ 20 & 06 & 51.9 & 5.566 & \mathrm{~S} & 149.786 & \mathrm{E} \\ 20 & 13 & 44.8 & 42.842 & \mathrm{~N} & 17.883 & \mathrm{E} \\ 20 & 38 & 18.38 & 25.650 & \mathrm{~N} & 124.684 & \mathrm{E} \\ 20 & 42 & 29.58 & 40.395 & \mathrm{~N} & 126.408 & \mathrm{~W}\end{array}$

$20 \mathrm{G} \quad 3.9$

$500 \mathrm{G} \quad 4.4$

$33 \mathrm{~N} 4.4$

$10 \mathrm{G}$

$20 \mathrm{G} \quad 5.1$

$33 \times 4.8$

$20 \mathrm{G} 3.9$

$33 \mathrm{~N} 5.35 .0$

$33 \mathrm{~N} 4.9$

$33 \mathrm{~N} 4.0$

$10 \mathrm{G}$

$33 \mathrm{~N}$

$150 \mathrm{G}$

$10 \mathrm{G}$

$33 \mathrm{~N}$

10

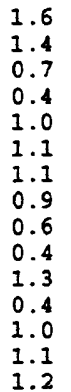

4.95 .1

5 TAIWAN REGION

9 FIJI ISLANDS REGION

10 SOLOMON ISLANDS

9 ADRIATIC SEA. MC 3.2 (ROM).

65 TAIWAN REGION

34 MINAEASSA PENINSULA, : SULAWESI

10 TAIWAN REGION

83 SOLOMON ISLANDS

10 SOLOMON ISLANDS

8 SOLOMON ISLANDS

47 ADRIATIC SEA. MI 3.6 (ROM).

8 SOLOMON ISLANDS

60 NEW BRITAIN REGION, P.N.G.

13 ADRIATIC SEA

5 NORTHEAST OF TAIWAN

195 OFF COAST OF NORTHERN CALIFORNIA. 〈GM-P). MW 5.5 (BRV). MD 4.5 (GM). ML 4.9 (BRK).

Centroid, Moment Tensor (HRV): Centroid origin time

$20: 42: 35.0$; Lat $40.42 \mathrm{~N}$; Ion $126.36 \mathrm{~W}$; Dep $15.0 \mathrm{Fix}$; Halfduration $1.3 \mathrm{sec}$; Principal axes (scale 10*17 Nm): (T) Val-2.17, Plg=6, Azm=48; (N) Val=-0.27, Plg=81, Azm=175; (P) Val=-1.90, Plg=8, Azm=318; Best double couple: MO-2.0*10**17 Nm; NP1: Strike-93, Dip-81, Slip=-179; NP2: Strike-3, Dip=89, Slip=-9.

31 SOLOMON ISLANDS

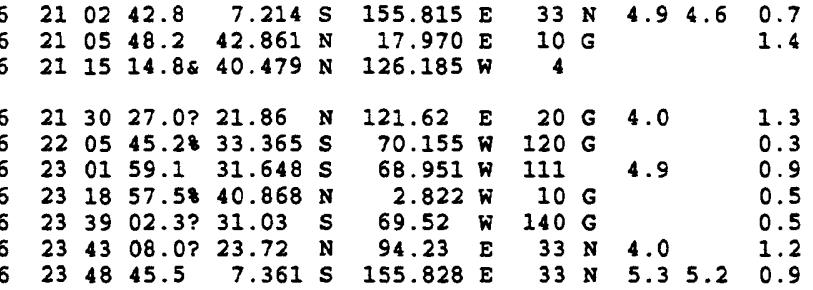

19 ADRIATIC SEA. ML 3.3 (ROM).

62 OFF COAST OF NORTHERN CALIFORNIA. LGM-P>. MD 3.3 (GM). ML 3.9 (GS).

5 TAIWAN REGION

10 CHILE-ARGENTINA BORDER REGION. MD 2.7 (SAN).

69 SAN JUAN PROVINCE, ARGENTINA. ND 4.7 (SAN).

7 SPAIN. MbIg 2.6 (MDD).

12 SAN JUAN PROVINCE, ARGENTINA. MD 3.8 (SAN).

9 MYANOAR-INDIA BORDER REGION

129 SOLOMON ISLANDS. 5.6 (BRV)

Centroid, Moment Tensor (BRV): Centroid origin time

$23: 48: 48.4$; Lat $7.52 \mathrm{~S}$; Lon $155.83 \mathrm{E}$; Dep 18.4 ; Half-

duration $1.5 \mathrm{sec}$; Principal axes (scale 10*17 $\mathrm{Nm}$ ): (T)

val=2.53, Plg=70, Azm-342; (N) val=-0.30, P1g=13, Azm-110;

(P) Val=-2.23, Plg=16, Azm=204; Best double couple: Mo=2.4*10**17 Nm; NP1: Strike-312, Dip=31, Slip-115; NP2: Strike-103, Dip-62, Silp=75.

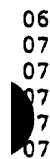

$\begin{array}{llllll}23 & 59 & 22.1 & 21.801 \mathrm{~N} & 121.294 \mathrm{E}\end{array}$ $002210.2 * 21.878 \times 121.490 \mathrm{E}$ $0027 \quad 53.4 \quad 21.813 \mathrm{~N} \quad 121.469 \mathrm{E}$ $015751.7 \quad 21.884 \mathrm{~N} 121.440 \mathrm{E}$ $022933.3 * 36.610 \mathrm{~N} 9.687$ W $0246 \quad 12.9 ? \quad 2.98$ N $126.93 \quad \mathrm{E}$ $\begin{array}{llllll}07 & 02 & 55 & 30.3 & 20.520 \mathrm{~S} & 174.360 \mathrm{~W}\end{array}$

$\begin{array}{lllll}20 & G & 4.4 & & 1.0 \\ 20 & G & 4.4 & & 1.5 \\ 20 & G & 5.0 & & 1.2 \\ 20 & G & 4.7 & & 1.2 \\ 10 & G & & & 1.0 \\ 33 & N & 4.0 & & 1.4 \\ 36 & D & 5.0 & 5.0 & 1.0\end{array}$

$\begin{array}{llll}36 & 5.0 & 5.0 & 1.4\end{array}$ $\begin{array}{llll}03 & 5713.9 \quad 42.929 & N\end{array}$ $040514.7832 .936 \mathrm{~S}$ 040949.3 ? $7.23 \mathrm{~S}$ $\begin{array}{lllll}04 & 17 & 56.0 & 42.870 \mathrm{~N}\end{array}$ 042311.2 ? $31.75 \mathrm{~s}$ $\begin{array}{lllll}04 & 25 & 39.4 ? & 23.55 & \mathrm{~S} \\ 04 & 29 & 55.2 & 7.240 & \mathrm{~S}\end{array}$
$17.761 \mathrm{E}$ 70.292 155.39 $17.762 \mathrm{~B}$ 179.14 $179.70 \mathrm{~B}$ $155.660 \mathrm{E}$
$10 \mathrm{G}$ $100 \mathrm{G}$ $33 \mathrm{~N}$ $10 \mathrm{G}$ $33 \mathrm{~N}$ $600 \mathrm{G}$

21
18
41
32
43
11
91

21 TAIWAN REGION

TAIWAN REGION

TAIWAN REGION

2 TAIWAN REGION

NORTHERN MOLOCCA SEA

TONGA ISLANDS. MW 5.3 (HRV)

Centroid, Moment Tensor (HRV): Centroid origin time

$02: 55: 34.7$; Lat $20.92 \mathrm{~S}$; Lon $173.80 \mathrm{~K}$; Dep $15.0 \mathrm{Fix}$; Half duration $1.1 \mathrm{sec}$; Principal axes (scale 10**17 Nm): (T) Val-1.02, Plg=70, Azm-298; (N) Val-0.20, Plg=0, Azm=207 (P) Val--1.22, Plg-20, Azm-117; Best double couple: Mo-1.1*10**17 Nm; NPI: Strike-206, Dip=25, S1ip=89; NP2: Strike-27, Dip-65, Slip-90.

38 ADRIATIC SEA

0.210 CHILE-ARGENIINA BORDER REGION. MD 2.9 (SAN).

4.6

6 SOLOMON ISLANDS

144 ADRIATIC SEA. ML 4.2 (ROM). Felt in the Ston area, Croatia. 6 KERMADEC ISLANDS REGION

10 SOUTH OF FIJI ISLANDS

61 SOLOMON ISLANDS. MW 5.1 (BRV).

Centroid, Moment Tensor (BRV): Centroid origin time 04:30:04.1; Lat 7.29 S; Lon 155.57 E; Dep 21.0; Halfduration $1.0 \mathrm{sec}$; Principal axes (scale 10**16 $\mathrm{Nm}$ ): (T) Val-7.37, Plg=71, Azm=237; (N) Val=-2.94, Plg=16, Azm=90; (P) Val--4.43, Plg-10, Azm-357; Best double couple: Mo-5.9*10*16 Nm; NP1: Strike-68, Dip-38, Slip-63; NP2 : Strike-281, Dip=57, Slip=110. 051732.8838 .198 $052020.3 * 7.322$ $052023.4 * 11.729 \mathrm{~S}$ $053825.8 \& 59.442 \mathrm{~N}$ $054157.7 \quad 51.664 \mathrm{~N}$ $\begin{array}{lllll}05 & 45 & 33.8 & 42.902\end{array}$ $\begin{array}{llll}05 & 53 & 26.3 * & 9.886 \mathrm{~S} \\ 06 & 22 & 30.3 * & 4.980 \mathrm{~S}\end{array}$ $06 \quad 43 \quad 49.5 \& 40.481 \mathrm{~N}$ $06 \quad 58 \quad 10.8 ? 20.58 \quad s$ $\begin{array}{lllll}07 & 19 & 47.23 & 22.07 \mathrm{~N}\end{array}$

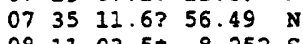
$081103.5 * 8.252 \mathrm{~S}$ $081525.6860 .550 \mathrm{~N}$ $\begin{array}{lllllll}08 & 15 & 33.5 & 21.731 & \mathrm{~N}\end{array}$ $090149.6 * 42.330 \mathrm{~N}$ $\begin{array}{lllll}09 & 06 & 00.8 \quad 7.178 \mathrm{~S}\end{array}$
$139.377 \mathrm{E} 33$

$155.978 \mathrm{E} \quad 33 \mathrm{~N}$

$33.085 \mathrm{E} 10$

$16.112 \mathrm{E} \quad 10 \mathrm{G}$

$17.811 \mathrm{E} \quad 10 \mathrm{G} \quad 4.5$

$119.178 \mathrm{E} \quad 33 \mathrm{~N} 4.6$

129.428 E $200 \mathrm{G} 4.7$

$126.078 \mathrm{~W}$

$174.30 \mathrm{~W}$

$121.94 \mathrm{E}$

$161.58 \mathrm{E}$

$121.177 \mathrm{E}$

$153.082 \mathrm{~W}$

$121.473 \mathrm{E}$
$17.678 \mathrm{E}$

$155.676 \mathrm{E}$
$153.352 \mathrm{~W} 116$
0

$33 \times 4.1$

$20 \mathrm{G} \quad 4.3$

$33 \mathrm{~N}$

$33 \mathrm{~N}$

141

20 G 4.7

$10 \mathrm{G}$

$\begin{array}{rrrr}5.4 & 5.6 & 0.8 & 15 \\ 1.0 & 141\end{array}$
1.254 ADRIATIC SEA. MC 3.8 (ROM).

0.85 NEAR WEST COAST OF HONSEO, JAPAN

0.713 SOLOMON ISLANDS

1.399 MALAWI

37 SOOTHERN ALASKA. LAEIC>

1.114 POLAND. ML 2.7 (MOX), 2.5 (CLL)

1.3154 ADRIATIC SEA. MI 4.3 (ROM). Felt in the Ston area, Croatia.

1.412 SOMBA REGION, INDONESIA

$1.1 \quad 17$ BANDA SEA

39 OFF COAST OF NORTHERN CALIFORNIA. 〈GM-P>. MD 3.0 (GM).

0.511 TONGA ISLANDS

0.26 TAIWAN REGION

1.45 NEAR EAST COAST OF KAMCHATKA

1.013 FLORES REGION, INDONESIA

53 SOUTHERN ALASKA. LAEIC>

20 TAIWAN REGION

ADRIATIC SEA

SOLOMON ISLANDS. MW 5.8 (BRV), 5.7 (GS)

Moment Tensor (GS): Dep 20; Principal axes (scale 10**17 $\mathrm{Nm}$ ): (T) Val=3.62, PIg=70, Azm-40; (N) Val-0.51, Plg-1, Azm-132; (P) Val=-4.13, Pig=20, Azm=223; Best double couple: Mo-3.9*10*17 Nm; NP1: Strike-314, Dip=25, Slip-92; NP2: Strike-132, Dip-65, Slip-89. 


$\begin{array}{lllllcccc}07 & 09 & 11 & 08.2 ? & 26.85 & \mathrm{~S} & 26.82 & \mathrm{E} \\ 07 & 09 & 14 & 45.4 & 7.214 & \mathrm{~S} & 155.612 & \mathrm{E} \\ 07 & 09 & 20 & 44.5 * & 12.271 & \mathrm{~N} & 125.466 & \mathrm{E} \\ 07 & 09 & 55 & 56.2 * & 16.250 & \mathrm{~N} & 97.882 & \mathrm{~W} \\ 07 & 10 & 04 & 12.2 ? & 7.26 & \mathrm{~S} & 155.64 & \mathrm{E} \\ 07 & 10 & 30 & 26.2 & 42.850 & \mathrm{~N} & 17.767 & \mathrm{E} \\ 07 & 10 & 31 & 58.4 & 32.653 & \mathrm{~S} & 178.530 & \mathrm{~W}\end{array}$

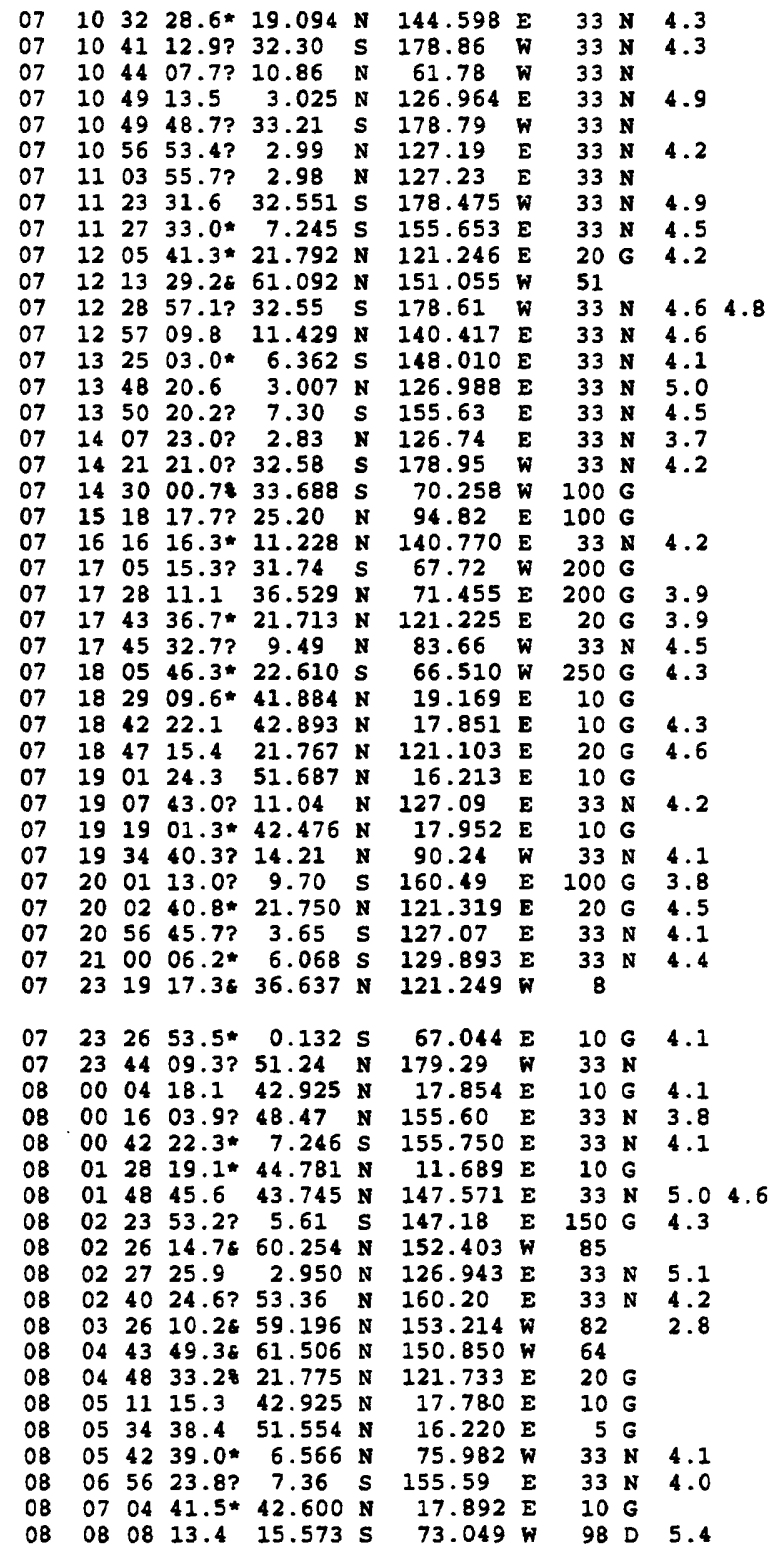

$\begin{array}{rrrrr}5 & G & 3.9 & & 1.5 \\ 33 & \mathrm{~N} & & & 0.9 \\ 33 & \mathrm{~N} & 4.9 & & 1.0 \\ 33 & \mathrm{~N} & 4.4 & & 1.0 \\ 33 & \mathrm{~N} & 4.0 & & 1.3 \\ 10 & \mathrm{G} & & 1.2 \\ 33 & \mathrm{~N} & 5.0 & 5.2 & 1.1\end{array}$

Centroid, Moment Tensor (HRV): Centroid origin time 09:06:08.2; Lat 7.45 S; Ion 155.78 E; Dep 17.0 Fix; Half duration $2.0 \mathrm{sec}$; Principal axes (scale 10*17 $\mathrm{Nm}$ ): (T) Val=6.12, $\mathrm{plg}=68, \mathrm{Azm}=7$; (N) Val=-0.25, $\mathrm{Plg}=8, \mathrm{~A}=1 \mathrm{~m}=16$; (P) Val=-5.87, Plg=21, Azm=209; Best double couple:

Mo-6.0*10*17 Non; NP1: Strike-313, Dip-25, Slip=108; NP2: Strike=113, Dip=66, S1ip-82.

6 REPUBLIC OF SOUTR AFRICA

15 SOLOMON ISLANDS

27 SAMAR, PEILIPPINE ISLANDS

30 OAXACA, MEXICO

8 SOLONON ISLANDS

54 ADRIATIC SEA. MD 3.6 (ROM).

48 SOUTE OF KERMADEC ISLANDS. HW 5.5 (HRV)

Centroid, Moment Tensor (HRV): Centroid origin time 10:32:09.0; Lat $31.85 \mathrm{~s}$; Ion 178.58 $\mathrm{W}$; Dep 35.4; Halfduration $1.0 \mathrm{sec}$; Principal axes (scale 10*17 Nm): (T) Val=1.62, $\mathrm{Plg}=47, \mathrm{Azm}=341$; (N) Val=0.39, $\mathrm{Plg}=31, \mathrm{Azm}=211$; (P) Val=-2.01, Plg=26, Azm-103; Best double couple: Mo=1.8*10*17 Nm; NP1: Strike=146, Dip=34, SIIp=22; NP2:

Strike-38, Dip=78, Slip-122.

1.316 MARIANA ISIANDS

0.810 SOUTH OF KERMADEC ISLANDS

0.2

1.2

1.2
0.9
0.6

1.3

0.9

1.3

1.0

1.0

1.2

1.0

0.6

1.2

0.6

0.1

0.5

1.1

0.5
0.7

0.7

1.2

1.4
1.1

1.2

1.3

1.2

1.0
1.1

0.9

0.9

0.7
1.4

1.1

1.5

1.3

TRINIDAD. ID 3.0 (TRN) .

6 TALAUD ISLANDS, INDONESIA

8 SOUTH OF KERMADEC ISLANDS

9 NORTHERN MOLUCCA SEA

5 NORTHERN MOLUCCA SEA

23 SOUTH OF KERMADEC ISLANDS

25 SOLONON ISLANDS

8 TAINAN REGION

52 SOUTHERN ALASKA. <AEIC>. ML 2.5 (AEIC).

14 SOUTH OF KERMADEC ISLANDS

17 WESTERN CAROLINE ISLANDS

12 NEW BRITAIN REGION, P.N.G.

43 TALAUD ISLANDS, INDONESIA

6 SOLOMON ISLANDS

8 NORTHERN MOLUCCA SEA

10 SOUTE OF KERMADEC ISLANDS

10 CHILE-ARGENTINA BORDER REGION. MD 2.5 (SAN).

10 MPANMAR-INDIA BORDER REGION

11 WESTERN CAROLINE ISLANDS

11 SAN JOAN PROVINCE, ARGENTINA. MD 4.1 (SAN).

25 AFGBANISTAN-TAJIKISTAN BORD REG.

13 TAIWAN REGION

12 COSTA RICA

16 JUJOY PROVINCE, ARGENTINA

10 ALBANIA

37 ADRIATIC SEA

25 TAIWAN REGION

14 POLAND. ML 2.5 (MOX), 2.3 (CLL).

14 PEILIPPINE ISIANDS REGION

12 ADRIATIC SEA

6 GOATEMALA

12 SOLOMON ISLANDS

26 TAIWAN REGION

8 SERAM, INDONESIA

13 BANDA SEA

96 CENTRAL CALIFORNIA. LGM-P>. MD 3.7 (GM). ML 3.8 (BRK), 3.8 (GS). Felt at salinas.

1.014 CARLSBERG RIDGE

0.855 ANDREANOF ISLANDS, ALEUTIAN IS.

$\begin{array}{lrl}0.8 & 5 & \text { ANDREANOF ISLAR } \\ 1.0 & 28 & \text { ADRIATIC SEA }\end{array}$

1.211 KURIL ISLANDS

1.013 SOLOMON ISLANDS

$\begin{array}{rrr}0.5 & 14 & \text { NORTEERN ITALY. ML } 3.1 \text { (VIE), } 2.9 \text { (LDG). } \\ 0.9 & 103 & \text { KURIL ISLANDS. Felt (II) at Yuzhno-KuIilsk. }\end{array}$

1.1 11 EASTERN NEW GUINEA REG., P.N.G.

19 SOUTHERN ALASKA. <AEIC>.

1.144 NORTHERN MOLUCCA SEA

0.811 NEAR EAST COAST OF RAMCHATKA

82 SOUTHERN ALASKA. LAEIC>.

71 SOUTHERN ALASKA. CAEIC>. MI 2.9 (AEIC).

1.37 TAIWAN REGION

$1.0 \quad 38$ ADRIATIC SEA. Felt slightly at ston, croatia.

0.612 POLAND. MU 3.0 (MOX).

1.011 NORTHERN COLOMBIA

1.215 SOLOMON ISLANDS

$0.8 \quad 20$ ADRIATIC SEA

1.0207 SOUTHERN PERO. MW 5.6 (GS), 5.6 (HRV). Felt (IV) at Mollendo and (III) at Arequipa and Camana.

Moment Tensor (GS): Dep 103; Principal axes (scale 10**17

$\mathrm{Nm}$ ): (T) Val-3.91, Plg=9, Azm=74; (N) Val=-1.49, PIg=0, Azm=344; (P) Val=-2.42, Plg=81, A2m=254; Best double couple: Mo-3.2*10**17 Nm; NP1: Strike-164, Dip=36, Slip--90; NP2: Strike-344, Dip=54, slip=-90.

Centroid, Moment Tensor (HRV): Centroid origin time 08:08:18.6; Lat $15.74 \mathrm{~s}$; Lon $73.18 \mathrm{~W}$; Dep 121.6; Halfduration $1.4 \mathrm{sec;}$ Principal axes (scale 10*17 Nm): (T) Val=2.91, Plg=11, Azm=74; (N) Val=0.33, Plg=7, Azm=165; (P) val=-3.24, Plg=77, Azm=288; Best double couple:

Mo=3.1*10**17 Nm; NP1: Strike-155, Dip=35, Slip=-103; NP2: strike=350, Dip=56, Silp=-81. 


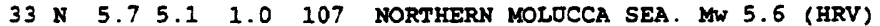

Centroid, Moment Tensor (HRV): Centroid origin time

$08: 12: 49.5$; Lat $2.86 \mathrm{~N}$ Fix; Lon 127.25 E Fix; Dep 33.0 Fix; half-duration 2.3 sec; principal axes (scale 10*\#17 Nm):

(T) Val=2.63, Plg=45, $A z m=210$; (N) Val=0.79, Plg=16,

Azm-317; (P) Val=-3.42, Plg=41, Azm=62; Best double couple: Mo-3.0*10*17 Nm; NP1: Strike-219, Dip-17, Slip=172; NP2: Strike=317, Dip=88, Slip=74.

$\begin{array}{llllllllll}08 & 09 & 32 & 53.2 & 42.864 & \mathrm{~N}\end{array}$

$113458.5 \quad 19.610$

$114251.8=19.520 \mathrm{~S}$

179.141

179.097 พ
$10 \mathrm{G} \quad 4.2$

$400 \mathrm{G} \quad 4.0$

$33 \mathrm{~N} 4.2$

$33 \mathrm{~N}$

$\begin{array}{lll}33 & N & 4.2\end{array}$

$10 \mathrm{G} \quad 4.5$

$60 \quad 4.9$
1.185 ADRIATIC SEA MI 3.9 (ROM)

1.216 FIJI ISILANDS REGION

1.499 CERAM SEA

0.99 PAKISTAN

1.015 ANDRBANOF ISLANDS, ALEUTIAN IS.

0.79 PACIFIC-ANTARCTIC RIDGE

0.9132 ANDREANOF ISLANDS, ALEUTIAN IS. WW 5.1 (HRV)

Centroid, Moment Tensor (ARV): Centroid origin time

11:07:18.2; Lat $51.63 \mathrm{~N}$; Lon $176.56 \mathrm{w}$; Dep 38.3 ; Half-

duration 1.0 sec: Principal axes (scale 10**16 Nm): (T)

Val-4.02, Plg-31, Azm=278; (N) Val-2.01, Plg-40, Azm-39; (P) Val=-6.03, P1g-34, Azm-164; Best double couple:

Mo-5. 0*10**16 Nm; NP1: Strike-312, Dip=40, Slip=-177; NP2:

Strike-221, Dip-88, slipm-50.

0.8146 FIJI ISIANDS REGION

1.215 FIJI ISLANDS REGION

1.3161 FIJI ISLANDS REGION. MW 5.6 (ERV).

$600 \mathrm{G} \quad 3.7$

600 G 4.9
67.076 W $146.425 \mathrm{~W}$ $143.719 \mathrm{E}$ 75.56 $127.646 \mathrm{E}$ $17.838 \mathrm{E}$ 152.349 $126.12 \mathrm{E}$ $70.509 \mathrm{~W}$ $179.910 \mathrm{E}$ 148.027 $96.389 \mathrm{E}$ $122.932 \mathrm{E}$ $153.004 \mathrm{~W}$ $6.200 \mathrm{E}$ $121.328 \mathrm{E}$ $17.831 \mathrm{E}$ $138.706 \mathrm{E}$ $17.863 \mathrm{E}$ $121.452 \mathrm{E}$ $17.878 \mathrm{E}$ $179.56 \quad \mathrm{E}$ 117.494 $27.25 \mathrm{E}$ $162.439 \mathrm{E}$ $130.647 \mathrm{E}$ $71.560 \mathrm{~W}$ 179.10 $17.246 \mathrm{E}$

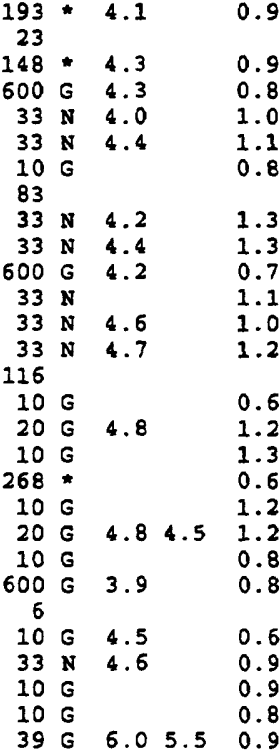

0.9

Centroid, Moment Tensor (ERV): Centroid origin time

11:43:04.0; Lat 19.72 S Fix; Lon 179.10 W Fix; Dep 684.4 Fix; Half-duration $1.3 \mathrm{sec}$; Principal axes (scale 10*17 Nim): (T) Val=2.74, Plg=68, Azm=166; (N) Val=0.01, Plg=8, Azm=56; (P) Val=-2.75, Plg-20, Azm-323; Best double couple: Mo-2.7*10*17 Nm; NP1: Strike-39, Dip-26, S1ip-72; NP2 : Strike-239, Dip=65, Slip=99.

0.926 SOUTH OF MARIANA ISLANDS

0.811 FIJI ISLANDS REGION

9 NORTEERN COLOMBIA

1.113 CERAM SEA

. 12 ADRIATIC SEA

103 SODTHERN ALASKA. 〈AEIC>.

1.312 PEILIPPINE ISLANDS REGION

1.317 . NEAR COAST OF NORTHERN CHILE

0.720 SOUTE OF FIJI ISLANDS

1.18 KORIL ISLANDS

1.049 MYANMAR

25 NEGROS, PEILIPPINE ISLANDS

48 SOUTHERN ALASKA. <AEIC>.

FRANCE. ML 1.8 (LDG).

TAIWAN REGION

ADRIATIC SEA

EASTERN SEA OF JAPAN

ADRIATIC SEA. MI 3.3 (ROM).

TAIWAN REGION

ADRIATIC SEA. MI 3.0 (ROM).

SOUTH OF FIJI ISLANDS

CENTRAL CALIFORNIA. LPAS-P>. MD 3.1 (PAS). MI 3.0 (GS).

SOUTH OF AFRICA

NEAR EAST COAST OF KAMCHATKA

KYUSHD, JAPAN

ADRIATIC SEA

NEAR COAST OF CENTRAL CHILE. MW 6.0 (GS), 6.0 (ERV), We 6.1

(GS). MS 5.4 (BRK). Some adobe houses damaged in the epicentral area. Felt (V) at Ia Calera, La Ligua, Los Andes, Los Vilos, Papudo, Quillota, Quintero, Valparaiso, Vina del Mar and zapallar; (IV) at San Antonio and

Santiago; (III) at La Serena. Felt (III) at Mendoza and San Juan, Argentina. Also felt (II) by people in high-rise buildings at Cordoba, Argentina.

Broadband Source Parameters (GS): Dep 40; NP1: Strike-351, Dip=52, Slip=63; Ne2: Strike=211, Dip=45, Slip=120;

Radiated energy $3.1 * 10 * * 13 \mathrm{Nm}$.

Homent Tensor (GS): Dep 42; Principal axes (scale 10**18 $\mathrm{Nm}$ ): (T) Val=1.09, Plg=69, Azm=175; (N) Val=0.07, Plg=20, Azm=6; (P) Val=-1.15, Plg=3, Azm=274; Best double couple: Mo-1.1*10*18 Nm; NP1: Strike-344, Dip=45, S1ip=61; NP2: Strike=203, Dip=52, Slip=116.

Centroid, Moment Tensor (ARV): Centroid origin time

00:20:45.2; Iat $32.06 \mathrm{~S}$; Lon $71.94 \mathrm{~W}$; Dep $49.0 \mathrm{Fix}$; Ealfduration $2.4 \mathrm{sec}$; Principal axes (scale 10**18 $\mathrm{Nm}$ ): (T) Val=0.92, Plg=66, Azm=156; (N) Val-0.11, Plg-21, Azm=2; (P) Val=-1.03, Plgm10, Azm-268; Best double couple:

Mo=9.7*10**17 Nm; NP1: Strike-334, Dip=40, Slip=55; NP2 :

Strike-196, Dip-58, Slip=116.

scalar yoment (PPT): Mo-1.1*10**18 Nm.

$09011419.6842 .876 \mathrm{~N} \quad 18.088 \mathrm{E} \quad 10 \mathrm{G}$ NORTHWESTERN BAIKAN REGION. ML 3.9 (ROM) FLORES REGION, INDONESIA

48 NEAR COAST OF PERO. Felt (IV) at Camana; (III) at Aplao, Arequipa and Mollendo; (II) at Atico.

\subsection{TAIWAN}

121.547
KORIL ISLANDS

ADRIATIC SEA

12 TAIWAN REGION

SOUTEERN PACIFIC OCEAN. NW 5.8 (ERV). 


$\begin{array}{lllllllrllllll}09 & 03 & 22 & 36.1 * & 24.767 & \mathrm{~S} & 179.878 & \mathrm{E} & 501 & \mathrm{D} & 4.5 & 0.9 & 23 \\ 09 & 04 & 11 & 42.0 & 42.864 & \mathrm{~N} & 17.692 & \mathrm{E} & 10 & \mathrm{G} & & & 1.3 & 31 \\ 09 & 04 & 29 & 13.0 & 45.132 & \mathrm{~N} & 6.508 & \mathrm{E} & 10 & \mathrm{G} & & & 0.3 & 9 \\ 09 & 04 & 34 & 20.5 & 30.434 & \mathrm{~N} & 130.726 & \mathrm{E} & 33 & \mathrm{~N} & 5.5 & 5.2 & 1.1 & 220\end{array}$

$\begin{array}{lllllclrl}04 & 40 & 07.8 ? & 31.61 & \mathrm{~S} & 179.31 & \mathrm{E} & 500 & \mathrm{G} \\ 06 & 05 & 14.4 ? & 40.61 & \mathrm{~N} & 35.66 & \mathrm{E} & 10 & \mathrm{G} \\ 07 & 02 & 16.3 & 44.310 & \mathrm{~N} & 6.440 & \mathrm{E} & 10 & \mathrm{G} \\ 07 & 13 & 04.2 ? & 3.24 & \mathrm{~S} & 139.21 & \mathrm{E} & 33 & \mathrm{~N} \\ 07 & 33 & 57.3 * & 49.666 & \mathrm{~S} & 127.291 & \mathrm{E} & 10 & \mathrm{G} \\ 08 & 04 & 47.8 & 42.828 & \mathrm{~N} & 17.808 & \mathrm{E} & 10 & \mathrm{G} \\ 08 & 06 & 35.2 * & 3.388 & \mathrm{~S} & 101.065 & \mathrm{E} & 33 & \mathrm{~N} \\ 08 & 13 & 23.68 & 44.482 & \mathrm{~N} & 6.864 & \mathrm{E} & 10 & \mathrm{G} \\ 09 & 32 & 11.3 & 21.217 & \mathrm{~S} & 179.167 & \mathrm{~W} & 625 & \mathrm{D}\end{array}$

4.

3.9

4.2

4.5

4.6

5.0

Centroid, Moment Tensor (HRV): Centroid origin time $03: 21: 53.2$; Lat $35.56 \mathrm{~s}$; Ion $104.50 \mathrm{~W}$; Dep $15.0 \mathrm{Fix}$; Halfduration $2.0 \mathrm{sec}$; Principal axes (scale 10*17 Nm): (T) Val-5.99, Plg-6, Azm-52; (N) Val=-0.15, Plg-77, Azm-169; (P) Val=-5.84, Plg-11, Azm=321; Best double couple: Mo-5.9*10*17 Nm; NP1: Strike-97, Dip=78, Slip=-176; NP2: Strike-6, Dip-86, SIip -12 .

SOUTH OF FIJI ISLANDS

ADRIATIC SEA. ML 3.3 (ROM).

FRANCE. ML 2.1 (GEN), 2.0 (IDG).

KYUSHU, JAPAN. MW 5.7 (BRV). Slight damage on Tanega-shima. Felt (IV JMA) at Sumiyoshi, Tanega-shima and (III JMA) on Kuchinoerabu-jima and Yaku-shima. Felt (II JMA) at Kagoshima, Miyakonojo and Miyezaki, Kyushu.

Centroid, Moment Tensor (HRV): Centroid origin time $04: 34: 21.7$; Lat $30.52 \mathrm{~N}$; Lon $130.81 \mathrm{E}$; Dep 25.5 ; Half duration $1.8 \mathrm{sec}$; Principal axes (scale 10**17 Nm): (T) Val-3.55, Plg=2, Azm-227; (N) Val-1.92, Plg-13, Azm-137; (P) Val--5.47, PIg-76, Azm-325; Best double couple: Mo-4.5*10**17 $\mathrm{Nm}_{i}$ NP1: Strike-331, Dip-45, Slip=-71; NP2: strike-125, Dip-48, Slip=-108.

1.011 KERMADEC ISLANDS REGION

1.07 TORKEY. Felt in Amasya and corum.

0.322 FRANCE. ML 2.4 (GEN), 2.3 (LDG).

1.27 IRIAN JAYA, INDONESIA

1.220 SOUTH OF AUSTRALIA

1.464 ADRIATIC SEA

1.126 SOOTEERN SUMATERA, INDONESIA

0.46 FRANCE. ML. 2.3 (GEN)

1.093 FIJI ISLANDS RBGION. MW 5.4 (BRV).

Centroid, Moment Tensor (ARV): Centroid origin time $09: 32: 18.8$; Lat $21.27 \mathrm{~s}$; Lon $179.30 \mathrm{w}$; Dep 622.2 ; Halfduration $1.1 \mathrm{sec}$; Principal axes (scale 10*17 $\mathrm{Nm}$ ): (T) Val=1.36, Plg=55, Azm-144; (N) Val--0.11, Plg-0, Azm-54; (P) Val--1.25, Plg-35, Azm-324; Best double Couple: Mo-1.3*10*17 Nm; NP1 : Strike-52, Dip-10, Slip-88; NP2 : strike-234, Dip=80, Slip-90.

$\begin{array}{lllllll}09 & 09 & 32 & 29.1 & 42.657 & \mathrm{~N} \\ 09 & 10 & 24 & 11.6 ? & 5.21 & \mathrm{~S} \\ 09 & 10 & 33 & 58.5 * & 2.291 & \mathrm{~N} \\ 09 & 10 & 11 & 43.68 & 36.632 & \mathrm{~N} \\ 09 & 11 & 23 & 14.4 ? & 2.10 & \mathrm{~S} \\ 09 & 11 & 51 & 26.8 & 42.716 & \mathrm{~N} \\ 09 & 12 & 05 & 52.28 & 38.597 & \mathrm{~N} \\ 09 & 12 & 23 & 03.2 & 43.650 & \mathrm{~N} \\ 09 & 13 & 55 & 53.1 ? & 22.19 & \mathrm{~S} \\ 09 & 14 & 02 & 18.88 & 44.360 & \mathrm{~N} \\ 09 & 14 & 24 & 50.68 & 60.021 & \mathrm{~N} \\ 09 & 14 & 46 & 59.88 & 60.481 & \mathrm{~N} \\ 09 & 15 & 38 & 21.8 & 12.557 & \mathrm{~N} \\ 09 & 15 & 57 & 05.1 & 42.774 & \mathrm{~N}\end{array}$
$17.625 \mathrm{E}$ $152.83 \mathrm{E}$ $126.618 \mathrm{E}$ $139.452 \mathrm{E}$ $119.56 \mathrm{E}$ $17.272 \mathrm{E}$ $6.951 \mathrm{~W}$ $147.255 \mathrm{E}$ $169.97 \mathrm{E}$ $7.330 \mathrm{E}$ $153.108 \mathrm{~W}$ $150.982 \mathrm{~W}$ $88.194 \mathrm{~W}$ $17.873 \mathrm{E}$
$10 \mathrm{G}$

$33 \times 4.0$

$33 \mathrm{~N} 4.4$

$10 \mathrm{G}$

$33 \mathrm{~N}$

$10 \mathrm{G}$

$10 \mathrm{G}$

15.

$100 \mathrm{G} 4.2$

$10 \mathrm{G}$

52 D 4.8

$10 \mathrm{G}$
0.9

1.8

1.1

0.7

1.3

0.9

0.4

0.9

1.5
0.4

67
59

1.1

12 ADRIATIC SEA. MG 3.5 (SKO)

8 NEW BRITAIN REGION, P.N.G.

5 NORTHERN MOLUCCA SEA

5 EASTERN BONSHO, JAPAN

16 SULAWESI, INDONESIA

10 ADRIATIC SEA

5 SPAIN. mbLg 2.9 (NDD).

0 KURII ISLANDS. Felt (II) on Shikotan.

11 LOYALTY ISLANDS REGION

8 NORTHERN ITALY. ML 2.1 (GEN).

7 SOUTHERN ALASRA. CAEIC>. ML 3.1 (AEIC).

KENAI PENINSDLA, ALASKA. <AEIC). ML 2.5 (AEIC)

OFF COAST OF CENTRAL AMERICA. Felt (III) at san salvador, El Salvador.

ADRIATIC SEA. MW 5.3 (HRV). MU 5.2 (VIE), 4.8 (ROM). Felt (V) at Herceg-Novi, Kotor and Tivat, Yugoslavia.

Centrojd, Moment Tensor (HRV): Centroid origin time $15: 57: 08.7$; Lat $43.03 \mathrm{~N}$; Ion $17.55 \mathrm{E}$; Dep 15.0 Fix; Halfduration $1.0 \mathrm{sec}$; Principal axes (scale 10*17 Nm): (T) Val-1.01, Plg-55, Azm=72; (N) Val-0.20, Plg-7, Azm-332; (P) Val=-1.21, PIg-34, Azm=238; Best double couple: MO=1.1*10*17 Nm; NP1: Strike-301, Dip-13, Slip-58; NP2: strike-154, Dip=79, Slip=97.

$09 \begin{array}{llll}16 & 21 & 07.6 * 42.571 \mathrm{~N}\end{array}$

$17.344 \mathrm{E}$ $17.616 \mathrm{E}$ $17.910 \mathrm{E}$ $110.812 \mathrm{~W}$ $143.697 \mathrm{E}$ $17.707 \mathrm{E}$ $93.769 \mathrm{E}$ $17.751 \mathrm{E}$ $20.433 \mathrm{E}$ $42.926 \mathrm{~W}$ $17.797 \mathrm{E}$ $143.712 \mathrm{E}$ $128.09 \mathrm{E}$ $79.97 \mathrm{~W}$ $69.981 \mathrm{E}$ $128.636 \mathrm{~W}$ $126.593 \mathrm{E}$ $126.159 \mathrm{E}$ $56.777 \mathrm{E}$ $22.715 \mathrm{E}$ $139.026 \mathrm{E}$ $12.068 \mathrm{E}$ $126.625 \mathrm{E}$ $76.576 \mathrm{~W} 111$ $120.098 \mathrm{E}$

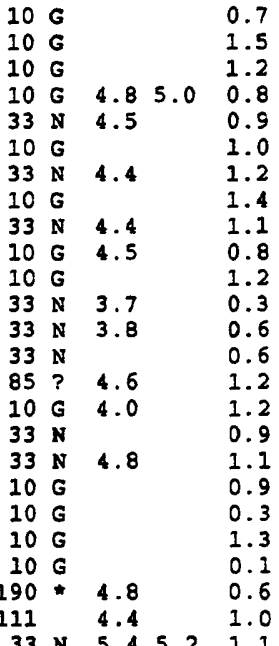

7 ADRIATIC SEA

8 ADRIATIC SEA

44 ADRIATIC SEA. MU 4.1 (VIE).

26 SOUTHERN EAST PACIFIC RISE

32 VOLCANO ISIANDS REGION

13 ADRIATIC SEA

6 NICOBAR ISIANDS, INDIA

22 ADRIATIC SEA. MI 3.3 (ROM).

37 IONIAN SEA

35 WESTERN GREENLAND

22 ADRIATIC SEA. ML 3.1 (ROM).

VOLCANO ISLANDS REGION

HALMAEERA, INDONESIA

10 NEAR COAST OF NORTHERN PERO

13 GINDD KUSE REGION, AFGHANISTAN

12 VANCOUVER ISLAND REGION

11 NORTEERN MOLOCCA SEA

39 MINDANAO, PHILIPPINE ISLANDS

aRABIAN SEA

7 GREECE

5 EASTERN BONSHO, JAPAN

6 NORTHERN ITALY. ML 2.2 (VIE).

13 BANDA SEA

25 COIOMBIA

94 MINAHASSA PENINSULA, SULAWESI. MW 5.8 (BRV)

Centroid, koment Tensor (HRV): Centroid origin time

$02: 34: 22.3$; Lat $1.43 \mathrm{~N}$; Ion $120.32 \mathrm{E}$; Dep $18.0 \mathrm{Fix}$; Half. duration 1.8 sec; Principal axes (scale 10*17 Nm): (T)

Val=6.03, Plg-60, Azm-177; (N) Val=-0.16, Plg=7, Azm=75 (P) Val--5.87, PIg-29, Azm-341; Best double couple: Mo-5.9*10*17 Nm; NP1: Strike-52, Dip-17, S1ip-66; NP2: Strike-257, Dip-74, SIip-97. 
$10045530.3 * 0.298 \mathrm{~N} \quad 121.710 \mathrm{E} \quad 100 \mathrm{G} \quad 4.4$ $\begin{array}{lllllllllll}10 & 05 & 09 & 25.8 & 45.436 \mathrm{~N} & 16.218 \mathrm{E} & 10 \mathrm{G} & 4.2\end{array}$

$\begin{array}{lllllllllllll}10 & 05 & 39 & 09.27 & 35.87 & \mathrm{~N} & 140.78 & \mathrm{E} & 10 \mathrm{G}\end{array}$ $\begin{array}{lllllll}10 & 06 & 20 & 53.2 & 1.119 \mathrm{~N} & 120.208 \mathrm{E}\end{array}$ $10 \quad 0542 \quad 37.9 * 13.880 \mathrm{~S} 75.573 \mathrm{~W}$

0.812 MINAHASSA PENINSULA, SULAWESI

1.1130 NORTHWESTERN BALKAN REGION. ML 4.7 (VIE) 4.6 (FUR) 4.3 (IUU), 4.1 (ROM), 4.0 (BRA). Some buildings damaged at Banovina, croatia. Felt (VII) at Petrinja and sisak; (VI) at zagreb, Croatia. Felt at Bjelovar, Koprivnica, Krizevci and as far as Dubrovnik, Croatia. Also felt in Slovenia.

NEAR EAST COAST OF HONSEO, JAPAN

$\begin{array}{rrl}0.1 & 5 & \text { NEAR EAST COAS } \\ 0.8 & 11 & \text { CENTRAL PERU }\end{array}$

$100 \mathrm{G} \quad 3.8, \quad 0.8$

WW 5.2 (HRV).

Moment Tensor (BRV): Centroid origin time

$06: 20: 59.2$; Lat $1.75 \mathrm{~N}$; Lon $120.58 \mathrm{E}$; Dep $33.0 \mathrm{Fix}$; Halfduration $1.1 \mathrm{sec}$; Principal axes (scale $10 * \star 16 \mathrm{Nm}$ ): (T) Val-8.97, PIg=65, Azm=199; (N) Val=-3.56, Plg=3, Azm=103 (P) val--5.42, Plg=25, Azm=11; Best double couple: Mo=7.2*10*16 Nm; NP1: Strike=95, Dip=21, Slip-82; NP2: strike-284, Dip $=70$, Silp $=93$. $\begin{array}{lllll}10 & 07 & 15 & 06.7835 .100 \mathrm{~N} \quad 117.493 \mathrm{~W}\end{array}$

$10 \quad 0743 \quad 15.8 ? \quad 43.94$ \& $147.21 \quad E$

$100748 \quad 40.9 * 20.658 \mathrm{~s}$

$10 \quad 083455.0 * 3.022 \mathrm{~N}$

$\begin{array}{llllll}10 & 08 & 52 & 59.1 & 2.942 & \mathrm{~N}\end{array}$

$\begin{array}{llllll}10 & 09 & 04 & 48.6 & 43.005\end{array}$

$10 \quad 0913 \quad 38.0 * 30.854 \mathrm{~N}$

$10 \quad 092937.0 * 2.838 \mathrm{~N}$

$\begin{array}{llllll}10 & 09 & 31 & 39.9 & 2.70 \quad \mathrm{~N}\end{array}$

$\begin{array}{llllll}10 & 10 & 12 & 15.73 & 60.53 \quad \mathrm{~s}\end{array}$

$\begin{array}{llllll}10 & 11 & 04 & 12.1 \% 58.442 \mathrm{~N} \\ 10 & 11 & 18 & 17.0 * 57.838 \mathrm{~s}\end{array}$

$\begin{array}{lllll}10 & 11 & 18 & 17.0 * & 57.838 \\ 10 & 11 & 33 & 56.2 * 21.642 \mathrm{~N}\end{array}$

$10 \quad 121724.73 \quad 35.15$ s

$10 \quad 124038.0 \quad 4.785 \mathrm{~N}$

$101323 \quad 39.7 * 7.493 \mathrm{~S}$

$\begin{array}{lllll}10 & 13 & 31 & 47.5 & 37.690 \mathrm{~N}\end{array}$

$\begin{array}{lllllll}10 & 13 & 34 & 23.88 & 31.769 & \mathrm{~s}\end{array}$

$\begin{array}{lllll}10 & 14 & 28 & 40.1827 .762 \mathrm{~N}\end{array}$

$\begin{array}{llllll}10 & 14 & 34 & 33.7 ? & 4.09\end{array}$

$10 \quad 14 \quad 38 \quad 54.5 \quad 7.943 \quad 5$

$10151611.1 * 0.993 \mathrm{~N}$

$10 \quad 155907.9 \quad 42.916 \mathrm{~N}$

$\begin{array}{lllll}10 & 16 & 02 & 00.0 * 13.902 \mathrm{~N}\end{array}$

$\begin{array}{lllllll}10 & 18 & 08 & 04.38 & 58.444 & \mathrm{~N}\end{array}$

$\begin{array}{lllllll}10 & 18 & 20 & 43.38 & 58.485 & \mathrm{~N}\end{array}$

$\begin{array}{lllllll}10 & 18 & 54 & 07.6 ? & 7.48 & \mathrm{~S} \\ 10 & 19 & 53 & 49.58 & 60.024 & \mathrm{~N}\end{array}$

$10 \quad 20 \quad 40 \quad 45.48 \quad 33.553 \mathrm{~N}$

$\begin{array}{lllllll}10 & 21 & 32 & 49.9 ? & 2.76 \mathrm{~s}\end{array}$

$\begin{array}{llllll}10 & 21 & 43 & 28.58 & 32.820 \mathrm{~S}\end{array}$

$\begin{array}{lllllll}10 & 22 & 54 & 50.3 & 42.764 & \mathrm{~N}\end{array}$

$\begin{array}{lllll}10 & 23 & 12 & 26.1859 .485 \mathrm{~N}\end{array}$

$\begin{array}{lllllll}10 & 23 & 13 & 56.28 & 9.682 & \mathrm{~N}\end{array}$

$\begin{array}{llllll}10 & 23 & 16 & 36.08 & 30.077 & \mathrm{~N}\end{array}$

$\begin{array}{lllllll}10 & 23 & 24 & 56.03 & 15.29 & \mathrm{~s}\end{array}$

$\begin{array}{lllllll}10 & 23 & 28 & 03.4 ? & 31.44 & \mathrm{~S}\end{array}$

$10232910.6 * 5.373 \mathrm{~S}$

$\begin{array}{lllllll}11 & 00 & 25 & 32.08 & 35.798 & \mathrm{~N} \\ 11 & 00 & 52 & 01.03 & 42.853 & \mathrm{~N}\end{array}$

$11022626.0 * 44.446 \mathrm{~N}$

$\begin{array}{lllllll}11 & 02 & 37 & 14.9 & 35.537 & \mathrm{~N}\end{array}$
$10 \quad 1010 \quad 20.3 * 7.145 \mathrm{~s}$

$10 \quad 1355 \quad 42.2 * 23.645 \mathrm{~s}$

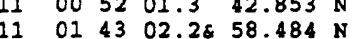

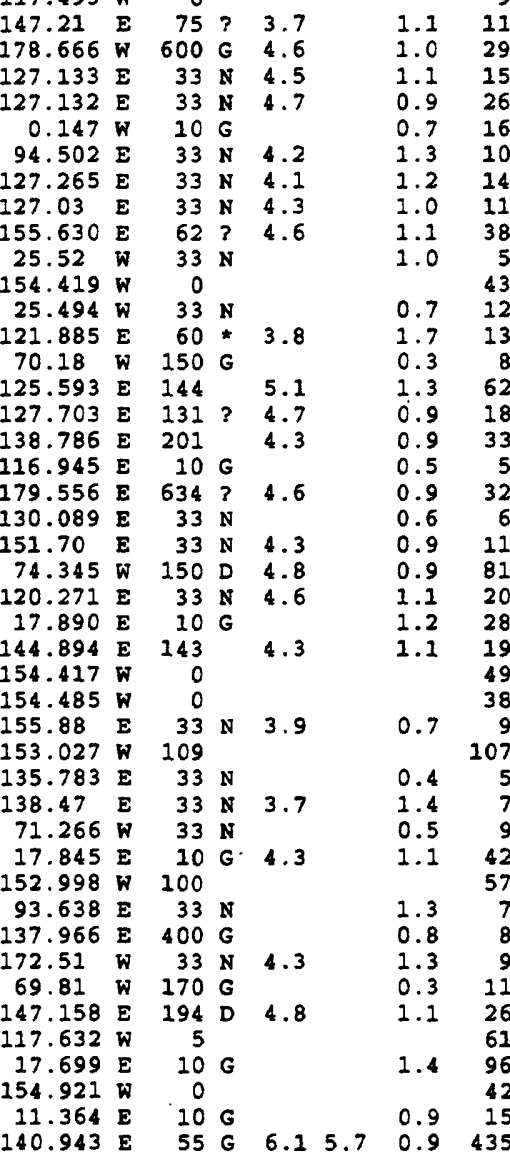

$\begin{array}{llllllll}11 & 02 & 49 & 57.9 ? & 46.39 & \mathrm{~N} & 148.12 \mathrm{E}\end{array}$ $110304 \quad 49.6 * 21.906 \mathrm{~N} \quad 121.306 \mathrm{E}$

$11.680 \mathrm{E}$

$33 \mathrm{~N}$

$33 \mathrm{~N} 4.3$

$10 \mathrm{G} \quad 4.9$

0.9

$1.2 \quad 12$

1.3200

$\begin{array}{lllllll}11 & 03 & 41 & 26.6 & 10.017 & \mathrm{~N}\end{array}$

$93.824 \mathrm{E}$ $131.505 \mathrm{E}$ $131.486 \mathrm{E}$ $124.201 \mathrm{E}$ 107.06 $121.166 \mathrm{E}$ 044121.5 ? $34.54 \mathrm{~S}$ $045312.3 * 13.169 \mathrm{~N}$ $\begin{array}{llll}05 & 21 & 32.5 & 35.452\end{array}$ $0540 \quad 38.1 \quad 44.360 \mathrm{~N}$
$7.323 \mathrm{E}$
$10 \mathrm{G}$

$10 \mathrm{G}$

$10 \mathrm{G} \quad 4.7$

$33 \mathrm{~N} 4.8$

$33 \mathrm{~N} 4.3$

$10 \mathrm{G}$
$0.4 \quad 6$

0.1

1.4

1. 4

1.3

0.5 $041106.9834 .276 \mathrm{~N}$ $043322.3 * 0.301 \mathrm{~s}$
CENTRAL CALIFO

FIJI ISLANDS REGION

TALAUD ISLANDS, INDONESIA

NORTEERN MOLUCCA SEA

PYRENEES. ML 3.2 (IDG), 2.6 (STR). mbLg 3.0 (NDD).

XIZANG

NORTHERN MOLUCCA SEA

NORTHERN MOLUCCA SER

SOLOMON ISLANDS

SOUTH SANDWICE ISLANDS REGION

ALASKA PENINSOLA. <AEIC>. MIL 3.2 (AEIC).

TAIWAN REGION

CHILE-ARGENTINA BORDER REGION

TALAUD ISLANDS, INDONESIA

BANDA SEA

NEAR WEST COAST OF HONSHO, JAPAN

WESTERN AUSTRAIIA

SOUTH OF FIJI ISLANDS

RYOKYO ISLANDS

NEW BRITAIN REGION, P.N.G

PERD-BRAZIL BORDER REGION

MINAEASSA PENINSULA, SULAWESI

ADRIATIC SEA. MI 3.3 (ROM).

MARIANA ISIAANDS

ALASKA PENINSULA. <AEIC>. ML 3.3 (AEIC).

SOLOMON ISLANDS

07 SOCTHERN ALASKA, 〈AEIC>.

NEAR S. COAST OF MTSTERN HONSEO

IRIAN JAYA, INDONESIA

NEAR COAST OF CENTRAL CHILE

ADRIATIC SEA

SOUTHERN ALASKA. LAEIC>.

NICOBAR ISLANDS, INDIA

SOUTH OF HONSHO, JAPAN

SAMOA ISLANDS REGION

SAN JUAN PROVINCE, ARGENTINA. MD 3.6 (SAN)

EASTERN NEW GOINEA REG., P.N.G.

CENTRAL CALIFORNIA. <PAS-P>. ND 3.7 (PAS). MI 3.6 (GS).

ADRIATIC SEA. ML 4.4 (VIE), 3.8 (ROM)

ALASKA PENINSULA. 〈AEIC>. MI 2.8 (AEIC).

NORTHERN ITALY. ML 2.5 (LDG).

NEAR EAST COAST OF HONSHD, JAPAN. MW 6.2 (HRV), 6.1 (GS). Me 6.1 (GS). Ms 5.6 (BRK). Felt (V JMA) at sawara; (IV JMA) in northern Chiba Prefecture; (III JMA) at Tokyo, parts of Kanagawa Prefecture and on the Iru Peninsula.

Broadband Source Parameters (GS): NP1: Strike=254, Dip=52, S1ip=-145; NP2: Strike-141, Dip-63, Slip-44; Radiated energy $3.7 * 10 * 13 \mathrm{Nm}$. Complex earthquake.

Moment Tensor (GS): Dep 35; Principal axes (scale 10*18 $\mathrm{Nm}$ ): (T) Val=1.45, Plg=22, Azm=205; (N) Val=-0.06, Plg=24, $A z m=305$; (P) Val=-1.39, Plg=56, Azm=77; Best double couple: Mo-1.4*10*18 Nm; NP1: Strike-258, Dip=31, Slip=-142; NP2: Strike=134, Dip=71, Slip=-65.

Centroid, Moment Tensor (HRV): Centroid origin time $02: 37: 16.7$; Lat $35.69 \mathrm{~N}$; Lon 141.01 E; Dep 38.0; Halfduration $3.0 \mathrm{sec;}$ Principal axes (scale $10 * * 18 \mathrm{Nm}$ ): (I) Val=1.92, Plg=9, Azm=204; (N) Val=0.10, Plg=28, Azm=299; (P) Val=-2.02, PIg=60, Azm=98; Best double couple: Mo-2.0*10*18 Nm; NP1: Strike=265, Dip=43, slip=-133; NP2 : Strike=137, Dip=60, Slip -57 .

Scalar Homent (PPT): MO=1.0*10**18 Nm.

NORTHWEST OF KORIL ISLANDS

TAIWAN REGION

GERMANY. ML 5.5 (STR) 5.4 (BNS), 5.2 (DBN) 5.2 (VIE), 5.1 (GRF), 5.0 (CLL), 5.0 (LDG). Slight damage at Halle and Zscherben.

$33 \mathrm{~N} 4.8 \quad 1.0 \quad 62$ ANDAMAN ISLANDS, INDIA

$33 \mathrm{~N} \quad 4.5$

ATSTERN ISLANDS, INDIA

WESTERN HONSHU, JAPAN

SOUTAERN WOLUCCA SEA

SOOTHERN EAST PACIFIC RISE

MINDORO, PHILIPPINE ISLANDS

NEAR EAST COAST OF EONSHO, JAPAN

NORTEERN ITALY. MU 2.6 (LDG), 2.4 (GEN).
SOUTH SANDWICE ISLANDS REGION

ALASKA PENINSULA. <AEIC>. ML 3.0 (AEIC). 

duration $1.0 \mathrm{sec}$; Principal axes (scale 10**17 Nm): (T) val-1.07, Plg=50, Azm=280; (N) Val-0.08, Plg=39, Azm-86; (P) Val=-1.15, Plg=7, Azm-181; Best double couple: Mo-1.1*10**17 km; NP1: Strike-306, Dip=51, Slip-144; NP2: Strike-61, Dip=63, Slip-45.

$11063955.1 * 9.079 \mathrm{~s} \quad 123.936 \mathrm{E}$ $1107 \quad 1708.28 \quad 37.597 \mathrm{~N}$ 074943.1 * $11.889 \mathrm{~N} 125.691 \mathrm{E}$ $081616.1 \% 61.717 \mathrm{~N} 146.616 \mathrm{~W}$ $082039.8836 .972 \mathrm{~N} \quad 71.220 \mathrm{E}$ $091507.0 * 13.393 \mathrm{~N} 140.732 \mathrm{E}$ $100427.6 ? 31.22 \mathrm{~S}$ $1038 \quad 55.66 \quad 63.220 \mathrm{~N}$ $110028.0 * 9.875 \mathrm{~s}$ $122539.7 * 35.686 \mathrm{~N}$ $\begin{array}{llll}12 & 55 & 54.6 * 23.177 \mathrm{~S} \\ 13 & 25 & 54.0 * 20.198 \mathrm{~s}\end{array}$ $141100.2661 .631 \mathrm{~N}$ $142126.9 * 7.509 \mathrm{~s}$ $151231.1 * 13.597 \mathrm{~N}$ $155212.5 * 9.947 \mathrm{~N}$ $164252.7 * 10.512 \mathrm{~S}$

$171713.8 \& 58.395$ N $154.821 \%$ $174951.3 * 5.282 \mathrm{~s} 129.981$ $183103.4 * 12.267$ N 143.772 $185415.9836 .876 \mathrm{~N} \quad 4.328 \mathrm{~N}$ $190247.9 * 21.616 \mathrm{~N} 121.699 \mathrm{E}$ $\begin{array}{lllll}20 & 06 & 00.1 * 8.936 \mathrm{~s} & 157.872 \mathrm{E}\end{array}$ 202229.8834 .138 N 117.061 \% $\begin{array}{llll}2039 & 31.7 & 42.837 \times & 17.809 \mathrm{E}\end{array}$ $230708.9 * 40.796 \mathrm{~N} 143.064 \mathrm{E}$ $231414.9 * 27.454 \mathrm{~N} 92.616 \mathrm{E}$ 234156.4 ? 19.16 s 178.22 \% $\begin{array}{lllllll}00 \quad 48 & 43.0 & 31.262 \mathrm{~S} & .73 .548 \mathrm{~W}\end{array}$ $011311.5 * 25.346 \mathrm{~S} 179.501 \mathrm{E}$ $\begin{array}{lllllll}01 & 47 & 32.4 * & 3.010 \mathrm{~N} & 126.947 & \mathrm{E} \\ 01 & 50 & 36.6 ? & 2.65 \mathrm{~N} & 127.35 & \mathrm{E}\end{array}$ $015210.1 * 3.021 \mathrm{~N} 126.762 \mathrm{E}$ $015338.3 * 2.978 \mathrm{~N} 127.103 \mathrm{E}$ $\begin{array}{lllllll}02 & 16 & 17.7 ? & 34.54 & \mathrm{~N} & 25.07 \mathrm{E}\end{array}$ 031728.1863 .490 N 150.938 \% $032418.5 * 44.440 \mathrm{~N} 147.676 \mathrm{E}$ $\begin{array}{llllll}03 & 32 & 29.9 & 42.868 \mathrm{~N} & 17.795 \mathrm{E}\end{array}$ $\begin{array}{ccccccc}03 & 47 & 39.7 ? & 3.03 & \mathrm{~N} & 126.80 & \mathrm{E} \\ 04 & 13 & 54.5 * & 42.323 & \mathrm{~N} & 17.482 & \mathrm{E}\end{array}$ $\begin{array}{lllll}04 & 18 & 43.4 & 42.863 & N\end{array}$ $043925.4834 .222 \mathrm{~s}$ $052531.6 \% 62.654 \mathrm{~N}$ $05 \quad 4105.3 * 19.725 \mathrm{~s}$ $06 \quad 1942.9860 .424 \mathrm{~N}$ $071008.0 * 5.384 \mathrm{~N}$ $072951.3 * 42.766 \mathrm{~N}$ 075909.3 ? $35.94 \mathrm{~N}$ $\begin{array}{lllll}08 & 16 & 59.2 & 55.713 \mathrm{~N}\end{array}$ $\begin{array}{llll}08 & 46 & 23.7 & 44.562 \mathrm{~N}\end{array}$ $\begin{array}{llll}09 & 22 & 02.58 & 33.624 \mathrm{~s}\end{array}$ $092226.46 .638 \mathrm{~s}$ 092433.2 ? $6.65 \mathrm{~s}$ $093730.4 \quad 10.152 \mathrm{~s}$ $095229.6 \& 34.630 \mathrm{~F}$ $\begin{array}{lll}10 & 16 & 42.1839 .124 \mathrm{~N}\end{array}$ $111515.0836 .283 \mathrm{~N}$

$1132 \quad 46.6 \quad 42.880 \mathrm{~N}$ $132058.3 * 44.342 \mathrm{~N}$ $140726.7832 .882 \mathrm{~S}$ $141403.4 * 10.452 \mathrm{~s}$ $141742.2863 .533 \mathrm{~N}$ $143929.7 * 21.606 \mathrm{~N}$ $\begin{array}{llll}16 & 50 & 25.16 & 33.181 \mathrm{~N}\end{array}$

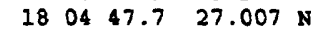
$\begin{array}{lllll}18 & 46 & 58.2 & 42.922 & \mathrm{~N}\end{array}$ $\begin{array}{llll}19 & 06 & 27.2 & 51.510 \mathrm{~N}\end{array}$ $191440.9 * 6.380 \mathrm{~s}$ $\begin{array}{llll}21 & 13 & 13.1 & 14.113 \mathrm{~s}\end{array}$ $211811.8 * 6.746 \mathrm{~s}$ $211818.3633 .906 \mathrm{~N}$

$12215628.2 * 21.938 \mathrm{~N}$ $12 \quad 22 \quad 13 \quad 50.88 \quad 33.739 \mathrm{~s}$

$\begin{array}{lllllll}12 & 22 & 20 & 31.87 & 56.17 & \mathrm{~S}\end{array}$

$\begin{array}{lllll}2 & 22 & 57 & 24.38 & 61.750\end{array}$

$\begin{array}{llllll}13 & 00 & 09 & 51.6 & 38.255 \mathrm{~N} & \\ 13 & 00 & 17 & 45.9 * & 43.174 \mathrm{~N}\end{array}$

$13002230.9 * 5.519 \mathrm{~s}$

$\begin{array}{llllll}13 & 00 & 56 & 49.7 & 6.709 \mathrm{~s}\end{array}$

$13011645.6 * 54.131$ N

$17.802 \mathrm{~B}$

$148.645 \mathrm{w}$

$177.897 \mathrm{~W}$

$147.752 \mathrm{~W}$

$126.394 \mathrm{E}$

$17.589 \mathrm{E}$

$140.29 \mathrm{E}$

$161.184 \mathrm{E}$

$6.872 \mathrm{E}$

$70.302 \mathrm{~W}$

$155.166 \mathrm{E}$

$155.04 \mathrm{E}$

$161.312 \mathrm{E}$

$116.665 \mathrm{~W}$

$4.959 \mathrm{w}$

$120.461 \mathrm{~W}$

$12.172 \mathrm{E}$

$70.753 \mathrm{~W}$

$120.408 \mathrm{E}$

$150.717 \mathrm{~W}$

$121.506 \mathrm{E}$

$115.606 \mathrm{~W}$

$92.492 \mathrm{E}$

$17.869 \mathrm{E}$

$16.591 \mathrm{E}$

$129.904 \mathrm{E}$

$167.182 \mathrm{E}$

$111.393 \mathrm{E}$

$117.145 \mathrm{~W}$

$121.595 \mathrm{E}$

$70.174 \mathrm{~W}$

$147.37 \mathrm{E}$

$151.625 \mathrm{w}$

$141.971 \mathrm{E}$

$18.038 \mathrm{E}$

$154.461 \mathrm{E}$
$129.726 \mathrm{E}$

163.288

$\begin{array}{rrrrr}33 & N & 4.1 & & 1 \\ 10 & G & & \\ 33 & N & & & 1 \\ 34 & & & & 1 \\ 150 & G & & & 1 \\ 33 & N & & & 1 \\ 300 & G & 4.2 & & 1.2 \\ 128 & & & & \\ 33 & N & & & 0 \\ 33 & N & & & 0 \\ 500 & G & 4.1 & & 1 \\ 33 & N & 4.7 & 4.6 & 1.0 \\ 32 & & & & \\ 150 & G & 4.1 & & 1.4 \\ 97 & D & 4.1 & & 1.1 \\ 33 & N & 4.7 & & 1.1 \\ 33 & N & 4.8 & & 1.0\end{array}$

$1.4 \quad 15$

TIMOR REGION, INDONESIA

1.19 SPAIN. mbLg 2.9 (MDD).

1.17 SAMAR, PHILIPPINE ISIAANDS

59 SOOTEERN ALASKA. 〈AEIC>. ML 2.6 (AEIC).

1.013 AFGEANISTAN-TAJIKISTAN BORD REG.

1.212 WEST OF MARIANA ISLANDS

1.310 RERMADEC ISIANDS REGION

53 CENTRAL ALASKA. LAEIC>

0.96 SODTA OF BAII, INDONESIA

0.86 NEAR EAST COAST OF HONSBD, JAPAN

1.118 SOOTH OF FIJI ISIANDS

20 TONGA ISIAANDS

75 SODTEERN ALASKA. CAEIC>. ML 2.8 (AEIC).

1.4

1.1

$1 \cdot 1$

15 BANDA SEA

10 NICOBAR ISIAANDS, INDIA

NEAR COAST OF PERU. Slight damage at Euaraz. Felt (III) at Buarmey and (II) at Casma and Paramonga. Also felt.in the Huaylas Valley.

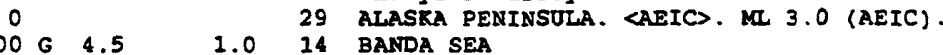

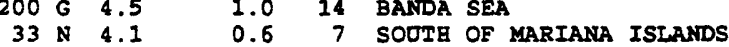

$33 \mathrm{~N}$

$33 \mathrm{~N} \quad 4.4$

$33 \mathrm{~N} 4.7$

8

$10 \mathrm{G}$

$33 \mathrm{~N} 4.6$

$33 \mathrm{~N} 4.6$

$550 \mathrm{G} \quad 4.5$

$33 \mathrm{~N} 4.6$

$500 \mathrm{G} 4.6$

$10 \mathrm{G} 4.6$

$33 \times 4.4$

$33 \times 4.4$

$33 \mathrm{~N} 4.5$

$33 \mathrm{~N} 4.5$

9

$\begin{array}{lll}33 & \mathbf{N} & \\ 10 & \mathrm{G} & 4.6\end{array}$

$\begin{array}{lll}10 & \mathrm{G} & 4.6 \\ 33 & \mathrm{~N} & 4.5\end{array}$

$10 \mathrm{G} 4.2$

E $10 \mathrm{G}$

$70.415 \mathrm{~W} 100 \mathrm{G}$

$17.895 \mathrm{E}$

100

550 G 4.5

$33 \mathrm{~N} 4.4$

$10 \mathrm{G} \quad 4.1$

$33 \mathrm{~N}$

$33 \mathrm{~N} 4.6$

$10 \mathrm{G}$

$100 \mathrm{G}$

$40 \mathrm{D} \quad 4.6$

$33 \mathrm{~N} 4.4$

$33 \mathrm{~N} 4.7$

6

$10 \mathrm{G}$

10

1.0

1.4

1.1

6
13 TTRAIT OF GIBR
2 TAN REGION

STRAIT OF GIBRALTAR. mbLg 2.5 (MDD)

1.5

1.0

1.5

0.8

1.0

1.3

1.2

1.1

0.6

1.4

$1.2 \quad 23$

$\begin{array}{rr}1.4 & 125 \\ 1.4 & 12\end{array}$

1.4

1.5

0.3

0.9

1.1

1.3

1.2

1.0

0.6

0.2

1.1

1.3

1.0

0.4

$10 \mathrm{G}$

$10 \mathrm{G}$

$65 \mathrm{G}$

$33 \mathrm{~N} 4.1$

15

$33 \times 4.3$

$49 \mathrm{D} \quad 4.6$

$10 \mathrm{G}$

$10 \mathrm{G}$

33 N 4.4

$200 \mathrm{G} 5.0$

$33 \mathrm{~N} 4.5$

14

$33 \mathrm{~N} 4.0$

$10 \mathrm{G}$

$10 \mathrm{G} \quad 4.5$

48 D 4.8

$\begin{array}{lll}48 & D & 4.8 \\ 10 & G & 3.7\end{array}$

200 G 5.2

$150 \mathrm{G} \quad 4.9$

SOJTEERN CALIFORNIA. <PAS-P>, MD 2.8 (PAS)

ADRIATIC SEA. ML 4.1 (VIE), 3.6 (ROM)

3 OFF EAST COAST OF HONSHU, JAPAN

13 EASTERN XIZANG-INDIA BORDER REG.

12 FIJI ISLANDS REGION

9 OFF COAST OF CENTRAL CHITE

7 SODTE OF FIJI ISLANDS

8 TALAOD ISLANDS, INDONESIA

NORTEERN MOLUCCA SEA

7 TAIAND ISLANDS, INDONESIA

MORTEERN MOLUCCA SEA

14 CRETE

Cemtral alaska. <aeico. yU 2.7 (AEIC).

KURIL ISLANDS

ADRIATIC SEA. ML 4.4 (VIE).

TAIAUD ISLANDS, INDONESIA

ADRIATIC SEA

O ADRIATIC SEA. ML 3.7 (ROM)

10 CHIIE-ARGENTINA BORDER REGION

73 CENTRAL ALASKA. CAEIC). ML 2.8 (AEIC).

21 FIJI ISLANDS REGION

69 SOCTHERN ALASKA. CAEIC>. ML 3.0 (AEIC).

15 MINDANAO, PEILIPPINE ISI.ANDS

8 ADRIATIC SEA

6 NEAR EAST COAST OF BONSHU, JAPAN

52 NEAR EAST COAST OF RAMCHATKA

22 FRANCE. MC 2.5 (LDG), 2.5 (GEN).

10 CHILE-ARGENTINA BORDER RDGION

34 SOLOMON ISLANDS

9 SOIOMON ISLANDS

29 SOLOMON ISLANDS

29 SOUTHERN CALIFORNIA. CPAS-PS. MD 2.8 (PAS). ML 3.0 (GS).

5 SPAIN. mbLg 2.6 (MDD).

62 CENTRAL CALIFORNIA. LGM-P). MD 3.3 (GM), 3.2 (PAS). ML 3.3 
$\begin{array}{llllllllll}13 & 01 & 44 & 43.9 ? & 51.37 & \mathrm{~N} & 178.89 & \mathrm{~W} & 33 & \mathrm{~N}\end{array}$

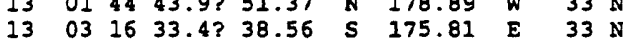
$\begin{array}{llllllllll}13 & 03 & 41 & 08.6 & 27.033 \mathrm{~N} & 88.234 \mathrm{E} & 33 \mathrm{~N} & 4.5\end{array}$ $\begin{array}{lllllllllllll}13 & 03 & 41 & 08.8 & 10.555 & \mathrm{~S} & 165.922 & \mathrm{E} & 157 & \mathrm{D} & 5.1\end{array}$

0.77 ANDREANOF ISLANDS, ALEUTIAN IS.

1.011 NORTE ISLAND, NEW ZEAIAND

0.821 SIKKIM, INDIA

0.9112 SANTA CRDZ ISLANDS. MW 5.5 (HRV)

Centroid, Moment Tensor (BRV): Centroid origin time $03: 41: 13.3$; Lat $10.66 \mathrm{~s}$; Lon $166.05 \mathrm{E}$; Dep 160.6; Halfduration 1.3 sec; Principal axes (scale 10*17 $\mathrm{km}$ ): (T) $\mathrm{Val}=2.02, \mathrm{Plg}=70, \mathrm{Azm}=94$; (N) $\mathrm{Val}=-0.20, \mathrm{Plg}=20, \mathrm{Azm}=262$; (P) Val=-1.82, Plg=4, Azm-353; Best double couple: MO=1.9*10*17 Nm; NP1: Strike-103, Dip=45, S1ip-119; NP2: Strike=245, Dip=52, Slip $=65$.

$1304 \quad 4253.28 \quad 37.924 \mathrm{~N} \quad 118.179 \mathrm{~W} \quad 1$

$13044925.0 * 7.108$ S $155.575 \mathrm{E}$ $\begin{array}{lllll}13 & 04 & 57 & 27.2 & 43.976 \mathrm{~N}\end{array}$ $\begin{array}{llllll}13 & 05 & 04 & 35.9 & 2.783 & \mathrm{~N}\end{array}$ $\begin{array}{rr}55.575 & \mathrm{E} \\ 7.618 & \mathrm{E}\end{array}$

$96.073 \mathrm{E}$
$33 \mathrm{~N} 4.1$

$10 \mathrm{G}$

$\begin{array}{llll}5.0 & 4 & 0.3 & 15\end{array}$ $\begin{array}{llllllll}13 & 06 & 28 & 44.18 & 35.849 & \mathrm{~N} & 121.342 \mathrm{~W} & 9\end{array}$

$13065947.9 * 12.285 \mathrm{~S} \quad 77.055 \mathrm{~W} 9 \mathrm{~g}$

$071340.7 * 2.863 \mathrm{~N}$ $0748 \quad 54.58 \quad 37.082 \mathrm{~N}$ $08 \quad 23 \quad 48.4 ? 28.42 N$ $\begin{array}{llll}1138 & 36.58 & 59.686 \mathrm{~N}\end{array}$ $\begin{array}{llll}13 & 41 & 12.3 & 51.547 \mathrm{~N}\end{array}$
$128.608 \mathrm{E}$ $5.204 \mathrm{~W} 150 \mathrm{G}$ $43.69 \mathrm{~W} \quad 10 \mathrm{G}$ 153.973 W 141 178.458 141 $33 \times 5.2$

39 (BRK)， 3.0 (GS)

SOLOMON ISLANDS

5 NEAR SOUTH COAST OF FRANCE. MU 2.4 (GEN), 1.8 (LDG).

78 NORTEERN SUMATERA, INDONESIA. MW 5.3 (HRV)

Centroid, Moment Tensor (HRV): Centrold origin time $05: 04: 40.8$; Lat $3.04 \mathrm{~N}$; Ion $95.52 \mathrm{E}$; Dep 34.6 ; Halfduration $1.1 \mathrm{sec}$; Principal axes.(scale $10 * 16 \mathrm{Nm}$ ): (T) Val-8.91, Plg=71, $A 2 m=19$; (N) Val=-0.66, $\mathrm{Plg}=7, \mathrm{Azm}=131$ (P) Val=-8.25, Plg-17, Azm=223; Best double couple: No-8.6*10*16 Nm; NP1: Strike-324, Dip-29, Slip=105; NP2: Strike-127, Dip-62, Slip-82.

32 CENTRAL CALIFORNIA. LGM-P>. MD 2.9 (GM). MI 3.0 (GS)

7 NEAR COAST OF PERU. Felt (IV) at Lima and (II) at Mala. Also felt at Chosica and Huaral. Iandslides occurred at lima.

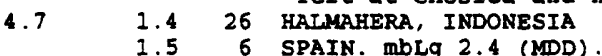

$4.74 .3 \quad 1.0 \quad 15$ NORTHERN MID-ATLANTIC RIDGE

49 SOUTHERN ALASKA. LAEIC>.

0.9209 ANDREANOF ISLANDS, ALEOTIAN IS. WW 5.1 (HRV). Felt on Adak. Centroid, voment Tensor (BRV): Centroid origin time $13: 41: 14.4$; Lat $51.55 \mathrm{~N}$; Lon $178.10 \mathrm{H}$; Dep 37.1 ; Halfduration $1.0 \mathrm{sec}$; Principal axes (scale $10 \star * 16 \mathrm{Nm}):(T)$ Val-6.32, Plg=62, Azm=310; (N) Val=-0.93, Plg=10, Azm=59; (P) Val=-5.39, Plg=26, Azm=154; Best double couple: Mo-5.9*10**16 Nm; NP1: Strike-267, Dip=21, Slip=119; NP2 : strike-56, Dip=72, slip=80.

$153.391 \mathrm{E}$ $132.22 \mathrm{E}$ $157.854 \mathrm{E}$ $78.26 \mathrm{E}$ $155.67 \mathrm{E}$ 135.84 H $126.757 \mathrm{E}$ $128.496 \mathrm{E}$ $98.05 \mathrm{E}$ $61.883 \mathrm{~W}$ $17.749 \mathrm{E}$ $17.833 \mathrm{E}$ $151.012 \mathrm{~N}$ $126.41 \mathrm{E}$ $71.004 \mathrm{~N}$ $174.069 \mathrm{~N}$ $126.645 \mathrm{E}$ $76.371 \mathrm{E}$ $75.156 \mathrm{~W}$ $92.638 \mathrm{E}$ $78.307 \mathrm{E}$ $96.645 \mathrm{E}$ $0.625 \mathrm{E}$ 122.795 E 10
4.6

4.

4.9
4.4

3.9

4.8

4.8

4.2

4.4

3.9

$4 \cdot 4$

5.0

4.5

4.6

4.9

4.7

4.8

4.5

5.5

$\begin{array}{ll}33 & \mathrm{~N} \\ 33 & \mathrm{~N} \\ 33 & \mathrm{~N} \\ 10 & \mathrm{G} \\ 33 & \mathrm{~N} \\ 10 & \mathrm{G} \\ 33 & \mathrm{~N} \\ 100 & \mathrm{G} \\ 33 & \mathrm{~N} \\ 10 & \mathrm{G} \\ 10 & \mathrm{G} \\ 10 & \mathrm{G} \\ 64 & \\ 33 & \mathrm{~N} \\ 60 & \mathrm{G} \\ 33 & \mathrm{~N} \\ 33 & \mathrm{~N} \\ 33 & \mathrm{~N} \\ 33 & \mathrm{~N} \\ 33 & \mathrm{~N} \\ 33 & \mathrm{~N} \\ 33 & \mathrm{~N} \\ 10 & \mathrm{G} \\ 181 & \end{array}$

1.3

0.3

0.8

1.4

1.2

1.0

1.1

0.9

0.9

5

1.2

1.1

1.0

0.8

0.8

1.1

1.1

0.9

0.9

1.4

1.0

0.9183
18 NEW IRELAND REGION, P.N.G.

4 SHIKOKU, JAPAN

86 KAMCHATKA

8 MID-INDIAN RIDGE

9 SOLONON ISLANDS

12 PACIFIC-ANTARCTIC RIDGE

22 NORTHERN MOLUCCA SEA

13 SERAY, INDONESIA

9 NORTHERN SULATERA, INDONESIA

7 WINDWARD ISLANDS. MD 3.3 (TRN).

10 ADRIATIC SEA

10 NORTEWESTERN BALKAN REGION

75 CENTRAL ALASKA. <AEIC>. MI 3.0 (AEIC).

10 NORTHERN MOLUCCA SEA

10 NEAR COAST OF CENTRAL CEILE. MD 2.5 (SAN).

107 ANDREANOF ISLANDS, ATEUTIAN IS.

15 NORTEERN YOLDCCA SEA

29 KASEMIR-INDIA BORDER REGION

9 NORTEERN PERD

48 EASTERN XIZANG-INDIA BORDER REG.

16 NORTEERN INDIA

16 MYAMMAR

8 SPAIN. mbLg 2.8 (MDD). III 2.4 (LDG).

MINAEASSA PENINSUIA, SULAKESI. MW 5.8 (HRV) Centroid, Moment Tensor (HRV): Centroid origin time

$02: 53: 28.7$; Lat $0.11 \mathrm{~N}$; Lon $123.01 \mathrm{E}$; Dep 177.6; Halfduration $2.0 \mathrm{sec}$; Principal axes (scale $10 * 17 \mathrm{Nm}$ ): (T) Val-7.64, $\mathrm{Plg}=61, \mathrm{Azm}=270$; (N) Val=-2.39, $\mathrm{Plg}=28, \mathrm{Azm}=73$; (P) Val=-5.26, Plg=7, Azm-167; Best double couple: Mo-6.4*10**17 Nm; NP1: Strike-285, Dip=45, Sl1p=131; NP2: Strike=54, Dip=58, Slip=57.

$\begin{array}{rllllrlrlrll}14 & 03 & 32 & 58.0 * & 3.075 & \mathrm{~N} & 127.275 & \mathrm{E} & 33 & \mathrm{~N} & 4.3 \\ 14 & 04 & 28 & 36.1 & 2.634 & \mathrm{~N} & 126.388 & \mathrm{E} & 100 & \mathrm{G} & 4.8 \\ 14 & 05 & 41 & 45.08 & 58.718 & \mathrm{~N} & 157.472 & \mathrm{E} & 33 & \mathrm{~N} & \\ 14 & 06 & 02 & 37.6 * & 42.753 & \mathrm{~N} & 17.453 & \mathrm{E} & 10 & \mathrm{G} & 4.0 \\ 14 & 06 & 32 & 37.48 & 38.282 & \mathrm{~N} & 122.092 & \mathrm{~W} & 9 & & \\ & & & & & & & & & & & \\ 14 & 07 & 55 & 00.4 & 4.645 & \mathrm{~S} & 152.659 & \mathrm{E} & 100 & \mathrm{G} & 5.1 \\ 14 & 08 & 01 & 03.7 & 36.046 & \mathrm{~N} & 70.706 & \mathrm{E} & 120 & \mathrm{D} & 5.1\end{array}$

1.3 12 TALAUD ISLANDS, INDONESIA

0.832 NORTHERN MOLUCCA SEA

0.47 KAMCHATKA

1.112 ADRIATIC SEA

44 NORTHERN CALIFORNIA. <GM-P>. MD 2.9 (GM). ML 2.9 (GS). Felt at Fairfield.

1.045 NEW BRITAIN REGION, P.N.G.

1.0219 HINDU KUSH REGION, AFGHANISTAN. MW 5.9 (GS), 5.9 (BRV). Felt at Islamabad and Rawalpindi, Pakistan.

Moment Tensor (GS): Dep 109; Principal axes (scale 10*:17 $\mathrm{Nm}$ ): (T) Val-7.88, Plg=49, $\mathrm{Azm}=26 ;$ (N) Val=-1.44, $\mathrm{Plg}=0$, Azm-116; (P) Val=-6.44, Plg-41, Azm-206; Best double Couple: Mo=7.2*10**17 Nm; NPI: Strike-296, Dip-4, Slip-90; NP2: Strike-116, Dip-86, Slip-90.

Centroid, Moment Tensor (BRV): Centroid origin time 08:01:09.0; Lat $35.96 \mathrm{~N}$; Lon $70.59 \mathrm{E}$; Dep 127.5; Halfduration 2.1 sec; Principal axes (scale $10 * * 17 \mathrm{Nm}$ ): (T) val-6.86, Plg=48, Azm-35; (N) Val-0.26, Plg=7, Azm-297; Val=-7.12, Plg=41, Azm=200; Best double couple: Mo-7.0*10*17 Nm; NP1: Strike=230, Dip-8, silp-23; NP2:

\section{Strike-118, DIp=87, Slip=97.
0.04 NORTHERN ITALY. MII 1.7 (GEN).}

1.155 SPAIN. mbIg 2.9 (MDD)

19 NEAR COAST OF NORTHERN CALIF. <GM-P>. MD 3.0 (GM). ML 3.0 
$14111528.4 \& 40.304 \mathrm{~N} 124.478 \mathrm{~W}$

$14121345.8 * 6.086$ S 130.276 E 33 N 4.0 $\begin{array}{lllllllll}14 & 12 & 46 & 04.2 & 29.060 \mathrm{~N} & 130.170 \mathrm{E} & 33 \mathrm{~N} & 4.5\end{array}$ 14124917.2 ? 15.31 N 92.10 W $100 \mathrm{G} 3.9$ $14125036.8 \quad 51.401 \mathrm{~N} 174.073 \mathrm{~W} 33 \mathrm{~N} 5.2$

(GS).

24 NEAR COAST OF NORTHERN CALIF. (GM-P>. MD 3.2 (GM). ML 3.3 (GS), 3.1 (BRK).

1.212 BANDA SEA

1.429 RYUKYU ISLANDS

1.413 MEXICO-GUATEMALA BORDER REGION

0.8195 ANDREANOF ISLANDS, ALEUTIAN IS. MW 5.2 (HRV).

Centroid, koment Tensor (HRV): Centroid origin time

$12: 50: 43.1$; Lat $51.47 \mathrm{~N}$; Lon $174.15 \mathrm{~W}$; Dep $15.0 \mathrm{Eix}$; Halfduration $1.1 \mathrm{sec}$; Principal axes (scale $10 * * 16 \mathrm{Nm}$ ): (T) Val=7.99, Plg=64, Azm=30; (N) Val=-1.23, Plg=18, Azm-258; (P) Val--6.76, Plg-18, Azm-162; Best double couple: Mo-7.4*10*16 Nm; NP1: Strike-226, Dip-31, Slip-54; NP2: Strike-87, Dip=65, Slip-110.

$\begin{array}{llllllllllll}14 & 13 & 03 & 33.9 & 9.986 & \mathrm{~N} & 125.358 \mathrm{E} & 201 \mathrm{D} & 5.0 & 1.0 & 79 & \text { MINDANAO, PBILIPPINE ISLANDS }\end{array}$

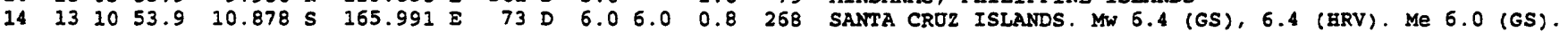
Broadband Source Parameters (GS): Dep 65; NP1: Strike-180, Dip=50, Slip=55; NP2: Strike=47, Dip=51, Slip=124; Radiated energy $2.4 * 10 * 13 \mathrm{Nm}$.

Moment Tensor (GS): Dep 70; Principal axes (scale 10*\$18 $\mathrm{Nm}$ ): (T) Val-4.86, Plg-64, Azm-23; (N) Val--0.02, Plg-26, Azm=209; (P) Val--4.B4, Plg=2, Azm-118; Best double couple: Mo-4.9*10*18 Nm; NP1: Strike-184, Dip=48, Slip=54; NP2: Strike-51, Dip=53, S1ip-123.

Centrold, Moment Tensor (HRV): Centroid origin time $13: 10: 59.5$; Lat $10.84 \mathrm{~s}$; Lon $165.94 \mathrm{E}$; Dep 64.6 ; Half duration $4.1 \mathrm{sec}$; Principal axes (scale 10*18 Nm): (T) $\mathrm{Val}=5.29, \mathrm{Plg}=52, \mathrm{Azm}=30$; (N) Val=-0.94, Plg=38, Azm=212; (P) Val--4.34, Plg-1, Azm-122; Best double couple: Mo-4. B*10*18 Nm; NP1: Strike=179, Dip-56, Slip-41; NP2: Strike=63, Dip $=57$, Slip=13B.

Scalar Moment (PPT): MO-5.0*10*\#18 Nm.

$134340.6 * 30.358 \times 131.135 \mathrm{E}$ $\begin{array}{llllll}13 & 56 & 56.6 & 30.527 \mathrm{~N} & 131.254 \mathrm{E}\end{array}$ $143247.7 * 27.670 \mathrm{~N} 139.801 \mathrm{E}$ $14 \quad 34 \quad 59.2 \quad 43.958 \quad \mathrm{~N}$ $\begin{array}{lllll}14 & 48 & 42.58 & 81.391 \mathrm{~N}\end{array}$ $\begin{array}{lllll}15 & 52 & 38.88 & 48.407 \mathrm{~N} & 148.508 \mathrm{E}\end{array}$ $\begin{array}{lllllll}16 & 27 & 19.5 & 43.282 & \mathrm{~N} & 146.713 \mathrm{E}\end{array}$ $\begin{array}{lllllll}16 & 37 & 59.7 ? & 44.36 & N & 7.38\end{array}$ $1728 \quad 57.9 * 40.675 \mathrm{~N} 143.193 \mathrm{E}$ $173918.1 * 21.687 \mathrm{~N} 121.578 \mathrm{E}$ $174838.3 * 52.086$ N 30.241 ผ $\begin{array}{lllll}18 & 13 & 30.4 * 5.445 \mathrm{~S} & 147.272 \mathrm{E}\end{array}$ $190938.6 * 29.842 \mathrm{~N} 51.647 \mathrm{E}$ $192944.0875 .443 \mathrm{~N} 110.473 \mathrm{E}$ 200135.0 ? 1.77 N 90.65 W $202840.3 * 3.075 \mathrm{~N} 127.255 \mathrm{E}$ $\begin{array}{lllll}21 & 17 & 28.6 & 10.664 \mathrm{~N} & 62.560 \mathrm{~N}\end{array}$ $\begin{array}{lllll}21 & 37 & 08.4 & 43.962 \mathrm{~N}\end{array}$ $\begin{array}{llllll}22 & 20 & 08.6 ? & 23.59 \mathrm{~S}\end{array}$ $\begin{array}{lllll}22 & 39 & 10.97 & 53.63 \quad \mathrm{~N}\end{array}$ $\begin{array}{llll}22 & 54 & 52.2 & 46.141 \mathrm{~N}\end{array}$

$\begin{array}{llll}23 & 10 & 01.88 & 45.983 \mathrm{~N}\end{array}$ $232000.9 * 72.252 \mathrm{~N}$ $\begin{array}{llllll}23 & 26 & 32.9 & 5.862 \mathrm{~S}\end{array}$ $\begin{array}{lllll}23 & 32 & 10.98 & 34.289 \mathrm{~s}\end{array}$ $001452.6 ? 53.78 \quad N$ $0016 \quad 07.8853 .483 \mathrm{~N}$ $0016 \quad 56.6 ? 53.03 \quad \mathrm{~N}$ $\begin{array}{llllll}00 & 21 & 22.1 & 72.206 & N\end{array}$ $00 \quad 22 \quad 02.96 \quad 67.417 \mathrm{~N}$ $004423.6 \quad 46.230 \mathrm{~N}$

$0049 \quad 35.38 \quad 43.685 \mathrm{~N}$ $015128.0 * 25.218 \mathrm{~s}$ $015213.2 * 12.774 \mathrm{~N}$ $022110.4 ? 29.04 \mathrm{~N}$ $\begin{array}{llll}02 & 25 & 55.2 & 46.630\end{array}$ 024054.5 ? $5.85 \mathrm{~s}$ $030130.6 \quad 6.438 \mathrm{~N}$ $030614.1 * 44.468 \mathrm{~N}$ $0323 \quad 42.7 * 45.124$ $033408.0 * 52.875 \mathrm{~N}$ $035504.2 * 42.692 \mathrm{~N}$ $053443.1 * 7.431 \mathrm{~s}$ $\begin{array}{lllll}06 & 37 & 43.6 & 43.946 \mathrm{~N}\end{array}$ $07 \quad 12 \quad 42.8 * 26.119 \mathrm{~s}$ $082515.7 \quad 46.147 \mathrm{~N}$

$082954.5 * 16.249 \mathrm{~s}$ $08 \quad 34 \quad 48.48 \quad 54.737$ $083751.3838 .487 \mathrm{~N}$ $084028.7 * 42.586$ $\begin{array}{llll}09 & 25 & 41.5 & 24.427\end{array}$ $103552.5 * 42.471$ $\begin{array}{llll}10 & 53 & 03.5 & 42.889 \mathrm{~N}\end{array}$ $105333.5 \& 58.498$ $114410.7 * 42.907$ $\begin{array}{llll}12 & 12 & 03.3 \& & 63.551\end{array}$ $\begin{array}{llll}12 & 13 & 28.1 .63 .587\end{array}$ $\begin{array}{llll}12 & 2651.1 & 2.919 \mathrm{~N}\end{array}$
$7.621 \mathrm{E}$

179.06

35.34

$6.755 \mathrm{E}$

$7.319 \mathrm{E}$

$126.786 \mathrm{E}$

$104.166 \mathrm{E}$

70.235

35.18

35.462 ต

34.99 W

$126.335 \mathrm{E}$

$146.098 \mathrm{~W}$

$14.325 \mathrm{E}$

$0.384 \mathrm{~W}$

E $500 \mathrm{G}$

$124.957 \mathrm{E} 33 \mathrm{~N}$

$35.00 \mathrm{E} \quad 10 \mathrm{G}$

$152.900 \mathrm{E} \quad 33 \mathrm{~N}$

145.17 E $100 \mathrm{G}$

33.827 W

$149.560 \mathrm{E}$

$146.699 \mathrm{E}$

$166.962 \mathrm{~W}$

$17.580 \mathrm{E}$

$126.571 \mathrm{E}$

$7.604 \mathrm{E}$

177.741

$14.888 \mathrm{E}$

$173.146 \mathrm{~W}$ $151.273 \mathrm{E}$ $71.409 \mathrm{E}$

$17.498 \mathrm{E}$

$179.919 \mathrm{~W}$

$17.227 \mathrm{E}$

$17.810 \mathrm{E}$

$150.350 \mathrm{~W}$

$17.688 \mathrm{E}$

$150.698 \mathrm{~W}$

$150.861 \mathrm{~W}$

$127.121 \mathrm{E}$

$10 \mathrm{G}$

$10 \mathrm{G}$

$5 \mathrm{G}$

$5 \mathrm{G}$
$33 \mathrm{~N}$

$100 \mathrm{G}$

$10 \mathrm{G}$
$10 \mathrm{G}$

$10 \mathrm{G}$

106

G

$10 \mathrm{G}$

$33 \mathrm{~N}$

$33 \mathrm{~N}$

$33 \mathrm{~N}$

$10 \mathrm{G}$

$5 \mathrm{G}$
$100 \mathrm{G}$

$10 \mathrm{G}$

$41 \mathrm{D}$

$33 \mathrm{~N}$

$10 \mathrm{G}$

$493 \mathrm{D}$

$10 \mathrm{G}$

$10 \mathrm{G}$

29

$10 \mathrm{G}$

9
19
$33 \mathrm{~N}$

$33 \mathrm{~N} 4$.

$10 \mathrm{G}$

$10 \mathrm{G}$

$57 \mathrm{G}$

$10 \mathrm{G}$

$33 \mathrm{~N}$

$3 \mathrm{~N} 3$.

$10 \mathrm{G} 3.7$

G

$33 \mathrm{~N}$

$10 \mathrm{G}$

6

$33 \times 4.6$

$19 \times 5.0$

4.4

4.4

4.0

4.9

$4 \cdot 4$

4.8
0.9

1.1

0.9

NEAR SOUTH COAST OF FRANCE. ML 2.1 (GEN), 1.8 (LDG).

0.65 EAST OF SEVERNAYA 2EMLYA, RUSSIA

$\begin{array}{llll}4.7 & 0.7 & 10 & \text { NORTHWEST OF K } \\ 4 & 0.9 & 31 & \text { KORIL ISLANDS }\end{array}$

0.14 NORTHERN ITALY. ML 1.6 (GEN).

1.221 OFF EAST COAST OF HONSHO, JAPAN

1.510 TAIWAN REGION

1.417 NORTHERN MID-ATIANTIC RIDGE

1.218 EASTERN NEW GUINEA REG., P.N.G.

1.313 SOUTHERN IRAN

1.012 NEAR COAST OF C. SIBERIA, ROSSIA

1.199 GALAPAGOS ISLANDS REGION

1.018 TALAOD ISIAANDS, INDONESIA

0.934 NEAR COAST OF VENEZUELA

$4.0 \quad 0.9$

0.4

1.5

0.9

1.2

1.1

1.1

0.4

1.1

0.4

1.4

$4.54 .8 \quad 1.0$

1.3

0.5

1.0

0.8

1.4

0.8

1.4

$4.74 .5 \quad 1.0$

3.51 .1

1.0
1.3

1.3
1.5

1.3

0.4

1.0

1.2

0.8
0.5

1.5

0.8

1.2

1.4

1.6

4 NEAR SOUTH COAST OF FRANCE. ML 2.0 (GEN), 1.6 (IDG).

13 SOTTH OF FIJI ISLANDS

9 NORTE ATLANTIC OCEAN

91 SWITZERLAND. MI 3.7 (GRF), 3.5 (LDG), 3.5 (STR), 3.4 (FOR), 3.2 (VIE).

11 NORTHERN ITALY. ML 2.0 (LDG)

10 NORTHCENTRAL SIBERIA, RUSSIA

36 SOUTHERN SUMATERA, INDONESIA

10 CHILE-ARGENTINA BORDER REGION. MD 3.6 (SAN)

9 NORTH ATLANTIC OCEAN

6 NORTH ATLANTIC OCEAN

5 NORTH ATLANTIC OCEAN

52 NORTHCENTRAL SIBERIA, RUSSIA

22 NORTHERN ALASKA. CAEIC>. ML 3.3 (AEIC).

7 NORTHWESTERN BALKAN REGION. ML 3.3 (EUR), 3.0 (VIE), 3.0 (IDG). Felt (IV) at Kranj and Preddvor, Slovenia.

5 PYRENEES. ML 2.0 (IDG)

19 SOUTE OF FIJI ISIAANDS

21 SAMAR, PHILIPPINE ISIANDS

23 EGYPI

19 KURIL ISLANDS

5 EASTERN NEW GOINEA REG., P.N.G.

72 CENTRAL MID-ATLANTIC RIDGE

11 KURIL ISLANDS

19 KURIL ISLANDS

21 FOX ISLANDS, ALEUTIAN ISLANDS

13 ADRIATIC SEA

14 BANDA SEA

NEAR SOUTH COAST OF FRANCE. ML 1.8 (GEN), 1.3 (LDG)

1 SOUTH OF FIJI ISLANDS

NORTHWESTERN BALKAN REGION. MU 3.7 (FUR), 3.6 (LJO), 3.5 (GRF) , 3.1 (VIE)

30 TONGA ISLANDS

13 NEAR EAST COAST OF KAMCHATKA

5 AFGHANISTAN-TAJIKISTAN BORD REG.

23 ADRIATIC SEA

15 SOUTH OF FIJI ISLANDS

ADRIATIC SEA

105 ADRIATIC SEA. ML 3.8 (SKO).

26 GUIF OF ALASKA. <AEIC>. MI 2.7 (AEIC)

28 ADRIATIC SEA. MI 3.4 (SKO), 3.3 (ROM)

18 CENTRAL ALASKA. <AEIC>. ML 2.7 (AEIC).

47 CENTRAL ALASKA. <AEIC>. ML 2.8 (AEIC).

1.028 NORTHERN MOLOCCA SEA 
$15 \quad 1255 \quad 52.4865 .166 \mathrm{~N} 148.536 \mathrm{w}$ $\begin{array}{lllllll}13 & 31 & 39.7 ? & 30.80 & \mathrm{~S} & 59.62 \mathrm{E}\end{array}$ $160739.1 * 31.613 \mathrm{~N} 132.001 \mathrm{E}$ $162947.8 * 20.016$ S 67.559 E $171049.1 * 2.628$ S $139.601 \mathrm{E}$ $171951.6 * 15.602 \mathrm{~N} 120.457 \mathrm{E}$

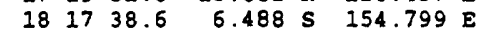

14

100 $33 \mathrm{~N}$ 21 D 4.4 $33 \mathrm{~N} 4.4$ $33 \times 4.8$

22 6 SOUTHEST INDIAN $R$ IDIC

UNEST INDIAN RIDGE

16 MID-INDIAN RIDGE

13 NEAR NORTH COAST OF IRIAN JAYA

30 LUZON, PHILIPPINE ISLANDS

268 SOLOMON ISLANDS. MW 5.9 (GS), 5.9 (HRV). Me 5.5 (GS). MS 5.8 (BRK).

Broadband Source Parameters (GS): Dep 33; NP1: Strike-130, Dip-52, Slip-90; NP2: Strike-310, Dip-38, Slip-90; Radiated energy $4.3 * 10 * * 12 \mathrm{Nm}$.

Moment Tensor (GS): Dep 34; Principal axes (scale 10**17 $\mathrm{Nm}$ ): (T) Val-6.71, PIg-87, Azm-214; (N) Val-0.09, Plg-1 Azm-323; (P) Val--6.79, Plg-3, Azm-54; Best double couple: Mo-6.8*10*17 Nm; NP1: Strike-145, Dip-42, S1ip-92; NP2: Strike-322, Dip $=48$, SIip-88.

Centroid, Moment Tensor (HRV): Centroid origin time

$18: 17: 42.2$; Lat $6.71 \mathrm{~S}$; Lon 154.92 E; Dep 40.0 Fix; Halfduration $2.2 \mathrm{sec}$; Principal axes (scale 10*17 $\mathrm{Nm}$ ): (T) Val-8.13, Plg-79, Azm-12; (N) Val=0.15, Plg-5, A2m-129; (P) Val=-8.28, Plg-10, Azm-220; Best double couple:

Mo-8.2*10**17 Nm; NP1: Strike-317, Dip-35, S1ip-99; NP2: Strike-125, Dip-55, Slip-84.

$\begin{array}{lllll}15 & 19 & 22 & 03.48 & 3.321\end{array}$ $\begin{array}{llll}19 & 46 & 07.8 ? & 36.65\end{array}$ $195129.1 * 52.721 \mathrm{~N}$ $210637.7839 .748 \mathrm{~N}$ $213236.8 * 39.105 N$ $\begin{array}{lllll}21 & 49 & 01.88 & 37.033 & N\end{array}$ $221714.5836 .266 \mathrm{~N}$

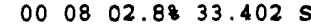
$004911.3 * 45.047 \mathrm{~N}$ $011331.8 * 28.340 \mathrm{~N}$ $\begin{array}{lllll}01 & 38 & 11.3 & 34.898 \mathrm{~N}\end{array}$ $\begin{array}{llllllll}01 & 56 & 04.7 ? & 15.46 \quad \mathrm{~s}\end{array}$ $\begin{array}{lllll}02 & 43 & 03.4 & 42.853\end{array}$ $\begin{array}{lllll}03 & 13 & 31.7 & 51.585 \mathrm{~N}\end{array}$ $\begin{array}{lllll}03 & 17 & 56.0 & 12.435 \mathrm{~N}\end{array}$ $03 \quad 4943.6 * 42.757$ $040202.4 * 1.854 \mathrm{~N}$ $054457.6 * 3.537 \mathrm{~s}$ $\begin{array}{llll}05 & 4732.3 & 3.420 \mathrm{~s}\end{array}$ $232238.9860 .403 \mathrm{~N}$

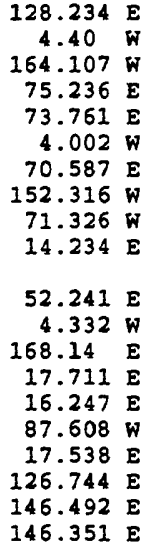

$100 \mathrm{G}$

$10 \mathrm{G}$

$33 \mathrm{~N}$

$33 \mathrm{~N}$

$33 \mathrm{~N}$

$5 \mathrm{G}$

$200 \mathrm{G}$

94

$50 \mathrm{G}$

$10 \mathrm{G}$

$33 \mathrm{~N}$

$10 \mathrm{G} \quad 3.9$

$10 \mathrm{G} 4.4$

$10 \mathrm{G}$

57 D 4.6

$10 \mathrm{G}$

33 N 4.8

$33 \mathrm{~N} 5.0$

$\begin{array}{llllll}33 & \mathrm{~N} & 5.0 & & 1.4 \\ 33 & \mathrm{~N} & 5.5 & 5.8 & 1.4\end{array}$
$200 \mathrm{G} 4.8$
$065413.6 * 3.357 \mathrm{~s}$ $\begin{array}{lllll}07 & 16 & 25.7 ? & 3.32 & 5\end{array}$ $073544.0833 .109 \mathrm{~s}$ $\begin{array}{lllllll}08 & 48 & 52.9 ? & 5.72 \quad S\end{array}$ $091455.5 * 25.100 \mathrm{~S}$ $094701.0859 .336 \mathrm{~N}$ $\begin{array}{lllll}10 & 00 & 20.8 & 33.145 & \mathrm{~N}\end{array}$ $101618.4834 .029 \mathrm{~s}$ $\begin{array}{lllll}12 & 16 & 14.8 & 13.370 \mathrm{~N}\end{array}$ $0655 \quad 46.7 * 3.244 \mathrm{~s}$
$146.298 \mathrm{E}$ $146.572 \mathrm{E}$ $146.38 \mathrm{E}$ $70.381 \mathrm{~W}$ $145.78 \mathrm{E}$ $175.269 \mathrm{~W}$ $151.673 \mathrm{~W}$ $137.553 \mathrm{E}$ $71.373 \mathrm{~W}$ $125.640 \mathrm{E}$

$\begin{array}{rrlrrr}33 & \mathrm{~N} & 4.9 & & 1.3 & 26 \\ 33 & \mathrm{~N} & 5.0 & & 1.3 & 24 \\ 33 & \mathrm{~N} & 4.3 & & 1.5 & 11 \\ 100 & \mathrm{G} & & & 0.3 & 9 \\ 33 & \mathrm{~N} & 4.1 & & 1.3 & 10 \\ 33 & \mathrm{~N} & 4.9 & 4.8 & 1.3 & 35 \\ 53 & & & & 55 \\ 357 & & 4.6 & & 0.9 & 54 \\ 50 & \mathrm{G} & & & 0.4 & 11 \\ 33 & \mathrm{~N} & 5.3 & 4.9 & 1.1 & 108\end{array}$

1 $123055.2 * 21.523$ $\begin{array}{llllll}13 & 23 & 47.0 ? & 26.71 & \mathrm{~s}\end{array}$ $1355 \quad 37.5 * 20.712 \mathrm{~N}$ 140223.9 ? $33.03 \mathrm{~S}$ $142249.5 * 23.712$ s

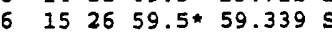

$121.188 \mathrm{E}$ $178.219 \mathrm{~W}$ $26.66 \mathrm{E}$ $144.842 \mathrm{E}$ $178.55 \mathrm{~W}$ $177.693 \mathrm{~W}$ 27.232 W
$33 \times 4.3$ $500 \mathrm{G} \quad 5.1$ $167 *$ $100 \mathrm{G} \quad 4.7$ $200 \mathrm{G} 4.7$

$1.0 \quad 15$

$1.4 \quad 128$

$1.0 \quad 8$

$0.7 \quad 17$ $125228.0 \quad 17.779 \mathrm{~s}$ 5 G 4.5
1.3

0.7

1.1

0.7

1.4

1.1

0.3

1.2

0.2

1.3

1.3

1.2

1.1

1.1
11 NORTB OF GALMABERA, INDONESIA

4 STRAIT OF GIBRALTAR

23 SOUTH OF ALASKA

8 SOUTHERN XINJIANG, CHINA

20

SPAIN. mbLg 1.9 (NDD).

9 HINDO KOSH REGION, AFGHANISTAN

72 SOUTHERN ALASKA. <AEIC>.

9 NEAR COAST OF CENTRAL CHILE

NORTHWESTERN BALKAN REGION. MI 2.7 (VIE), 2.7 (LDG), 2.5 (INU).

10 SOUTHERN IRAN

92 MOROCCO. mbLg 3.8 (MDD).

18 VANUATO ISLANDS

15 ADRIATIC SEA. ML 4.2 (LJO), 4.1 (ROM).

13 POIAND. ML 3.2 (GRF), 2.9 (MOX).

48 NEAR COAST OF NICARAGUA

12 ADRIATIC SEA

26 NORTHERN MOLJCCA SEA

27 BISMARCK SEA

73 BISMARCK SEA. MW 5.9 (GS), 5.9 (HRV). MS 5.9 (BRK).

Moment Tensor (GS): Dep 35; Principal axes (scale 10 1 ; Nm): (T) Val-7.82, Plg-6, Azm-326; (N) Val-1.46, Plg-83 Azm-185; (P) Val- -9.27 , Plg-4, Azm-57; Best double couple: Mo-8.5*10**17 Nm; NP1: Str1ke-102, Dip-83, slip-1; NP2: Strike-12, Dip-89, Slip-173.

Centroid, Moment Tensor (HRV): Centroid origin time 05:47:33.7; Lat 3.54 S; Lon 146.89 E; Dep 15.0 Fix; Halfduration $2.3 \mathrm{sec}$; Principal axes (scale 10*17 $\mathrm{Nm}$ ): (T) Val-8.62, Plg-6, Azm-319; (N) Val-0.49, Plg-81, Azm-90; (P) val--9.12, Plg-7, Azm-228; Best double couple:

Mo-8.9*10**17 Nm; NP1: Strike-4, Dip-81, S1ip--179; NP2: Strike-274, Dip-89, Slip--9.

BISMARCK SEA

BISMARCK SEA

BISMARCK SEA

CHILE-ARGENTINA BORDER REGION. MD 2.2 (SAN)

EASTERN NEW GOINEA REG., P.N.G.

SOUTH OF TONGA ISIANDS

KENAI PENINSULA, ALASKA. CAEIC>. MC 3.0 (AEIC).

NEAR $S$. COAST OF BONSHU, JAPAN

NEAR COAST OF CENTRAL CHILE. MD 3.7 (SAN).

PHILIPPINE ISLANDS REGION. NW 5.3 (HRV).

Centroid, Moment Tensor (HRV): Centroid origin time

$12: 16: 13.8$; Lat $13.47 \mathrm{~N}$; Lon $125.84 \mathrm{E}$; Dep $15.0 \mathrm{Fix}$; Half-

duration 1.0 sec; Principal axes (scale 10**16 Nm): (T)

Val-8.35, Plg-12, Azm-235; (N) Val-1.36, Plg-22, Azm-330;

(P) Val--9.71, P1g-64, Azm-120; Best double couple:

Mo-9.0*10*16 Nm; NPI: Strike-299, Dip-39, Slip=-128; NP2 :

Strike-164, Dip=60, Slip--64.

TAIWAN REGION

FIJI ISLANDS REGION

REPUBLIC OF SOOTH AFRICA

MARIANA ISLANDS

SOUTH OF KERMADEC ISLANDS

SOUTH OF FIJI ISIANDS

SOUTH SANDWICH ISLANDS REGION. MW 5.3 (BRV).

Centroid, Moment Tensor (HRV): Centroid origin time

15:27:03.5: Lat $59.63 \mathrm{~S}$; Lon $26.37 \mathrm{w}$; Dep $15.0 \mathrm{Fix}$; Halfduration 1.0 sec; Principal axes (scale $10 * * 17 \mathrm{Nm}$ ): (T) Val-1.03, P1g-68, Azm-254; (N) Val--0.08, Plg-20, Azm-99; (P) Val--0.94, Plg-8, Azm-5; Best double couple:

Mo-9.9*10**16 Nm; NP1: Strike-74, Dip-41, Slip-59; NP2: Strike-292, Dip-56, Slip-114.

$\begin{array}{lllll}16 & 15 & 43 & 24.48 & 37.027 \mathrm{~N}\end{array}$

$3.404 \mathrm{~W} \quad 10 \mathrm{G}$

$69.190 \mathrm{~W} 122 * 4.6$

$\begin{array}{lllllllllll}16 & 17 & 07 & 16.13 & 17.70 & \mathrm{~S} & 178.29 & \mathrm{~W} & 500 & \mathrm{G} & 4.8 \\ 16 & 17 & 08 & 14.1 * & 17.812 & \mathrm{~S} & 178.342 & \mathrm{~W} & 600 & \mathrm{G} & 4.6\end{array}$

$16 \quad 170814.1 * 17.812 \mathrm{~S}$

$\begin{array}{rrrrr}16 & 18 & 59 & 14.8 \% 63.276 \mathrm{~N} \\ 16 & 19 & 32 & 40.0 * 9.982 \mathrm{~s}\end{array}$

178.342

$151.046 \mathrm{~W}$

$160.707 \mathrm{E}$
11

$33 \times 4.6$
0.611 SPAIN. mbIg 2.7 (MDD).

1.415 NORIHERN CHILE

1.8106 FIJI ISLANDS REGION

1.132 FIJI ISLANDS REGION

65 CENTRAL ALASKA. LAEIC>. ML 3.0 (AEIC).

1.029 SOLOMON ISLANDS 
$\begin{array}{llllllll}20 & 52 & 03.5 & 67.324 & \mathrm{~N} & 166.225 \mathrm{~W}\end{array}$ $211240.4 * 7.319 \mathrm{~s} 155.416 \mathrm{E}$ $213808.8 * 23.142 \mathrm{~s} 68.132 \mathrm{w}$ $\begin{array}{lllllll}21 & 45 & 16.7 & 1.244 & S & 102.219 \mathrm{E}\end{array}$ $\begin{array}{lllll}21 & 58 & 08.0 & 42.402 \mathrm{~N} & 1.385 \mathrm{E}\end{array}$ $215822.8 * 42.675 \mathrm{~N} \quad 1.267 \mathrm{E}$ $\begin{array}{lllll}22 & 07 & 31.6 & 42.349 \mathrm{~N} & 1.433 \mathrm{E}\end{array}$ $\begin{array}{lllll}22 & 15 & 07.28 & 46.267 \mathrm{~N} & 2.917 \mathrm{E}\end{array}$ $\begin{array}{llllll}22 & 16 & 17.2 & 9.312 \mathrm{~N} & 126.409 \mathrm{E}\end{array}$ $\begin{array}{lllllll}22 & 37 & 07.8 & 42.608 & \mathrm{~N} & 17.558 \mathrm{E}\end{array}$ $\begin{array}{lllllll}23 & 04 & 39.2 & 3.324 & \mathrm{~S} & 143.452 \mathrm{E}\end{array}$ $\begin{array}{lllll}10 & G & 4.6 & 4.3 & 1.0\end{array}$ $33 \times 4.7$ 125 G 4.1 262 D 5.0 $5 \mathrm{G}$ $5 \mathrm{G}$

\section{$10 \mathrm{G}$}

$33 \mathrm{~N}$

$10 \mathrm{G}$

$33 \mathrm{~N} \quad 4.9$

\section{BERING STRAIT}

1.341 SOLONON ISLANDS

0.814 NORTHERN CHILE

1.197 SOUTHERN SUMATERA, INDONESIA

1.218 PYRENEES. mbLg 3.0 (MDD). ML 2.9 (STR), 2.8 (LDG)

1.511 PYRENEES. mbLg 3.1 (MDD). ML 2.9 (LDG).

1.131 PYRENEES. mbLg 3.3 (MDD). ML 3.2 (LDG). Felt (III) in the epicentral area.

0.49 FRANCE. MI 1.5 (IDG).

1.137 MINDANAO, PHILIPPINE ISLANDS

0.715 ADRIATIC SEA

1.038 NEAR N COAST OF NEW GUINEA, PNG. MW 5.2 (HRV). Centroid, Moment Tensor (BRV) : Centroid origin time 23:04:38.9; Lat $3.53 \mathrm{~S}$; Lon $143.76 \mathrm{E}$; Dep $15.0 \mathrm{Fix}$; Halfduration $1.1 \mathrm{sec}$ : Principal axes (scale 10*16 Nm): (T) Val-7.83, Plg-72, Azm-106; (N) Val--0.18, Plg=13, Azm-332; (P) Val=-7.66, Plg-13, Azm-239; Best double couple: Mo-7.8*10*16 Nm; NP1: Strike-313, Dip-34, Slip-67; NP2: Strike-160, Dip-59, Slip-105.

$\begin{array}{lllll}16 & 23 & 05 & 09.7 * 20.423 & 5\end{array}$ $\begin{array}{llllll}17 & 00 & 25 & 41.9 & 46.097 & \mathrm{~N}\end{array}$ $\begin{array}{lllllll}17 & 01 & 58 & 02.7 & 38.641 & \mathrm{~N}\end{array}$ $\begin{array}{llllll}17 & 05 & 32 & 48.98 & 61.786 \quad \mathrm{~N}\end{array}$ $\begin{array}{lllll}17 & 05 & 59 & 44.1 & 12.689 \mathrm{~N}\end{array}$

68.745 พ $136 ?$ 6.772 E $5 \mathrm{G}$ $20.838 \mathrm{E}$ $151.857 \mathrm{~W} 107 \mathrm{G}$ $88.115 \mathrm{~W}$ $0733.05 .1 \quad 42.934 \times 17.904 \mathrm{E}$ $0906 \quad 28.5828 .958$ N $103.862 \mathrm{E}$ $091855.7 * 42.839 \times 17.806 \mathrm{E}$ $092117.0 * 38.741 \mathrm{~N} 142.015 \mathrm{E}$ $100414.3853 .477 \mathrm{~N} 159.880 \mathrm{E}$ $103001.0845 .249 \mathrm{~N} \quad 6.482 \mathrm{E}$ $\begin{array}{lllllll}10 & 50 & 56.0 ? & 7.11 & \mathrm{~N} & 73.14 & \text { W } \\ 13 & 45 & 22.8 & 42.866 & \mathrm{~N} & 17.820 & \mathrm{E}\end{array}$

73 :

0.79 CEILE-BOLIVIA BORDER REGION

0.839 SWITZERLAND. ML 2.6 (IDG), 2.6 (STR)

1.033 GREECE

79 SODTHERN ALASKA. LAEIC>. Dsulutan, El Salvador.

1.0 9 ADRIATIC SEA

1.06 SICBOAN, CHINA

$1.5 \quad 8$ ADRIATIC SEA

$\begin{array}{lrl}1.5 & 8 & \text { ADRIATIC SEA } \\ 1.1 & 16 & \text { NEAR EAST COAST OF HONSHU, JAPAN }\end{array}$

1.17 NEAR EAST COAST OF KAMCHATKA

1.38 FRANCE. ML 2.1 (IDG).

1.48 NORTMERN COLOMBIA
$10 \mathrm{G}$ 107 $56 \quad 0 \quad 4.9$

$10 G$

$33 \mathrm{~N}$

$10 \mathrm{G}$

$5 G$

$150 \mathrm{G}$

$\begin{array}{llll}10 G & 5.4 & 5.1 & 1.4 \\ 1.2\end{array}$
1.1121 OFF COAST OF CENTRAI AMERICA. Felt (III) at San Salvador and

248 ADRIATIC SEA. MW 5.5 (HRV). KC 4.9 (ROM). Additional damage in the slano and Ston areas, Croatia. Felt on the Peljesac Peninsula and on Korcula. Croatia. Also felt at Dubrovnik, Croatia. Felt (V) at Herceg-Novi, Kotor and Tivat; (IV) at Bar, Budva, Niksic and Podgorica, Yugoslavia. Felt (III) in the northwestern part of the former Yugoslav Republic of Macedonia.

Centroid, Moment Tensor (HRV): Centroid origin time $13: 45: 27.9$; Lat $42.59 \mathrm{~N}$; Lon $17.53 \mathrm{E}$; Dep 15.0 Fix; Half duration $1.3 \mathrm{sec}$; Principal axes (scale 10*17 $\mathrm{Nm}$ ): (T) Val-1.54, Plg-77, Azm-72; (N) Val-0.25, Plg-8, Azm=306; (P) val-1.80, Plg-11, Azm-215: Best double couple: Mo-1.7*10*17 Nm; NP1: Strike-295, Dip-35, Slip-76; NP2 : Strike-132, Dip-56, Slip-99.

$14 \quad 18 \quad 26.4 \quad 51.687 \mathrm{~N}$

$16.224 \mathrm{E}$ $143046.1 * 42.933 \mathrm{~N}$ $150128.6829 .175 \mathrm{~N}$ $\begin{array}{lllll}15 & 06 & 40.6 & 42.855 \mathrm{~N}\end{array}$ $\begin{array}{lllll}15 & 35 & 49.1 & 46.824 & N\end{array}$ $1554 \quad 46.7 * 28.819 \mathrm{~S}$ $162256.3 * 18.962 \mathrm{~N}$ $153818.8 * 1.302 \mathrm{~N}$ $164801.9 * 24.113 \mathrm{~S}$ 171236.2 ? $\quad 0.24 \mathrm{~N}$ $\begin{array}{lllll}18 & 19 & 22.7 & 21.517 & \mathrm{~N}\end{array}$ $\begin{array}{llllll}18 & 45 & 33.5 & 42.885 \mathrm{~N}\end{array}$ $190321.8 * 30.083 \mathrm{~N}$ $192714.2862 .119 \mathrm{~N}$ $193847.8862 .603 \times$ $194819.8 * 1.280 \mathrm{~s}$ $2004 \quad 40.2846 .799 \mathrm{~N}$ $202257.5 \& 59.992 \mathrm{~N}$ $204501.7 * 13.671 \mathrm{~N} 144.864 \mathrm{E}$

$10 \mathrm{G}$ $17.622 \mathrm{E}$ $130.609 \mathrm{E}$ $17.941 \mathrm{E}$ $1.932 \mathrm{w}$ 69.915 พ $145.416 \mathrm{E}$ $122.989 \mathrm{E}$ 66.699 $98.71 \mathrm{E}$ $121.419 \mathrm{E}$ $17.905 \mathrm{E}$ $81.675 \mathrm{E}$ $149.754 \mathrm{~W}$ $149.472 \mathrm{~W}$ $127.641 \mathrm{E}$ $2.026 \mathrm{~W}$ $212500.4 * 15.637 \mathrm{~N} 144.975 \mathrm{E}$ $221156.1 * 54.919 \mathrm{~s} 129.001 \mathrm{~W}$

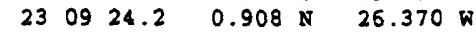

$10 \mathrm{G}$

$33 \mathrm{~N}$

$10 \mathrm{G}$

$5 \mathrm{G}$

$106 \mathrm{D}$

$250 \mathrm{G}$

50 G 4.7

$200 * 4.1$

$33 \times 3.9$

$33 \times 4.6$

$10 \mathrm{G}$

$33 \mathrm{~N}$

50

64

$10 \mathrm{G}$

139

114

$33 \mathrm{~N}$

$10 \mathrm{G} \quad 4.6$

10 G $5.3 \quad 5.2 \quad 0.3 \quad 19$

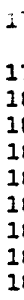$$
23
$$

$224707.143 .648 \times 147.213 \mathrm{E}$

$\begin{array}{llllll}33 & \mathrm{~N} & 5.5 & 4.6 & 0.9 & 259\end{array}$ $\begin{array}{llllll}23 & 53 & 39.08 & 32.867 & \mathrm{~s} \\ 00 & 08 & 07.28 & 31.959 & \mathrm{~s}\end{array}$ $70.949 \mathrm{~W}$ $\begin{array}{lllllll}00 & 08 & 07.28 & 31.959 \mathrm{~S} & 116.858 \mathrm{E}\end{array}$ $\begin{array}{llllll}00 & 13 & 07.0 & 43.010 \mathrm{~N} & 146.870 \mathrm{E}\end{array}$ $004114.28 \quad 31.965 \mathrm{~s} \quad 116.709 \mathrm{E}$ $\begin{array}{llllll}01 & 41 & 01.98 & 47.259 \mathrm{~N} & 2.421 \mathrm{~W}\end{array}$ $020923.3 * 25.557 \mathrm{~s} \quad 179.445 \mathrm{E}$ $021057.4 * 5.643 \mathrm{~s} 102.780 \mathrm{E}$ $\begin{array}{lllllll}02 & 13 & 40.48 & 33.862 & \mathrm{~S} & 69.989 \mathrm{~W} \\ 02 & 16 & 27.7 * & 33.738 & \mathrm{~N} & 82.099 \mathrm{~W}\end{array}$

$70 \mathrm{G}$

$10 \mathrm{G}$

$53 \mathrm{D}$

$10 \mathrm{G}$

$550 \mathrm{G}$

$43 \mathrm{D}$

$10 G$
$5 G$
0.916

$1.27 \%$

$0.6 \quad 5$ RYUKYU ISLANDS

1.175 ADRIATIC SEA. ML 3.6 (BRA)

1.135 FRANCE. ME 3.6 (LDG) 3.0 (STR)

1.218 CHILE-ARGENTINA BORDER REGION. MD 4.6 (SAN).

1.124 MARIANA ISLANDS

1.226 MINAHASSA PENINSULA, SULAWESI

1.216 SALTA PROVINCE, ARGENTINA

1.415 NORTHERN SUMATERA, INDONESIA

1.227 TAIWAN REGION

1.344 ADRIATIC SEA. MI 3.5 (ROM).

1.011 XIZANG

52 CENTRAL ALASKA. <AEIC>. ML 2.9 (AEIC).

47 CENTRAI ALASKA. LAEIC>. MI 2.6 (AEIC).

16 EALMAEBRA, INDONESIA

0.97 BAY OF BISCAY. NL 2.0 (LDG)

44 SOOTEERN ALASXA. <AEIC>.

17 MARIANA ISLANDS. Felt (III) by people in high-rise buildings at Dededo and Tamuning, Guam.

814 MARIANA ISLANDS REGION

CENTRAC MID-ATLANTIC RIDGE. MW 5.6 (HRV).

Centroid, Moment Tensor (HRV): Centroid origin time 23:09:29.6; Lat $1.07 \mathrm{~N}$; Lon $26.56 \mathrm{~W}$; Dep $15.0 \mathrm{Fix}$; Ealf duration $1.6 \mathrm{sec}$; Principal axes (scale 10**17 $\mathrm{Nm}$ ): (T) Val-2.63, Plg=0, Azm-213; (N) Val-0.30, Plg-90, Azm-180; (P) Val=-2.33, Plg=0, Azm-123; Best double couple: Mo-2.5*10**17 Nm; NP1: Strike-258, Dip-90, S1ip=-180; NP2: Strike-348, Dip-90, slip=0.

KURIL ISLANDS. Felt (V) on Shikotan and (IV) at YuzhnoKurilsk, Runashir.

0.39 CHILE-ARGENTINA BORDER REGION. MD 2.9 (SAN).

1.57 WESTERN AUSTRALIA

$5.0 \quad 0.9 \quad 157$ KURIL ISLANDS

1.25 WESTERN AOSTRALIA

0.68 FRANCE. MC 2.5 (LDG).

$4.7 \quad 0.7 \quad 19$ SOUTH OF FIJI ISLANDS

$4.6 \quad 0.8 \quad 16$ SOUTHERN SOMATERA, INDONESIA

0.110 CEILE-ARGENTINA BORDER RBGION MD 3.5 (SAN)

0.65 GEORGIA, USA. mbLg 2.8 (GS). Felt at Edgefield and GEORGIA, USA. mbLg 2.8 (GS)
MCCormick, South Carolina.

SOUTH OF EONSEO, JAPAN. MW 5.7 (HRV).

Centroid, Moment Tensor (HRV): Centroid origin time $03: 25: 48.8$; Lat $32.35 \mathrm{~N}$; Ion $141.65 \mathrm{E}$; Dep 22.9 ; Halfduration $1.6 \mathrm{sec}$; Principal axes (scale 10*17 Nm): (T) val=3.55, Plg=74, Azm=231; (N) Val=0.54, Plg=10, Azm=358; 
(P) Val--4.09, Plg=12, Azm=90; Best double couple:

Mo-3.8*10**17 Nm; NP1: Strike-193, Dip-34, Slip-108; NP2: Strike-352, Dip-58, Slip-78.

Centroid, Moment Tensor (HRV): Centroid origin time

$04: 11: 47.2$; Lat $20.26 \mathrm{~s}$; Lon $168.85 \mathrm{E}$; Dep $34.0 \mathrm{Fix}$; Half -

duration $1.6 \mathrm{sec}$; Principal axes (scale $10 * 17 \mathrm{Nm}$ ): (T)

Val=4.19, Plg=76, Azm=2B; (N) Val=0.30, Plg=10, Azm=160;

(P) Val=-4.49, Plg=11, Azm-252; Best double couple:

Mo-4.3*10**17 Nm; NP1: Strike-354, Dip=35, slip-107; KP2: Strike=154, Dip=56, Slip=7B.

$\begin{array}{lllllllll}18 & 04 & 13 & 18.9 & 52.739 & \mathrm{~N} & 154.097 & \mathrm{E}\end{array}$ $\begin{array}{lllllllll}18 & 04 & 43 & 42.1 ? & 21.8 B & 5 & 112.63 \quad \mathrm{~W}\end{array}$ $\begin{array}{lllll}18 & 05 & 16 & 40.2 * 39.607 \mathrm{~N} \quad 41.050 \mathrm{E}\end{array}$ $\begin{array}{lllllllll}18 & 05 & 29 & 03.6 & 9.553 \mathrm{~N} & 126.256 \mathrm{E}\end{array}$ $\begin{array}{llllll}18 & 05 & 53 & 27.0 * & 9.552 & \mathrm{~N} \\ 18 & 06 & 15 & 46.2 & 32.493 & \mathrm{~N} \\ 18 & 07 & 01 & 36.08 & 31.975 & \mathrm{~S} \\ 18 & 07 & 29 & 17.58 & 41.792 & \mathrm{~N} \\ 18 & 08 & 41 & 50.7 * & 3.307 & \mathrm{~S} \\ 18 & 09 & 08 & 49.9 * & 23.009 & \mathrm{~S} \\ 18 & 09 & 15 & 45.4 * & 21.388 & \mathrm{~N} \\ 18 & 09 & 51 & 46.7 & 68.017 & \mathrm{~N} \\ 18 & 09 & 54 & 47.88 & 53.774 & \mathrm{~N} \\ 18 & 10 & 35 & 48.1 * & 23.044 & \mathrm{~S} \\ 18 & 13 & 14 & 40.5 ? & 14.35 & \mathrm{~S} \\ 18 & 14 & 36 & 40.7 * & 26.028 & \mathrm{~N} \\ 18 & 17 & 06 & 38.8 * & 21.264 & \mathrm{~N} \\ 18 & 17 & 25 & 06.8 & 29.326 & \mathrm{~N} \\ 18 & 17 & 34 & 20.6 & 11.435 & \mathrm{~N}\end{array}$

$\begin{array}{llll}18 & 18 & 21 & 34.8 * 43.112\end{array}$

$18 \quad 18 \quad 23 \quad 36.0 * 53.803 \mathrm{~N}$

$\begin{array}{llllll}18 & 19 & 09 & 35.9 ? & 18.90 & N\end{array}$

$18 \quad 1914 \quad 52.0 * 5.856 \mathrm{~S}$

$\begin{array}{llllll}18 & 19 & 57 & 20.38 & 48.133\end{array}$

$\begin{array}{llllll}18 & 20 & 30 & 11.6 & 43.915 & \mathrm{~N}\end{array}$

$\begin{array}{llllll}18 & 20 & 32 & 19.7 ? & 72.18 & \mathrm{~N}\end{array}$

$\begin{array}{lllllll}18 & 21 & 23 & 54.5 & 51.509 & \mathrm{~N} \\ 18 & 21 & 25 & 14.38 & 36.744 & \mathrm{~N}\end{array}$

$18 \quad 221048.5 * 2.973 \mathrm{~N}$

$\begin{array}{lllll}18 & 22 & 15 & 45.0 * 30.606 \mathrm{~N}\end{array}$

$\begin{array}{llllllll}18 & 22 & 34 & 57.4 ? \quad 45.63\end{array}$

$\begin{array}{lllllll}18 & 22 & 46 & 24.9 ? & 4 B . B B & S\end{array}$

$\begin{array}{lllllll}18 & 22 & 56 & 59.28 & 65.718 & \mathrm{~N}\end{array}$

$\begin{array}{lllll}18 & 23 & 35 & 51.8 * 31.827 \mathrm{~S}\end{array}$

$\begin{array}{llllllll}18 & 23 & 37 & 27.4 ? & 72.06 \quad \mathrm{~N}\end{array}$

$\begin{array}{llll}18 & 23 & 42 & 13.8 * 18.834 \mathrm{~N}\end{array}$

$\begin{array}{llllll}18 & 23 & 50 & 36.0 & 12.754 & \mathrm{~N}\end{array}$

$1900 \quad 14 \quad 29.4 \quad 38.672 \quad N$

$\begin{array}{lllllll}19 & 02 & 52 & 29.98 & 46.679 & N\end{array}$

$\begin{array}{lllll}19 & 04 & 45 & 31.0 \quad 32.519 & \mathrm{~N}\end{array}$

$\begin{array}{rrrrrr}400 & G & 3.9 & & 0.9 & 22 \\ 10 & G & & & 1.2 & 7 \\ 10 & G & 4.1 & & 1.3 & 11 \\ 33 & N & 5.5 & 5.3 & 1.1 & 111\end{array}$

$\begin{array}{lllll}33 & 5.5 & 5.3 & 1.1 & 111\end{array}$
$126.234 \mathrm{E}$ $141.332 \mathrm{E}$ $116.736 \mathrm{E}$ $126.400 \mathrm{~W}$ $169.647 \mathrm{E}$ $121.073 \mathrm{E}$ $139.830 \mathrm{E}$ $35.672 \mathrm{~h}$ $169.675 \mathrm{E}$ $171.73 \mathrm{E}$ $61.018 \mathrm{E}$ $99.443 \mathrm{E}$ $130.142 \mathrm{E}$ $85.471 \mathrm{~W}$ $140.867 \mathrm{E}$

$\begin{array}{rrrrr}33 & \mathrm{~N} & 4.8 & & 1.0 \\ 22 & \mathrm{D} & 4.9 & 4.8 & 1.0 \\ 10 & \mathrm{G} & & & 1.0 \\ 5 & & & & \\ 33 & \mathrm{~N} & 4.3 & & 1.2 \\ 33 & \mathrm{~N} & 4.2 & & 1.4 \\ 33 & \mathrm{~N} & 4.4 & & 0.9 \\ 33 & \mathrm{~N} & 4.7 & 4.7 & 0.8 \\ 10 & \mathrm{G} & & & 0.8 \\ 33 & \mathrm{~N} & 4.6 & & 1.7 \\ 600 & \mathrm{G} & 4.6 & & 0.6 \\ 10 & \mathrm{G} & 4.8 & & 1.3 \\ 33 & \mathrm{~N} & & & 1.0 \\ 75 & & 4.6 & & 1.2 \\ 193 & \mathrm{D} & 5.3 & & 1.0\end{array}$
193 D 5.3
NORTEWEST OF KURIL ISLANDS

SOUTHERN EAST PACIFIC RISE

TURREY. Felt in Bingol.

MINDANAO, PHILIPPINE ISLANDS. MW 5.7 (HRV).

Centroid, Moment Tensor (FRV): Centroid origin time

$05: 29: 08.3$; Lat $9.49 \mathrm{~N}$; Lon $126.64 \mathrm{E}$; Dep 20.5; Halfduration $1.7 \mathrm{sec;}$ Principal axes (scale 10*17 $\mathrm{Nm}$ ): (T) Val-4.49, Plg=72, $A z m=289$; (N) Val=-0.40, Plg-6, Azm=180; (P) Val=-4.09, Plg-17, Aam=BB; Best double couple:

Mo-4.3*10*17 km; NP1: Strike=168, Dip=28, Slip=77; NP2: Strike-3, Dip-63, slip=97.

\section{MINDANAO, PEILIPPINE ISLANDS}

SOUTH OF BONSEU, JAPAN

WESTERN AUSTRALIA

3 OFF COAST OF NORTHERN CALIFORNIA. <GM-P>. ID 3.0 (GM).

IRIAN JAYA, INDONESIA

LOYALTY ISLANDS REGION

TAIWAN REGION

EASTERN SIBERIA, ROSSIA

NORTE ATLANTIC OCEAN

IOYALTY ISLANDS REGION

VANOATU ISLANDS REGION

SOUTHERN IRAN

MYANDAR-CHINA BORDER REGION

RYUKYO ISLANDS

294 NICARAGUA. NW 5.6 (GS), 5.6 (ERV)

Moment Tensor (GS): Dep 191; Principal axes (scale 10**17

$\mathrm{Nm}$ ): (T) Val-2.71, Plg-68, $A 2 m=48$; (N) Val=0.47, Plg=1,

Azm=140; (P) Val=-3.18, Plg-22, Azm=230; Best double

couple: Mo-2.9*10*17 Nm; NP1: strike-321, Dip=23, slip-91; NP2: Strike=140, Dip=67, slip-89.

centroid, Moment Tensor (HRV): Centrold origin time $17: 34: 26.4$; Lat $11.45 \mathrm{~N}$; Lon $85.82 \mathrm{w}$; Dep 184.2; Halfduration $1.5 \mathrm{sec}$; Principal axes (scale 10**17 $\mathrm{Nm}$ ): (T) Val=2.65, Plg=66, Azm=66; (N) Val=0.04, Plg=4, Azm=327; (P) val=-2.69, Plg=23, Azm=235; Best double couple:

Mo-2.7*10**17 Nin; NP1: Strike-316, Dip=22, Slip=78; NP2:

Strike-148, Dip=69， Slip=95. $35.416 \mathrm{~F}$

NORTE ATLANTIC OCEAN
$18210406.5 * 2.855 \mathrm{~N}$

$\begin{array}{lllllll}19 & 03 & 53 & 42.28 & 30.771 & N\end{array}$

$147.530 \mathrm{E}$ $67.23 \mathrm{~W}$ $153.197 \mathrm{E}$

$2.411 \mathrm{~W}$ $7.670 \mathrm{E}$ $126.34 \mathrm{E}$ $126.843 \mathrm{E}$ $175.010 \mathrm{~W}$ $3.464 \mathrm{~W}$ $128.048 \mathrm{E}$ $130.892 \mathrm{E}$ $6.01 \mathrm{E}$ $121.53 \mathrm{E}$ $174.397 \mathrm{E}$ $16.859 \mathrm{E}$ $125.97 \mathrm{E}$ $145.260 \mathrm{E}$ $40.515 \mathrm{E}$ $0.494 \mathrm{~W}$ $4.90 \mathrm{~B} \mathrm{~W}$ $132.691 \mathrm{E}$ $141.434 \mathrm{E}$

$\begin{array}{rrrrrr}67 & \mathrm{D} & 3.7 & & 1.4 & 18 \\ 10 & \mathrm{G} & 4.7 & 4.7 & 1.3 & 16 \\ 33 \mathrm{~N} & & & 0.1 & 5 \\ 33 & \mathrm{~N} & 4.6 & & 1.0 & 21 \\ 10 \mathrm{G} & & & 0.5 & 6 \\ 10 \mathrm{G} & & & 1.2 & 18 \\ 10 \mathrm{G} & & & 1.5 & 6 \\ 100 \mathrm{G} & 4.2 & & 0.8 & 19 \\ 51 \mathrm{D} & 4.6 & & 0.9 & 24 \\ 10 \mathrm{G} & & & 1.0 & 6 \\ 150 \mathrm{G} & 4.6 & & 1.1 & 19 \\ 45 & & & & 1.3 & 11 \\ 10 \mathrm{G} & & & 0.8 & 4 \\ 10 \mathrm{G} & & & 1.3 & 6 \\ 33 & \mathrm{~N} & & & 0.8 & 10 \\ 10 \mathrm{G} & & & 1.5 & \mathrm{~B} \\ 10 \mathrm{G} & & & 1.4 & 5 \\ 600 \mathrm{G} & 4.2 & & 0.5 & 11 \\ 10 \mathrm{G} & 5.0 & 4.4 & 1.0 & 57 \\ 10 \mathrm{G} & & & 0.9 & 16 \\ 10 & \mathrm{G} & & & 0.6 & 22 \\ 33 & \mathrm{~N} & & & 0.6 & 5 \\ 20 & & 5.4 & 4.9 & 1.0 & 119\end{array}$

$\begin{array}{lllllll}19 & 05 & 29 & 40.3 & 43.953 & \mathrm{~N}\end{array}$ $\begin{array}{lllllll}19 & 07 & 13 & 01.9 ? & 6.38 & \mathrm{~s}\end{array}$ $\begin{array}{lllllll}19 & 07 & 35 & 36.38 & 36.279 & \mathrm{~N}\end{array}$ 19091729.2 ? $6.76 \mathrm{~s}$ $19 \quad 121744.3 * 4.791 \mathrm{~s}$ $\begin{array}{llllll}19 & 12 & 37 & 07.4 & 32.969 & \mathrm{~s}\end{array}$ $19 \quad 1404 \quad 40.6 * 19.6845$ $19 \quad 14 \quad 18 \quad 54.6 * 50.995 \mathrm{~N}$ $19 \quad 14 \quad 44 \quad 32.7 ? 45.41 \mathrm{~N}$ $\begin{array}{llll}19 & 1752 \quad 59.5 \& 65.014 \quad \mathrm{~N}\end{array}$

$\begin{array}{llllll}19 & 18 & 44 & 27.38 & 59.090 & \mathrm{~N}\end{array}$

$\begin{array}{lllll}19 & 18 & 45 & 05.1 * 6.277 \mathrm{~s}\end{array}$

$19205253.4 * 31.274 \mathrm{~S}$ 130.27 E 172 $120.464 \mathrm{~W}$ $154.41 \mathrm{E}$ $135.438 \mathrm{E}$ $72.149 \mathrm{~W}$ $170.028 \mathrm{E}$ 177.736 W

$\begin{array}{ccrc}177.736 & \text { W } & 33 & \mathrm{~N} \\ 26.51 & \mathrm{E} & 100 & \mathrm{G}\end{array}$ $146.900 \mathrm{~W} 15$
$7.626 \mathrm{E} \quad 10 \mathrm{G}$

$33 \mathrm{~N} \quad 4.7$

$33 \times 4.7$

$33 \mathrm{~N} 4.9$

$33 \mathrm{~N} 4.8$

4.3

0.9

\section{KORII ISLANDS}

MONA PASSAGE

NEW IRELAND REGION, P.N.G.

FRANCE. ML 1.7 (LDG)

NEAR SOUTE COAST OE FRANCE. ML 2.2 (LDG), 2.2 (GEN)

NORTECENTRAL SIBERIA, RUSSIA

NORTEERN MOLUCCA SEA

ANDREANOF ISLANDS, ALEUTIAN IS.

STRAIT OF GIBRALTAR. IUbLg 2.5 (MDD).

HAIMAHERA, INDONESIA

KYUSHU, JAPAN

FRANCE. MC 2.2 (LDG)

SOUTH OF AUSTRALIA

EASTERN SIBERIA, ROSSIA

WESTERN ADSTRALIA

NORTHCENTRAI SIBERIA, RUSSIA

MARIANA ISIANDS

ETHIOPIA

SPAIN. mbIg 3.1 (MDD). MI 2.7 (LDG).

BAY OF BISCAY. NC 3.1 (LDG)

SOUTHEAST OF SHIKORD, JAPAN

SOUTH OF HONSHU, JAPAN. MW 5.4 (HRV).

Centroid, Moment Tensor (BRV): Centroid origin time

$04: 45: 34.8$; Lat $32.39 \mathrm{~N}$; Lon $141.70 \mathrm{E}$; Dep 33.8 ; Half -

duration 1.2 sec; Principal axes (scale $10 * 17 \mathrm{Nm}$ ): (T)

Val=1.41, Plg=72, Azm=250; (N) Val=0.29, Plg=6, Azm-357;

(P) Val=-1.71, Plg=17, Azm=89; Best double couple:

MO-1.6*10*17 $\mathrm{Nm}$; NP1: Strike-187, Dip=28, Slip-102; NP2: Strike-354, Dip=63, Slip=B4.

0.211 NEAR SOOTH COAST OF FRANCE. IIL 2.4 (LDG), 2.1 (GEN).

$4.6 \quad 0.9 \quad 16$ BANDA SEA

CENTRAL CALIFORNIA. 〈GM-P>. MD 3.0 (GM). ML 3.0 (BRK).

1.314 SOLOMON ISLANDS

1.49 IRIAN JAYA REGION, INDONESIA.

OFF COAST OF CENTRAL CHIIE. ND 4.9 (SAN).

20 VANUATO ISLANDS

1.416 ANDREANOF ISLANDS, ALEUTIAN IS.

ROMANIA

42 NORTHERN ALASRA, <AEIC>. MC 3.1 (AEIC). FeIt at Fairbanks, North Pole and along Chena hot Springs Road.

75 SOUTHERN ALASKA. <AEIC>.

$4.7 \quad 1.5 \quad 10$ BANDA SEA

0.913 SAN JUAN PROVINCE, ARGENTINA. MD 4.3 (SAN). 

duration $1.3 \mathrm{sec}$; Priacipal axes (scale 10*\#17 Nm): (T) Val-2.31, Plg=41, Azm=77; (N) Val=-0.32, Plg=10, Azm-176; (P) Val=-1.99, Plg=47, Azm=277; Best double couple: Mo-2.2*10**17 Nm; NP1: Strike-101, Dip-11, slip=-165; NP2: Strike-356, Dip=87, SIip=-80.

$\begin{array}{lllllll}19 & 21 & 54 & 30.48 & 33.921 \mathrm{~s}\end{array}$

$\begin{array}{llllll}19 & 22 & 15 & 23.38 & 62.804 & N\end{array}$

$\begin{array}{llllll}20 & 00 & 03 & 18.3 & 9.597 \mathrm{~N}\end{array}$
$71.177 \mathrm{~W}$ 150.519 W $126.293 \mathrm{E}$
$50 \mathrm{G}$ $85 \quad \begin{array}{lll}0.3 & 50\end{array}$

NEAR COAST OF CENTRAL CHILE

CENTRAL AIASKA. <AEIC>

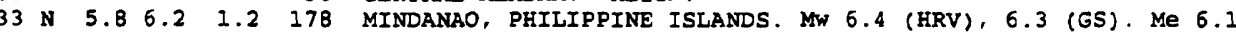
(GS). Ms 6.3 (BRK). Felt at Butuan and surigao. Also felt on Leyte.

Broadband Source Parameters (GS): Dep 20; NP1: Strike=15,

Dip=70, Slip-80; NP2: Strike-222, Dip=22, Slip=116; Radiated energy $3.3 * 10 * \star 13 \mathrm{Nm}$.

Moment Tensor (GS): Dep 24; Principal axes (scale 10**18 $\mathrm{Nm}$ ): (T) Val=3.69, Plg=63, Azm=295; (N) Val=-0.03, Plg=6, Azm-192; (P) Val=-3.66, Plg-26, Azm-99; Best double couple: MO-3.7*10**18 Nin; NP1: Strike-175, Dip=20, Slip=71; NP2: strike-14, Dip-71, Slip-96.

Centroid, Moment Tensor (HRV) : Centroid origin time

00:03:22.9; Lat $9.57 \mathrm{~N}$; Lon $126.68 \mathrm{E}$; Dep 22.9; Half duration $4.2 \mathrm{sec}$; Principal axes (scale 10**18 Nm): ( $\mathrm{T}$ ) Val=4.74, Plg=67, Azm=295; (N) Val=0.08, Plg=8, Azm=1B5; (P) val=-4.82, Plg=21, Azm=92; Best double couple:

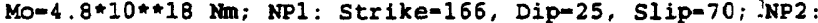
strike-8, Dip $=67$, Slip=99.

scalar Moment (PPT): MO-8.0*10*18 Nm.

$0000729.0 * 18.966 \mathrm{~s}$ $001142.9 * 9.593 \mathrm{~N}$ $\begin{array}{lllll}00 & 23 & 04.7 & 9.548 \mathrm{~N}\end{array}$ $011950.9 \quad 42.850 \mathrm{~N}$ $012504.0 * 6.218 \mathrm{~s}$ $\begin{array}{lllll}02 & 02 & 49.6 & 29.821 & 5\end{array}$ $03 \quad 3424.7$ ? $23.30 \mathrm{~N}$ $0352 \quad 34.8 * 9.534 \mathrm{~N}$ $041027.6 \quad 9.463 \mathrm{~N}$
0.6

$68.729 \mathrm{~W} 150 \mathrm{G}$ $126.204 \mathrm{E} 33 \mathrm{~N}$ $126.457 \mathrm{E}$ $17.681 \mathrm{E}$ $154.996 \mathrm{E}$ $73.011 \mathrm{E}$ $144.43 \mathrm{E}$ $126.411 \mathrm{E}$ $126.284 \mathrm{E}$

CHILE-BOLIVIA BORDER REGION

ADRIATIC SEA. ML 3.3 (ROM).

ADRIATIC SEA. MI
SOLOMON ISLANDS

MID-INDIAN RIDGE

VOLCANO ISLANDS REGION

MINDANAO, PHILIPPINE ISLANDS

MINDANAO, PHILIPPINE ISIANDS. MW 6.6 (HRV), 6.5 (GS). Me 5.9 (GS). MS 6.4 (BRK). Felt at Butuan and Surigao. Also felt on Leyte.

Broadband Source Parameters (GS): Dep 24; NPI: Strike-25, Dip=66, S1ip-110; NP2: Strike-163, Dip-31, Slip-52;

Radiated energy $1.6 * 10 * 13 \mathrm{~km}$.

yoment Tensor (GS): Dep 26; Principal axes (scale 10**18 $\mathrm{Nm}$ ): (T) Val=5.40, Plg=65, Azm=305; (N) Val=-0.01, Plg-11, Azm=189; (P) Val=-5.39, Plg=22, Azm=95; Best double couple: Mo-5.4*10*18 Nm; NP1: Strike-165, Dip=25, SIip=63; NP2: Strike-14, Dip-67, slip=102.

Centroid, Moment Tensor (HRV): Centroid origin time $04: 10: 33.6$; Lat $9.53 \mathrm{~N}$; Lon $126.66 \mathrm{E}$; Dep $22.0 \mathrm{Fix}$; Half duration $2.4 \mathrm{sec}$; Principal axes (scale 10*18 Nm): (T) Val=7.42, $\mathrm{Plg}=64, \mathrm{Azm}=307$; (N) Val=0.18, Plg=13, Azm=187; (P) Val=-7.60, Plg-21, Azm-92; Best double couple: MO=7.5*10**18 Nm; NPI: Strike=158, Dip=26, S1ip=58; NP2: Strike=13, Dip=68, slip=105.

scalar Moment (PPT): Mo-1.3*10*19 Nm.

MINDANAO, PHILIPPINE ISLANDS. MW 6.6 (HRV).

Centroid, Moment Tensor (HRV): Centroid origin time $04: 11: 03.0$; Lat 9.45 N Fix; Lon 126.33 E Fix; Dep 15.0 Fix; Half-duration $4.9 \mathrm{sec}$; Principal axes (scale 10**19 $\mathrm{Nm}$ ): (T) Val=0.95, Plg=45, Azm=102; (N) Val=0.10, Plg-1, Azm-193; (P) Val=-1.05, Plg-45, Azm-283; Best double couple: Mo-1.0*10*19 Nm; NP1: Strike-105, Dip-1,

Slip=-178; NP2: Strike=13, Dip=90, Slip=-89.

$\begin{array}{llllllll}20 & 04 & 24 & 54.6 & 9.495 & \mathrm{~N} & 126.384 & \mathrm{E}\end{array}$

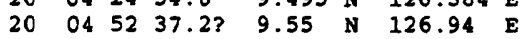
$20045356.2 * 9.418 \mathrm{~N} 126.509 \mathrm{E}$ $20 \quad 0504 \quad 36.1 ? 50.76$ N $96.64 \quad \mathrm{E}$

$\begin{array}{llllllll}20 & 05 & 30 & 22.08 & 44.267 & \mathrm{~N} & 7.127 & \mathrm{E}\end{array}$ $\begin{array}{lllllll}20 & 05 & 47 & 27.3 & 43.937 & \mathrm{~N}\end{array}$ $20060546.8 * 9.479 \mathrm{~N}$ $20 \quad 06 \quad 34 \quad 21.9 ? \quad 9.60 \quad N$

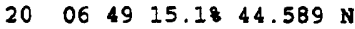
$\begin{array}{llllll}20 & 07 & 03 & 03.7 * 9.632 \mathrm{~N}\end{array}$ $20 \quad 0705 \quad 34.2 * 9.567 \mathrm{~N}$ $\begin{array}{llllllll}0 & 07 & 12 & 43.6 & 2.468 & \mathrm{~N}\end{array}$ $\begin{array}{llllllll}20 & 07 & 13 & 02.3 \& & 37.310 \mathrm{~N}\end{array}$ $20 \quad 07 \quad 46 \quad 57.9 ? \quad 9.58 \quad N$ $20080110.5 * 45.899 \mathrm{~N}$ $\begin{array}{lllll}20 & 09 & 48 & 01.1 & 9.595\end{array}$ $200950 \quad 47.4 * 9.483$ $20 \quad 1138 \quad 02.7 * 54.293 \mathrm{~N}$ 20 $7.637 \mathrm{E}$ $126.501 \mathrm{E}$ $126.69 \mathrm{E}$ $7.229 \mathrm{E}$ $126.522 \mathrm{E}$ $126.622 \mathrm{E}$ $128.665 \mathrm{E}$ $121.049 \mathrm{~W}$ $126.15 \mathrm{E}$ $10.671 \mathrm{E}$ $126.555 \mathrm{E}$ $126.464 \mathrm{E}$ $126.544 \mathrm{E}$ $164.202 \mathrm{~W}$

\begin{tabular}{|c|c|c|c|}
\hline 33 & $\mathbf{N}$ & 5.1 & 0.9 \\
\hline 33 & $\mathbf{N}$ & & 1.0 \\
\hline 33 & $\mathbf{N}$ & 5.2 & 1.1 \\
\hline 33 & $\mathbf{N}$ & & 1.4 \\
\hline 10 & G & & 0.1 \\
\hline 10 & G & & 0.8 \\
\hline 33 & $\mathbf{N}$ & 5.1 & 1.1 \\
\hline 33 & $\mathbf{N}$ & & 0.9 \\
\hline 10 & G & & 0.4 \\
\hline 33 & $\mathbf{N}$ & 4.7 & 0.9 \\
\hline 33 & $\mathbf{N}$ & 4.6 & 0.9 \\
\hline $\begin{array}{r}05 \\
0\end{array}$ & $D$ & 5.1 & 1.2 \\
\hline 33 & $\mathbf{N}$ & & 1.2 \\
\hline 10 & G & & 1.1 \\
\hline 33 & $\mathbf{N}$ & 5.2 & 1.0 \\
\hline 33 & $\mathbf{N}$ & 4.9 & 1.1 \\
\hline 33 & $\mathbf{N}$ & & 1.2 \\
\hline 33 & $\mathbf{N}$ & 5.46 .0 & 1.3 \\
\hline
\end{tabular}

MINDANAO, PHIIIPPINE ISLANDS

MINDANAO, PHIIIPPINE ISLANDS MINDANAO, PHILIPPINE ISLANDS ROSSIA-MONGOLIA BORDER REGION NORTHERN ITALY. MII 1.6 (GEN) . NEAR SOOTH COAST OF FRANCE. MI 2.7 (LDG), 2.4 (GEN). MINDANAO, PHILIPPINE ISLANDS

MINDANAO, PHILIPPINE ISIAANDS NORTEERN ITALY. MI 1.6 (GEN). MINDANAO, PHILIPPINE ISIANDS MINDANAO, PHILIPPINE ISLANDS HALMABERA, INDONESIA CENTRAL CALIFORNIA. <GM-P>. MD 3.0 (GM). ML 3.0 (BRK). MINDANAO, PHILIPPINE ISLANDS NORTHERN ITALY. ML 2.6 (VIE).

MINDANAO, PEILIPPINE ISIANDS MINDANAO, PHILIPPINE ISLANDS 'UNIMAK' 'ISLAANTD 'REGION

MINDANAO, PHILIPPINE ISIAANDS. WW 6.1 (GS), 6.1 (HRV). Me 5.6 (GS). MS 6.0 (BRK)

Broadband Source Parameters (GS): Radiated energy $6.2 * 10 * 12$ $\mathrm{Nm}$

Moment Tensor (GS): Dep 7; Principal axes (scale 10**18 Nm): (T) Val=1.84, Plg=45, $\mathrm{Azm}=316$; (N) $\mathrm{Val}=-0.01, \mathrm{PIg}=39$ Azm=174; (P) Val=-1.B3, Plg=20, Azm=67; Best double couple: Mo-1.8*10**18 Nm; NPI: Strike=113, Dip-43, Slip-22; NP2: Strike=6, Dip=75, Sip-130.

Centroid, Moment Tensor (HRV): Centroid origin time

$12: 24: 58.5$; Lat $9.52 \mathrm{~N}$; Lon $126.82 \mathrm{E}$; Dep $15.0 \mathrm{Fix}$; Half- 
duration $2.5 \mathrm{sec}$; Principal axes (scale 10*18 Nm): (T)

Val=1.33, Plg-69, Azm=307; (N) Val=0.10, Plg=14, Azm-179 (P) Val=-1.43, Plg-16, Azm-85; Best double couple: MO=1.4*10**18 Nm; NP1: Strike-155, Dip-31, Slip-63; NP2: Strike-6, Dip-63, Slip-105.

$\begin{array}{lllllll}20 & 12 & 34 & 04.7 & 9.463 & \mathrm{~N} & 126.566 \mathrm{E}\end{array}$ $20124730.8 * 9.519$ N $126.654 \mathrm{E}$ $20 \quad 1306 \quad 36.5 * 9.567 \mathrm{~N} 126.545 \mathrm{E}$ $20132442.6 * 9.565 \mathrm{~N} 126.649 \mathrm{E}$ $20133311.6 * 9.463 \mathrm{~N} 126.686 \mathrm{E}$ $20 \quad 13 \quad 4125.9 ? \quad 9.42$ N $126.47 \quad E$ $\begin{array}{llllllll}20 & 13 & 53 & 59.18 & 33.767 & \mathrm{~N} & 118.117 \mathrm{~W}\end{array}$ $20141047.8 * 9.630 \mathrm{~N} 126.445 \mathrm{E}$ $\begin{array}{lllllll}20 & 14 & 15 & 11.28 & 9.471 & \mathrm{~N} & 126.487 \mathrm{E}\end{array}$ $201436 \quad 00.1$ ? 9.75 N $126.56 \quad$ E $\begin{array}{llllllll}20 & 14 & 58 & 20.6 & 43.948 & \mathrm{~N} & 7.629 \mathrm{E}\end{array}$ $\begin{array}{lllllll}20 & 14 & 59 & 16.0 & 43.963 & \mathrm{~N} & 7.620 \mathrm{E}\end{array}$ $20151831.6 * 9.660 \mathrm{~N} 126.729 \mathrm{E}$ $20 \quad 1528 \quad 27.4$ ? 9.47 N 126.65 E $\begin{array}{lllllllll}20 & 15 & 30 & 24.7 & 9.618 & \mathrm{~N} & 126.608 \mathrm{E}\end{array}$ $\begin{array}{lllllllll}20 & 15 & 37 & 11.8 ? & 9.55 & \mathrm{~N} & 126.51 & \mathrm{E}\end{array}$ $20 \quad 16 \quad 18 \quad 31.4 * 9.676 \mathrm{~N} \quad 126.596 \mathrm{E}$ $2016 \quad 3407.6 ? \quad 9.69$ N 126.65 E $20163653.8 * 51.399$ N 175.879 \% $201653 \quad 44.4 * 9.558 \times 126.413 \mathrm{~N}$ $\begin{array}{lllllll}20 & 17 & 01 & 12.1 & 9.624 & \mathrm{~N} & 126.694 \mathrm{E}\end{array}$

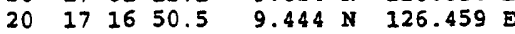
$20 \quad 132128.0 ? \quad 9.44 \quad \mathrm{~N} \quad 126.77 \quad$

\begin{tabular}{|c|c|c|c|}
\hline 33 & $\mathbf{N}$ & 5.1 & 0.8 \\
\hline 33 & N & 4.7 & 1.0 \\
\hline 33 & N & 5.1 & 1.0 \\
\hline 33 & $\mathbf{N}$ & & 1.3 \\
\hline 33 & $\mathbf{N}$ & & 0.8 \\
\hline 33 & $\mathbf{N}$ & 4.7 & 1.1 \\
\hline $\begin{array}{l}33 \\
13\end{array}$ & $\mathbf{N}$ & & 1.2 \\
\hline 33 & $\mathbf{N}$ & & 0.8 \\
\hline 33 & $\mathbf{N}$ & & 1.0 \\
\hline 33 & $\mathbf{N}$ & & 1.3 \\
\hline 10 & G & & 0.3 \\
\hline 10 & $\mathbf{G}$ & & 0.4 \\
\hline 33 & $\mathbf{N}$ & & 0.7 \\
\hline 33 & $\mathbf{N}$ & & 0.9 \\
\hline 33 & $\mathbf{N}$ & 5.1 & 1.0 \\
\hline 33 & $\mathbf{N}$ & & 1.2 \\
\hline 33 & $\mathbf{N}$ & 4.8 & 1.1 \\
\hline 33 & N & 4.4 & 1.2 \\
\hline 33 & $\mathbf{N}$ & & 1.1 \\
\hline 33 & $\mathbf{N}$ & 4.7 & 1.2 \\
\hline 33 & $\mathrm{~N}$ & 5.1 & 1.0 \\
\hline 33 & $\mathbf{N}$ & 5.05 .3 & 1.0 \\
\hline
\end{tabular}

$201730 \quad 03.4 * 43.139 \mathrm{~N} 146.868 \mathrm{E}$ $20173155.9 * 8.801 \mathrm{~N} 126.628 \mathrm{E}$ $20 \quad 1737 \quad 06.3 * 53.083 \mathrm{~s} \quad 9.695 \mathrm{E}$

$33 \mathrm{~N} 4.4$ $33 \mathrm{~N} 4.9$ $10 \mathrm{G} 5.6$

Scalar Moment (PPT): MO=4.1*10*\#18 Nan.

MINDANAO, PHIIIPPINE ISLANDS

MINDANAO, PHILIPPINE ISLANDS

MINDANAO, PHILIPPINE ISLANDS

MINDANAO, PHILIPPINE ISLANDS

MINDANAO, PHILIPPINE ISLANDS

MINDANAO, PHILIPPINE ISLANDS

MINDANAO, PHILIPPINE ISLANDS

SOUTHERN CALIFORNIA. <PAS-P>. MD 2.5 (PAS). Felt.

MINDANAO, PHILIPPINE ISLANDS

MINDANAO, PHILIPPINE ISLANDS

MINDANAO, PHILIPPINE ISLANDS

NEAR SOUTH COAST OF FRANCE. MII 2.1 (LDG), 2.1 (GEN).

NEAR SOUTH COAST OF FRANCE. ML 2.1 (LDG), 1.9 (GEN).

MINDANAO, PEILIPPINE ISLANDS

MINDANAO, PEILIPPINE ISIANDS

MINDANAO, PEILIPPINE ISIANDS

MINDANAO, PEILIPPINE ISLANDS

MINDANAO, PEILIPPINE ISILANDS

MINDANAO, PEILIPPINE ISLANDS

ANDREANOF ISLANDS, ALEUTIAN IS

MINDANAO, PHILIPPINE ISLANDS

MINDANAO, PEILIPPINE ISLANDS

MINDANAO, PHILIPPINE ISLANDS. NW 5.3 (HRV).

Centroid, Moment Tensor (HRV): Centroid origin time

17:16:54.3; Lat 9.23 N; Lon 127.03 E; Dep 15.0 Fix; Halfduration 1.1 sec; Principal axes (scale $10 * 16 \mathrm{Nm}$ ): (T)

Val=9.35, Plg=69, Azm=250; (N) Val=-0.58, Plg-6, Azm=355; (P) Val=-8.77, Plg-20, Azm-87; Best double couple:

Mo=9.1*10*16 Nm; NP1: Strike=187, Dip=25, Slip=103; NP2: Strike-353, Dip-65, S1ip-84.

KURIL ISIANDS

MINDANAO, PHILIPPINE ISLANDS

SOUTHWEST OF AFRICA. MW 5.8 (BRV)

Centroid, Moment Tensor (BRV): Centroid origin time

17:37:10.4; Lat $53.04 \mathrm{~s}$; Lon $9.89 \mathrm{E}$; Dep 15.0 Fix; Balf-

duration $1.9 \mathrm{sec}$; Principal axes (scale 10**17 Nm): (T)

Val-7.60, Plg-10, Azm=0 i (N) Val--3.56, Plg=16, Azm=267;

(P) Val=-4.04, Plg-71, Azm-122; Best double couple:

Mo $=5.8 * 10 * 17 \mathrm{Nm}$; NP1: Strike-109, Dip-37, slip=-63; NP2 : Strike-256, Dip-57, Slip=-109.

$\begin{array}{llllllll}20 & 17 & 37 & 27.2 & 46.126 & \mathrm{~N} & 6.796 \mathrm{E}\end{array}$

$20 \quad 1753 \quad 03.6 * 9.683 \mathrm{~N} \quad 126.257 \mathrm{E}$

$20180205.5 * 9.591 \mathrm{~N} 126.527 \mathrm{E}$

2018 05 $03.8 * 9.770 \mathrm{~N} \quad 126.595 \mathrm{E}$

$201808 \quad 47.9 * 56.479$ N 156.255 \%

$\begin{array}{lllllllll}20 & 18 & 16 & 28.5 & 9.661 & \mathrm{~N} & 126.430 \mathrm{~F}\end{array}$

$20 \quad 18 \quad 23 \quad 31.5 * 9.662 \mathrm{~N} \quad 126.559 \mathrm{E}$

$\begin{array}{llllllll}20 & 18 & 27 & 47.7 ? & 9.55 & N & 126.70 & E\end{array}$

$\begin{array}{lllllllll}20 & 18 & 51 & 09.7 & 9.698 & \mathrm{~N} & 126.418 & \mathrm{E}\end{array}$

$20 \quad 18 \quad 53 \quad 45.2 * 9.960 \mathrm{~N} \quad 126.806 \mathrm{E}$

$20191950.7 * 9.456 \mathrm{~N} 126.655 \mathrm{E}$

$\begin{array}{lllllll}20 & 19 & 24 & 57.3 & 9.570 & \mathrm{~N} & 126.643 \mathrm{E}\end{array}$

$10 \mathrm{G}$

$33 \mathrm{~N}$

$33 \mathrm{~N}$

$33 \mathrm{~N} 4.6$

57 D

$33 \mathrm{~N} 5.2$

$33 \mathrm{~N} 5.0$

$33 \mathrm{~N}$

$33 \mathrm{~N} 4.8$

$33 \mathrm{~N} 5.0$

$33 \mathrm{~N}$

$\begin{array}{lllll}33 & \mathrm{~N} & 5.5 & 5.6 & 1.1\end{array}$
20

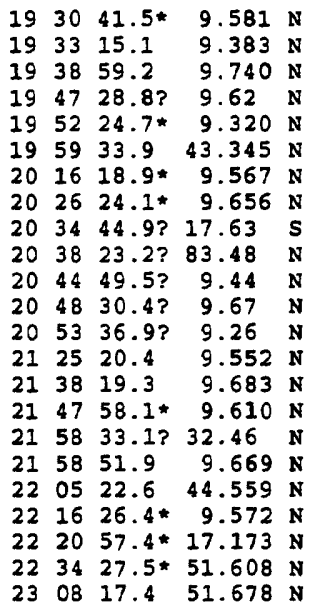

$126.610 \mathrm{E}$ $126.430 \mathrm{E}$ $126.512 \mathrm{E}$ $126.79 \mathrm{E}$ $126.310 \mathrm{E}$ $1.589 \mathrm{~W}$ 126.837 $126.587 \mathrm{E}$ 178.49 W 0.82 W $126.43 \mathrm{E}$ $126.71 \mathrm{E}$ $126.81 \mathrm{E}$ $126.529 \mathrm{E}$ $126.701 \mathrm{E}$ $126.552 \mathrm{E}$ $49.96 \mathrm{E}$ $126.545 \mathrm{E}$ $7.298 \mathrm{E}$ $126.523 \mathrm{E}$ $101.520 \mathrm{H}$ $174.732 \mathrm{~K}$ $16.123 \mathrm{E}$
$33 \mathrm{~N}$

$33 \mathrm{~N} 5.0$

$33 \mathrm{~N} 5.1$

$33 \mathrm{~N}$

10

$33 \mathrm{~N} 5.0$

$33 \mathrm{~N} 4.9$

600 G 4.7

$10 \mathrm{G} 4.2$

$33 \mathrm{~N}$

$33 \mathrm{~N}$

$33 \mathrm{~N}$

$33 N \quad 5.25 .7 \quad 1.1$

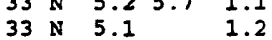

$33 \mathrm{~N} 5.1$

$33 \mathrm{~N} 5.2$

$10 \mathrm{G}$

$33 \mathrm{~N} 4.9$

$33 \mathrm{~N}$

$10 \mathrm{G}$
1.0

1.1
0.9

0.5

0.9

1.2

1. 3

1. 3

1.1

0.9

1.0

0.8

1.1

0.9

0.5

1.0
1.156

1.024

$0.6 \quad 17$

1.322

0.933

1.331

0.510

1.144

$1.1 \quad 17$

14

98

(ITIT), 3.0 (STR), 2.7 (VIE)

MINDANAO, PHILIPPINE ISLANDS

MINDANAO, PHILIPPINE ISLANDS

MINDANAO, PHIIIPPINE ISLANDS

ALASKA PENINSUTA

MINDANAO, PHILIPPINE ISIANDS

MINDANAO, PHIIIPPINE ISLANDS

MINDANAO, PHILIPPINE ISIAANDS

MINDANAO, PHILIPPINE ISLANDS

MTNDANAO, PEIIIPPINE ISIANDS

MINDANAO, PHILIPPINE ISIAANDS

MINDANAO, PHILIPPINE ISLANDS. MW 5.8 (GS), 5.8 (HRV)

Moment Tensor (GS): Dep 12; Principal axes (scale 10**17 $\mathrm{Nm}$ ): (T) Val-5.85, Plg=47, Azm-318; (N) Val=-0.08, Plg=36, Azm=176; (P) Val--5.77, Plg=20, Azm=71; Best double couple: Mo-5.8*10**17 Nm; NP1: Strike=118, Dip-41, Slip=25; NP2: Strike-8, Dip-74, slip-128.

Centroid, Moment Tensor (HRV): Centroid origin time 19:25:01.9; Lat 9.46 N; Lon 127.02 E; Dep 15.0 Fix; Halfduration 2.0 sec; Principal axes (scale $10 * * 17 \mathrm{Nm}$ ): (T) Val-5.29, Plg-71, Azm=267; (N) Val-0.64, Plg-1, Azm=0; (P) Val-5.93, Plg-19, Azm=90; Best double couple:

Mo-5.6*10*17 Nm; NP1: Strike=182, Dip=26, Slip-93; NP2: Strike-359, Dip-64, Slip=89.

MINDANAO, PHILIPPINE ISLANDS

18

38

10

11

39

21

11

10

9

43

24

29

19

28

14

16
MINDANAO PHIIIPPINE ISLANDS

MINDANAO, PHILIPPINE ISLANDS

MINDANAO, PHILIPPINE ISLANDS

MINDANAO, PHILIPPINE ISLANDS

PYRENEES. MC 3.1 (LDG), 2.9 (STR). MbLg 2.9 (MDD)

MINDANAO, PHIIIPPINE ISLANDS

MINDANAO, PHILIPPINE ISLANDS

FIJI ISLANDS REGION

NORTH OF SVALBARD

MINDANAO, PHILIPPINE ISLANDS

MINDANAO, PEILIPPINE ISLANDS

MINDANAO, PHILIPPINE ISIAANDS

MTNDANAO， PEILIPPINE ISLANDS

MINDANAO, PEILIPPINE ISLANDS

MINDANAO, PEILIPPINE ISLANDS

WESTERN IRAN

MINDANAO, PEILIPPINE ISIANDS

NORTHERN ITALY. ML 2.4 (GEN), 2.1 (LDG).

MINDANAO, PEILIPPINE ISLANDS

NEAR COAST OF GUERRERO, MEXICO

ANDREANOF ISLANDS, ALEUTIAN IS.

POLAND. MI 3.1 (MOX). 


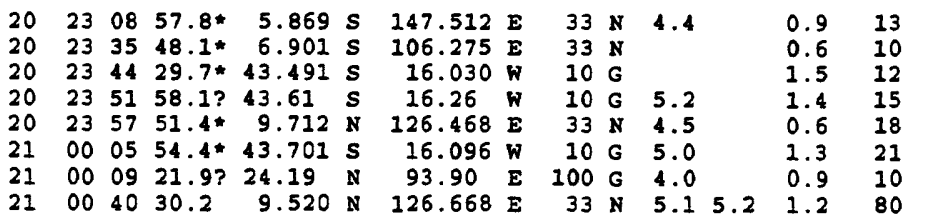

$\begin{array}{lllll}21 & 01 & 01 & 20.6\end{array}$ $\begin{array}{lllll}21 & 01 & 04 & 24.1\end{array}$ $\begin{array}{llll}21 & 01 & 23 & 33.3\end{array}$
$9.706 N \quad 126.497 \mathrm{E}$ $9.562 \mathrm{~N} 126.530 \mathrm{E}$ $6.155 \mathrm{~S} 146.174 \mathrm{E}$
$33 \times 4.7$

$33 \mathrm{~N} 5.0$ $\begin{array}{llllr}10 \mathrm{G} & 5.7 & 5.8 & 1.1 & 48 \\ & 1.2 & 113\end{array}$

3 EASTERN NEW GUINEA REG., P.N.G.

10 JAWA, INDONESIA

2 SOUTHERN MID-ATLANTIC RIDGE

5 SOUTHERN MID-ATLANTIC RIDGE

8 MINDANAO, PHILIPPINE ISIANDS

1 SOUTHERN MID-ATLANTIC RIDGE

MINDANAO, PHILIPPINE ISILANDS. WW 5.3 (HRV)

Centroid, Moment Teasor (HRV): Centroid origin time $00: 40: 32.3$; Lat $9.03 \mathrm{~N}$; Lon $127.02 \mathrm{E}$; Dep 37.1 ; Balf duration $1.0 \mathrm{sec;}$ Principal axes (scale $10 * 17 \mathrm{Nm}$ ): (T) Val=0.91, Plg-72, Azm-198; (N) Val-0.10, Plg-17, Azm=354; (P) Val=-1.01, PIg=7, Azm-86; Best double couple: MO=9.6*10**16 Nm; NP1: Strike-194, Dip=41, Slip-116; NP2 : strike-341, Dip-54, Slip-69.

MINDANAO, PAILIPPINE ISLANDS

MINDANAO, PUILIPPINE ISLANDS

EASTERN NEW GUINEA REG., P.N.G. MW 5.9 (GS), 5.8 (BRV). MS 5.9 (BRK).

Moment Tensor (GS): Dep 6; Principal axes (scale 10**17 Nm): (T) Val-7.64, Plg-52, $\mathrm{Azm}=36$; (N) Val=0.00, Plg=14 Azm-288; (P) Val=-7.64, Plg-35, Azm-188; Best double couple: Mo-7.6*10*17 Nm; NP1: Strike=232, Dip=16, Slip-33 ; NP2: Strike-110, Dip=81, Slip-104.

Centroid, Moment Tensor (HRV): Centroid origin time 01:23:39.9; Lat $6.49 \mathrm{~S}$; Lon $146.57 \mathrm{E}$; Dep $15.0 \mathrm{Fix}$; Halfduration $1.9 \mathrm{sec}$; Principal axes (scale $10 \star 17 \mathrm{Nm}$ ): (T) Val-5.31, Plg=71, Azm=39; (N) Val=-0.04, Plg=4, Azm=297; (P) Val=-5.28, Plg-18, Azm-205; Best double couple: Mo-5.3*10*17 Nm; NP1: Strike-289, Dip=27, Slip-81; NP2: Strike-119, Dip-64, Slip-95.

$21012400.08 \quad 35.700 \mathrm{~N} \quad 84.000 \mathrm{~N}$ 21013111.5 ? $9.45 \mathrm{~N} 126.23 \mathrm{E}$ $21 \quad 0134 \quad 30.5 \quad 42.837$ N $17.795 \mathrm{E}$

$21013535.3 * 9.718$ N $126.444 \mathrm{E}$ $21015628.9 ? \quad 8.48$ S $122.49 \quad \mathrm{E}$ $21015812.3 * 9.494 \mathrm{~N} 126.169 \mathrm{E}$ $21021257.0 * 65.006 \mathrm{~N} 133.956 \mathrm{~W}$ $\begin{array}{lllll}21 & 02 & 21 & 43.6 * 24.844 \mathrm{~N} & 125.012 \mathrm{E}\end{array}$ $\begin{array}{llllllll}21 & 02 & 45 & 56.8 ? & 9.56 & \mathrm{~N} & 126.34\end{array}$ $\begin{array}{llllll}21 & 02 & 53 & 18.6 & 18.999 \mathrm{~s}\end{array}$
5 G

$33 \mathrm{~N} 4.5$

$10 \mathrm{G} \quad 4.2$

$33 \times 4.9$

$33 \mathrm{~N} 4.1$

$33 \mathrm{~N} 4.6$

$10 \mathrm{G}$

$33 \mathrm{~N} 4.1$

$33 \mathrm{~N} 4.6$

3 TENNESSEE. CMACRO>. mbLg 2.0 (GS). Felt at Maryvale.

1.010 MINDANAO, PBILIPPINE ISLANDS

1.296 ADRIATIC SEA. ML 4.5 (LJU), 4.0 (ROM). Additional damage in the slano and ston areas, Croatia.

0.929 MINDANAO, PHILIPPINE ISLANDS

0.912 FLORES REGION, INDONESIA

1.116 MINDANAO, PHILIPPINE ISLANDS

0.97 NORTHERN YUKON TERRITORY, CANADA

0.610 SOUTHWESTERN RYUKYO ISLANDS

0.814 MINDANAO, PBILIPPINE ISLANDS

1.1165 CENTRAL BOLIVIA. WW 5.7 (BRV)

Centroid, Moment Tensor (ERV): Centroid origin time $02: 53: 22.5$; Lat $18.76 \mathrm{~s}$; Lon $67.22 \mathrm{~W}$; Dep 215.5; Half duration $1.5 \mathrm{sec}$; Principal axes (scale $10 * 17 \mathrm{Na}$ ): (T) Val-4.25, Plg-21, Azm-118; (N) Val--0.34, Plg=58, Azm-350; (P) Val--3.90, Plg-23, Azm=218; Best double couple: Mo-4.1*10*17 Nm; NP1: Strike-258, Dip-58, slip--1; NP2: strike-348, Dip-89, slip=-148.

$\begin{array}{llllllll}21 & 03 & 01 & 37.5 ? & 9.81 \quad \mathrm{~N} & 126.87 \quad \mathrm{E}\end{array}$

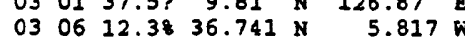
$\begin{array}{lllll}030749.6 & 9.632 \mathrm{~N} \quad 126.494 \mathrm{E}\end{array}$ $\begin{array}{lllll}03 & 1649.8 & 9.789 & \mathrm{~N} & 126.541 \mathrm{E}\end{array}$ $1032756.6 \quad 9.695 \mathrm{~N} \quad 126.549 \mathrm{E}$

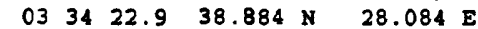
$\begin{array}{lllll}03 & 3758.5 & 9.660 \mathrm{~N} & 126.529 \mathrm{E}\end{array}$ $\begin{array}{lllllll} & 43 & 50.7 ? & 9.66 & \mathrm{~N} & 126.88 \mathrm{E}\end{array}$ $\begin{array}{lllllll}04 & 06 & 01.1 ? & 9.47 \mathrm{~N} \quad 126.62 \mathrm{E}\end{array}$ $040614.9 * 51.387 \mathrm{~N} 174.840 \mathrm{~W}$ $0410 \quad 37.9865 .902 \mathrm{~N} \quad 149.894 \mathrm{~W}$ $\begin{array}{llllll}04 & 32 & 26.7 & 55.968 \mathrm{~s} & 24.721 \mathrm{~W}\end{array}$ $1044646.8 * 32.458 \mathrm{~N} 141.520 \mathrm{E}$ $051617.3 * 51.652 \mathrm{~N} 174.704 \mathrm{~W}$ $051849.4750 .99 \%$ $\begin{array}{llll}05 & 36 & 13.8 & 1.124 \mathrm{~N}\end{array}$ $\begin{array}{lllll}07 & 10 & 54.3 & 42.695 & \mathrm{~N} \\ 08 & 27 & 55.9 & 31.025 & \mathrm{~N}\end{array}$ $085152.1 * 36.185 \mathrm{~N}$ $\begin{array}{llll}10 & 36 & 05.9 & 9.662 \mathrm{~N}\end{array}$ 1038 27.7? $6.34 \mathrm{~s}$ $\begin{array}{llllll}11 & 00 & 32.08 & 37.074 & \mathrm{~N} \\ 11 & 32 & 32.0 ? & 32.43 & \mathrm{~S}\end{array}$ $\begin{array}{lllll}11 & 34 & 19.4 & 9.656 \mathrm{~N}\end{array}$

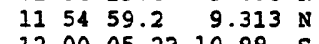
120005.2 ? $10.99 \mathrm{~s}$ $120125.8843 .916 \mathrm{~N}$ $\begin{array}{llll}12 & 05 & 50.9 & 53.827\end{array}$ $\begin{array}{llllll}12 & 14 & 56.6 ? & 36.49 & N\end{array}$ $121832.5 * 9.669 \mathrm{~N}$ $125411.9 * 47.075 \mathrm{~N}$ $135004.6 ? 6.53 \mathrm{~S}$ $\begin{array}{lllll}14 & 03 & 33.8 ? & 42.69 & N\end{array}$ $\begin{array}{lllll}14 & 18 & 44.9 ? & 8.45 & 5\end{array}$ $\begin{array}{llll}15 & 41 & 21.6 & 21.841 \mathrm{~N}\end{array}$ $162125.4 * 9.688 \mathrm{~N}$ $164704.2 * 9.434 \mathrm{~N}$ $165704.2 * 21.799 \mathrm{~N}$

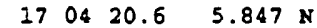

$33 \mathrm{~N}$

$10 \mathrm{G}$

$33 \mathrm{~N} 5.0$

$33 \mathrm{~N} 5.0$

$33 \mathrm{~N} 5.3$

10 G 4.1

$33 \mathrm{~N} 5.24$

$33 \mathrm{~N} 4.5$

$33 \mathrm{~N} 4.5$

$33 \mathrm{~N} 4.1$

30

$51 \mathrm{D} 5.1$

$51 \mathrm{D} 4.7$

$33 \mathrm{~N}$

$33 \mathrm{~N}$

$44 ? 5.1$

$10 \mathrm{G} 4.0$

$33 \mathrm{~N} 4.6$

$17.877 \mathrm{E}$

$141.331 \mathrm{E}$

$70.837 \mathrm{E}$

$126.512 \mathrm{E}$

$146.47 \mathrm{E}$

$139.371 \mathrm{E}$

$71.14 \mathrm{~W}$

$126.486 \mathrm{E}$

$126.609 \mathrm{E}$

74.42 W

$7.561 \mathrm{E}$

$167.131 \mathrm{~W}$

$140.96 \mathrm{E}$

$126.485 \mathrm{E}$

$144.593 \mathrm{E}$

$146.09 \mathrm{E}$
17.95

130.30

$121.593 \mathrm{E}$

$126.560 \mathrm{E}$

$126.685 \mathrm{E}$

$121.413 \mathrm{E}$
139

$33 \mathrm{~N} 5.1$

$33 \mathrm{~N} 4.5$

$10 \mathrm{G}$

$60 \mathrm{G}$

$33 \mathrm{~N} 5.0$

$33 \mathrm{~N} 4.8$

$150 \mathrm{G} \quad 4.6$

$10 \mathrm{G}$

100
$33 \mathrm{~N}$

$33 \mathrm{~N}$

$400 \mathrm{G} 4.8$

$33 \mathrm{~N} 4.0$

$10 \mathrm{G}$

$100 \mathrm{G} 4.2$

$33 \mathrm{~N} 4.9$

$33 \mathrm{~N} 4.5$

$33 \mathrm{~N} 4.5$

$33 \times 4.3$

$33 \times 4.94$
1.315 MINDANAO, PHILIPPINE ISIANDS

0.97 STRAIT OF GIBRALTAR. mbIg 2.9 (MDD).

1.037 MINDANAO, PHILIPPINE ISLANDS

0.841 MINDANAO, PHILIPPINE ISLANDS

1.067 MINDANAO, PHILIPPINE ISIANDS

1.126 TURKEY

66 MINDANAO, PHILIPPINE ISLANDS

16 MINDANAO, PHILIPPINE ISIANDS

16 MINDANAO, PHILIPPINE ISILANDS

19 ANDREANOF ISLANDS, ALEUTIAN IS.

19 NORTHERN ALASRA. $\angle A E I C)$. ML 2.9 (AEIC).

34 SOUTH SANDWICH ISLANDS REGION

1.019 SOUTH OF HONSHO, JAPAN

0.814 ANDREANOF ISLANDS, ALEUTIAN IS.

I.4 7 ANDREANOF ISLANDS, ALEUTIAN IS.

1.232 MINAHASSA PENINSOLA, SULAWESI

1.237 ADRIATIC SEA. ML 3.9 (VIE).

0.617 SOUTH OF HONSHD, JAPAN

1.016 HINDO KUSA REGION, AFGHANISTAN

1.145 MINDANAO, PBILIPPINE ISLANDS

1.57 EASTERN NEW GUINEA REG., P.N.G.

1.05 EASTERN HONSHO, JAPAN

0.410 NEAR COAST OF CENTRAL CHILE. ND 2.8 (SAN).

1.044 MINDANAO, PBILIPPINE ISLANDS

0.934 MINDANAO, PHILIPPINE ISLANDS

0.511 CENTRAT PERO

1.15 NEAR SOUTH COAST OF FRANCE. MU 1.5 (GEN).

0.892 FOX ISIANDS, AIEUTIAN ISLANDS. Felt slightly at Daalaska.

0.46 NEAR EAST COAST OF BONSHO, JAPAN

1.132 MINDANAO, PHILIPPINE ISLANDS

0.812 SEA OF OKHOTSK

1.59 EASTERN NEW GUINEA REG., P.N.G.

1.412 ADRIATIC SEA

1.5 B TANIMBAR ISLANDS REG., INDONESIA

0.938 TAIWAN REGION

1.120 MINDANAO, PHILIPPINE ISLANDS

0.823 MINDANAO, PHILIPPINE ISLANDS

0.917 TATWAN REGION

45 NORTHERN SUMATERA, INDONESIA, NW 5.2 (HRV).

Centroid, moment Tensor (BRV): Centroid origin time 17:04:21.9; Lat 5.85 N; Lon 95.38 E; Dep 33.0 Fix; Halfduration $1.0 \mathrm{sec}$; Principal axes (scale $10 * 16 \mathrm{Nm}$ ): (T) Val-6.96, Plg-33, Azm-273; (N) Val=-0.76, Plg-40, Azm=150; (P) Val--6.20, PIg-32, Azm-28; Best double couple: 
21
21
21
21 $170708.4 * 5.754 \mathrm{~N} \quad 95.004 \mathrm{E}$ $\begin{array}{lllllll}17 & 43 & 17.47 & 36.77 \quad \mathrm{~N} & 2.97\end{array}$ $\begin{array}{lllllll}18 & 17 & 22.4 & 9.360 \mathrm{~N} & 126.593 \mathrm{E}\end{array}$ $172316.8 * 39.672 \mathrm{~N} 144.668 \mathrm{E}$ $173813.8 * 9.507 \mathrm{~N} 127.017 \mathrm{E}$
33

$33 \mathrm{~N}$

$10 \mathrm{G}$

33
Mo-6.6*10**16 Nm; NP1: Str1ke-61, D1p-40, S11p-1; NP2: Strike-330, Dip-89, Slip-130.

1.213 NORTEERN SUMATERA, INDONESIA

1.213 NORTEERN SUMATERA, INDONESIA

0.713 PHILIPPINE ISLANDS REGION

0.14 STRAIT OF GIBRALTAR. MbIg 2.5 (MDD).

MINDANAO, PEILIPPINE ISLANDS. NW 5.6 (BRV)

Centrold, Moment Tensor (HRV): Centroid origin time $18: 17: 25.6$; Lat $9.29 \mathrm{~N}$; Lon $126.96 \mathrm{E}$; Dep $15.0 \mathrm{Fix}$; Half duration $1.6 \mathrm{sec}$ : Principal axes (scale 10**17 Nm): (T) Val-2.76, Plg=73, Azm=261; (N) Val-0.10, Plg=3, $A z m=0 ;$ (P) Val--2.86, Plg-17, Azm=91; Best double couple Mo=2.8*10**17 Nm; NP1: Strike-185, Dip-28, Slip-96; NP2 Strike-358, Dip-62, Slip=87

$\begin{array}{lllllllrl}18 & 52 & 52.6 * & 9.397 & \mathrm{~N} & 126.931 & \mathrm{E} & 33 & \mathrm{~N} \\ 19 & 25 & 59.4 & 51.640 & \mathrm{~N} & 16.261 & \mathrm{E} & \mathrm{S} & \mathrm{G} \\ 19 & 36 & 23.8 ? & 51.84 & \mathrm{~N} & 174.85 & \mathrm{~W} & 33 & \mathrm{~N} \\ 19 & 55 & 29.5 ? & 9.3 .8 & \mathrm{~N} & 126.74 & \mathrm{E} & 33 & \mathrm{~N} \\ 20 & 37 & 04.0 * & 9.293 & \mathrm{~N} & 126.494 & \mathrm{E} & 33 & \mathrm{~N} \\ 20 & 54 & 13.3 * & 9.795 & \mathrm{~N} & 126.709 & \mathrm{E} & 33 & \mathrm{~N} \\ 21 & 18 & 42.7 * & 6.267 & \mathrm{~S} & 146.236 & \mathrm{E} & 33 & \mathrm{~N} \\ 21 & 18 & 55.3 * & 9.505 & \mathrm{~N} & 127.025 & \mathrm{E} & 33 & \mathrm{~N} \\ 21 & 35 & 25.2 * & 6.222 & \mathrm{~S} & 146.344 & \mathrm{E} & 33 & \mathrm{~N} \\ 21 & 54 & 19.0 * & 9.640 & \mathrm{~N} & 126.452 & \mathrm{E} & 33 & \mathrm{~N} \\ 22 & 15 & 00.3 ? & 6.49 & \mathrm{~S} & 129.98 & \mathrm{E} & 150 & \mathrm{G} \\ 22 & 27 & 41.6 & 42.868 & \mathrm{~N} & 17.694 & \mathrm{E} & 10 & \mathrm{G} \\ 22 & 38 & 54.4 & 6.203 & \mathrm{~S} & 146.212 & \mathrm{E} & 33 & \mathrm{~N} \\ 22 & 53 & 16.3 & 26.456 & \mathrm{~N} & 128.938 & \mathrm{E} & 33 & \\ 23 & 34 & 46.7 ? & 5.09 & \mathrm{~S} & 102.74 & \mathrm{E} & 33 & \mathrm{~N} \\ 23 & 40 & 15.1 & 17.550 & \mathrm{~S} & 178.835 & \mathrm{~W} & 533 & \mathrm{D} \\ 00 & 35 & 17.2 ? & 5.71 & \mathrm{~S} & 133.57 & \mathrm{E} & 33 & \mathrm{~N} \\ 00 & 51 & 54.3 * & 9.604 & \mathrm{~N} & 126.456 & \mathrm{E} & 33 & \mathrm{~N} \\ 02 & 21 & 33.3 & 15.903 & \mathrm{~S} & 71.727 & \mathrm{~W} & 140 & \mathrm{D}\end{array}$

\section{.4}

4

$4 \cdot 4$

4.5
4.6

4.5

4.6

4.8

4.0

5.0
4.0

4.7

4.6

3.9

4.4

5.4 .7

$0.7 \quad 15$

1.113 POLAND. ML 2.7 (MOX).

1.25 ANDREANOF ISLANDS, ALEUTIAN IS.

1.413 MINDANAO, PBILIPPINE ISLANDS

1.319 MINDANAO, PEILIPPINE ISLANDS

0.816 MINDANAO, PEILIPPINE ISLANDS

$0.6 \quad 17$ EASTERN NEW GUINEA REG., P.N.G.

$1.0 \quad 10$ PAILIPPINE ISLANDS REGION

1.423 EASTERN NEW GUINEA REG., P.N.G.

1.025 MINDANAO, PHILIPPINE ISLANDS

1.46 BANDA SEA

1.164 ADRIATIC SEA. ME 3.6 (ROM)

0.742 EASTERN NEW GUINEA REG., P.N.G.

0.822 RYUKYO ISLANDS

0.997 FIJI ISLANDS REGION

1.77 ARU ISIANDS REGION, INDONESIA

1.023 MTNDANAO, PEILIPPINE ISLANDS

0.976 SOUTEERN PERU. Felt (II) at Arequipa

$02 \quad 2907.6 \quad 34.040 \mathrm{~N} 140.627 \mathrm{E} 55$
228
1.419 SOUTEERN SUMATERA, INDONESIA

$\begin{array}{lllcl}03 & 23 & 13.0 ? & 21.41 & \mathrm{~S} \\ 04 & 08 & 11.3 & 42.732 & \mathrm{~N} \\ 05 & 24 & 58.28 & 44.557 & \mathrm{~N} \\ 06 & 11 & 22.7 * & 9.537 & \mathrm{~N} \\ 06 & 52 & 58.8 * & 5.764 & \mathrm{~S} \\ 08 & 05 & 04.3 ? & 9.41 & \mathrm{~N} \\ 08 & 05 & 24.8 ? & 34.52 & \mathrm{~N} \\ 08 & 12 & 37.8 & 32.143 & \mathrm{~N} \\ 08 & 18 & 30.9 ? & 9.74 & \mathrm{~N} \\ 08 & 25 & 19.48 & 59.890 & \mathrm{~N} \\ 08 & 29 & 03.0 ? & 9.68 & \mathrm{~N} \\ 08 & 45 & 54.0 ? & 34.49 & \mathrm{~S} \\ 09 & 04 & 50.0 * & 9.195 & \mathrm{~N} \\ 09 & 06 & 45.6 & 12.008 & \mathrm{~N} \\ 09 & 49 & 50.1 ? & 16.99 & \mathrm{~N} \\ 09 & 56 & 39.9 ? & 22.76 & \mathrm{~S} \\ 09 & 57 & 38.28 & 62.953 & \mathrm{~N} \\ 10 & 43 & 25.2 & 9.476 & \mathrm{~N}\end{array}$

174.37 ผ $17.735 \mathrm{E}$ $7.482 \mathrm{E}$ $126.843 \mathrm{E}$ $127.957 \mathrm{E}$ $126.62 \mathrm{E} 365$ ? $24.64 \mathrm{E} 33 \mathrm{~N}$ $137.776 \mathrm{E} \quad 387$ $126.94 \mathrm{E}$ $153.392 \mathrm{~W} 127$ 126.51 $70.40 \mathrm{~W}$ $126.457 \mathrm{E}$ $143.399 \mathrm{E}$ 100.57 $178.96 \mathrm{E} 600$ $151.080 \mathrm{~W} 113$

$\begin{array}{rrlll}33 & \mathrm{~N} & 4.5 & & 0.7 \\ 10 & \mathrm{G} & & & 1.1 \\ 10 & \mathrm{G} & & & 0.5 \\ 33 & \mathrm{~N} & 4.5 & & 1.4 \\ 365 & \mathrm{P} & 4.7 & & 0.9 \\ 33 & \mathrm{~N} & 4.4 & & 1.1 \\ 33 & \mathrm{~N} & & & 1.1 \\ 387 & & 3.9 & & 0.9 \\ 33 & \mathrm{~N} & 4.9 & & 1.4 \\ 127 & & & & \\ 33 & \mathrm{~N} & 4.1 & & 0.9 \\ 10 & \mathrm{G} & & & 0.1 \\ 33 & \mathrm{~N} & 4.6 & & 1.1 \\ 33 & \mathrm{~N} & 5.1 & 4.5 & 1.0 \\ 33 & \mathrm{~N} & & & 1.5 \\ 600 & \mathrm{G} & 4.2 & & 1.1 \\ 113 & & & & \\ 33 & \mathrm{~N} & 5.1 & 5.0 & 1.1\end{array}$

0.7

1.1

1.4

1.1

0.9

1.4

0.9

1.1

1.0

.1

$126.831 \mathrm{E}$

\section{Cente} Centrold Monent 02:29:11.0; Lat $33.87 \mathrm{~N} \mathrm{Fix;} \mathrm{Lon} 140.05 \mathrm{EFix}$; Dep 51.2; Balf-duration $1.1 \mathrm{sec}$; Principal axes (scale $10 *+16 \mathrm{~m}$ ): (T) Val-4.98, Plg=35, Azm-215; (N) Val-4.05, Plg-20, Azm-319; (P) Val--9.02, Plg-48, Azm-73; Best double couple: Mo=7.0*10**16 $\mathrm{Nm}$; NP1: Strike-250, Dip-21, S11p--160; NP2: Strike-142, Dip $=83$, Silp $=-70$.

12 TONGA ISLANDS

33 ADRIATIC SEA

7 NORTEERN ITALY. ML 1.8 (GEN).

20 MINDANAO, PHIIIPPINE ISLANDS

18 BANDA SEA

11 MINDANAO, PGILIPPINE ISLANDS

11 CRETE

33 SOUTE OF BONSEU, JAPAN

18 MINDANAO, PBILIPPINE ISIANDS

51 SOUTHERN ALASKA. <AEIC>

14 MINDANAO, PGILIPPINE ISLANDS

6 CHILE-ARGENTINA BORDER REGION

23 MINDANAO, PBILIPPINE ISLANDS

71 SOUTE OF MARIANA ISLANDS

8 NEAR COAST OF GUERRERO, MEXICO

17 SOUTE OF FIJI ISLANDS

23 CENTRAL ALASKA. <AEIC>.

67 MINDANAO, PEILIPPINE ISLANDS. 5.4 (HRV).

Centroid, Moment Tensor (BRV): Centroid origin time

$10: 43: 28.5$; Lat $9.47 \mathrm{~N}$; Lon $126.94 \mathrm{E}$; Dep 15.0 Fix; Balfduration $1.1 \mathrm{sec}$; Principal axes (scale 10*17 $\mathrm{Nm}$ ): (T) Val-1.39, Plg-66, Azm-304; (N) Val--0.24, Plg-16, Azm-175; (P) Val-1.15, Plg-18, Azm-79; Best double couple: Mo-1.3*10**17 Nm; NP1: Strike-146, D1p-31, Slip-57; NP2 Strike-3, Dip $=65$, Slip-108. 
.813 CHILE-BOLIVIA BORDER REGION

0.599 FIJI ISLANDS REGION

1.218 MINDANAO, PAIIIPPINE ISLANDS

8 SOOTE OF JAWA, INDONESIA

15 TAIWAN REGION

13 TAIWAN REGION

39 MINDANAO, PAILIPPINE ISLANDS

23 MINDANAO, PBIIIPPINE ISLANDS

12 CENTRAL BOLIVIA

16 SODTH OF FIJI ISLANDS

12 CARLSBERG RIDGE

32 MINDANAO, PAILIPPINE ISLANDS

12 MINDANAO, PHIIIPPINE ISLANDS

11 ANDAMAN ISLANDS, INDIA

17 SOUTH OF SUMBA, INDONESIA

66 SOUTBERN ALASKA. CAEIC>.

11 LOZON, PAILIPPINE ISLANDS

16 SOUTHERN SUMATERA, INDONESIA

6 PYRENEES. ML 3.5 (STR), 2.9 (LDG).

17 MINDANAO, PAILIPPINE ISILANDS

82 NEW IRELAND REGION, P.N.G.

11 MINDANAO, PEIIIPPINE ISIANDS

9 AFGEANISTAN-TAJIKISTAN BORD REG.

65 CENTRAL ALASKA. CAEIC). ML 2.8 (AEIC).

0.926 MINDANAO, PHILIPPINE ISLANDS

0.833 MINDANAO, PHILIPPINE ISLANDS

0.821 MINAHASSA PENINSULA, SULAWESI

6 RYUKYD ISLANDS

25 MINABASSA PENINSULA, SULAFESI

6 EASTERN KASHMIR

9 TIMOR REGION, INDONESIA

7 STRAIT OF GIBRALTAR. mbIg 2.6 (MOD).

20 MINDANAO, PHILIPPINE ISLANDS

13 XIZANG

5 CENTRAL CALIFORNIA. 〈PAS-P>. MD 2.8 (PAS).

40 SOUTHERN SOMATERA, INDONESIA

23 PEILIPPINE ISLANDS REGION

12 MARIANA ISLANDS

9 NEAR COAST OF CENTRAL CHILE

45 KURII ISLANDS

8 TANIMBAR ISLANDS REG., INDONESIA

20 SOUTHERN EAST PACIFIC RISE

8 SOUTHWESTERN RYOKYO ISIANDS

11 SOUTHWESTERN RYOKYO ISI.ANDS

16 SOUTEERN SUMATERA, INDONESIA

100 SOUTEERN ALASKA. <AEIC>.

1.120

NORTBERN MOLJCCA SEA

1.020 MINDANAO, PHILIPPINE ISLANDS

18 FIJI ISLANDS REGION

7 SOUTB OF SUMBAWA, INDONESIA

16 ROSSIA-MONGOLIA BORDER REGION

47 NORTHERN MOLOCCA SEA

27 ADRIATIC SEA. ML 3.5 (VIE). Felt at Biograd and zadar, Croatia.

$002751.924 .941 \times 123.478$ E $\quad 25$ D $5.35 .4 \quad 1.4$

$46^{\circ}$ SOUTHWESTERN RYOKYU ISLANDS. MW 5.6 (BRV)

Centroid, Moment Tensor (HRV): Centroid origin time

00:28:01.9; Lat $25.05 \mathrm{~N}$; Lon $123.33 \mathrm{E}$; Dep 15.0 Fix; Halfduration $1.6 \mathrm{sec}$; Principal axes (scale 10*17 Nm): (T) Val=3.52, Plg=6, Azm-357; (N) Val=-0.47, Plg=10, Azm=266; (P) Val--3.06, Plg-78, Azm-118; Best double couple: Mo-3.3*10*17 Nm; NP1: Strike-98, Dip=40, Slip=-74; NP2: Strike-258, Dip-52, S1ip=-103.

$24003050.4862 .350 \mathrm{~N} 152.734$ พ 158

$24005429.3 * 24.941$ N 123.444 .

$240110 \quad 47.5 * 4.855 \mathrm{~S} \quad 138.609 \mathrm{E}$

$24012659.5 * 37.393$ S $138.776 \mathrm{E}$

24. $013027.4 \quad 24.937$ N $123.464 \mathrm{E}$

$\begin{array}{llllllll}24 & 02 & 45 & 20.4 & 43.958 & \mathrm{~N} & 15.544 \mathrm{E}\end{array}$

$24032801.8 * 23.342$ N 88.586 E

$24042012.8 * 9.765 \mathrm{~N} \quad 126.549 \mathrm{E}$

$24042626.5 * 9.688$ N $126.472 \mathrm{E}$

$24044116.5 * 19.715 \mathrm{~S} 178.518$ พ

$2404 \quad 4241.0840 .794 \mathrm{~N} 123.718 \mathrm{~W}$

$$
\begin{aligned}
& 24 \\
& 24 \\
& 24 \\
& 24 \\
& 24 \\
& 24 \\
& 24 \\
& 24
\end{aligned}
$$

\section{$\begin{array}{llll}06 & 02 & 23.58 & 72.799 \mathrm{~N}\end{array}$}

$5.728 \mathrm{E}$ $073242.7 * 6.539$ s $103.843 \mathrm{E}$ $\begin{array}{rrrrrrr}07 & 49 & 39.9 ? & 36.12 & S & 178.69 & E \\ 08 & 34 & 28.7 ? & 9.48 & N & 126.90 & E\end{array}$ $093005.8845 .760 \mathrm{~N} \quad 26.792 \mathrm{E}$ $\begin{array}{lllllll}09 & 46 & 16.6 ? & 22.26 & \mathrm{~S} & 178.23 & \mathrm{w}\end{array}$ $104656.6 * 43.171 \mathrm{~N} 126.177$ พ

$33 \times 4.6$

$33 \mathrm{~N} 4.6$

$10 \mathrm{G} \quad 4.7$

$33 \mathrm{~N} \quad 4.9$

$10 \mathrm{G}$

$36 \mathrm{D} \quad 4.6$

$33 \mathrm{~N} 4.5$

$33 \mathrm{~N}$

600 G 4.8

34

$10 \mathrm{G}$

$55 \mathrm{D} \quad 4.2$

200 G 4.3

$33 \mathrm{~N} 4.3$

$120 \mathrm{G}$

$400 \mathrm{G} 4.0$

$10 \mathrm{G}$

$147 \mathrm{D} 6.0$
108

1.0 12

1.2

1.2
0.9

1.1

1. 5

1.0

0.5

0.9

0.9

0.9

1.112

0.813

0.6

$\begin{array}{ll}0.6 & 14 \\ 0.7 & 54\end{array}$

0.7417
CENTRAL ALASKA. 〈AEIC>

SOUTHWESTERN RYOKYU ISLANDS

IRIAN JAYA, INDONESIA

OFF SOUTH COAST OF AUSTRAIIA

24 SOUTHWESTERN RYUKYO ISLANDS

3 ADRIATIC SEA, Ma 3.3 (ROM). Felt at Biograd, Croatia.

5 INDIA-BANGILADESB BORDER REGION

24 MINDANAO, PHILIPPINE ISLANDS

8 MINDANAO, PHILIPPINE ISLANDS

9 FIJI ISLANDS REGION

31 NORTHERN CALIFORNIA. 〈GM-P>. MD 3.5 (GM). MI 3.4 (BRK), 3.4 (GS). Felt in the Arcata-Eureka area.

6 NORWEGIAN SEA

SOUTHWEST OF SUMATERA, INDONESIA

OFF E. COAST OF N. ISLAND, N.Z.

MINDANAO, PHILIPPINE ISLANDS

ROMANIA

SOOTH OF FIJI ISIANDS

OFF COAST OF OREGON

LEEWARD ISLANDS. NW 5.8 (GS)， 5.7 (GRV). mb 5.7 (BRK). Moment Tensor (GS): Dep 136; Principal axes (scale 10*t17 $\mathrm{Nm}$ ): (T) Val=6.58, Plg-11, Azm=163; (N) Val=-1.72, Plg=42, Azm-263; (P) Val=-4.86, Plg-46, Azm=62; Best double couple: Mo-5.7*10*17 Nm; NP1: Strike-214, Dip=50, S1ip=-151; NP2: strike-105, Dip $=68$, Slip $=-43$.

Centroid, Moment Tensor (HRV): Centroid origin time

$11: 42: 23.4$; Lat $15.39 \mathrm{~N}$; Lon $61.22 \mathrm{~W}$; Dep 138.7 ; Halfduration $1.6 \mathrm{sec}$; Principal axes (scale 10*17 $\mathrm{Nm}$ ): (T) Val-3.72, Plg=9, Azm-157; (N) Val=-0.29, Plg-24, Azm-251; (P) Val--3.43, Plg-65, Azm-47; Best double couple:

Mo-3.6*10**17 Nm; NP1: Strike=221, Dip=41, Slip=-127; NP2: 


$\begin{array}{lllrlrl}11 & 44 & 45.8 * & 9.534 & \mathrm{~N} & 126.578 & \mathrm{E} \\ 11 & 52 & 46.3 & 9.341 & \mathrm{~S} & 113.487 & \mathrm{E} \\ 12 & 22 & 24.7 * & 17.766 & \mathrm{~N} & 95.188 & \mathrm{~W} \\ 12 & 45 & 47.08 & 47.718 & \mathrm{~N} & 122.966 & \mathrm{~W} \\ 13 & 02 & 40.6 * & 17.836 & \mathrm{~N} & 146.186 & \mathrm{E} \\ 13 & 16 & 49.1 & 20.187 & \mathrm{~S} & 176.326 & \mathrm{~W} \\ 13 & 26 & 06.4 * & 72.420 & \mathrm{~N} & 126.215 & \mathrm{E} \\ 13 & 26 & 12.48 & 33.684 & \mathrm{~S} & 70.235 & \mathrm{~W} \\ 14 & 06 & 13.98 & 37.207 & \mathrm{~N} & 71.292 & \mathrm{E} \\ 14 & 32 & 22.7 & 9.470 & \mathrm{~N} & 126.453 & \mathrm{E} \\ 15 & 14 & 10.7 ? & 5.85 & \mathrm{~S} & 103.15 & \mathrm{E} \\ 15 & 19 & 28.88 & 32.384 & \mathrm{~N} & 132.125 & \mathrm{E} \\ 17 & 51 & 32.3 & 35.447 & \mathrm{~N} & 141.242 & \mathrm{E} \\ 19 & 10 & 02.7 & 43.539 & \mathrm{~N} & 126.268 & \mathrm{~W} \\ 19 & 24 & 49.5 & 27.222 & \mathrm{~N} & 100.373 & \mathrm{E}\end{array}$

Strike=86, Dip=58, Slip=-62.

1.128 MINDANAO, PHIIIPPINE ISLANDS

1.325 SOUTH OF JAWA, INDONESIA

$33 \mathrm{~N} \quad 4.6$

1.515 OAXACA, MEXICO

34 WASHINGTON. 〈SEA-P>. MD 3.5 (SEA). Felt.

I9 MARIANA ISLANDS

48

$150 \mathrm{G} \quad 3.9$

$220 \mathrm{D} 5.0$

0.961 FIJI ISLANDS REGION

0.566 NORTHCENTRAI SIBERIA, RUSSIA

$\begin{array}{rr}33 & \mathrm{~N} \\ 110 & \mathrm{G}\end{array}$

$103 \mathrm{D}$

$33 \mathrm{~N} \quad 4.8$

0.2

0.8

1.0

1.4

$33 \mathrm{~N}$

$33 \mathrm{~N}$

$33 \mathrm{~N}$

$10 \mathrm{G}$

$33 \times 5.35 .20 .5$

10 CHILE-ARGENTINA BORDER REGION. MD 3.3 (SAN).

9 AFGHANISTAN-TAJIKISTAN BORD REG.

41 MINDANAO, PHILIPPINE ISLANDS

5 SOUTHERN SUMATERA, IKDONESIA

5 SHIKORD, JAPAN

19 NEAR EAST COAST OF HONSHU, JAPAN

79 OFF COAST OF OREGON

189 YUNNAN, CHINA. MW 5.5 (HRV). Damage and a landslide occurred in the Lijiang area.

Centroid, Moment Tensor (HRV): Centroid origin time

$19: 24: 54.5$; Lat $27.18 \mathrm{~N}$; Ion $100.70 \mathrm{E}$; Dep 15.0 Fix; halfduration $1.2 \mathrm{sec}$; Principal axes (scale $10 * * 17 \mathrm{Nm}$ ): (T) Val=2.10, Plg=5, Azm=236; (N) Val=-0.28, Plg=5, Azm=327; (P) Val=-1.81, Plg-83, Azm=104; Best double couple: Mo-2.0*10**17 Nm; NP1: Strike-321, Dip=41, Slip=-98; NP2: Strike=151，Dip=50， Slipe-83.

\begin{tabular}{|c|c|c|c|c|c|c|c|c|c|c|}
\hline & $\begin{array}{l}03 \\
06 \\
02 \\
43 \\
43 \\
19 \\
48 \\
53\end{array}$ & $\begin{array}{l}00.1 \\
19.4 ? \\
48.9 * \\
05.9 ? \\
14.6 \\
05.1 ? \\
25.3 * \\
10.8\end{array}$ & $\begin{array}{l}51.681 \\
36.97 \\
27.044 \\
21.94 \\
48.222 \\
15.00 \\
24.257 \\
47.100\end{array}$ & $\begin{array}{l}N \\
N \\
N \\
S \\
N \\
S \\
S \\
N\end{array}$ & $\begin{array}{c}16.218 \\
5.81 \\
100.220 \\
178.34 \\
153.123 \\
173.04 \\
179.655 \\
10.364\end{array}$ & $\begin{array}{l}\mathbf{E} \\
\mathbf{W} \\
\mathbf{E} \\
\mathbf{W} \\
\mathbf{E} \\
\mathbf{W} \\
\mathbf{E} \\
\mathbf{E}\end{array}$ & $\begin{array}{r}10 \\
10 \\
33 \\
500 \\
33 \\
33 \\
600 \\
5\end{array}$ & $\begin{array}{l}\mathbf{G} \\
\mathbf{W} \\
\mathbf{G} \\
\mathbf{N}\end{array}$ & $\begin{array}{l}4.7 \\
4.7 \\
5.0\end{array}$ & $\begin{array}{l}0.6 \\
0.1 \\
1.0 \\
1.1\end{array}$ \\
\hline 2 & $\begin{array}{l}41 \\
56 \\
14 \\
16 \\
11 \\
16 \\
53 \\
24 \\
08 \\
11 \\
12 \\
20 \\
39 \\
41\end{array}$ & $\begin{array}{l}16.8 * \\
28.9 \\
37.9 * \\
53.7 ? \\
01.2^{\star} \\
56.8 \\
31.8 \\
56.8 * \\
17.27 \\
44.2^{\star} \\
08.6 ? \\
23.0 * \\
21.1 \\
16.8 * \\
29.6 ? \\
55.2 \star\end{array}$ & $\begin{array}{c}51.523 \\
27.231 \\
4.412 \\
9.95 \\
51.144 \\
51.581 \\
51.591 \\
37.371 \\
42.95 \\
2.112 \\
2.03 \\
30.440 \\
9.411 \\
21.486 \\
9.42 \\
37.888 \\
9.694\end{array}$ & $\begin{array}{l}N \\
N \\
N \\
N \\
N \\
N \\
N \\
S \\
N \\
S \\
S \\
N \\
N \\
N \\
N \\
S\end{array}$ & $\begin{array}{c}16.041 \\
100.378 \\
126.918 \\
126.48 \\
176.084 \\
16.267 \\
16.285 \\
146.007 \\
0.67 \\
99.716 \\
99.70 \\
78.584 \\
126.461 \\
122.591 \\
127.08 \\
146.203 \\
126.406\end{array}$ & $\begin{array}{l}\mathbf{E} \\
\mathbf{E} \\
\mathbf{E} \\
\mathbf{E} \\
\mathbf{W} \\
\mathbf{E} \\
\mathbf{E} \\
\mathbf{E} \\
\mathbf{W} \\
\mathbf{E} \\
\mathbf{E} \\
\mathbf{E} \\
\mathbf{E} \\
\mathbf{E}\end{array}$ & $\begin{array}{r}10 \\
33 \\
100 \\
33 \\
33 \\
10 \\
10 \\
10 \\
10 \\
33 \\
33 \\
33 \\
33 \\
33 \\
33 \\
10 \\
33\end{array}$ & $\begin{array}{l}\text { G } \\
\text { N } \\
\text { G } \\
\text { N } \\
\text { N } \\
\text { G } \\
\text { G } \\
\text { G } \\
\text { G } \\
\text { N } \\
N \\
N \\
N \\
N \\
N \\
\text { G } \\
\text { N }\end{array}$ & $\begin{array}{l}4.7 \\
4.8 \\
4.4 \\
4.6 \\
4.9 \\
4.2 \\
4.7 \\
4.3 \\
5.5 \\
5.7\end{array}$ & $\begin{array}{l}0.8 \\
0.8 \\
1.0 \\
1.8 \\
1.2 \\
1.3 \\
1.2 \\
1.0 \\
1.2 \\
1.5 \\
1.3\end{array}$ \\
\hline
\end{tabular}

13 POLAND. ML 3.3 (GRF), 2.8 (MOX).

4.14 STRAIT OF GIBRALTAR. mbLg 2.2 (MDD).

20 YUNRAN, CEINA

25 FIJI ISLANDS REGION

91 KURIL ISLANDS

18 SAMOA ISLANDS REGION

27 SOUTH OF FIJI ISLANDS

17 ADSTRIA. ML 2.7 (STR), 2.4 (IDG), 2.4 (FOR), 2.2 (VIE). Felt (IV) at Lech and Warth.

0.611 POIAND. ML 3.2 (GRF), 3.1 (MOX), 2.5 (CLL).

. 48 YUMNAN, CHINA

15 TALAUD ISLANDS, INDONESIA

10 MINDANAO, PEILIPPINE ISLANDS

20 ANDREANOF ISLANDS, ALEUTIAN IS.

23 POLAND. MIL 3.6 (GRF), 3.3 (VIE), 2.9 ( (NOX).

11 POLAND. MCL 2.7 (MOX).

6 NEAR S.E. COAST OF AUSTRALIA

7 PYRENEES. mbLg 2.9 (MDD). ML 2.7 (IDG).

20 SOUTHERH SUMATERA, INDONESIA

10 SOUTHERH SUMATERA, INDONESIA

20 NORTHERN INDIA

43 MINDANAO, PEILIPPINE ISLANDS

10 TAIWAN REGION

17 PEIIIPPINE ISLANDS REGION

17 NEAR S.E. COAST OF AUSTRALIA

82 MINDANAO, PEILIPPINE ISLANDS. NW 5.8 (HRV).

Centroid, Moment Tensor (ERV): Centroid origin time

09:26:49.4; Lat $9.61 \mathrm{~N}$; Lon $126.77 \mathrm{E}$; Dep $15.0 \mathrm{Fix}$; Half duration $1.9 \mathrm{sec}$; Principal axes (scale 10**17 $\mathrm{Nm}$ ): (T) Val=6.14, Plg=69, Azm=251; (N) Val=0.22, Plg=7, Azm=0; (P) Val=-6.35, Plg=20, Azm=93; Best double couple: Mo $=6.2 * 10 * 17 \mathrm{Nm} ;$ NP1: Strike-196, Dip=26, Slip=107; NP2: Strike=357, Dip=65, Siip=82.

120.878 E 100 G 5.0 $126.624 \mathrm{E} 33 \mathrm{~N} 5.0$ $129.452 \mathrm{E} 144 * 4.9$ 126.16 E 33 N 4.5 $176.580 \mathrm{~W} 300 \mathrm{G} 4.4$ $99.186 \mathrm{E} \quad 33 \mathrm{~N} \quad 5.0$ $166.619 \mathrm{E} \quad 33 \mathrm{~N} 4.6$ $92.057 \mathrm{~W} 78 * 4.8$ 179.182 W 600 G 4.8 $\begin{array}{lllll}143.722 \mathrm{E} & 33 \mathrm{~N} & 4.8\end{array}$ 17.87 E $10 \mathrm{G} 4.2$ $128.655 \mathrm{E} 100 \mathrm{G} 3.7$ $123.237 \mathrm{E} \quad 33 \mathrm{~N} \quad 4.5$ $29.417 \mathrm{~W} \quad 10 \mathrm{G}$ $126.297 \mathrm{E} \quad 33 \mathrm{~N} \quad 4.6$ $44.382 \mathrm{E} \quad 10 \mathrm{G} \quad 4.6$ $126.135 \mathrm{E} 33 \mathrm{~N} 5.1$ $166.378 \mathrm{~W} 10 \mathrm{G}$ $51.247 \mathrm{E} \quad 10 \mathrm{G} \quad 4.4$ $138.604 \mathrm{E} 391 * 3.9$ 142.71 E 143 ? 3.9 $68.653 \mathrm{~W} 127$ ? $79.219 \mathrm{~W} \quad 100$ D 4.8
1.5

1.1

0.9

0.7

1.4
35 SERAM, INDONESIA

14 EAST OF KURIL ISIANDS

24 MINDANAO, PHILIPPINE ISLANDS

15 MINAHASSA PENINSULA, SULAWESI

10 EAST OF KURIL ISLANDS

56 NORTH ISLAND, NEW ZEALAND. MW 5.4 (BRV).

Centroid, Moment Tensor (BRV): Centroid origin time $10: 35: 21.4$; Lat $38.76 \mathrm{~s}$; IOD $174.86 \mathrm{E}$; Dep 216.3; Halfduration $1.2 \mathrm{sec}$; Principal axes (scale 10**17 $\mathrm{Nm}$ ): (T) val=1.37, $\mathrm{Plg}=38, A z m=24$; (N) Val=-0.07, Plg=43, Azm=160; (P) Val=-1.30, Plg=24, Azm=274; Best double couple: Mo-1.3*10**17 $\mathrm{Nm}$; NP1: Strike-53, Dip=44, Slip-168; NP2: Strike=152, Dip -82 , Slip $=46$.

1.048 MINDORO, PHILIPPINE ISIANDS

1.141 MINDANAO, PHILIPPINE ISLANDS

1.421 BANDA SEA

0.914 MINDANAO, PHILIPPINE ISLANDS

1.021 FIJI ISLANDS REGION

1.315 YUNAAN, CHINA

1.121 VANOATU ISLANDS

1.218 MEAR COAST OF CHIAPAS, MEXICO

0.522 FIJI ISLANDS REGION

1.064 VOLCANO ISLANDS REGION

1.412 ADRIATIC SEA

0.910 BANDA SEA

1.120 MINAHASSA PENINSULA, SULAWESI

1.311 AZORES ISIANDS. Felt (III) or Faial.

0.917 MINDANAO, PEILIPPINE ISLANDS

0.814 SOUTH INDIAN OCEAN

1.129 PHILIPPINE ISLANDS REGION

1.26 BERING STRAIT

$1.0 \quad 37$ PERSIAN GULF

0.210 SOUTH OF HONSHU; JAPAN

0.99 NEW GOINEA, PAPUA NEW GUINEA

1.510 CHILE-BOIIVIA BORDER REGION

0.971 PERU-ECUADOR BORDER REGION. Felt (V) at Gonzanama, Loja, and 


$\begin{array}{rrrllllrlrllll}25 & 17 & 41 & 17.2 & 27.433 & \mathrm{~N} & 88.552 & \mathrm{E} & 33 & \mathrm{~N} & 5.0 & 4.5 & 1.1 & 58 \\ 25 & 17 & 49 & 35.4 * & 17.553 & \mathrm{~N} & 95.571 & \mathrm{~N} & 116 & * & 4.3 & & 1.4 & 22 \\ 25 & 17 & 49 & 53.2 * & 21.826 & \mathrm{~N} & 144.697 & \mathrm{E} & 33 & \mathrm{~N} & 4.4 & & 1.0 & 10 \\ 25 & 17 & 56 & 30.2 & 46.049 & \mathrm{~N} & 14.285 & \mathrm{E} & 10 \mathrm{G} & & & 1.5 & 33\end{array}$

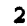
$\begin{array}{llllllllll}25 & 18 & 49 & 06.7 ? & 23.75 & S & 179.99 & E\end{array}$ $25 \quad 18 \quad 4917.0 ?-5.76$ (

$25190738.5 * 39.227 \mathrm{~N} \quad 71.476 \mathrm{E}$ 25
$33 \mathrm{~N}$

$33 \mathrm{~N}$

$33 \times 4.9$

500 G 4.5

148.51 E $100 \mathrm{G} \quad 4.6$

6

50 G 4.3

1364.3

$222 * 4.4$

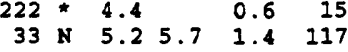

$\begin{array}{llllll}25 & 19 & 07 & 13.5 \% & 36.698 \mathrm{~N}\end{array}$

Hacara, Ecuador

SIKKIM, INDIA

OAXACA, MEXICO

MARIANA ISLANDS REGION

NORTHWESTERN BALKAN REGION. MU 3.5 (IJU), 3.3 (FUR), 3.2 (VIE), 3.2 (LDG). Felt (V) in the Ljubljana and Smledinik areas, Slovenia.

6 EASTERN SEA OF JAPAN

1.15 NEAR N COAST OF NEW GOINEA, PNG.

1.240 MINDANAO, PHILIPPINE ISLANDS

1.224 SOUTH OF FIJI ISLANDS

0.611 NEW BRITAIN REGION, P.N.G.

60 CENTRAL CAIIFORNIA. LGM-P>. MD 3.3 (GM). ML 3.4 (BRK), 3.1 (GS).

1.012 TAJIKISTAN

54 NORTHERN CAIIFORNIA. LGM-P>. MD 3.1 (GM). MI 3.2 (GS), 3.1 (BRK)

MARIANA ISLANDS. Felt (III) at Dededo and Potts Junction, Guam.

SOUTH OF MARIANA ISLANDS

SOUTH OF JAWA, INDONESIA. WW 5.6 (GS), 5.6 (HRV).

Moment Tensor (GS): Dep 14; Principal axes (scale 10*\#17 $\mathrm{Nm}$ ): (T) Val-2.89, Plg-51, Azm-97; (N) Val--0.11, Plg=39, $A 2 m=283$; (P) Val=-2.78, Plg-3, Azm-191; Best double couple: Mo-2.8*10**17 $\mathrm{Nm}$; NP1: Strike-248, Dip=54, Slip=39; NP2 : Strike-132, Dip=59, Slip=137.

Centroid, Moment Tensor (HRV) : Centroid origin time $21: 16: 10.9$; Lat $9.74 \mathrm{~s}$; Lon $108.32 \mathrm{E}$; Dep 38.8 ; Half duration $1.6 \mathrm{sec}$; Principal axes (scale 10*\#17 $\mathrm{Nm}$ ): (T) val=2.78, Plg=39, Azm=106; (N) val=0.52, Plg=50, Azm=273; (P) Val--3.30, Plgm, Azm=11; Best double couple: Mo-3.0*10*\#17 Nm; NP1: Strike-141, Dip-58, slip-155; NP2: Strike-245, Dip=69, Slip=34.

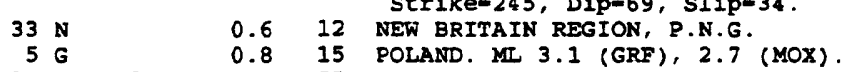

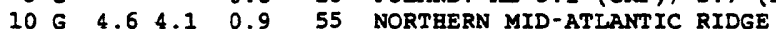

$33 \mathrm{~N} 4.4 \quad 1.0 \quad 11$ MINDANAO, PHILIPPINE ISLANDS

$33 \mathrm{~N} 4.0 \quad 0.3 \quad 7$ TAINAN REGION

$33 \mathrm{~N} 4.1 \quad 0.9 \quad 16$ YONNAN, CHINA

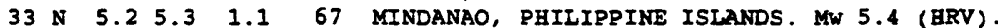

Centroid, moment Tensor (HRV): Centroid origin time

$03: 45: 32.3$; Lat $9.64 \mathrm{~N}$; Lon $127.04 \mathrm{E}$; Dep $15.0 \mathrm{Fix}$; Halfduration $1.2 \mathrm{sec}$; Principal axes (scale 10**17 Nm): (T)

Val-1.10, Plg-61, Azm=203; (N) Val-0.43, Plg-26, Azm=357 (P) val=-1.53, Plg-11, Azm-92; Best double couple:

Mo-1.3*10*17 Nm; NP1: Strike-211, Dip=41, Slip-133; NP2: Strike-341, Dip-61, Slip-60.

$\begin{array}{llllll}26 & 04 & 31 & 29.18 & 47.000 & \end{array}$

$6.827 \mathrm{E}$ $050003.4 \quad 9.728 \mathrm{~N} 126.736 \mathrm{E}$ $\begin{array}{lllll}05 & 23 & 17.9 & 9.712 & \mathrm{~N} \\ 0 & 126.812 \mathrm{E}\end{array}$ $055256.0 * 44.144 \mathrm{~N} 148.710 \mathrm{E}$ $26055902.1 * 9.906 \mathrm{~N} 126.076 \mathrm{E}$ $26061158.4 * 11.446 \mathrm{~N} 86.970 \mathrm{~W}$ 26070512.8 ? 9.31 N $126.79 \mathrm{E}$ $\begin{array}{lllll}26 & 07 & 06 & 52.6 * 39.736 & N\end{array}$ $\begin{array}{llllllll}26 & 07 & 17 & 33.2 & 52.186 & \mathrm{~N}\end{array}$ $26073939.5 * 9.447 \mathrm{~N}$ 26074733.4 * $9.507 \mathrm{~N}$ $\begin{array}{lllllll}26 & 08 & 13 & 45.2 & 54.019 & \mathrm{~N}\end{array}$ $\begin{array}{lllllll}26 & 08 & 17 & 36.4 ? & 9.41 \mathrm{~N}\end{array}$ $\begin{array}{lllllll}26 & 09 & 15 & 57.9 & 35.448 & \mathrm{~N}\end{array}$ $26094604.1 * 4.866 \mathrm{~s}$ $26095524.4 ? 29.65 \quad N$ $\begin{array}{llllllll}26 & 10 & 17 & 42.4 \text { ? } 32.51 & 5\end{array}$ $\begin{array}{lllll}26 & 10 & 26 & 27.2 * 43.518 \mathrm{~N}\end{array}$ $\begin{array}{llllll}26 & 11 & 27 & 04.7 & 51.657\end{array}$ $\begin{array}{llllllll}26 & 11 & 30 & 44.4 ? & 23.27 & N\end{array}$ $\begin{array}{llllll}26 & 12 & 31 & 49.1 & 40.030\end{array}$ $\begin{array}{llllll}26 & 12 & 35 & 45.5 ? & 9.23\end{array}$ $26 \cdot 143635.5 * 20.7375$ $26144956.9 * 7.150 \mathrm{~s}$ $\begin{array}{lllll}26 & 15 & 29 & 07.9 & 6.155\end{array}$ $171.491 \mathrm{H}$ $126.605 \mathrm{~B}$ $126.722 \mathrm{E}$ $159.689 \mathrm{E}$ $126.43 \mathrm{E}$ $140.402 \mathrm{E}$ $151.145 \mathrm{E}$ $52.25 \mathrm{E}$ $71.60 \mathrm{~W}$ $128.289 \mathrm{~W}$ $16.253 \mathrm{E}$ $143.85 \mathrm{E}$ $20.796 \mathrm{E}$ $126.70 \mathrm{E}$ $68.439 \mathrm{w}$ $129.635 \mathrm{E}$
$20 \mathrm{G}$

$33 \mathrm{~N} 4.7$

$33 \mathrm{~N} 4.7$

$33 \mathrm{~N}$

$33 \mathrm{~N} 4.8$

$33 \mathrm{~N} 4.6$

$33 \mathrm{~N} 4.3$

$84 ? 3.8$

$33 \mathrm{~N} 4.8$

$33 \mathrm{~N} 4.5$

$33 \mathrm{~N} 4.6$

$33 \mathrm{~N} 4.94 .5$

$33 \mathrm{~N}$

$50 \mathrm{G}$

$150 \mathrm{G}$

$33 \mathrm{~N}$

$20 \mathrm{G}$

$10 \mathrm{G}$

$5 \mathrm{G}$

$33 \mathrm{~N} 4.3$

$10 \mathrm{G} 4.0$

$33 \mathrm{~N} 4.4$

$164 \mathrm{D} 4.0$

$100 \mathrm{G} 4.3$

$33 \mathrm{~N} 5.1$
1.2

31 MINDANAO, PHILIPPINE ISLANDS

1.28 KURIL ISLANDS

1.030 MINDANAO, PHILIPPINE ISLANDS

$1.3 \quad 34$ NEAR COAST OF NICARAGUA

1.016 MINDANAO, PHILIPPINE ISIANDS

1.013 SOUTHERN XINJIANG, CHINA

1.048 FOX ISLANDS, ALEUTIAN ISLANDS

1.018 MINDANAO, PHILIPPINE ISLANDS

1.063 NEAR EAST COAST OF KAMCHATKA

0.98 MINDANAO, PHILIPPINE ISLANDS

0.710 NEAR EAST COAST OF BONSHU, JAPAN

1.217 NEW BRITAIN REGION, P.N.G.

1.1 11 SOUTHERN IRAN. Felt in the Shiraz area.

0.510

0.349

$0.7 \quad 15$

1.07

1.236

$\begin{array}{ll}1.3 & 12 \\ 0.9 & 16\end{array}$

0.3

OFF COAST OF OREGON

POLAND. MIL 3.4 (GRF), 2.9 (MOX).

VOLCANO ISLANDS REGION

GREECE-ALBANIA BORDER REGION

MINDANAO, PHILTPPINE ISLANDS

CHILE-BOLIVIA BORDER REGION

BANDA SEA
1.131

0.919 MINDANAO, PHILIPPINE ISLANDS

1.4 $\begin{array}{lllll}16 & 48 & 26.0 & 12.14 \mathrm{~N}\end{array}$ $\begin{array}{llll}17 & 00 & 18.3 & 9.462 \mathrm{~N}\end{array}$ $\begin{array}{lllll}17 & 17 & 20.1 & 23.981 & \mathrm{~N}\end{array}$ $183505.6 * 47.338$ $184618.133 .540 \mathrm{~s}$ $190112.7 \& 62.544 \mathrm{~N}$ $1940 \quad 57.4 * 38.905 \mathrm{~N}$ $194404.7 ? 5.88 \mathrm{~S}$ $\begin{array}{llllll}20 & 13 & 31.9 ? & 18.57 \quad \mathrm{~S}\end{array}$ $202110.3844 .310 \mathrm{~N}$ $\begin{array}{llllll}20 & 29 & 34.5 & 44.115 & \mathrm{~N}\end{array}$ $\begin{array}{llll}21 & 37 & 34.8 & 43.936 \mathrm{~N}\end{array}$ $214338.5 * 9.274 \mathrm{~N}$
$70.785 \mathrm{~W}$

86.23 W $126.420 \mathrm{E}$ $125.211 \mathrm{E}$ $12.860 \mathrm{~W}$ $71.803 \mathrm{~W}$ 151.154 W $140.724 \mathrm{E}$ $147.42 \mathrm{E}$ 179.85 W $7.547 \mathrm{E}$ $21.017 \mathrm{E}$ $7.778 \mathrm{E}$ $126.554 \mathrm{E}$
$80 \mathrm{G}$

$33 \mathrm{~N} 4.7$

$33 \mathrm{~N} 5.0$

$33 \mathrm{~N} 4.5$

$10 \mathrm{G} \quad 4.6$

$40 \mathrm{G}$

91

42 *

$100 \mathrm{G} 4.3$

$600 \mathrm{G} \quad 4.3$

$5 \mathrm{G}$

$10 \mathrm{G}$

4.5

$33 \mathrm{~N} 4.7$
TEAR COAST OF CENTRAL CHILE. MD 3.4 (SAN).

47 EASTERN NEW GOINEA REG., P.N.G. MW 5.2 (HRV)

Centroid, Moment Tensor (HRV): Centroid origin time

15:29:11.2; Lat 6.51 S; Lon 146.24 E; Dep 26.4; Balf-

duration 1.0 sec; Principal axes (scale 10*\#16 Nm): (T)

Val-5.87, Plg-8, Azm=108; (N) Val-2.22, Plg=75, Azm=232; (P) Val=-8.09, Plg-12, Azm-16; Best double couple:

Mo-7.0*10**16 Nm; NPI: Strike-153, Dip-75, Slip=-177; NP2: Strike-62, Dip=87, Slip=-15.

0.38 CIILE-ARGENTINA BORDER REGION. MD 2.2 (SAN).

1.512 NICARAGDA

1.046 MINDANAO, PHILIPPINE ISLANDS

1.016 SOUTHWESTERN RYUXYO ISLANDS

1.417 SOOTEERN MID-ATIANTIC RIDGE

0.5

1.0

1.4

1.2

0.5

1.3
NEAR COAST OF CENTRAL CHILE. MD 3.1 (SAN).

91 CENTRAL ALASKA. <AEIC>.

EASTERN HONSHU, JAPAN

EASTERN NEW GUINEA REG., P.N.G.

FIJI ISLANDS REGION

NORTEERN ITALY. ML 2.2 (LDG)

NORTEWESTERN BALKAN REGION. MIL 4.1 (VIE).

23 NEAR SOUTH COAST OF FRANCE. ML 2.6 (LDG), 2.5 (STR).

20 MINDANAO, PHILIPPINE ISLANDS 


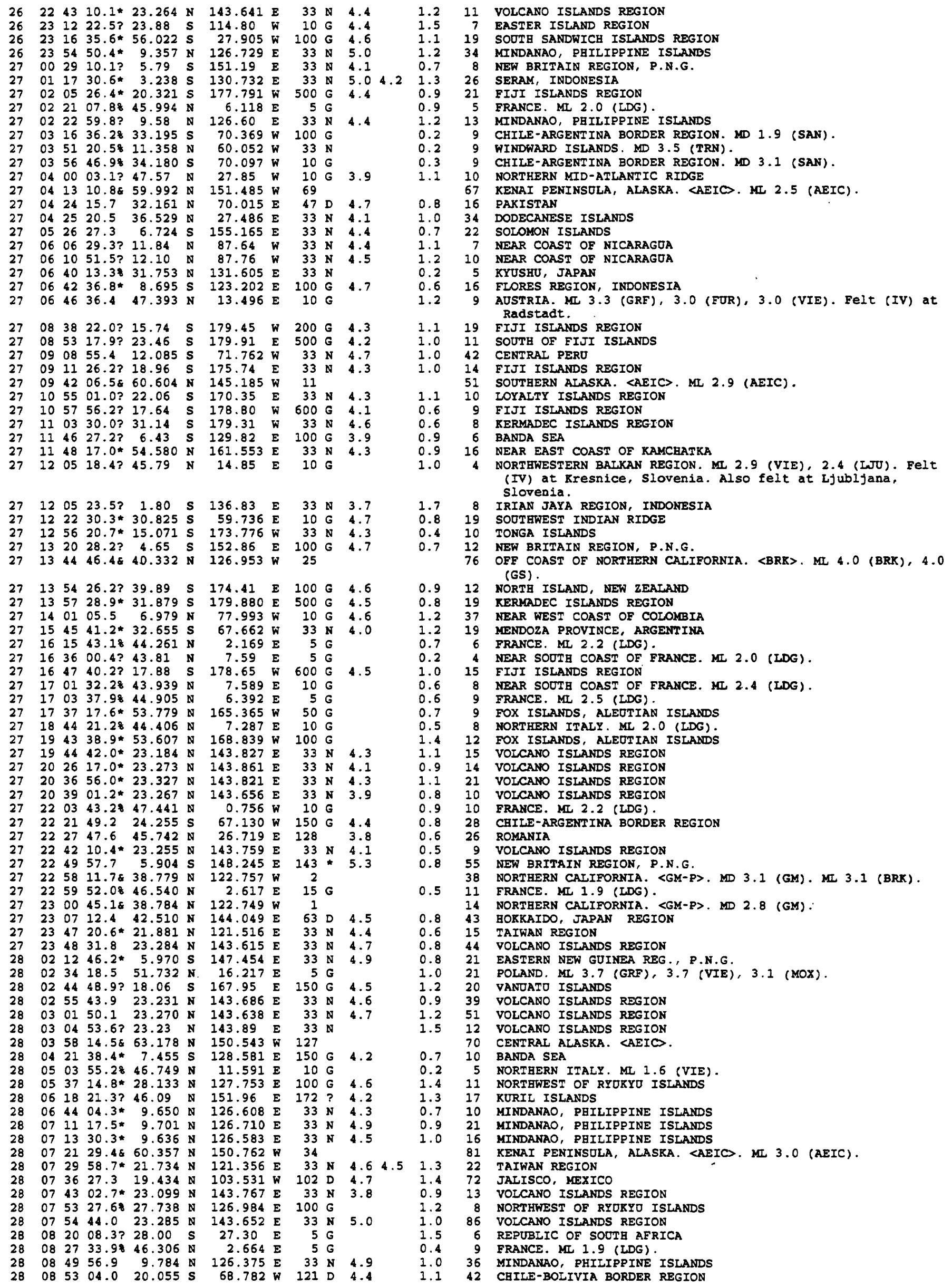


$\begin{array}{llllll}28 & 08 & 55 & 33.88 & 46.317\end{array}$ $\begin{array}{llllll}28 & 09 & 04 & 24.6 & 23.434 & \mathrm{~N}\end{array}$ $280908 \quad 28.1 \quad 23.333 \mathrm{~N}$ $\begin{array}{lllllll}8 & 09 & 13 & 10.5 ? & 23.25 \mathrm{~N}\end{array}$ $\begin{array}{llllll}8 & 09 & 31 & 21.8 ? & 23.29 & N\end{array}$ $28095135.7 * 4.241 \mathrm{~s}$ $\begin{array}{llllll}28 & 10 & 07 & 53.38 & 44.683\end{array}$ $101722.9 * 27.904 \mathrm{~N}$ $\begin{array}{llllll}11 & 02 & 46.8 & 35.075\end{array}$ $\begin{array}{llllll}8 & 11 & 02 & 55.3 & 56.057\end{array}$ $28111023.2 * 34.929 \mathrm{~N}$ $\begin{array}{lllllll}8 & 11 & 18 & 34.4 & 23.264 & \mathrm{~N}\end{array}$ $\begin{array}{lllll}11 & 47 & 37.8 * 23.364\end{array}$ $\begin{array}{lllll}28 & 12 & 10 & 52.7 * 41.117\end{array}$ $2812 \quad 1759.5 \quad 11.682 \mathrm{~N}$
$2.666 \mathrm{E}$ $143.682 \mathrm{E}$ $143.858 \mathrm{E}$ $143.52 \mathrm{E}$ $143.46 \quad \mathrm{E}$ $127.116 \mathrm{E}$ $7.670 \mathrm{E}$ $127.180 \mathrm{E}$ $23.865 \mathrm{E}$ $163.070 \mathrm{E}$ $23.800 \mathrm{E}$ $143.777 \mathrm{E}$ $143.760 \mathrm{E}$ $50.326 \mathrm{E}$ $86.470 \mathrm{~W}$
50

$33 \mathrm{~N} 4.6$

$33 \mathrm{~N} 4.5$

$33 \mathrm{~N}$

$33 \mathrm{~N}$

$327 * 4.5$

$10 \mathrm{G}$

$100 \mathrm{G} \quad 4.0$

$33 \mathrm{~N} 4.7$

$33 \mathrm{~N} 5.14 .5$

$33 \mathrm{~N} 3.4$

$33 \mathrm{~N} 4.5$

$33 \mathrm{~N} 4.2$

$64 \mathrm{D} \quad 4.3$

$\begin{array}{rr}1.0 & 17 \\ 1.0 & 167\end{array}$
0.68 FRANCE. MI 1.7 (IDG).

0.837 VOLCANO ISLANDS REGION

1.137 VOLCANO ISLANDS REGION

0.86 VOLCANO ISLANDS REGION

1.27 VOLCANO ISLANDS REGION

1.514 BANDA SEA

0.610 NORTHERN ITALY. ML 2.1 (LDG).

0.76 RYOKYD ISLANDS

1.3160 CRETE. Felt throughout western Crete.

1.073 NEAR EAST COAST OF KAMCHATKA

1.422 CRETE

.9 32 VOLCANO ISLANDS REGION

VOLCANO ISLANDS REGION

CASPIAN SEA

NEAR COAST OF NICARAGUA, MW 5.7 (HRV)

Centroid, Moment Tensor (HRV): Centroid origin time

12:18:07.9; Lat $11.68 \mathrm{~N} \mathrm{Fix;} \mathrm{Lon} 86.47$ W Fix; Dep 15.0 Fix; Half-duration $1.4 \mathrm{sec;}$ Principal axes (scale 10**17 Nm): (T) Val-4.57, P1g-50, Azm-54; (N) Va1--0.16, Plg-9,

Azm-312; (P) Val--4.41, Plg-38, Azm=214; Best double couple: Mo-4.5*10*17 Nm; NP1: Strike-256, Dip-11, Slip=34; NP2: Strike-133, Dip-84, Slip=100.

$28123325.6 * 47.129 \mathrm{~N} 153.034 \mathrm{E}$

$33 \mathrm{~N} \quad 3.7$

$33 \mathrm{~N} 4.2$

$33 \times 4.8$

$\begin{array}{lllll}13 & 14 & 19.8 & 19.531 \mathrm{~N} & 102.616 \mathrm{E}\end{array}$

131738.4 ? $23.13 \mathrm{~N} 143.63 \mathrm{E}$

$\begin{array}{llllllll}13 & 20 & 41.4 ? & 28.08 & S & 177.72 & W\end{array}$

$\begin{array}{lllll}13 & 51 & 17.5 & 3.680 \mathrm{~S} & 102.371 \mathrm{E}\end{array}$

33

$102 \mathrm{D}$

$92 \mathrm{D} \quad 4.5$

$33 \times 4.8$

$8 \begin{array}{llllllll}14 & 08 & 51.5 \& & 59.802 & \mathrm{~N} & 152.253 & \mathrm{~W}\end{array}$

$28 \quad 14 \quad 1042.4 \quad 10.038 \mathrm{~N}$

$125.369 \mathrm{E}$
67

235

5.6

\subsection{KURIL ISLANDS}

1.027 VOLCANO ISLANDS REGION

$1.3 \quad 30$ SOUTHEAST ASIA

0.57 VOLCANO ISLANDS REGION

1.39 KERYADEC ISLANDS REGION

1.032 SOUTHERN SDMATERA, INDONESIA

0.995 SOUTHERN IRAN

$1.0 \quad 177$
59 SOUTHERN ALASRA. CAEIC). ML 2.8 (AEIC).

IEYTE, PHILIPPINE ISLANDS. MW 6.3 (GS), 6.3 (HRV). Me 6.3 (GS).

Broadband Source Parameters (GS): Dep 218; NPI: Strike-25, Dip-90, S1ip--180; NP2: Strike-115, Dip-90, S1ip-0;

Radiated energy $7.3 * 10 * \star 13 \mathrm{Nm}$.

voment Tensor (GS): Dep 237; Principal axes (scale 10*18 $\mathrm{Nm}$ ): (T) Val-3.21, Plg-9, Azm=154; (N) Val-0.08, Plg-79, Azm-8; (P) Val--3.29, Plg-6, Azm-245; Best double couple: Mo-3.2*10**18 Nm; NP1: Strike=290, Dip-79, Slip=2; NP2: Strike-199, Dip=88, Slip=169.

Centroid, Moment Tensor (HRV): Centroid origin time

$14: 10: 46.6$; Lat $10.19 \mathrm{~N}$; Ion $125.33 \mathrm{E}$; Dep 237.6 ; Halfduration $3.5 \mathrm{sec}$; Principal axes (scale 10**18 Nm): (T) Val-3.65, Plg-5, Azm-156; (N) Val--0.28, Plg-80, Azm-32; (P) Val--3.38, Plg-8, Azm-247; Best double couple: Mo-3.5*10**18 Nm; NP1: Strike-291, Dip-81, Slip--2; NP2: Strike-21, Dip-88, slip--171.

$2814 \quad 40 \quad 31.0 * 23.361 \mathrm{~N}$ $\begin{array}{lllll}28 & 15 & 21 & 00.8 & 42.992\end{array}$ $28 \quad 1535 \quad 46.0 * 24.810 \mathrm{~s}$ $\begin{array}{llllll}28 & 15 & 48 & 07.48 & 44.600\end{array}$ $\begin{array}{llllllll}28 & 16 & 02 & 09.1 ? & 34.32 & \mathrm{~s}\end{array}$ $\begin{array}{lllll}28 & 16 & 12 & 45.1 & 42.864\end{array}$

$\begin{array}{llllllll}28 & 16 & 46 & 42.2 ? & 23.26 & N\end{array}$ $\begin{array}{llllll}28 & 17 & 07 & 04.6 & 51.503\end{array}$ $\begin{array}{llllll}28 & 17 & 07 & 30.62 & 42.421\end{array}$ $\begin{array}{llllll}28 & 18 & 24 & 12.2 & 18.165 & \mathrm{~s}\end{array}$ $\begin{array}{lllllll}28 & 18 & 34 & 27.0 & 43.425 & \mathrm{~N}\end{array}$ $28 \quad 191220.6 * 34.870 \mathrm{~N}$ $\begin{array}{llllll}28 & 19 & 28 & 54.1 & 51.702 & \mathrm{~N}\end{array}$ $\begin{array}{llllll}28 & 19 & 34 & 47.5 \& 37.531 & N\end{array}$

$28 \quad 194417.3 * 11.761 \mathrm{~N}$ $\begin{array}{lllllll}28 & 20 & 00 & 22.1 & 34.467 & \mathrm{~N}\end{array}$ $\begin{array}{llllll}28 & 20 & 24 & 33.08 & 34.282 \mathrm{~N}\end{array}$ $\begin{array}{llllllll}28 & 21 & 04 & 29.98 & 61.268 & \mathrm{~N}\end{array}$ $\begin{array}{lllll}28 & 21 & 09 & 01.0 * 23.016 \mathrm{~s}\end{array}$ $\begin{array}{lllll}28 & 21 & 52 & 12.5 \& 62.362 & \mathrm{~N}\end{array}$ $28 \quad 2251 \quad 46.1 \quad 23.233 \mathrm{~N}$ $28230609.2 * 47.918 \mathrm{~N}$ $28234928.8 * 19.4825$ $\begin{array}{llllllll}29 & 00 & 40 & 06.7 & 43.187 & N\end{array}$ $2900 \quad 48 \quad 54.0 \quad 47.027 \mathrm{~N}$ $29011614.5 * 8.352 \mathrm{~N}$ $290145 \quad 57.5 * 11.283 \mathrm{~N}$ $\begin{array}{lllllll}29 & 02 & 22 & 02.6 & 51.618 & \mathrm{~N}\end{array}$ $2903 \quad 37 \quad 15.3 * 8.316 \mathrm{~N}$ $\begin{array}{lllllll}29 & 03 & 44 & 25.68 & 12.322 & \mathrm{~N}\end{array}$ $\begin{array}{rrrrrr}29 & 05 & 09 & 26.9 & 8.288 & \mathrm{~N} \\ 29 & 05 & 52 & 37.4 & 51.716 & \mathrm{~N}\end{array}$ $\begin{array}{llllll}29 & 05 & 52 & 37.4 & 51.716 & N \\ 29 & 06 & 15 & 43.6 & 10.549 & \mathrm{~S}\end{array}$
$143.727 \mathrm{E}$ $175.120 \mathrm{~W}$ $20.683 \mathrm{E}$ $179.709 \mathrm{E}$ $7.192 \mathrm{E}$ 71.19 $17.855 \mathrm{E}$

$143.93 \mathrm{E}$ $161.505 \mathrm{E}$ 8.077 พ $70.037 \mathrm{~W}$ $146.832 \mathrm{E}$

$139.863 \mathrm{E}$ $16.162 \mathrm{E}$ 118.438 พ

86.401 W $29.349 \mathrm{E}$ $118.534 \mathrm{~W}$ $150.614 \mathrm{~W}$ $179.036 \mathrm{E}$ $149.594 \mathrm{~W}$ $143.634 \mathrm{E}$ $147.350 \mathrm{E}$

$67.445 \mathrm{~K}$

$0.555 \mathrm{~W}$ $7.211 \mathrm{E}$ $126.854 \mathrm{E}$

87.127 W

$16.227 \mathrm{E}$ $126.767 \mathrm{E}$ 60.786 $126.785 \mathrm{E}$ $175.397 \mathrm{~W}$ $165.867 \mathrm{E}$
$33 \mathrm{~N} 4.1$

$267 \mathrm{D} 4.9$

$10 \mathrm{G} 4.2$

$500 \mathrm{G} 4.2$

$10 \mathrm{G}$

$10 \mathrm{G} \quad 4.1$

$33 \mathrm{~N}$

$\begin{array}{llllllll}33 & \mathrm{~N} & 5.0 & 4.7 & 1.0 & 145\end{array}$

$10 \mathrm{G}$

$56 \mathrm{D} 5.2$

$33 \mathrm{~N}$

$92 * 4.4$

5 G

10

$33 \mathrm{~N} 4.3$

$33 \mathrm{~N} 4.2$

11

43

600 G 4.1

66

$33 \mathrm{~N} 4.6$

400 G 4.4

$224 * 3.9$

$5 \mathrm{G}$

$5 \mathrm{G}$

$33 \mathrm{~N} 4.4$

$33 \mathrm{~N} 4.5$

56

$33 \mathrm{~N} 4.6$

$70 \mathrm{G}$

$33 \mathrm{~N} 4.9$

$33 \mathrm{~N} 4.8$

$185 * 5.2$

$\begin{array}{rr}0.5 & 6 \\ 1.0 & 156\end{array}$

0.617

$0.7 \quad 12$

0.914

39

1.152

30

58

0.915

75

0.811

.47

$\begin{array}{ll}0.9 & 7 \\ 0.9 & 11\end{array}$

1.119

1.320

0.915

$0.7 \quad 21$

0.28

1.136

$0.7 \quad 147$
1.114 VOLCANO ISLANDS REGION

0.9168 TONGA ISILANDS

1.259 NORTHWESTERN BALKAN REGION. ML 4.0 (ROM)

1.019 SOUTH OF FIJI ISLANDS

0.38 NORTHERN ITALY. MI 2.5 (LDG).

0.47 NEAR COAST OF CENTRAL CAILE

1.448 ADRIATIC SEA. ML 3.9 (VIE). Felt (V) in the ston area ADRIATIC

\section{VOLCANO ISLANDS REGION}

$1.0 \quad 25$

$0.9 \quad 42$

1.171

SPAIN mbLg 2.9 (MDD).

NEAR COAST OF NORTHERN CAILE

KURIL ISLANDS

NEAR S. COAST OF HONSHU, JAPAN

POLAND. MIL 3.4 (GRF)

CALIFORNIA-NEVADA BORDER REGION. <GM-P>. MD 3.3 (GM). ML 3.4 (BRK), 3.2 (GS)

NEAR COAST OF NICARAGDA

EASTERN MEDITERRANEAN SEA

SOUTHERN CALIFORNIA. <PAS-P>. MD 2.5 (PAS). MI 2.8 (GS).

SOUTHERN ALASKA. 〈AEIC>. ML 2.5 (AEIC).
$290616 \quad 45.9 * 34.274 \mathrm{~N}$ $2906 \quad 45 \quad 25.7 * 28.059 \mathrm{~N}$ 29
$26.650 \mathrm{E}$ $128.555 \mathrm{E}$
$33 \mathrm{~N}$

$33 N 4.8$

33
0.7

1.3 
$071109.3 * 28.063$ $07 \quad 1942.4 \quad 42.691$ $072346.7 * 23.266 \mathrm{~N}$ $073406.3 * 28.043 \mathrm{~N}$ $\begin{array}{llllll} & 45 & 07.07 & 42.47\end{array}$ $090044.2 \quad 0.866 \mathrm{~N}$
$127.924 \mathrm{E}$ $17.620 \mathrm{E}$ $143.657 \mathrm{E}$ $128.686 \mathrm{E}$ 8.09 W $126.477 \mathrm{E}$ $\begin{array}{lll}33 & \mathrm{~N} & 4.7\end{array}$

$10 \mathrm{G}$

$33 \mathrm{~N} 4.5$

$10 \mathrm{G}$

$33 \mathrm{~N}$
$33 \mathrm{~N} 4.7$

1.217 NORTHWEST OF RYUKYO ISLANDS

0.812 ADRIATIC SEA

0.919 VOLCANO ISIANDS REGION

1.311 RYUKYO ISLANDS

0.14 SPAIN. mbLg 2.4 (MDD).

52

NORTHERN MOLUCCA SEA. MW 5.4 (HRV).

Centroid, Moment Tensor (HRv): Centroid origin time

09:00:49.5; Lat $1.07 \mathrm{~N}$; Lon $126.40 \mathrm{E}$; Dep $33.0 \mathrm{Fix}$; घalfduration 1.1 sec; Principal axes (scale 10*17 Nm): (T)

Val-1.27, Plg-72, Azm=96; (N) Val--0.14, Plg=11, Azm=221; (P) Val--1.13, Plg-15, Azm-314; Best double couple: Mo-1. 2*10*17 Nm; NP1: Strike-59, Dip-32, Slip-111; NP2: Strike-215, Dip=61, Slip=77.

$0959 \quad 47.0 * 27.892 \mathrm{~N}$

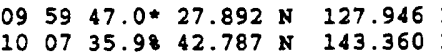
$17.566 \mathrm{~N} 10 \mathrm{G}$

1.018 RYORYO ISLANDS

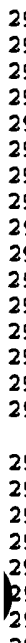

29 $122005.2 ? 21.58 \quad 5$ $\begin{array}{lllll}12 & 21 & 44.6 & 19.948 & \mathrm{~N}\end{array}$ $\begin{array}{llll}12 & 47 & 18.1 & 64.589\end{array}$ $\begin{array}{lllll}13 & 01 & 59.8 & 64.426 & \end{array}$ $130920.4 * 25.0545$ $1318 \quad 17.6 * 64.450 \mathrm{~N}$

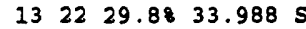
$132856.0 * 64.391 \mathrm{~N}$ $\begin{array}{llll}13 & 39 & 14.8 & 64.437 \mathrm{~N}\end{array}$ $135408.7 \& 36.451 \mathrm{~N}$

$\begin{array}{llll}13 & 56 & 29.48 & 63.324 \mathrm{~N}\end{array}$ $144011.2859 .578 \mathrm{~N}$ $\begin{array}{llll}14 & 43 & 26.4 & 21.949\end{array}$ $\begin{array}{llll}15 & 04 & 35.2 ? & 10.79\end{array}$ $\begin{array}{llll}15 & 25 & 49.5 * 6.384 \mathrm{~S}\end{array}$ $\begin{array}{lllll}16 & 26 & 15.7 ? & 9.45 \mathrm{~N}\end{array}$ $\begin{array}{lllll}17 & 12 & 25.2 & 52.198\end{array}$ $\begin{array}{llllll}17 & 41 & 03.48 & 66.162 & \end{array}$ $\begin{array}{lll}17 & 48 & 57.4 * 22.585 N\end{array}$ $\begin{array}{llll}18 & 01 & 14.3 & 8.993 \mathrm{~s}\end{array}$ $\begin{array}{lllll}18 & 18 & 59.68 & 38.251 & \end{array}$ $1820 \quad 58.3866 .168 \mathrm{~N}$ $\begin{array}{llll}18 & 36 & 29.48 & 45.779\end{array}$ $\begin{array}{lllll}18 & 45 & 03.28 & 42.922 & N\end{array}$ $\begin{array}{lllll}19 & 10 & 59.6 & 32.442\end{array}$ $1932 \quad 51.0 ? 36.04$ $\begin{array}{llll}19 & 45 & 06.3 & 35.707\end{array}$ $\begin{array}{llll}20 & 27 & 00.78 & 34.199 \mathrm{~s}\end{array}$ $\begin{array}{lllll}20 & 45 & 41.0 ? & 5.32 \quad 5\end{array}$ $\begin{array}{lllll}21 & 00 & 46.7 ? & 31.40\end{array}$ $21 \quad 1950.2 * 33.365$ $213641.9 * 9.401 \mathrm{~N}$ $224701.5 * 25.092$

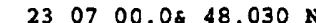

$232707.8 * 26.344 \mathrm{~S}$ $2339 \quad 12.6$ ? 36.34 $000655.6 * 8.705 \mathrm{~s}$ $\begin{array}{llllll}00 & 10 & 36.68 & 49.119 \mathrm{~N}\end{array}$ $\begin{array}{lllll}00 & 12 & 38.1 & 23.337 & \mathrm{~N}\end{array}$ $001303.5 * 23.414 \mathrm{~N}$ $\begin{array}{llllll}00 & 26 & 39.9 ? & 23.36 & \mathrm{~N} \\ 00 & 32 & 03.6 & 0.150 & \mathrm{~s}\end{array}$
29. $105328.8 \% 17.61$ $\begin{array}{llll}11 & 45 & 26.6 & 45.430\end{array}$ $\begin{array}{llll}11 & 58 & 13.9 & 51.646\end{array}$ $\begin{array}{llll}12 & 12 & 35.1 & 38.401\end{array}$ $132129.6 * 64.463 \mathrm{~N}$ $\begin{array}{llll}14 & 04 & 10.7 ? & 20.32 \mathrm{~s}\end{array}$
179.09 W 600 G 4.6

$130.520 \mathrm{E} \quad 33 \mathrm{~N} \quad 4.5$

$151.465 \mathrm{E} \quad 33 \mathrm{~N} 4.8$

$16.340 \mathrm{E} 5 \mathrm{G}$

$73.141 \mathrm{E} 106 * 4.6$

$173.95 \mathrm{~W} 33 \mathrm{~N}$

$145.490 \mathrm{E} \quad 97 * 4.9$

$17.849 \mathrm{~W}$

$17.929 \mathrm{~W}$

$179.822 \mathrm{E}$

$18.087 \mathrm{~F}$

17.866 W

70.956 W

$17.905 \mathrm{~W}$

$17.761 \mathrm{~W}$

$120.203 \mathrm{~W}$

149.094 ผ 87

175.59

$152.563 \mathrm{~W}$

$121.808 \mathrm{E}$

61.68 W

$154.809 \mathrm{E}$

$126.63 \mathrm{E}$

$30.058 \mathrm{~W}$

$148.332 \mathrm{~W}$

$142.232 \mathrm{E}$

$157.438 \mathrm{E}$

$69.133 \mathrm{E}$

$148.503 \mathrm{~W}$

$0.313 \mathrm{E}$

$0.013 \mathrm{E}$

$141.292 \mathrm{E}$

3.89

$140.545 \mathrm{E}$

$71.339 \mathrm{~W}$

$151.95 \mathrm{E}$

69.82 W

$46.002 \mathrm{E}$

$126.464 \mathrm{E}$

$179.715 \mathrm{E} 500 \mathrm{G}$

122.700 พ 56

$27.485 \mathrm{E}$ $4.09 \mathrm{~W}$ $130.052 \mathrm{E}$ 125.897 พ $143.789 \mathrm{E}$

$143.662 \mathrm{E}$ $143.64 \mathrm{E}$ $125.268 \mathrm{E}$

97 G
$10 \mathrm{G}$

$10 \mathrm{G} 4$

$500 \mathrm{G} \quad 4.3$

$10 \mathrm{G} 4.0$

$10 \mathrm{G} \quad 4.1$

$10 \mathrm{G} 4.2$

$10 \mathrm{G} 4.4$

18

$20 \mathrm{G}$

44 ?

30

$5 G$

$10 \mathrm{G}$

$33 \mathrm{~N}$

$50 \mathrm{G}$

$170 \mathrm{G}$

$33 \mathrm{~N} 4.5$

$33 \mathrm{~N} 4.6$
HORKAIDO, JAPAN REGION

ICELAND. WW 5.6 (HRV).

Centroid, Moment Tensor (URV): Centroid origin time

20:48:24.3; Lat $64.66 \mathrm{~N}$; Lon $17.27 \mathrm{~W}$; Dep $15.0 \mathrm{Fix}$; HalEduration $1.7 \mathrm{sec}$; Principal axes (scale 10*17 $\mathrm{Nm}$ ): (T) Val=3.17, Plg-72, Azm-304; (N) Val=-1.10, Plg=18, Azm=123; (P) Val=-2.07, Plg=0, Azm-213; Best double couple:

MO-2.6*10*\#17 Nm; NP1: Strike-320, Dip-47, Slip=115; NP2: Strike-106, Dip-48, Slip-65.

8756 CENTRAL ALASRA. CAEIC).

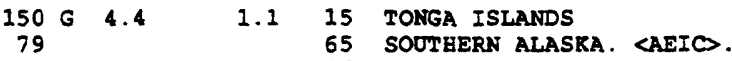

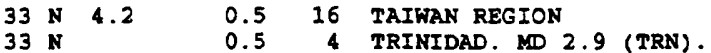

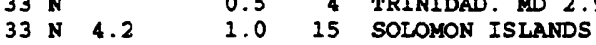

$33 \mathrm{~N} 4.6 \quad 1.2 \quad 15$ MINDANAO, PHILIPPINE ISLANDS

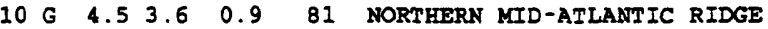

$276 * 3.7 \quad 0.9 \quad 15$

$\begin{array}{llll}33 & \times .8 & 0.8 & 35\end{array}$

$33 \mathrm{~N} 4.7 \quad 1.0$

NORTHERN ALASKA. LAEIC>. YIL 2.7 (AEIC).

VOLCANO ISLANDS REGION

SOIOMON ISLANDS

TAUIKISTAN

46 NORTHERN AIASKA. CAEIC>. MII 3.5 (AEIC).

FRANCE. ML 2.4 (LOG)

PYRENEES. MIL 2.4 (LDG)

46 SOUTH OF HONSHU, JAPAN

STRAIT OF GIBRALTAR. mbIg 2.5 (MDD).

10 NEAR EAST COAST OF HONSHU, JAPAN

7 NEAR COAST OF CENTRAL CHILE

11 NEW BRITAIN REGION, P.N.G.

10 SAN JUAN PROVINCE, ARGENTINA. MD 3.5 (SAN).

1.229 IRAN-IRAQ BORDER REGION. Felt at Salehabad, Iran.

$\begin{array}{lll}1.2 & 29 & \text { IRAN-IRAQ BORDER REGION. FEI } \\ 1.3 & 23 & \text { MINDANAO, PHILIPPINE ISLANDS }\end{array}$

0.712 SOUTH OF FIJI ISLANDS

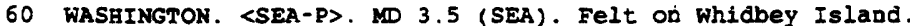
Also felt on vancouver Island from victoria to sooke. British Columbia.

$5 \mathrm{G} \quad 4.4 \quad 1.1 \quad 21$ REPUBLIC OF SOUTH AFRICA

$20 \mathrm{G} \quad 0.74$ STRAIT OF GIBRALTAR. mbIg 2.4 (MDD).

$33 \mathrm{~N} 4.2 \quad 1.5 \quad 16$ TANIMBAR ISIANDS REG., INDONESIA

$30 \quad 37$

$36 \mathrm{D} \quad 1.014$

$33 \mathrm{~N} 1.4$

$33 \mathrm{~N} \quad 5.25 .4 \quad 1.2$

VANCOUVER ISLAND REGIÓN. <PGC-P>. ML 3.2 (PGC).

VOLCANO ISLANDS REGION

VOLCANO ISLANDS REGION

VOLCANO ISLANDS REGION

63 SOUTHERN MOLUCCA SEA. MW 5.4 (ERV). $\begin{array}{llllll}33 & D & 5.1 & 4.9 & 1.0 & 116\end{array}$
$30 \quad 01 \quad 16 \quad 44.1861 .457 \times 146.679 \mathrm{~W}$

30011918.3 ? 23.31 N $144.50 \mathrm{E}$

$30 \quad 013722.3 ? 24.77$ S 178.90 H

30 01 $53 \quad 38.8$ ? 59.13 s 25.10 W

$30020724.8 * 59.115 \mathrm{~S} \quad 24.906 \mathrm{~W}$

$\begin{array}{lllllllll}30 & 02 & 50 & 39.58 & 65.641 \mathrm{~N} & 145.172 \mathrm{~W}\end{array}$

30032004.410 .984 N 124.885 E $\begin{array}{llllllll}30 & 02 & 23 & 00.1 ? & 23.20 & \mathrm{~N} & 144.18 \mathrm{E}\end{array}$
17

$33 \times 4.9$

500 G 4.5

$33 \mathrm{~N} 4.4$

$33 \mathrm{~N} 4.7$

$33 \mathrm{~N}$

14

$33 \times 5.2 \quad 4.5 \quad 0.9$
Centroid, yoment Tensor (HRV): Centroid origin time

$00: 32: 12.3$; Lat $0.16 \mathrm{~N}$; Lon $125.86 \mathrm{E}$; Dep 45.2; Half -

duration 1.2 sec: Principal axes (scale 10*17 $\mathrm{kin}$ ): (T)

Val=1.62, Plg-72, Azm-292; (N) Val=-0.45, Plg-6, Azm=41;

(P) Val=-1.17, Plg-17, Azm-133; Best double couple:

MO-1.4*10**17 $\mathrm{km}$; NP1: Strike-232, Dip-28, Slip-103; NP2: strike=38, Dip-63, silp -83 .

66 SOUTEERN ALASKA. CAEIC>. MI 2.7 (AEIC).

$\begin{array}{lllllllllllll}30 & 03 & 36 & 03.6 ? & 6.29 & S & 129.80 & E & 200 & G & 4.2 & 0.8 & 10\end{array}$

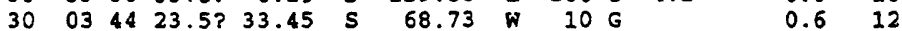

VOLCANO ISLANDS REGION

322 SOTTR OF FTJI ISIMANDS

1.515

.234

SOUTE SANDWICH ISLANDS REGION

VOLCANO ISLANDS REGION

26

LEYTE, PEILIPPINE ISIANDS. Felt (III RF) at Taclaban. Also felt (II RF) at Lapu-Lapu, Cebu.

BANDA SEA

MENDOZA PROVINCE, ARGEMTINA. MD 4.1 (SAN).
SOUTE SANDWICH ISLANDS REGION

NORTHERN ALASKA. $\angle A E I C>$. MC 3.1 (AEIC) 
$30 \quad 04 \quad 14 \quad 47.6 ? \quad 15.34 \quad s$ $054950.5 \quad 54.047$

$062539.5 * 25.831 \mathrm{~N}$ $\begin{array}{llllll}07 & 09 & 08.08 & 34.073 \mathrm{~s}\end{array}$ $\begin{array}{llll}07 & 39 & 57.2 & 36.027\end{array}$ $081717.0 * 33.256 \mathrm{~N}$ $083701.9 \quad 5.929 \mathrm{~s}$ $\begin{array}{lllll}08 & 44 & 44.18 & 53.275 \mathrm{~N}\end{array}$ $\begin{array}{llllll}08 & 46 & 42.6 & 23.263 \mathrm{~N}\end{array}$ $085447.1 ? \quad 9.41 \mathrm{~N}$ $090727.4 * 63.830 \mathrm{~N}$ $09 \quad 14 \quad 46.48 \quad 36.236 \mathrm{~N}$ $\begin{array}{lllll}09 & 22 & 13.1 & 38.014 & \mathrm{~N}\end{array}$ $\begin{array}{llll}10 & 13 & 28.2 & 6.844 \mathrm{~s}\end{array}$ $103303.3 * 9.900 \mathrm{~N}$ $\begin{array}{lllll}11 & 19 & 49.08 & 60.192\end{array}$ $112751.945 .533 \mathrm{~N}$
172.41 W $33 \mathrm{~N} 4.5$ 179.826 E 400 G 4.5 $7.320 \mathrm{E} \quad 10 \mathrm{G}$ $16.512 \mathrm{E} \quad 10 \mathrm{G}$ $160.005 \mathrm{E} 102 \mathrm{D} 5.5$

$143.478 \mathrm{E} \quad 33 \mathrm{~N} 4.6$ $70.098 \mathrm{~W} \quad 10 \mathrm{G}$ $70.893 \mathrm{E} \quad 108 \mathrm{D} \quad 4.5$ $141.409 \mathrm{E} \quad 33 \mathrm{~N} 4.9$ $148.134 \mathrm{E} 100 \mathrm{G} 5.2$ $142.739 \mathrm{E} \quad 33 \mathrm{~N}$ $143.602 \mathrm{E} \quad 33 \mathrm{~N} 4.7$ $126.28 \mathrm{E} \quad 33 \mathrm{~N} 4.5$ $18.395 \mathrm{~W} \quad 10 \mathrm{G} 4.2$ 120.795 . $14.045 \mathrm{E}$ $126.885 \mathrm{E}$ $126.334 \mathrm{E}$

$141.045 \mathrm{~W}$ $151.828 \mathrm{E}$
9 $10 \mathrm{G}$ $400 \mathrm{G} 4$. 33

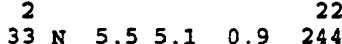

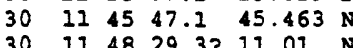
$30 \quad 114829.3 ? 11.01 \quad N$ $\begin{array}{llllll}30 & 14 & 04 & 39.0 * & 2.160 \mathrm{~N}\end{array}$ $\begin{array}{llllll}30 & 15 & 01 & 40.9 ? & 32.98 & 5\end{array}$ $\begin{array}{llllll}30 & 15 & 53 & 33.2 & 8.063\end{array}$ $\begin{array}{lllll}30 & 16 & 04 & 02.1 * 23.217 & \mathrm{~N}\end{array}$ $\begin{array}{lllllll}30 & 16 & 15 & 13.58 & 60.183 & \mathrm{~N}\end{array}$ $\begin{array}{llllll}30 & 16 & 21 & 20.4 * 7.594 \mathrm{~N}\end{array}$ $30 \quad 16 \quad 3720.6 * 45.480 \mathrm{~N}$ $30 \quad 174723.6 * 23.049 \mathrm{~N}$

$\begin{array}{llllll}30 & 18 & 17 & 00.3 & 44.903\end{array}$

$\begin{array}{lllll}30 & 18 & 31 & 03.3 * 19.842 \mathrm{~s}\end{array}$

$\begin{array}{llllll}30 & 18 & 42 & 16.2 & 22.304 & \mathrm{~s}\end{array}$

$\begin{array}{lllll}30 & 18 & 52 & 03.5 & 45.537\end{array}$
$169.859 \mathrm{E}$ $151.739 \mathrm{E}$ $61.87 \mathrm{~W}$ $128.666 \mathrm{E}$ $72.14 \mathrm{~W}$ $120.542 \mathrm{E}$ $94.048 \mathrm{E}$ $152.337 \mathrm{~W}$ $35.603 \mathrm{~W}$ $151.779 \mathrm{E}$ $93.992 \mathrm{E}$ $6.378 \mathrm{E}$ $69.011 W$ $68.406 \mathrm{~W}$ $151.811 \mathrm{E}$

$\begin{array}{rrrrr}33 & N & 4.6 & 1.5 \\ 33 & N & 4.9 & & 1.1 \\ 70 & G & & & 1.3 \\ 100 & G & 4.3 & & 0.8 \\ 10 & G & & & 0.6 \\ 33 & N & 4.3 & & 0.8 \\ 33 & N & 4.2 & & 1.2 \\ 85 & & & & \\ 10 & G & & & 0.8 \\ 33 & N & 4.6 & & 1.1 \\ 33 & N & 4.0 & & 0.5 \\ 5 & G & & & 0.7 \\ 134 & * & 4.7 & & 1.1 \\ 126 & \mathrm{D} & 4.9 & & 1.0 \\ 33 & \mathrm{~N} & 5.5 & 5.1 & 0.9\end{array}$

1.530

1.153

1.3

0.8

0.68

815

15
9

66

29

7

34

12

47 258
$30 \quad 193132.8 \& 60.270$ N 151.997 W 67

$\begin{array}{lllllllll}30 & 19 & 33 & 26.9 & 51.627 & \mathrm{~N} & 16.288 & \mathrm{E}\end{array}$

$\begin{array}{llllllll}30 & 20 & 34 & 02.5 * 9.762 \mathrm{~N} & 126.369 \mathrm{E}\end{array}$

$302040 \quad 17.4 * 33.673 \mathrm{~N} 25.486 \mathrm{E}$

$302050 \quad 43.6 * 23.222 \mathrm{~N} \quad 143.684 \mathrm{E}$

$3022 \quad 25 \quad 43.7 * 9.266 \mathrm{~N} \quad 125.279 \mathrm{E}$

$30225733.9 * 19.711$ S $169.668 \mathrm{E}$
67

$\begin{array}{lll}10 & \mathrm{G} & \\ 33 & \mathrm{~N} & 4.5\end{array}$

$10 \mathrm{G}$

$33 \mathrm{~N} 4.6$

$33 \mathrm{~N} 4.5$

$300 \mathrm{G} \quad 4.8$
26

8 NORTEERN ITALY. NU 2.1 (GEN).

IONIAN SEA. UI 3.1 (ROM).

NEAR EAST COAST OF KAMCHATKA. Felt (II) at PetropaviovskKamchatskiy.

VOLCANO ISLANDS REGION

CHILE-ARGENTINA BORDER REGION. MD 3.3 (SAN).

HINDO KOSH REGION, AFGHANISTAN

OFF EAST COAST OF HONSHU, JAPAN

NEW BRITAIN REGION, P.N.G.

SARHALIN ISLAND

VOLCANO ISLANDS REGION

MINDANAO, PHILIPPINE ISILANDS

ICELAND

CENTRAL CALIFORNIA. 〈GM-P>. MD 2.8 (GM)

SICILY. ML 3.3 (ROM).

BANDA SEA

MINDANAO, PHILIPPINE ISIANDS

SOTTHEASTERN ALASKA. <AEIC>. ML 2.7 (AEIC).

KURIL ISLANDS. NW 5.5 (HRV). Felt (III) on Simushir.

Centroid, Moment Tensor (HRV): Centroid origin time

$11: 27: 55.5$; Lat $45.65 \mathrm{~N}$; Lon $152.22 \mathrm{E}$; Dep 26.8; Half-

duration 1.4 sec; Principal axes (scale 10**17 Nm): (T) Val=2.39, $\mathrm{Plg}=68, \mathrm{Azm}=275 ;$ (N) Val=-0.08, $\mathrm{Plg}=12, \mathrm{Azm}=36$;

(P) val--2.31, Plg=18, Azm=130; Best double couple: Mo-2.3*10**17 Nm; NP1: Strike=238, Dip=29, Siip=115; NP2:

Strike=31, Dip-64, Slip=77.

LOYALTY ISLANDS REGION

KURIL ISLANDS

WINDWARD ISLANDS. MD 3.1 (TRN)

HALMAHERA, INDONESIA

OFF COAST OF CENTRAL CHILE. MD 3.3 (SAN)

FLORES REGION, INDONESIA

MYANMAR-INDIA BORDER REGION

SOUTHERN ALASKA, 〈AEIC>.

CENTRAL MID-ATLANTIC RIDGE

KURIL ISIANDS

MYANMAR-INDIA BORDER REGION

FRANCE. MU 2.7 (LDG) 2.6 (STR).

NORTHERN CHILE

NORTHERN CHILE

RORIL ISLANDS. MW 5.5 (ERV).

Centrold, Moment Tensor (HRV): Centroid origin time

$18: 52: 09.0$; Lat $45.29 \mathrm{~N}$; Ion $152.12 \mathrm{E}$; Dep 15.0 Fix; Half-

duration $1.4 \mathrm{sec}$; Principal axes (scale 10**17 $\mathrm{Nm}$ ): (T) Val=2.04, $\mathrm{Plg}=76, \mathrm{Azm}=133$; (N) Val=-0.12, $\mathrm{Plg}=0, \mathrm{Azm}=224$ (P) Val=-1.92, Plg=14, Azm=314; Best double couple: Mo-2.0*10*17 Nm; NP1: Strike-44, Dip=31, S1ip=90; NP2: Strike-224, Dip=59, S1ip-90.

RENAI PENINSULA, ALASKA. CAEIC>. ML 2.6 (AEIC).

POLAND. ML 3.0 (MOX).

MINDANAO, PHILTPPINE ISLANDS

EASTERN MEDITERRANEAN SEA

VOLCANO ISLANDS REGION

MINDANAO, PHILIPPINE ISLANDS

VANOATU ISLANDS

Compiled by Francis w. Baldwin, Pamela J. Benfield, Don L. Blakeman, George I. Choy, Stuart K. Koyanagi, John H. Minsch, Waverly J. Person, Stuart A. Sipkin and Madeleine D. Zirbes. 


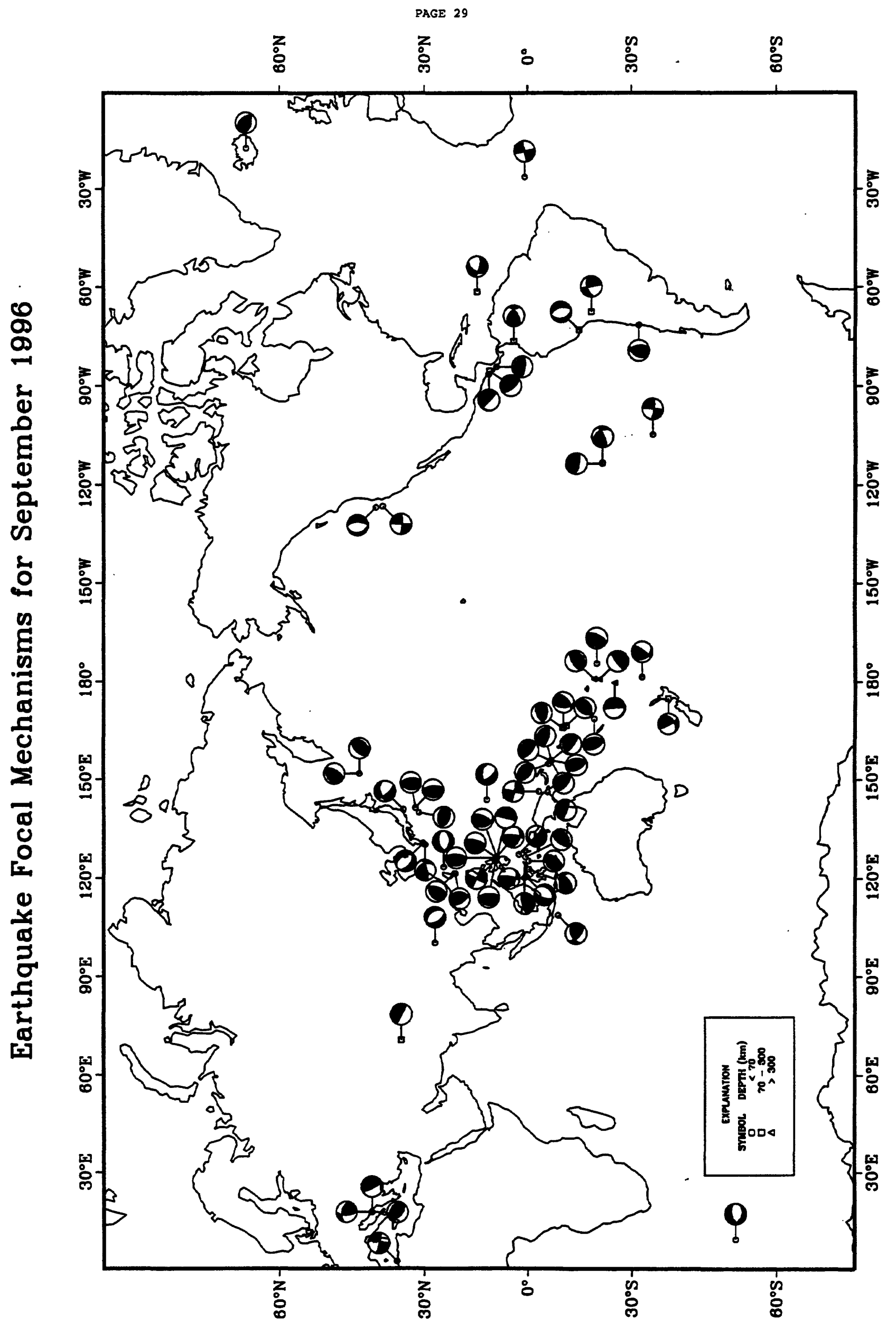




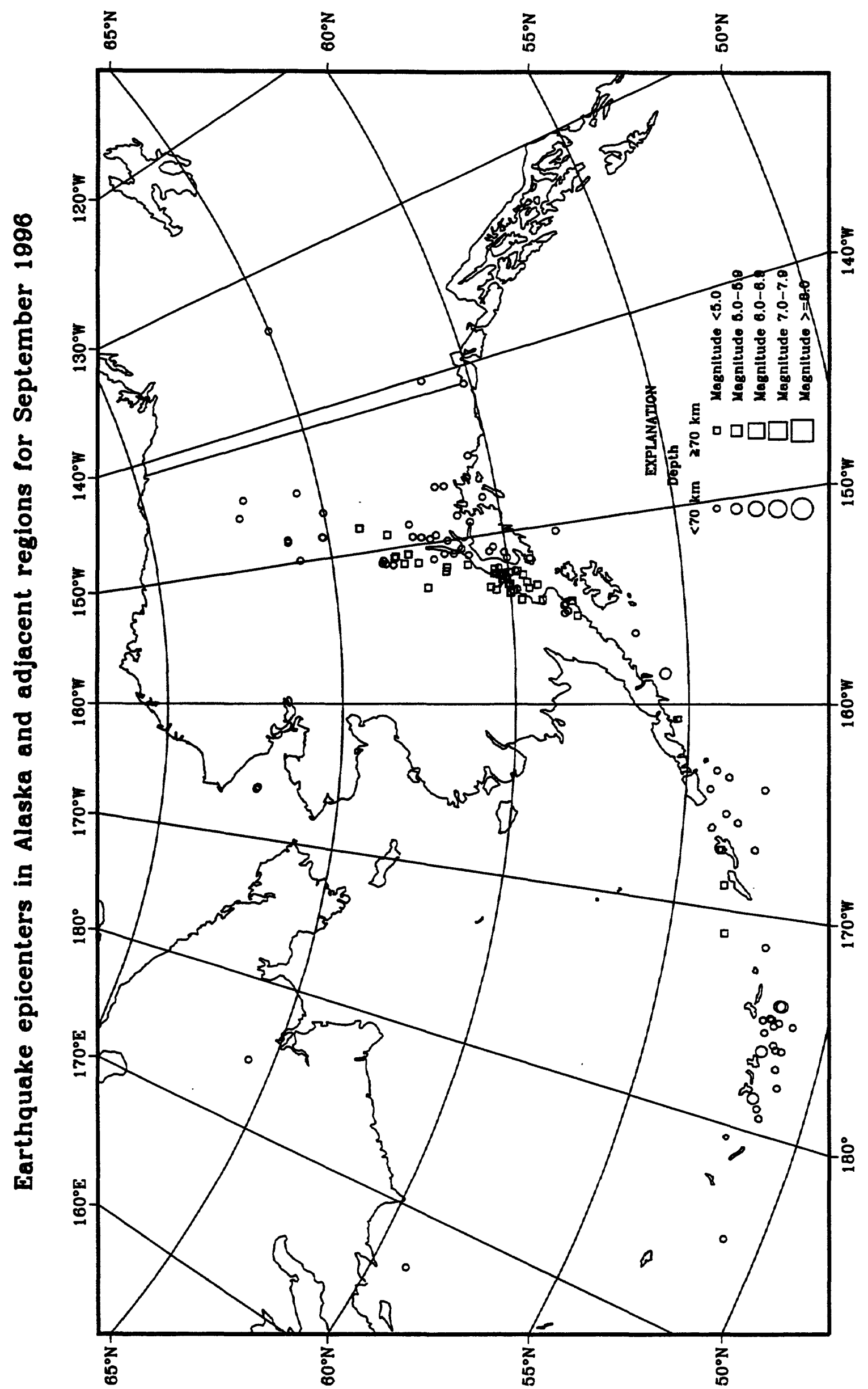


PAGE 31

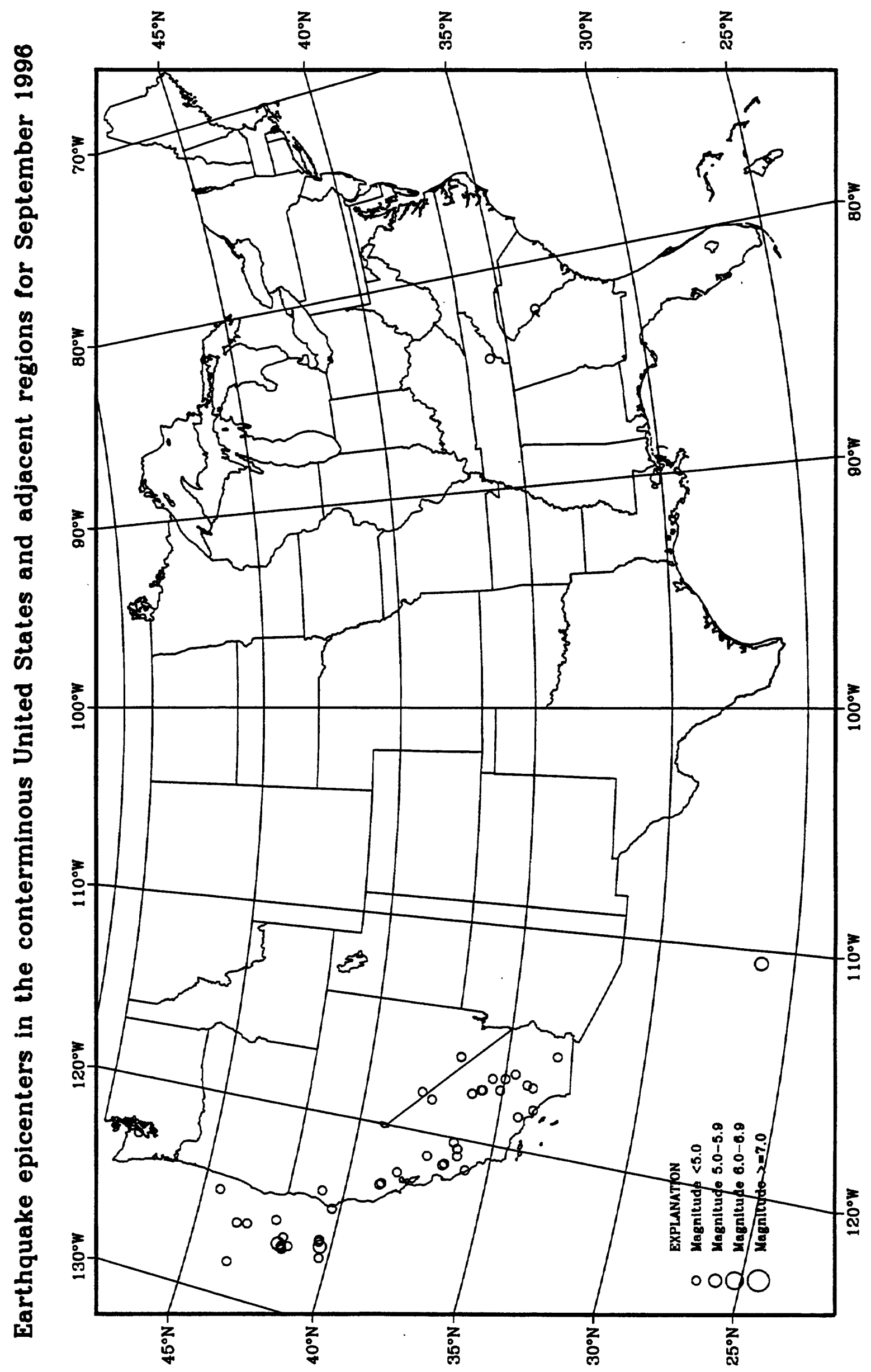




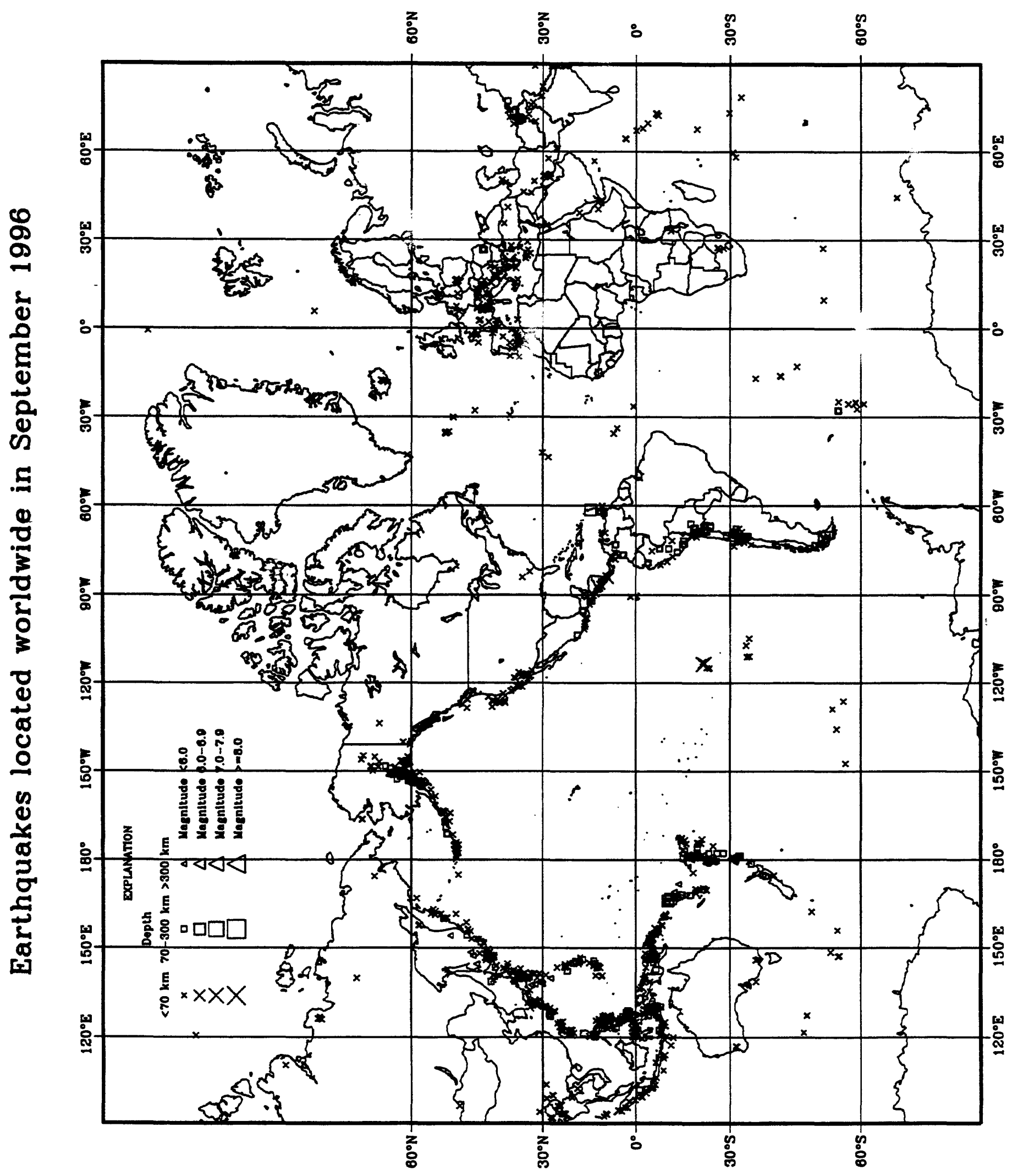

